\title{
COAL ASH CORROSION RESISTANT MATERIALS TESTING
}

\section{Topical Report: Removal of Section "C"}

April 1, 1999 thru August 31, 2003

Prepared by:

\section{THE BABCOCK \& WILCOX COMPANY}

Power Generation Group

20 South Van Buren

Barberton, Ohio 44203-0351

D. K. McDonald

Phone: (330) 860-6175, Fax: (330) 860-2348

P. L. Daniel

Phone: (330) 860-1953, Fax: (330) 860-2348

D. J. DeVault

Phone: (330) 860-2435, Fax: (330) 860-2348

and

Research and Development Division

1562 Beeson Street

Alliance, Ohio 44601-2196

E.S. Robitz, Jr.

Phone: (330) 860-6636, Fax: (330) 860-6676

Submitted by:

Contract Research Division

Date published: June 2004

This report does not contain any Trade Secret/Proprietary Information.

This project was funded in part by the U. S. Department of Energy and the Ohio Coal Development Office, Department of Development, State of Ohio

U.S. Department of Energy Contract:

Ohio Coal Development Office Grant Agreement:

The Babcock \& Wilcox Company Contract:
DE-FC26-99FT40525

CDO/D-97-02

CRD 1392 


\section{Legal Notice/Disclaimer}

"This report was prepared by The Babcock \& Wilcox Company $(\boldsymbol{B} \& W)$ pursuant to a contract partially funded by the U. S. Department of Energy (DOE) and the Ohio Coal Development Office /Ohio Department of Development (OCDO/ODOD). Neither the State of Ohio nor any of its agencies, the DOE, nor $\mathrm{B} \& \mathrm{~W}$, nor any of its subcontractors, nor any person acting on behalf of either:

(a) makes any warranty or representation, express or implied, with respect to the accuracy, completeness, or usefulness of information contained in this report, or that the use of any information, apparatus, method, or process disclosed in this report may not infringe privately owned rights; or

(b) assumes any liabilities with respect to the use of, or for damages resulting from the use of, any information, apparatus, method or process disclosed in this report.

Reference herein to any specific commercial product, process, or service by trade name, trademark, manufacturer, or otherwise, does not necessarily constitute or imply its endorsement, recommendation, or favoring nor do the view and Opinions of authors expressed herein necessarily state or reflect those of the State of Ohio nor the DOE.

NOTICE to JOURNALISTS AND PUBLISHERS: Please feel free to quote and borrow from this report, however, please include a statement noting the Ohio Coal Development Office's support for this project. 


\begin{abstract}
The "Coal Ash Corrosion Resistant Materials Testing Program" is being conducted by The Babcock \& Wilcox Company (B\&W), the U.S. Department of Energy (DOE) and the Ohio Coal Development Office (OCDO) at Reliant Energy's Niles plant in Niles, Ohio to provide full-scale, in-situ testing of recently developed boiler superheater materials. Fireside corrosion is a key issue for improving efficiency of new coal fired power plants and improving service life in existing plants.

In November 1998, B\&W began development of a system to permit testing of advanced tube materials at metal temperatures typical of advanced supercritical steam temperatures $\left(1100^{\circ} \mathrm{F}\right.$ and higher $)$ in a boiler exhibiting coal ash corrosive conditions. Several materials producers including Oak Ridge National Laboratory (ORNL) contributed advanced materials to the project.

In the spring of 1999 a system consisting of three identical sections, each containing multiple segments of twelve different materials, was installed. The sections are cooled by reheat steam, and are located just above the furnace entrance in Niles' Unit \#1, a 110 MWe unit firing high sulfur Ohio coal. In November 2001 the first section was removed for thorough metallurgical evaluation after 29 months of operation. The second section was removed in August of 2003. Its evaluation has been completed and is the subject of this report.. The final section remains in service and is expected to be removed in the spring of 2005. This paper describes the program; its importance, the design, fabrication, installation and operation of the test system, materials utilized, and experience to date. This report briefly reviews the results of the evaluation of the first section and then presents the results of the evaluation of the second section.
\end{abstract}




\section{TABLE OF CONTENTS}

Section

$\underline{\text { Pg. }}$

TITLE PAGE

DISCLAIMER

2

ABSTRACT

TABLE OF CONTENT

3

LIST OF TABLES

4

LIST OF FIGURES

1.0 INTRODUCTION

2.0 EXECUTIVE SUMMARY

3.0 EXPERIMENTAL

4.0 RESULTS AND DISCUSSION

5.0 CONCLUSION

6.0 REFERENCE

ANNEX 1 - Program Timeline: 2002 - 2003

53

ACRONYMS AND ABBREVIATIONS

APPENDICES

$\underline{\text { Tab }}$

A. Save 25

A-1

B. Modified $800 \mathrm{H}$

B-1

C. $347 \mathrm{HFG}$

C-1

D. NF 709

D-1

E. $\mathrm{Fe}_{3} \mathrm{Al}$

E-1

F. $310 \mathrm{Ta}$

F-1

G. $310 \mathrm{HCbN}(\mathrm{HR} 3 \mathrm{C})$

G-1

H. HR 120

H-1

I. Thermie

I-1

J. IN52 Weld Overlay

$\mathrm{J}-1$

K. Incoclad 671

K-1 


\section{LIST OF TABLES}

\begin{tabular}{|c|l|c|}
\hline$\#$ & \multicolumn{1}{|c|}{ Description } & Pg. \\
\hline 1 & $\begin{array}{l}\text { Tube Sample Designation, Average Surface Metal Temperature, and } \\
\text { Exposure Time Based on Position in Test Section C }\end{array}$ & 17 \\
\hline 2 & Representative Analysis of Coal Burned in Niles Unit-1 & $\mathbf{1 8}$ \\
\hline 3 & Deposit Analysis & $\mathbf{1 8}$ \\
\hline 4 & Deposit Analyses (X-Ray Diffraction Results) & 19 \\
\hline 5 & Carbon and Chlorine Analysis Results & 19 \\
\hline 6 & Maximum Wastage Rate as a Function of Position in Test Section C & $\mathbf{3 6}$ \\
\hline 7 & Rankings Per Material Based on Rate of Metal Loss in Test Section C & $\mathbf{3 7}$ \\
\hline 8 & Maximum Rate of Metal Loss for Each Candidate Material & $\mathbf{3 8}$ \\
\hline 9 & Maximum Wastage Rate as a Function of Time and Position & $\mathbf{3 9}$ \\
\hline
\end{tabular}

\section{LIST OF FIGURES}

\begin{tabular}{|c|c|c|}
\hline \# & Description & Pg. \\
\hline 1 & $\begin{array}{l}\text { Schematic of Test Section Showing Position of Welds and Location of } \\
\text { Dutchmen (i.e. samples removed early) }\end{array}$ & 20 \\
\hline 2 & Daily Average Surface Metal Temperatures (F) for Tube Segment "R-S & 21 \\
\hline 3 & Appearance of Test Section C After Removal From Service (Part 1) & 22 \\
\hline 4 & Appearance of Test Section C After Removal From Service (Part 2) & 23 \\
\hline 5 & $\begin{array}{l}\text { Typical Documentation of As-Received Appearance for Tube Sample Y-Z } \\
\text { (Modified 800) }\end{array}$ & 24 \\
\hline 6 & $\begin{array}{l}\text { Schematic Depicting Sectioning to Remove Tube Sample From the Test } \\
\text { Section }\end{array}$ & 25 \\
\hline 7 & Schematic Depicting Sectioning of Tube Sample & 25 \\
\hline 8 & Typical Semi-Quantitative Composition Verification for Modified $800 \mathrm{H}$ & 26 \\
\hline 9 & Typical Example of OD Surface Appearance After Grit Blast & 27 \\
\hline 10 & $\begin{array}{l}\text { A Typical Silhouette with Wall Thickness Measurements Showing Metal } \\
\text { Loss as a Function of Position }\end{array}$ & 28 \\
\hline 11 & $\begin{array}{l}\text { Weld Profile Through Tube Sample Showing Good Corrosion Resistance } \\
\text { in the Vicinity of the Weld }\end{array}$ & 29 \\
\hline 12 & $\begin{array}{l}\text { Weld Profile Through Clad Tube Sample Incoclad } 671 \text { Adjacent to Weld } \\
\text { "V" in Section C(at } 1121 \text { F) }\end{array}$ & 29 \\
\hline 13 & $\begin{array}{l}\text { Weld Profile Through Clad Tube Sample Incoclad } 671 \text { Adjacent to Weld } \\
\text { "P" (at 1203F) in Section C }\end{array}$ & 30 \\
\hline 14 & Maximum Wastage Rate as a Function of Position within Section C & 40 \\
\hline 15 & Maximum Wastage Rate for Each Candidate Material in Both Sections & 41 \\
\hline 16 & $\begin{array}{l}\text { Wastage as a Function of Average Surface Metal Temperature for the } \\
\text { Twelve Candidate Alloys in Test Section C }\end{array}$ & 42 \\
\hline 17 & $\begin{array}{l}\text { Wastage as a Function of Average Surface Metal Temperature for the } \\
\text { Twelve Candidate Alloys in Test Section A }\end{array}$ & 43 \\
\hline 18 & Weld Cracks Intersecting External Corrosion for 72 WO Test Segment & 44 \\
\hline 19 & Internal Cracking in 72 Weld Overlay Test Segment from Section C & 44 \\
\hline 20 & Internal Cracking in 72 Weld Overlay Archive Material & 45 \\
\hline 21 & Maximum Wastage as a Function of Time for the Candidate Materials & 45 \\
\hline
\end{tabular}




\begin{tabular}{|c|c|c|}
\hline 22 & $\begin{array}{l}\text { Chromium Content Versus Wastage Rate for Both Sections A and C With } \\
\text { Logarithmic Trend Line }\end{array}$ & 46 \\
\hline 23 & $\begin{array}{l}\text { Chromium Plus Nickel Content Versus Wastage Rate With Linear } \\
\text { Regression Fit Trend Line }\end{array}$ & 46 \\
\hline 24 & Common Characteristics at External Scale/Metal Interface & 47 \\
\hline 25 & $\begin{array}{l}\text { Illustrates the Characteristic Microstructural Regions Found at the } \\
\text { External Scale/Metal Interface for Many of the Candidate Materials }\end{array}$ & 48 \\
\hline 26 & Characteristics of External Scale/Metal Interface for IN52 Weld Overlay & 49 \\
\hline 27 & $\begin{array}{l}\text { Typical Examples of the Condition of the Internal Scale/Metal Interface } \\
\text { after Service }\end{array}$ & 50 \\
\hline
\end{tabular}




\subsection{INTRODUCTION}

This report represents the second in a series of three topical reports that document progress toward completion of the DOE-sponsored program to compare and rank the coal ash corrosion resistance of twelve candidate superheater tube materials (and/or coatings). (Contract No. DEFC26-99FT40525).

The first topical report, issued on October 31, 2002, addressed the evaluation of Section A which was the first test section to be removed from the boiler. The Section A report provided the full details of the project background, material selection, fabrication of test materials and sections, description of the test system, and system installation and startup. The report also provided the operating history through 2001 along with the results of the evaluation of the candidate materials within the section. That information will not be repeated here. The reader is directed to "Topical Report: Removal of Section A" if this information is needed.

This second topical report addresses herein the evaluation of test Section $\mathrm{C}$ which was removed for evaluation in August 2003. Section $C$ was the second test section to be removed from the boiler. The results of the evaluation of each candidate material in Section $\mathrm{C}$ are contained in the twelve separate appendices that accompany this report. The main body of this report integrates this information and draws preliminary observations and conclusions based on the results to date. Annex 1 of this report documents the operating history of the test sections beginning where the Section A report left off at the end of 2001. This annex thus provides a timeline of activities throughout 2002 and 2003. Annex 1 can be found following the "Observations and Conclusions section of this report.

At this point in time, test Section B continues in service, and likely will be removed for evaluation within the next twelve months. At that time a third topical report will be issued to cover the evaluation of test Section B after it has been removed from service. Once all three sections have been evaluated, and the topical reports issued, the program will be brought to completion, and a final report will be issued that integrates and summarizes observations and conclusions that can be drawn from all of the data collected in this program. 


\subsection{EXECUTIVE SUMMARY}

In April 1999, three identical superheater test sections were installed into the Niles Unit \#1 for the purpose of testing and ranking the coal ash corrosion resistance of candidate superheater alloys. The Niles boiler burns high sulfur coal (3\% to $3.5 \%)$ that has a reasonably high alkali content, thus the constituents necessary for coal ash corrosion are present in the ash. The test sections were controlled to operate with an average surface metal temperature from approximately $1060^{\circ} \mathrm{F}$ to $1210^{\circ} \mathrm{F}$ which was well within the temperature range over which coal ash corrosion occurs. Thus, this combination of aggressive environment and high temperature was appropriate for testing the performance of candidate corrosion-resistant tube materials. Analyses of the deposit and scale confirmed that the aggressive alkali-iron-trisulfate constituent was present at the metal surface and active in tube metal wastage. The test sections were constructed so that the response of twelve different candidate tube and/or coating materials could be studied. The plan was to remove and evaluate one of the three test sections at time intervals of 1 year, 3 years, and 5 years. This would permit an assessment of performance of the candidate materials as a function of time.

Test Section A was removed in November 2001 after about 24 months of service at the desired steam temperature set point, with about 15.5 months of exposure at full temperature. A progress report, issued in October 2002, was written to document the performance of the candidate alloys in that test section. The evaluation described the condition of each tube sample after exposure. It involved a determination of the rate of wall thickness loss for these samples. In cases where there was more than one sample of a candidate material in the test section, an assessment was made of the performance of the alloy as a function of temperature.

Test Sections B and C were examined during the November 2001 outage, and it was decided that, due to excessive wastage, certain tube samples needed to be removed and replaced in order to ensure that Test Sections B and $\mathrm{C}$ would have a chance of remaining in the boiler for their intended exposure period. These suspect tube samples were replaced and the two remaining test sections were put back into service. The tube samples that were removed from Test Sections B and $\mathrm{C}$ were set aside for later analysis at the end of the planned exposure period.

Test Sections $\mathrm{B}$ and $\mathrm{C}$ were again examined approximately six months later. At that time, measured wall thickness losses raised concerns about additional tube samples. These suspect samples were also removed, set aside for later analysis, and replaced. The test sections then went back into service until the end of the second exposure period, which was concluded in May 2003 when, due to evidence of excessive wastage, the valves were opened effectively stopping corrosion. In August 2003, Test Sections B and C were removed for closer examination. Section $\mathrm{C}$ had experienced about 42 months of service at the desired team temperature set point with 28.5 months at full temperature. Test Section B was re-installed into the boiler (at the location originally occupied by Section $\mathrm{C}$ ), and will remain in service until the end of the program.

This report provides the results of the evaluation of Test Section $\mathrm{C}$, including the samples that remained in the Test Section for the full exposure period as well as those that were removed early. The analysis of Test Section $\mathrm{C}$ followed much the same protocol that was employed in the assessment of Test Section A. Again, the focus was on determining and documenting the relative corrosion rates of the candidate materials. The detailed results of the investigation are included in 
this report as a series of twelve appendices. Each appendix is devoted to the performance of one of the candidate alloys. The table below summarizes metal loss rate for the worst case sample of each of the candidate materials for both Test Sections A and C. The body of this report compares these for all of the samples in the test section.

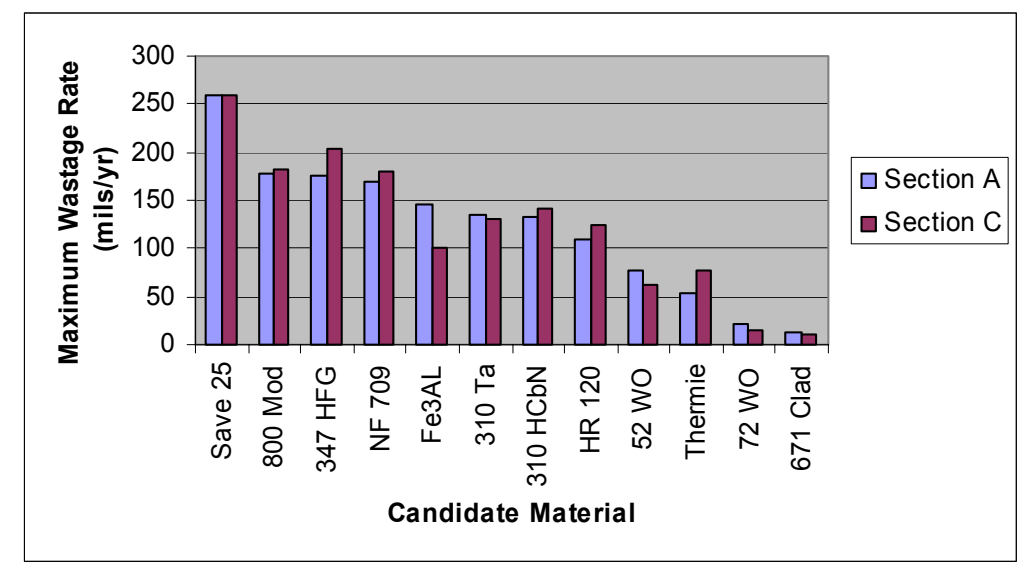

\section{Maximum Wastage Rate for Each Candidate Material In Test Sections A and C}

Review of this chart reveals that, with few exceptions, the results for Test Section C $(20,832$ hour exposure at corrosive temperatures) were very similar to those earlier determined for Test Section A (11,288 hour exposure). In general, it can be stated that, while the sampling represented by these tests is small, the relative performance of the candidate alloys appears to have been strongly influenced by their total alloy content, in particular, the sum of their chromium plus nickel contents.

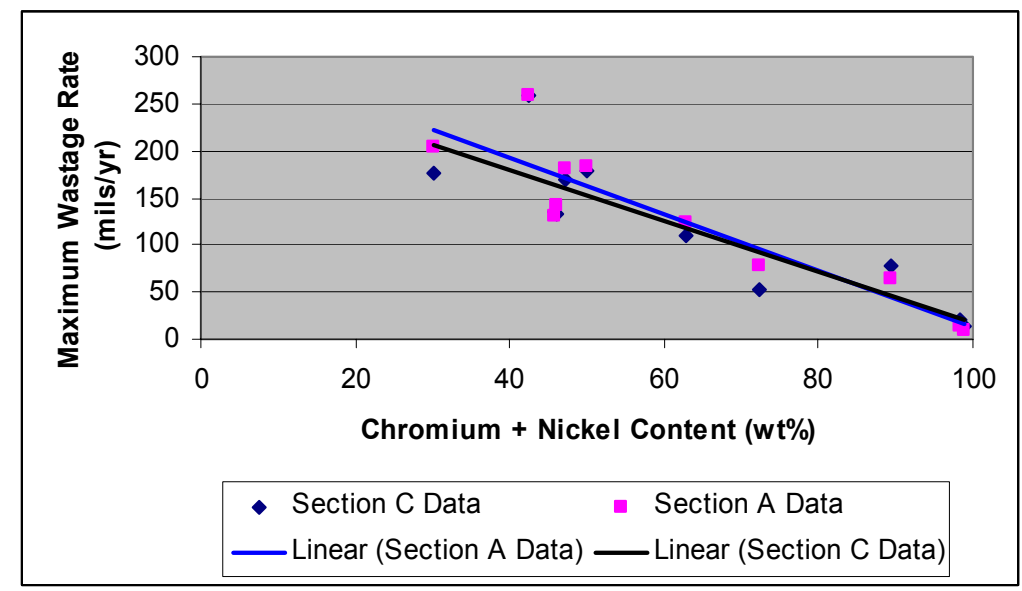

\section{Maximum Wastage Rate as a Function of Chromium Plus Nickel}

Two areas of caution also were identified with regard to some of the more resistant materials. First, the IN 52 Weld Overlay samples went into service with an 80 mil thick cladding for corrosion protection, but by the end of the Test Section $\mathrm{C}$ exposure period, the cladding was completely wasted away, and the underlying substrate was exposed to the corrosive environment. Given the fact that the $800 \mathrm{H}$ substrate is much less corrosion resistant than the cladding, the wastage rate is expected to increase significantly for this material. Second, it was determined that the IN 72 Weld Overlay, while being very corrosion resistant also had experienced a weld 
cracking phenomenon that may impact its service life. Preliminary investigations suggest that the cladding may have contained small, and difficult to detect, internal cracks prior to service exposure. Preliminary investigations also suggest that the extent of cracking within the IN 72 Weld Overlay may increase in quantity and in length as a function of time at temperature. This issue however has not yet been fully resolved. So, at this point, for the sake of comparison, the data illustrated in the charts above only take corrosion wastage into account. If the wall thickness lost due to the observed cracking had been included in the wastage rate calculations, then the wastage rate would have increased by about 50\%. However, if this additional $50 \%$ were taken into account, the total wall thickness lost for the IN 72 Weld Overlay still would be small relative to some of the other materials. Nonetheless, it must be remembered that the IN 72 Weld Overlay samples initially had only 80 mils of cladding to act as a corrosion barrier. Once the cladding is breached, as was noted for the IN 52 Weld Overlay, the wastage rate is expected to increase considerably.

Of particular concern regarding the damage found in IN 72 Weld Overlay, is the fact that, while these samples are in service, the surface deposit and scale may hide the fact that the underlying metal is not sound. The extent of damage is normally monitored by visual inspection and dimensional measurements in situ within the boiler without disturbing the surface condition of the tubes. If the cladding is breached by a cracking mechanism, and the underlying metal is corroding away beneath the cladding, then, this condition may not be discernable by standard inspection methods. Obviously, this could lead to a false sense of security regarding the soundness of a test section. Given this concern, the otherwise good corrosion resistance of this material, and the usefulness of weld cladding as an inexpensive means of achieving this corrosion resistance, suggests that elimination of this cracking is an area worthy of further study.

\subsection{EXPERIMENTAL}

\section{Procedures \& Results}

This section of the report documents the procedures and sequence of activities used to assess the condition of Test Section $\mathrm{C}$ after service. The primary focus is on determining the relative coal ash corrosion resistance of the candidate superheater tube alloys. General results also are offered in this section of the report. Detailed results are provided on a material-by-material basis in the attached appendices.

\section{Background:}

At the beginning of the program, three identical test sections were installed side-by-side in the Niles Unit-1 boiler. These were identified as Test Sections A, B, and C. The intent was to remove and evaluate a section after each of three increasingly longer exposure increments. The purpose was to demonstrate how the candidate tube alloys performed as a function of time. Test Section A was to be removed after one year. Section B was to be removed after three years of exposure, and Test Section $\mathrm{C}$ was to be removed at the end of the program, after approximately 5 years of exposure.

Due to various practical issues, and due to the fact that some of the candidate alloys corroded much more rapidly than expected, as the program progressed, it became necessary to deviate from the original plan. Test Section A was removed and analyzed first as planned. However, 
when it came time to remove the second test section for analysis, examination revealed that Section C was distorted (due to in situ maintenance), while Section B was relatively unaffected. Based on this, it was decided to evaluate Section $\mathrm{C}$ and to reinstall Section B. The results of the analysis of Section C are the subject of this report. Test Section B remains in the boiler, and will continue in service until the end of the program.

Test Section A was removed from Niles Unit-1 in November 2001, after 28.5 calendar months (approximately 21,200 hours) from start-up, 24 months with the desired steam temperature set point, and an equivalent of 11,288 hours (15.5 months) of exposure under conditions likely to cause coal ash corrosion. At the time that Section A was removed for analysis, it became apparent that some of the tube samples in Sections B and C (particularly the Save 25 samples) would not survive the additional time required to reach the next planned exposure increment. In an effort to ensure that Sections B and C would not fail prior to their scheduled removal time, it was decided that questionable tube samples should be removed from the test sections and replaced with "dutchmen" tube lengths. The dutchmen were intended to provide the wall thickness and corrosion resistance needed to extend the life of the section. Six contiguous tubes (between welds $G$ and $M$ ) were removed from both Sections B and $C$ and replaced. The dutchman for Section B was made from a single length of Alloy $800 \mathrm{H}$ which was clad with Inco 52 weld metal. The dutchman for Section C was made from two lengths of $310 \mathrm{HCbN}$ that were joined together to cover the width of the gap.

Sections B and C went back into service with the dutchmen in place. When they were reexamined about six months later, it was noted that additional tube samples needed to be replaced due to excessive corrosion. So, in June 2002, for both sections, three contiguous tube samples (between welds D and G) were removed and replaced with Inco 52 clad dutchmen. When these repairs were made both sections went back into service until the outage in May 2003 when, due to evidence of excessive wastage, the valves were opened effectively stopping corrosion.

In August 2003, both sections were removed and visually evaluated. This examination revealed that Section $\mathrm{C}$ had become distorted due to field maintenance difficulties while Section B was relatively unaffected, see figures below for comparison.

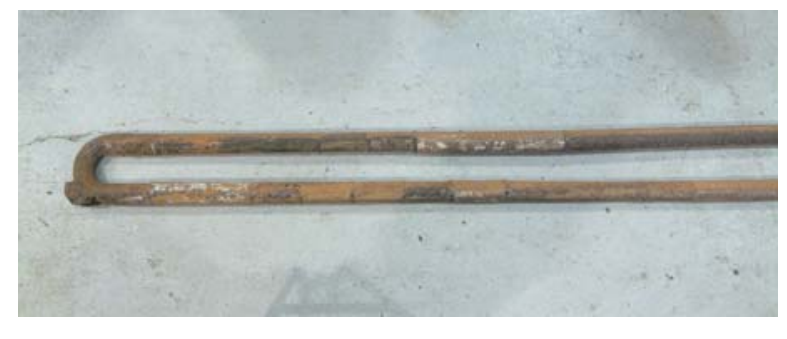

Section B - As Removed

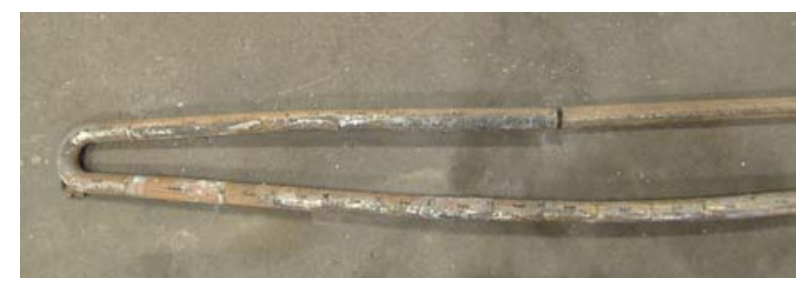

Section C - As Removed

Furthermore, inspection of both sections revealed evidence of tube wastage that suggested that additional tube samples would need to be replaced if either section were to survive to the completion of the program. Based on this, it was decided to remove and replace all of the questionable samples from Section B, then, to put it back into service. It further was decided to use the samples from Section C for the current evaluation. It should be noted that Section B was re-installed into the boiler at the location previously occupied by Section C. 


\section{$\underline{\text { Section C Lay-Out }}$}

Figure 1 provides a schematic showing the position of the welds that join samples to one another to form Test Section C. Note that each weld has been given a letter designation. Using these, the tube sample positions are indicated by the weld letter designations at either end of the sample. As was mentioned earlier, the three test sections were identical, so, the same positions were occupied by the same candidate materials in all three test sections.

Figure 1 also shows the positions of the dutchmen in Section $\mathrm{C}$ at the time of its' removal from service. As was mentioned above, the tube samples at these locations were removed early due to excessive corrosion, so they did not experience the full exposure interval for Section C. Nonetheless, the samples from these locations were set aside (at the time of their removal) and their assessment will be included in the analysis of Section C. The only sample that could not be fully analyzed was the Save 25 sample at location D-E. This was due to the fact that at some point, the D-E sample leaked, was repaired with a weld pad, and put back into service. Otherwise the full sequence of tube samples is included in this analysis.

Table 1 provides the key to the sequence of candidate materials within the three test sections. In this table, the candidate alloys are listed in the sequence in which they are encountered in the test section as one progresses from the hottest location toward the coolest. This table includes the tube sample letter designations that are based on the weld lettering shown in Figure 1. Included in this table are calculated Test Section $\mathrm{C}$ average surface metal temperature at each location and the total exposure time for each sample.

\section{Exposure Time}

Initial exposure of the three test sections to flue gas occurred when Niles Unit-1 resumed operation in June of 1999, following a maintenance outage during which the sections were installed. Test Section C was removed from service in May of 2003, after a period of 47 calendar months in the boiler, 42 months at desired steam temperature set point and about 28.5 months at full temperature under conditions likely to cause coal ash corrosion. During this time the boiler shifted load and cycled on and off line in normal response to power demand and boiler maintenance requirements.

It is well understood that the rapid metal loss associated with coal ash corrosion of austenitic materials occurs over a temperature range between approximately $1,000 \mathrm{~F}$ and 1,300 $\mathrm{F}$. The intent of this work was to control the test sample average surface metal temperatures to fall within this range over the full length of the test section. This required that the boiler be operated at sufficiently high load to assure reasonably constant gas temperatures. At low loads, reheat steam pressure and/or furnace exit gas temperature is too low for good temperature control. Through the initial phase of each startup, final stage of shutdowns, and other times when the unit load was low, steam flow through the sections was maximized to keep surface metal temperatures below $1000^{\circ} \mathrm{F}$. In this way, coal ash corrosion was retarded during these periods when test conditions could not be controlled.

For the purpose of this test, $1000^{\circ} \mathrm{F}$ surface metal temperature at the hot end of the test section (Point-T in Figure 22) was taken as the transition temperature above which the rate of coal ash 
corrosion is significant and below which it is negligible. For Test Section $\mathrm{C}$ the accumulative time at full temperature under conditions likely to cause coal ash corrosion was 20,832 hours.

\section{$\underline{\text { Test Section Surface Metal Temperatures }}$}

As indicated above, desired test section temperatures are maintained by controlling the temperature of steam exiting the test section. It is constantly monitored by thermocouple measurement, and an arithmetic average outlet steam temperature is recorded at five minute intervals.

The external surface temperature of each alloy test segment is calculated at five minute intervals using the measured outlet steam temperatures, the average gas temperature, and B\&W's heat transfer model for the test section. In the following appraisals of coal ash corrosion, an average surface metal temperature is determined for each test specimen. The indicated average surface metal temperatures are arithmetic averages of the calculated segment external surface metal temperatures for the time that the sample was exposed to the coal ash corrosion temperature range. Most of the tube samples within Section C experienced the full 20,832 hours at full temperature. The average surface metal temperatures for samples that were removed early were calculated based on the exposure temperatures during their time in the boiler. For all of these samples, the indicated arithmetic average provides a reasonable and practical approximation of the "isothermal equivalent temperature", i.e. the isothermal temperature at which exposure over time would produce the observed extent of corrosion. The average surface metal temperature approximates the isothermal equivalent temperature to the extent that the corrosion rate is a linear function of temperature. This simplification is justified at this point due to the limited amount of available data, but the data seems to suggest that the corrosion rate may be departing from linearity for some of the materials under investigation. Table 1 shows the estimated average surface metal temperatures for test segments in Section C.

Temperatures were not constant. As gas, steam, and metal temperatures rose and fell at the beginning and end of each high-temperature boiler operating cycle, samples accumulated a few hours of coal ash corrosion below the target exposure surface metal temperature. Also, test section surface metal temperatures were low throughout an initial six month shakedown period. The full target section outlet steam temperature of $1075^{\circ} \mathrm{F}$ was only maintained after November of 1999. The temperature history of Section C, Segment "R-S" (Figure 2) illustrates the surface metal temperature variability. For this segment the average surface metal temperature over the duration of the test is $1208^{\circ} \mathrm{F}$. As shown in Figure 2, surface metal temperatures are initially low. Then from July through December 1999, they climb to an average surface metal temperature appropriate for testing, and remain near this temperature whenever the section is operational until it is removed in from service May of 2003. Figure 2 also illustrates the daily surface metal temperature variability. The wide spread in values is generally caused by transients when the unit is taken off line.

\section{$\underline{\text { Environment }}$}

The Niles cyclone boiler is fired with Ohio bituminous coal having a sulfur content of approximately $3 \%$ to $3.5 \%$. The sulfur in this coal is pyretic and organic, with little sulfate. Chlorine concentrations are typically less than $0.05 \%$. Table 2 shows a representative coal analysis. This same coal has been used to date throughout the program. 


\section{Documentation:}

Test Section Appearance Upon Receipt After Exposure: Figures 3 and 4 show the appearance of Test Section $\mathrm{C}$ when it was received at the Research Center. Two figures were needed to document the entire length of the test section; however, there is some overlap between them. The weld lettering designations are noted in these figures. The schematic provides a representation of how each tube sample was originally situated within the test section.

Tube Sample Surface Appearance: Close-up photographs were taken to document the condition of each tube sample. Figure 5 provides an example of this documentation for one tube sample. Three views were documented. The 12 o'clock position faced downward* into the oncoming gas flow. The 6 o'clock position was at the top of the tube on the side downstream of the gas flow direction. The 3 o'clock position was halfway between the two. These figures typically document the extent of deposit and scaling. In some cases where the scale and deposit have fallen away, evidence also is seen of the chalky white deposit associated with alkali-irontrisulfate hot corrosion. The rest of these are included in separate appendices to this report. Each appendix is devoted to a different candidate material.

\section{Tube Sectioning:}

Figure 6 provides a schematic that depicts how the tube sample was sectioned to extract specimens for evaluation. Figure 7 shows the sectioning plan for each tube sample. All cuts were made dry using a saw. As they were cut, samples were marked to maintain sample identity and tube orientation (i.e. 12 o'clock, 3 o'clock, and 6 o'clock).

\section{Composition Verification:}

For Test Section A, removed and analyzed earlier, a portion of the "extra material", identified as RS-4 in Figure 7, was sent to a third-party laboratory for confirmatory chemical analysis, primarily by optical emission spectrometry. The results of these analyses were compared with the reported heat analyses from the various providers of materials for test. They were also compared with material specifications if they were available. Otherwise, a "nominal" or "target" tube metal composition was used.

The results of these spectroscopic analyses for Test Section A, confirmed that the correct tube material had been used at each location in the test section, and that the tube material complied with its' intended chemical composition. The scanning electron microscope (SEM) also was used to perform a semi-quantitative chemical analysis (using the energy dispersive $\mathrm{x}$-ray spectrometry (EDS)) for each of the twelve different material types in Test Section A. These results compared well with the somewhat more accurate optical emission spectrometry analyses as can be seen from reviewing the data in the appendices to the final report covering the Section A analysis.

\footnotetext{
* By selecting the 12 o'clock orientation as the one facing directly into the gas flow direction, the resultant orientation yields an "upside-down" clock reference system relative to the physical position of the tube within the boiler, i.e. 12 o'clock faces downward.
} 
With alloy identification confirmed for Test Section A, it was decided that only representative sampling would be used to verify that the correct materials were at their proper location within Test Section C. It also was decided to use EDS as the analytical method, and that OES would be used as a fall-back if problems arose. To this end, twelve samples were chosen for analysis. Each of the twelve represented the most aggressively attacked sample of each material type. The EDS analyses confirmed that all twelve samples were of the proper material type.

The results of these analyses are included in the appendices of this report which provide detail on a material-by-material basis. Figure 8 shows a typical example.

\section{Surface Condition:}

Dimension rings (identified as "RS-2" in Figure 7) were removed from the approximate centerline of each tube sample. These were grit blasted with aluminum oxide grit to remove deposit and scale. The surface condition of each ring was photographed, and is included in the appendices of this report. Figure 9 shows a typical example. These are intended to show and

record the level and type of attack that was occurring at the external surface of these tubes (i.e. beneath the deposit/scale that are shown in Figure 5).

Figure 10 shows a typical example of a dimension ring cross section and associated measurements. Such figures provide, as a reference, the amount of wall thickness measured at the position indicated. Dimensioning is discussed in more detail below. In general, it was the side of the tube that faced the gas flow that experienced most of the metal loss. The maximum wall thinning was typically between the 12 o'clock and 9 o'clock (or 3 o'clock) positions.

\section{Dimensioning:}

The grit blasted rings were measured with certified micrometers that were accurate to +/- 0.001 ". The measurements were taken at eight predetermined circumferential locations that were indicated by the "o'clock" positions: 12:00, 1:30, 3:00, 4:30, 6:00, 7:30, 9:00, and 10:30 o'clock. The "o'clock" positions were the same as those used above to express the orientation of the tube within the test section. Prior to service, measurements had been taken and recorded for each of these orientations for each of the tube samples. The measurements taken after service were compared with these and a "delta" calculated to express the wall thickness losses at each position. These are recorded for each candidate material in the appendices that follow.

Note that an attempt was made to determine the maximum wall thickness loss for each tube sample. In some cases, it was clear that the minimum wall thickness was not at one of the eight predetermined locations, so, there was no "before service" measurement for use as a reference. In these cases, the average "before service" wall thickness measurement was determined and used as the reference measurement for determining the wall thickness lost. The location and value of the maximum are also documented in the appendices in tables such as the one shown in Figure 10.

Note also that it was not possible to take the "after service" measurements at the same axial position as the "before service" measurements, since these were taken at the tube ends, and the tube ends now were part of the welds that joined the tube samples to one another. Given the short 
lengths of the tube samples, and the overall consistency of the wall thickness measurements, it was judged that this 2 to 3 -in. difference in axial location would have only a minor impact on the accuracy of the wall thickness measurements.

\section{Metallography:}

A full cross-section ring was polished and etched for one tube sample for each material type, (i.e. the ring identified as RS-1 in Figure 7). The same electrolytic etching procedure was used for each material. It involved using a $10 \%$ oxalic acid etchant with a 9 volt open circuit potential. These then were examined using the scanning electron microscope (SEM).

\section{$\underline{\mathrm{SEM} / \mathrm{EDS}}$ :}

One tube sample from each candidate material was selected for a detailed assessment of the microstructures of both the external and internal interfaces. Typically the sample selected was the one that experienced the greatest amount of metal loss. Typically the examination was along the tube radius that extended through the thinnest wall for a given tube sample. Given the high level of resistance to steam-side oxidation for all of the candidate alloys, the focus of this analysis primarily was on the deposit/scale/metal interface at the external surface of the tubes. Nonetheless, the condition at the scale/metal interface also was documented for the internal surface of the tube. For both surfaces, photographs were taken to record the nature of the scale and the condition of the underlying metal. These are recorded in the appendices along with comments regarding distinctive and/or unique features.

While in the SEM, EDS(energy dispersive spectroscopy) analyses were made of selected regions of the deposit, scale, and underlying tube metal. These also are recorded in the appendices.

\section{Weld Profile:}

Welds of every material type were cross sectioned (as per sample RS-3 in Figure 7) and evaluated. The purpose of this was to investigate the condition of the welds and weld heat affected zones relative to the remainder of the tube. The weld cross sections were polished, etched and photographed to record their appearance. Typical results are shown in Figures 11, 12 and 13 .

\section{Deposit Analysis:}

Deposit was scraped and removed from outside surfaces and submitted for elemental and compound identification by chemical analysis and x-ray diffraction respectively. The deposit was tight and adherent and did not contain the heavy outer deposit that is sometimes seen on superheater tubes. Deposits were subjected to inductively coupled plasma - optical emission spectrographic (ICP-OES) analysis. The results are provided in Tables 3 and 4.

Lastly, EDS analyses, performed during the SEM investigation, indicated the potential presence of carbon in both the deposit and in the tube metal adjacent to the scale/metal interface, see Table 5. Because carbon analysis by EDS is not accurate, and because of the potential for contamination by the epoxy resin used as the mounting compound, a combustion-gravimetric analysis was performed to determine the presence of carbon in the deposit. The result of this 
analysis also is reported in Table 5 which also provides the chlorine content of the inner and outer layers of the deposit.

Table 1: $\quad$ Tube Sample Designation, Average Surface Metal Temperature, and Exposure Time Based on Position in Test Section C

\begin{tabular}{|c|c|c|c|}
\hline Sample Designation & Material & $\begin{array}{c}\text { Average Surface } \\
\text { Metal Temperature } \\
\text { (F) }\end{array}$ & $\begin{array}{c}\text { Exposure Time } \\
\text { (hrs) }\end{array}$ \\
\hline S-T & Incoclad 671 & 1219 & 20,832 \\
\hline R-S & IN52 Weld Overlay & 1208 & " \\
\hline Q-R & IN72 Weld Overlay & 1208 & " \\
\hline P-Q & Incoclad 671 & 1203 & " \\
\hline O-P & HR 120 & 1194 & “" \\
\hline $\mathrm{N}-\mathrm{O}$ & $310 \mathrm{HCbN}(\mathrm{HR} 3 \mathrm{C})$ & 1190 & " \\
\hline M-N & $310 \mathrm{Ta}$ & 1188 & " \\
\hline L-M & Modified $800 \mathrm{H}$ & 1185 & 13,590 \\
\hline K-L & Save 25 & 1180 & “ \\
\hline $\mathrm{J}-\mathrm{K}$ & NF 709 & 1178 & "“ \\
\hline I-J & $347 \mathrm{HFG}$ & 1174 & “" \\
\hline H-I & Thermie & 1171 & “" \\
\hline G-H & $310 \mathrm{HCbN}(\mathrm{HR} 3 \mathrm{C})$ & 1167 & “" \\
\hline F-G & $310 \mathrm{Ta}$ & 1166 & 15,807 \\
\hline E-F & Modified $800 \mathrm{H}$ & 1162 & " \\
\hline D-E & Save 25 & 1158 & “" \\
\hline C-D & NF 709 & 1153 & 20,832 \\
\hline $\mathrm{B}-\mathrm{C}$ & $\mathrm{Fe} 3 \mathrm{Al}$ & 1149 & " \\
\hline A-B & $347 \mathrm{HFG}$ & 1147 & " \\
\hline Return Bend & TP 310 & $* * * *$ & \\
\hline $\mathrm{U}-\mathrm{V}$ & $671 \mathrm{Clad}$ & 1121 & “" \\
\hline V-W & HR 120 & 1110 & “" \\
\hline W-X & $310 \mathrm{HCbN}(\mathrm{HR} 3 \mathrm{C})$ & 1106 & “" \\
\hline $\mathrm{X}-\mathrm{Y}$ & $310 \mathrm{Ta}$ & 1103 & “" \\
\hline $\mathrm{Y}-\mathrm{Z}$ & Modified $800 \mathrm{H}$ & 1099 & “" \\
\hline Z-AA & Save 25 & 1096 & 15,807 \\
\hline AA-BB & $310 \mathrm{HCbN}$ & 1092 & 20,832 \\
\hline BB-CC & NF 709 & 1088 & " \\
\hline CC-DD & $347 \mathrm{HFG}$ & 1086 & “" \\
\hline DD-EE & HR 120 & 1088 & " \\
\hline EE-FF & Incoclad 671 & 1086 & "6 \\
\hline FF-GG & IN72 Weld Overlay & 1079 & “" \\
\hline GG-HH & IN52 Weld Overlay & 1073 & “" \\
\hline HH-II & Thermie & 1067 & “" \\
\hline II-JJ & Save 25 & 1058 & “" \\
\hline JJ-KK & NF709 & 1048 & " \\
\hline
\end{tabular}


Table 2. Representative Analysis of Coal Burned in Niles Unit-1

\begin{tabular}{|c|c|}
\hline Coal Analysis & \\
\hline Moisture & $5.0 \%$ \\
\hline Carbon & $66.5 \%$ \\
\hline Hydrogen & $4.9 \%$ \\
\hline Nitrogen & $1.4 \%$ \\
\hline Sulfur & $3.1 \%$ \\
\hline Alkali (as $\left.\mathrm{Na}_{2} \mathrm{O}\right)$ & $0.2 \%$ \\
\hline Ash & $12.3 \%$ \\
\hline & \\
\hline Ash Analysis & \\
\hline $\mathrm{SiO}_{2}$ & $41.5 \%$ \\
\hline $\mathrm{Al}_{2} \mathrm{O}_{3}$ & $19.5 \%$ \\
\hline $\mathrm{TiO}_{2}$ & $1.2 \%$ \\
\hline $\mathrm{Fe}_{2} \mathrm{O}_{3}$ & $26.8 \%$ \\
\hline $\mathrm{CaO} \mathrm{O} \%$ & $3.8 \%$ \\
\hline $\mathrm{MgO}_{\mathrm{K}_{2} \mathrm{O}}$ & $0.7 \%$ \\
\hline $\mathrm{Na}_{2} \mathrm{O}$ & $1.6 \%$ \\
\hline $\mathrm{SO}_{3}$ & $0.5 \%$ \\
\hline $\mathrm{P}_{2} \mathrm{O}_{5}$ & $3.3 \%$ \\
\hline $\mathrm{BaO}_{2}$ & $0.6 \%$ \\
\hline $\mathrm{Undetermined}$ & $0.1 \%$ \\
\hline & $0.3 \%$ \\
\hline
\end{tabular}

Table 3: Deposit Analysis

\begin{tabular}{|l|c|c|c|c|}
\hline Constituent & $\begin{array}{c}\text { Section A } \\
\text { Outer Layers } \\
\text { (wt\%) }\end{array}$ & $\begin{array}{c}\text { Section A } \\
\text { Inner Layers } \\
\text { (wt\%) }\end{array}$ & $\begin{array}{c}\text { Section C } \\
\text { Outer Layers } \\
\text { (wt\%) }\end{array}$ & $\begin{array}{c}\text { Section C } \\
\text { Inner Layers } \\
\text { (wt\%) }\end{array}$ \\
\hline Silicon, $\mathrm{SiO}_{2}$ & 31.84 & 28.64 & 23.39 & 20.51 \\
\hline Aluminum, $\mathrm{Al}_{2} \mathrm{O}_{3}$ & 14.11 & 9.66 & 11.49 & 7.90 \\
\hline Iron, $\mathrm{Fe}_{2} \mathrm{O}_{3}$ & 22.99 & 12.85 & 14.39 & 12.26 \\
\hline Titanium, $\mathrm{TiO} 0_{2}$ & 1.06 & 0.60 & 0.66 & 0.53 \\
\hline Calcium, $\mathrm{CaO}$ & 3.60 & 4.06 & 4.26 & 7.61 \\
\hline Magnesium, $\mathrm{MgO}$ & 0.35 & 0.17 & 0.84 & 0.76 \\
\hline Sodium, $\mathrm{Na}_{2} \mathrm{O}$ & 0.70 & 0.96 & 2.06 & 1.88 \\
\hline Potassium, $\mathrm{K}_{2} \mathrm{O}$ & 1.82 & 3.82 & 6.92 & 5.64 \\
\hline Phosphorous, $\mathrm{P}_{2} \mathrm{O}_{5}$ & 0.88 & 0.91 & 1.02 & 0.89 \\
\hline Sulfur, $\mathrm{SO} \mathrm{O}_{3}$ & 11.64 & 20.00 & -- & - \\
\hline Sulfur, $\mathrm{SO} \mathrm{O}_{4}$ & -- & -- & 34.79 & 41.84 \\
\hline Strontium, $\mathrm{SrO}$ & -- & -- & 0.07 & 0.05 \\
\hline Manganese, $\mathrm{MnO}$ & -- & -- & 0.06 & 0.06 \\
\hline Barium, $\mathrm{BaO}$ & -- & -- & & 0.07 \\
\hline
\end{tabular}


Table 4:Deposit Analyses (X-Ray Diffraction Results)

\begin{tabular}{|c|c|}
\hline Level & Scale Analysis \\
\hline Major & $\begin{array}{ll}\text { - } & \text { Cristobalite, syn, } \mathrm{SiO}_{2} \\
\text { - } & \alpha Q \text { Quartz, } \mathrm{SiO}_{2}\end{array}$ \\
\hline Minor & $\begin{array}{ll}\text { - } & \text { Ferrinatrite }\left(\mathrm{Na}_{3} \mathrm{Fe}\left(\mathrm{SO}_{4}\right)_{3}\left(\mathrm{H}_{2} \mathrm{O}\right)_{3}\right. \\
\text { - } & \text { Potassium Dihydrogen Phosphate } \\
& (\mathrm{KH} 2 \mathrm{PO} 3) \\
\text { - } & \text { Tamarugite }\left(\mathrm{NaAl}\left(\mathrm{SO}_{4}\right)_{2}\left(\mathrm{H}_{2} \mathrm{O}\right)_{6}\right.\end{array}$ \\
\hline Trace & $\begin{array}{ll}- & \text { Hohmannite } \\
& \left(\mathrm{Fe}_{2}\left(\mathrm{H}_{2} \mathrm{O}\right)_{4}\left(\mathrm{SO}_{4}\right)_{2} \mathrm{O}\left(\mathrm{H}_{2} \mathrm{O}\right)_{4}\right.\end{array}$ \\
\hline
\end{tabular}

Table 5: Carbon and Chlorine Analysis Results

\begin{tabular}{|l|c|c|}
\hline Constituent & Outer Layers, (wt\%) & Inner Layers, (wt\%) \\
\hline Carbon & 0.27 & 0.58 \\
\hline Chlorine & 0.05 & 0.05 \\
\hline
\end{tabular}




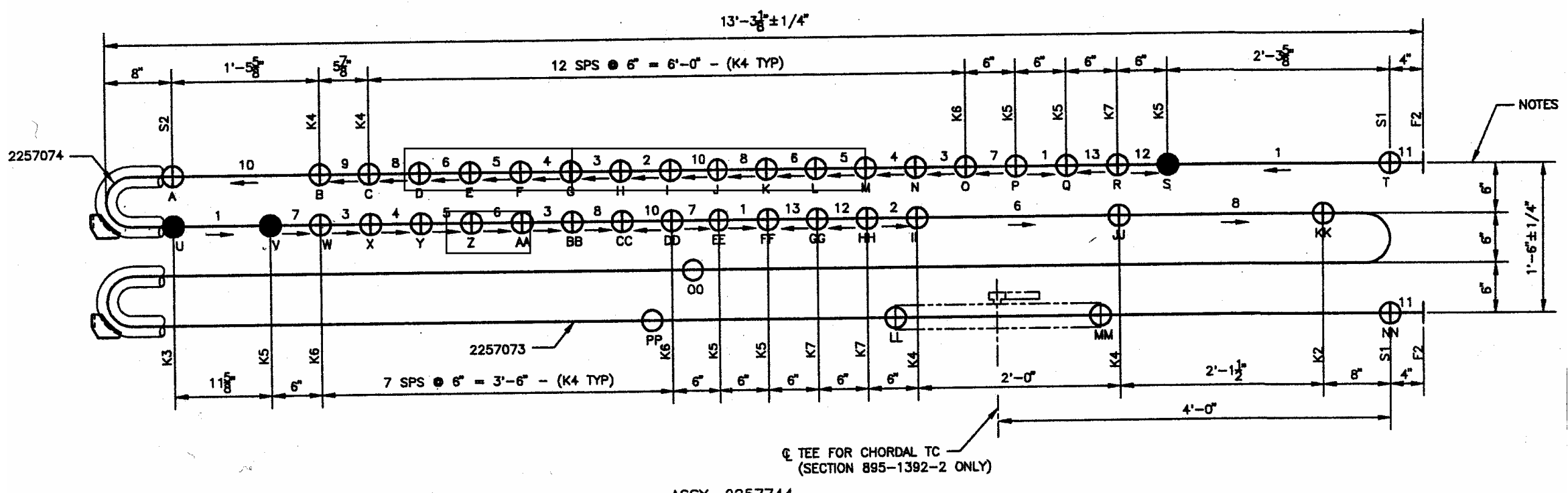

ASSY 2257744

Figure 1: Schematic of Test Section Showing Position of Welds and Location of Dutchmen (i.e. samples removed early)

Note:

1. The welds are indicated as circles in the schematic above. A letter designation is associated with each weld. The candidate tube samples lie between the welds and are designated by the weld at either end of the sample.

2. The "hot leg" of this test section is toward the top in this schematic. The hottest position in the test section is at position S-T. Accordingly, the coolest position in the test section is at position JJ-KK at the inlet to the "cold leg" of the test section.

3. Tube samples removed and replaced by "dutchmen" in November 2001 are highlighted in red.

4. Tube samples removed and replaced by "dutchmen" in June 2002 are highlighted in yellow. 


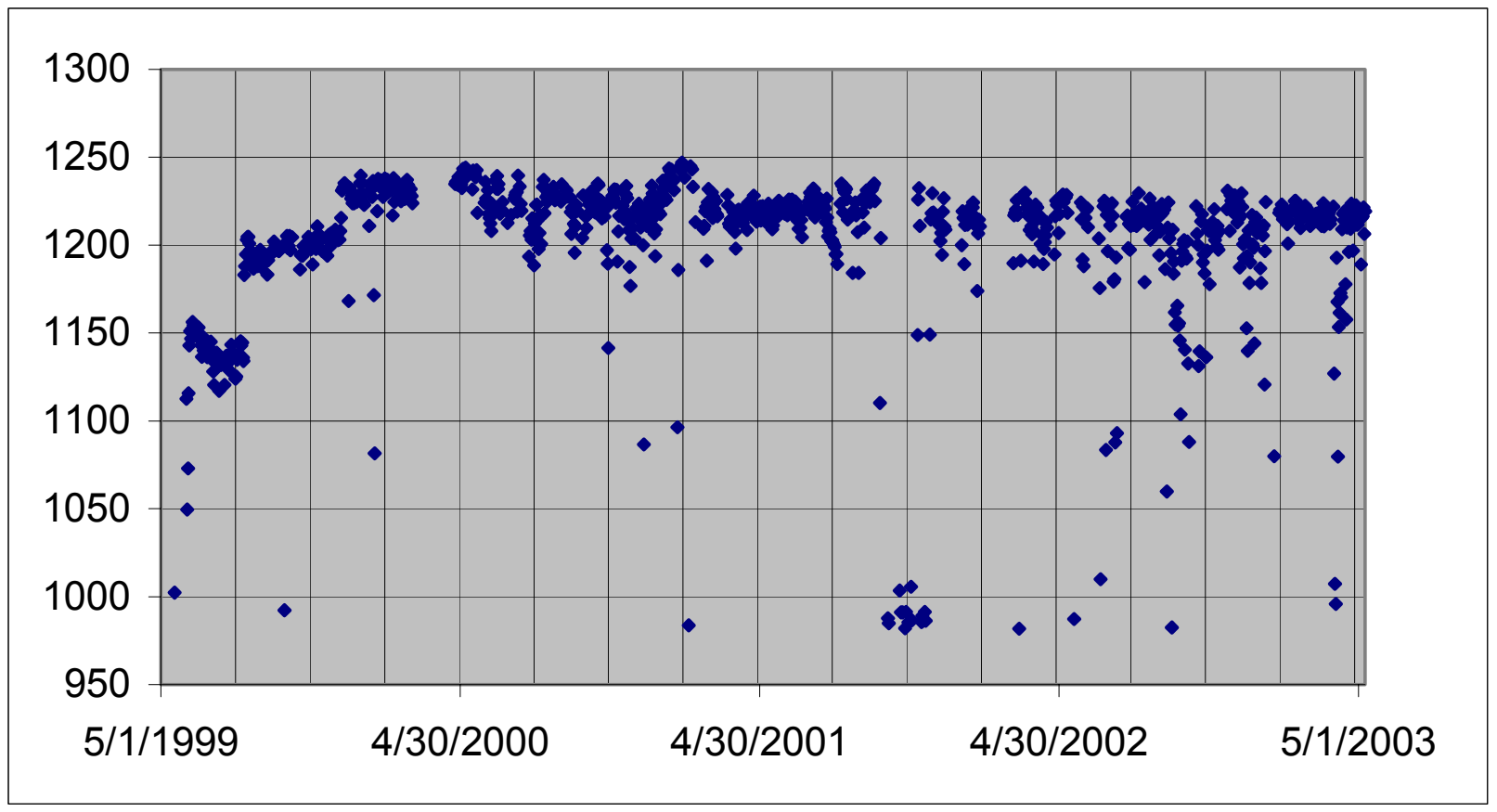

Figure 2: Daily Average Surface Metal Temperatures (F) for Tube Segment "R-S 


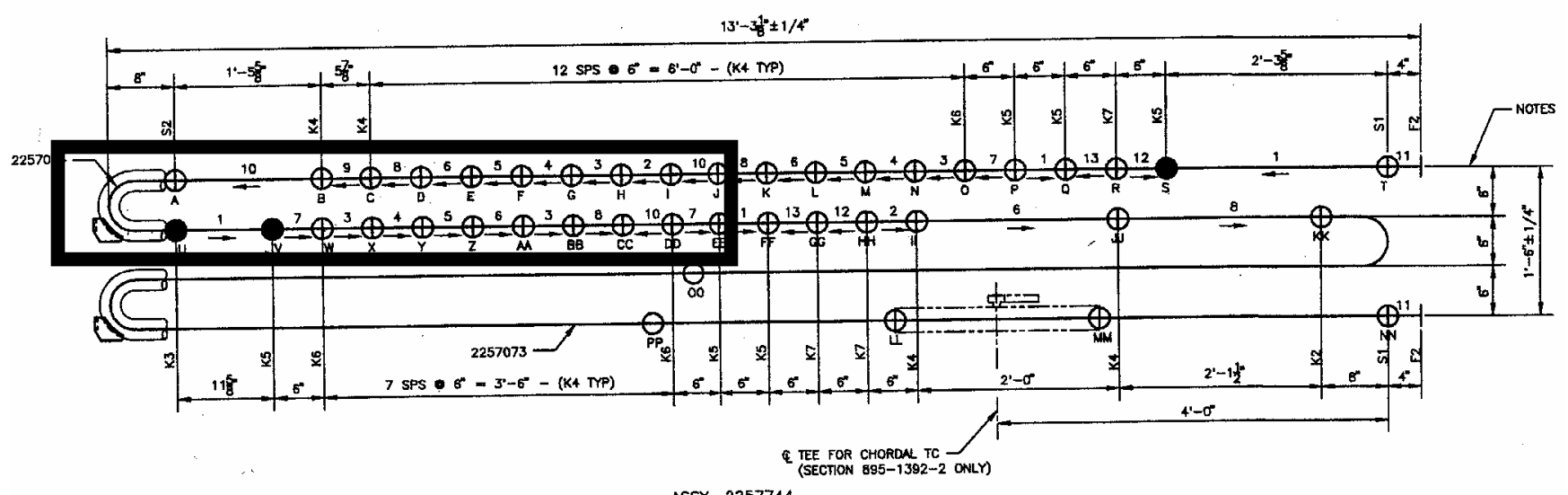

ASSY 2257744

B $\quad$ C $\quad$ D

G

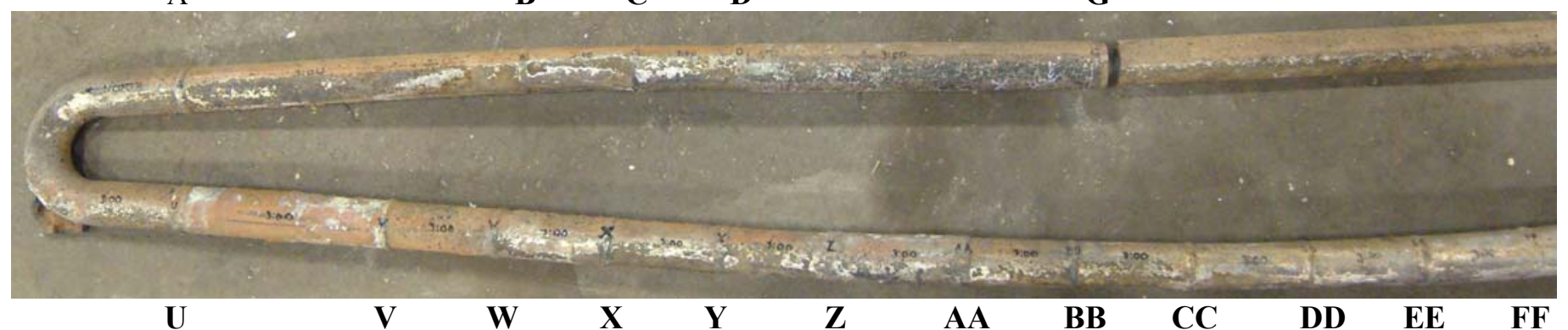

Figure 3: Appearance of Test Section C After Removal From Service (Part 1)

Note: 1) The region highlighted in the schematic drawing of the test section is shown in the photograph below.

2) The letters adjacent to the photograph indicate the positions of the various welds used to construct the test section. These letters also are used to identify the candidate metal samples that lie between them. For instance, Sample A-B lies between welds A and B. It was manufactured from 347 HFG. Sample B-C was manufactured from $\mathrm{Fe}_{3} \mathrm{Al}$, etc. 


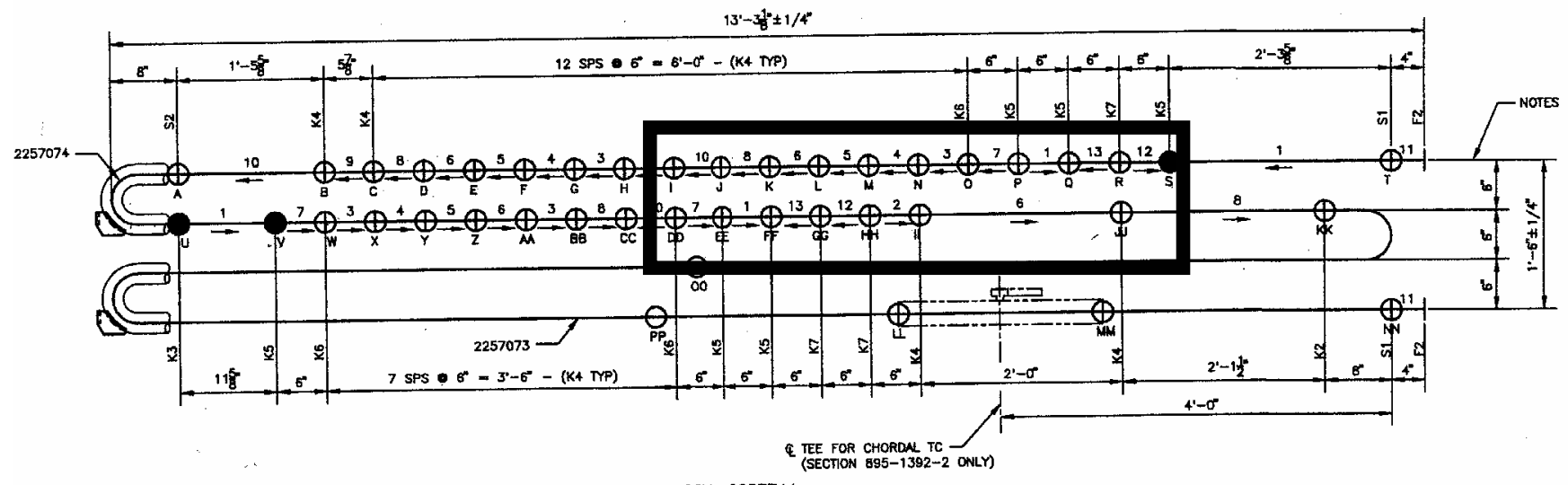

ASSY 2257744

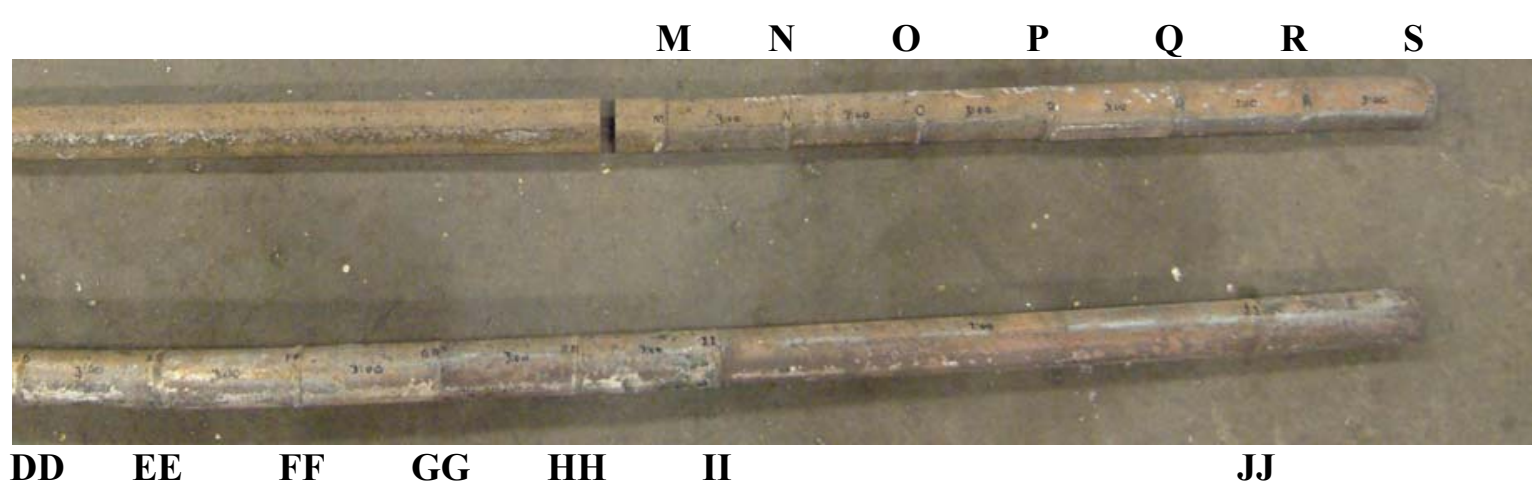

Figure 4: $\quad$ Appearance of Test Section C After Removal From Service (Part 2)

Note: 1) The region highlighted in the schematic drawing of the test section is shown in the photograph below 


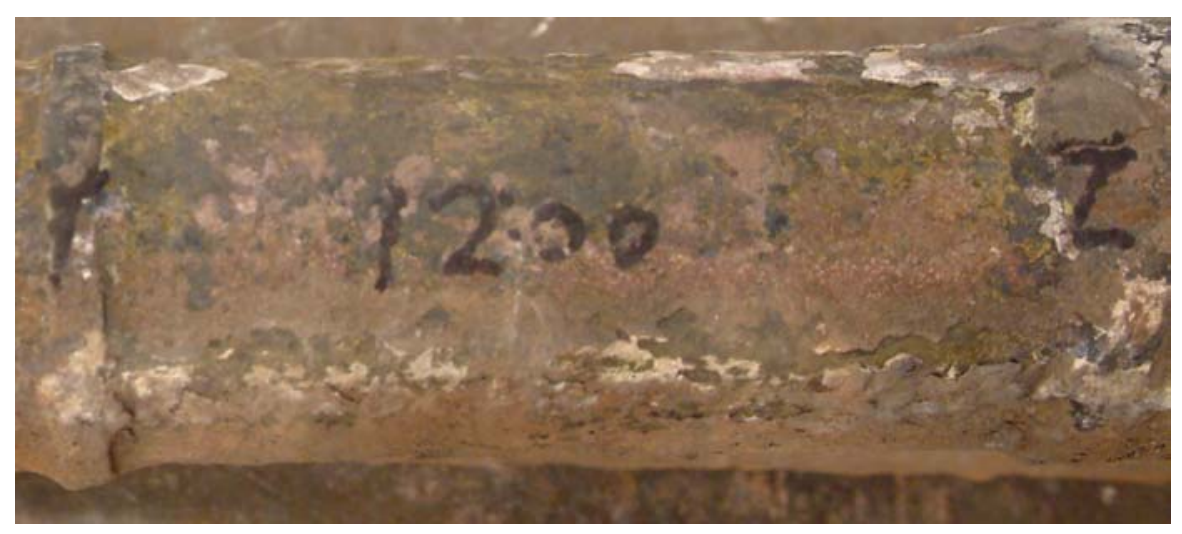

12 O'Clock - Facing Gas Flow (Note: 3 o'clock is up in this image)

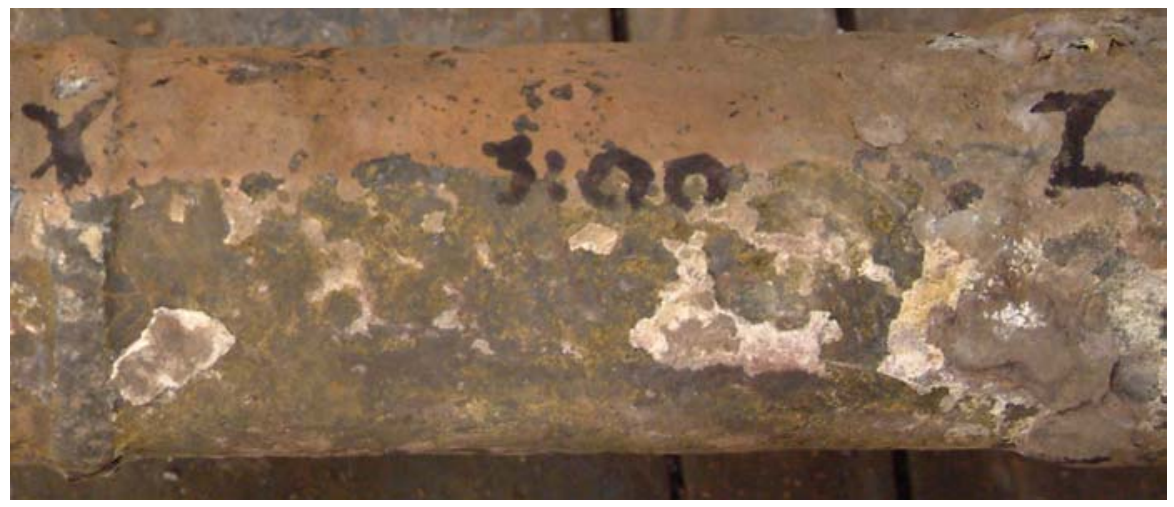

3 O'Clock

(Note: 6 o'clock is up in this image)

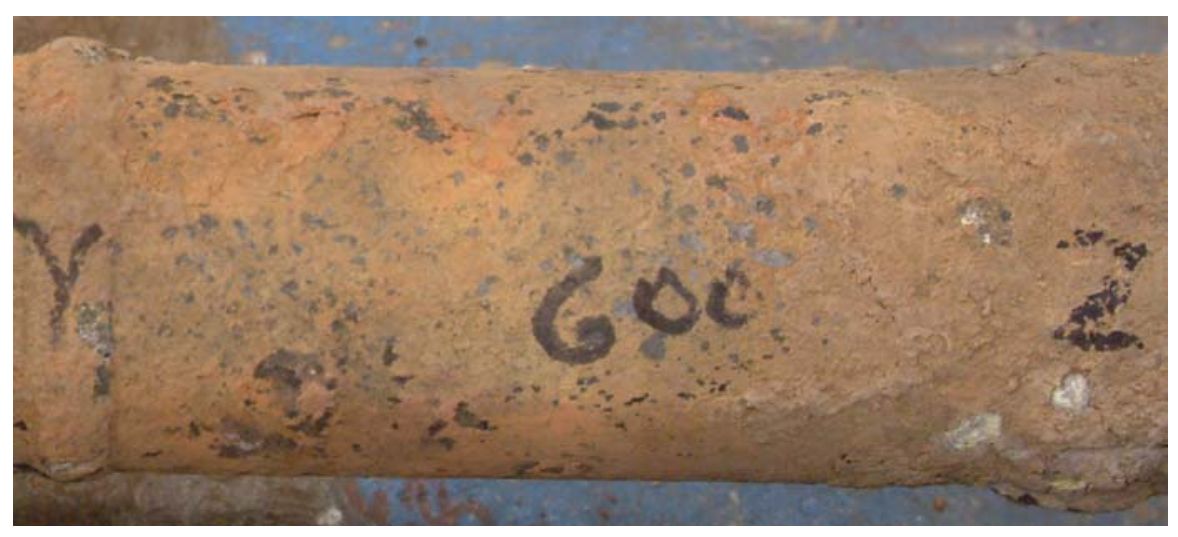

6 O'Clock - Opposite Gas Flow

Figure 5: Typical Documentation of As-Received Appearance for Tube Sample Y-Z (Modified 800H)

Note: The chalky alkali-iron trisulfate scale is noted in all three orientations. Differences in coloration are primarily due to lighting effects, with the 3 o'clock orientation being the most accurate in this regard. 


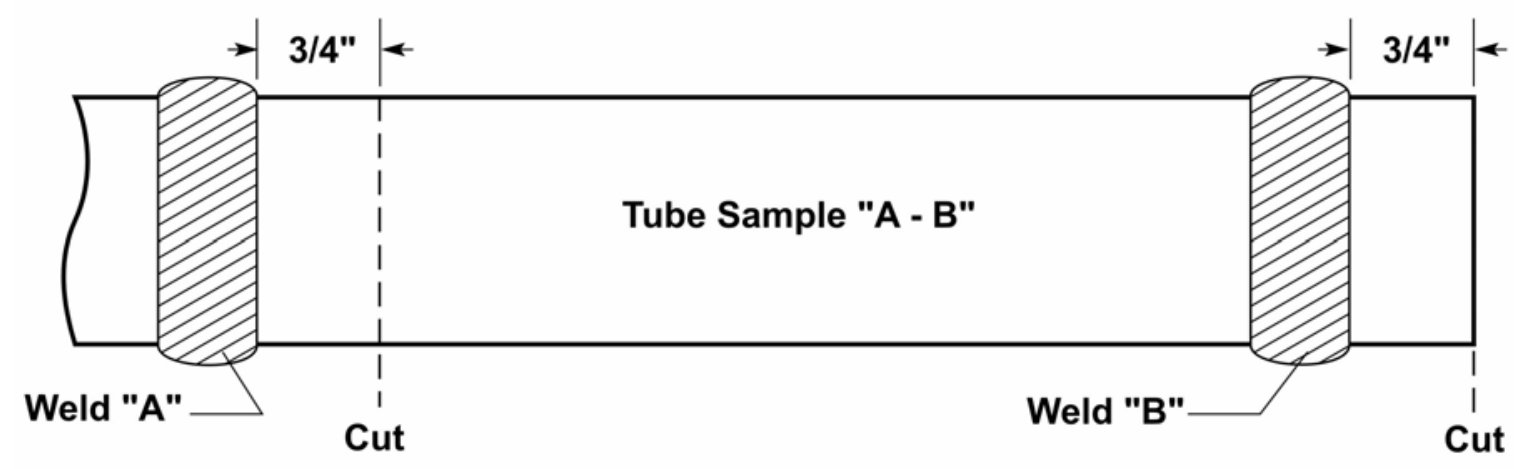

Figure 6: $\quad$ Schematic Depicting Sectioning to Remove Tube Sample From the Test Section

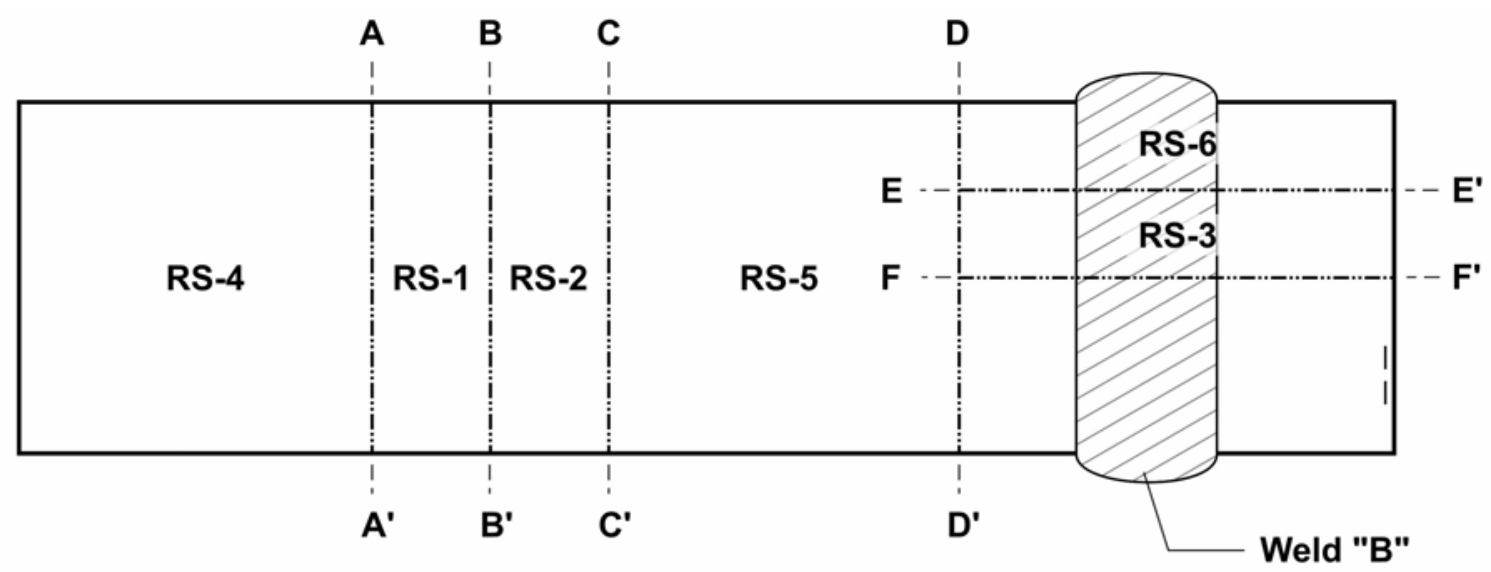

\begin{tabular}{|lll|}
\hline & Key \\
\hline Sample & & Purpose \\
\cline { 1 - 1 } RS-1 & & Metallography ring \\
RS-2 & & Dimension ring \\
RS-3 & & Weld profile \\
RS-4, 5, 6 & Extra material \\
\hline
\end{tabular}

Note:

1) Cut $B-B^{\prime}$ is at the approximate center of the tube sample

2) Specimens RS-1, RS-2, RS-3 are all about $1 / 2$ "thick

Figure 7: $\quad$ Schematic Depicting Sectioning of Tube Sample 


\section{Figure 8: Typical Semi-Quantitative Composition Verification for Modified 800H}

Description:

Source:

Reference Composition:
Nominal $20 \mathrm{Cr} / 30 \mathrm{Ni}$ steel with improved creep strength due to addition of $\mathrm{V}, \mathrm{Nb}, \mathrm{Ti}$, and $\mathrm{B}$

Oak Ridge National Laboratory (ORNL)

"Target" Chemistry per ORNL (Reference 1).

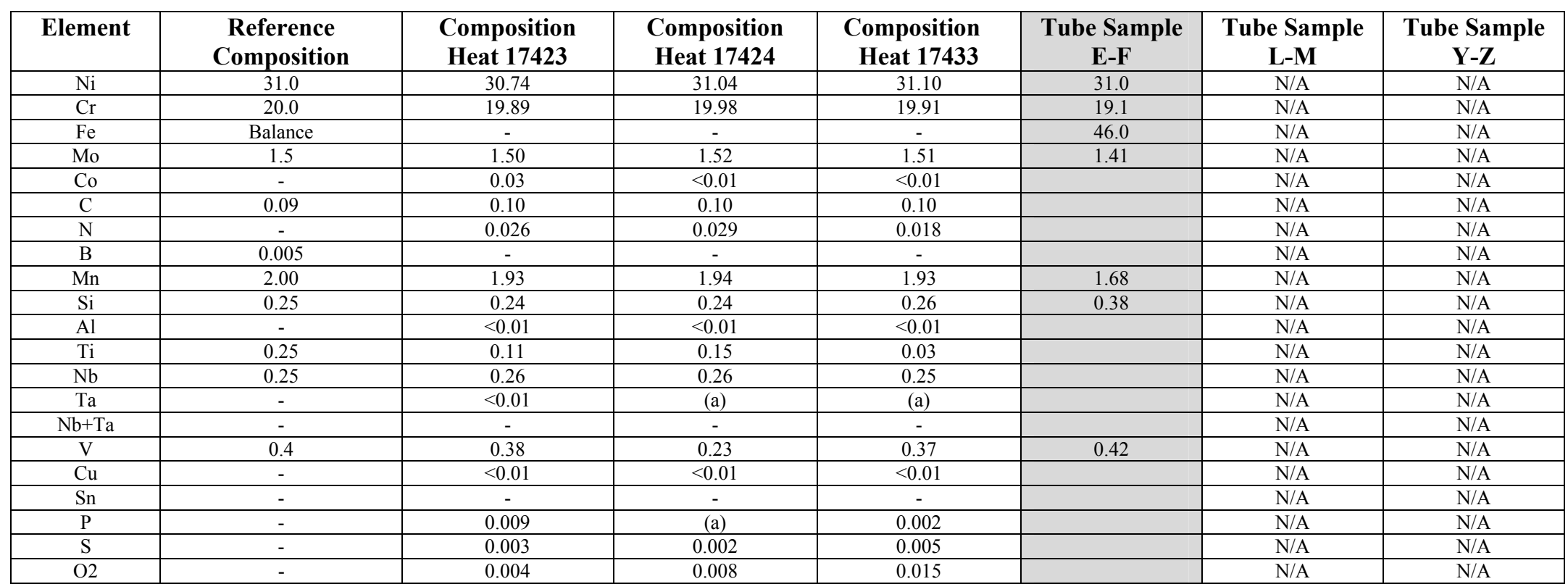

a- Not analyzed.

b- By difference.

Reference 1:

"Fabrication of Test Tubes for Coal Ash Corrosion Testing", Sikka, V.K., Swindeman, R.W., Wright, I.G., Judkins, R.R., Johnson, R., Proc, $13^{\text {th }}$ Annual Conf. on Fossil Energy Mat'ls, May 1999.

Note: $\quad$ Sample E-F was from Heat 17433

Sample L-M was from Heat 17423

Sample Y-Z was from Heat 17424 


\section{O'Clock}

(N/A mils lost)

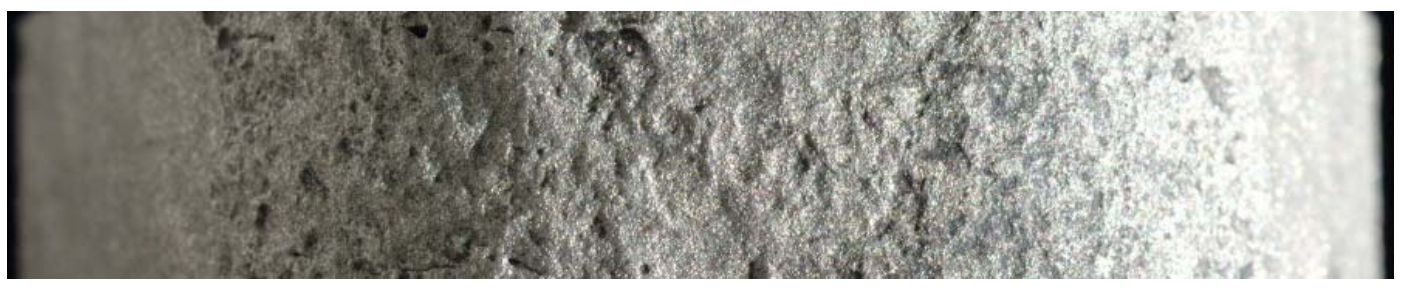

3 O'Clock

(106 mils lost)

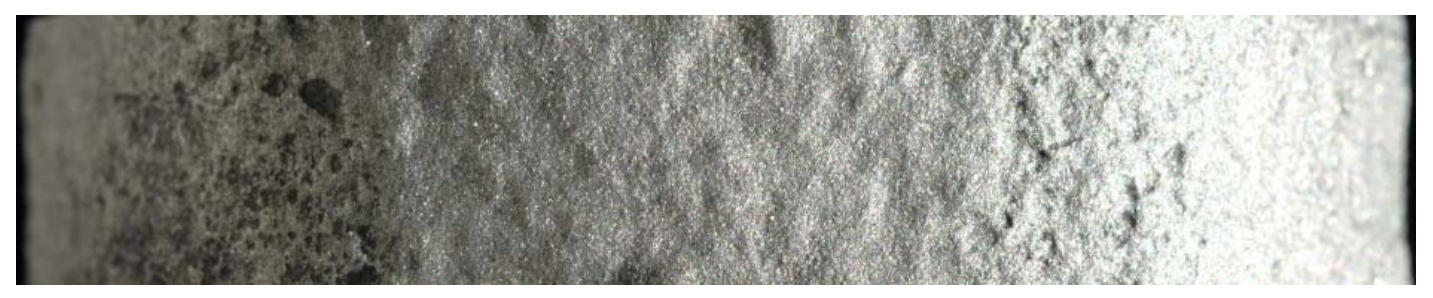

6 O'Clock

(0 mils lost)

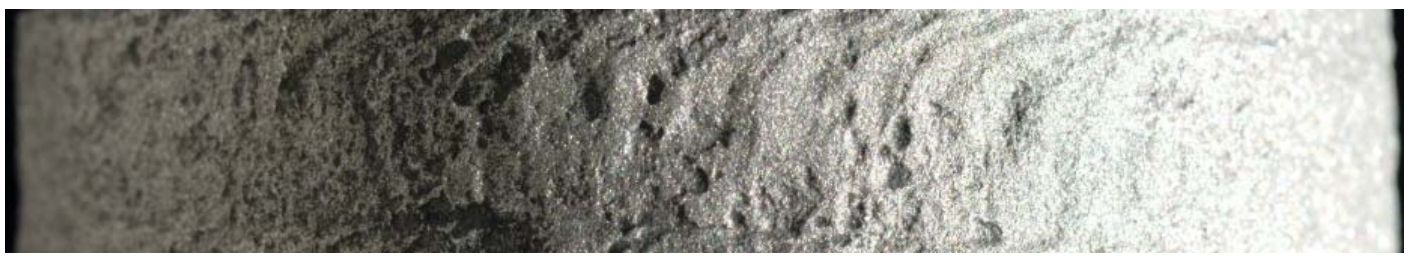

Figure 9: Typical Example of OD Surface Appearance After Grit Blast

Note: This particular example is for IN52 Weld Overlay, Sample GG-HH. 
Material:

Sample Location:

Maximum Rate:

Maximum Loss Orientation:

Average metal Temperature:

\section{Modified 800H}

Y-Z

79 mils/yr

Approx. 9:30 o'clock

$1099 \mathrm{~F}$

\section{O’Clock}

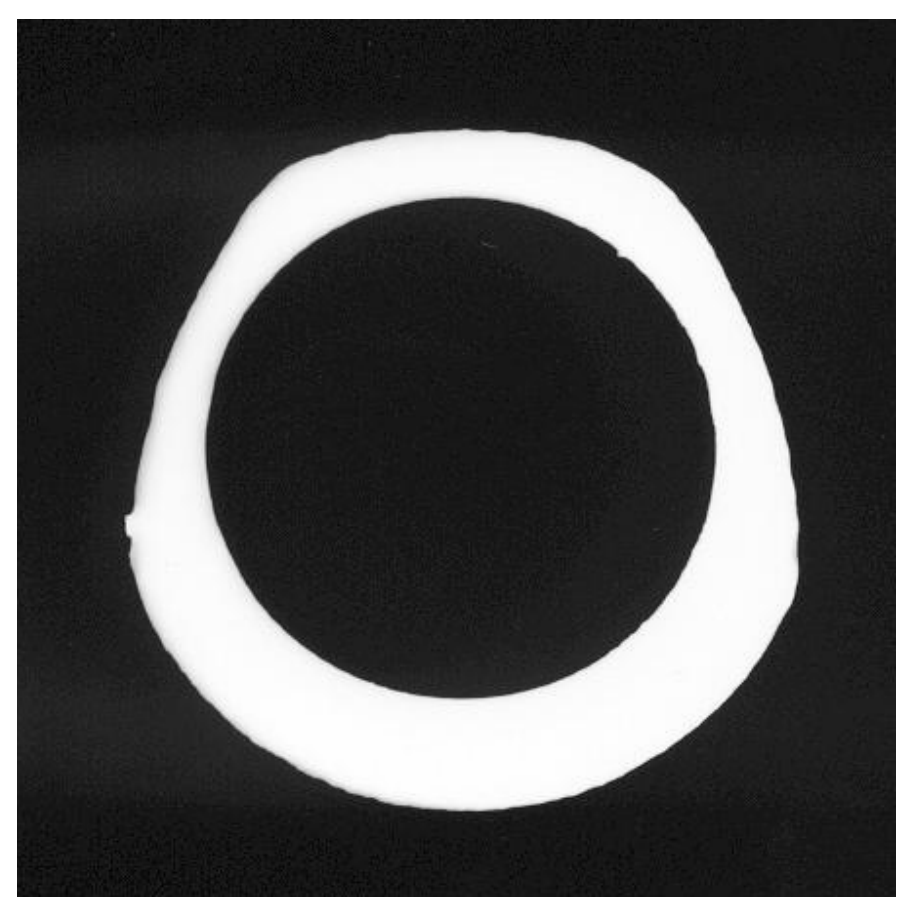

3 O'Clock

Approx. 1.2X

Wall Reduction by Position (in.)

\begin{tabular}{|l|c|c|c|c|c|c|c|c|c|c|}
\hline & $\begin{array}{c}\mathbf{1 2} \\
\text { o'clock }\end{array}$ & $\mathbf{1 : 3 0}$ & $\mathbf{3 : 0 0}$ & $\mathbf{4 : 3 0}$ & $\mathbf{6 : 0 0}$ & $\mathbf{7 : 3 0}$ & $\mathbf{9 : 0 0}$ & $\mathbf{1 0 : 3 0}$ & AVG & $\begin{array}{c}\text { Max }^{*} \\
\text { Loss }\end{array}$ \\
\hline Before & 0.402 & 0.401 & 0.401 & 0.400 & 0.400 & 0.401 & 0.401 & 0.401 & 0.401 & 0.401 \\
\hline After & 0.253 & 0.226 & 0.294 & 0.389 & 0.390 & 0.387 & 0.233 & 0.294 & & 0.212 \\
\hline Delta & 0.149 & 0.175 & 0.107 & 0.011 & 0.010 & 0.014 & 0.168 & 0.107 & & 0.189 \\
\hline
\end{tabular}

Figure 10: A Typical Silhouette with Wall Thickness Measurements Showing Metal Loss as a Function of Position

* Measurement taken at "maximum loss orientation" noted above. The average "before" wall thickness was used to calculate the "delta". 


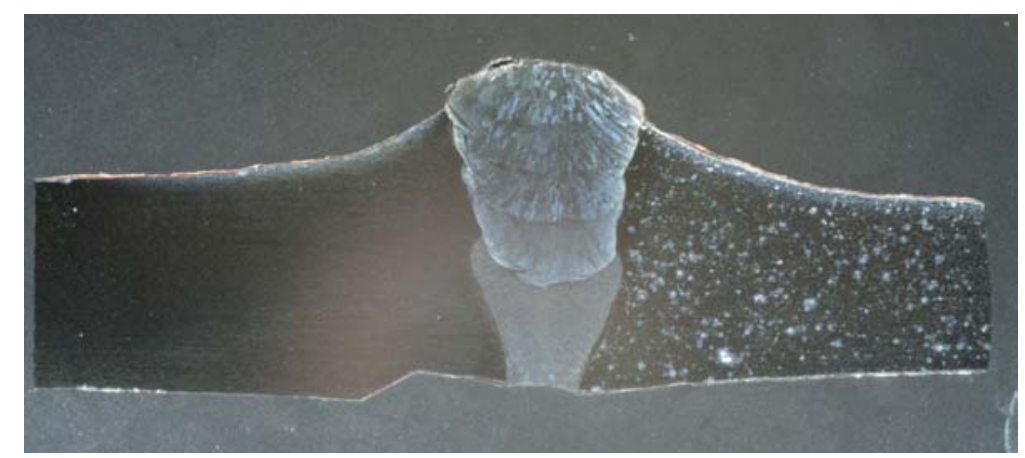

Note: $\mathrm{Fe}_{3} \mathrm{Al}$ material to the right of the weld in this image and $347 \mathrm{HFG}$ to the left.

Figure 11: Weld Profile Through Tube Sample Showing Good Corrosion Resistance in the Vicinity of the Weld

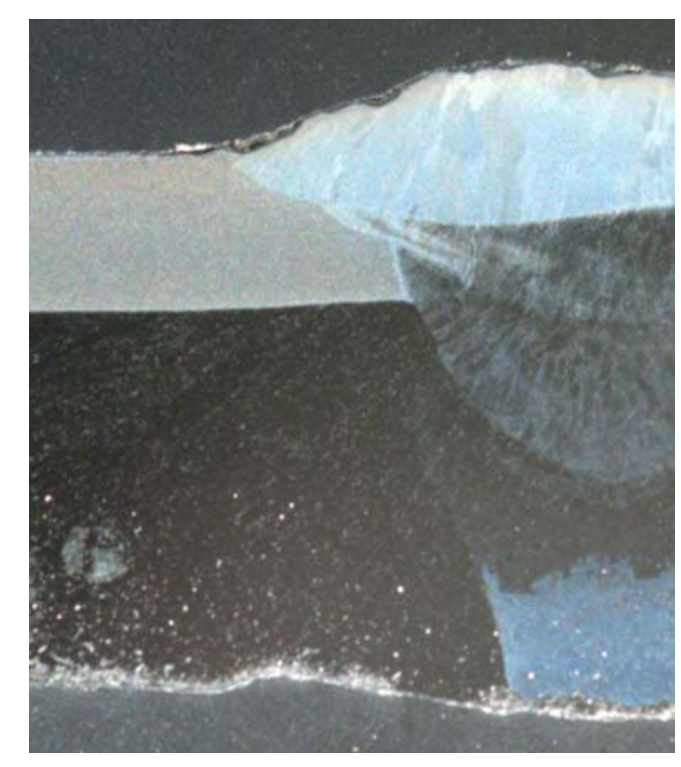

Figure 12: Weld Profile Through Clad Tube Sample

Incoclad 671 Adjacent to Weld "V" in Section C (at 1121 F) 


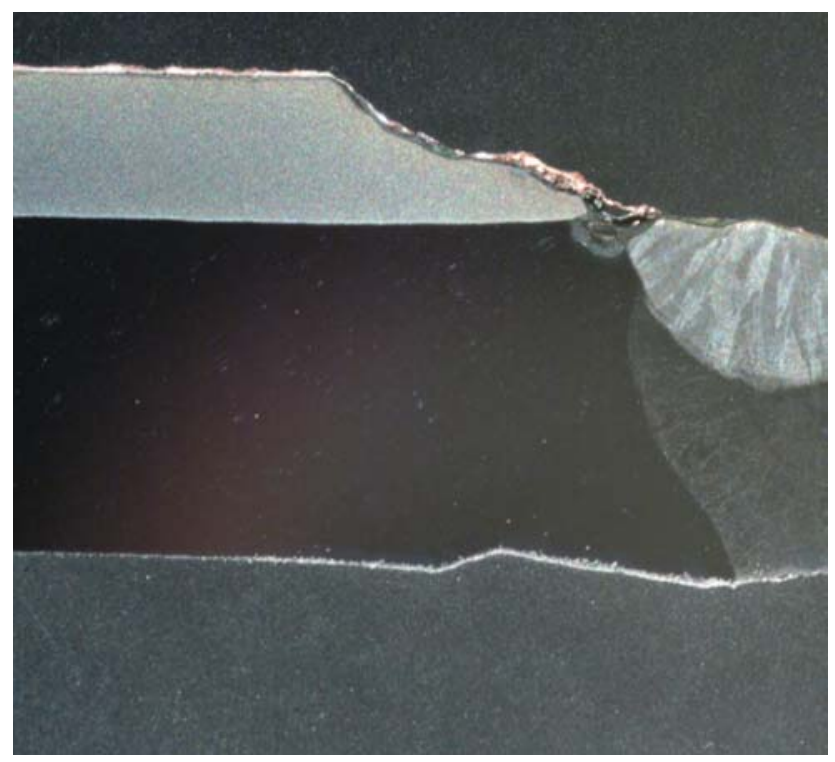

Figure 13: Weld Profile Through Clad Tube Sample Incoclad 671 Adjacent to Weld "P" (at 1203 F) in Section C 


\subsection{RESULTS \& DISCUSSION}

The primary goal of this program is to rank the relative performance of candidate superheater tube alloys for use in boilers fired with corrosive high-sulfur coals. The operational history for Niles Unit \#1 demonstrates that the service conditions provide an environment within the boiler that is aggressive enough to test the ability of these materials to resist coal ash corrosion, and to provide an adequate ranking of their relative performance.

Surface Metal Temperature: The average surface metal temperature, from the hot to the cooler end of the test section, ranged from about $1210^{\circ} \mathrm{F}$ to $1060^{\circ} \mathrm{F}$. As was discussed earlier, over this temperature range, the coal ash corrosion rate for a given austenitic alloy is generally expected to increase with increasing temperature. Recall that at about $1000^{\circ} \mathrm{F}$, alkali-iron trisulfates in the coal ash become molten, and this molten constituent is very aggressive. The upper limit to this is about $1300^{\circ} \mathrm{F}$ where the stability of the complex sulfates decreases and the corrosion rate ebbs. The exact temperature range over which the sulfates are molten depends on the ash, gas and scale chemistries.

Flue Gas Temperature: The flue gas temperature has a significant impact on the wastage rate for superheater tube materials in that it directly influences the average surface metal temperature. The flue gas temperature in the region of interest in Niles Unit \#1 was estimated to be $2114^{\circ} \mathrm{F}$.

Internal Pressure: It is understood that the internal pressure for the test section was low, at approximately $300 \mathrm{psig}$. This has the benefit that almost the full wall thickness of the tube was available as a "corrosion allowance". Though it does not impose pressure stresses typical of a supercritical boiler, it offered the advantage that a candidate tube sample would not need to be pulled from service almost up to the point that its full wall thickness had wasted away.

Candidate Alloy Rankings Based on Corrosion Resistance: Based on the above, and considering the fact that the boiler was burning high-sulfur coal, the service of Test Section C provided an aggressive environment that was suitable for ranking candidate superheater materials with respect to their resistance to coal ash corrosion. That the environment is aggressive is further confirmed by the fact that some of the candidate tube materials corroded at a very high rate, and that this resulted in the need to replace tube segments in Section $\mathrm{C}$ before the full exposure period was completed.

Table 6 provides the wastage rate (mils of wall thickness lost per year) as a function of position within the Test Section C. Figure 14 presents this same information graphically. Table 7 provides the wastage rate with all of the samples of a given material grouped separately. Examination of these results allows one to determine the maximum wastage rate for each of the candidate materials. These maximum rates, shown in Table 8, are the limiting case for any of the candidate materials when exposed to the coal ash corrosion conditions for Section C. Thus, these rates can be used to rank the performance of these materials relative to one another. From this it can be seen that Incoclad 671 and IN 72 Weld Overlay exhibited the lowest maximum corrosion rates based on Section $\mathrm{C}$ exposure. These same materials also exhibited the best corrosion resistance based on Section A exposure. It should also be noted that Save 25 exhibited the highest maximum corrosion rate for both test sections. The other materials performed between these two extremes. However, almost all of these candidate materials exhibited a maximum 
corrosion rate in excess of 50 mils per year. Figure 15 provides a graphical comparison of these maximum wastage rates for both Sections A and C.

Wastage Rate as a Function of Temperature: Figure 16 shows a plot of wastage as a function of average service temperature for each of the twelve candidate materials in Section C. Interpretation of these data is complicated by the fact that, while some materials behaved as one would have expected, others did not. The data show that for some materials the wastage rate increased with temperature, e.g. Save 25 and 800 Mod. Some materials exhibited an apparent decrease in wastage rate at intermediate temperatures, e.g. $347 \mathrm{HFG}$ and HR 120. Other materials exhibited an apparent increase in wastage rate at an intermediate temperature, e.g. 310 $\mathrm{HCbN}$ and $310 \mathrm{Ta}$. Finally, the more resistant high-chromium, high-nickel alloys appeared to exhibit a decrease in rate as temperature increased, e.g. Incoclad 671, IN 72 WO, and IN 52 WO. It further should be understood that, for the most part, performance was consistent in that the candidate materials exhibited this same sort of performance in both Sections A and C, compare Figures 16 and 17.

It is tempting, but risky at this point, to draw inferences from the corrosion rate versus temperature data. For instance, the data that showed a reduced corrosion rate at an intermediate temperature might be taken to infer that two corrosion mechanisms were active, one mechanism acting to accelerate corrosion at the lower temperature, and the other mechanism acting to accelerate corrosion at the highest temperature. However, before drawing such inferences, one must also take into consideration the fact that the normal operating conditions within this region of the boiler are not as controllable as might attain during laboratory testing. As an example, when the location of tube samples are "mapped" to show their position within the test section, it becomes apparent that those samples that are located at either end of the test section tend to evidence a lower than expected wastage rate. This suggests the possibility that the physical location of the samples within the test section may influence their wastage rate. It has been speculated, but not confirmed, that the lower than expected wastage rate for the samples at either end of the section may be due to the possibility that the actual surface metal temperatures in these locations are cooler than the calculated average surface metal temperatures that have been used in this investigation. Several practical boiler operational issues can be postulated that might account for this. To date this issue has not been fully explored. It is, however, interesting to note that the Section $\mathrm{C}$ analysis revealed that the candidate materials performed similarly to those from Section A, e.g. $347 \mathrm{HFG}$ experienced a decrease in wastage rate at an intermediate temperature in both cases. This and the results for the other materials suggest that the test sections appear to be performing in a consistent manner.

\section{Additional Issues for Consideration:}

There are a few issues that should be taken into account in assessing the wastage data in the tables and figures above. First, some of the candidate materials relied on a surface coating that had been breached at some point, perhaps early on, during the Section C exposure period. With the coating breached, it can no longer provide resistance to corrosion, and the remaining tube life would be determined by the corrosion resistance of the underlying substrate material.

Analysis of Section A showed that the coating on the $\mathrm{Fe}_{3} \mathrm{Al}$-coated specimen was breached early during the exposure period, and that corrosion was wasting away the underlying Type $304 \mathrm{H}$ 
stainless steel substrate. This also was true for Section C, in fact, no evidence could be found of the original $\mathrm{Fe}_{3} \mathrm{Al}$ coating.

Analysis of Section C revealed that IN 52 Weld Overlay tube segments appear to have done well based on the calculated wastage rate. However, it should be noted that within the timeframe of the Section $\mathrm{C}$ exposure, the original 80 mil thick protective cladding was completely breached, and that only the $800 \mathrm{H}$ substrate remained to provide corrosion resistance for these specimens. Note from Table 8 that $800 \mathrm{H}$ has to date demonstrated one of the higher maximum wastage rates. Thus, now that the protective coating has been wasted away, one would expect that the calculated wastage rate would increase for the IN 52 Weld Overlay samples that remain in the boiler.

Finally, based on calculated wastage rates, the IN 72 Weld Overlay tube segments consistently have demonstrated excellent corrosion resistance, see Table 8. However, this material appears also concurrently to be suffering from a time-dependent cracking problem. The cracking problem does not appear related to the hot-side corrosion mechanism for this material; however, in at least one case, corrosion had removed enough material to reveal and penetrate along an underlying crack. If this additional loss of wall thickness had been taken into account, the calculated corrosion rate would have increased by more than fifty percent. The extent of the IN 72 Weld Overlay cracking problem was first discovered when the material was examined in the SEM along with the other eleven candidate materials. Figure 18 shows an SEM image of one region where underlying cracks intersected the tube surface.

The cracking shown in Figure 18 spurred further investigation. Figure 19 shows evidence of cracking at other locations in this tube segment. Note that the cracking appears to be at roughly the mid-point of the 72 Weld Overlay cladding, and does not contain a corrosion product. Similar cracking was found for both 72 Weld Overlay test segments in Section C. Closer examination of Section A samples revealed the same sort of sub-surface cracking, but to a somewhat lesser extent. Finally, examination of an archive 72 Weld Overlay tube sample revealed a few similar internal cracks, see Figure 20.

The implications of the 72 Weld Overlay cracking problem are not clear. The fact that Incoclad 671, which has a very similar composition to that of 72 Weld Overlay, and appears to be performing well without cracking, suggests that this may be more of a process-related problem than a material chemistry issue. Additional work would be required to resolve this.

\section{Wastage Rate as a Function of Time}

Recall that one goal of this program was to characterize wastage as a function of time for each of the candidate materials. To facilitate this, the three test sections were built to be identical in terms of the placement of the candidate alloy tube samples. Then, they were installed side-byside within Niles Unit \#1, on the assumption that they would experience essentially the same exposure conditions. The tube samples in Test Section A were removed and examined after 15.5 months of exposure at full temperature. For Section C, samples were removed after 18.6, 21.6 and 28.5 months at full temperature. As was explained earlier, excessive corrosion caused the need for early removal of samples from Section C. 28.5 months is considered to be the full exposure period. 
Table 9 presents the corrosion rate data for both Section A and Section $\mathrm{C}$ together. It should be noted from this that the wastage rates at higher temperatures (above about $1150 \mathrm{~F}$ ) remained nearly constant of increased as a function of time. At lower temperatures, rates stayed the same or decreased with time. This decrease in rate with time suggests the possibility that built-up scale is providing some level of protection against further wastage.

The chart in Figure 21 was constructed by plotting the maximum wall thickness lost as a function of time for the materials from Section A and Section C. Then a straight-line was fit to the two data points for each material. As was indicated above, this is not to suggest that the corrosion rate was linear for these materials. Inspection of this chart, and given the fact that most of these materials started with an initial 400 mil wall thickness, the reason for the early removal of some of these materials becomes readily apparent.

\section{$\underline{\text { Rationale for Relative Alloy Performance: }}$}

While this has not been considered in depth, the analysis of the data for Section A provided some insight as to why one candidate alloy performed better than another. The Section A analysis provided further confirmation of the initial assumption that high chromium content would result in good performance under coal ash corrosion conditions. Section $\mathrm{C}$ data now also are available, and Figure 22 shows the maximum corrosion rate plotted against chromium content for both test sections. Logarithmic fit lines are plotted for both sets of data. Examination of these suggests that the materials behaved similarly in both test sections. While a relatively good fit is indicated, the data spread at lower chromium contents appears to be excessive.

Figure 23 plots the maximum corrosion rate against the sum of the chromium plus nickel contents. In this case, the linear fit lines almost completely overlap, and the data spread appears to be more reasonable, particularly at the lower chromium plus nickel contents.

This leads once again to the observation that the wastage response of these materials appeared to be inversely related to the total alloy content in these alloys, in particular, the sum of the chromium plus nickel contents. While it is granted that the sampling in question is not statistically significant, the data suggest that the correlation between chromium plus nickel content and wastage rate merits additional study.

\section{$\underline{\text { Scale/Metal Interface Issues: }}$}

Both the internal and external scale/metal interfaces were examined in detail for each candidate material. The focus typically centered on the one sample for each candidate material that experienced the greatest amount of wastage. The results of these analyses are recorded in the separate appendices for each candidate material.

Examination of the microstructure revealed that the less corrosion resistant materials seemed to have characteristics in common at the external scale/metal interface. These are illustrated in the sketch shown in Figure 24. Figure 25 shows an example of the five regions typically found for corrosion sensitive materials. These would include a high-chromium scale adhering to the tube surface, and a distinct chromium-depleted diffusion zone in the metal below the scale.

It should be noted that in some cases the appearance of the scale/metal interface was somewhat more complicated. Figure 26 shows the appearance of the interface at the external surface of an 
IN52 Weld Overlay sample. Here, once again, an adherent high-chromium scale is found along the interface (Region 1 in Figure 26). However, below the interface is a fairly homogeneous band of material that is somewhat depleted in chromium (Region 2), and beyond that, one can see evidence of internal corrosion penetration into and along interdendritic regions. The significance of this is beyond the scope of this effort and has not fully been explored. However, it should be noted that the IN72 Weld Overlay, also developed similar interface characteristics.

For all of the candidate alloys, examination of the internal scale/metal interfaces revealed minimal conclusive evidence of scale or corrosion penetration. In most cases, the internal scale thickness and corrosion penetration were non-existent or less than 1 mil. This may have been due to some minimal exfoliation after these scales had formed. Figure 27 shows typical examples of the appearance of these interfaces. 


\begin{tabular}{|c|c|c|c|}
\hline Material & Tube Sample & $\begin{array}{c}\text { Rate Metal Lost* } \\
\text { (mils/yr) }\end{array}$ & $\begin{array}{c}\text { Average Surface Meta } \\
\text { Temperature, (F) }\end{array}$ \\
\hline 52 Weld Overlay & R-S & 48 & 1208 \\
\hline 72 Weld Overlay & Q-R & 6 & 1208 \\
\hline Incoclad 671 & $\mathrm{P}-\mathrm{Q}$ & 2 & 1203 \\
\hline HR 120 & $\mathrm{O}-\mathrm{P}$ & 124 & 1194 \\
\hline $310 \mathrm{HCbN}$ & $\mathrm{N}-\mathrm{O}$ & 133 & 1190 \\
\hline $310 \mathrm{Ta}$ & M-N & 131 & 1188 \\
\hline $800 \mathrm{Mod}$ & L-M & 183 & 1185 \\
\hline Save 25 & K-L & $>259$ & 1180 \\
\hline NF 709 & $\mathrm{~J}-\mathrm{K}$ & 181 & 1178 \\
\hline $347 \mathrm{HFG}$ & I-J & 204 & 1174 \\
\hline Thermie & H-I & 77 & 1171 \\
\hline $310 \mathrm{HCbN}$ & G-H & 141 & 1167 \\
\hline $310 \mathrm{Ta}$ & $\mathrm{F}-\mathrm{G}$ & 127 & 1166 \\
\hline 800 Mod & E-F & 176 & 1162 \\
\hline Save 25 & D-E & $\mathrm{N} / \mathrm{A} * *$ & 1158 \\
\hline NF 709 & C-D & 114 & 1153 \\
\hline Fe3Al & B-C & 100 & 1149 \\
\hline $347 \mathrm{HFG}$ & A-B & 76 & 1147 \\
\hline Incoclad 671 & $\mathrm{U}-\mathrm{V}$ & 1 & 1121 \\
\hline HR 120 & V-W & 46 & 1110 \\
\hline $310 \mathrm{HCbN}$ & $\mathrm{W}-\mathrm{X}$ & 52 & 1106 \\
\hline $310 \mathrm{Ta}$ & $\mathrm{X}-\mathrm{Y}$ & 64 & 1103 \\
\hline $800 \mathrm{Mod}$ & $\mathrm{Y}-\mathrm{Z}$ & 79 & 1099 \\
\hline Save 25 & Z-AA & 148 & 1096 \\
\hline $310 \mathrm{HCbN}$ & AA-BB & 79 & 1092 \\
\hline NF 709 & $\mathrm{BB}-\mathrm{CC}$ & 100 & 1088 \\
\hline $347 \mathrm{HFG}$ & CC-DD & 120 & 1086 \\
\hline HR 120 & DD-EE & 79 & 1088 \\
\hline Incoclad 671 & EE-FF & 10 & 1086 \\
\hline 72 Weld Overlay & FF-GG & $14 * * *$ & 1079 \\
\hline 52 Weld Overlay & GG-HH & 63 & 1073 \\
\hline Thermie & HH-II & 34 & 1067 \\
\hline Save 25 & II-JJ & 56 & 1058 \\
\hline
\end{tabular}

Table 6: Maximum Wastage Rate as a Function of Position in Test Section C

* $\quad$ Metal loss rate is based on time at temperature, not total time within the boiler.

** $\quad \mathrm{N} / \mathrm{A}=$ Sample overlain with weld metal, thus no data available.

*** The calculated rate is based solely on wall thickness measurements and does not take into account that corrosion had removed sufficient material to connect an underlying weld crack with the surface.

Key: Sample removed from service November 2001

Sample removed from service June 2002

Sample remained in service until the end of Section C exposure in May 2003 -----

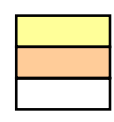




\begin{tabular}{|c|c|c|c|}
\hline Material & Tube Sample & $\begin{array}{l}\text { Maximum Rate } \\
\text { Metal Lost* (mils/yr) }\end{array}$ & $\begin{array}{c}\text { Average Surface Metal } \\
\text { Temperature, (F) }\end{array}$ \\
\hline Save 25 & K-L & $>259$ & 1180 \\
\hline Save 25 & D-E & $\mathrm{N} / \mathrm{A}^{* *}$ & 1158 \\
\hline Save 25 & Z-AA & 148 & 1096 \\
\hline Save 25 & II-JJ & 56 & 1058 \\
\hline $800 \mathrm{Mod}$ & L-M & 183 & 1185 \\
\hline 800 Mod & E-F & 176 & 1162 \\
\hline 800 Mod & $\mathrm{Y}-\mathrm{Z}$ & 79 & 1099 \\
\hline 347 HFG & I-J & 204 & 1174 \\
\hline 347 HFG & CC-DD & 120 & 1086 \\
\hline 347 HFG & A-B & 76 & 1147 \\
\hline NF 709 & $\mathrm{~J}-\mathrm{K}$ & 181 & 1178 \\
\hline NF 709 & C-D & 114 & 1153 \\
\hline NF 709 & $\mathrm{BB}-\mathrm{CC}$ & 100 & 1088 \\
\hline Fe3Al & $\mathrm{B}-\mathrm{C}$ & 100 & 1149 \\
\hline 310 Ta & M-N & 131 & 1188 \\
\hline 310 Ta & F-G & 127 & 1166 \\
\hline $310 \mathrm{Ta}$ & $\mathrm{X}-\mathrm{Y}$ & 64 & 1103 \\
\hline $310 \mathrm{HCbN}$ & G-H & 141 & 1167 \\
\hline $310 \mathrm{HCbN}$ & $\mathrm{N}-\mathrm{O}$ & 133 & 1190 \\
\hline $310 \mathrm{HCbN}$ & AA-BB & 79 & 1092 \\
\hline $310 \mathrm{HCbN}$ & W-X & 52 & 1106 \\
\hline HR 120 & O-P & 124 & 1194 \\
\hline HR 120 & DD-EE & 79 & 1088 \\
\hline HR 120 & $\mathrm{~V}-\mathrm{W}$ & 46 & 1110 \\
\hline Thermie & H-I & 77 & 1171 \\
\hline Thermie & HH-II & 34 & 1067 \\
\hline $52 \mathrm{WO}$ & GG-HH & 63 & 1073 \\
\hline $52 \mathrm{WO}$ & R-S & 48 & 1208 \\
\hline $72 \mathrm{WO}$ & FF-GG & $14 * * *$ & 1079 \\
\hline $72 \mathrm{WO}$ & Q-R & 6 & 1208 \\
\hline 671 Clad & EE-FF & 10 & 1086 \\
\hline 671 Clad & $\mathrm{P}-\mathrm{Q}$ & 4 & 1203 \\
\hline 671 Clad & $\mathrm{U}-\mathrm{V}$ & 1 & 1121 \\
\hline
\end{tabular}

Table 7: Rankings Per Material Based on Rate of Metal Loss in Test Section C

* $\quad$ Metal loss rate is based on time at temperature, not total time within the boiler.

** $\quad \mathrm{N} / \mathrm{A}=$ Sample overlain with weld metal, thus no data available.

*** The calculated rate is based solely on wall thickness measurements and does not take into account that corrosion had removed sufficient material to connect an underlying weld crack with the surface.

Key: $\quad$ Sample removed from service November 2001

Sample removed from service June 2002

Sample remained in service until the end of Section C exposure in May 2003 ----

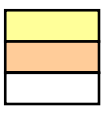




\begin{tabular}{|c|c|c|c|}
\hline Material & Tube Sample & $\begin{array}{c}\text { Surface Metal } \\
\text { Temperature (F) }\end{array}$ & $\begin{array}{c}\text { Section C } \\
\text { Maximum Rate of } \\
\text { Metal Loss (mils/yr) }\end{array}$ \\
\hline Save 25 & K-L & 1180 & $>259$ \\
\hline 347 HFG & I-J & 1174 & 204 \\
\hline 800 Mod & L-M & 1185 & 183 \\
\hline NF 709 & J-K & 1178 & 181 \\
\hline 310 HCbN & G-H & 1167 & 141 \\
\hline 310 Ta & M-N & 1188 & 131 \\
\hline HR 120 & O-P & 1194 & 124 \\
\hline Fe3Al & B-C & 1149 & 77 \\
\hline Thermie & H-I & 1171 & 63 \\
\hline IN 52 WO & GG-HH & 1073 & $14 *$ \\
\hline IN 72 WO & FF-GG & 1079 & 10 \\
\hline 671 Clad & EE-FF & 1086 & \\
\hline
\end{tabular}

* Note: this rate does not take wall loss due to internal cracking in this weld overlay material.

Table 8: Maximum Rate of Metal Loss for Each Candidate Material 


\begin{tabular}{|c|c|c|c|c|c|}
\hline Material & $\begin{array}{c}\text { Tube } \\
\text { Sample }\end{array}$ & $\begin{array}{c}\text { Section A } \\
\text { Rate Metal } \\
\text { Lost* } \\
\text { (mils/yr) }\end{array}$ & $\begin{array}{c}\text { Section A } \\
\text { Exposure } \\
\text { Temperature } \\
\text { (F) }\end{array}$ & $\begin{array}{c}\text { Section C } \\
\text { Rate Metal } \\
\text { Lost* } \\
\text { (mils/yr) }\end{array}$ & $\begin{array}{c}\text { Section C } \\
\text { Exposure } \\
\text { Temperature } \\
\text { (F) } \\
\end{array}$ \\
\hline $\begin{array}{l}52 \text { Weld } \\
\text { Overlay }\end{array}$ & R-S & 44 & 1198 & 48 & 1208 \\
\hline $\begin{array}{l}72 \text { Weld } \\
\text { Overlay }\end{array}$ & Q-R & 10 & 1198 & 6 & 1208 \\
\hline Incoclad 671 & P-Q & 4 & 1193 & 2 & 1203 \\
\hline HR 120 & O-P & 102 & 1184 & 124 & 1194 \\
\hline $310 \mathrm{HCbN}$ & $\mathrm{N}-\mathrm{O}$ & 108 & 1180 & 133 & 1190 \\
\hline $310 \mathrm{Ta}$ & M-N & 123 & 1178 & 131 & 1188 \\
\hline $800 \mathrm{Mod}$ & L-M & 169 & 1174 & 183 & 1185 \\
\hline Save 25 & K-L & 259 & 1170 & $>259$ & 1180 \\
\hline NF 709 & $\mathrm{~J}-\mathrm{K}$ & 170 & 1167 & 181 & 1178 \\
\hline $347 \mathrm{HFG}$ & I-J & 174 & 1164 & 204 & 1174 \\
\hline Thermie & H-I & 53 & 1160 & 77 & 1171 \\
\hline $310 \mathrm{HCbN}$ & G-H & 133 & 1157 & 141 & 1167 \\
\hline $310 \mathrm{Ta}$ & F-G & 136 & 1154 & 127 & 1166 \\
\hline $800 \mathrm{Mod}$ & E-F & 178 & 1150 & 176 & 1162 \\
\hline Save 25 & D-E & 224 & 1146 & $\mathrm{~N} / \mathrm{A}^{* *}$ & 1158 \\
\hline NF 709 & C-D & 136 & 1142 & 114 & 1153 \\
\hline $\mathrm{Fe} 3 \mathrm{Al}$ & B-C & 145 & 1139 & 100 & 1149 \\
\hline 347 HFG & A-B & 83 & 1137 & 76 & 1147 \\
\hline Incoclad 671 & $\mathrm{U}-\mathrm{V}$ & 2 & 1110 & 1 & 1121 \\
\hline HR 120 & V-W & 61 & 1100 & 46 & 1110 \\
\hline $310 \mathrm{HCbN}$ & $\mathrm{W}-\mathrm{X}$ & 98 & 1095 & 52 & 1106 \\
\hline $310 \mathrm{Ta}$ & $X-Y$ & 120 & 1092 & 64 & 1103 \\
\hline $800 \mathrm{Mod}$ & Y-Z & 154 & 1088 & 79 & 1099 \\
\hline Save 25 & Z-AA & 208 & 1084 & 148 & 1096 \\
\hline $310 \mathrm{HCbN}$ & AA-BB & 129 & 1081 & 79 & 1092 \\
\hline NF 709 & BB-CC & 145 & 1077 & 100 & 1088 \\
\hline $347 \mathrm{HFG}$ & CC-DD & 176 & 1075 & 120 & 1086 \\
\hline HR 120 & DD-EE & 109 & 1077 & 79 & 1088 \\
\hline Incoclad 671 & EE-FF & 13 & 1075 & 10 & 1086 \\
\hline $\begin{array}{l}72 \text { Weld } \\
\text { Overlay }\end{array}$ & FF-GG & 21 & 1068 & $14 * * *$ & 1079 \\
\hline $\begin{array}{l}52 \text { Weld } \\
\text { Overlay }\end{array}$ & GG-HH & 77 & 1062 & 63 & 1073 \\
\hline Thermie & HH-II & 31 & 1056 & 34 & 1067 \\
\hline Save 25 & II-JJ & 67 & 1047 & 56 & 1058 \\
\hline
\end{tabular}

Table 9: Maximum Wastage Rate as a Function of Time and Position

Note: Those test segments highlighted in blue experienced an increase in wastage rate with time.

\footnotetext{
* Metal loss rate is based on time at temperature (up to 11,288 hours for Section A and up to 20,832 hours for Section C, not total time within the boiler.

** $\quad$ N/A = Sample overlain with weld metal, thus no data available

*** The calculated rate is based solely on wall thickness measurements and does not take into account that corrosion had removed sufficient material to connect an underlying weld crack with the surface.
} 


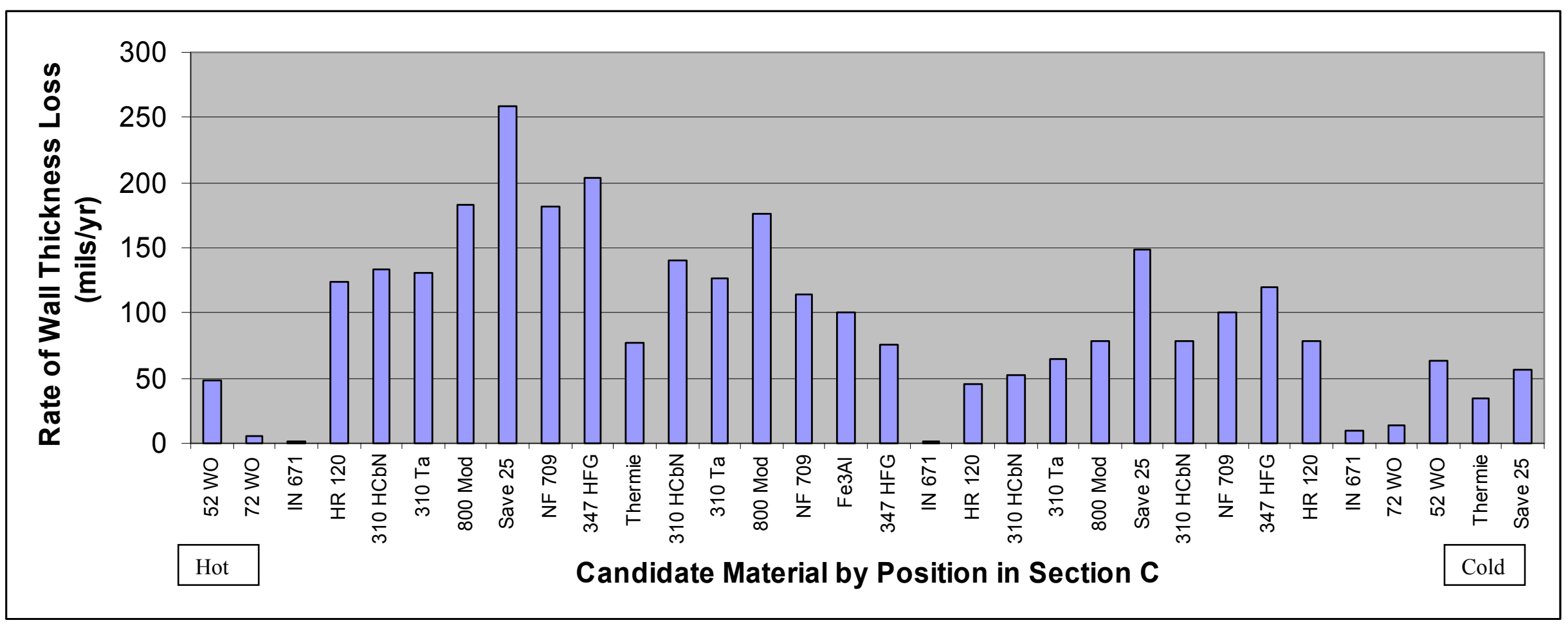

Figure 14: Maximum Wastage Rate as a Function of Position within Section C

Note:

1. The materials are listed in sequence according to their position within the test section, with the hottest end of the test section to the left, and the coolest end of the test section to the right.

2. No data provided for Save 25 tube segment at position D-E because this segment had been overlain with weld metal 


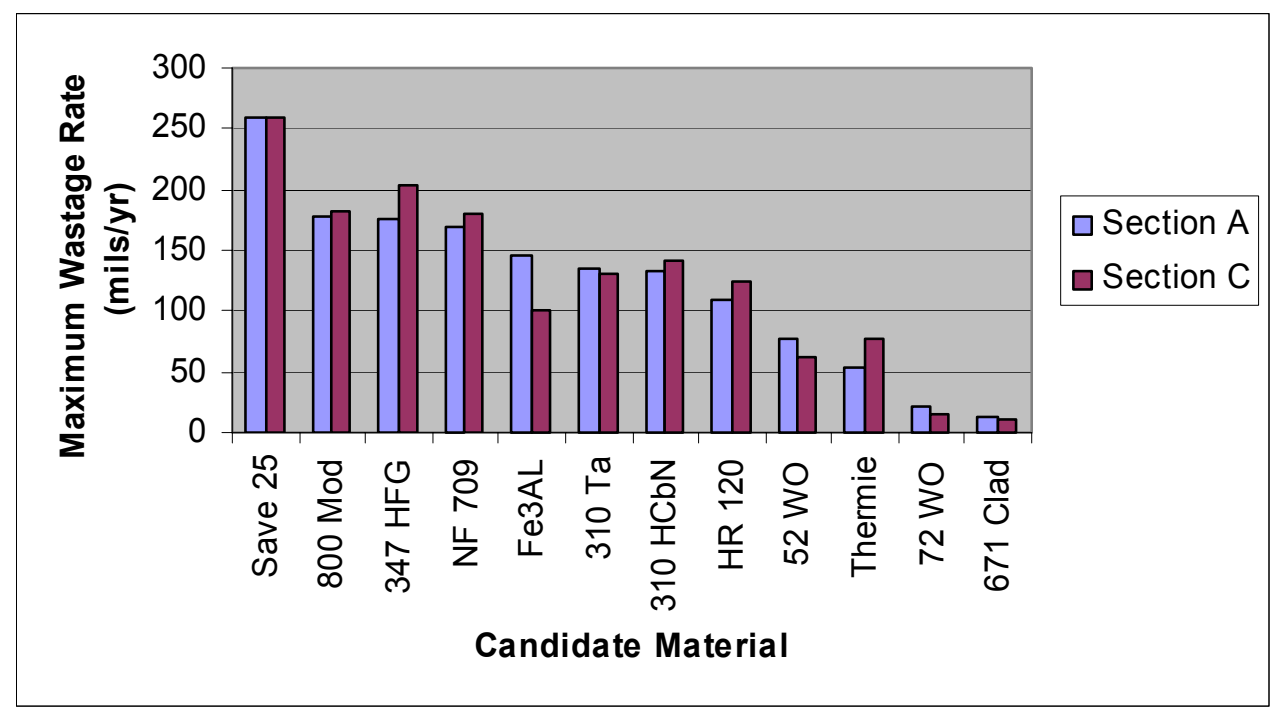

Figure 15: Maximum Wastage Rate for Each Candidate Material in Both Sections 


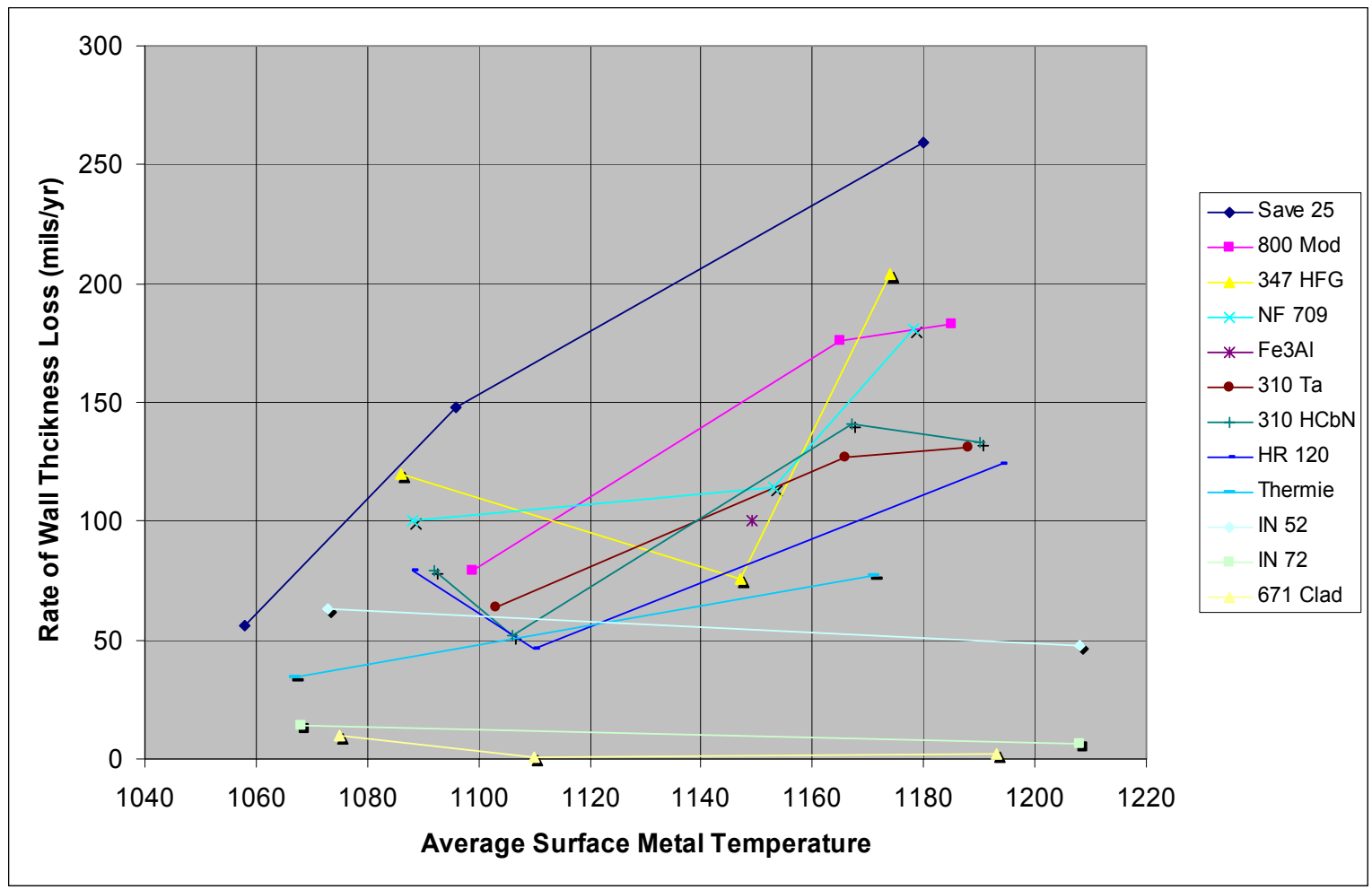

Figure 16: Wastage as a Function of Average Surface Metal Temperature for the Twelve Candidate Alloys in Test Section C 
Metal Loss vs Average Temperature

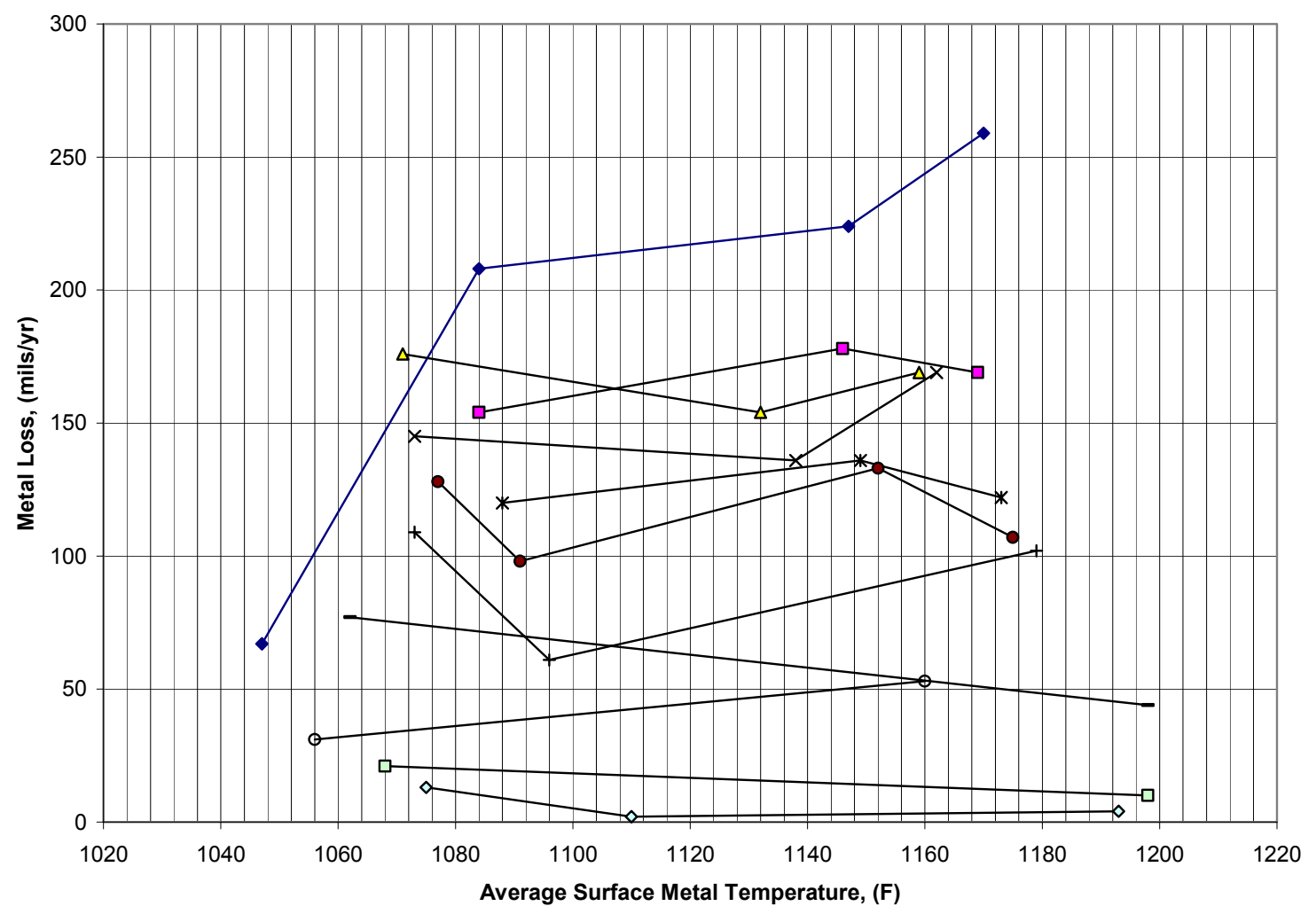

Figure 17: Wastage as a Function of Average Surface Metal Temperature for the Twelve Candidate Alloys in Test Section A 


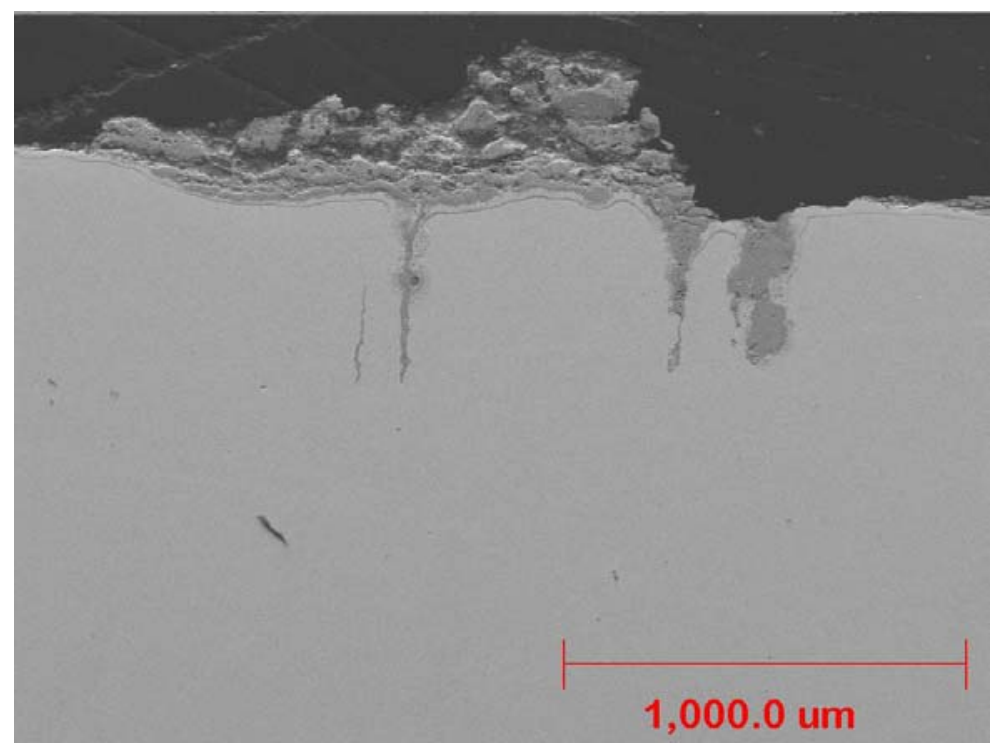

Figure 18: Weld Cracks Intersecting External Corrosion for 72 WO Test Segment

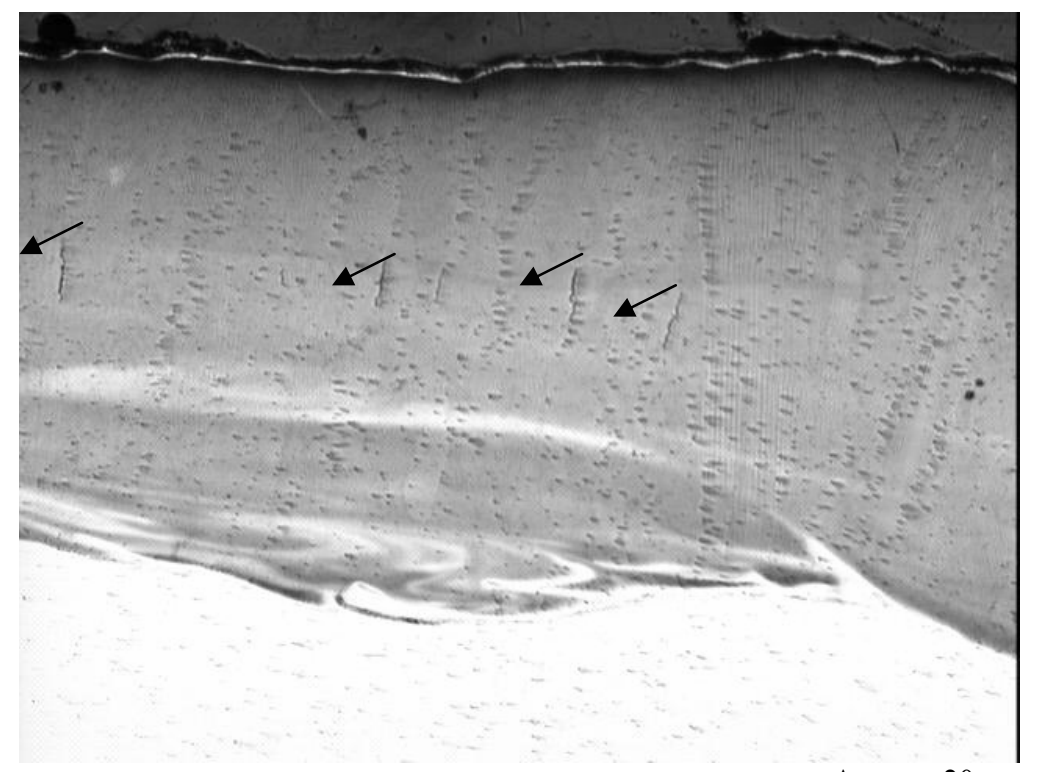

Approx. 20x

Figure 19: Internal Cracking in 72 Weld Overlay Test Segment from Section C 


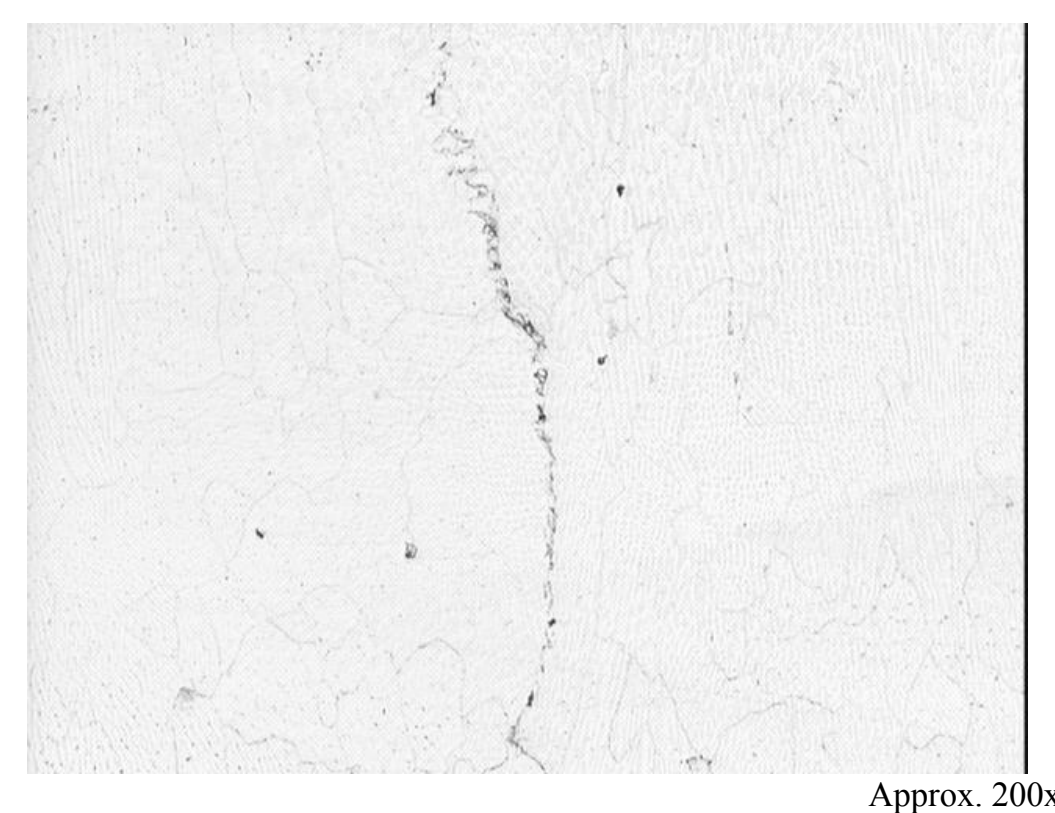

Figure 20: Internal Cracking in 72 Weld Overlay Archive Material

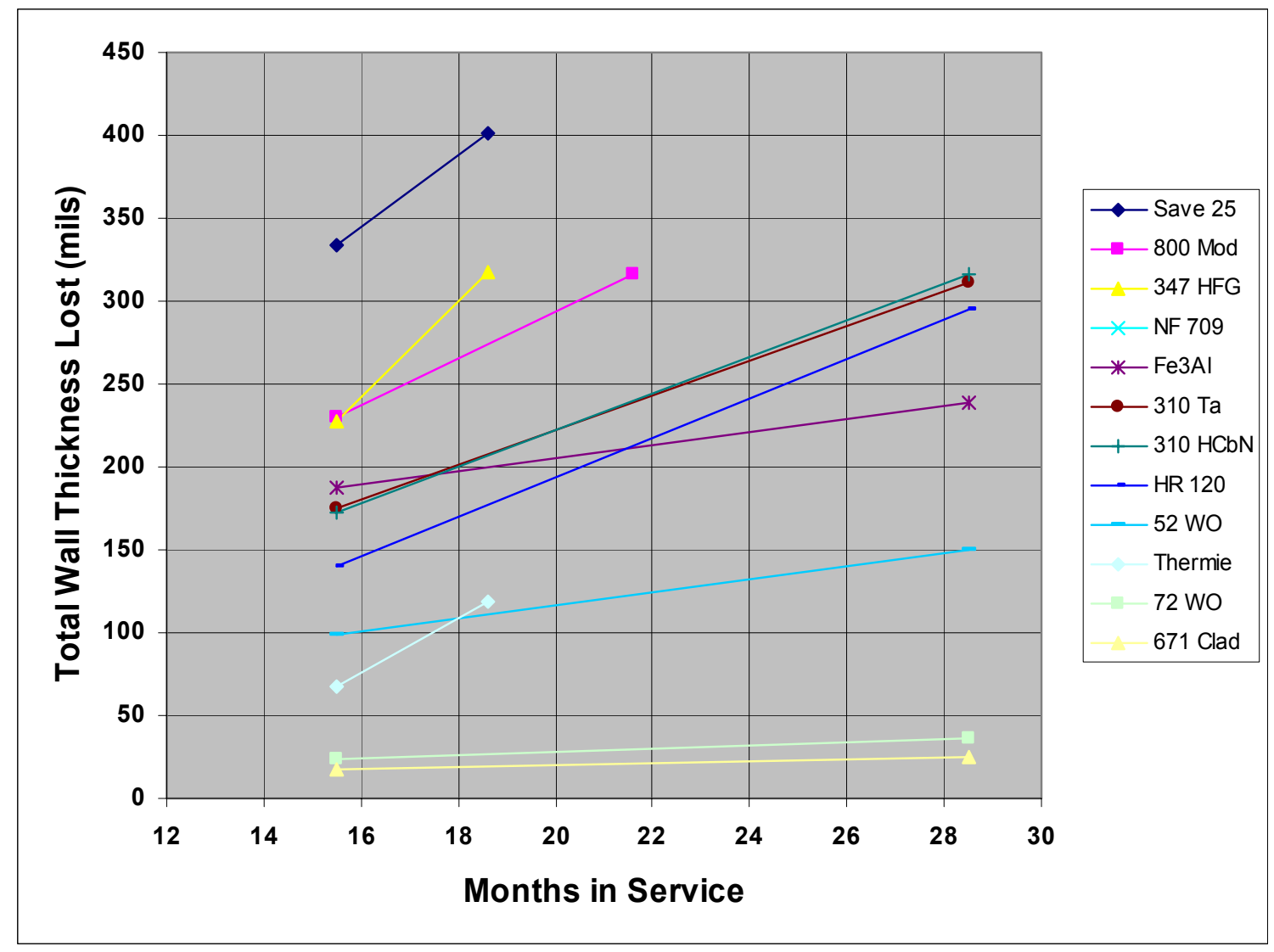

Figure 21: Maximum Wastage as a Function of Time for the Candidate Materials 


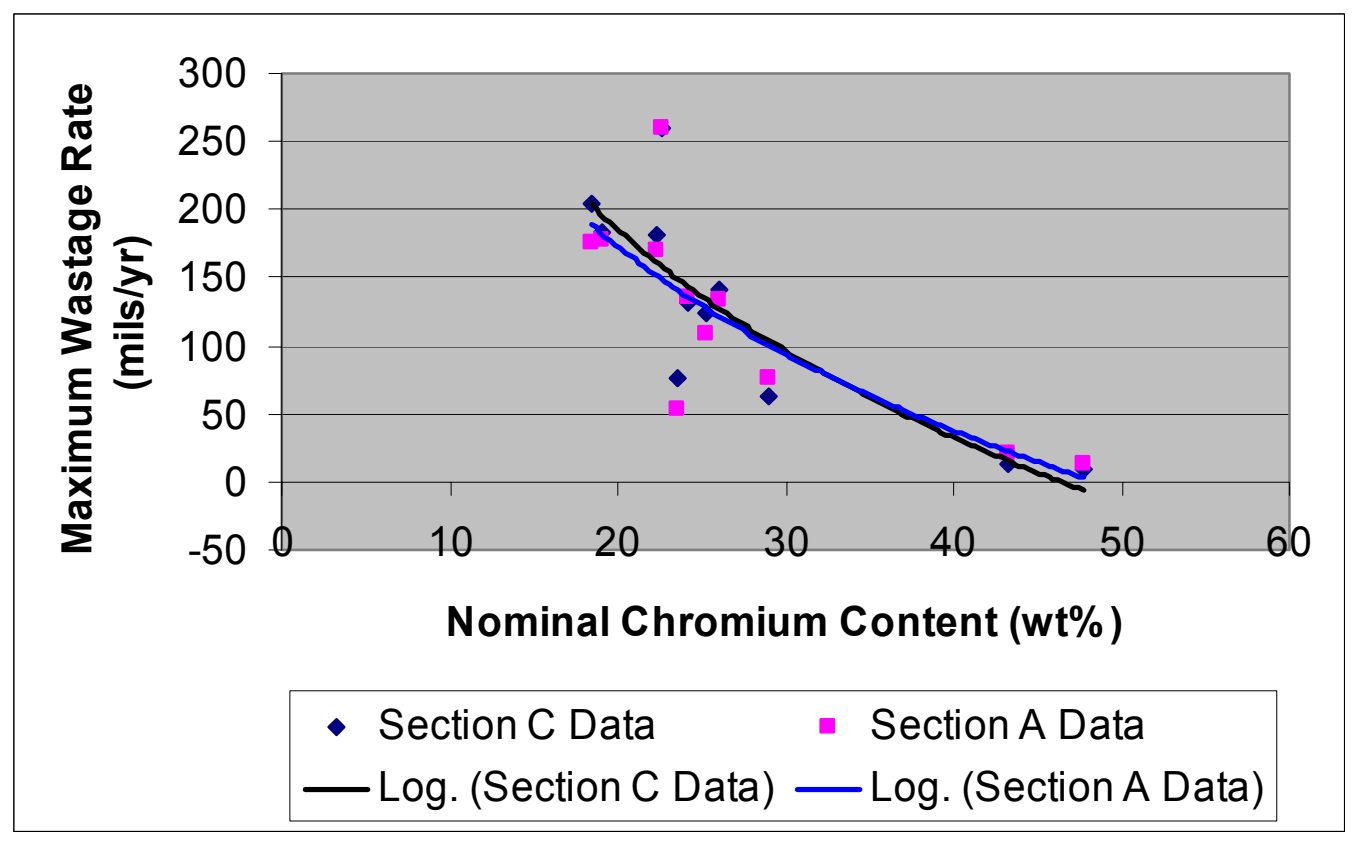

Figure 22: Chromium Content Versus Wastage Rate for Both Sections A and C With Logarithmic Trend Line

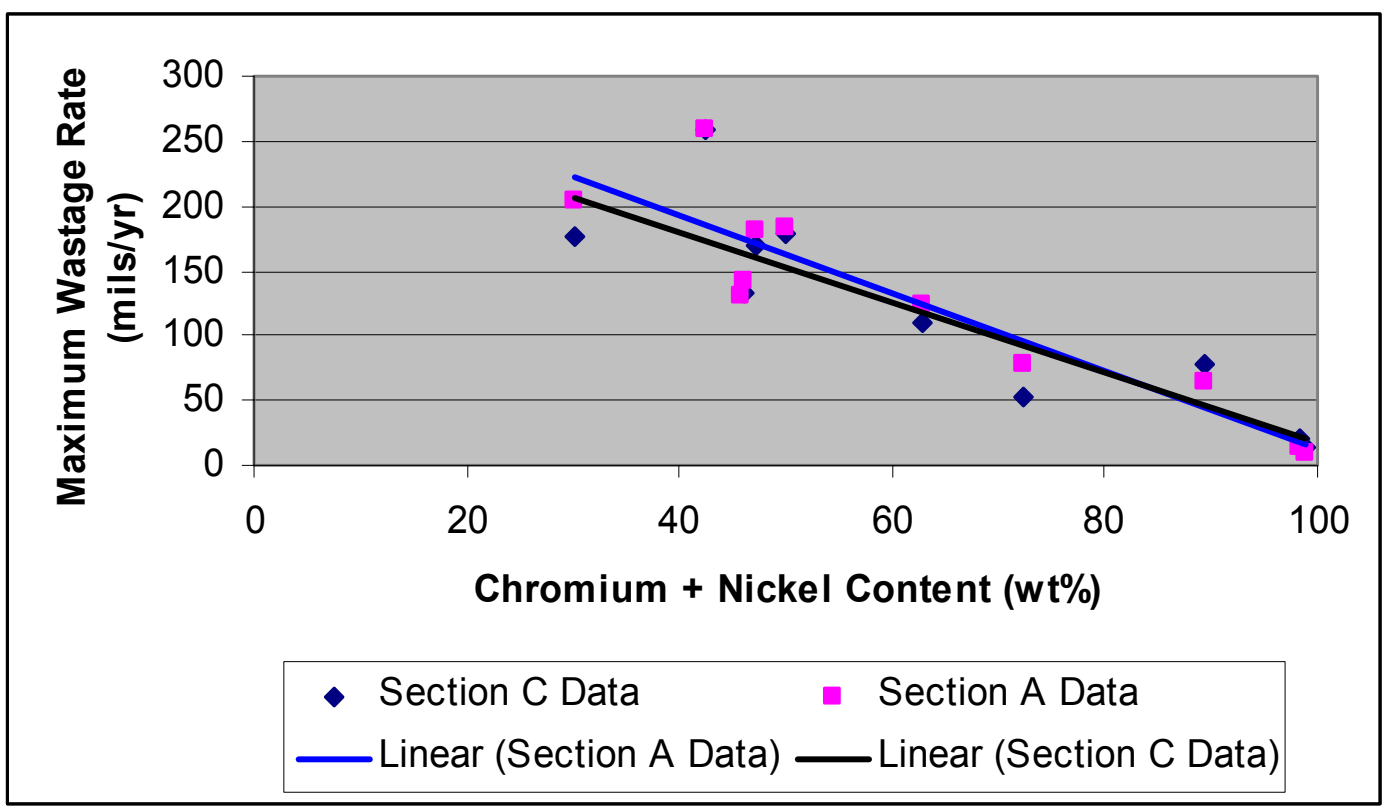

Figure 23: Chromium Plus Nickel Content Versus Wastage Rate With Linear Regression Fit trend Line 


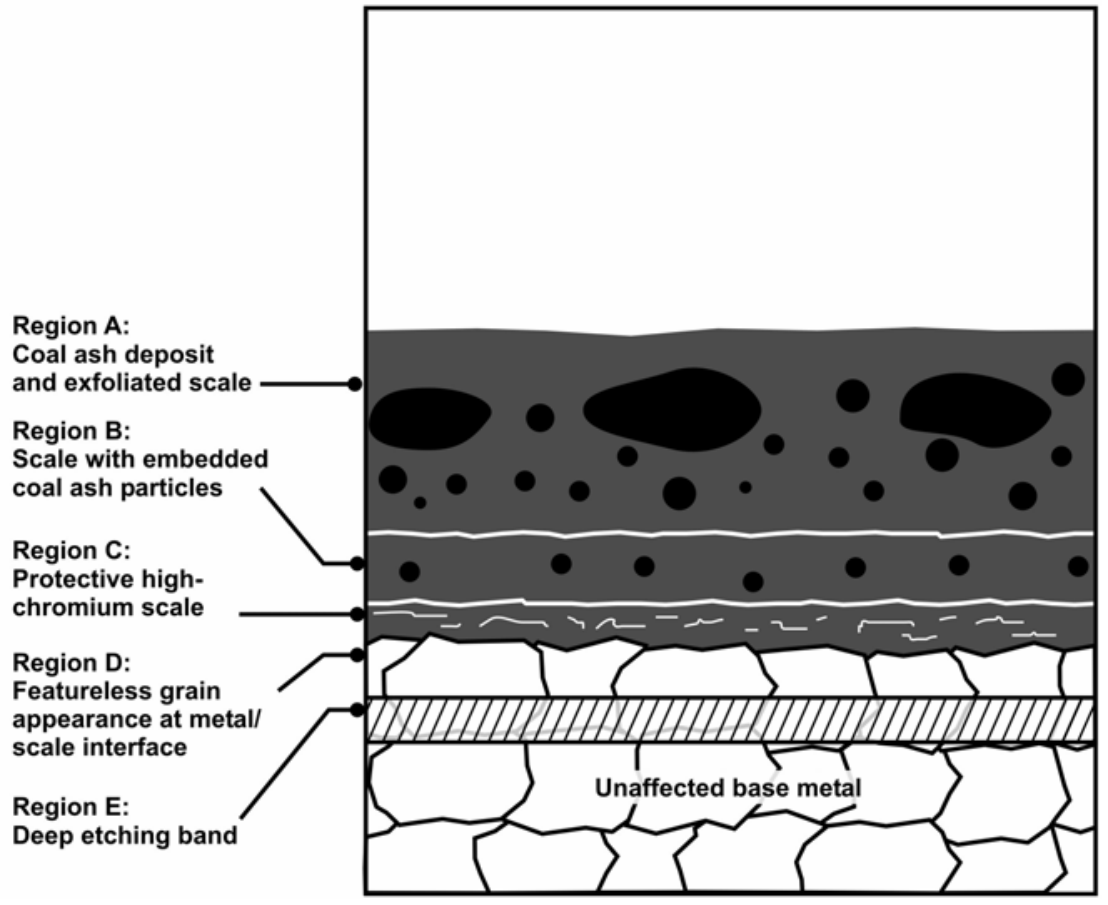

Figure 24: Common Characteristics at External Scale/Metal Interface 


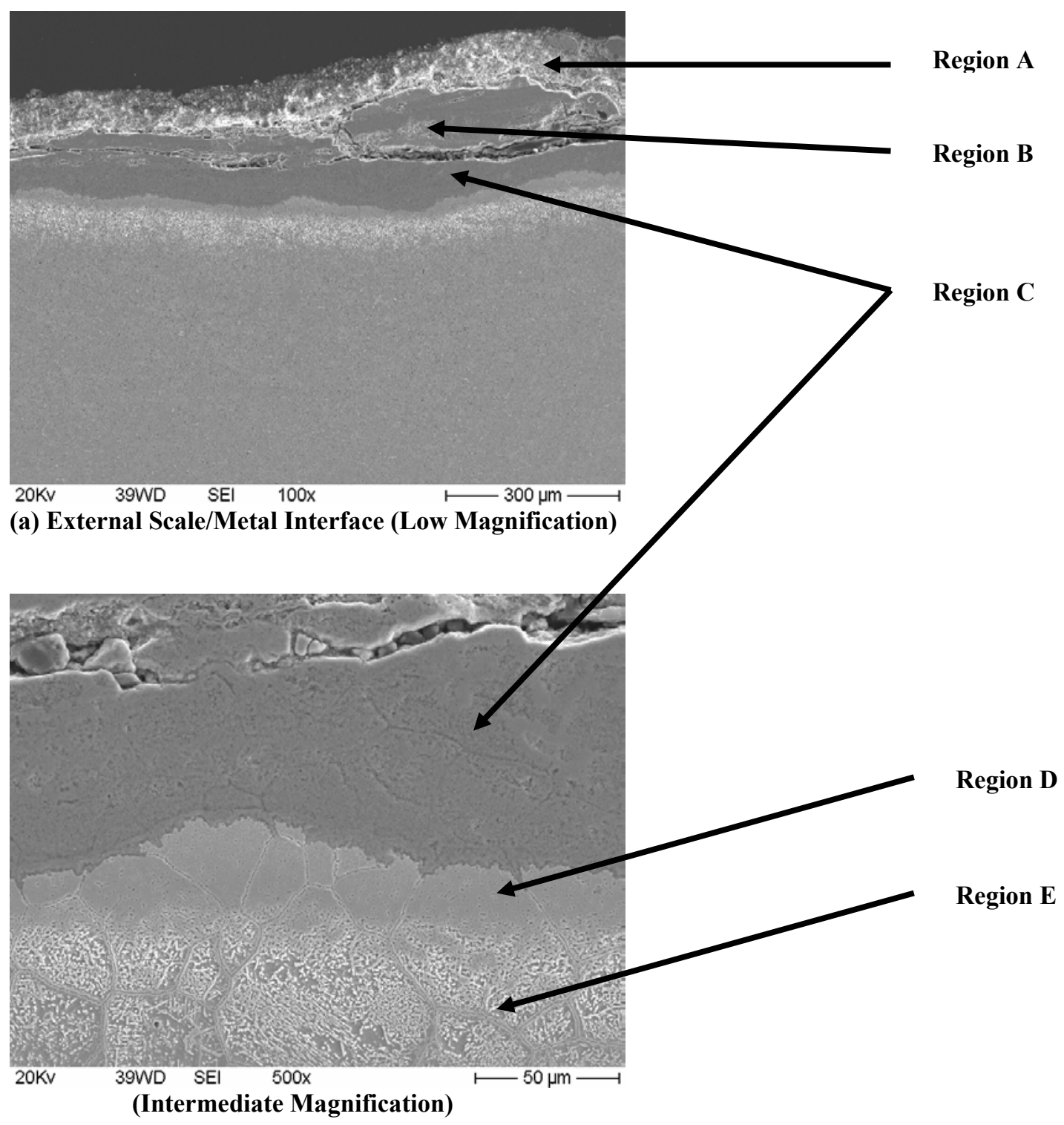

Figure 25: Illustrates the Characteristic Microstructural Regions Found at the External Scale/Metal Interface for Many of the Candidate Materials.

Note: These photographs are of the scale/metal interface for NF 709. 


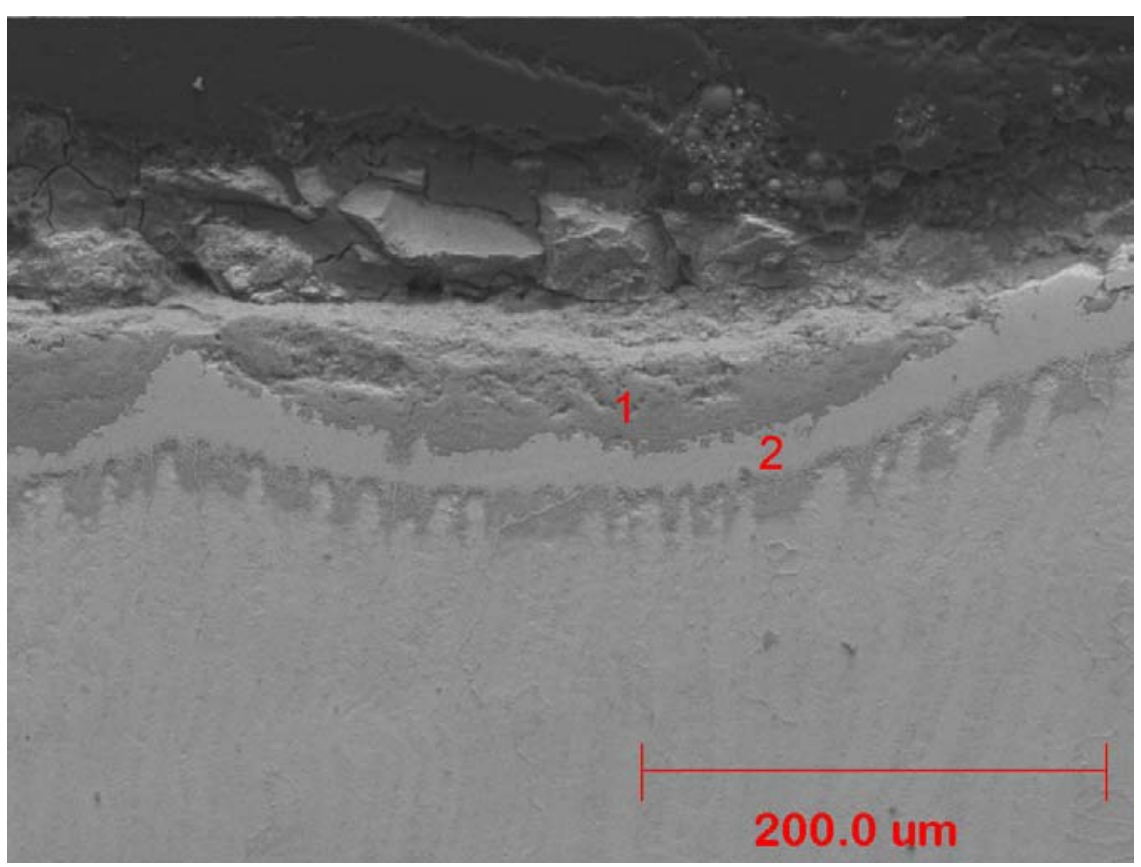

Figure 26: Characteristics of External Scale/Metal Interface for IN52 Weld Overlay 


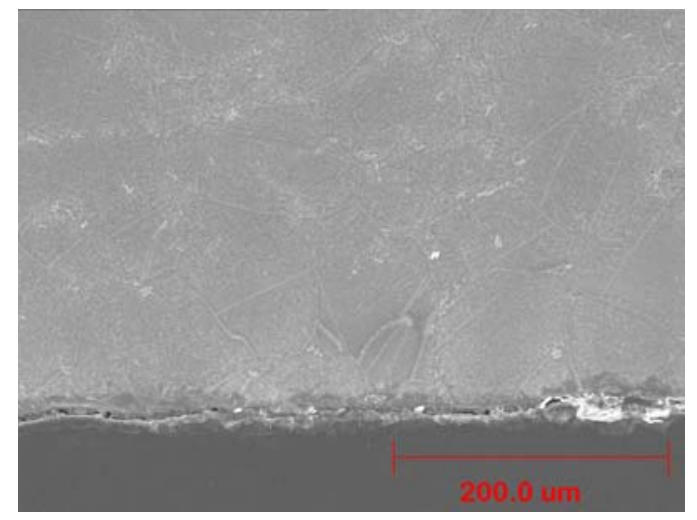

Internal Scale/Metal Interface for Type 310 Ta

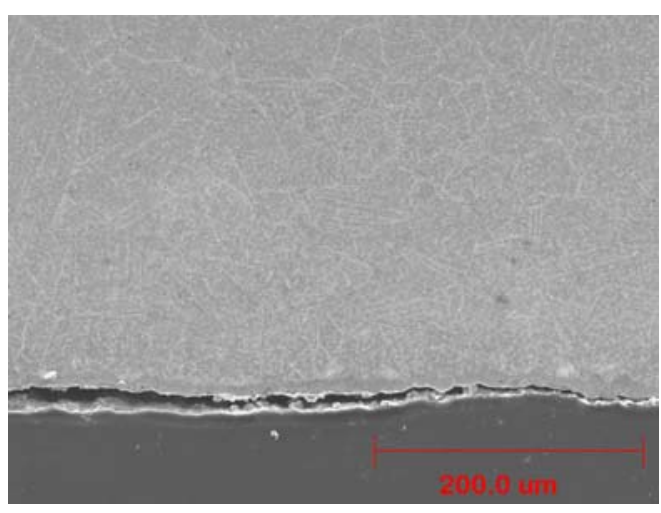

Internal Scale/Metal Interface for HR 120

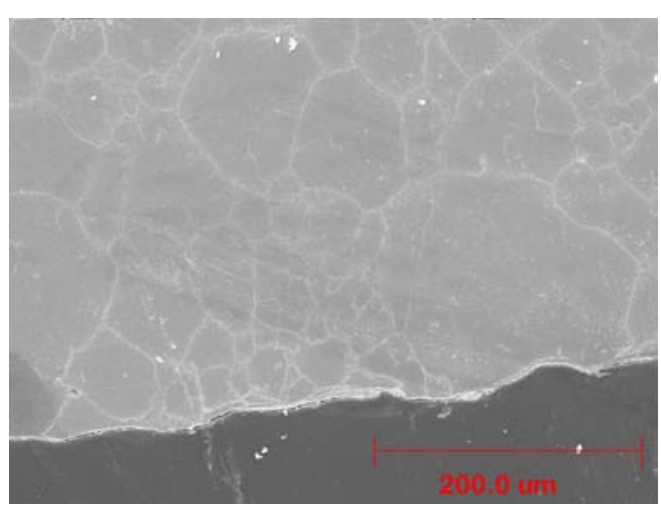

Internal Scale/Metal Interface for Save 25

Figure 27 Typical Examples of the Condition of the Internal Scale/Metal Interface after Service 


\subsection{CONCLUSION}

Evaluation of tubes removed from Section $C$ suggests the observation that the candidate materials performed very similarly in both Sections A and C.

- The relative rankings of the candidate materials (based on wastage rate) are very similar for Section A (with 15.5 months of exposure) and Section C (after 28.5 months of exposure).

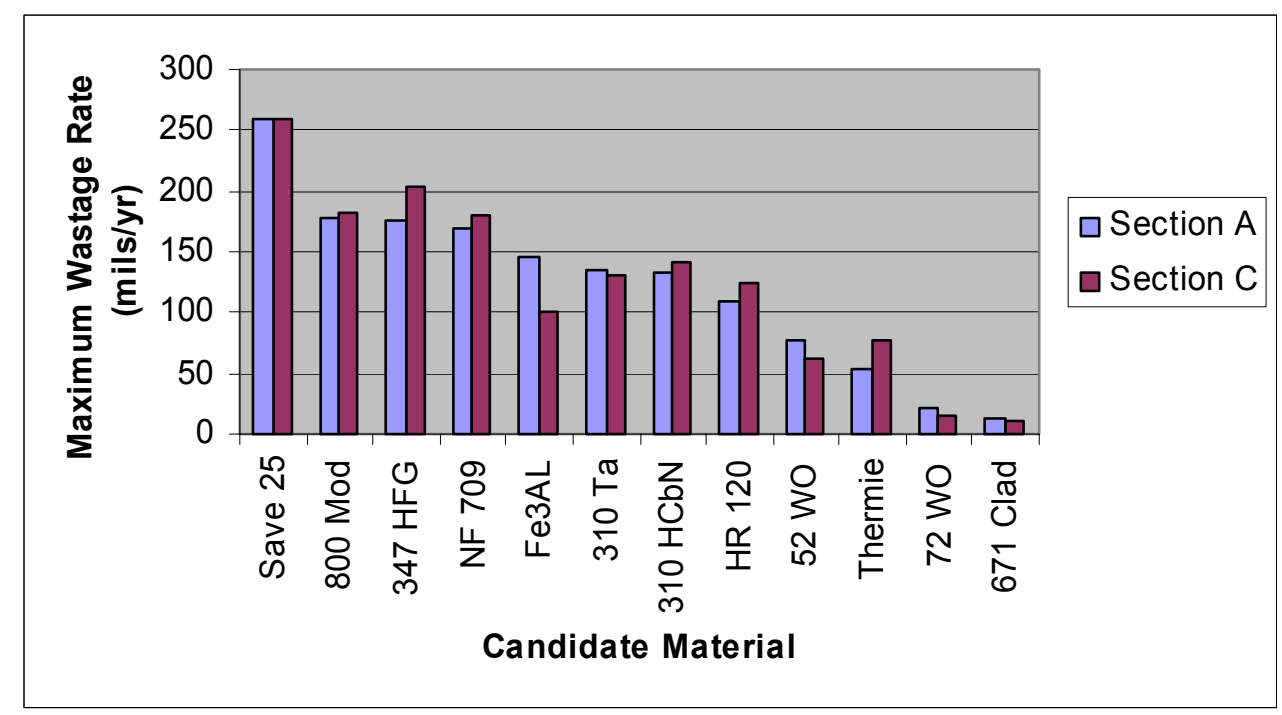

- The same materials showed the best resistance to coal ash corrosion: Incoclad 671, IN72 Weld Overlay. Thermie, and IN52 Weld Overlay.

- The same materials performed poorly: Save 25, Modified 800H, 347 HFG, and NF 709.

- The same materials demonstrated a reduced wastage rate at intermediate temperatures: 347 HFG, NF 709, and HR 120.

Evaluation of the materials which performed well suggests the following:

- Incoclad 671 continued to exhibit the best resistance to coal ash corrosion. This good performance was attributed to its' high levels of chromium and nickel (and low levels of iron).

- IN72 Weld Overlay exhibited good resistance to coal ash corrosion; however, it also showed evidence of an internal cracking problem that may increase in extent with increased time at temperature. The fact that IN72 has a chromium and nickel content similar to that of Incoclad 671 suggests that this problem may be process-related. However, it raises a serious concern in that the cracking is sub-surface and thus may not be visible during in situ visual examination of these tubes.

- It was noted that Incoclad 671 and IN72 Weld overlay both exhibited a decrease in corrosion rate with additional time at temperature. This suggests that a coherent protective scale is formed for these materials, and that this scale slows further wastage. 
- Thermie also exhibited a increased wastage rate as a function of time. The rate for this material was ten-fold greater than that of either the Incoclad 671 or the IN 72 Weld Overlay.

- IN52 Weld Overlay exhibited moderate resistance to coal ash corrosion; however, at this point (i.e. after 28.5 months at temperature in the corrosive environment of this boiler), the full 80 mil thickness of the weld overlay has been breached. With this corrosion barrier removed the wastage rate is expected to increase with continued exposure.

For the purpose of discussion, a line was drawn to define materials as moderate (or poor) performers if they demonstrated a wastage rate in excess of 100 mils/year, for at least one exposure temperature. The following comments apply to these materials:

- With the exception of $347 \mathrm{HFG}$, these materials are all highly alloyed, with a chromium content in the range of 20 to $25 \mathrm{wt} \%$ and a with a nickel content that ranged from as low as 17 to as high as 37 . Type 347 HFG stainless steel is less highly alloyed.

- Preliminary plots of alloy content versus wastage show a good correlation between chromium content and performance, and a better correlation between chromium plus nickel content and performance.

- From this it is not clear why Save 25, with a chromium content of 21-24 wt $\%$, and a total alloy content of between 36 and $46 \mathrm{wt} \%$ would perform more poorly than the less highly alloyed 347 HFG. However, it has been speculated (but not confirmed) that the high copper content of Save 25 may have had an adverse effect on its' ability to develop and/or maintain a protective chromium oxide scale.

Tube-to-tube weld investigations suggest the following:

- In general, the Inconel 625 weld metal (used to join the tubes to one another) performed well, and better than most of the candidate alloys in this investigation. Weld cross sections revealed that most tubes were thicker in the vicinity of these welds and decreased in wall thickness toward the mid-point of the tube segment. For these materials, there was no evidence that either the weld metal or the adjacent heat affected zone suffered from preferential corrosion.

- For the case of the best performing materials, at higher temperatures the Inconel 625 provided less resistance than the candidate material (i.e. Incoclad 671 and IN 72 Weld Overlay). In at least one case, the Inconel 625 weld metal had wasted to the extent that it exposed the material under the cladding to coal ash corrosion conditions, and corrosion was progressing inward at that point. 


\subsection{REFERENCE}

\section{Annex 1 Program Timeline: 2002 - 2003}

On October 31, 2002 a Topical Report providing the full details of the project background, material selection, fabrication of the test materials and sections, description of the test system, and system installation, start-up and operating history through 2001 was provided along with the results of evaluation of Section A removed in November of 2001. That information will not be repeated herein and the reader is referred to "Topical Report: Removal of Section A" for that information. The following provides the operating history of the sections beginning where the Section A report left off through the end of 2003. It is followed by the results of evaluation of the second section, Section C, removed in August 2003.

\section{$\underline{2002}$}

During January Section C ran as intended with $1075^{\circ} \mathrm{F}$ outlet steam temperature while B control valve was kept open due to a failed position indicator and evaluation of the samples from the top two rows of Section A was initiated.

During the annual maintenance outage from February 17 through March 3, a mechanical positioner was installed on Section C. The position indicator on B was tested and worked properly during the outage. Changes were also made to the control logic to accommodate this new equipment. The unusual pressure drop across the outlet stop valves, discovered in November, is believed to be transmitter calibration and will be investigated further when the unit is operating. Evaluation of the samples from the top two rows of Section A continued.

After restart in early March the position indicator on Section B was not working (presumably related to heat) so the inlet valve was opened until the indicator could be replaced. Tuning of the temperature control logic will be necessary because the slower mechanical positioners caused the valves to 'hunt' to maintain the outlet temperature resulting in outlet temperature fluctuations of $\pm 25 \mathrm{~F}$ (maximum temperature of $1100 \mathrm{~F}$ ).

Samples from Section A were prepared and documentation (photographs) of the as-received condition was completed in April. Ring sections were cut for dimensioning and metallographic examination were polished, etched and their condition was documented.. The dimension rings were de-scaled and measured at eight locations. The appearance of the de-scaled surfaces was photographed. The metallographic rings were mounted and ground flat and the chemistry was determined for all 33 tube samples. After some tinkering, the position indicator for Section B began working properly. Arrangements were made for the first week in May to adjust the control logics for all three valves to minimize the outlet temperature fluctuations caused by the more sluggish mechanical positioners. In May they were adjusted and the system ran as intended without incident.

Metallographic specimens through welds, chemical analysis and hardness for each ring specimen, dimensional comparison to as-received measurements and SEM evaluation of deposit and scale from selected samples began in May. Specimen photography, preparation of dimension rings, dimensional measurements, and light metallography were completed. 
Specimen alloy chemistries were verified by spectroscopic analyses. SEM work was initiated for one area on each alloy, a total of 12 specimens. Energy dispersive X-ray analyses were used to document chemical compositions of the alloy, scale near the alloy scale interface, the inner layer of deposit, and the outermost layer of deposit that is still in-place on the tube surface and report writing began. In June SEM work was completed and report writing and data evaluation continued. A heavier sample of deposit from one area of the surface will be sent for analysis at a chemistry laboratory next month. Examination of metallographic sections through selected welds is yet to begin.

The unit was in a forced outage from July 10 to July 18 (not due to the sections). Otherwise, the system ran as intended during July and Topical report writing continued.

The unit experienced two forced outages from August 12 to August 18 and another from August 28 to August 31, neither due to the test sections. Otherwise, the system ran as intended during August. Examination of metallographic sections through selected welds was completed. A draft of the Topical Report for Section A was completed and released for internal review and some minor issues for further examination were identified as a result of analysis report writing.

Internal review of the draft report was completed in September and the missing and incomplete sections were written. A meeting is planned for the first week in October to review and consolidate comments with the objective of releasing the draft Topical Report to DOE and OCDO by the end of October. The sections continued to run at temperature without problems. The intermediate thermocouple on section " $\mathrm{A}$ " (which no longer contains any test materials) was reading abnormally high and inconsistent with adjacent thermocouples. On occasion it caused the control valve to switch to manual-open at low load but it was returned to auto by the operator.

The system ran as intended during October. The Topical Report for Section A was completed, internally reviewed, was reproduced for transmittal in early November. On October 8, the superheater test sections were briefly inspected (visually). All six return bends were dislodged from their support lugs. All three sections were carefully inspected for evidence of steam leakage, and there were no leaks. One alloy segment in Section B and in Section $\mathrm{C}$ had lost about 250 mils (about $60 \%$ ) of the tube wall thickness. This is the 347HFG segment in the next to top leg of the test sections. Two other alloy segments in Sections B and C had lost about 200 mils from the wall thickness. One of these was the hottest HR120 segment adjoining the Incoclad at the hot end of the outlet leg of the sections. The other was the NF709 near the lower temperature end of the top leg. The Topical Report for Section A was released on October 31, 2002 for review and comment.

The system ran as intended during November and December.

\section{$\underline{2003}$}

The system ran as intended January through June with no incidents. A visit was made to the plant on May 27 to inspect the existing tubes while the boiler was down. One tube sample in both B \& $\mathrm{C}$ Sections was found to be severely corroded. Due to the ash on the tubes and the access and lighting conditions during the inspection, some confusion existed as to which alloy was corroded but it was decided that the samples would be replaced at the next opportunity and the corroded 
specimens returned for examination. Meanwhile the control valves were locked open to keep the sections cool and minimize further corrosion until then.

A visit was made to the plant on June 19 to inspect the existing tubes in more detail. Both supports on the baffle wall of all three Sections had moved off the support lugs. The lower test row of Section $\mathrm{C}$ was bowed downward and was touching the adjacent $3^{\text {rd }}$ row (from the top). The upper row of Section $\mathrm{C}$ had significant wastage in two of the samples (310Ta and $310 \mathrm{HCbN}$ ). Most of the unclad samples in Section B showed significant wastage as well. There was concern that the thinning walls of Sections B and C would not last until the fall outage so two bends were weld prepped and plans were made so the plant could remove the top two rows of both Sections, ship them to Alliance, Ohio for evaluation, and install the bends to connect the top two rows. Once the top two rows were removed the valves would be left open (run cool) until the fall outage when the two new Sections will be installed for the USC Materials Project (DE-FG26-01NT41175 and OCDO D-00-20). If Section B could be sufficiently 'repaired' by replacing the corroded samples with new ones to be confident it could operate for another 1.5 to 2 years it would be reinstalled.

The system ran through July with the control valves locked open to keep the sections cool and minimize further corrosion. As planned, the top two rows of both of the remaining sections $(\mathrm{B} \& \mathrm{C})$ were removed in August during a brief unscheduled outage and replaced with the bends sent in early July. Both Sections were transported to the B\&W Research Center (BWRC) and metal loss was preliminarily assessed using calipers. Based on those measurements the remaining life of each specimen was considered in regard to operating an additional 18 months. Since the Section taken from the $\mathrm{C}$ position (farthest from the boiler sidewall) was badly distorted but the one from the $\mathrm{B}$ (middle) location is in good condition, it was decided to replace the specimens in Section B whose remaining life would not be expected to achieve 18 more months and reinstall it in the boiler in the $\mathrm{C}$ location during the fall outage to obtain additional operating time.

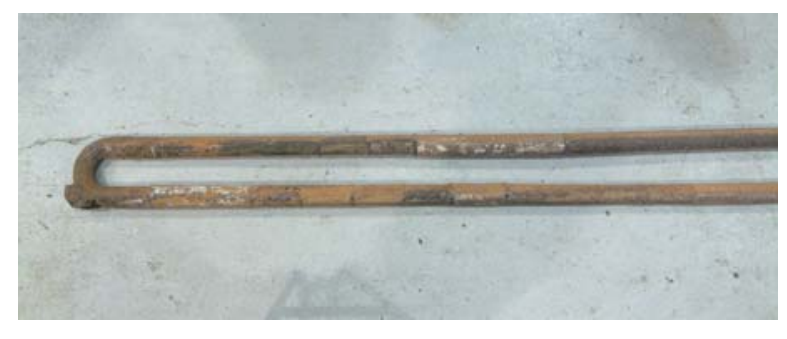

Section B - As Removed

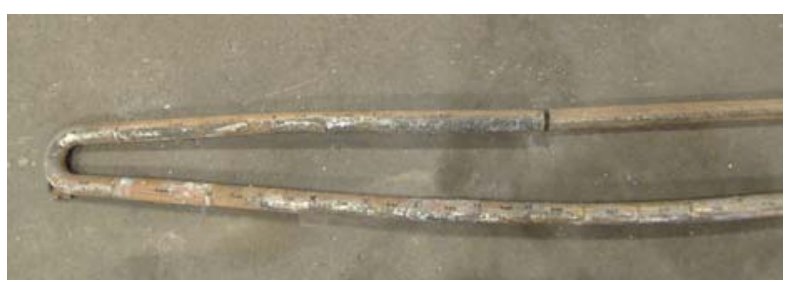

Section C - As Removed

The Niles unit came down for its fall outage on September $24^{\text {th }}$. Plans for evaluation of the specimens in Section $\mathrm{C}$ were initiated and the unacceptable specimens in Section B were removed and the remaining portions weld prepped. After further review of the corrosion, one additional sample was marked for removal leaving (15) of the original (33) specimens for further exposure. Segments of left over IncoClad and some HR3C material were used to replace the removed specimens. These were installed in the two-row portion of Section B in early October and it was returned to the plant for reinstallation in location $\mathrm{C}$ during the outage.

In November the repaired Section B was re-installed in the C location. Section C segments were disassembled and measurements and sample preparations made at BWRC. All (33) thirty-three 
of the tube samples from Section $\mathrm{C}$ were sectioned to yield dimension rings, and specimens for metallography and composition verification including the specimens that were part of the original installation, and those that were added later to replace samples that had evidenced excessive wall losses. The dimension rings were lightly grit-blasted, thickness was measured at eight different o'clock positions, and the data was compared with the original wall thickness at these locations to determine the amount metal lost. In Section A, it was noted in some cases that the weld heat affected zones may have been more aggressively attached than the remainder of the tube sample. To evaluate this, weld cross sections have been prepared to evaluate the weld heat affected zone for each of the candidate materials.

In December, the insulation damaged when accessing some instrumentation was repaired and the unit restarted on December 22 and controls verification planned for early January 2004 after which Section B (in the C location) will be operated at full temperature. Full cross section rings from Section $\mathrm{C}$ were polished and are prepared for evaluation using the scanning electron microscope at the First Energy Beta Labs. First Energy Beta Labs have been contracted to perform an analysis of the deposit for comparison with the analysis for Section A. It is also planned to use the energy dispersive (semi-quantitative) analysis capabilities of the scanning electron microscope at First Energy Beta Labs to verify that the correct alloys were in their proper locations.

Note that removal of the last Section is currently shown as October (fall) of 2004. The actual removal date will depend on the outage dates set by Reliant Energy and may extend to the spring of 2005 which will delay the Report from the current schedule. It was also discovered after restart (in January 2004) that the section outlet thermocouples (which are used for control of the outlet steam temperature) were not properly installed. Actual operation at desired temperatures must be delayed until they can be repaired. Once repaired and the system is operating properly, the operating time and schedule will be reevaluated. 


\section{Acronyms and Abbreviations}

$\begin{array}{ll}\text { Approx. } & \text { Approximately } \\ \text { ANSI } & \text { American National Standards Institute } \\ \text { Avg } & \text { Average } \\ \text { AWS } & \text { American Welding Society } \\ \text { BEI } & \text { Backscattered Electron Image } \\ \text { B\&W } & \text { The Babcock \& Wilcox Company } \\ \text { BWRC } & \text { Babcock \& Wilcox Research Center } \\ \text { Cont. } & \text { Continued } \\ \text { DOE } & \text { Department of Energy } \\ \text { EDAX } & \text { Energy Dispersive Analysis by X-Ray } \\ \text { EDS } & \text { Energy Dispersive X-Ray Spectrometry } \\ \text { ICP } & \text { Inductively Coupled Plasma } \\ \text { ID } & \text { Inside Diameter } \\ \text { IN } & \text { Inconel } \\ \text { Max } & \text { Maximum } \\ \text { Mod } & \text { Modified } \\ \text { N/A } & \text { Not analyzed } \\ \text { NSC } & \text { Nippon Steel Corporation } \\ \text { OCDO } & \text { Ohio Coal Development Office } \\ \text { OCP } & \text { Open Circuit Potential } \\ \text { OD } & \text { Outside Diameter } \\ \text { OES } & \text { Optical Emission Spectrometry } \\ \text { ORNL } & \text { Oak Ridge National Laboratory } \\ \text { PNNL } & \text { Pacific Northwest National Laboratory } \\ \text { SEI } & \text { Secondary Electron Image } \\ \text { SEM } & \text { Scanning Electron Microscope } \\ \text { SMC } & \text { Special Metals Company } \\ \text { SMI } & \text { Sumitomo Metal Industries } \\ \text { TP } & \text { Type (a stainless steel designator) } \\ \text { WO } & \text { Weld Overlay } \\ & \end{array}$




\title{
COAL ASH CORROSION RESISTANT MATERIALS TESTING
}

\section{Topical Report: Removal of Section "C"}

\author{
APPENDICES
}

U.S. Department of Energy Contract:

DE-FC26-99FT40525

Ohio Coal Development Office Grant Agreement:

$\mathrm{CDO} / \mathrm{D}-97-02$

The Babcock \& Wilcox Company Contract:

CRD 1392 


\section{INDEX TO APPENDICES}

\begin{tabular}{cl} 
Appendix & \multicolumn{1}{c}{ Material } \\
A & Save 25 \\
B & Modified 800H \\
C & 347 HFG \\
D & NF 709 \\
E & Fe 3 Al \\
F & 310 Ta \\
G & 310 HCbN (HR3C) \\
H & HR 120 \\
I & Thermie \\
J & IN52 Weld Overlay \\
K & Incoclad 671 \\
L & IN72 Weld Overlay
\end{tabular}




\section{Appendix A}

\section{Analysis of Performance}

\section{Test Section C}

Save 25 


\section{Overview of Results: Save 25}

The metal loss rate for the Save 25 samples increased with average surface metal temperature over the range of exposure for Section C, i.e. from $1058 \mathrm{~F}$ to $1180 \mathrm{~F}$. The rate of metal loss at the highest temperature, in excess of 259 mils per year*, was adequate to perforate the tube wall within 18.6 months of exposure. This was the highest rate of metal loss for any of the candidate alloys. Save 25 continued to demonstrate poor resistance to corrosion down to an average surface metal temperature of about 1096 F. Sample II-JJ, which saw exposure at 1058 F, experienced a moderate rate of metal loss of 58 mils per year. More than half of the wall thickness remained at the end of the Section C exposure period for this sample.

As was stated in the Section A report, typically, Save 25 would be expected to perform similar to the candidate alloys that have a similar level of chromium, i.e. approximately 20 to $25 \% \%$.

Candidate alloys having this chromium level include 310 Ta and $310 \mathrm{HCbN}$ (HR3C). These Type 310 stainless steel based alloys performed near in the middle of the pack relative to the other candidate alloys. The most significant difference between Save 25 and the Type 310-based alloys appears to be that Save 25 includes the addition of a significant amount of copper (up to 4\%). It has been speculated that copper may act to either inhibit formation of the protective chromium oxide scale, or it may facilitate de-cohesion between the scale and the underlying metal. Neither of these hypotheses has been proven.

Analysis of deposit on the surface of tube sample II-JJ revealed that the deposit had the known aggressive combination of species, namely iron, oxygen, the alkali metals, and sulfur. The presence of significant amounts of arsenic was unexpected, and its' significance in unclear. Examination and analysis of the scale adjacent to the metal surface revealed the chalky-white appearance associated with Alkali-iron-sulfates. Thus, the required "bad actor" was available to test the performance limits of this alloy.

The analysis of the deposit and the metal surface immediately adjacent to the interface revealed the presence of carbon. While carbon determinations using EDS are unreliable, the implications of carbon in the deposit are worthy of additional consideration, as is the potential for carbon ingress from the deposit into the abutting metal.

Finally, while it was not the focus of this investigation, the internal surface condition was briefly examined for sample II-JJ. * Note throughout this appendix, the reported metal loss rate was calculated based on
time at temperature rather than total time within the boiler. 


\section{Material: Save 25}

\section{Summary of Findings}

\begin{tabular}{|c|c|}
\hline Issue & Discussion \\
\hline $\begin{array}{l}\text { Sample } \\
\text { Location and } \\
\text { Orientation. }\end{array}$ & $\begin{array}{l}\text { - In assessing the data for Section C, it is important to understand that } \\
\text { some tube samples had to be removed from the section early due to } \\
\text { excessive thinning. } \\
\text { The schematic in Figure A-1 shows the positions of the tube lengths } \\
\text { that were removed early. In November 2001, a length was removed } \\
\text { that comprised six tube specimens. In June 2002, two lengths were } \\
\text { removed. One length comprised three tube specimens. The other } \\
\text { involved only one specimen. } \\
\text { - Figure A-1 also shows the positions of the four Save } 25 \text { samples } \\
\text { within Section C when it was initially installed. Examination of this } \\
\text { figure reveals that three of the four Save } 25 \text { specimens were removed } \\
\text { early, Sample K-L in November 2001, Samples D-E and Z-AA in June } \\
\text { 2002. The lowest temperature sample, Sample II-JJ, survived until the } \\
\text { end of the Section C exposure period. } \\
\text { The samples that were removed early were set aside for later analysis, } \\
\text { and have been included in the current evaluation along with the other } \\
\text { tube samples that made up Section C. However, it should be noted that } \\
\text { it was not possible to analyze Sample D-E extensively due to the fact } \\
\text { that it was overlaid with weld metal during its service life. } \\
\text { Also shown in Figure A-1 is the calculated average surface metal } \\
\text { temperature at each location. }\end{array}$ \\
\hline $\begin{array}{l}\text { Composition } \\
\text { Verification. }\end{array}$ & $\begin{array}{l}\text { - The Save } 25 \text { tube samples for this program were manufactured from } \\
\text { one heat of material (Heat D621907) by Sumitomo Metals Industries } \\
\text { (SMI). The table in Figure A-2 provides a "specified" composition and } \\
\text { ladle analysis per the SMI-provided materials certificates. } \\
\text { Previous analysis of the Save } 25 \text { samples from Section A showed that } \\
\text { for three of the four tube samples, the actual tube composition did not } \\
\text { comply with the specified composition in that it is slightly high in } \\
\text { copper relative to the maximum allowable of } 4 \mathrm{wt} \% \text {. } \\
\text { - However, for Section C, it was decided that it would be unnecessary to } \\
\text { analyze each and every tube sample for composition verification. } \\
\text { Rather, it was decided to select a representative sample from each tube } \\
\text { material type for confirmatory analysis. } \\
\text { During the Section A evaluation, the energy dispersive spectrographic } \\
\text { (EDS) capabilities of the scanning electron microscope (SEM) were } \\
\text { used to perform semi-quantitative analyses of each of the twelve } \\
\text { different candidate tube materials. Comparison of these EDS results } \\
\text { showed reasonably good correlation with results obtained using } \\
\text { quantitative analytical techniques. } \\
\text { Based on this, it was decided that the accuracy provided by EDS } \\
\text { would suffice for composition verification, but that more detailed } \\
\text { analyses would be undertaken if questions arose. } \\
\text { The results of the EDS analysis of tube sample II-JJ confirmed that the } \\
\text { correct material was used at this location. }\end{array}$ \\
\hline
\end{tabular}




\section{Material: Save 25}

\section{Summary of Findings, (continued)}

\begin{tabular}{|c|c|}
\hline Issue & Discussion \\
\hline $\begin{array}{l}\text { As-Received } \\
\text { Appearance }\end{array}$ & $\begin{array}{l}\text { - Figure A-3 documents the appearance of the Save } 25 \text { tube prior to } \\
\text { exposure. } \\
\text { - Figures A-4 through A-7 document the as-received appearance of the } \\
\text { Save } 25 \text { tube samples after service. Three views are provided for each } \\
\text { tube: } 12 \text { o'clock (the view that faces on-coming gas flow), } 3 \text { o'clock } \\
\text { (the view where the gas flow is tangent to the surface), and } 6 \text { o'clock } \\
\text { (the view that shows the back-side of the tube relative to the gas flow } \\
\text { direction). } \\
\text { - The reader should be alerted to the fact that, within the boiler, the gas } \\
\text { flows upward over these tubes. This implies that for the "o'clock" } \\
\text { conventions described above, (with the } 12 \text { o'clock orientation facing } \\
\text { the gas flow direction), the actual physical orientation of these tubes is } \\
\text { such that the } 12 \text { o'clock side of the tube faces downward, and the } 6 \\
\text { o'clock orientation faces upward within the boiler. } \\
\text { The } 12 \text { and } 3 \text { o'clock positions for Sample K-L show that this tube } \\
\text { was perforated by corrosion near mid-length. } \\
\text { Figure A-5 shows the weld cladding repair to Sample D-E and } \\
\text { provides the reason why further analysis of this sample would be } \\
\text { fruitless. } \\
\text { Sample II-JJ shows evidence of the gray/white scale associated with } \\
\text { molten alkali-iron sulfate attack. }\end{array}$ \\
\hline $\begin{array}{l}\text { Grit-Blasted } \\
\text { Surface } \\
\text { Appearance }\end{array}$ & $\begin{array}{l}\text { - Figures A- } 8 \text { through A-11 show the appearance of the external surface } \\
\text { of the Save } 25 \text { tube samples after grit-blasting to remove deposit and } \\
\text { scale. The } 12 \text { o'clock and } 3 \text { o'clock positions for all four samples } \\
\text { show the appearance of general wastage due to fireside corrosion. } \\
\text { - With the exception of Sample II-JJ, all of the Save } 25 \text { samples showed } \\
\text { significant pitting at the } 6 \text { o'clock position. }\end{array}$ \\
\hline $\begin{array}{l}\text { Wall } \\
\text { Thickness } \\
\text { Loss } \\
\text { Determined }\end{array}$ & $\begin{array}{l}\text { - Tube cross-sections were prepared to allow for measurement of wall } \\
\text { thickness to determine metal loss due to exposure. These are shown in } \\
\text { silhouette in Figures A-12 through A-15. } \\
\text { The same relative "o'clock" positions were maintained as discussed } \\
\text { earlier. Measurements were taken at eight evenly spaced locations } \\
\text { about the tube circumference. These were then compared with } \\
\text { measurements taken at these same locations prior to exposure. } \\
\text { - The before and after measurements were documented in the table that } \\
\text { accompanies each figure. These form the basis for the "delta" } \\
\text { calculation to determine the amount of wall thickness lost. . } \\
\text { - In some cases, the minimum wall thickness in the cross section was } \\
\text { not at one of the eight predetermined locations, ( Samples K-L and II- } \\
\text { JJ are examples). In these cases, there was no "before" measurement } \\
\text { for comparison. So, the average "before" wall thickness was used to } \\
\text { calculate the wall thickness lost at these off locations. } \\
\text { For Sample K-L, the wall was completely perforated, so, it was not } \\
\text { possible to determine an accurate maximum rate of metal loss. }\end{array}$ \\
\hline
\end{tabular}




\section{Material: Save 25}

\section{Summary of Findings, (continued)}

\begin{tabular}{|c|c|}
\hline Issue & Discussion \\
\hline $\begin{array}{l}\text { Wall } \\
\text { Thickness } \\
\text { Loss Versus } \\
\text { Average Metal } \\
\text { Temperature }\end{array}$ & $\begin{array}{l}\text { - The chart in Figure A-16 plots the maximum wall thickness lost } \\
\text { against the calculated average surface metal temperature for each of } \\
\text { the four Save } 25 \text { samples. } \\
\text { As a general trend, it is apparent that the rate of attack increases } \\
\text { continuously as the temperature is raised over the interval of roughly } \\
1040 \mathrm{~F} \text { to } 1180 \mathrm{~F} \text {. } \\
\text { - It also was noted that Save } 25 \text { suffers minimal wall reduction at } 1040 \\
\mathrm{~F} \text {, but that the rate of metal loss increases dramatically when the } \\
\text { temperature is raised to } 1080 \mathrm{~F} \text {. } \\
\text { - The observed high rate of metal loss provides the reason that all of the } \\
\text { Save } 25 \text { samples that saw an exposure of } 1080 \mathrm{~F} \text { and above have been } \\
\text { removed from service. At this point only the II-JJ sample (at } 1040 \mathrm{~F} \text { ) } \\
\text { remains in service for Section B. }\end{array}$ \\
\hline $\begin{array}{l}\text { Wall } \\
\text { Thickness } \\
\text { Loss as a } \\
\text { Function of } \\
\text { Time and } \\
\text { Location }\end{array}$ & $\begin{array}{l}\text { - The chart in Figure A-17 plots metal loss as a function of time for } \\
\text { three of the four sample locations Sections A and C. with samples } \\
\text { from Section C having experienced generally longer exposure times. } \\
\text { Note: Sample D-E data was not plotted because this sample had been } \\
\text { overlain with weld metal in Section C, so, no data was available for } \\
\text { this sample. } \\
\text { - The ( } 401 \text { mils) data point for Sample K-L represents complete } \\
\text { penetration of the wall. } \\
\text { - While the data is very limited, data for Sample Z-AA suggest that the } \\
\text { corrosion rate slowed with time after an initial rapid loss of wall } \\
\text { thickness. }\end{array}$ \\
\hline $\begin{array}{l}\text { Metallurgical } \\
\text { Analysis - } \\
\text { SEM }\end{array}$ & $\begin{array}{l}\text { - Sample II-JJ was selected for a detailed SEM/EDS assessment of the } \\
\text { constituents and structure in the vicinity of the external and internal } \\
\text { surfaces. This sample was selected because it was the only Save } 25 \\
\text { sample to survive for the full exposure period of Section C. } \\
\text { - Figures A-18a and b show the appearance of the scale/metal interface } \\
\text { for Sample K-L. Six distinct regions are in evidence. These regions } \\
\text { are, starting within the metal and working out: } \\
\circ \text { Base metal - etch shows grain boundaries and carbides. } \\
\circ \text { Diffusion zone - light etching - This region is obvious as a } \\
\text { light-appearing band that is parallel to the metal surface, } \\
\text { perhaps see most clearly in Figure A-18b. } \\
\text { Diffusion zone - dark etching - This constitutes the leading } \\
\text { edge of the grains as they interface with the scale. As EDS } \\
\text { analysis later shows, this zone is depleted in chromium and } \\
\text { possibly enriched in carbon. } \\
\text { Thin porous, loosely adhering scale - this is seen continuous } \\
\text { along the surface. EDS analysis will later show that this is } \\
\text { predominantly chromium-oxide. } \\
\text { Bulk deposit - EDS analysis will show that this region } \\
\text { contains know aggressive species, including iron, sulfur, } \\
\text { chlorine, and alkali metals. }\end{array}$ \\
\hline
\end{tabular}




\section{Summary of Findings, (continued)}

\section{Material: $\quad$ Save 25}

\begin{tabular}{|c|c|}
\hline Issue & Discussion \\
\hline $\begin{array}{l}\text { Metallurgical } \\
\text { Analysis - } \\
\text { SEM, } \\
\text { Continued. }\end{array}$ & $\begin{array}{l}\text { - Figure A-19 shows the appearance of the microstructure at mid-wall. } \\
\text { - Figure A-20 shows the appearance of the internal surface for Sample } \\
\text { II-JJ. Little evidence of an oxide scale was found. While it cannot be } \\
\text { stated conclusively without further investigation, the irregular surface } \\
\text { contour at the internal surface suggest that this surface had } \\
\text { experienced metal loss due to steam-side oxidation, but that the scale } \\
\text { subsequently had spalled away from the surface. }\end{array}$ \\
\hline $\begin{array}{l}\text { EDS Analysis } \\
\text { Results }\end{array}$ & $\begin{array}{l}\text { - The table in Figure A-21 provides a summary of the EDS analyses of } \\
\text { the locations numbered in Figure A-18b. Also shown in this table is a } \\
\text { summary of the spectrographic analysis reported earlier, in Figure A-2. } \\
\text { - Review of this table suggests the following: } \\
\text { - Area 1: Bulk deposit/scale analysis - Alkali metals are present. } \\
\text { Sulfur and phosphorous are present in an increased amount } \\
\text { compared to the base metal. An iron oxide is likely, as are } \\
\text { aluminum, silicon and titanium oxides. Carbon content is } \\
\text { relatively high, however, EDS is reliable qualitatively but not } \\
\text { quantitatively for light elements such as carbon. This suggests } \\
\text { that carbon is present, but the exact amount is unknown. } \\
\text { - Area 2: Adherent scale - This appears to be comprised of an } \\
\text { chromium-, iron-, and nickel-rich oxide. Again of significance } \\
\text { here is the presence of sulfur and carbon. } \\
\text { - Area 3: Diffusion zone - dark etching - This is leading edge of } \\
\text { the metal adjacent to the scale/metal interface. This region is } \\
\text { depleted in chromium. Oxygen and perhaps carbon ingress are } \\
\text { suggested by this analysis. } \\
\text { Area 4: Diffusion zone - light etching - The analysis here is } \\
\text { practically indistinguishable from that of the bulk tube alloy. } \\
\text { Area 5: Tube metal composition - Given its limitations this EDS } \\
\text { analysis compares favorably with that of the reference } \\
\text { spectrographic analysis below it. }\end{array}$ \\
\hline
\end{tabular}




\section{Gas Flow}

Direction

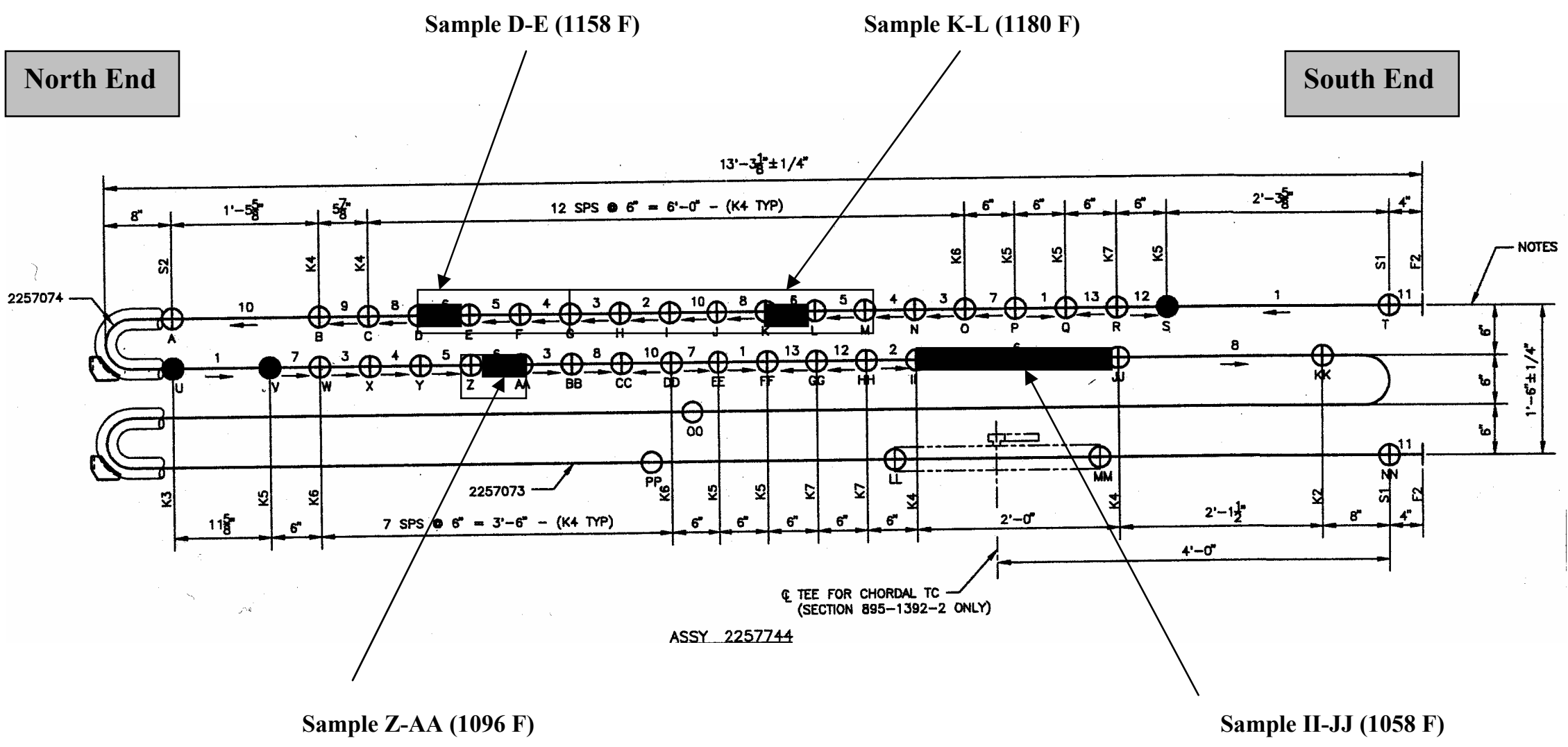

Figure A-1: Schematic Drawing Showing Position of Save 25 Tube Samples Within Section C

1. Drawing shown in same relative orientation as per the actual installation.

2. This group of tube samples was removed from service in November 2001 - highlighted in red.

3. This group of tube samples was removed from service in June 2002 - highlighted in yellow 


\section{Figure A-2 Semi-Quantitative Composition Verification for Save 25}

Description:

Source:

Reference Composition:
Nominal $22 \mathrm{Cr} / 18 \mathrm{Ni}$ steel with $\mathrm{Nb}$ and $\mathrm{N}$ added to provide creep strength.

Sumitomo Metal Industries (SMI)

"Specified" chemistry - per SMI certificate.

\begin{tabular}{|c|c|c|c|c|c|c|}
\hline Element & $\begin{array}{c}\text { Reference } \\
\text { Composition }\end{array}$ & $\begin{array}{c}\text { Composition (a) } \\
\text { Heat D621907 }\end{array}$ & $\begin{array}{c}\text { Tube Sample } \\
\text { D-E }\end{array}$ & $\begin{array}{c}\text { Tube Sample } \\
\text { K-L }\end{array}$ & $\begin{array}{c}\text { Tube Sample } \\
\text { Z-AA }\end{array}$ & $\begin{array}{c}\text { Tube Sample } \\
\text { II-JJ }\end{array}$ \\
\hline $\mathrm{Ni}$ & $15.00-22.00$ & 19.80 & $\mathrm{~N} / \mathrm{A}$ & N/A & $\mathrm{N} / \mathrm{A}$ & 19.6 \\
\hline $\mathrm{Cr}$ & $21.00-24.00$ & 22.60 & N/A & N/A & N/A & 22.0 \\
\hline $\mathrm{Fe}$ & Balance & Balance & $\mathrm{N} / \mathrm{A}$ & $\mathrm{N} / \mathrm{A}$ & $\mathrm{N} / \mathrm{A}$ & 52.8 \\
\hline Mo & - & - & N/A & $\mathrm{N} / \mathrm{A}$ & $\mathrm{N} / \mathrm{A}$ & - \\
\hline $\mathrm{Co}$ & - & - & N/A & N/A & N/A & - \\
\hline $\mathrm{C}$ & $0.05-0.12$ & 0.07 & N/A & $\mathrm{N} / \mathrm{A}$ & N/A & - \\
\hline $\mathrm{N}$ & $0.15-0.30$ & 0.22 & N/A & N/A & N/A & - \\
\hline $\mathrm{B}$ & - & - & $\mathrm{N} / \mathrm{A}$ & N/A & $\mathrm{N} / \mathrm{A}$ & - \\
\hline $\mathrm{Mn}$ & $2.0 \max$ & 0.64 & N/A & $\mathrm{N} / \mathrm{A}$ & N/A & 0.44 \\
\hline $\mathrm{Si}$ & $1.5 \max$ & 0.23 & $\mathrm{~N} / \mathrm{A}$ & $\mathrm{N} / \mathrm{A}$ & $\mathrm{N} / \mathrm{A}$ & 0.18 \\
\hline $\mathrm{Al}$ & - & - & $\mathrm{N} / \mathrm{A}$ & N/A & $\mathrm{N} / \mathrm{A}$ & - \\
\hline $\mathrm{Ti}$ & - & - & N/A & N/A & N/A & - \\
\hline $\mathrm{Al}+\mathrm{Ti}$ & - & - & $\mathrm{N} / \mathrm{A}$ & $\mathrm{N} / \mathrm{A}$ & $\mathrm{N} / \mathrm{A}$ & - \\
\hline $\mathrm{Nb}$ & $0.30-0.60$ & 0.45 & N/A & $\mathrm{N} / \mathrm{A}$ & N/A & - \\
\hline $\mathrm{Ta}$ & - & - & $\mathrm{N} / \mathrm{A}$ & $\mathrm{N} / \mathrm{A}$ & $\mathrm{N} / \mathrm{A}$ & - \\
\hline $\mathrm{Nb}+\mathrm{Ta}$ & - & - & N/A & $\mathrm{N} / \mathrm{A}$ & $\mathrm{N} / \mathrm{A}$ & - \\
\hline $\mathrm{V}$ & - & - & N/A & $\mathrm{N} / \mathrm{A}$ & N/A & - \\
\hline $\mathrm{W}$ & $0.80-2.80$ & 1.31 & N/A & N/A & N/A & 1.86 \\
\hline $\mathrm{Cu}$ & $2.00-4.00$ & 3.31 & $\mathrm{~N} / \mathrm{A}$ & $\mathrm{N} / \mathrm{A}$ & $\mathrm{N} / \mathrm{A}$ & 3.24 \\
\hline $\mathrm{Sn}$ & - & - & N/A & N/A & N/A & - \\
\hline $\mathrm{P}$ & $0.030 \max$ & 0.022 & N/A & N/A & N/A & - \\
\hline $\mathrm{S}$ & $0.010 \max$ & (c) & $\mathrm{N} / \mathrm{A}$ & $\mathrm{N} / \mathrm{A}$ & $\mathrm{N} / \mathrm{A}$ & - \\
\hline $\mathrm{O} 2$ & - & - & $\mathrm{N} / \mathrm{A}$ & $\mathrm{N} / \mathrm{A}$ & N/A & - \\
\hline
\end{tabular}

a - Ladle analysis

b- By difference

$\mathrm{N} / \mathrm{A}=$ Sample not analyzed .

c- Not detected 


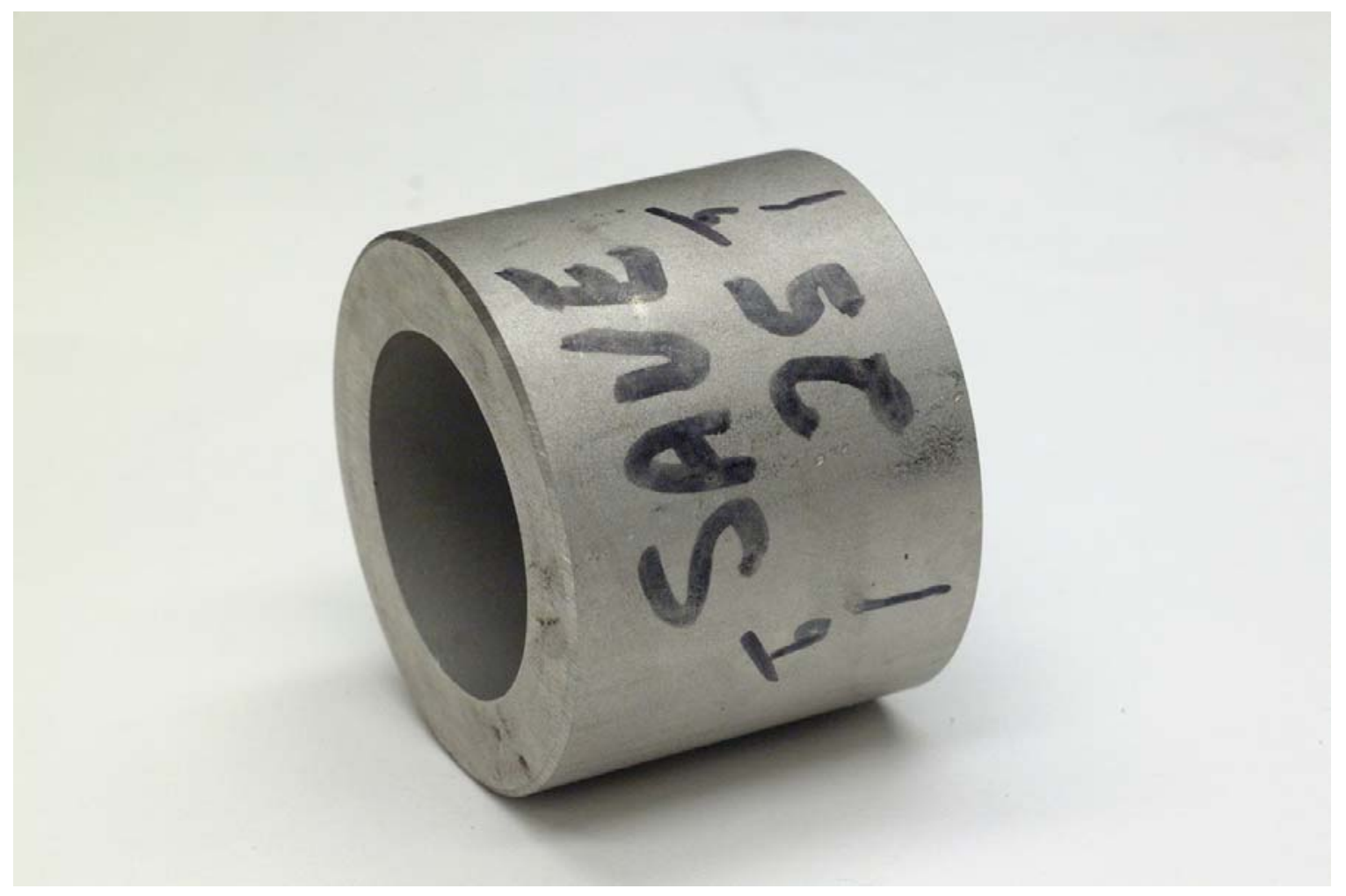

Figure A-3: Appearance of Save 25 Tube Sample Prior to Service 


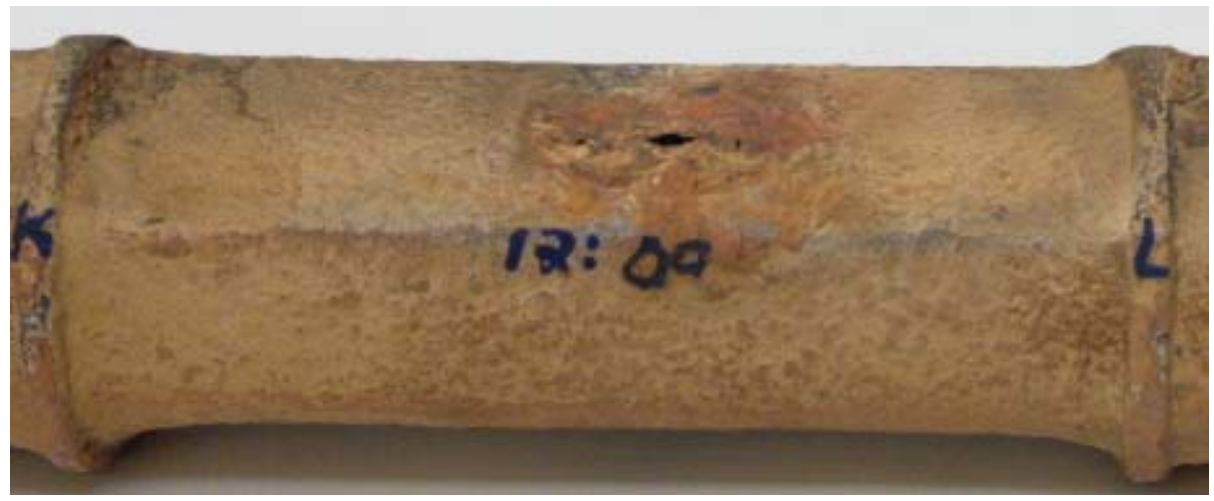

12 O'Clock - Facing Gas Flow

(Note: 3 o'clock is up in this image)

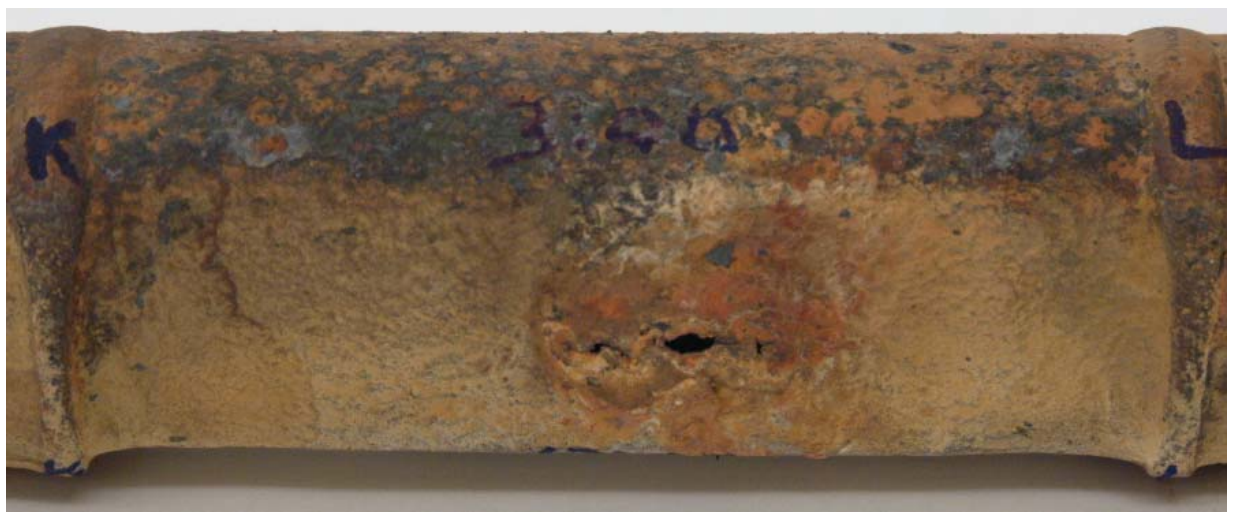

3 O'Clock

(Note: 6 o'clock is up in this image)

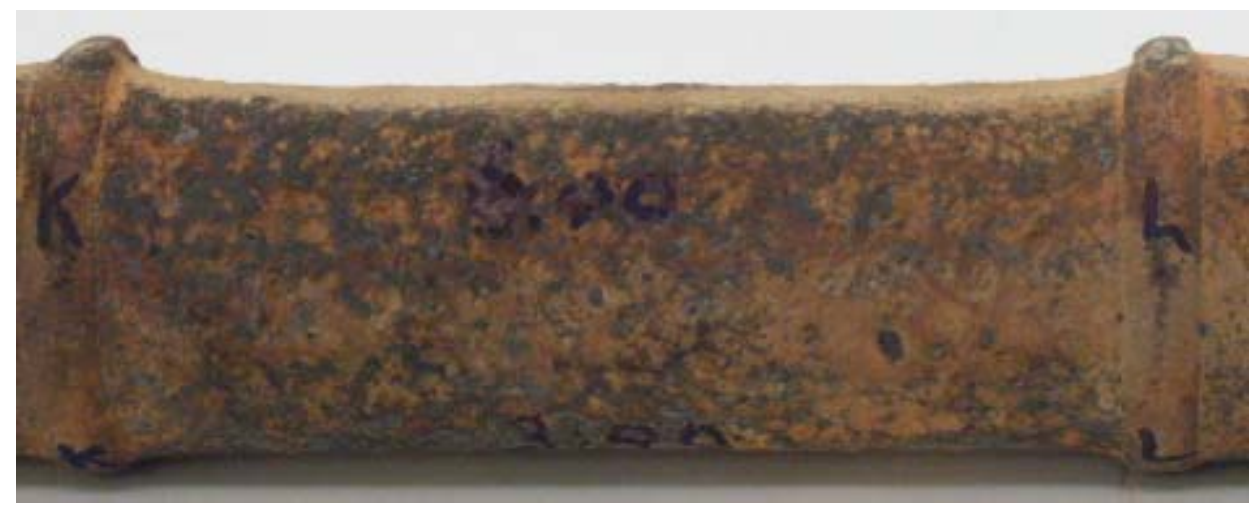

6 O'Clock - Opposite Gas Flow

Figure A-4: As-Received Appearance of Sample K-L (Save 25)

(Average metal temperature: $1180 \mathrm{~F}$ )

Note: this tube sample was removed from service on 11/6/01 


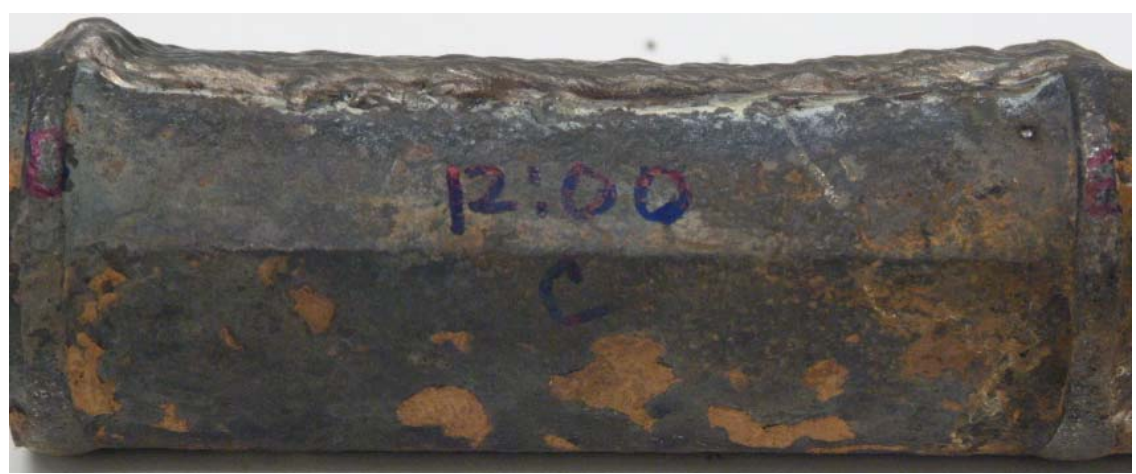

12 O'Clock - Facing Gas Flow

(Note: 3 o'clock is up in this image)

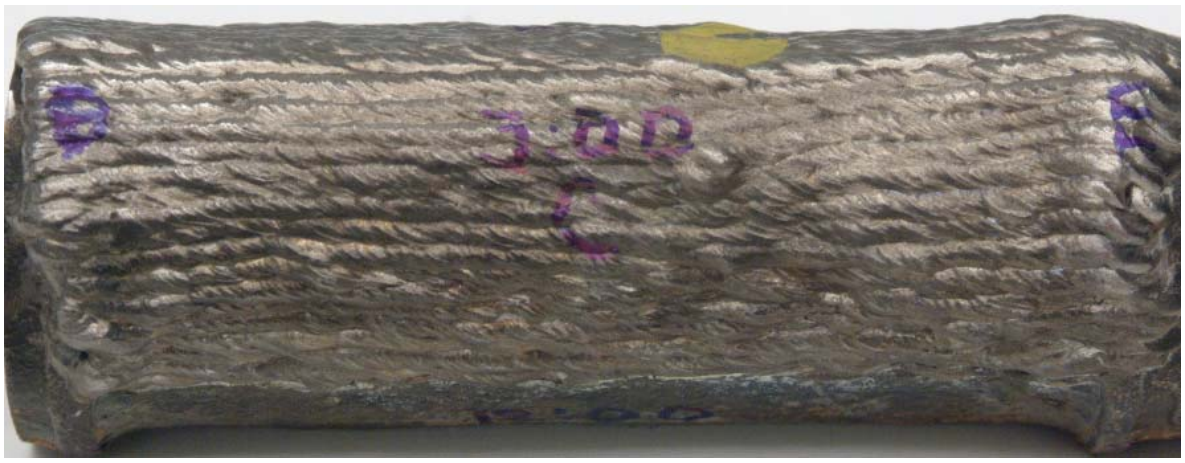

3 O'Clock

(Note: 6 o'clock is up in this image)

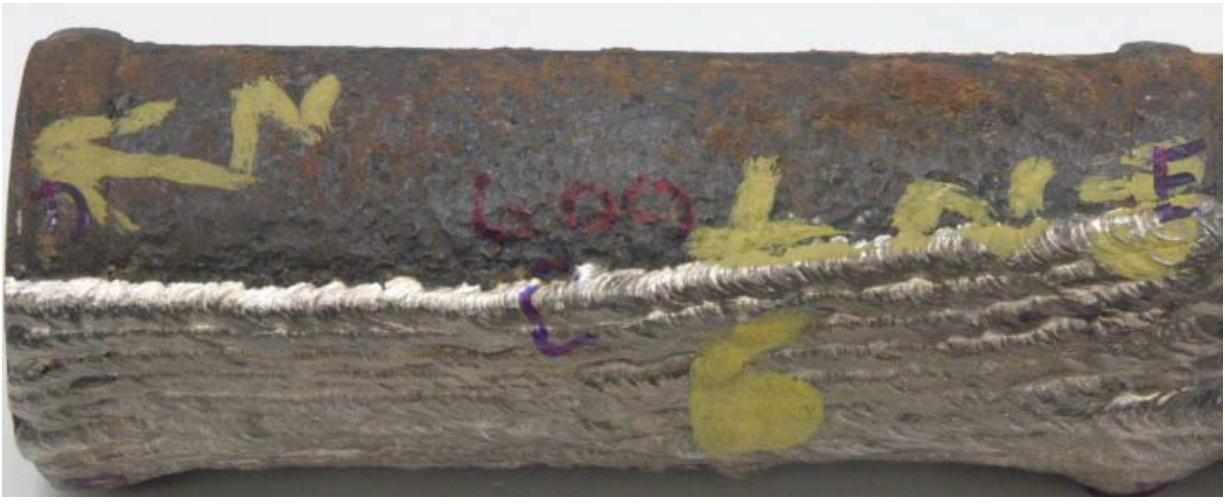

6 O'Clock - Opposite Gas Flow

Figure A-5: As-Received Appearance of Sample D-E (Save 25)

\section{(Average metal temperature: 1158 F)}

Note: this tube sample was surface clad to extend life.

This tube sample was removed from service on $6 / 10 / 02$ 


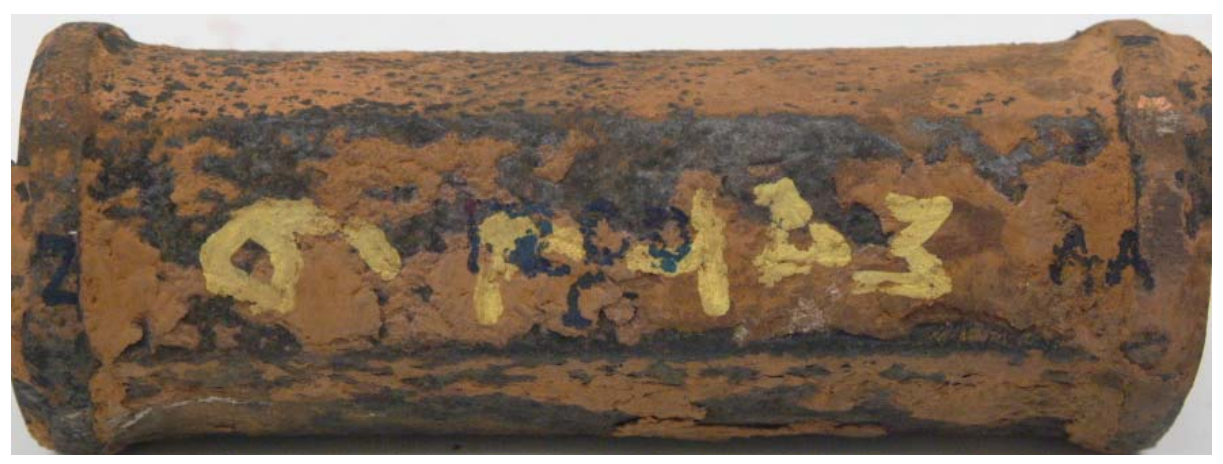

12 O'Clock - Facing Gas Flow

(Note: 3 o'clock is up in this image)

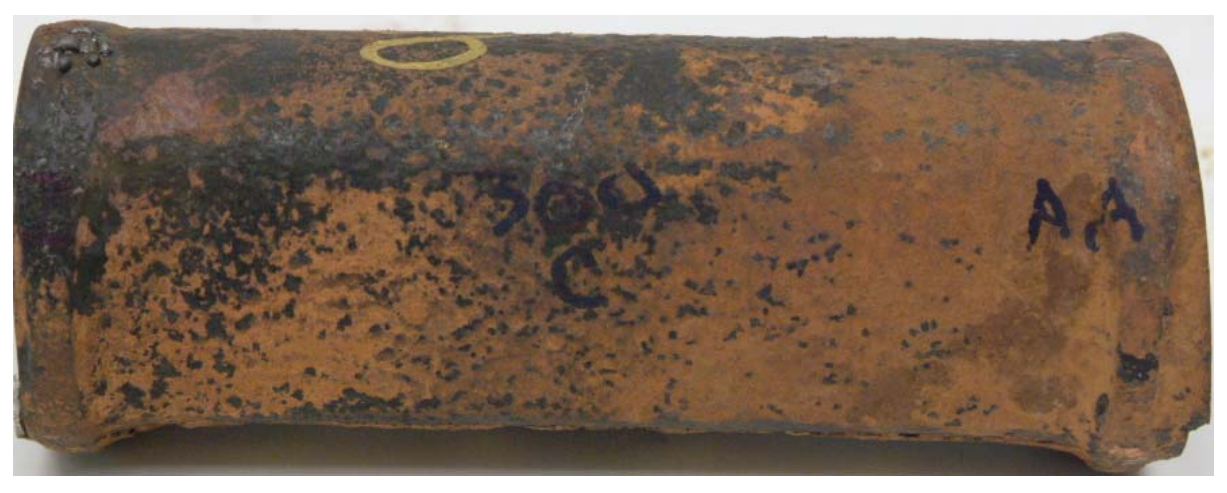

3 O'Clock

(Note: 6 o'clock is up in this image)

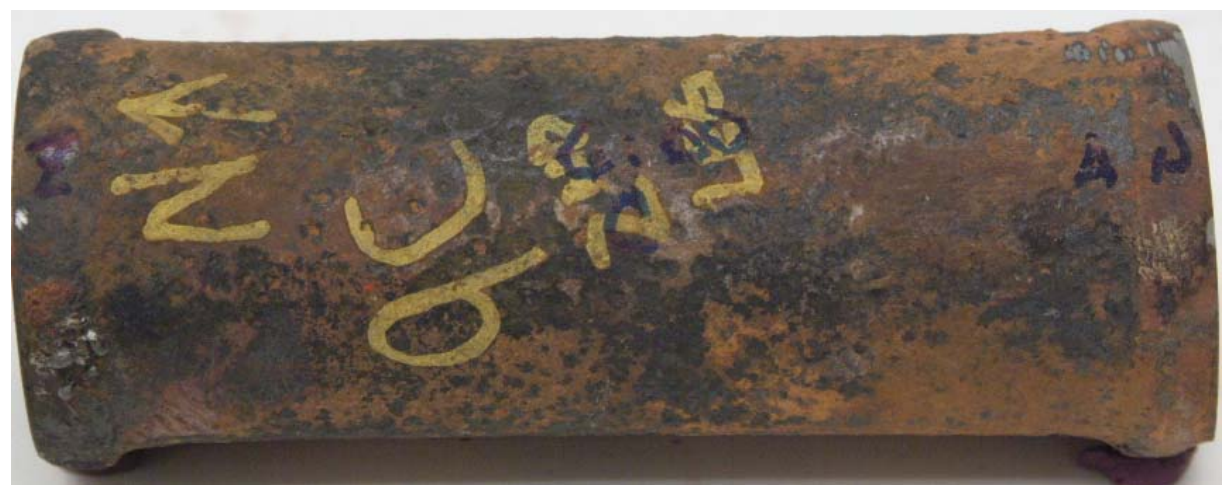

6 O'Clock - Opposite Gas Flow

Figure A-6: As-Received Appearance of Sample Z-AA (Save 25)

(Average metal temperature: 1096 F)

Note: this sample was removed from service prematurely on 6/10/02 


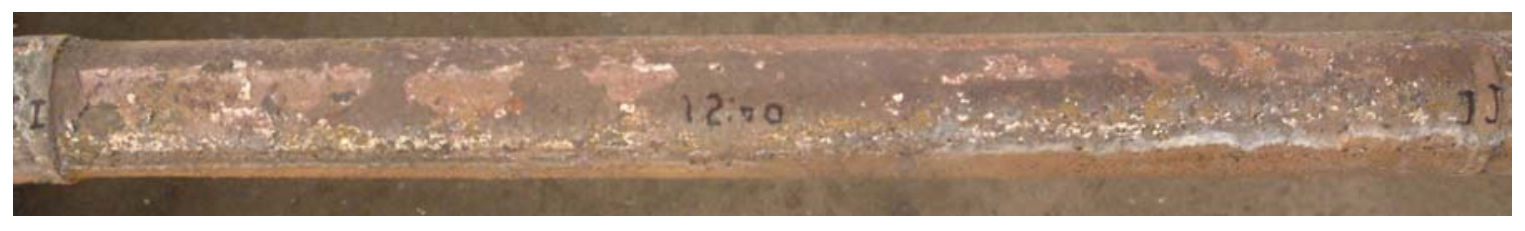

12 O'Clock - Facing Gas Flow

(Note: 3 o'clock is up in this image)

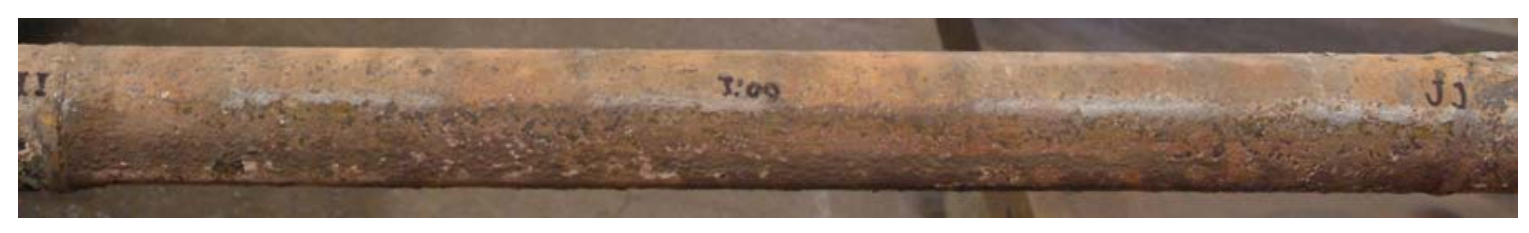

3 O'Clock

(Note: 6 o'clock is up in this image)

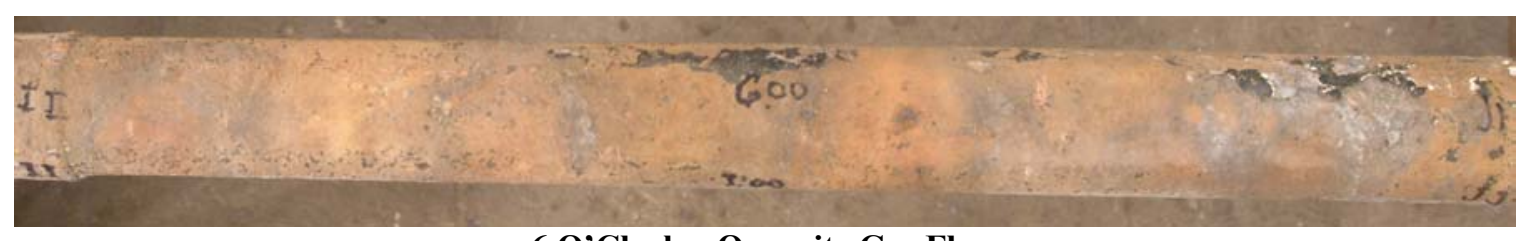

6 O'Clock - Opposite Gas Flow

Figure A-7: As-Received Appearance of Sample II-JJ (Save 25)

(Average metal temperature: 1058 F) 
12 O'Clock

(289 mils)

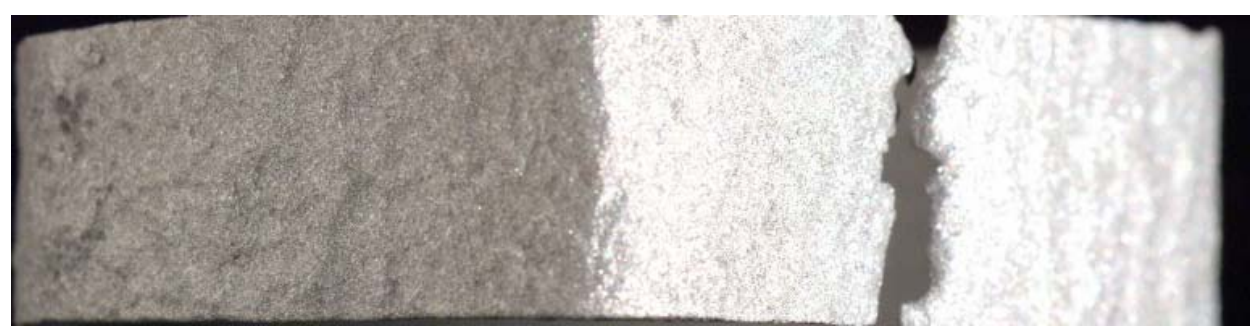

3 O'Clock

(274 mils)

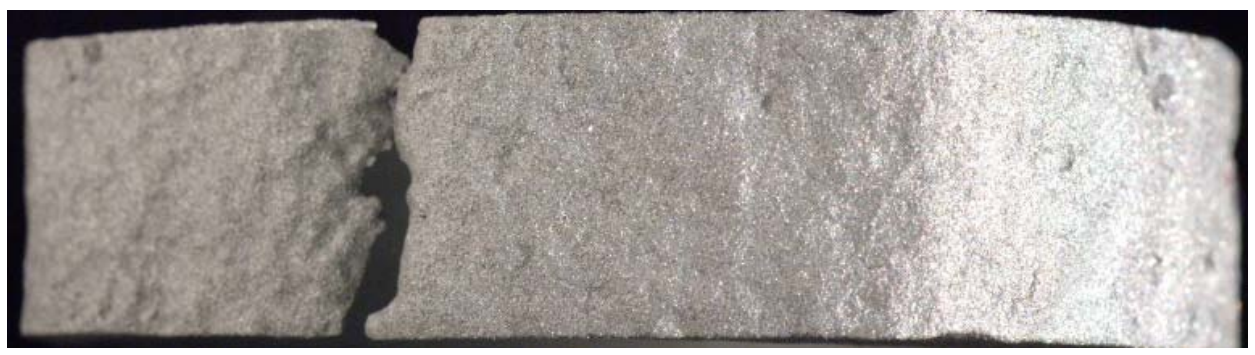

6 O'Clock

(2 mils lost)

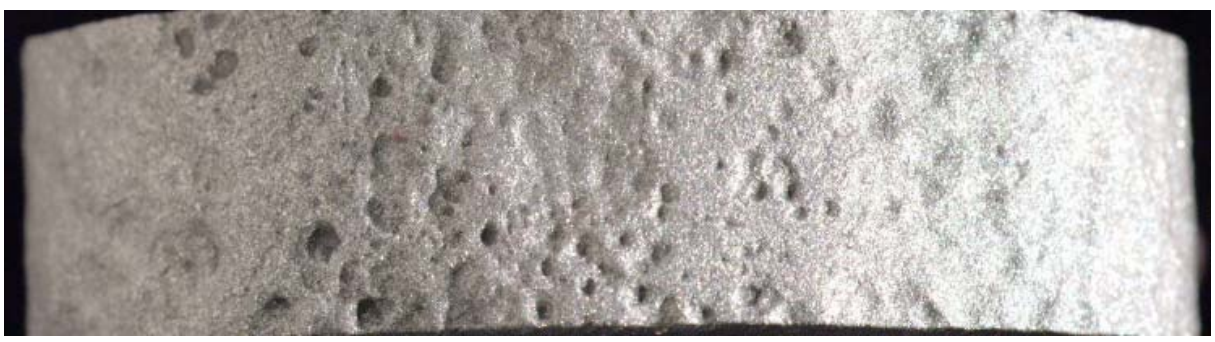

Figure A-8: OD Surface Appearance After Grit Blast

Material: Save 25

Sample: K-L 


\section{O'Clock}

( N/A)

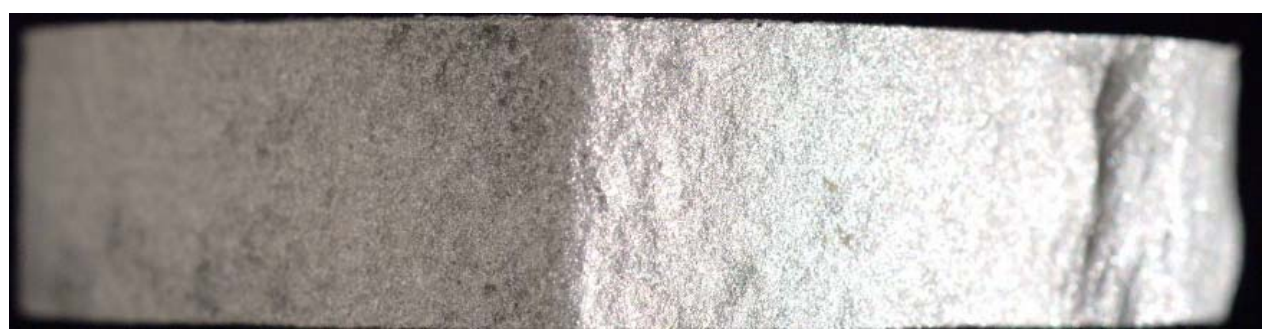

\section{O'Clock}

(Not this shows the condition of the weld overlay build-up, not the original surface.)

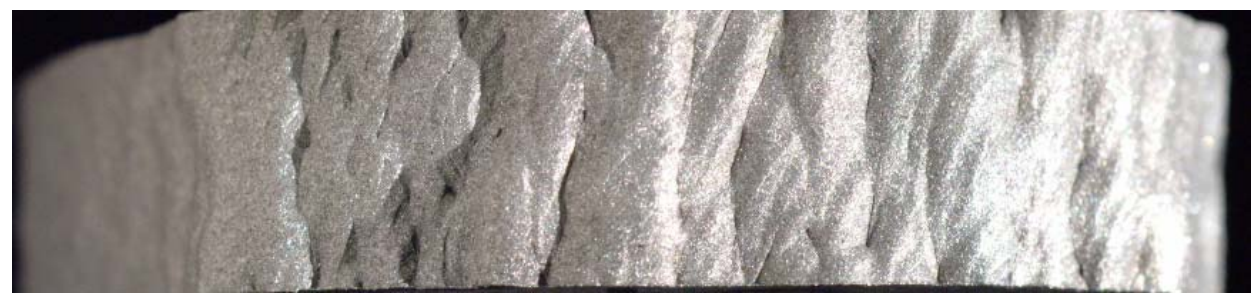

\section{O'Clock}

(The weld overlay can be seen to the left in this image. The original surface is to the right.)

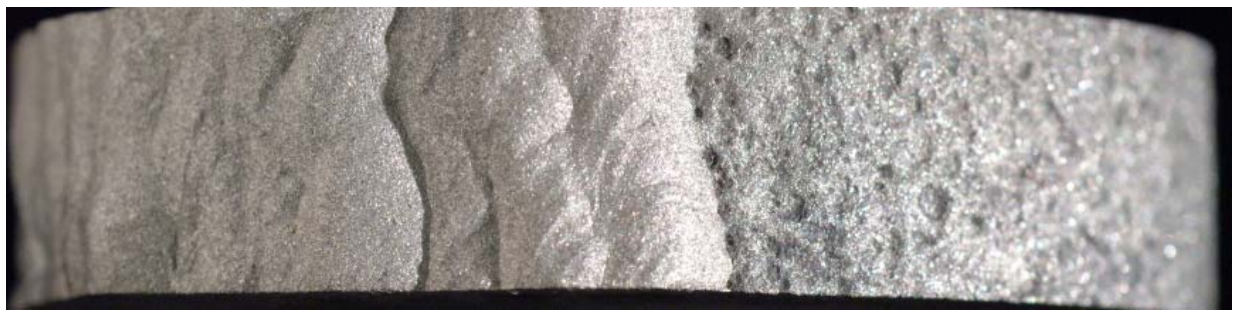

Figure A-9: OD Surface Appearance After Grit Blast

Material: Save 25

Sample: D-E 
12 O'Clock

(253 mils lost)

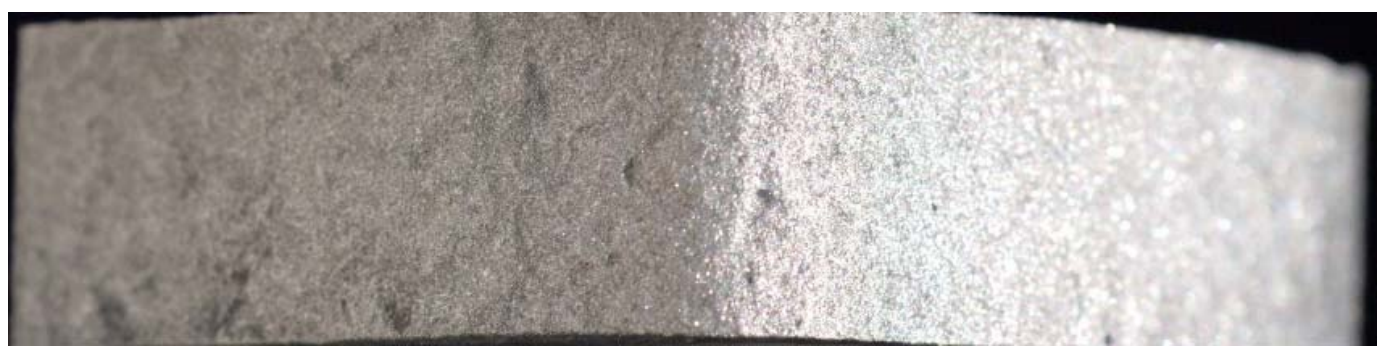

3 O'Clock

(207 mils lost)

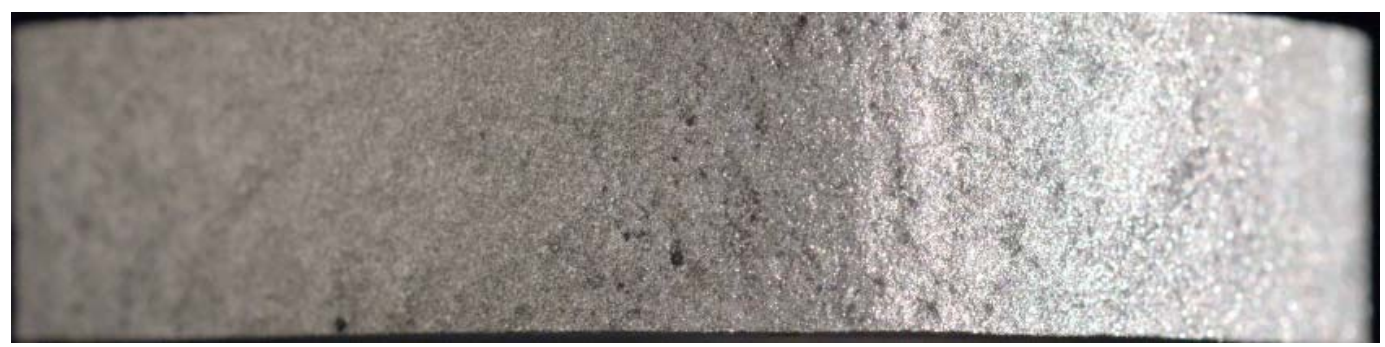

6 O'Clock

(0 mils lost)

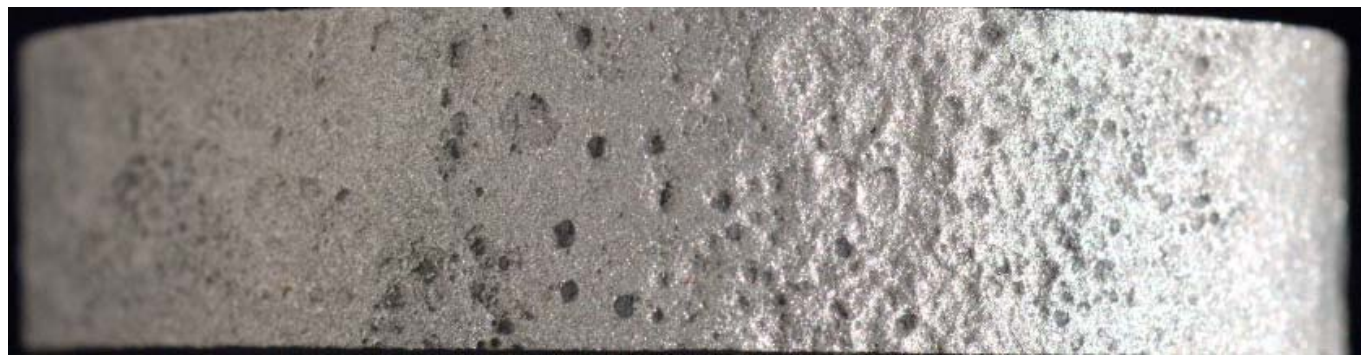

Figure A-10: OD Surface Appearance After Grit Blast

Material: Save 25

Sample: Z-AA 
12 O'Clock

(106 mils lost)

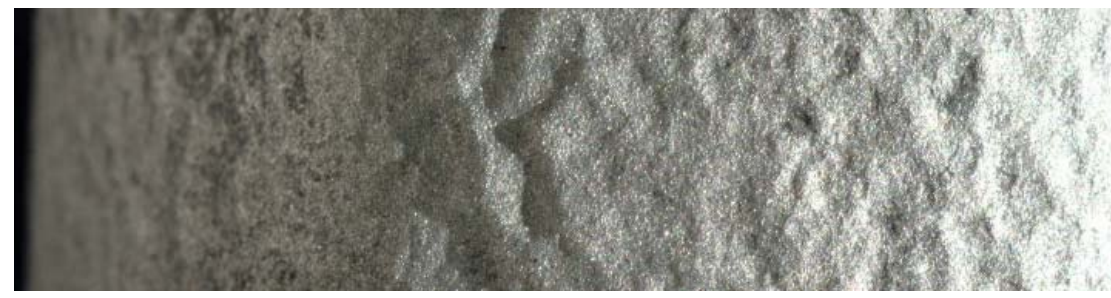

3 O'Clock

(74 mils lost)

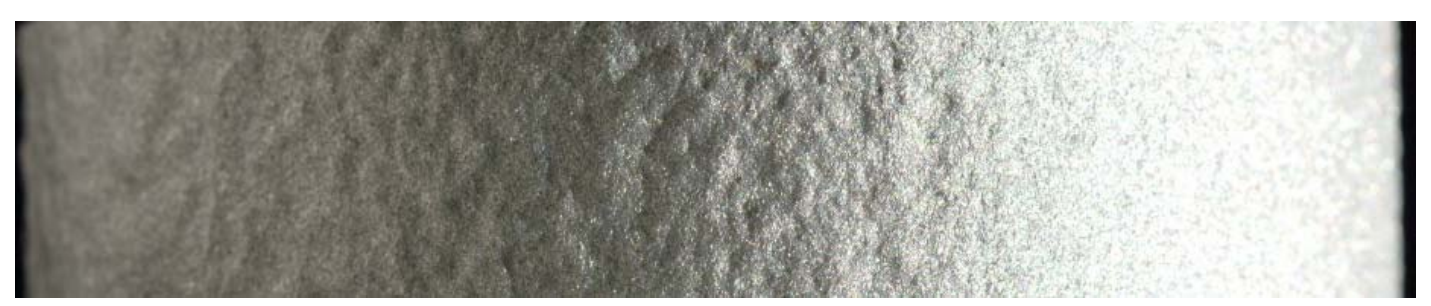

6 O'Clock

(6 mils lost)

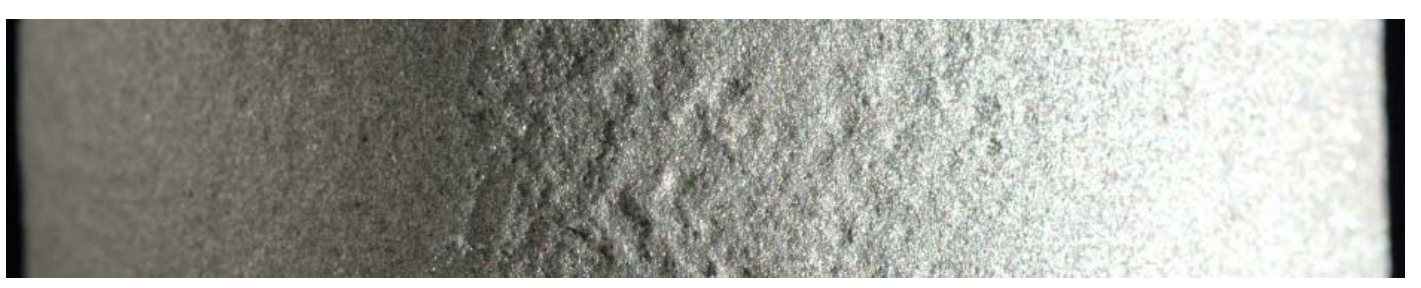

Figure A-11: OD Surface Appearance After Grit Blast

Material: Save 25

Sample: II-JJ

Material:

SAVE 25 
Sample Location:

Maximum Rate:

Maximum Loss Orientation: Average metal Temperature:
K-L

$>259$ mils/yr

Approx. 2:30 o'clock

$1180 \mathrm{~F}$

\section{O’Clock}

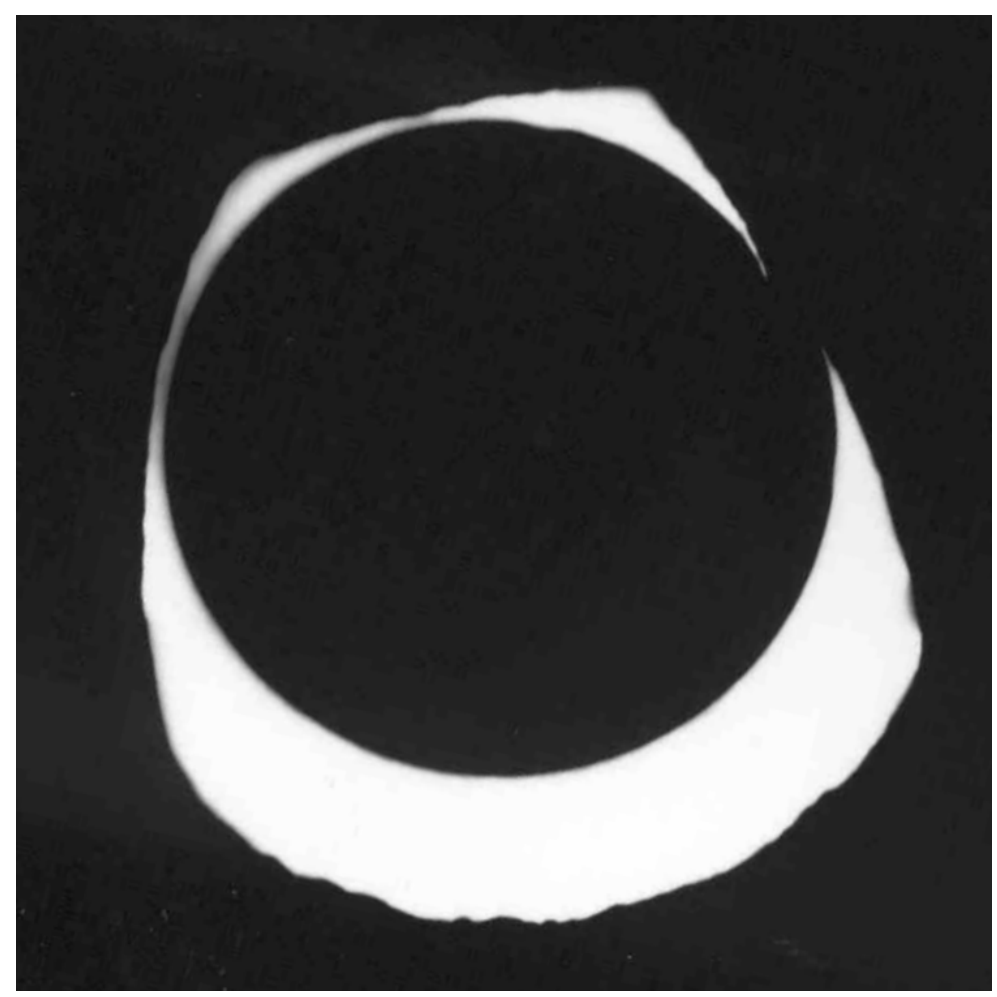

3 O'Clock

Approx. 1.2X

\section{Wall Reduction by Position (in.)}

\begin{tabular}{|l|c|c|c|c|c|c|c|c|c|c|}
\hline & $\begin{array}{c}\mathbf{1 2} \\
\text { o'clock }\end{array}$ & $\mathbf{1 : 3 0}$ & $\mathbf{3 : 0 0}$ & $\mathbf{4 : 3 0}$ & $\mathbf{6 : 0 0}$ & $\mathbf{7 : 3 0}$ & $\mathbf{9 : 0 0}$ & $\mathbf{1 0 : 3 0}$ & AVG & $\begin{array}{c}\text { Max* } \\
\text { Loss }\end{array}$ \\
\hline Before & 0.398 & 0.402 & 0.404 & 0.402 & 0.403 & 0.404 & 0.399 & 0.397 & 0.401 & 0.401 \\
\hline After & 0.109 & 0.029 & 0.130 & 0.389 & 0.401 & 0.287 & 0.040 & 0.130 & & 0.000 \\
\hline Delta & 0.289 & 0.373 & 0.274 & 0.013 & 0.002 & 0.117 & 0.359 & 0.267 & & 0.401 \\
\hline
\end{tabular}

Figure A-12: Silhouette and Wall Thickness Measurements for Sample K-L

Note: this tube sample was removed from service on 11/6/01

* Measurement taken at "maximum loss orientation" noted above. The average

"before" wall thickness was used to calculate the "delta". 


\section{Material:}

Sample Location:

Maximum Rate:

Maximum Loss Orientation:

Average metal Temperature:

\section{SAVE 25}

D-E

N/A mils/yr

N/A

$1158 \mathrm{~F}$

12 O’Clock

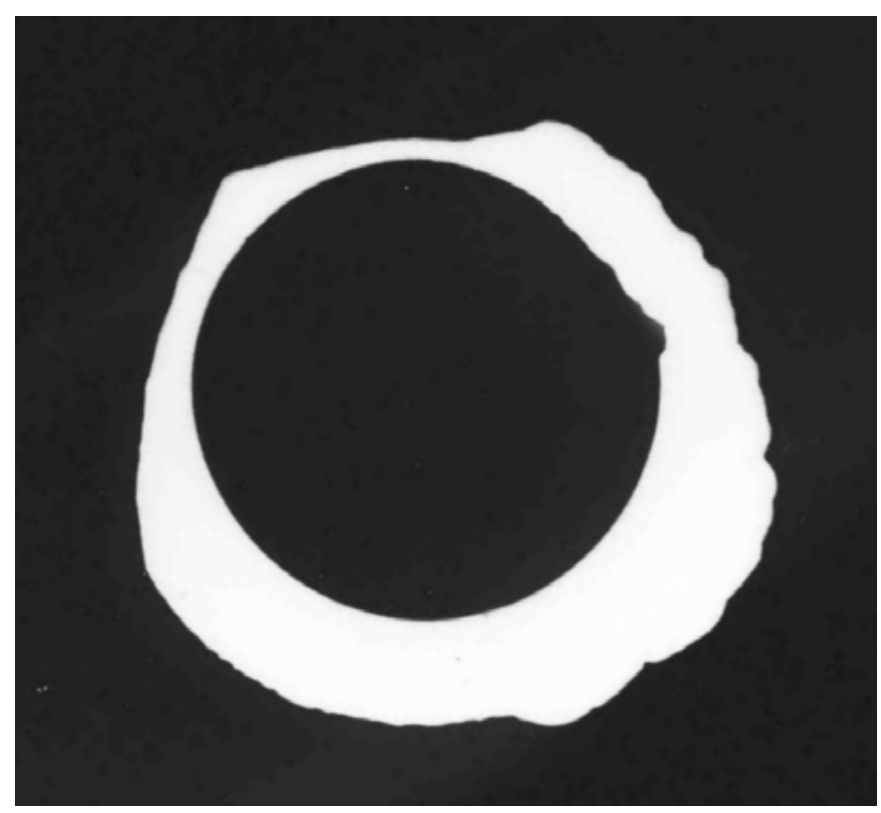

3 O'Clock

Approx. 1.2X

Wall Reduction by Position (in.)

\begin{tabular}{|l|c|c|c|c|c|c|c|c|c|c|}
\hline & $\begin{array}{c}12 \\
\text { o'clock }\end{array}$ & $\mathbf{1 : 3 0}$ & $\mathbf{3 : 0 0}$ & $\mathbf{4 : 3 0}$ & $\mathbf{6 : 0 0}$ & $\mathbf{7 : 3 0}$ & $\mathbf{9 : 0 0}$ & $\mathbf{1 0 : 3 0}$ & AVG & $\begin{array}{c}\text { Max } \\
\text { Loss }\end{array}$ \\
\hline Before & 0.399 & 0.399 & 0.401 & 0.403 & 0.404 & 0.401 & 0.401 & 0.404 & 0.402 & 0.402 \\
\hline After & N/A & N/A & N/A & N/A & N/A & N/A & N/A & N/A & & N/A \\
\hline Delta & N/A & N/A & N/A & N/A & N/A & N/A & N/A & N/A & & N/A \\
\hline
\end{tabular}

Figure A-13: Silhouette and Wall Thickness Measurements for Sample D-E

Note: this tube sample had a weld build-up applied in an effort to extend service; however, it ultimately was removed from service on 6/10/02. No measurements taken due to weld build-up. 


\section{Material:}

Sample Location:

Maximum Rate:

Maximum Loss Orientation:

Average metal Temperature:

\section{SAVE 25}

\section{Z-AA}

$148 \mathrm{mils} / \mathrm{yr}$

Approx. 2:30 o'clock

$1096 \mathrm{~F}$

\section{O’Clock}

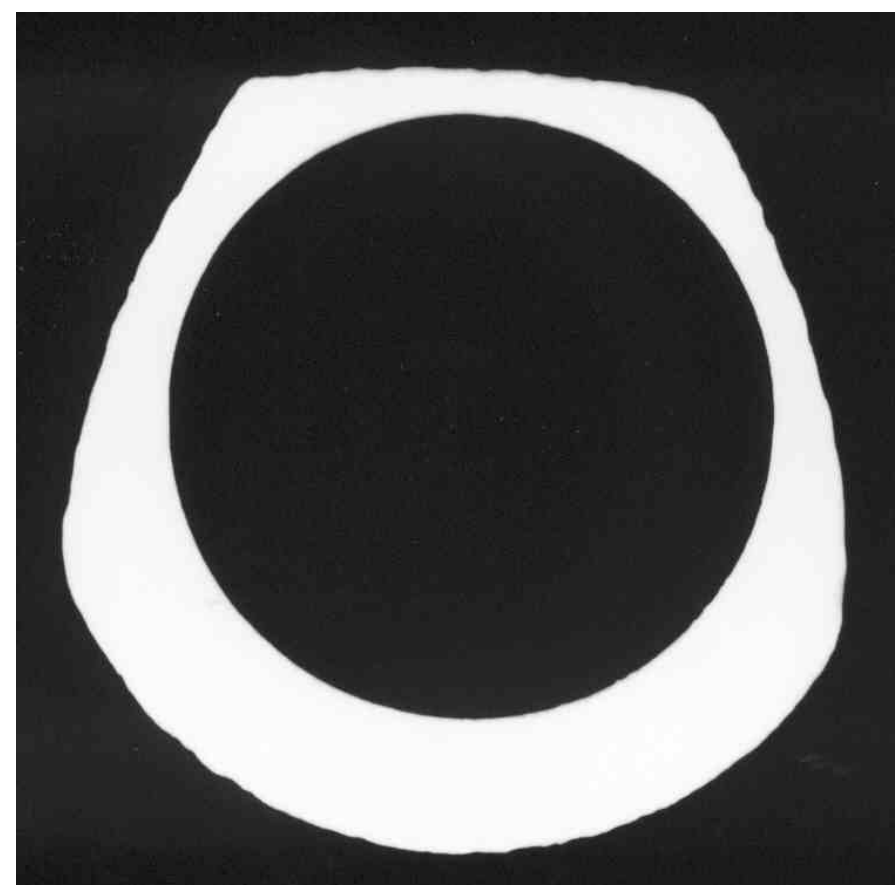

3 O’Clock

Approx. 1.2X

Wall Reduction by Position (in.)

\begin{tabular}{|l|c|c|c|c|c|c|c|c|c|c|}
\hline & $\begin{array}{c}\mathbf{1 2} \\
\text { o'clock }\end{array}$ & $\mathbf{1 : 3 0}$ & $\mathbf{3 : 0 0}$ & $\mathbf{4 : 3 0}$ & $\mathbf{6 : 0 0}$ & $\mathbf{7 : 3 0}$ & $\mathbf{9 : 0 0}$ & $\mathbf{1 0 : 3 0}$ & AVG & $\begin{array}{c}\text { Max }^{*} \\
\text { Loss }\end{array}$ \\
\hline Before & 0.397 & 0.398 & 0.397 & 0.400 & 0.398 & 0.396 & 0.398 & 0.399 & 0.398 & 0.398 \\
\hline After & 0.144 & 0.191 & 0.190 & 0.386 & 0.398 & 0.387 & 0.219 & 0.246 & & 0.130 \\
\hline Delta & 0.253 & 0.209 & 0.207 & 0.014 & 0.000 & 0.009 & 0.179 & 0.153 & & 0.268 \\
\hline
\end{tabular}

Figure A-14: Silhouette and Wall Thickness Measurements for Sample Z-AA

Note: this tube sample was removed from service on $6 / 10 / 02$

* Measurement taken at "maximum loss orientation" noted above. The average

"before" wall thickness was used to calculate the "delta". 


\section{Material:}

Sample Location:

Maximum Rate:

Maximum Loss Orientation:

Average metal Temperature:
SAVE 25

\section{II-JJ}

56 mils/yr

Approx. 2:30 o'clock $1058 \mathrm{~F}$

\section{O'Clock}

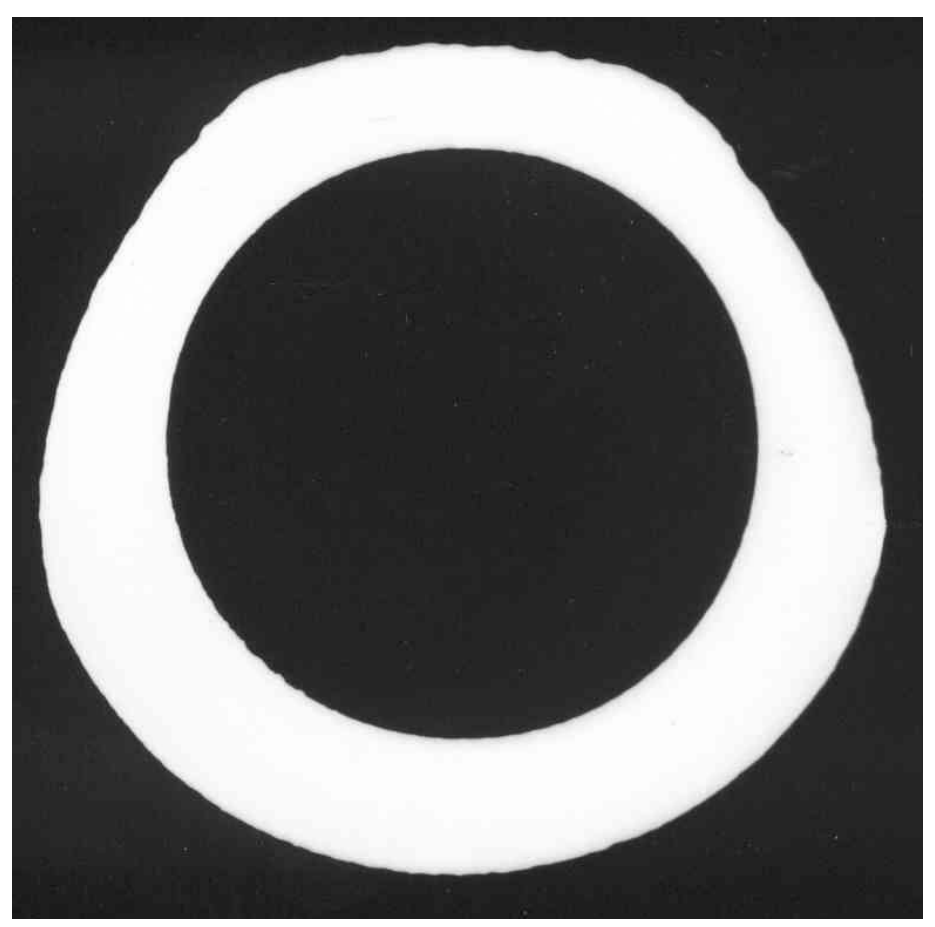

3 O'Clock

Approx. 1.2X

Wall Reduction by Position (in.)

\begin{tabular}{|l|c|c|c|c|c|c|c|c|c|c|}
\hline & $\begin{array}{c}\mathbf{1 2} \\
\text { o'clock }\end{array}$ & $\mathbf{1 : 3 0}$ & $\mathbf{3 : 0 0}$ & $\mathbf{4 : 3 0}$ & $\mathbf{6 : 0 0}$ & $\mathbf{7 : 3 0}$ & $\mathbf{9 : 0 0}$ & $\mathbf{1 0 : 3 0}$ & AVG & $\begin{array}{c}\text { Max }^{*} \\
\text { Loss }\end{array}$ \\
\hline Before & 0.420 & 0.424 & 0.426 & 0.428 & 0.425 & 0.423 & 0.421 & 0.420 & 0.423 & 0.423 \\
\hline After & 0.314 & 0.311 & 0.352 & 0.416 & 0.419 & 0.421 & 0.370 & 0.306 & & 0.290 \\
\hline Delta & 0.106 & 0.113 & 0.074 & 0.012 & 0.006 & 0.002 & 0.051 & 0.114 & & 0.133 \\
\hline
\end{tabular}

Figure A-15: Silhouette and Wall Thickness Measurements for Sample II-JJ

* Measurement taken at "maximum loss orientation" noted above. The average

"before" wall thickness was used to calculate the "delta". 


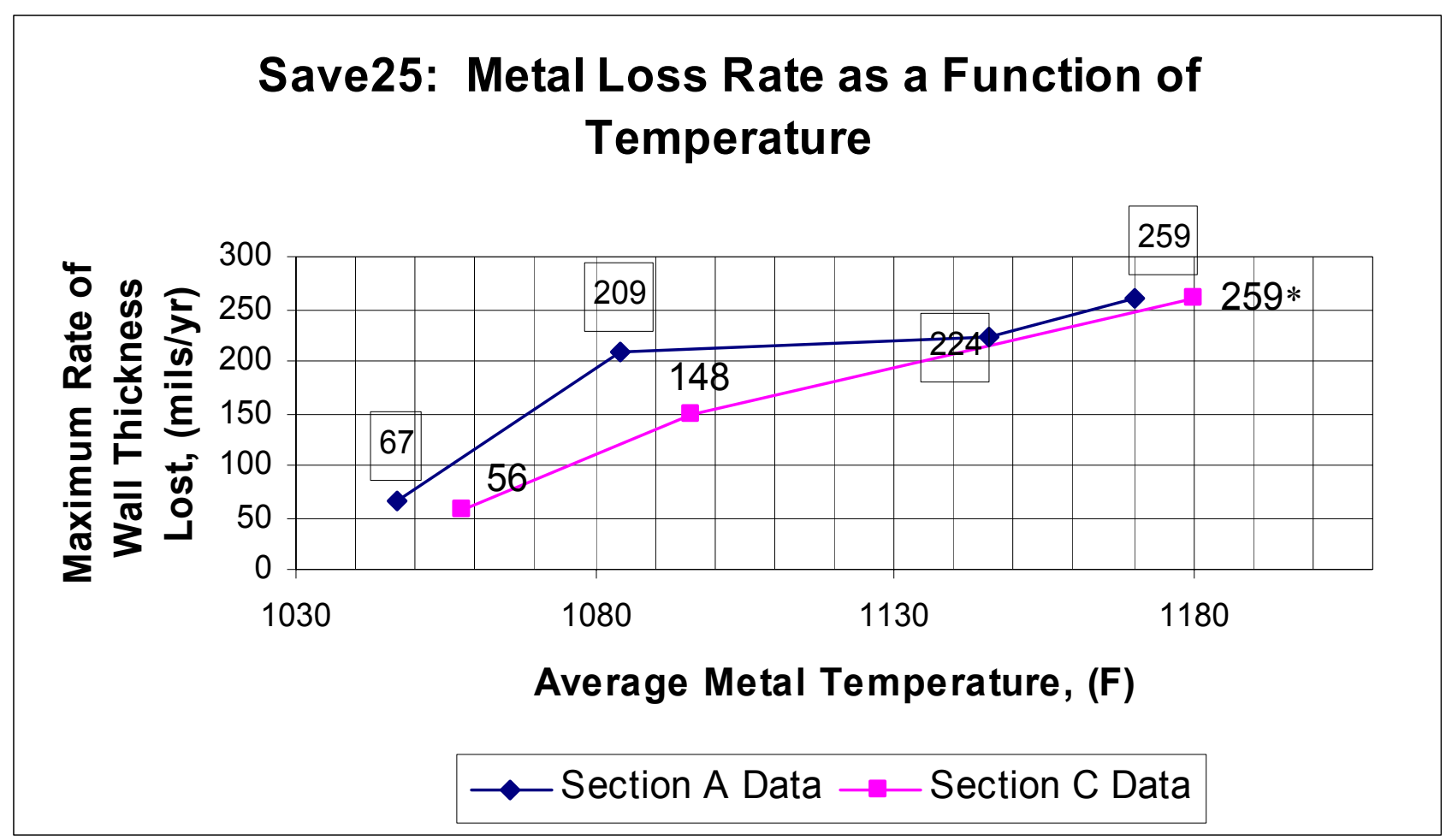

Figure A-16: Metal Loss Rate as a Function of Temperature

\section{Material: Save 25}

Note: In analyzing these data it is important to understand that the three of the four Section C samples were removed early due to excessive corrosion losses. Sample K-L (1180 F) was removed in November 2001, shortly after the Section A samples. At the same time, Sample D-E (1158 F) had its' wall thickness builtup using a weld overlay. It then was removed from service at the same time as Sample Z-AA in June 2002. The weld overlay destroyed wall thickness data for Sample D-E and accounts for the fact that no data is plotted for this sample for Section C. Sample II-JJ (1058 F) was removed with the remaining Section C samples in May 2003.

* - The wall thickness for this sample was completely penetrated; therefore, this data point represents a minimum rate of loss. 


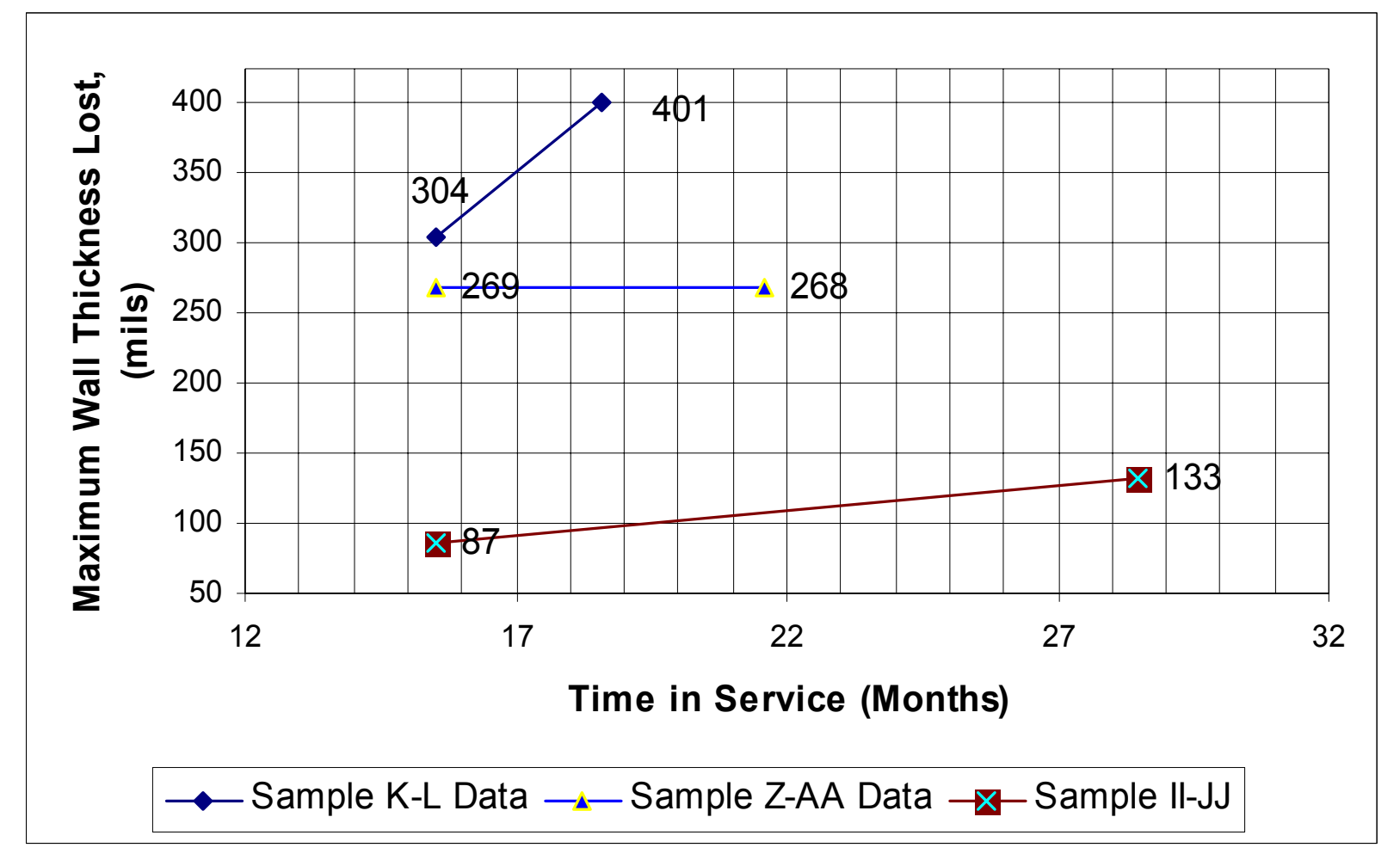

Figure A-17: Metal Loss as a Function of Time

\section{Material: Save 25}

Note: From the plot above it can be seen that Sample K-L from Section C, which was completely perforated, had somewhat more exposure than the Section A samples, even though it was removed at roughly the same time, i.e. November 2001. There was no data for the D-E sample from Section C due to the fact that it was overlain with weld metal. Sample Z-AA from Section C was removed in June 2002. Sample II-JJ experienced the full exposure life for both Sections A and C. 


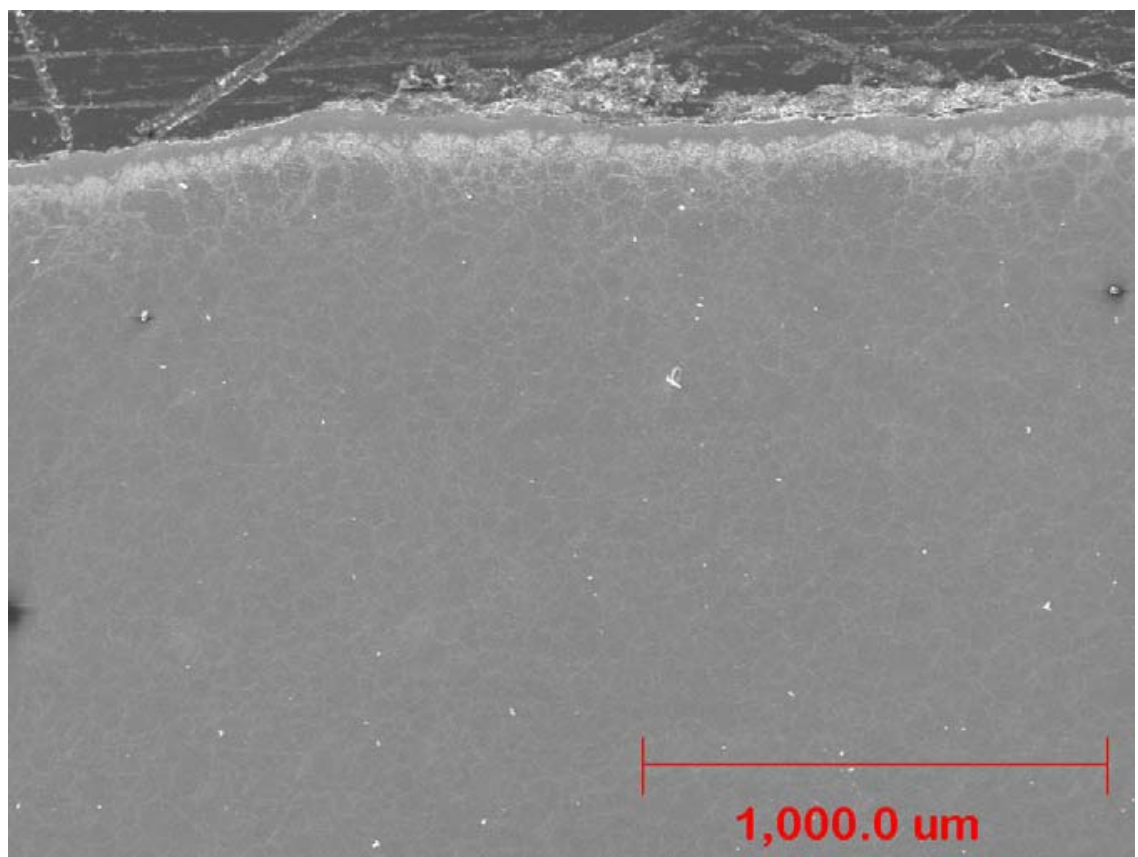

(a) External Scale/Metal Interface (SEI - Low Magnification)

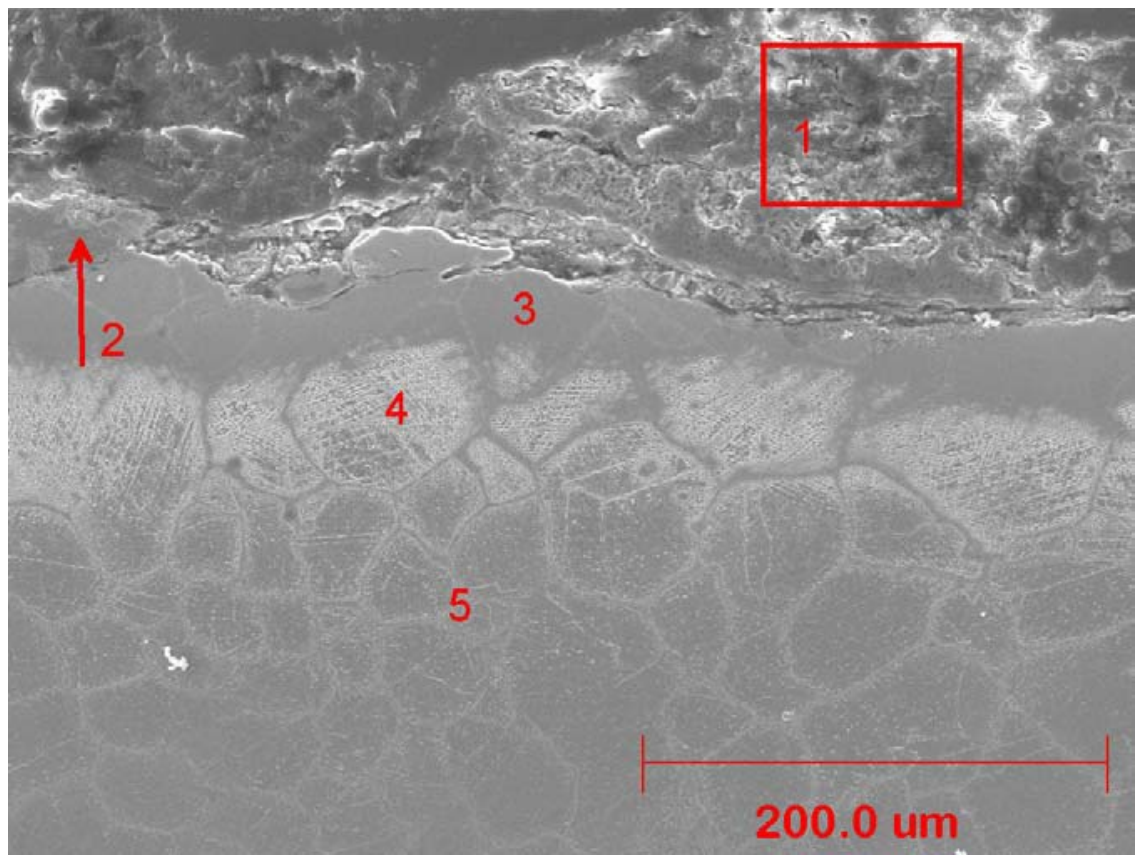

(b) External Scale/Metal Interface (SEI - Intermediate Magnification)

Figure A-18: SEM Microstructure: Save 25

Note: Numbers on Figure (b) indicate distinct areas characterized by the visual assessment and EDS analyses that follow. 


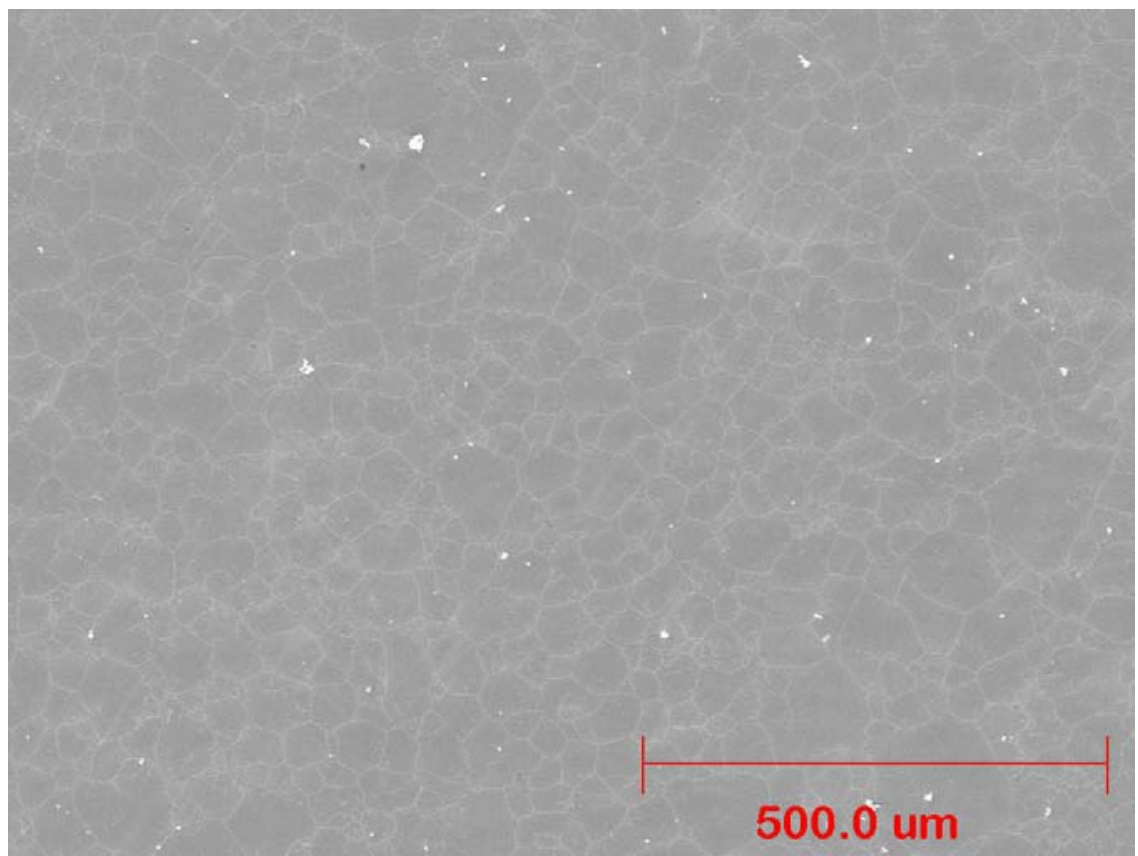

Figure A-19: SEM Mid-Wall Microstructure: Save 25

Note: Photomicrograph at approximately 200X. Electrolytic 10\% oxalic acid etch, 9V OCP 


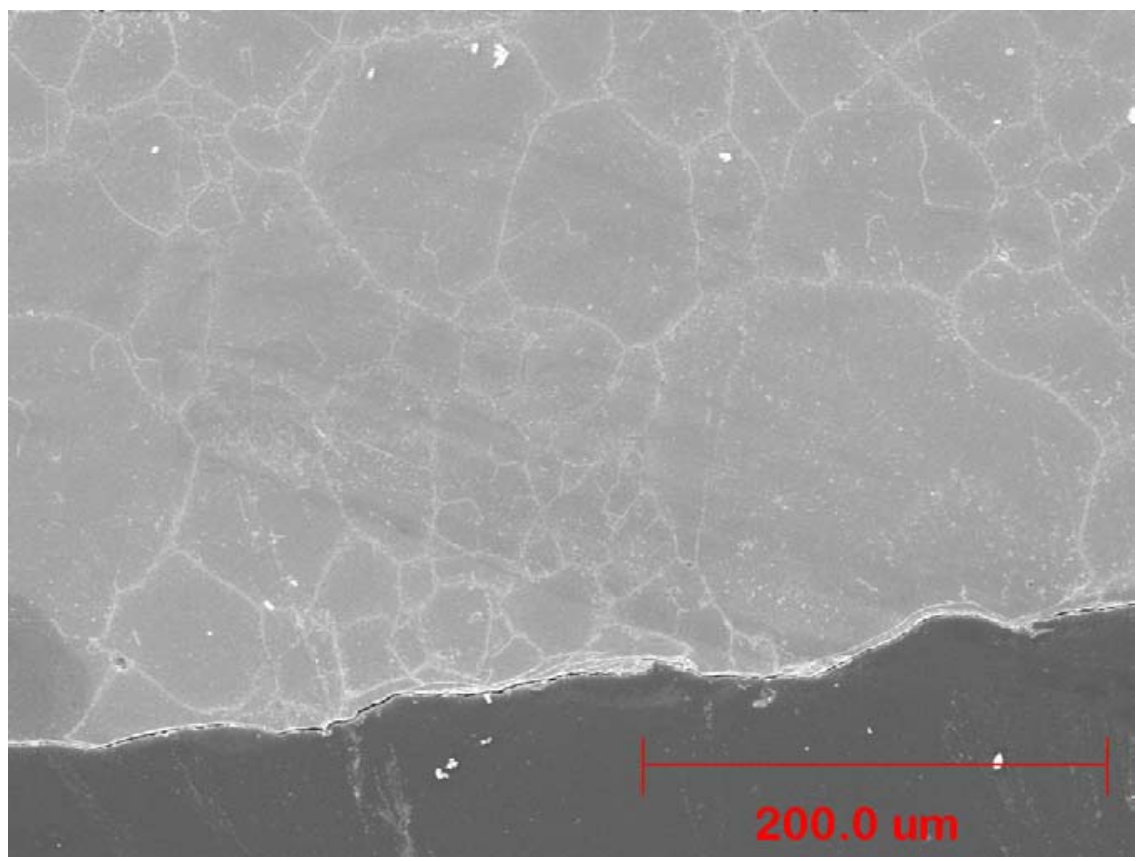

(a) Internal Surface (SEI - Intermediate Magnification)

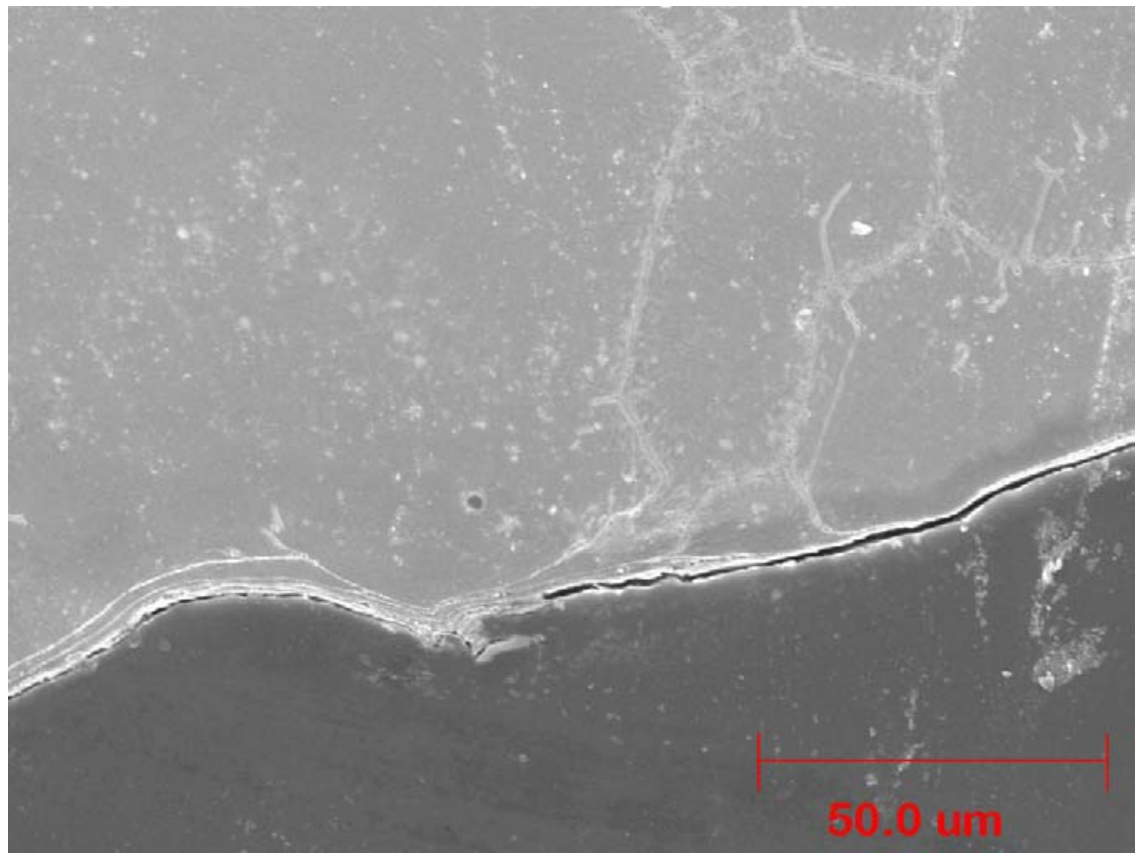

(b) Internal Surface (SEI - High Magnification)

Figure A-20: SEM Microstructure: Save 25 
Figure A-21: EDS Analysis of Deposit/Scale/Metal Interface for Save 25 (Sample II-JJ)

\begin{tabular}{|c|c|c|c|c|c|c|c|c|c|c|c|c|c|c|c|c|c|c|}
\hline Area & Description & $\mathbf{N i}$ & $\mathrm{Cr}$ & $\mathbf{F e}$ & $\mathbf{C u}$ & Mn & $\mathbf{W}$ & $\mathbf{T i}$ & Al & $\mathbf{S i}$ & $\mathrm{C}$ & $\mathbf{O}$ & $\mathbf{C a}$ & $\mathbf{K}$ & As & $\mathrm{Cl}$ & $\mathbf{P}$ & $\mathbf{S}$ \\
\hline 1 & $\begin{array}{l}\text { Bulk deposit/ } \\
\text { scale analysis }\end{array}$ & 1.88 & 0.30 & 13.2 & -- & - & -- & 0.24 & 5.11 & 4.87 & 50.0 & 16.8 & 0.49 & 0.73 & 5.09 & 0.29 & -- & 1.14 \\
\hline 2 & $\begin{array}{l}\text { Adherent } \\
\text { scale. }\end{array}$ & 11.8 & 32.4 & 28.8 & -- & 0.54 & -- & -- & 0.69 & 1.17 & 3.56 & 14.6 & -- & -- & -- & -- & -- & 6.38 \\
\hline 3 & $\begin{array}{l}\text { Diffusion } \\
\text { zone - dark } \\
\text { etching along } \\
\text { scale/metal } \\
\text { interface. }\end{array}$ & 24.5 & 11.1 & 56.6 & 2.79 & 0.36 & -- & -- & -- & 0.52 & 2.30 & 1.85 & -- & -- & -- & -- & -- & -- \\
\hline 4 & $\begin{array}{l}\text { Diffusion } \\
\text { zone - light } \\
\text { etching. }\end{array}$ & 20.3 & 22.4 & 53.3 & 2.75 & 0.36 & -- & -- & -- & 0.82 & -- & -- & -- & -- & -- & -- & -- & -- \\
\hline 5 & Base Metal & 19.6 & 22.0 & 52.8 & 3.24 & 0.44 & 1.86 & -- & -- & 0.18 & -- & -- & -- & -- & -- & -- & -- & -- \\
\hline
\end{tabular}




\section{Appendix B}

\section{Analysis of Performance}

\section{Test Section C}

Modified 800H 


\section{Overview of Results: Modified 800H}

Analysis of Section A revealed that Modified $800 \mathrm{H}$ had sustained a maximum rate of 178 mils per year* of exposure to the corrosion-inducing service conditions within the Niles boiler. This resulted in a maximum measured wall reduction of 0.230 inches after 1.29 years of exposure within Section A. Time of exposure is based on time above a minimum average surface metal temperature of $1000 \mathrm{~F}$, not calendar time. So, given an initial wall thickness of about 0.400 inches, Section A results showed that one Modified $800 \mathrm{H}$ tube sample had lost almost 60 percent of its initial wall thickness after a relatively short exposure time. Consistent with this observation, shortly after Section A was removed from the boiler, the Modified $800 \mathrm{H}$ samples at location L-M were removed from both Sections B and C and replaced with a more corrosion resistant material. The Modified $800 \mathrm{H}$ samples at location E-F were replaced for both sections approximately six months later. For Section C, only one Modified $800 \mathrm{H}$ tube sample survived for the full length of time until the section was removed for evaluation, that was the sample at location Y-Z. This corresponded to the location within Section $\mathrm{C}$ having lowest average surface metal temperature for a Modified $800 \mathrm{H}$ sample.

It should be noted that the metal loss rates determined to date for both Section A and Section C all were based on a ring sample that was extracted from mid-length of each tube sample. In this analysis additional attention was focused on metal losses in the vicinity of welds. Representative samples were taken and it was determined that, for the Modified $800 \mathrm{H}$ samples evaluated, the Inconel 625 weld filler metal is reasonably resistant to coal ash corrosion and that the tube material immediately adjacent to the weld (i.e. the weld heat affected zone) was somewhat less affected by corrosion than was the remainder of the tube. Indeed the tube wall appeared to be progressively thinner toward the mid-point of the tube sample.

Due to the lack of deposit in the section examined, no deposit analysis was performed on modified $800 \mathrm{H}$, however, it is reasonable to infer that the same deposit was found everywhere on Section C. This being the case, it is likely that the same alkali-iron-sulfate that gave rise to attack elsewhere in the section caused the wastage observed for the modified $800 \mathrm{H}$.

Evaluation of the microstructure at the external scale/metal interface revealed that Modified $800 \mathrm{H}$ had developed a continuous adherent chromium-oxide scale covering the external surface of the tube. Similar results were found during the evaluation of the Modified $800 \mathrm{H}$ sample removed from Section A. The results of this assessment suggested that this material suffered rapid general metal loss that may be related to the degree of chromium depletion in the vicinity of the metal surface (as low as 9\%). The evidence suggested also that progressive spalling of the chromium oxide scale that adheres to the surface may also have played an important role in influencing the poor performance of this material.

* $\quad$ Note throughout this appendix, the reported metal loss rate was calculated based on time at temperature rather than total time within the boiler. 


\section{Material: Modified 800H}

\section{Summary of Findings}

\begin{tabular}{|c|c|}
\hline Issue & Discussion \\
\hline $\begin{array}{l}\text { Sample } \\
\text { Location and } \\
\text { Orientation. }\end{array}$ & $\begin{array}{l}\text { - In assessing the data for Section C, it is important to understand that } \\
\text { some tube samples had to be removed from the section early due to } \\
\text { excessive thinning. The schematic in Figure B-1 shows the relative } \\
\text { positions of the Modified } 800 \mathrm{H} \text { samples in all three sections. Also, } \\
\text { shown are the positions of all of the excessively thinned tubes that } \\
\text { were removed and replaced in order to extend the life of Section C. } \\
\text { Two modified } 800 \mathrm{H} \text { samples were removed early. Sample L-M was } \\
\text { removed in November } 2001 \text {. Sample E-F was removed in June } 2002 \text {. } \\
\text { Sample Y-Z survived for the full Section C exposure period. } \\
\text { The samples that were removed early were set aside for later analysis, } \\
\text { and have been included in the current evaluation along with the other } \\
\text { tube samples that made up Section C. } \\
\text { Also shown in this figure is the calculated average metal temperature } \\
\text { at each location. }\end{array}$ \\
\hline $\begin{array}{l}\text { Composition } \\
\text { Verification. }\end{array}$ & $\begin{array}{l}\text { - The table in Figure B-1 provides a reference composition from Oak } \\
\text { Ridge National Laboratory (ORNL) literature for this alloy. } \\
\text { ORNL provided material from three different heats of Modified } 800 \mathrm{H} \\
\text { for assessment in this program. The composition for each of the three } \\
\text { heats also is reported. } \\
\text { During the Section A evaluation, detailed composition verification was } \\
\text { undertaken for each tube sample that was removed from service. This } \\
\text { served, in part, to verify that the each tube material was located at its' } \\
\text { proper position within the test section. } \\
\text { Based on the good results for Section A, it was decided that it would } \\
\text { be unnecessary to analyze each and every tube sample for composition } \\
\text { verification. Rather, it was decided to select a representative sample } \\
\text { from each tube material type for confirmatory analysis. } \\
\text { During the Section A evaluation, the energy dispersive spectrographic } \\
\text { (EDS) capabilities of the scanning electron microscope (SEM) were } \\
\text { used to perform semi-quantitative analyses of each of the twelve } \\
\text { different candidate tube materials. Comparison of these EDS results } \\
\text { showed reasonably good correlation with results obtained using } \\
\text { quantitative analytical techniques, including optical emission } \\
\text { spectrometry. } \\
\text { Based on this, it was decided that the accuracy provided by EDS } \\
\text { would suffice for composition verification, but that more detailed } \\
\text { analyses would be undertaken if questions arose. } \\
\text { The results of the EDS analysis of tube sample E-F confirmed that the } \\
\text { correct material was used at this location. }\end{array}$ \\
\hline
\end{tabular}




\section{Material: Modified 800H}

\section{Summary of Findings, (continued)}

\begin{tabular}{|c|c|}
\hline Issue & Discussion \\
\hline $\begin{array}{l}\text { As-Received } \\
\text { Appearance }\end{array}$ & $\begin{array}{l}\text { - Figure B-3 documents the typical appearance of this material when it } \\
\text { was received from ORNL. } \\
\text { - Figures B-4 through B-6 document the as-received appearance of the } \\
\text { external surface of the tube samples after service exposure. Three } \\
\text { views are provided for each tube: } 12 \text { o'clock (the view that faces on- } \\
\text { coming gas flow), } 3 \text { o'clock (the view where the gas flow is tangent to } \\
\text { the surface), and } 6 \text { o'clock (the view that shows the back-side of the } \\
\text { tube relative to the gas flow direction. } \\
\text { - The reader should be alerted to the fact that, within the boiler, the gas } \\
\text { flows upward over these tubes. This implies that for the "o'clock" } \\
\text { conventions described above, (with the } 12 \text { o'clock orientation facing } \\
\text { the gas flow direction), the actual physical orientation of these tubes is } \\
\text { such that the } 12 \text { o'clock side of the tube faces downward, and the } 6 \\
\text { o'clock orientation faces upward within the boiler. } \\
\text { External tube wastage is visible for all three samples; however, this is } \\
\text { more clearly shown in the tube silhouette images that follow. } \\
\text { A relatively thick rust-red scale and deposit build-up is seen on } \\
\text { Samples L-M and Y-Z. This build up is not in evidence on Sample E- } \\
\text { F. It is believed that the build-up was removed (perhaps for tube } \\
\text { inspection purposes) after the tube was removed from service. } \\
\text { The gray/white scale associated with molten alkali-iron sulfate attack } \\
\text { can be found at the } 12 \text { and } 3 \text { o'clock positions for Sample Y-Z. }\end{array}$ \\
\hline $\begin{array}{l}\text { Grit-Blasted } \\
\text { Surface } \\
\text { Appearance }\end{array}$ & $\begin{array}{l}\text { Figures B-7 through B-9 show the appearance of the external surface } \\
\text { of the Modified } 800 \mathrm{H} \text { tube samples after grit-blasting to remove } \\
\text { deposit and scale. The } 12 \text { o'clock and } 3 \text { o'clock positions for all three } \\
\text { samples show the appearance of general wastage due to fireside } \\
\text { corrosion. } \\
\text { - The } 6 \text { o'clock position for all three tube samples shows that evidence } \\
\text { of shallow but extensive pitting on the back-side. }\end{array}$ \\
\hline $\begin{array}{l}\text { Wall } \\
\text { Thickness } \\
\text { Loss } \\
\text { Determined }\end{array}$ & $\begin{array}{l}\text { - Tube cross-sections were prepared to allow for measurement of wall } \\
\text { thickness to determine metal loss due to exposure. These are shown in } \\
\text { silhouette in Figures B-10 through B-12. } \\
\text { The same relative "o'clock" positions were maintained as discussed } \\
\text { earlier. Measurements were taken at eight evenly spaced locations } \\
\text { about the tube circumference. These were then compared with } \\
\text { measurements taken at these same locations prior to exposure. } \\
\text { - The before and after measurements were documented in the table that } \\
\text { accompanies each figure. These form the basis for the "delta" } \\
\text { calculation to determine the amount of wall thickness lost. } \\
\text { For all three tube samples, the minimum wall thickness in the cross } \\
\text { section was not at one of the eight predetermined locations. In these } \\
\text { cases, there was no "before" measurement for comparison. So, the } \\
\text { average "before" wall thickness was used to calculate the wall } \\
\text { thickness lost at these off locations. }\end{array}$ \\
\hline
\end{tabular}




\section{Material: Modified 800H}

\section{Summary of Findings, (continued)}

\begin{tabular}{|c|c|}
\hline Issue & Discussion \\
\hline $\begin{array}{l}\text { Rate of Wall } \\
\text { Thickness } \\
\text { Loss Versus } \\
\text { Average } \\
\text { Surface Metal } \\
\text { Temperature }\end{array}$ & $\begin{array}{l}\text { - The chart in Figure B-13 plots the maximum rate of wall thickness lost } \\
\text { against the calculated average surface metal temperature for the three } \\
\text { Modified } 800 \mathrm{H} \text { samples in Section C. Similar data from Section A } \\
\text { also are provided on this chart. } \\
\text { - Review of these data reveals a relatively high rate of metal loss for all } \\
\text { locations, with one sample (Sample Y-Z from Section C) evidencing a } \\
\text { somewhat lower rate. } \\
\text { - For Section C, the rate of metal loss increased with temperature. These } \\
\text { are similar to the results for Section A with the exception that, for } \\
\text { Section A, the rate at } 1150 \mathrm{~F} \text { was somewhat higher that that at } 1174 \mathrm{~F} \text {. } \\
\text { It also was noted that Sample Y-Z experienced a much higher rate of } \\
\text { metal loss in Section A than it did in Section C. } \\
\text { These inconsistencies are noted, however, they are not surprising } \\
\text { given the relatively limited sampling of this program. Furthermore, it's } \\
\text { pointed out that all of these data suggest an objectionably high rate for } \\
\text { Modified } 800 \mathrm{H} \text { over the temperature range in question. }\end{array}$ \\
\hline $\begin{array}{l}\text { Wall } \\
\text { Thickness } \\
\text { Loss as a } \\
\text { Function of } \\
\text { Time and } \\
\text { Location }\end{array}$ & $\begin{array}{l}\text { - The chart in Figure B-14 plots metal loss as a function of time for each } \\
\text { sample location for Sections A and C. with samples from Section C } \\
\text { having experienced generally longer exposure times. } \\
\text { - Shortly after Section A was removed for analysis, the tube Sample L- } \\
\text { M was also removed and replaced in Section C due to excessive metal } \\
\text { loss. } \\
\text { Metal losses in Sample E-F as a function of time provide the reason } \\
\text { that it was removed early from Section C. } \\
\text { - In Section C, Sample Y-Z lost less wall thickness in } 28.5 \text { months of } \\
\text { exposure, than it did in Section A in } 18.6 \text { months. This was in spite of } \\
\text { the fact that the average surface metal temperature for Sample Y-Z in } \\
\text { Section C exceeded that of the same sample location in Section A } \\
\text { (1099 F vs } 1088 \mathrm{~F}) \text {. }\end{array}$ \\
\hline $\begin{array}{l}\text { Metallurgical } \\
\text { Analysis - } \\
\text { SEM }\end{array}$ & $\begin{array}{l}\text { Sample E-F was selected for a detailed SEM/EDS assessment of the } \\
\text { constituents and structure in the vicinity of the external and internal } \\
\text { surfaces. This sample was selected based on the fact that it had lost the } \\
\text { most wall thickness of all of the Modified } 800 \mathrm{H} \text { samples. The focus of } \\
\text { this investigation was in the region of the tube where the maximum } \\
\text { amount of material was lost. }\end{array}$ \\
\hline
\end{tabular}




\section{Material: Modified 800H}

\section{Summary of Findings, (continued)}

\begin{tabular}{|c|c|}
\hline Issue & Discussion \\
\hline $\begin{array}{l}\text { Metallurgical } \\
\text { Analysis - } \\
\text { SEM, } \\
\text { Continued. }\end{array}$ & $\begin{array}{l}\text { - Figures B-15a, and -15b show the secondary electron images of the } \\
\text { scale/metal interface for Sample E-F. Three distinct regions are in } \\
\text { evidence. These regions are, starting within the metal and working out: } \\
\text { Base metal - oxalic acid etch delineates grain boundaries and } \\
\text { carbides. } \\
\text { Deeply etching base metal - this region is obvious as a light- } \\
\text { appearing band that is parallel to the metal surface, perhaps } \\
\text { see most clearly in Figure B-15b (see the area marked with a } \\
\text { "2" in this photo). This band is much less than one-half grain } \\
\text { wide, and affects only those grains contiguous with the } \\
\text { surface. This deeper etch likely reflects composition } \\
\text { differences due to diffusion in this region. EDS will later show } \\
\text { that this zone is depleted in chromium. } \\
\text { Thin adhering scale - this is seen continuous along the } \\
\text { surface. EDS analysis will later show that this is } \\
\text { predominantly chromium-oxide. } \\
\text { - Figure B-16 shows the microstructure at mid-wall in Sample E-F. } \\
\text { Figure B-17 shows the appearance of the internal metal surface for } \\
\text { Sample E-F. A very thin oxide scale is noted along the surface. This } \\
\text { region seems relatively unaffected due to service exposure. }\end{array}$ \\
\hline $\begin{array}{l}\text { Metallurgical } \\
\text { Analysis - } \\
\text { Weld Cross } \\
\text { Section. }\end{array}$ & $\begin{array}{l}\text { - Figure B-18 shows a longitudinal profile (at the } 12 \text { o'clock } \\
\text { orientation) of a Modified } 800 \mathrm{H} \text { sample adjacent to weld " } \mathrm{Z} \text { " in } \\
\text { Section C, see Figure F-1 for locations } \\
\text { An Inconel } 625 \text { weld metal was used throughout Section C. The cross } \\
\text { sections show that the TP } 310 \text { Ta heat affected zones are not } \\
\text { preferentially attached, in fact, the wall thickness for the tube sample } \\
\text { tapers from the weld toward the middle of the tube length. }\end{array}$ \\
\hline $\begin{array}{l}\text { EDS Analysis } \\
\text { Results }\end{array}$ & $\begin{array}{l}\text { - The table in Figure B-19 provides a summary of the EDS analyses of } \\
\text { the locations numbered in Figure B-15b. Review suggests: } \\
\text { - Area 1: For the scale at the out surface (See Figure 15b) - There } \\
\text { was no evidence of the alkali metals. Nor were sulfur and } \\
\text { phosphorous present. The scale is comprised primarily of a } \\
\text { chromium-rich oxide. } \\
\text { - Area 2: For the deeply diffusion zone in the metal at the interface } \\
\text { (See Figure 15b) - This zone is depleted in chromium relative to } \\
\text { the matrix (roughly } 9 \% \text { as opposed to } 20 \% \text { ). This zone also was } \\
\text { noted to be depleted in vanadium and to some extent silicon. } \\
\text { Area 3: Base metal - The EDS base metal analysis were used to } \\
\text { confirm that the correct alloy had been used in this location. The } \\
\text { results compared well with the results of the spectrographic } \\
\text { analysis of this grade of material (as per the Section A report). }\end{array}$ \\
\hline
\end{tabular}




\section{Gas Flow}

Direction

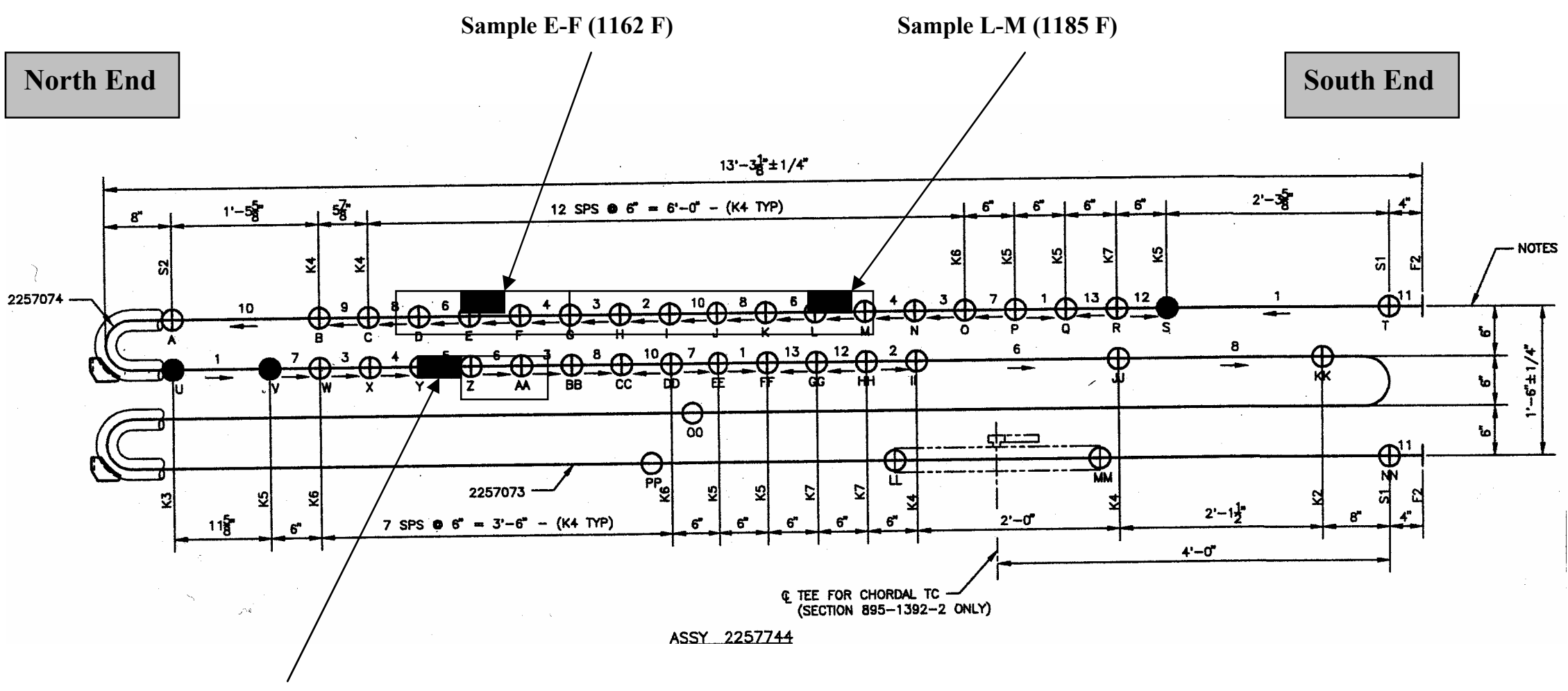

Sample Y-Z (1099 F)

\section{Figure B-1: Schematic Drawing Showing Position of Modified 800H Tube Samples Within Section C}

1. Drawing shown in same relative orientation as per the actual installation.

2. This group of tube samples was removed from service in November 2001 - highlighted in red.

3. This group of tube samples was removed from service in June 2002 - highlighted in yellow 


\section{Figure B-2 Semi-Quantitative Composition Verification for Modified 800H}

Description: $\quad$ Nominal $20 \mathrm{Cr} / 30 \mathrm{Ni}$ steel with improved creep strength due to addition of V, Nb, Ti, and B

Source:

Reference Composition: "Target" Chemistry per ORNL (Reference 1).

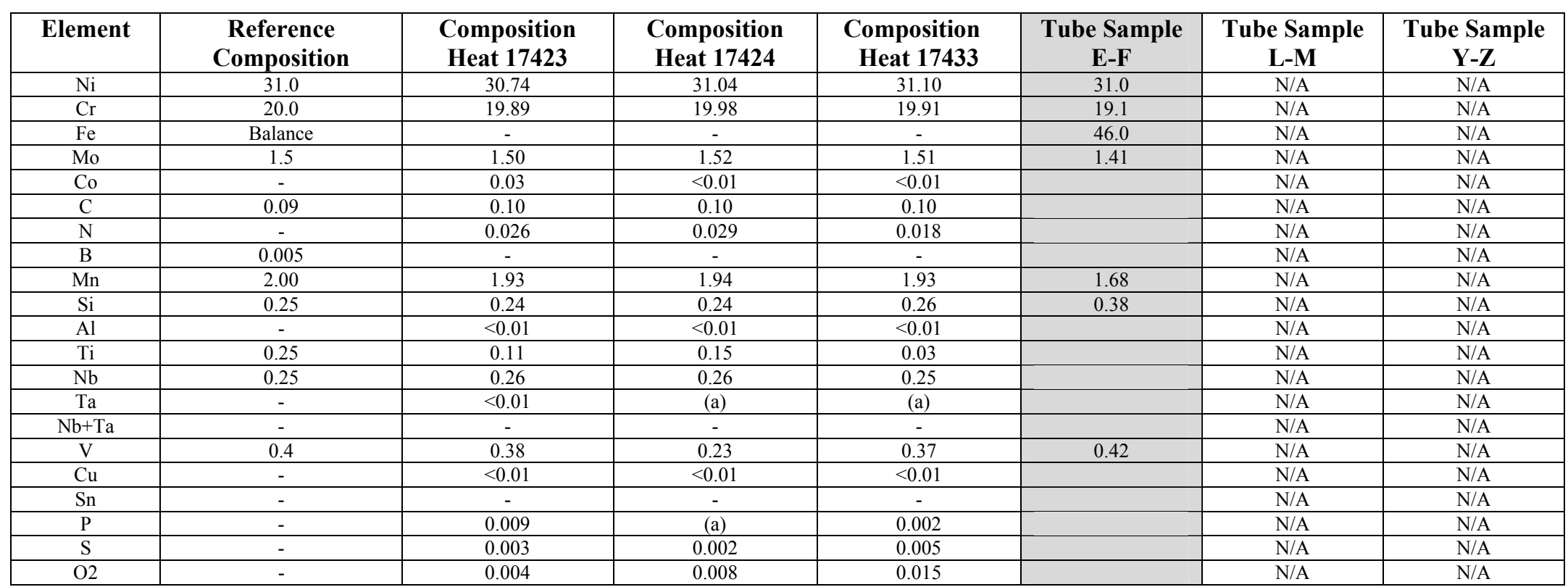

a- Not analyzed.

b- By difference.

Reference 1:

"Fabrication of Test Tubes for Coal Ash Corrosion Testing”, Sikka, V.K., Swindeman, R.W.,

Wright, I.G., Judkins, R.R., Johnson, R., Proc, $13^{\text {th }}$ Annual Conf. on Fossil Energy Mat'ls, May 1999.

Note: $\quad$ Sample E-F was from Heat 17433

Sample L-M was from Heat 17423

Sample Y-Z was from Heat 17424

$\mathrm{N} / \mathrm{A}=$ Tube sample not analyzed. 


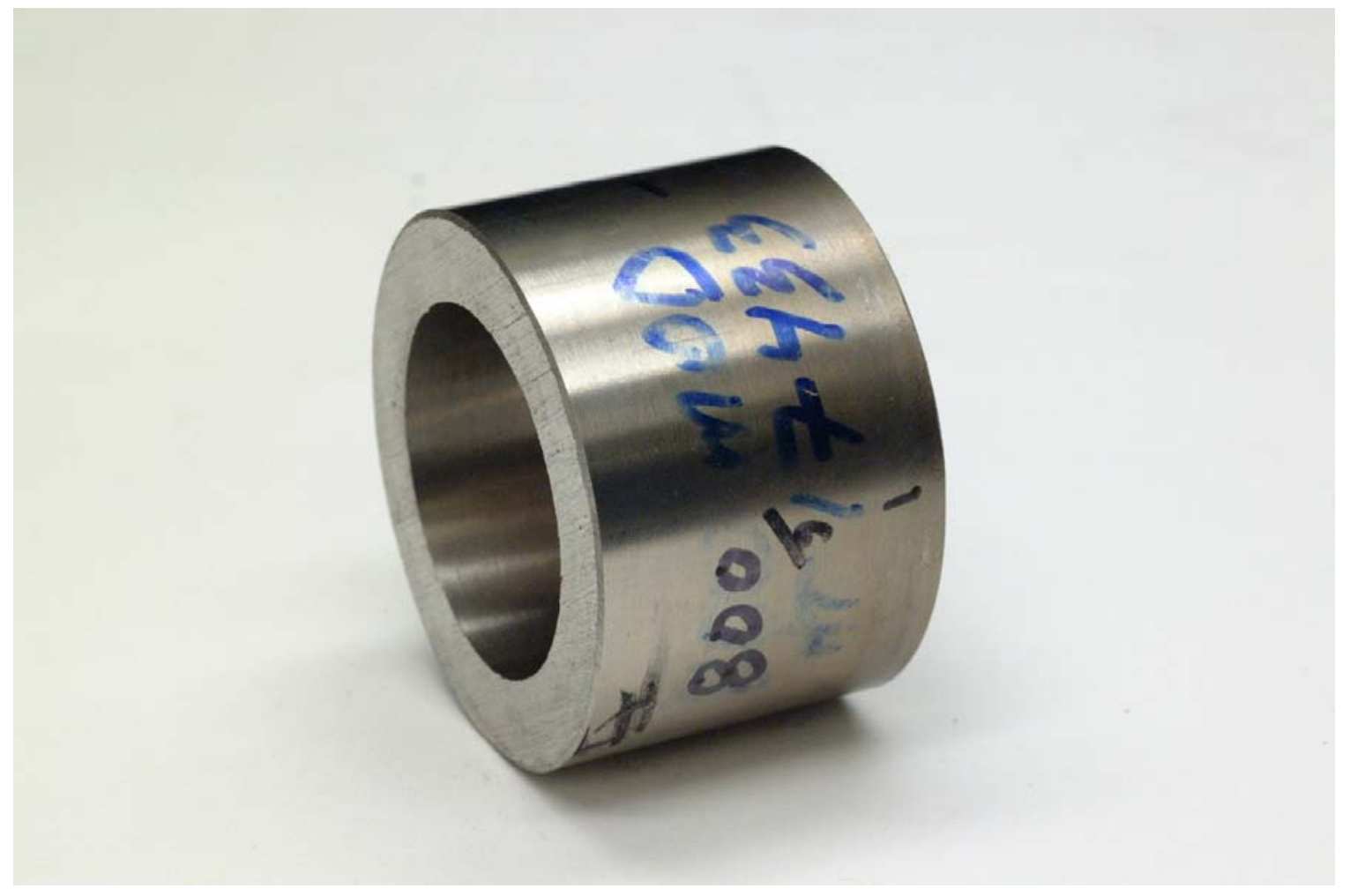

Figure B-3: Appearance of Modified $800 \mathrm{H}$ Tube Sample Prior to Service 


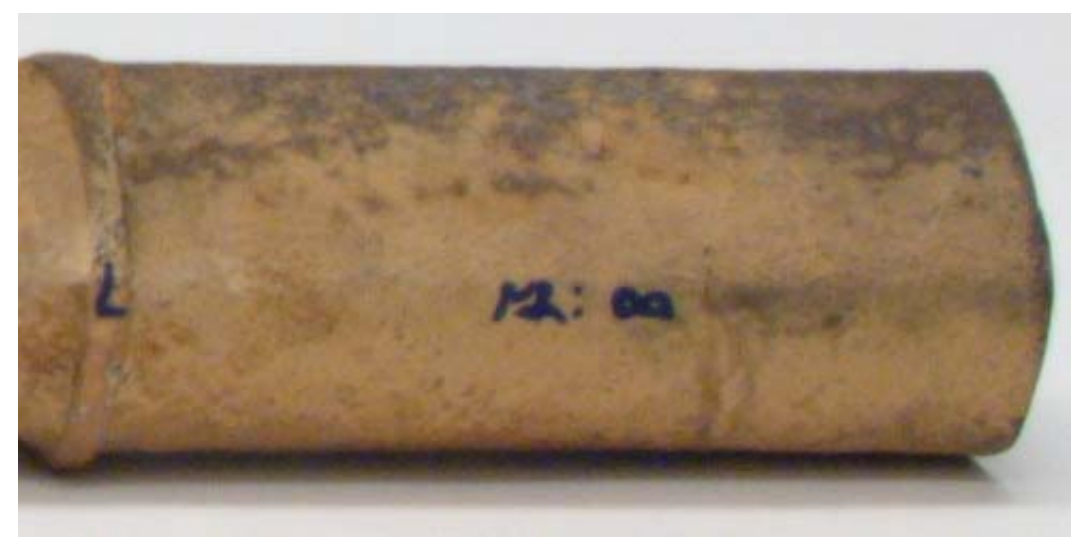

12 O'Clock - Facing Gas Flow

(Note: 3 o'clock is up in this image)

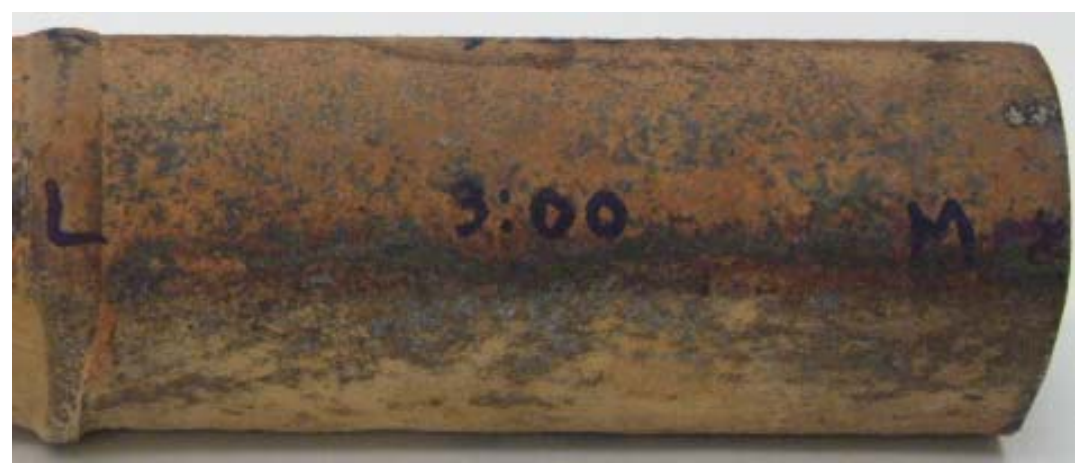

3 O'Clock

(Note: 6 o'clock is up in this image)

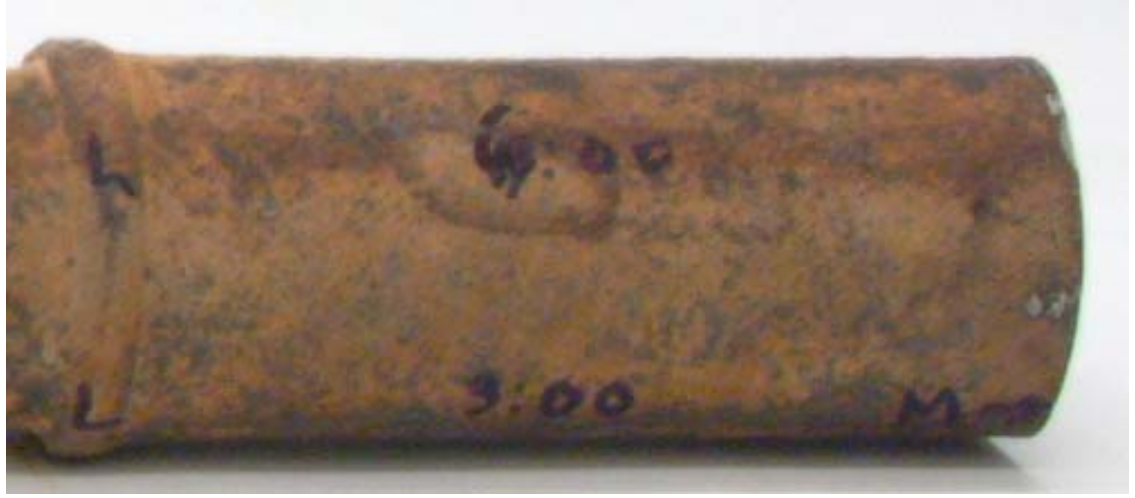

6 O'Clock - Opposite Gas Flow

Figure B-4 As-Received Appearance of Sample L-M (Modified 800H)

(Average metal temperature: 1185 F)

Note: this tube sample was removed from service on 11/6/01 


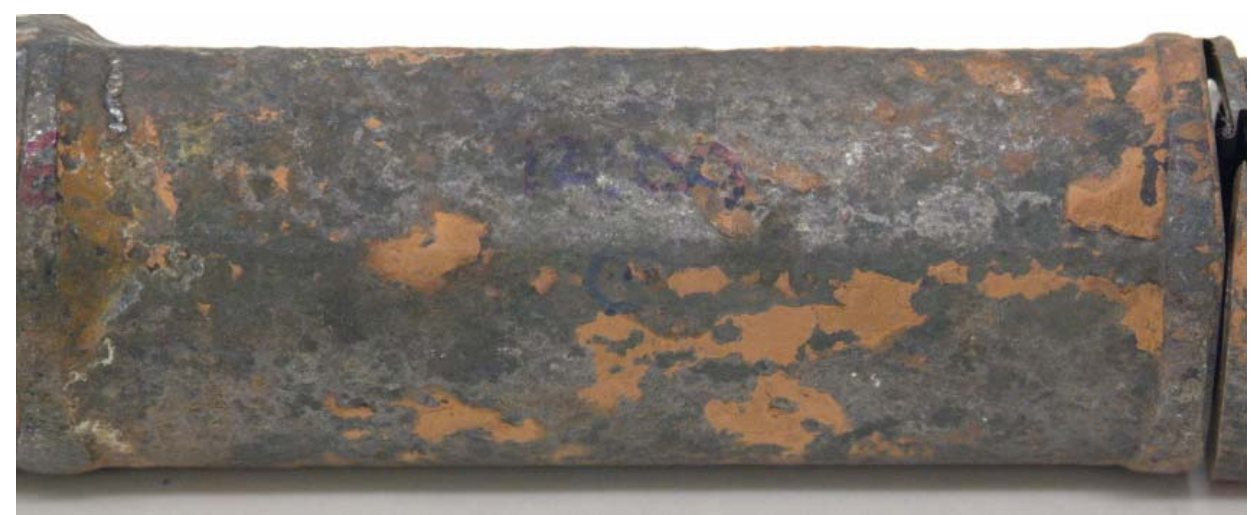

12 O'Clock - Facing Gas Flow

(Note: 3 o'clock is up in this image)

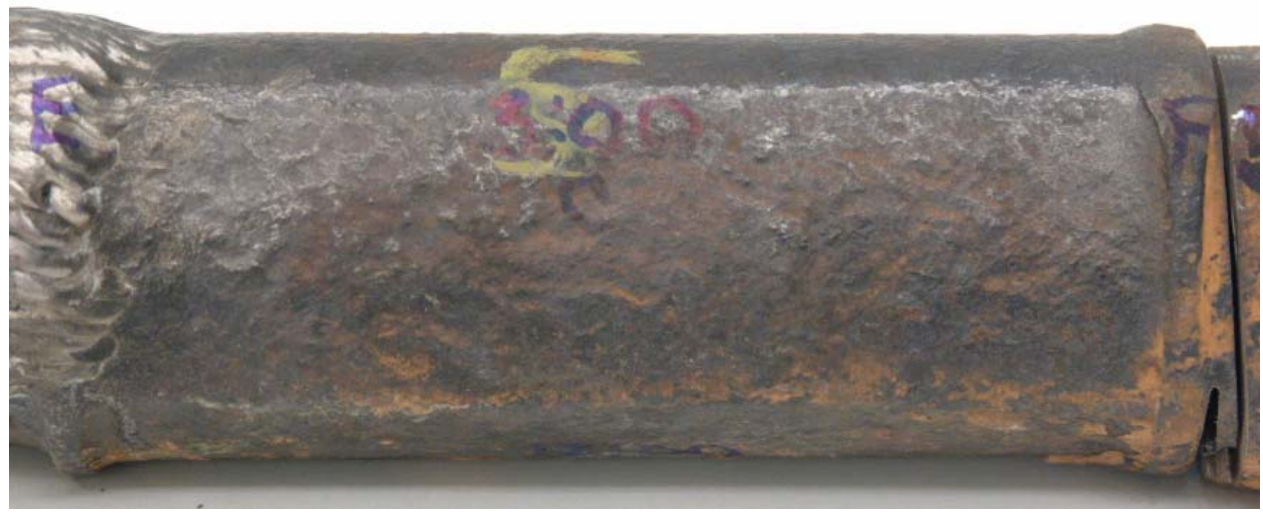

3 O'Clock

(Note: 6 o'clock is up in this image)

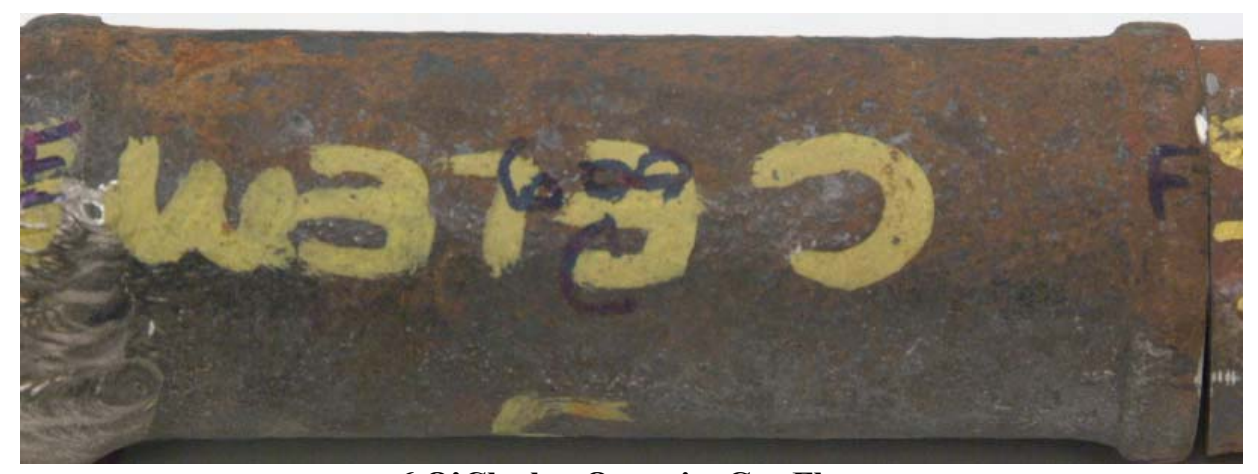

6 O'Clock - Opposite Gas Flow

Figure B-5 As-Received Appearance of Sample E-F (Modified 800H)

(Average metal temperature: $1162 \mathrm{~F}$ )

Note: this tube sample was removed from service on 6/10/02) 


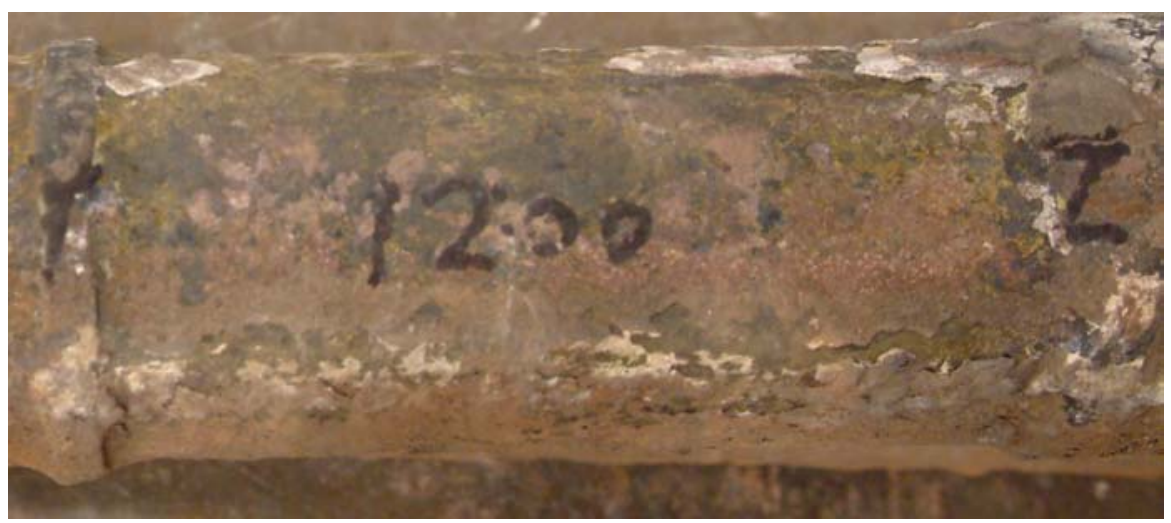

12 O'Clock - Facing Gas Flow

(Note: 3 o'clock is up in this image)

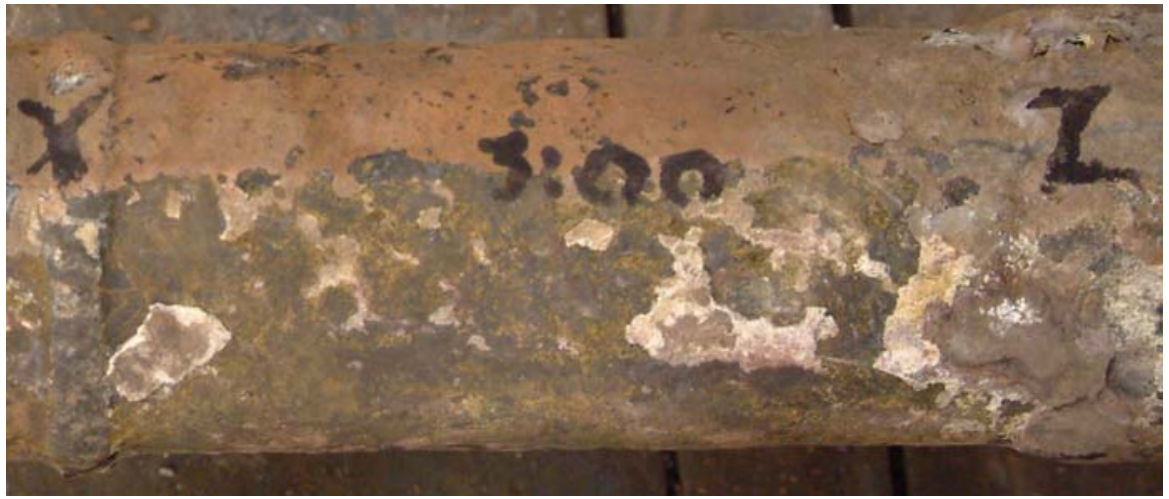

3 O'Clock

(Note: 6 o'clock is up in this image)

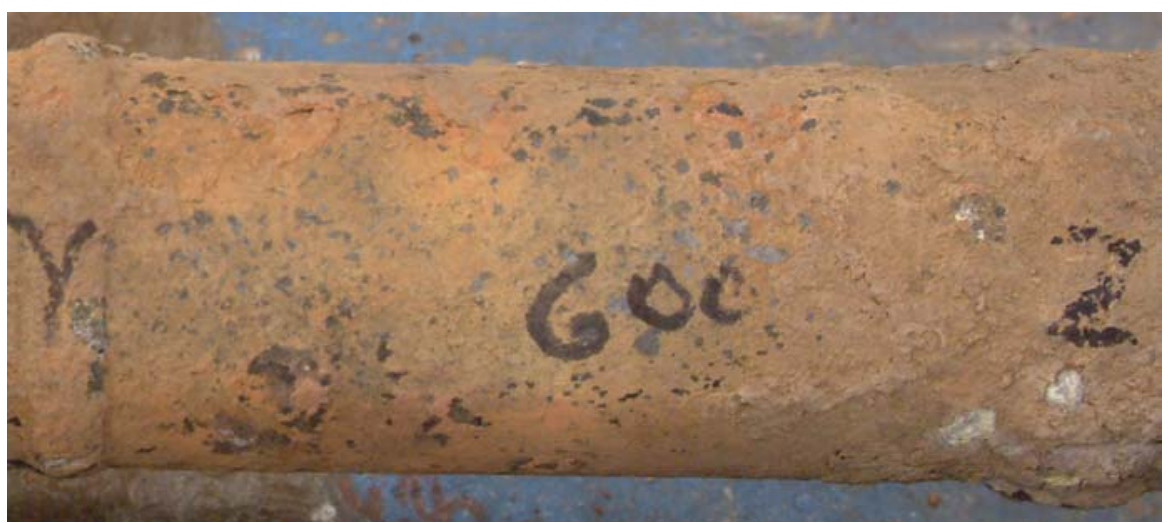

6 O'Clock - Opposite Gas Flow

Figure B-6 As-Received Appearance of Sample Y-Z (Modified 800H)

(Average metal temperature: 1099 F) 
12 O'Clock

(198 mils lost)

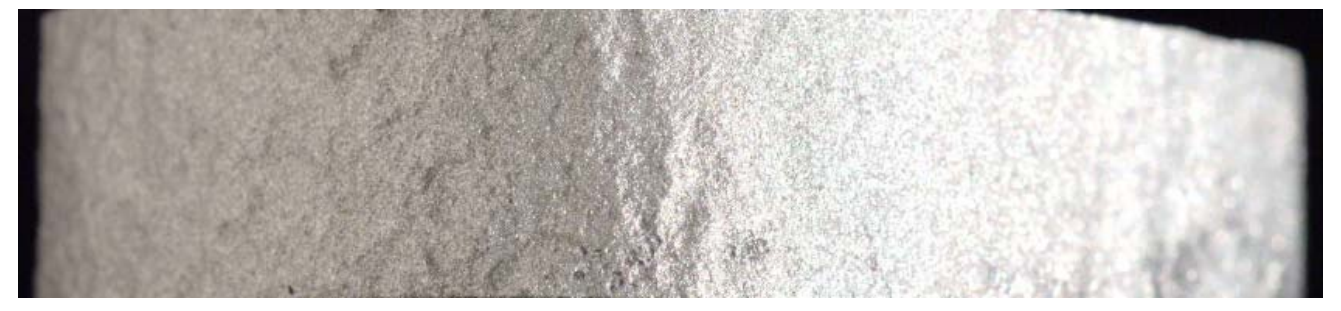

3 O'Clock

(200 mils lost)

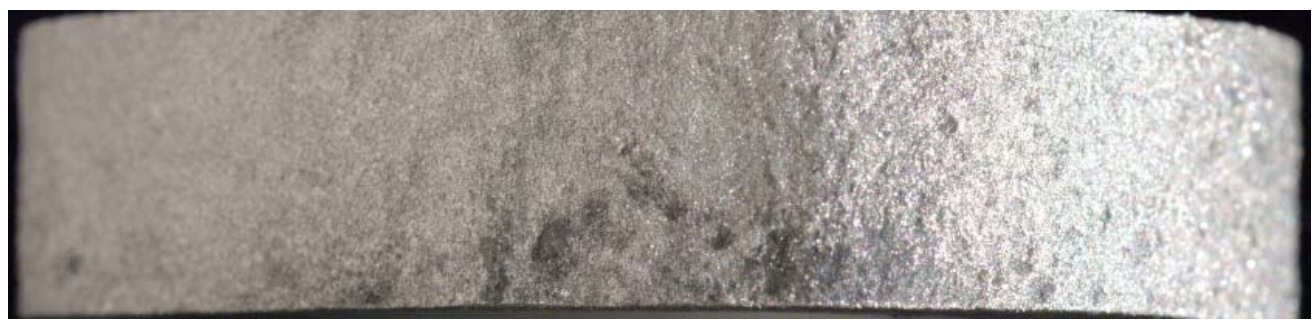

6 O'Clock

(5 mils lost)

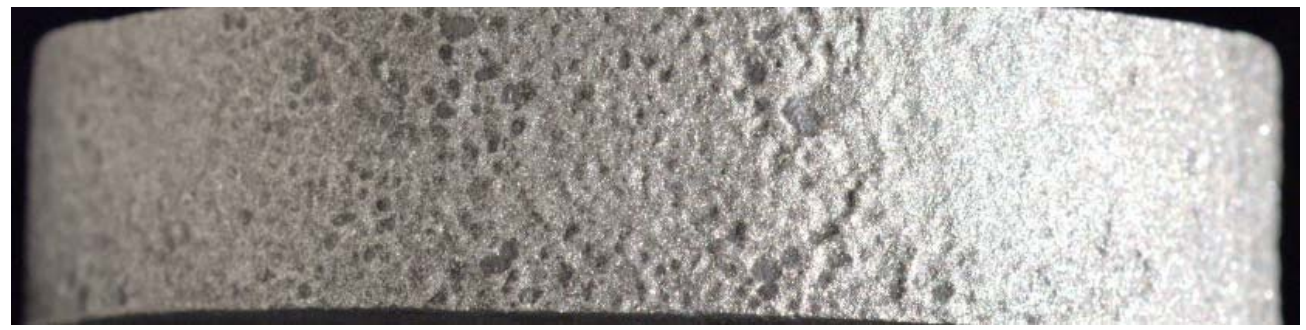

Figure B-8 OD Surface Appearance After Grit Blast

Material: $\quad$ Modified 800H

Sample: $\quad$ L-M 
12 O'Clock

(220 mils lost)

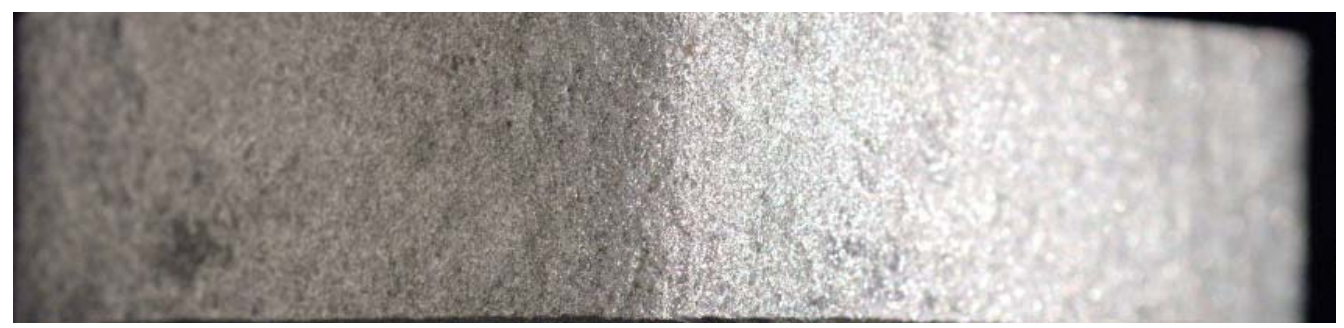

3 O'Clock

(251 mils lost)

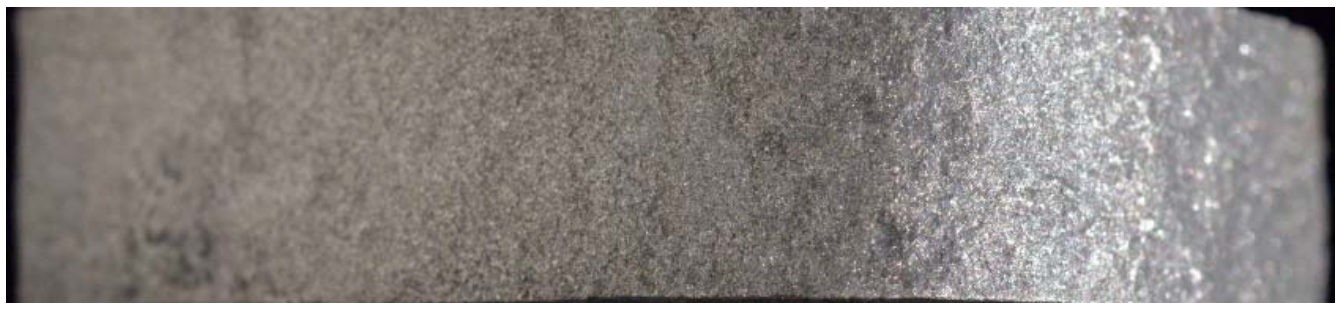

6 O'Clock

(12 mils lost)

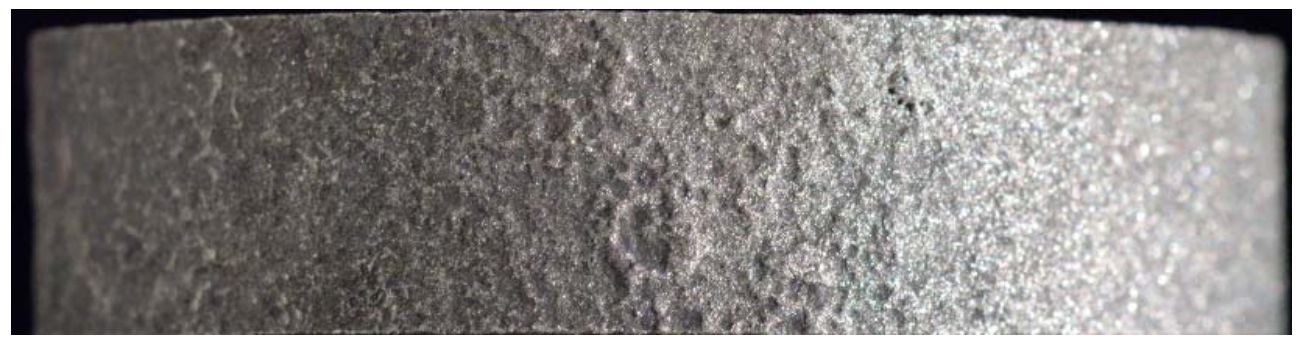

Figure B-8 OD Surface Appearance After Grit Blast

Material: $\quad$ Modified 800H

Sample: E-F 
12 O'Clock

(149 mils lost)

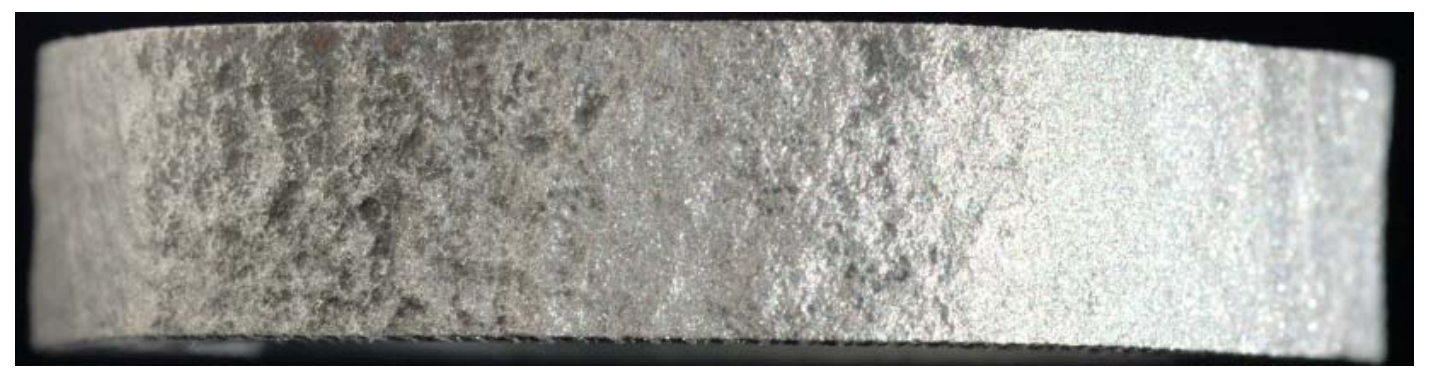

3 O'Clock

(107 mils lost)

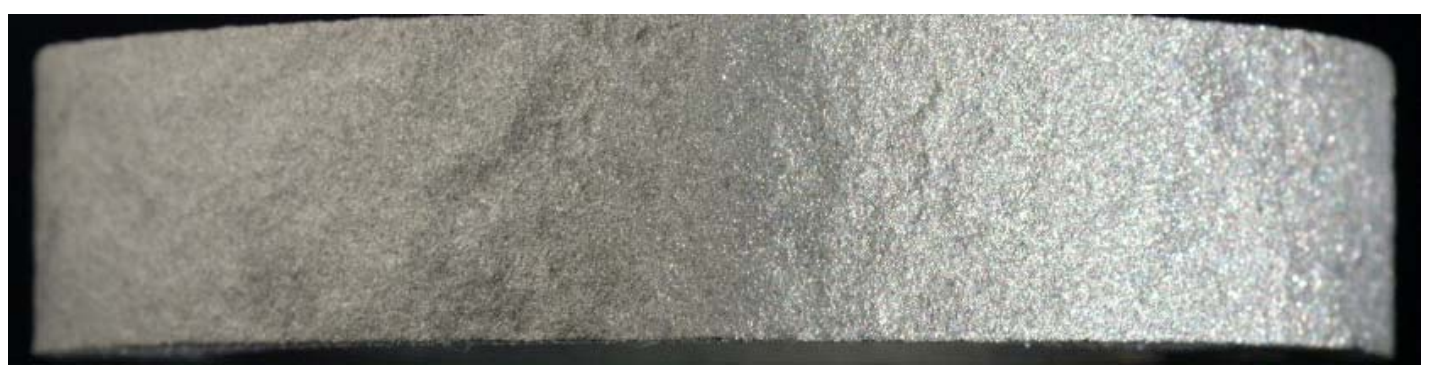

6 O'Clock

(10 mils lost)

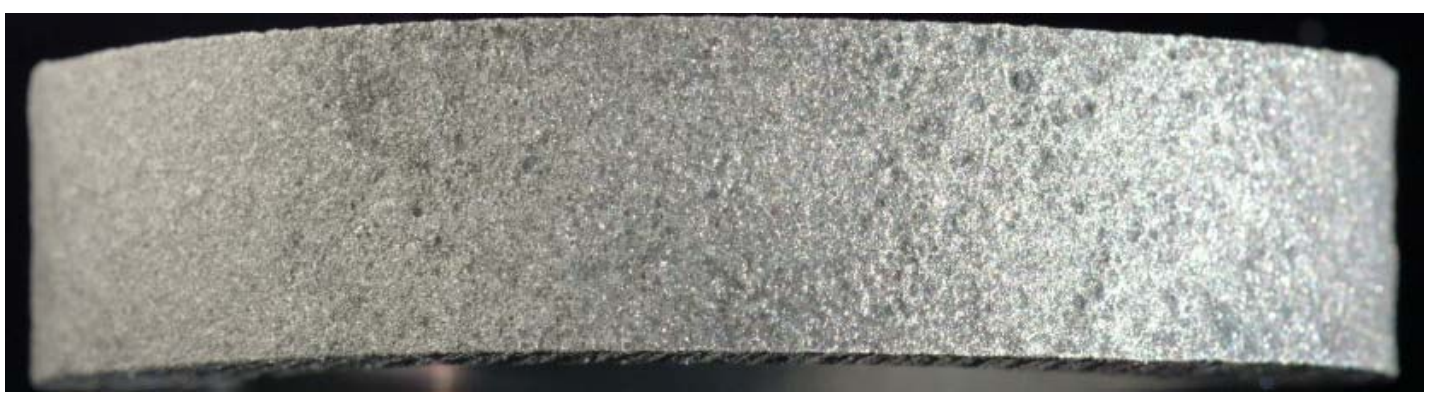

Figure B-9 OD Surface Appearance After Grit Blast

Material: Modified 800H

Sample: $\quad$ Y-Z 


\section{Material:}

Sample Location:

Maximum Rate:

Maximum Loss Orientation:

Average metal Temperature:

\section{Modified 800H}

L-M

$183 \mathrm{mils} / \mathrm{yr}$

Approx. 2:30 o'clock

$1185 \mathrm{~F}$

\section{O’Clock}

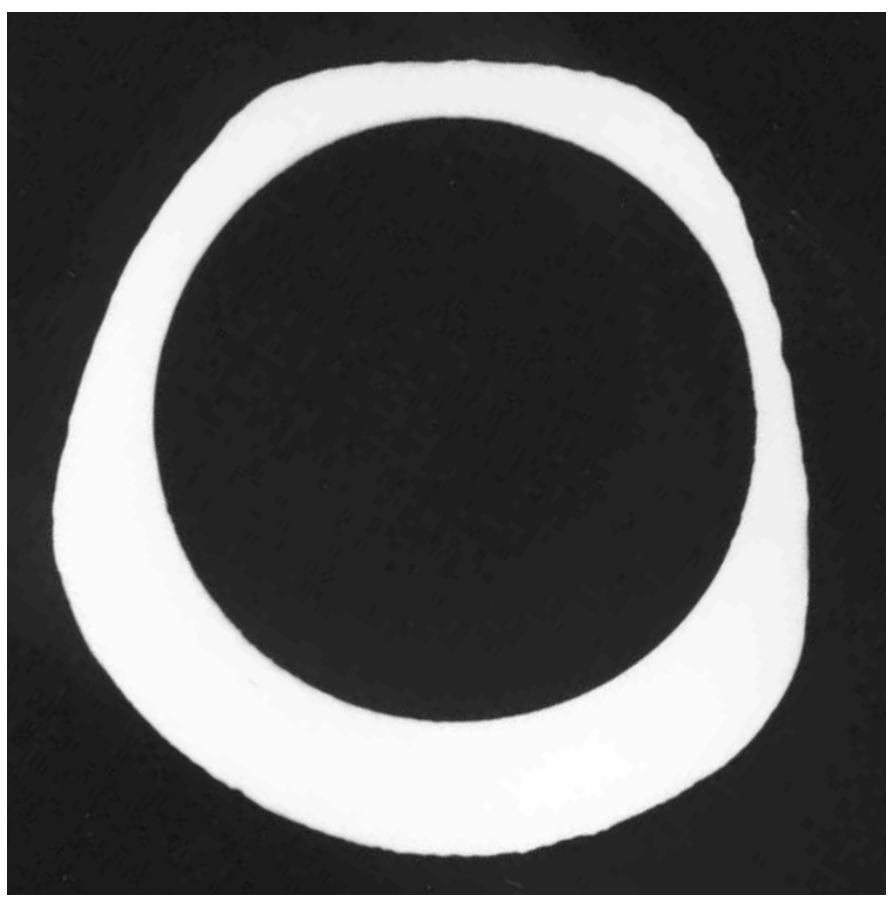

3 O'Clock

Approx. 1.2X

Wall Reduction by Position (in.)

\begin{tabular}{|l|c|c|c|c|c|c|c|c|c|c|}
\hline & $\begin{array}{c}\mathbf{1 2} \\
\text { o'clock }\end{array}$ & $\mathbf{1 : 3 0}$ & $\mathbf{3 : 0 0}$ & $\mathbf{4 : 3 0}$ & $\mathbf{6 : 0 0}$ & $\mathbf{7 : 3 0}$ & $\mathbf{9 : 0 0}$ & $\mathbf{1 0 : 3 0}$ & AVG & $\begin{array}{c}\text { Max } \\
\text { Los }^{*}\end{array}$ \\
\hline Before & 0.400 & 0.399 & 0.398 & 0.398 & 0.398 & 0.399 & 0.400 & 0.400 & 0.399 & 0.399 \\
\hline After & 0.202 & 0.134 & 0.198 & 0.380 & 0.393 & 0.375 & .0190 & 0.233 & & 0.115 \\
\hline Delta & 0.198 & 0.265 & 0.200 & 0.018 & 0.005 & 0.024 & 0.210 & 0.167 & & 0.284 \\
\hline
\end{tabular}

Figure B-10: Silhouette and Wall Thickness Measurements for Sample L-M

Note: this sample was removed from service on 11/6/01.

* Measurement taken at "maximum loss orientation" noted above. The average

"before" wall thickness was used to calculate the "delta". 


\section{Material:}

Sample Location:

Maximum Rate:

Maximum Loss Orientation:

Average metal Temperature:

\section{Modified 800H}

E-F

$176 \mathrm{mils} / \mathrm{yr}$

Approx. 2:30 o'clock

$1162 \mathrm{~F}$

\section{O’Clock}

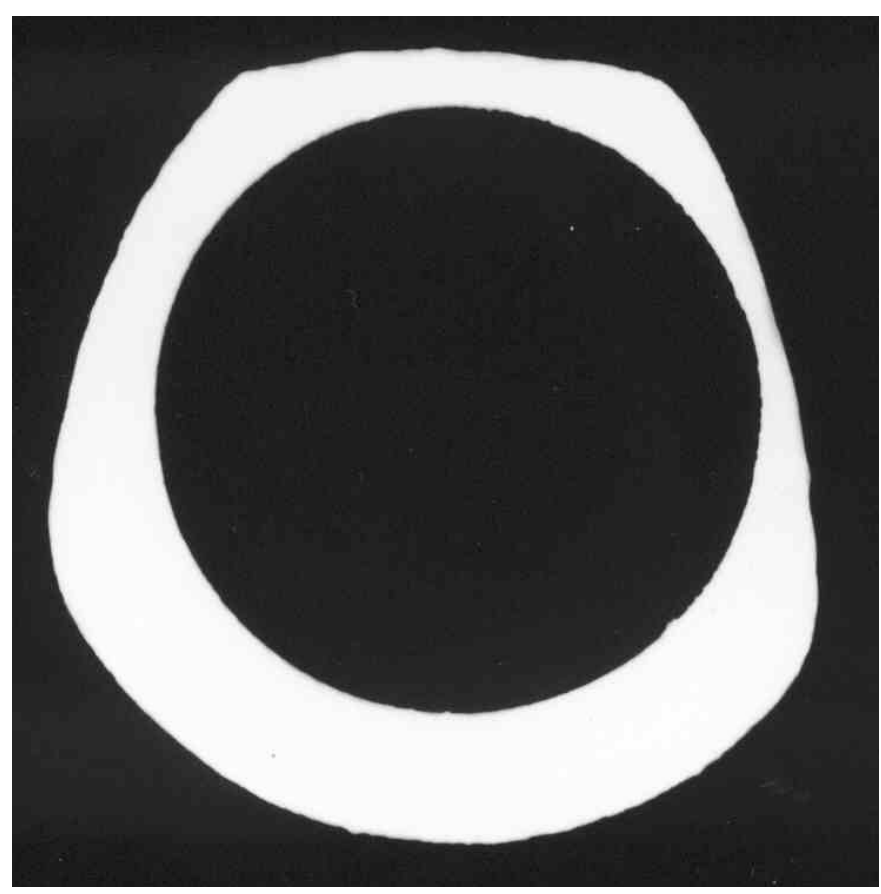

3 O'Clock

Approx. 1.2X

\section{Wall Reduction by Position (in.)}

\begin{tabular}{|l|c|c|c|c|c|c|c|c|c|c|}
\hline & $\begin{array}{c}12 \\
\text { o'clock }\end{array}$ & $\mathbf{1 : 3 0}$ & $\mathbf{3 : 0 0}$ & $\mathbf{4 : 3 0}$ & $\mathbf{6 : 0 0}$ & $\mathbf{7 : 3 0}$ & $\mathbf{9 : 0 0}$ & $\mathbf{1 0 : 3 0}$ & AVG & $\begin{array}{c}\text { Max } \\
\text { Loss }\end{array}$ \\
\hline Before & 0.396 & 0.398 & 0.400 & 0.400 & 0.400 & 0.403 & 0.400 & 0.398 & 0.399 & 0.399 \\
\hline After & 0.176 & 0.132 & 0.149 & 0.382 & 0.388 & 0.383 & 0.236 & 0.281 & & 0.083 \\
\hline Delta & 0.220 & 0.266 & 0.251 & 0.018 & 0.012 & 0.020 & 0.164 & 0.117 & & 0.316 \\
\hline
\end{tabular}

Figure B-11: Silhouette and Wall Thickness Measurements for Sample E-F

Note: this tube sample was removed from service on 6/10/02.

* Measurement taken at "maximum loss orientation" noted above. The average

"before" wall thickness was used to calculate the "delta". 


\section{Material:}

Sample Location:

Maximum Rate:

Maximum Loss Orientation:

Average metal Temperature:

\section{Modified 800H}

Y-Z

79 mils/yr

Approx. 9:30 o'clock 1099 F

\section{O'Clock}

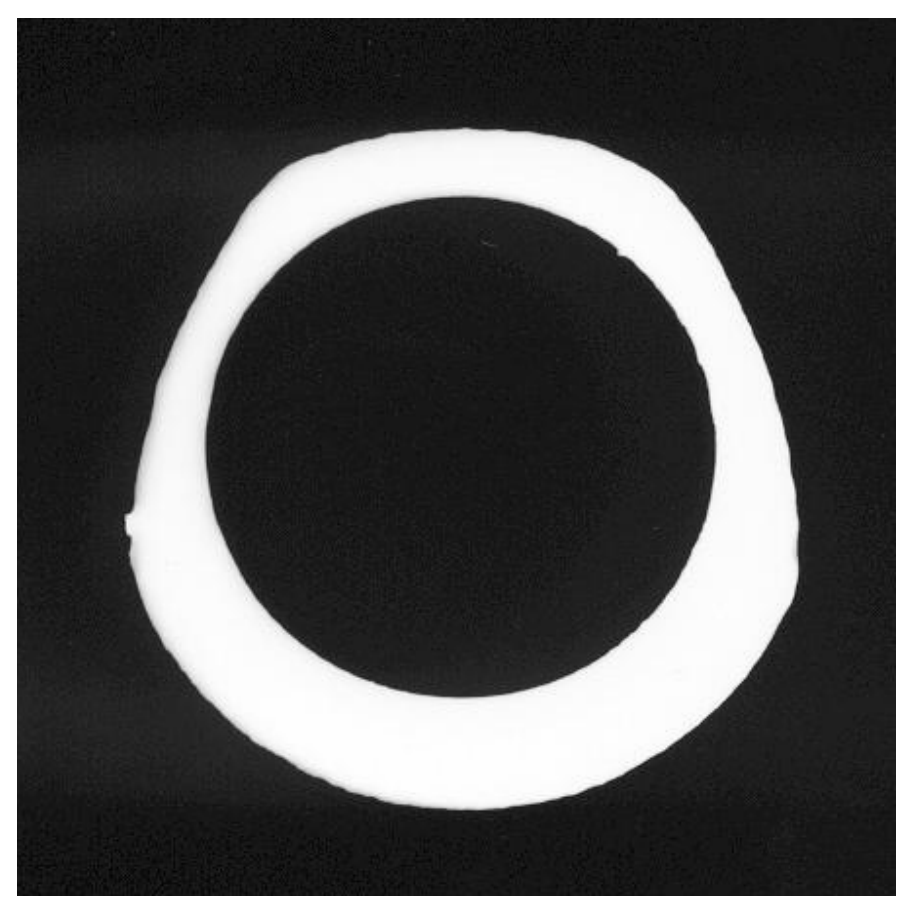

3 O’Clock

Approx. 1.2X

Wall Reduction by Position (in.)

\begin{tabular}{|l|c|c|c|c|c|c|c|c|c|c|}
\hline & $\begin{array}{c}\mathbf{1 2} \\
\text { o'clock }\end{array}$ & $\mathbf{1 : 3 0}$ & $\mathbf{3 : 0 0}$ & $\mathbf{4 : 3 0}$ & $\mathbf{6 : 0 0}$ & $\mathbf{7 : 3 0}$ & $\mathbf{9 : 0 0}$ & $\mathbf{1 0 : 3 0}$ & AVG & $\begin{array}{c}\text { Max }^{*} \\
\text { Loss }\end{array}$ \\
\hline Before & 0.402 & 0.401 & 0.401 & 0.400 & 0.400 & 0.401 & 0.401 & 0.401 & 0.401 & 0.401 \\
\hline After & 0.253 & 0.226 & 0.294 & 0.389 & 0.390 & 0.387 & 0.233 & 0.294 & & 0.212 \\
\hline Delta & 0.149 & 0.175 & 0.107 & 0.011 & 0.010 & 0.014 & 0.168 & 0.107 & & 0.189 \\
\hline
\end{tabular}

Figure B-12: Silhouette and Wall Thickness Measurements for Sample Y-Z

* Measurement taken at "maximum loss orientation" noted above. The average

"before" wall thickness was used to calculate the "delta". 


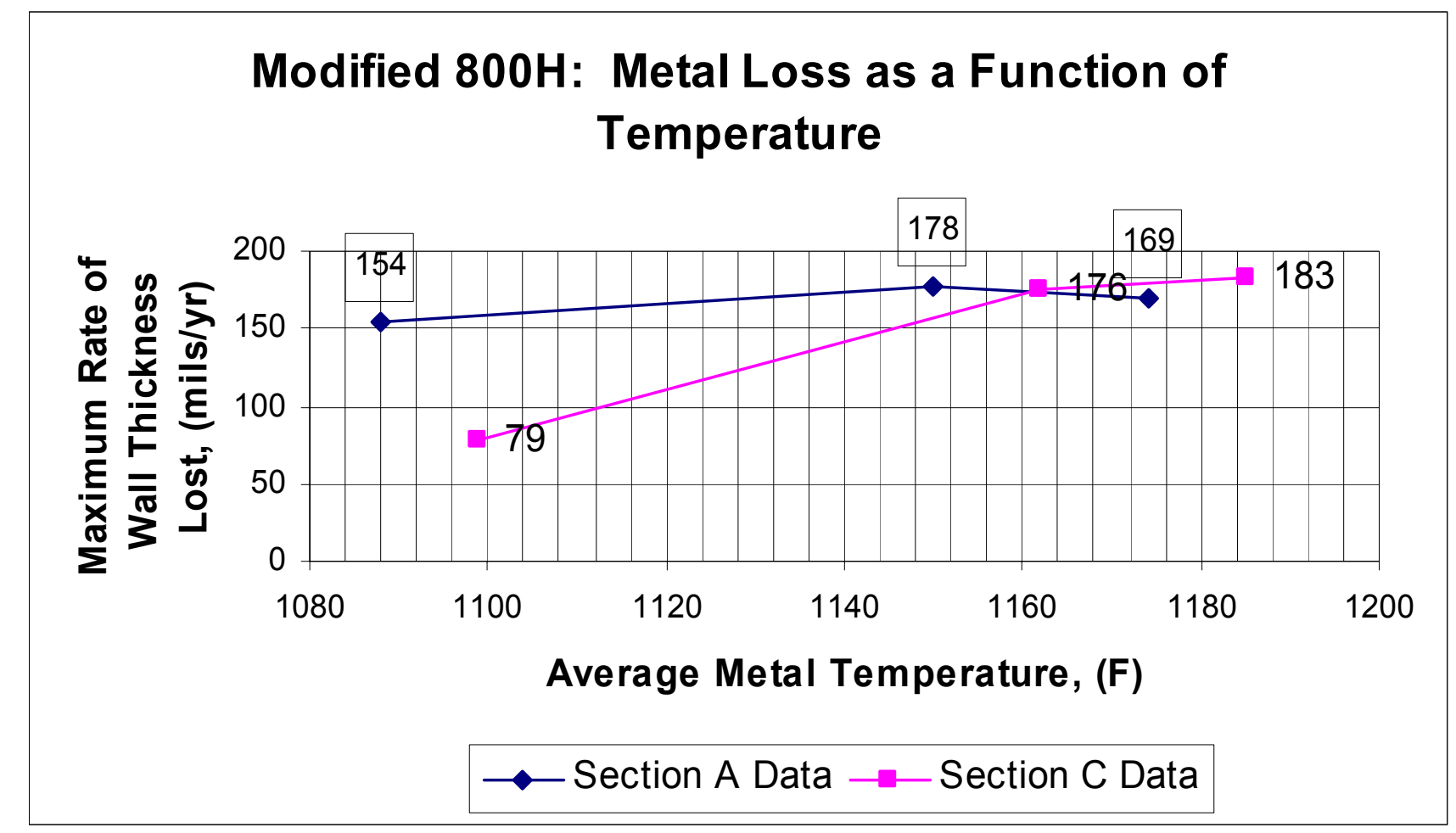

Figure B-13: Metal Loss Rate as a Function of Temperature

\section{Material: Modified 800H}

Note: In analyzing these data it is important to understand that the three Section $\mathrm{C}$ samples were removed at different times, and so they experienced different lengths of exposure. Sample L-M (1185 F) was removed November 2001, shortly after the Section A samples. Sample E-F (1162 F) was removed in June 2002. Sample Y-Z (1099 F) saw was removed with the remaining Section C samples in May 2003. 


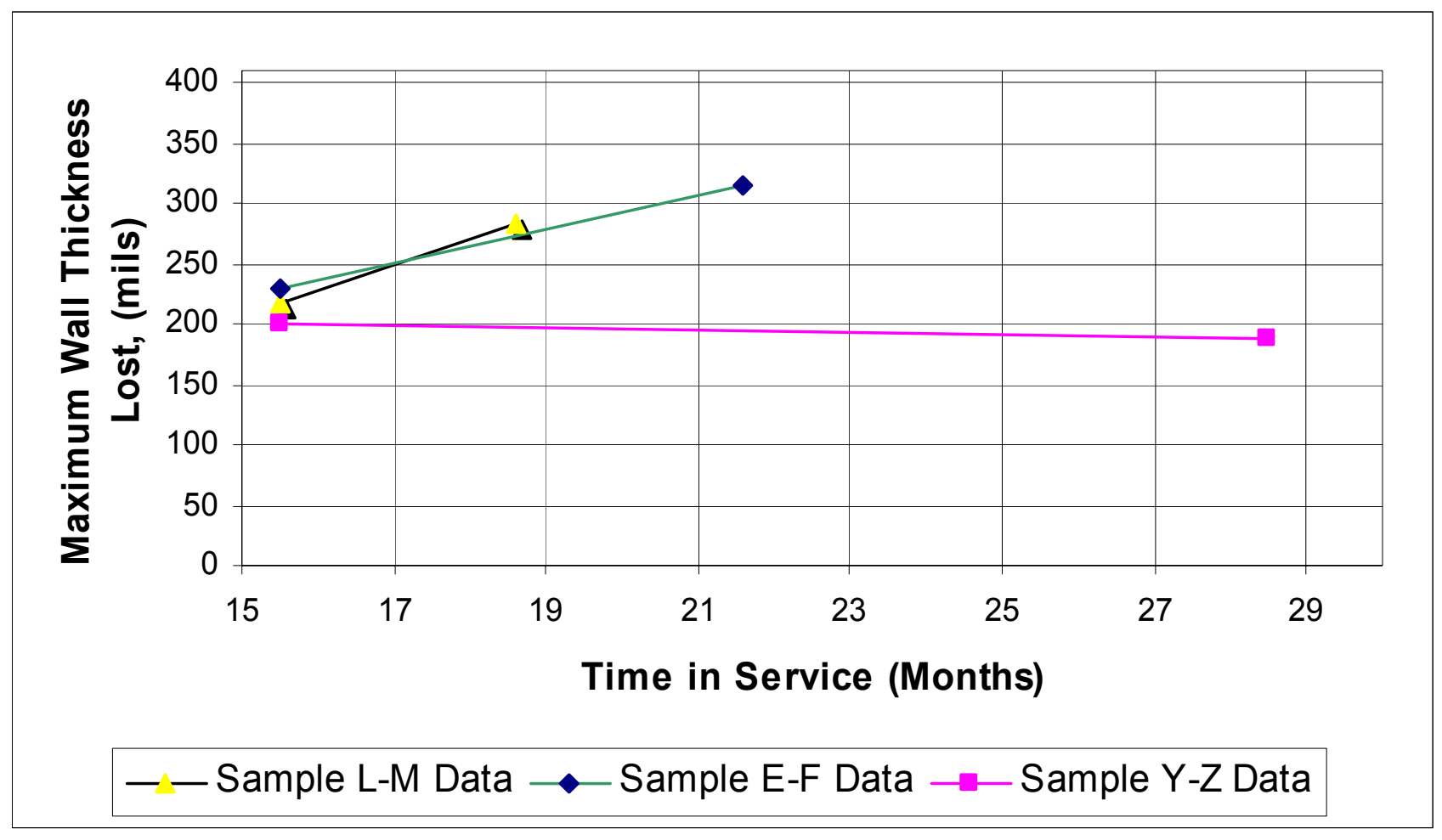

Figure B-14: Metal Loss as a Function of Time

\section{Material: Modified 800H}

Note: Sample L-M was removed from Section C shortly after the Section A samples were removed. For Section C, Sample E-F was removed early due to excessive wall thickness losses (316 mils). Sample Y-Z experienced the full exposure life for both Sections A and C. 


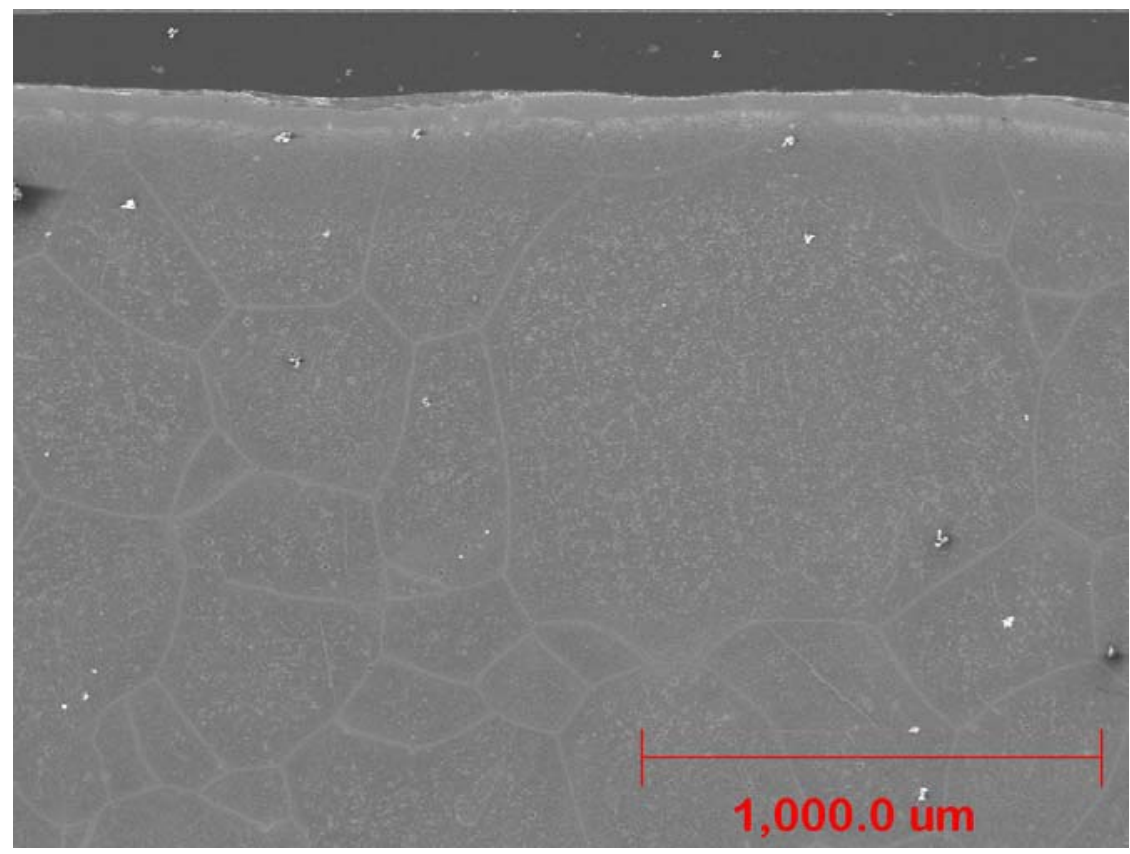

(a) External Scale/Metal Interface (Low Magnification)

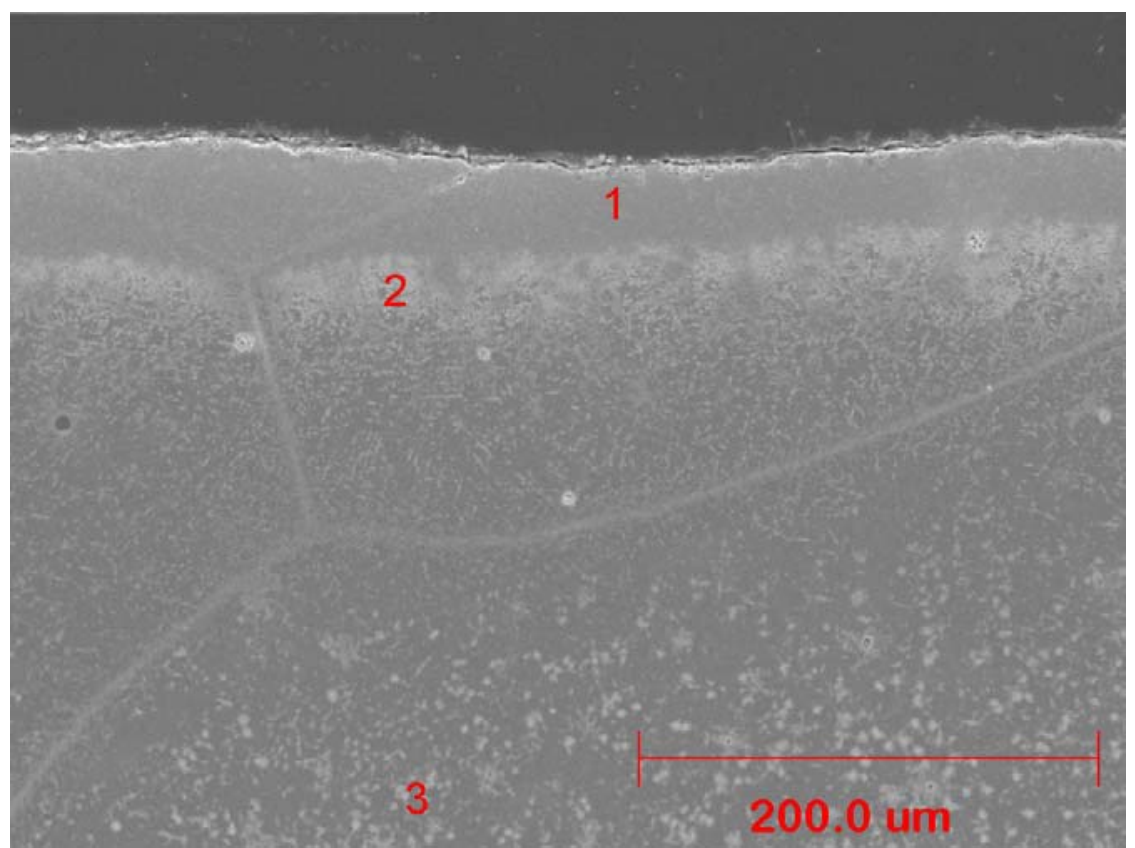

(b) External Scale/Metal Interface (Intermediate Magnification)

\section{Figure B-15: Microstructure: Modified 800H}

Note: EDS analysis was performed at numbered locations. See Figure B-16 for results. 


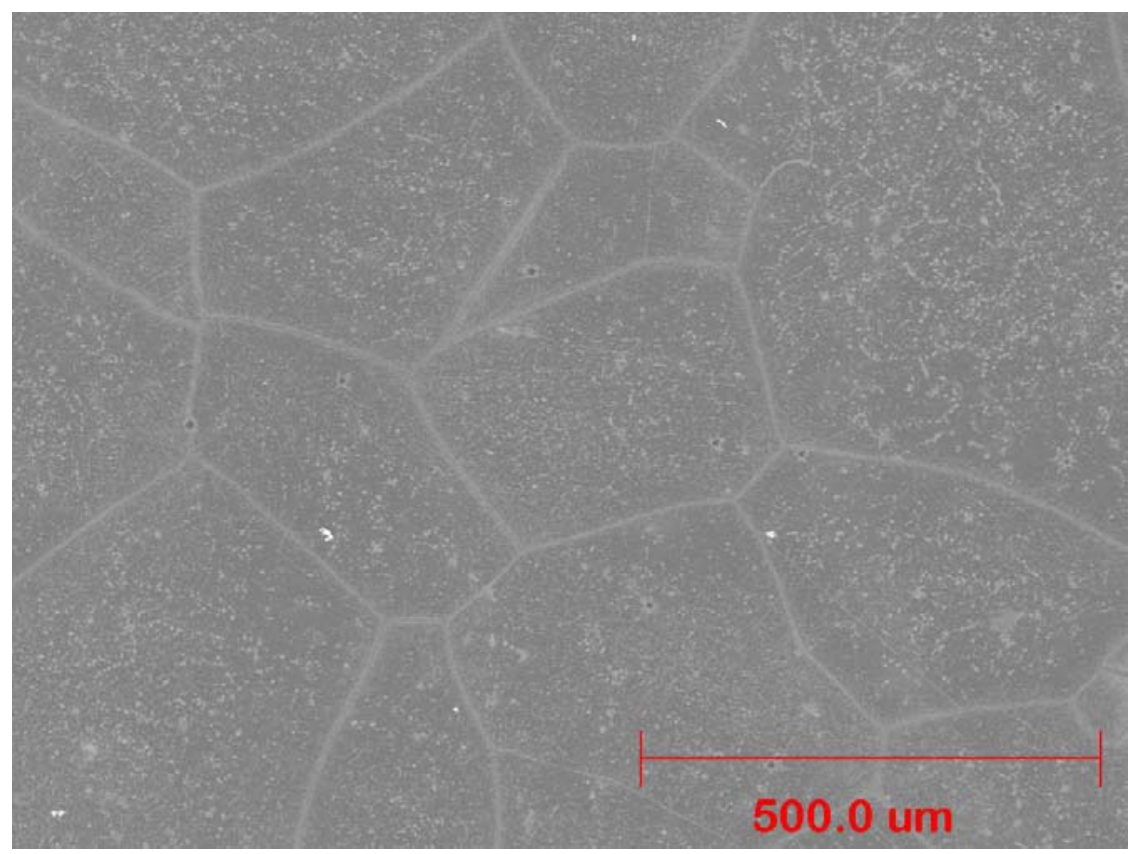

Midwall Microstructure

Figure B-16: Microstructure: Modified 800H 


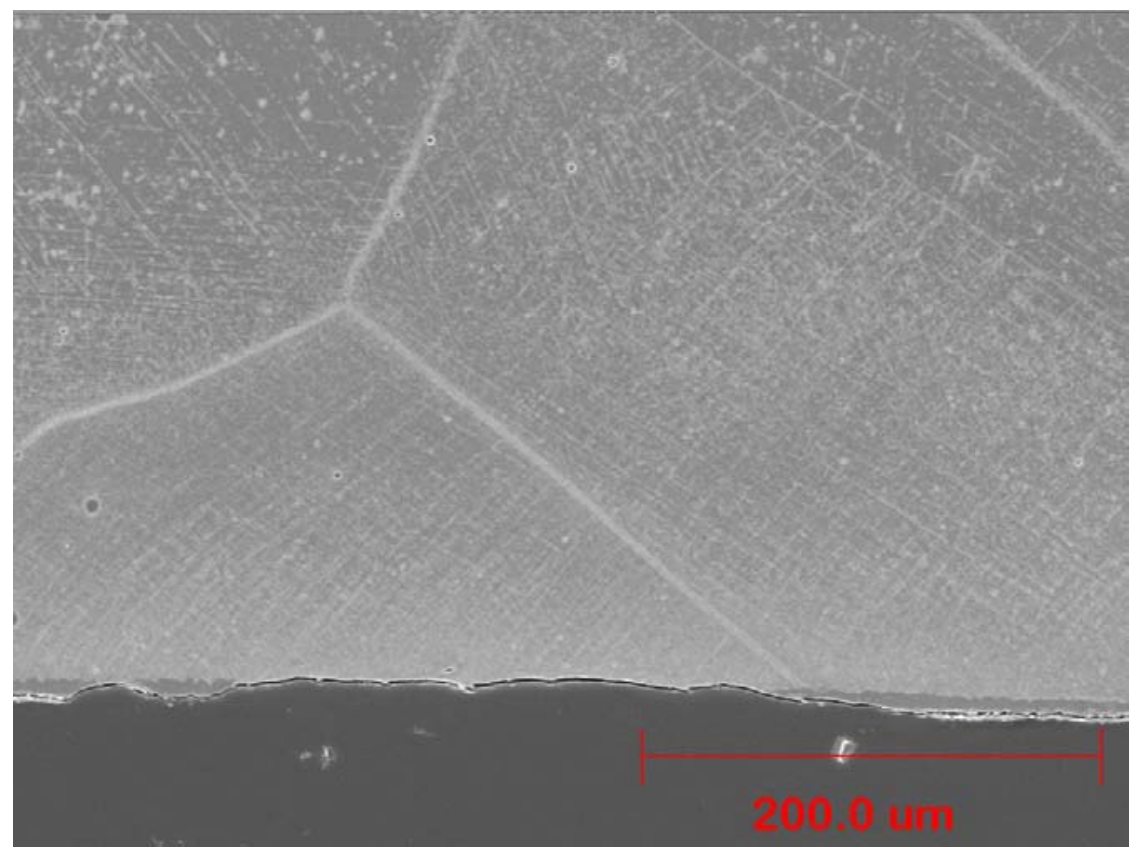

Internal Surface Appearance

Figure B-17: Microstructure: Modified 800H

Note: Photomicrograph at approximately 200X. Electrolytic 10\% oxalic acid etch, 9V OCP 


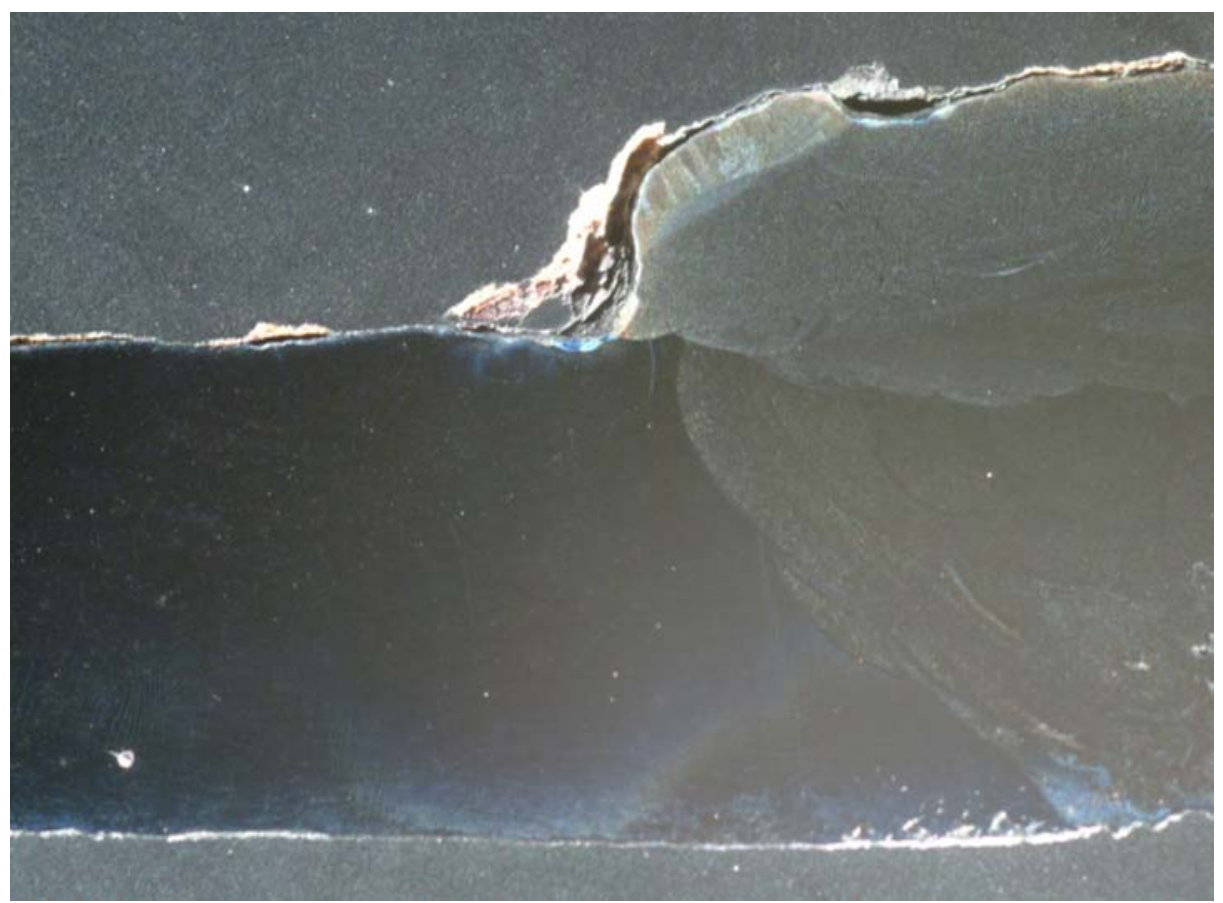

Modified 800H Adjacent to Weld "Z"

Figure B-18: Longitudinal Cross-Section: Modified 800H Adjacent to Weld

Note: The weld shown above is not original with Section C. It was used in inserting a "dutchman" between welds Z and AA on June 10, 2002. 
Figure B-19: EDS Analysis of Deposit/Scale/Metal Interface for Modified 800H (Sample E-F)

\begin{tabular}{|c|l|l|c|c|c|c|c|c|c|c|c|c|}
\hline Area & Description & $\begin{array}{l}\text { Visual } \\
\text { Appearance }\end{array}$ & Ni & Cr & Fe & Mn & Mo & V & Al & Si & C & O \\
\hline 1 & $\begin{array}{l}\text { Adherent } \\
\text { chromium-oxide } \\
\text { scale. }\end{array}$ & $\begin{array}{l}\text { Generally } \\
\text { featureless, } \\
\text { however, grain } \\
\text { boundaries } \\
\text { continuous } \\
\text { with } \\
\text { underlying } \\
\text { metal. }\end{array}$ & 3.78 & 42.5 & 14.9 & 4.27 & -- & -- & -- & 0.54 & -- & 31.4 \\
\hline 2 & $\begin{array}{l}\text { Diffusion zone } \\
\text { adjacent to } \\
\text { scale/metal } \\
\text { interface, } \\
\text { depleted in Cr, } \\
\text { Si, and V. }\end{array}$ & $\begin{array}{l}\text { Featureless, } \\
\text { dark etching } \\
\text { grains. }\end{array}$ & 38.9 & 9.06 & 50.1 & 1.55 & & -- & -- & 0.33 & -- & -- \\
\hline 3 & $\begin{array}{l}\text { Base Metal } \\
\text { Maimary grain }\end{array}$ & 31.0 & 19.1 & 46.0 & 1.68 & -- & 0.42 & -- & 0.38 & -- & -- \\
\hline structure. & $\begin{array}{l}\text { Reference bulk } \\
\text { composition per } \\
\text { spectrographic } \\
\text { analysis. }\end{array}$ & & 30.6 & 20.4 & 44.4 & 2.13 & 1.33 & 0.36 & 0.02 & 0.28 & -- & -- \\
\hline
\end{tabular}


Appendix C

Analysis of Performance

Test Section C

347 HFG 


\section{Overview of Results: 347 HFG}

The three Section C 347 HFG tube samples experienced rates of metal loss that ranged from 204, 120, and 76 mils per year*. Taking into consideration the initial 400 mil wall thickness tube Sample I-J, which experienced the highest rate of loss, was removed from service and replaced after only 1.55 years of exposure to corrosive conditions. The other two samples survived until the end of the Section C exposure period (about 2.38 years).

Comparison of the results for Sections $\mathrm{A}$ and $\mathrm{C}$ revealed that, for both sections, the sample which saw an intermediate temperature exposure (Sample A-B, at $1147 \mathrm{~F}$ in Section C) experienced a lower rate of metal loss that the samples in the section that were at a higher temperature (Sample $\mathrm{I}-\mathrm{J}$ at $1174 \mathrm{~F}$ ) and at a lower temperature (Sample CC-DD at $1086 \mathrm{~F}$ ). The reason for this is not clear, but if it is real and not due to scatter, then, it suggests that more than one corrosion mechanism may be operating over the service temperature range. In any case, based on the Section A assessment, these differences in performance, do not appear to be related to alloying and/or microstructural differences in that all three samples appeared to be identical.

It should be noted that the metal loss rates determined to date for both Section A and Section C all were based on a ring specimen that was extracted from mid-length of each tube sample. In this analysis additional attention was focused on metal losses in the vicinity of welds. Representative samples were taken and it was determined that, for the 347 HFG samples evaluated, the Inconel 625 weld filler metal is reasonably resistant to coal ash corrosion and that the tube material immediately adjacent to the weld (i.e. the weld heat affected zone) was somewhat less affected by corrosion than was the remainder of the tube. Indeed the tube wall appeared to be progressively thinner toward the mid-point of the tube sample.

Analysis of deposit on the surface revealed that it contained the known aggressive combination of species, namely iron, oxygen, the alkali metals, chlorine and sulfur. Examination and analysis of the scale adjacent to the metal surface revealed the chalky-white appearance associated with Alkali-iron-sulfates. Thus, the required "bad actor" was available to test the performance limits of this alloy.

Finally, the analysis of the deposit and the metal surface immediately adjacent to the interface revealed the presence of carbon. While carbon determinations using EDS are unreliable, the implications of carbon in the deposit are worthy of additional consideration, as is the potential for carbon ingress from the deposit into the abutting metal. 


\section{Material: 347 HFG}

\section{Summary of Findings}

\begin{tabular}{|c|c|}
\hline Issue & Discussion \\
\hline $\begin{array}{l}\text { Sample } \\
\text { Location and } \\
\text { Orientation. }\end{array}$ & $\begin{array}{l}\text { - In assessing the data for Section C, it is important to understand that } \\
\text { some tube samples had to be removed from the section early due to } \\
\text { excessive thinning. The schematic in Figure C-1 shows the relative } \\
\text { positions of the } 347 \text { HFG samples in all three sections. Also, shown } \\
\text { are the positions of all of the excessively thinned tubes that were } \\
\text { removed and replaced in order to extend the life of Section C. } \\
\text { - One } 347 \text { HFG sample was removed early. Sample I-J was removed in } \\
\text { November 2001, (i.e. about the same time that Section A was removed } \\
\text { from service for evaluation). The other two } 347 \text { HFG samples survived } \\
\text { for the full Section C exposure period. } \\
\text { The sample that was removed early was set aside for later analysis, } \\
\text { and has been included in the current evaluation along with the other } \\
\text { tube samples that made up Section C. } \\
\text { Also shown in this figure is the calculated average surface metal } \\
\text { temperature at each location. }\end{array}$ \\
\hline $\begin{array}{l}\text { Composition } \\
\text { Verification. }\end{array}$ & $\begin{array}{l}\text { - The table in Figure C-2 provides the specified composition from } \\
\text { ASTM A213 (TP347 HFG). } \\
\text { - Sumitomo supplied from one heat all of the } 347 \text { HFG tubing used in } \\
\text { this program. The table in Figure C-2 provides the heat analysis } \\
\text { reported by Sumitomo. The analysis compares well with the } \\
\text { specification. } \\
\text { During the Section A evaluation, detailed composition verification was } \\
\text { undertaken for every tube sample that was removed from service. This } \\
\text { served, in part, to verify that the each tube material was located at its' } \\
\text { proper position within the test section. } \\
\text { Based on the good results for Section A, it was decided that it would } \\
\text { be unnecessary to analyze each and every tube sample for composition } \\
\text { verification. Rather, it was decided to select a representative sample } \\
\text { from each tube material type for confirmatory analysis. } \\
\text { During the Section A evaluation, the energy dispersive spectrographic } \\
\text { (EDS) capabilities of the scanning electron microscope (SEM) were } \\
\text { used to perform semi-quantitative analyses of each of the twelve } \\
\text { different candidate tube materials. Comparison of these EDS results } \\
\text { showed reasonably good correlation with results obtained using } \\
\text { quantitative analytical techniques, including optical emission } \\
\text { spectrometry. } \\
\text { Based on this, it was decided that the accuracy provided by EDS } \\
\text { would suffice for composition verification, but that more detailed } \\
\text { analyses would be undertaken if questions arose. } \\
\text { The results of the EDS analysis of tube sample CC-DD confirmed that } \\
\text { the correct material was used at this location. }\end{array}$ \\
\hline
\end{tabular}




\section{Material: 347 HFG}

\section{Summary of Findings, (continued)}

\begin{tabular}{|c|c|}
\hline Issue & Discussion \\
\hline $\begin{array}{l}\text { As-Received } \\
\text { Appearance }\end{array}$ & $\begin{array}{l}\text { - Figure C-3 shows the typical appearance of the } 347 \text { HFG material } \\
\text { prior to service. } \\
\text { - Figures C-4 through C- } 6 \text { document the as-received appearance of the } \\
\text { external surface of the } 347 \text { HFG tube samples after they had been } \\
\text { removed from service. Three views are provided for each tube: } 12 \\
\text { o'clock (the view that faces on-coming gas flow), } 3 \text { o'clock (the view } \\
\text { where the gas flow is tangent to the surface), and } 6 \text { o'clock (the view } \\
\text { that shows the back-side of the tube relative to the gas flow direction. } \\
\text { - The reader should be alerted to the fact that, within the boiler, the gas } \\
\text { flows upward over these tubes. This implies that for the "o'clock" } \\
\text { conventions described above, (with the } 12 \text { o'clock orientation facing } \\
\text { the gas flow direction), the actual physical orientation of these tubes is } \\
\text { such that the } 12 \text { o'clock side of the tube faces downward, and the } 6 \\
\text { o'clock orientation faces upward within the boiler. } \\
\text { Wastage is visible for all three samples in all three orientations; } \\
\text { however, it may most clearly be seen at the } 12 \text { o'clock position where } \\
\text { the profile of the weld metal provides a reference. } \\
\text { A relatively thick rust-red back-side scale and deposit build-up is seen } \\
\text { at the } 6 \text { o'clock position on all three samples. } \\
\text { - A relatively thin red-brown deposit is seen on all other surfaces. } \\
\text { - There is no clear evidence in these figures of the gray/white scale } \\
\text { associated with molten alkali-iron sulfate attack. However, scraping to } \\
\text { remove the red scale revealed evidence of a gray/white scale } \\
\text { immediately adjacent to the metal surface. }\end{array}$ \\
\hline $\begin{array}{l}\text { Grit-Blasted } \\
\text { Surface } \\
\text { Appearance }\end{array}$ & $\begin{array}{l}\text { - Figures C-7 through C-9 show the appearance of the external surface } \\
\text { of the } 347 \text { HFG tube samples after grit-blasting to remove deposit and } \\
\text { scale. The } 12 \text { o'clock and } 3 \text { o'clock positions for all three samples } \\
\text { show the appearance of general wastage due to fireside corrosion. } \\
\text { - No significant pitting was found on the back-side of these samples. }\end{array}$ \\
\hline $\begin{array}{l}\text { Wall } \\
\text { Thickness } \\
\text { Loss } \\
\text { Determined }\end{array}$ & $\begin{array}{l}\text { - Tube cross-sections were prepared to allow for measurement of wall } \\
\text { thickness to determine metal loss due to exposure. These are shown in } \\
\text { silhouette in Figures C-10 through C-12. } \\
\text { - The same relative "o'clock" positions were maintained as discussed } \\
\text { earlier. Measurements were taken at eight evenly spaced locations } \\
\text { about the tube circumference. These were then compared with } \\
\text { measurements taken at these same locations prior to exposure. } \\
\text { - The before and after measurements were documented in the table that } \\
\text { accompanies each figure. These form the basis for the "delta" } \\
\text { calculation to determine the amount of wall thickness lost. } \\
\text { - For all three samples, the minimum wall thickness in the cross section } \\
\text { was not at one of the eight predetermined locations. In these cases, } \\
\text { there was no "before" measurement for comparison. So, the average } \\
\text { "before" wall thickness was used to calculate the wall thickness lost at } \\
\text { these off-locations. }\end{array}$ \\
\hline
\end{tabular}




\section{Material: 347 HFG}

\section{Summary of Findings, (continued)}

\begin{tabular}{|c|c|}
\hline Issue & Discussion \\
\hline $\begin{array}{l}\text { Rate of Wall } \\
\text { Thickness } \\
\text { Loss Versus } \\
\text { Average Metal } \\
\text { Temperature }\end{array}$ & $\begin{array}{l}\text { - The chart in Figure C-13 plots the maximum wall thickness lost } \\
\text { against the calculated average surface metal temperature for each of } \\
\text { the three } 347 \text { HFG samples in Section C. Similar data for Section A } \\
\text { also are plotted on this chart. } \\
\text { - It was noted that, for both sections, the maximum rate of metal loss } \\
\text { was greater at the low and high temperatures, and dipped significantly } \\
\text { at an intermediate temperature. The reason for this is not clear; } \\
\text { however, it may suggest the role of different corrosion mechanisms. } \\
\text { - It should be noted that, given an initial wall thickness of } 400 \text { mils, then } \\
\text { given the measured maximum rate of metal loss of between } 204 \text { and } \\
174 \text { mils per year, the samples at the highest exposure temperature (i.e. } \\
\text { the samples at position I-J) would not be expected to survive for three } \\
\text { years of exposure. This provides further justification as to why these } \\
\text { samples were removed early from all three sections. }\end{array}$ \\
\hline $\begin{array}{l}\text { Wall } \\
\text { Thickness } \\
\text { Loss as a } \\
\text { Function of } \\
\text { Time and } \\
\text { Location }\end{array}$ & $\begin{array}{l}\text { - The chart in Figure C-14 plots metal loss as a function of time for each } \\
\text { sample location for both Sections A and C, with samples from Section } \\
\text { C having experienced generally longer exposure times. } \\
\text { - Sample I-J was removed from Section C and replaced at about the } \\
\text { same time that Section A was removed for analysis, i.e. November } \\
\text { 2001. It was noted that the rate of metal loss appears to be greater for } \\
\text { the Sample I-J from Section C as compared with the same sample in } \\
\text { Section A. This may have been due to the somewhat higher } \\
\text { temperatures for the I-J position in Section C (1174 F vs } 1164 \mathrm{~F}) \text {, but } \\
\text { due to limited sampling it is difficult to draw this conclusion this with } \\
\text { certainty. } \\
\text { The chart shows a straight line fit to the maximum metal loss data } \\
\text { points for Samples A-B and CC-DD for both Sections A and C. } \\
\text { Evaluation of the rate of metal loss data depicted in Figure C-13 } \\
\text { suggests that a straight line fit might well be appropriate for the } \\
\text { samples at position A-B in that the rate of metal loss was the same at } \\
\text { this location for both Section A and Section C. } \\
\text { For the samples at position CC-DD, the rate of metal loss drops off } \\
\text { between Section A (176 mils/yr) and Section C (120 mils/yr), so, a } \\
\text { straight-line fit would likely be less appropriate. }\end{array}$ \\
\hline
\end{tabular}




\section{Material: 347 HFG}

\section{Summary of Findings, (continued)}

\begin{tabular}{|c|c|}
\hline Issue & Discussion \\
\hline $\begin{array}{l}\text { Metallurgical } \\
\text { Analysis - } \\
\text { SEM, } \\
\text { Continued. }\end{array}$ & $\begin{array}{l}\text { - Sample CC-DD was selected for a detailed SEM/EDS assessment of } \\
\text { the constituents and structure in the vicinity of the external and } \\
\text { internal surfaces. This sample was selected based on the fact that, } \\
\text { between the two samples that survived the full Section C exposure } \\
\text { period, Sample CC-DD had lost material at the maximum rate. The } \\
\text { focus of this investigation was in the region of the tube where the } \\
\text { maximum amount of material was lost, i.e. in the thinnest section. } \\
\text { - Figures C-15a and b show the appearance of the external scale/metal } \\
\text { interface for Sample CC-DD. Five distinct regions are in evidence. } \\
\text { These are highlighted by the numbered locations shown in Figure C- } \\
\text { 15b. These same locations were analyzed using the EDS capabilities of } \\
\text { the SEM. The results are provided later. } \\
\text { A description of the regions is provided below, starting within the } \\
\text { metal and working out: } \\
\text { Base metal - oxalic acid etch delineates grain boundaries and } \\
\text { carbides. } \\
\text { Deeply etching base metal - It can be seen as the relatively } \\
\text { light-appearing band that is parallel to the metal surface in } \\
\text { Figure C-15b. Figure C-15b shows that this band is } \\
\text { approximately one-half grain wide, and appears related to } \\
\text { etchant attack along the grain boundaries of those grains that } \\
\text { are near to the interface with the scale. EDS analysis will } \\
\text { show that this etching response reflects composition } \\
\text { differences due to diffusion (primarily chromium) in this } \\
\text { region. } \\
\text { Figure C-16 documents the appearance of the mid-wall } \\
\text { Deep etching base metal (darker gray) - EDS analysis showed } \\
\text { that this region (2 to } 3 \text { grains wide) also is within the diffusion } \\
\text { zone adjacent to the external surface. } \\
\text { An adherent scale - this is shown to be continuous along the } \\
\text { interface with the underlying metal. EDS analysis will show } \\
\text { that this is a chromium-rich oxide. } \\
\text { Thick and very porous deposit/scale conglomerate - many } \\
\text { spherical particles are noted along with what appears to be } \\
\text { islands of scale (metal oxides). } \\
\text {-Ditle or no scale or metal loss was in evidence. }\end{array}$ \\
\hline
\end{tabular}




\section{Summary of Findings, (continued)}

\section{Material: $\quad 347$ HFG}

\begin{tabular}{|c|c|}
\hline Issue & Discussion \\
\hline $\begin{array}{l}\text { Metallurgical } \\
\text { Analysis - } \\
\text { Weld Cross } \\
\text { Section. }\end{array}$ & $\begin{array}{l}\text { - Figure C-18 shows a longitudinal profile (at the } 12 \text { o'clock } \\
\text { orientation) of the } 347 \text { HFG samples adjacent to the welds in Section } \\
\text { C. The welds in question are those identified as welds "B" and "DD", } \\
\text { see Figure C-1 for locations } \\
\text { - An Inconel } 625 \text { weld metal was used throughout Section C. The cross } \\
\text { sections show that the } 347 \text { HFG heat affected zones are not } \\
\text { preferentially attached, in fact, the wall thickness for the tube sample } \\
\text { tapers from the weld toward the middle of the tube length. }\end{array}$ \\
\hline $\begin{array}{l}\text { EDS Analysis } \\
\text { Results }\end{array}$ & $\begin{array}{l}\text { - The table in Figure C-19 provides a summary of the EDS analyses of } \\
\text { the locations numbered in Figure C-15b. The "areas" below } \\
\text { correspond to the numbered locations indicated in the C-15 figures. } \\
\text { Review of this table suggests the following: } \\
\text { - Area 1: For the bulk deposit analysis - Potassium, sulfur, and } \\
\text { chlorine are present as constituents of corrosion species. An iron- } \\
\text { rich oxide is likely, as are aluminum and silicon oxides. Carbon } \\
\text { content is relatively high, however, EDS is reliable qualitatively } \\
\text { but not quantitatively for light elements such as carbon. } \\
\text { - } \text { Area 2: Adherent scale - this region appears to be primarily a } \\
\text { chromium / iron oxide. } \\
\text { - Area 3: Deep etching diffusion zone - this region which is } \\
\text { contiguous with the adherent scale appears to be depleted in } \\
\text { chromium (5 wt\% vs } 20 \mathrm{wt} \% \text { in the base metal). This of course is } \\
\text { consistent with the formation of the chromium-rich oxide scale. } \\
\text { - Area 4: - Gray etching diffusion zone - this analysis confirms } \\
\text { that the diffusion zone is approximately } 2 \text { grains wide for this } \\
\text { sample. The chromium content in this region was measured to be } \\
\text { 12 wt\% versus } 20 \mathrm{wt} \% \text { in the base metal. } \\
\text { Area 5: - Base metal - Area } 5 \text { was used to determine the base } \\
\text { metal composition. }\end{array}$ \\
\hline
\end{tabular}




\section{Gas Flow}

Direction

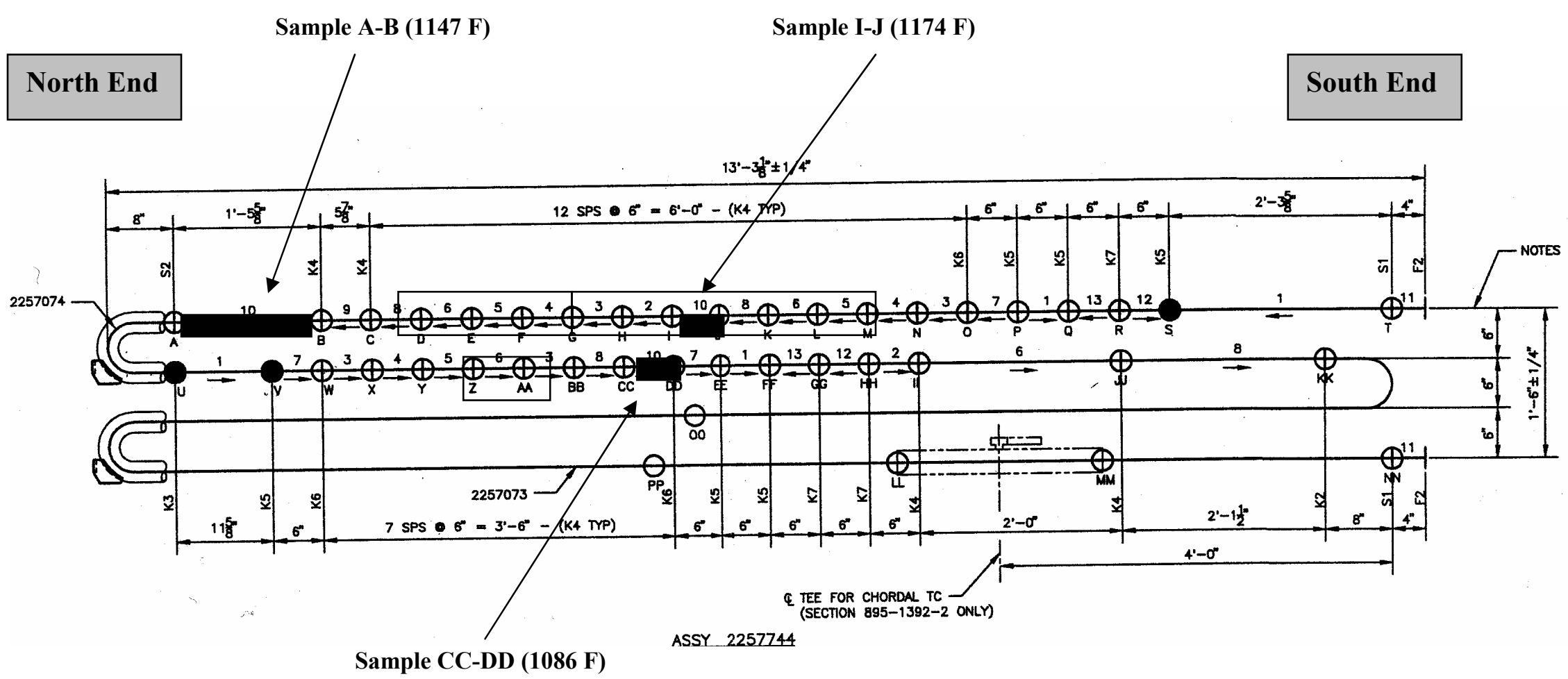

Figure C-1: Schematic Drawing Showing Position of 347 HFG Tube Samples Within Section C

1. Drawing shown in same relative orientation as per the actual installation.

2. This group of tube samples was removed from service in November 2001 - highlighted in red.

3. This group of tube samples was removed from service in June 2002 - highlighted in yello 


\section{Figure C-2 Semi-Quantitative Composition Verification for 347 HFG}

Description:

Source:

Reference Composition:
Nominal $18 \mathrm{Cr} / 11 \mathrm{Ni}$ steel with $\mathrm{Nb}$ and Ta added to provide creep strength.

Sumitomo Metal Industries (SMI)

"Specified" chemistry - per ASTM A213 (TP347 HFG).

\begin{tabular}{|c|c|c|c|c|c|}
\hline Element & $\begin{array}{c}\text { Reference } \\
\text { Composition }\end{array}$ & $\begin{array}{c}\text { Composition (a) } \\
\text { Heat D892703 }\end{array}$ & $\begin{array}{c}\text { Tube Sample } \\
\text { A-B }\end{array}$ & $\begin{array}{c}\text { Tube Sample } \\
\text { I-J }\end{array}$ & $\begin{array}{c}\text { Tube Sample } \\
\text { CC-DD }\end{array}$ \\
\hline $\mathrm{Ni}$ & $9.00-13.0$ & 11.98 & N/A & N/A & 12.3 \\
\hline $\mathrm{Cr}$ & $17.0-20.0$ & 18.4 & N/A & N/A & 18.0 \\
\hline $\mathrm{Fe}$ & Balance & Balance & N/A & N/A & -- \\
\hline $\mathrm{Mo}$ & - & - & N/A & N/A & -- \\
\hline $\mathrm{Co}$ & - & - & N/A & N/A & -- \\
\hline $\mathrm{C}$ & $0.06-0.10$ & 0.09 & N/A & N/A & -- \\
\hline $\mathrm{N}$ & - & - & N/A & N/A & -- \\
\hline $\mathrm{B}$ & - & - & N/A & N/A & -- \\
\hline $\mathrm{Mn}$ & $2.00 \max$ & 1.46 & N/A & N/A & 1.05 \\
\hline $\mathrm{Si}$ & $0.75 \max$ & 0.41 & N/A & N/A & 0.57 \\
\hline $\mathrm{Al}$ & - & - & N/A & N/A & -- \\
\hline $\mathrm{Ti}$ & - & - & N/A & N/A & -- \\
\hline $\mathrm{Al}+\mathrm{Ti}$ & - & - & N/A & N/A & -- \\
\hline $\mathrm{Nb}$ & - & - & N/A & N/A & -- \\
\hline $\mathrm{Ta}$ & - & - & N/A & N/A & -- \\
\hline $\mathrm{Nb}+\mathrm{Ta}$ & $(8 \times \mathrm{C}) \min -1.0 \max$ & 0.9 & N/A & N/A & N/D \\
\hline $\mathrm{V}$ & - & - & N/A & N/A & -- \\
\hline $\mathrm{W}$ & - & - & N/A & N/A & -- \\
\hline $\mathrm{Cu}$ & - & - & N/A & N/A & -- \\
\hline $\mathrm{Sn}$ & - & - & N/A & N/A & -- \\
\hline $\mathrm{P}$ & $0.040 \max$ & 0.027 & N/A & N/A & -- \\
\hline $\mathrm{S}$ & $0.030 \max$ & 0.001 & N/A & N/A & -- \\
\hline $\mathrm{O} 2$ & - & - & N/A & N/A & -- \\
\hline
\end{tabular}

$\mathrm{N} / \mathrm{D}=$ EDS technique (gold coating) precluded an accurate niobium determination.

$\mathrm{N} / \mathrm{A}=$ Tube sample not analyzed. 


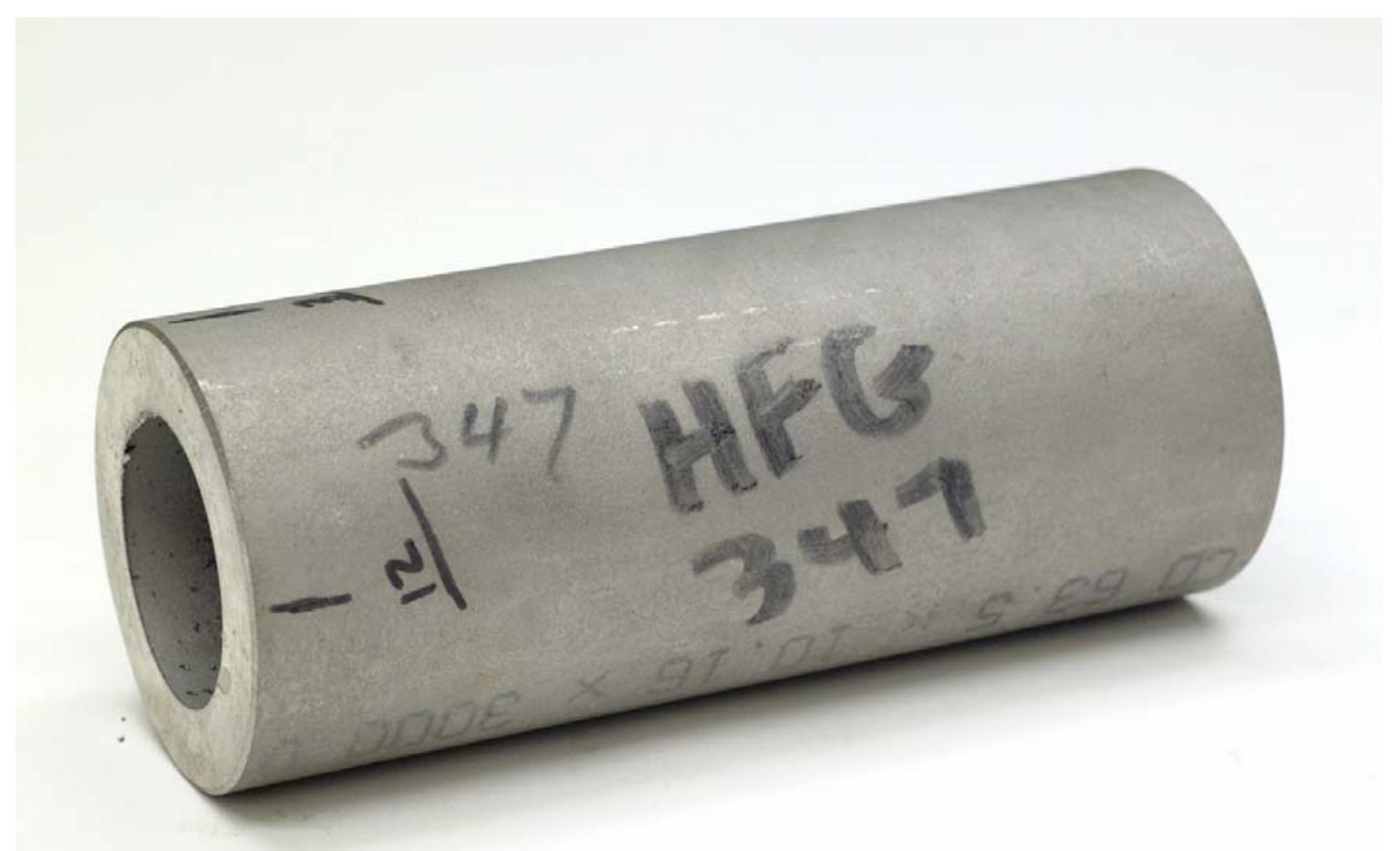

Figure C-3: Appearance of 347 HFG Tube Sample Prior to Service 


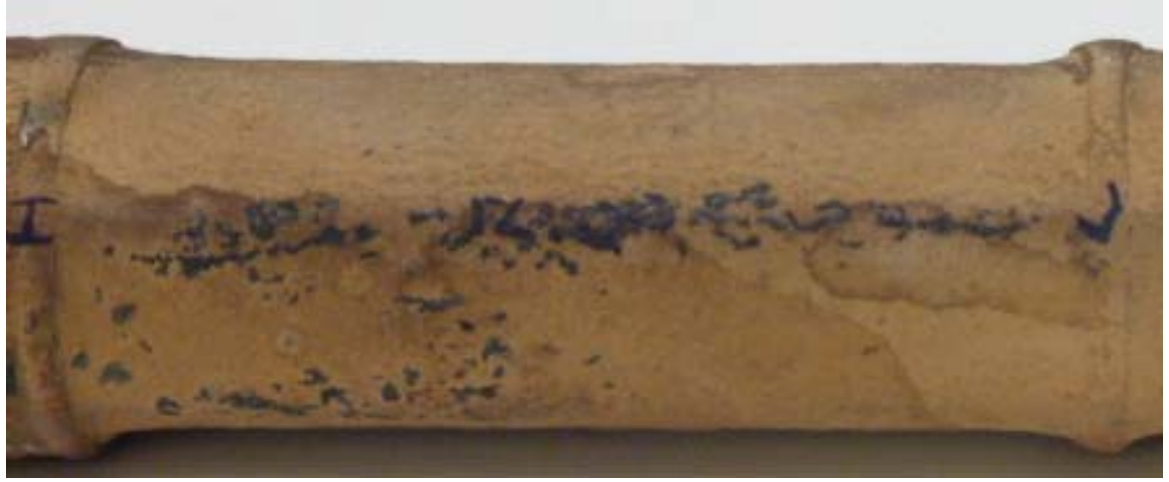

12 O'Clock - Facing Gas Flow

(Note: 3 o'clock is up in this image)

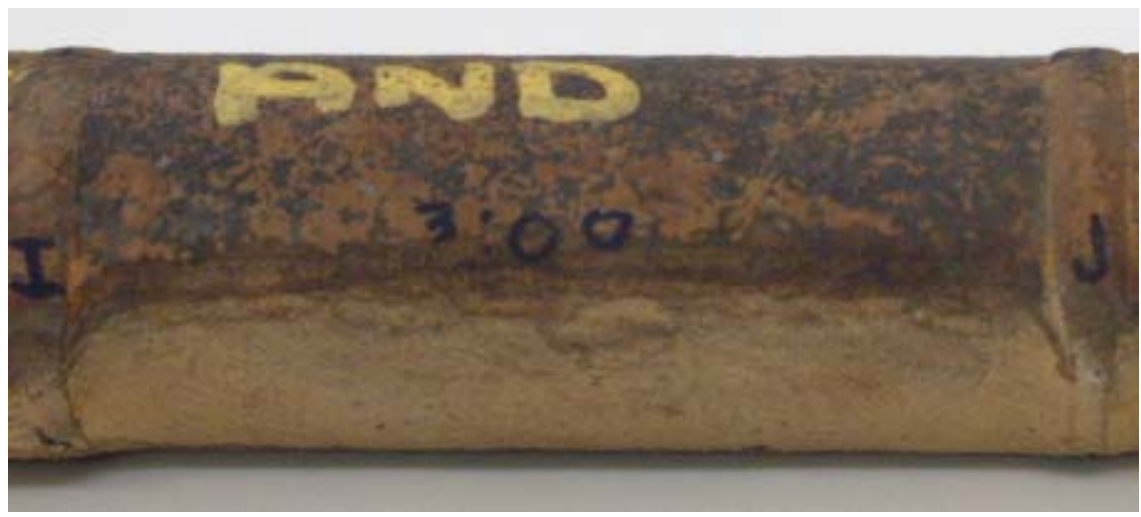

3 O'Clock

(Note: 6 o'clock is up in this image)

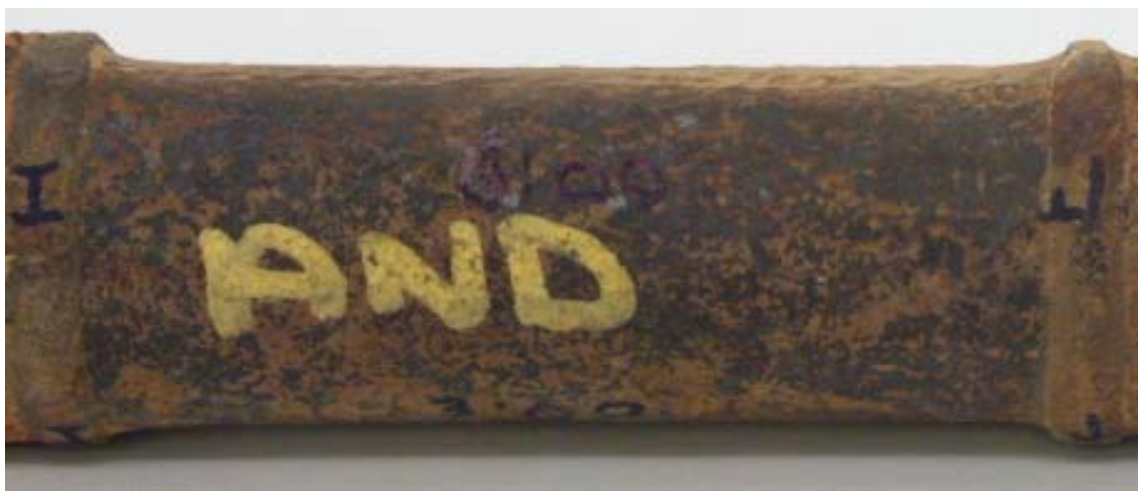

6 O’Clock - Opposite Gas Flow

Figure C-4 As-Received Appearance of Sample I-J (347 HFG)

(Average metal temperature: $1174 \mathrm{~F}$ )

Note: this tube sample was removed from service on 11/6/01. 


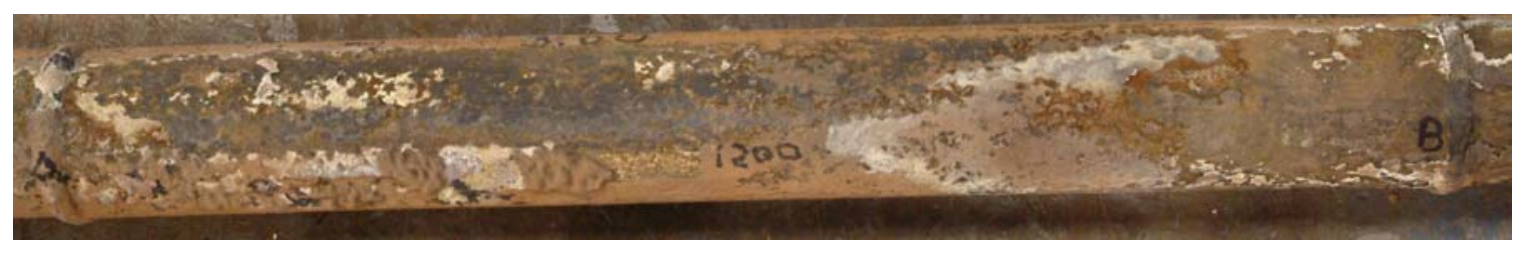

12 O'Clock - Facing Gas Flow

(Note: 3 o'clock is up in this image)

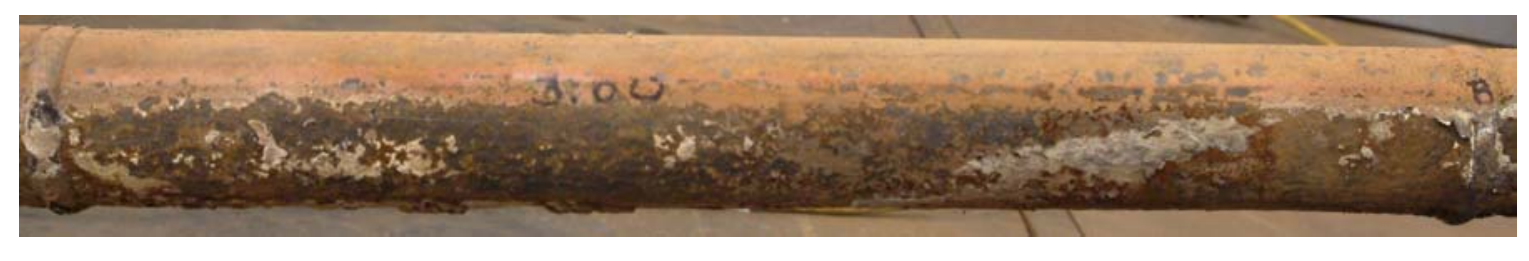

\section{O'Clock}

(Note: 6 o'clock is up in this image)

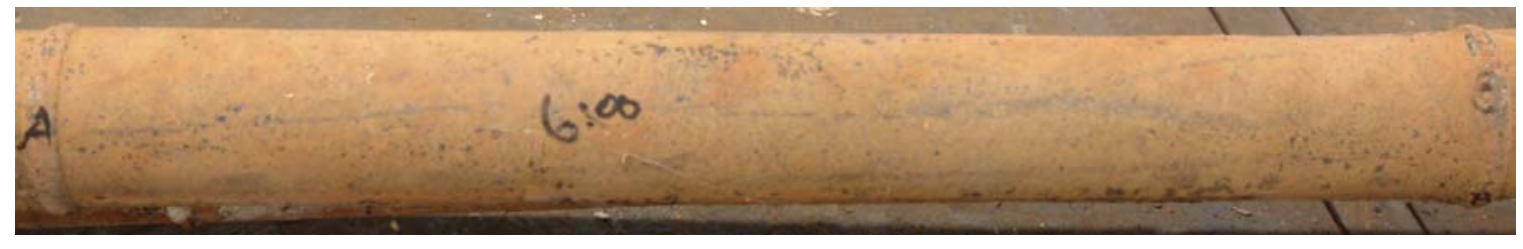

6 O'Clock - Opposite Gas Flow

Figure C-5 As-Received Appearance of Sample A-B (347 HFG)

(Average metal temperature: 1147 F) 


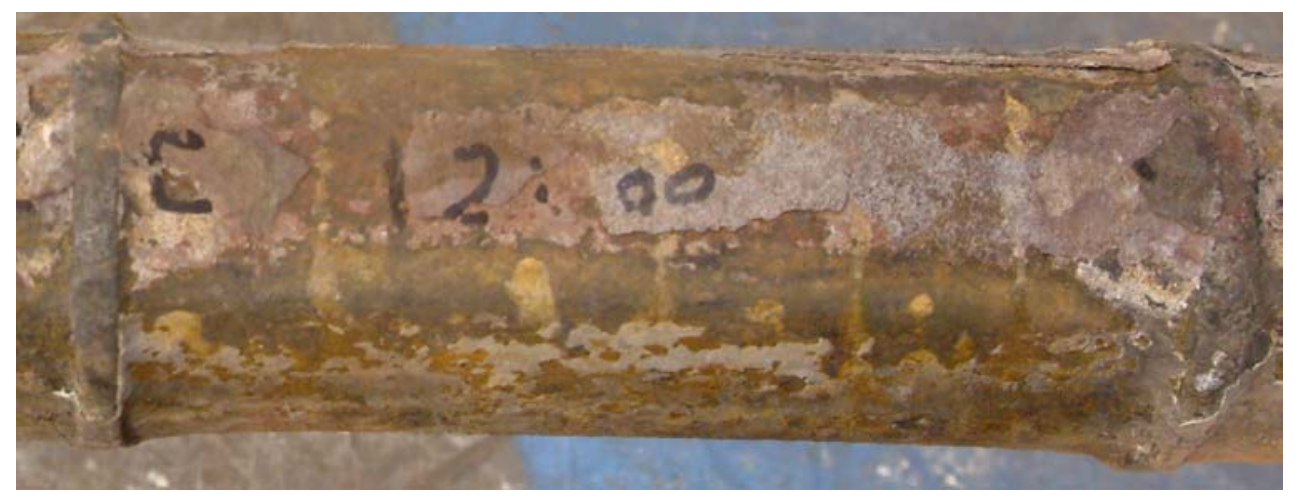

12 O'Clock - Facing Gas Flow

(Note: 3 o'clock is up in this image)

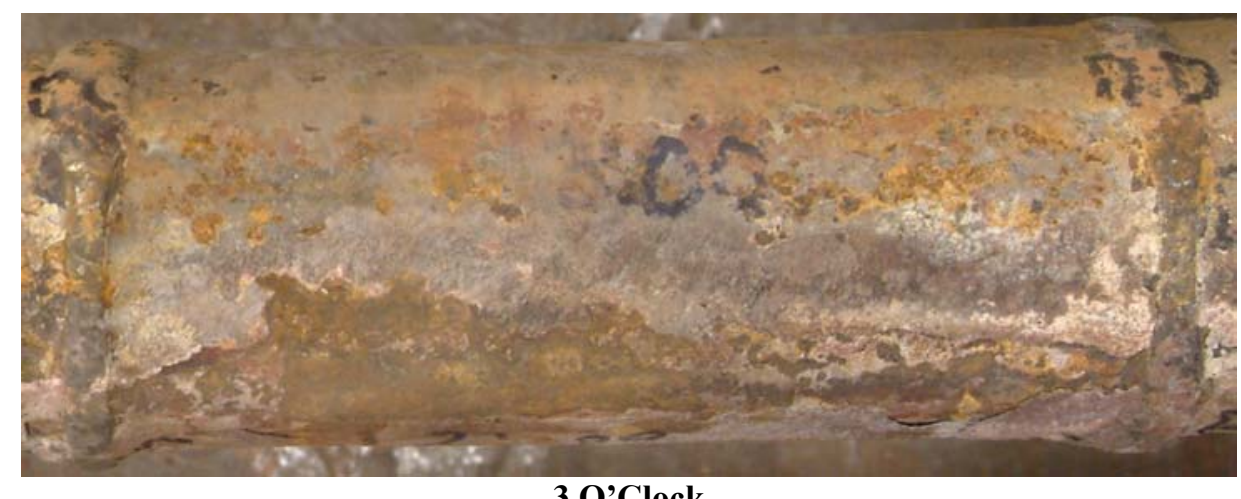

\section{O'Clock}

(Note: 6 o'clock is up in this image)

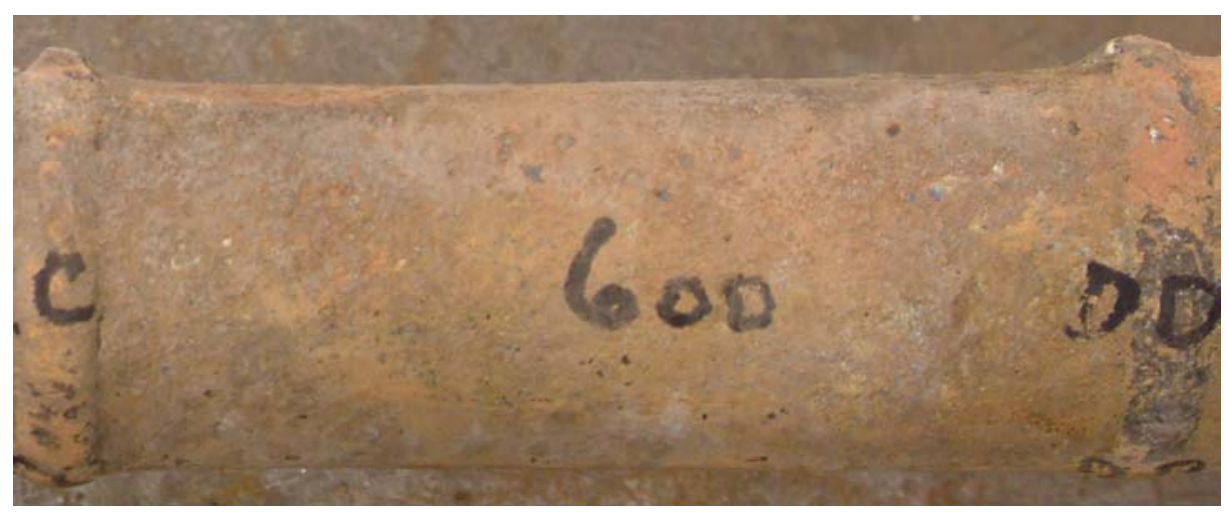

6 O’Clock - Opposite Gas Flow

Figure C-6 As-Received Appearance of Sample CC-DD (347 HFG)

(Average metal temperature: 1086 F) 
12 O'Clock

(205 mils lost)

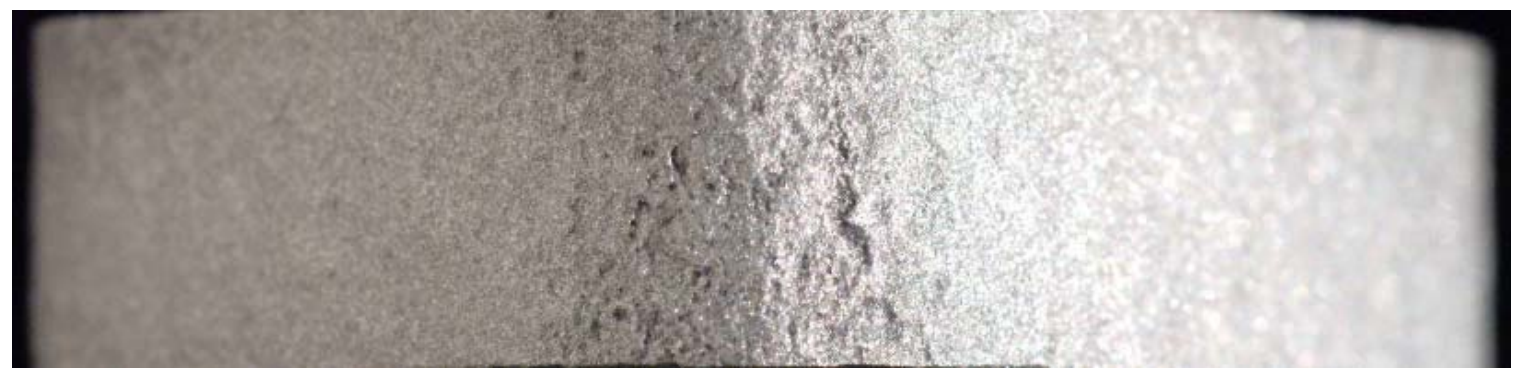

3 O'Clock

(281 mils lost)

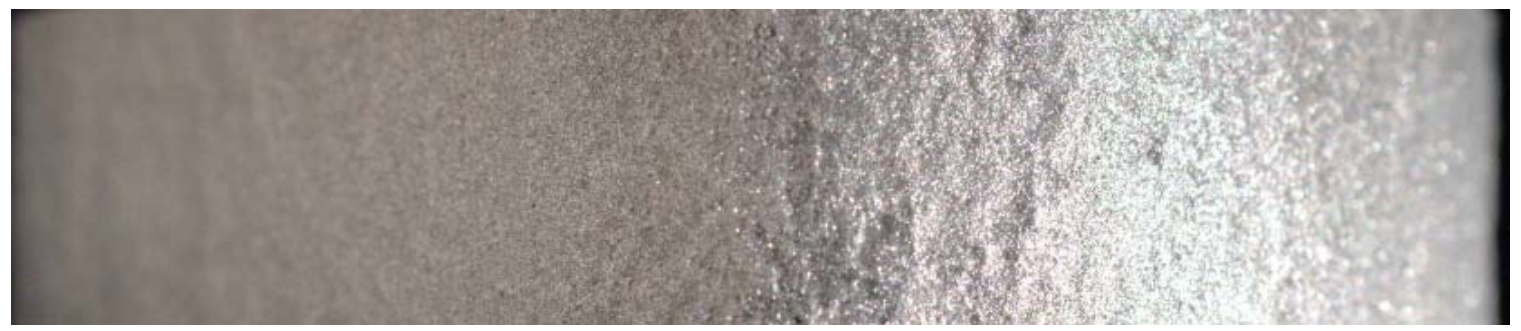

6 O'Clock

(15 mil lost)

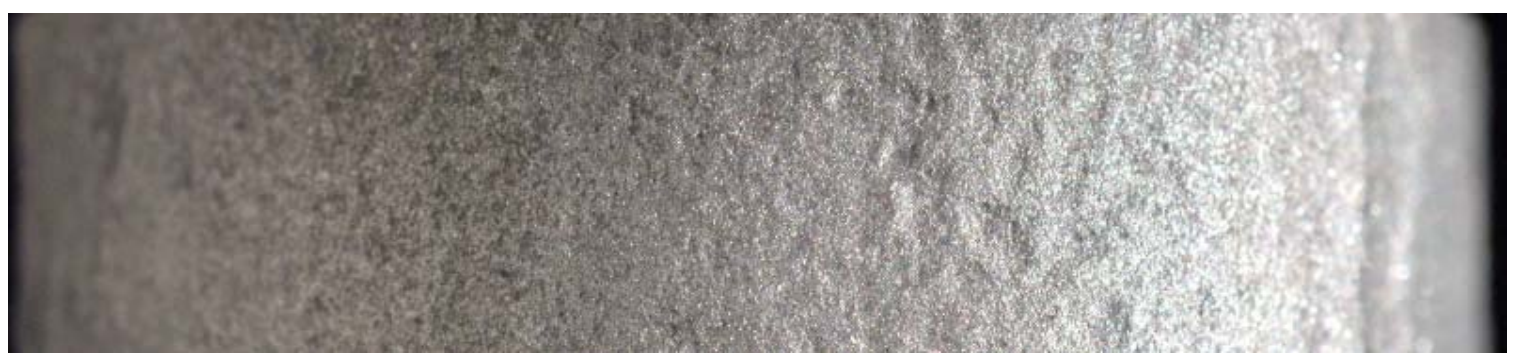

Figure C-7: OD Surface Appearance After Grit Blast

Material: $\quad 347$ HFG

Sample: I-J

C-14 
12 O'Clock

(142 mils lost)

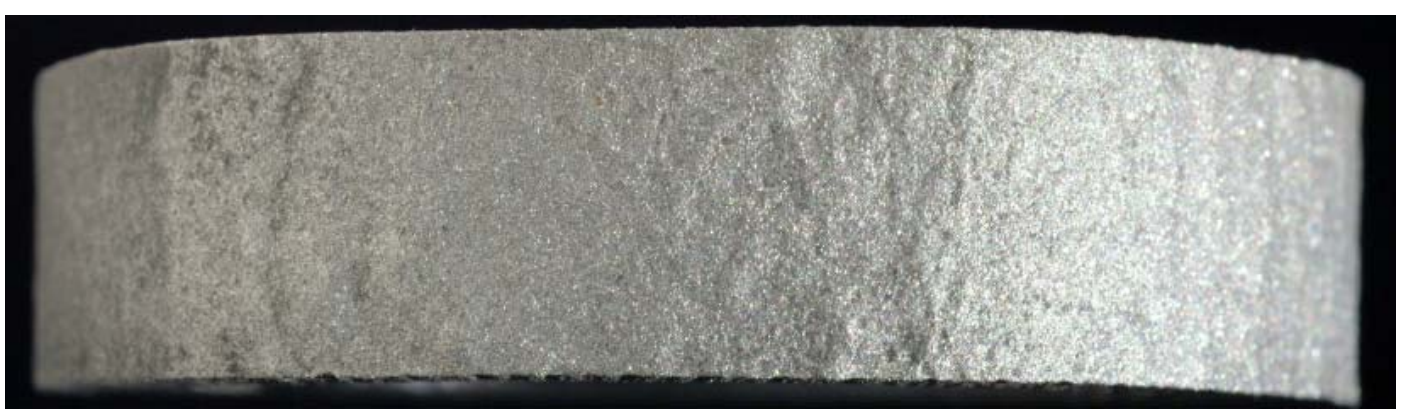

3 O'Clock

(60 mils lost)

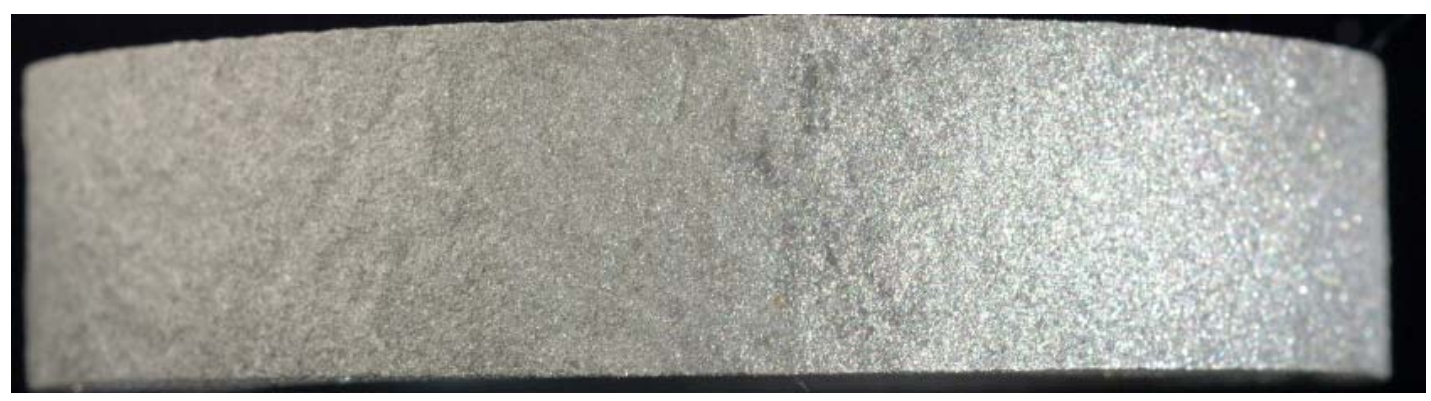

6 O'Clock

(15 mil lost)

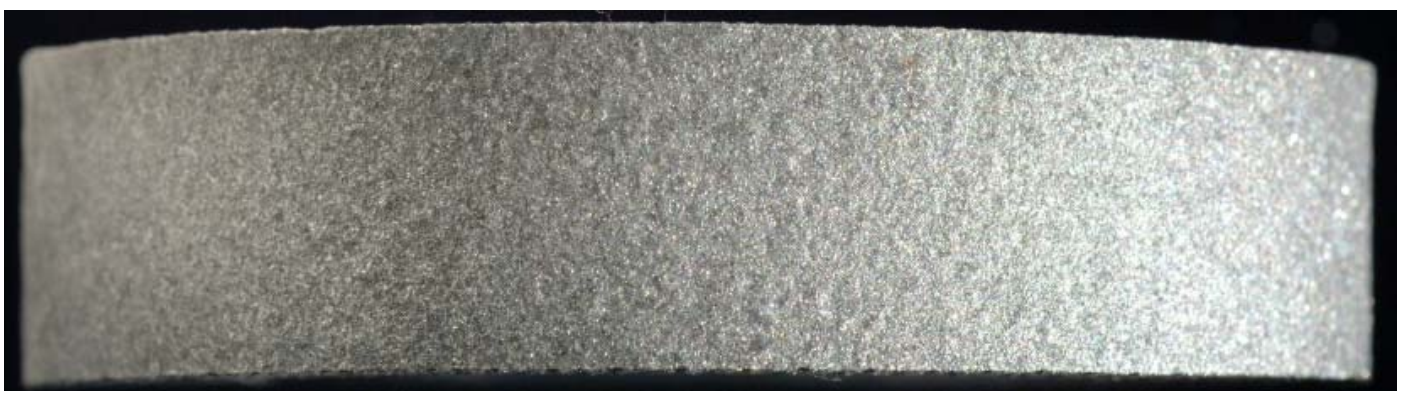

Figure C-8: OD Surface Appearance After Grit Blast

Material: 347 HFG

Sample: A-B

C-15 
12 O'Clock

(205 mils lost)

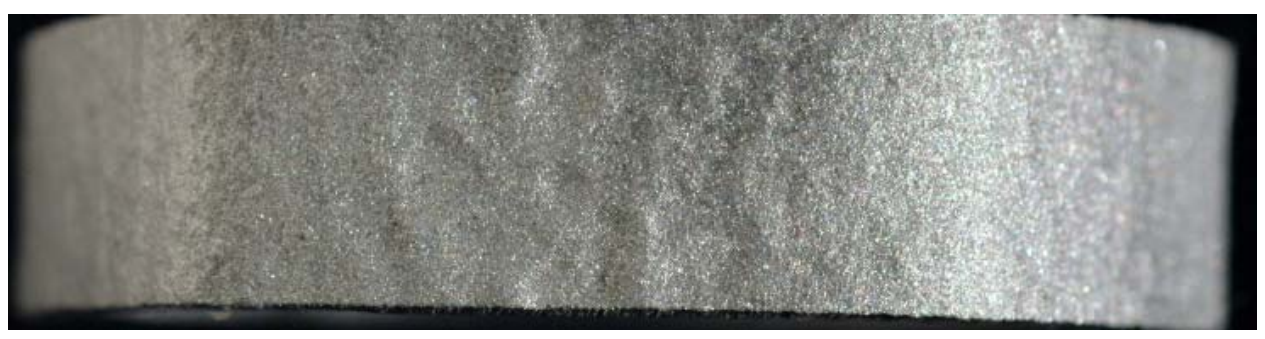

3 O'Clock

(229 mils lost)

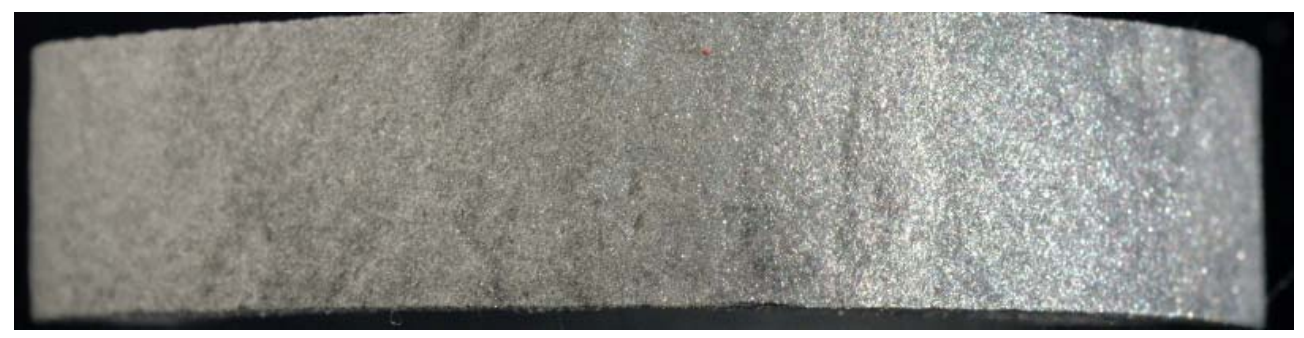

6 O'Clock

(8 mils lost)

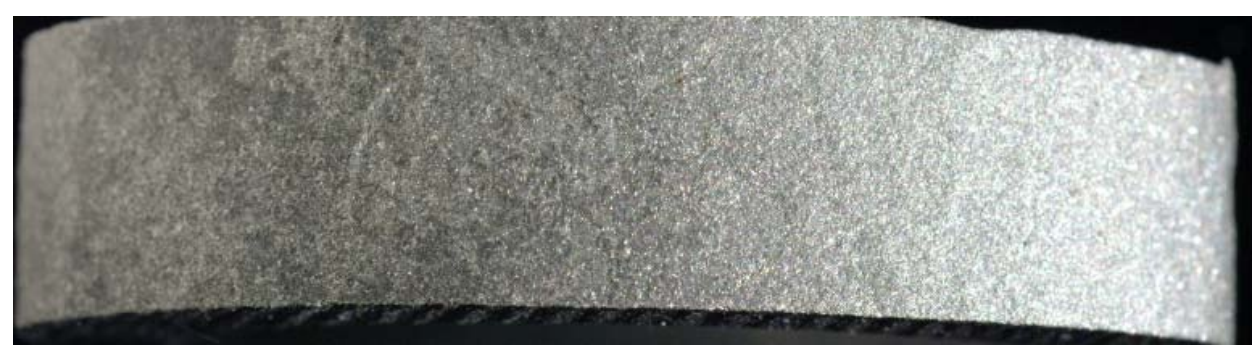

Figure C-9: OD Surface Appearance After Grit Blast

Material: $\quad 347$ HFG

Sample: $\quad$ CC-DD 


\section{Material:}

Sample Location:

Maximum Rate:

Maximum Loss Orientation:

Average metal Temperature:

\section{HFG}

I-J

$204 \mathrm{mils} / \mathrm{yr}$

Approx. 2:30 o'clock

$1174 \mathrm{~F}$

\section{O’Clock}

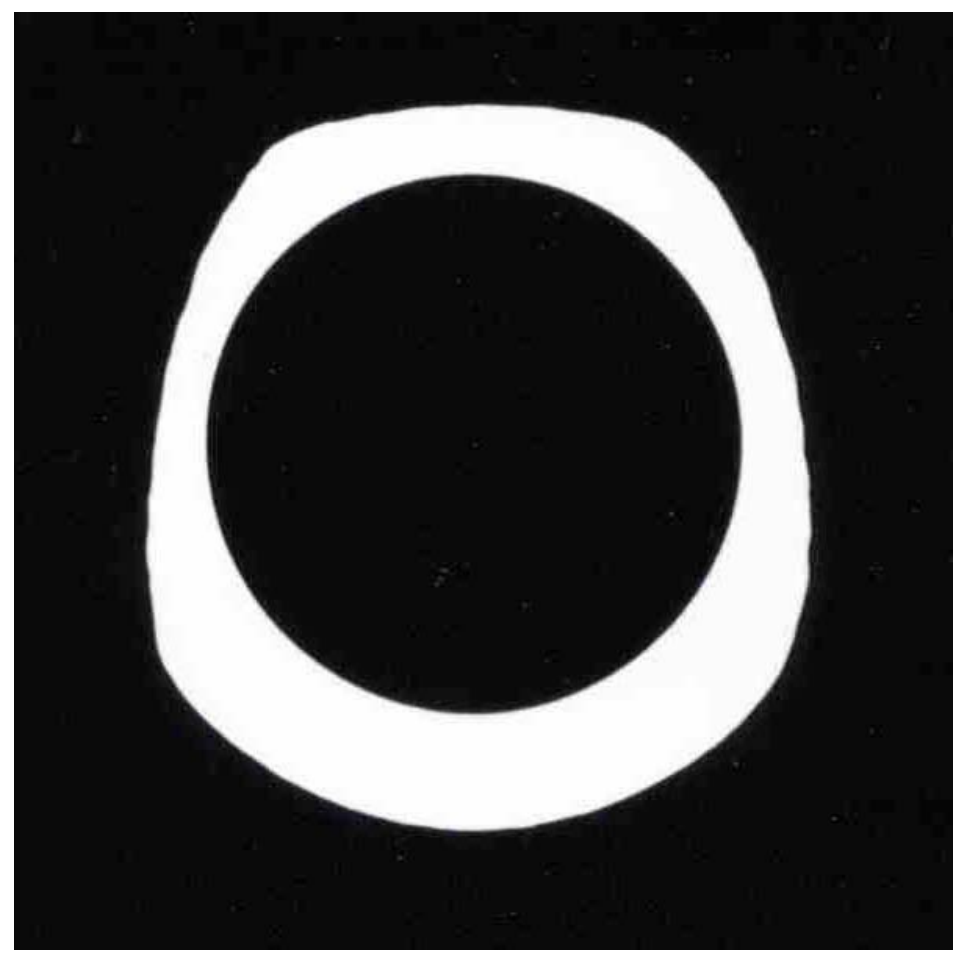

3 O'Clock

Approx. 1.2X

Wall Reduction by Position (in.)

\begin{tabular}{|l|c|c|c|c|c|c|c|c|c|c|}
\hline & $\begin{array}{c}12 \\
\text { o'clock }\end{array}$ & $\mathbf{1 : 3 0}$ & $\mathbf{3 : 0 0}$ & $\mathbf{4 : 3 0}$ & $\mathbf{6 : 0 0}$ & $\mathbf{7 : 3 0}$ & $\mathbf{9 : 0 0}$ & $\mathbf{1 0 : 3 0}$ & AVG & $\begin{array}{c}\text { Max }^{*} \\
\text { Loss }\end{array}$ \\
\hline Before & 0.397 & 0.401 & 0.402 & 0.403 & 0.403 & 0.405 & 0.404 & 0.398 & 0.402 & 0.402 \\
\hline After & 0.192 & 0.157 & 0.121 & 0.369 & 0.388 & 0.360 & 0.128 & 0.236 & & 0.085 \\
\hline Delta & 0.205 & 0.244 & 0.281 & 0.034 & 0.015 & 0.045 & 0.276 & 0.162 & & 0.317 \\
\hline
\end{tabular}

Figure C-10: Silhouette and Wall Thickness Measurements for Sample I-J

Note: this tube sample was removed from service on 11/6/01.

* Measurement taken at "maximum loss orientation" noted above. The average

"before" wall thickness was used to calculate the "delta". 


\section{Material:}

Sample Location:

Maximum Rate:

Maximum Loss Orientation:

Average metal Temperature:

\section{HFG}

A-B

$76 \mathrm{mils} / \mathrm{yr}$

Approx. 10:00 o'clock

1147 F

12 O’Clock

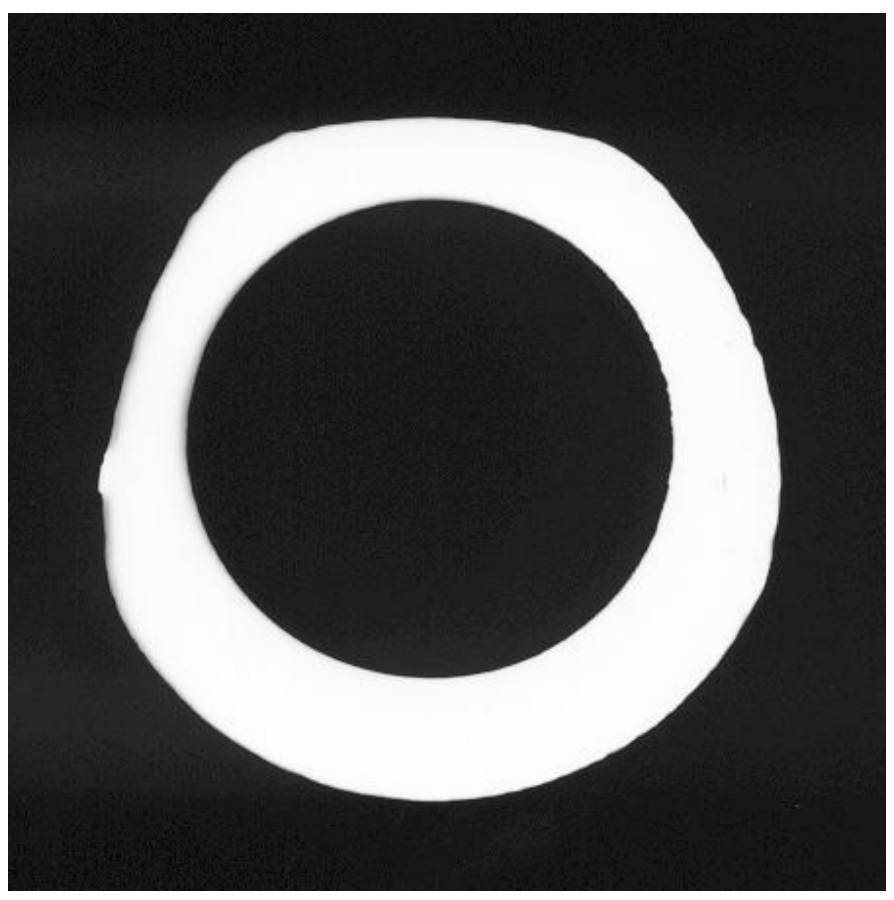

3 O’Clock

Approx. 1.2X

Wall Reduction by Position (in.)

\begin{tabular}{|l|c|c|c|c|c|c|c|c|c|c|}
\hline & $\begin{array}{c}\mathbf{1 2} \\
\text { o'clock }\end{array}$ & $\mathbf{1 : 3 0}$ & $\mathbf{3 : 0 0}$ & $\mathbf{4 : 3 0}$ & $\mathbf{6 : 0 0}$ & $\mathbf{7 : 3 0}$ & $\mathbf{9 : 0 0}$ & $\mathbf{1 0 : 3 0}$ & $\begin{array}{c}\text { MVG } \\
\text { Los }\end{array}$ \\
\hline Before & 0.441 & 0.438 & 0.436 & 0.436 & 0.439 & 0.441 & 0.443 & 0.443 & 0.440 & 0.440 \\
\hline After & 0.299 & 0.327 & 0.376 & 0.423 & 0.424 & 0.424 & 0.274 & 0.355 & & 0.259 \\
\hline Delta & 0.142 & 0.111 & 0.060 & 0.013 & 0.015 & 0.017 & 0.169 & 0.088 & & 0.181 \\
\hline
\end{tabular}

Figure C-11: Silhouette and Wall Thickness Measurements for Sample A-B

* Measurement taken at "maximum loss orientation" noted above. The average

"before" wall thickness was used to calculate the "delta". 


\section{Material:}

Sample Location:

Maximum Rate:

Maximum Loss Orientation:

Average metal Temperature:

\section{HFG}

CC-DD

$120 \mathrm{mils} / \mathrm{yr}$

Approx. 9:30 o'clock

$1086 \mathrm{~F}$

\section{O’Clock}

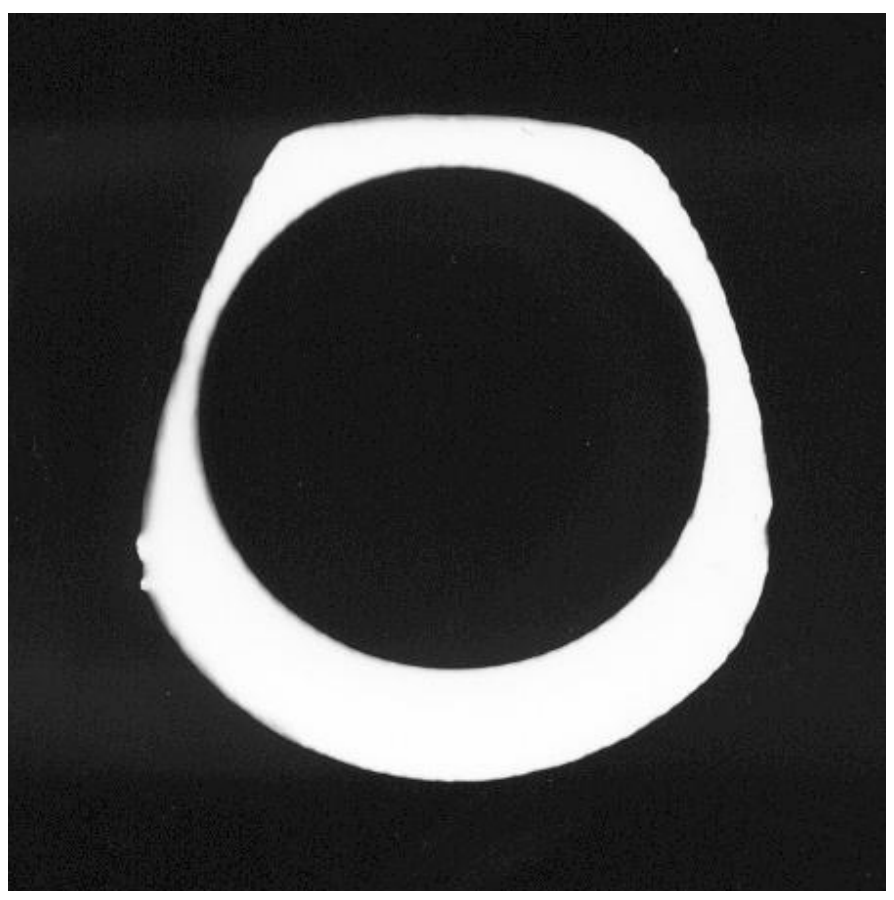

3 O’Clock

Approx. 1.2X

\section{Wall Reduction by Position (in.)}

\begin{tabular}{|l|c|c|c|c|c|c|c|c|c|c|}
\hline & $\begin{array}{c}\mathbf{1 2} \\
\text { o'clock }\end{array}$ & $\mathbf{1 : 3 0}$ & $\mathbf{3 : 0 0}$ & $\mathbf{4 : 3 0}$ & $\mathbf{6 : 0 0}$ & $\mathbf{7 : 3 0}$ & $\mathbf{9 : 0 0}$ & $\mathbf{1 0 : 3 0}$ & AVG & $\begin{array}{c}\text { Max } \\
\text { Loss }^{*}\end{array}$ \\
\hline Before & 0.398 & 0.402 & 0.402 & 0.399 & 0.397 & 0.397 & 0.398 & 0.397 & 0.399 & 0.399 \\
\hline After & 0.193 & 0.224 & 0.173 & 0.381 & 0.391 & 0.382 & 0.155 & 0.179 & & 0.114 \\
\hline Delta & 0.205 & 0.178 & 0.229 & 0.018 & 0.008 & 0.015 & 0.243 & 0.218 & & 0.285 \\
\hline
\end{tabular}

Figure C-12: Silhouette and Wall Thickness Measurements for Sample CC-DD

* Measurement taken at "maximum loss orientation" noted above. The average

"before" wall thickness was used to calculate the "delta". 


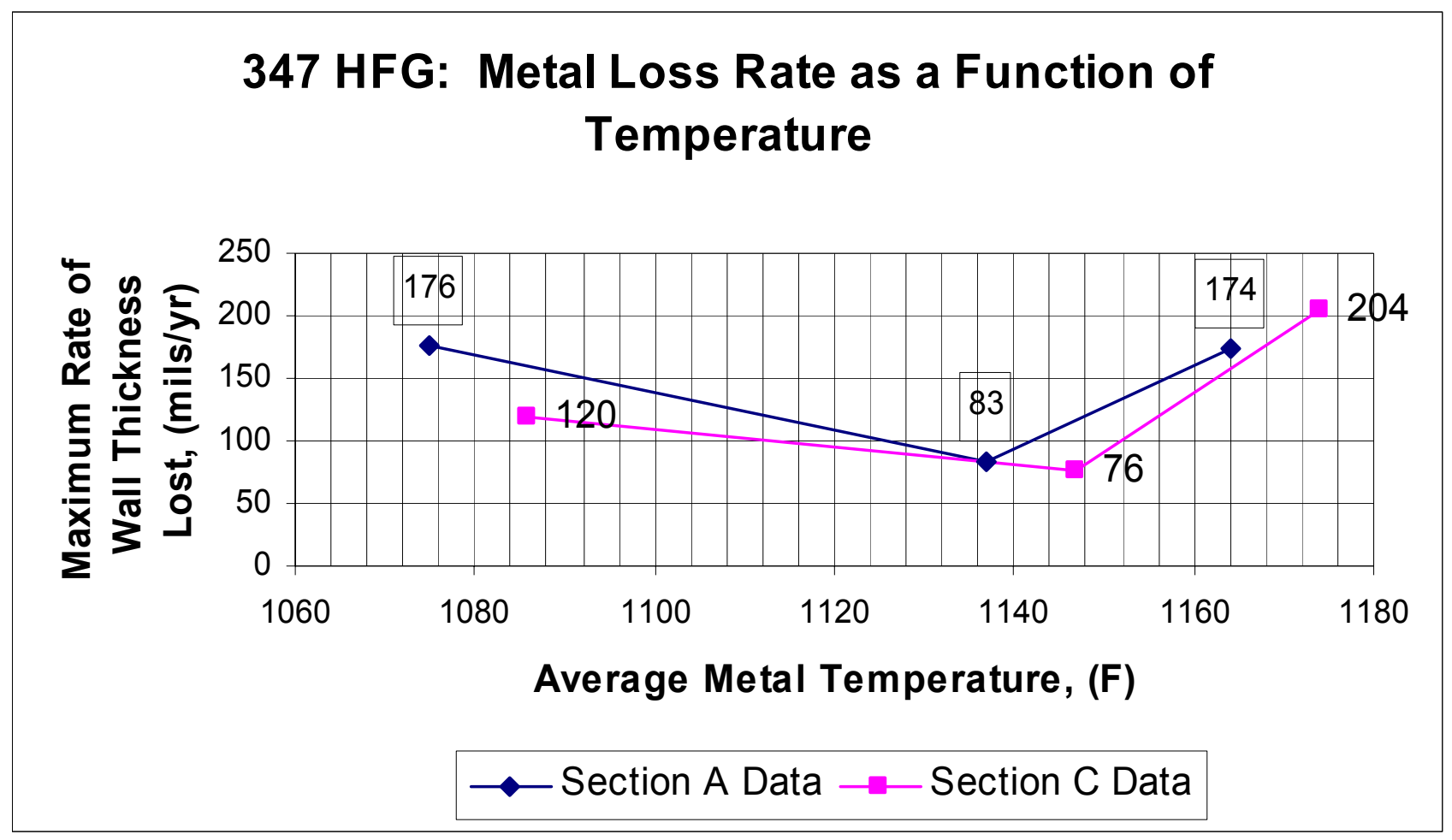

Figure C-13: Metal Loss Rate as a Function of Temperature

Material: 347 HFG

Note: In analyzing these data it is important to understand that one of the three Section C samples was removed at an earlier date than the other two., and so they experienced different lengths of exposure. Sample I-J (1175 F) was removed in November 2001, at about the same time as the Section A samples. Sample A-B (1147 F) and Sample CC-DD were removed with the remaining Section C samples in May 2003. 


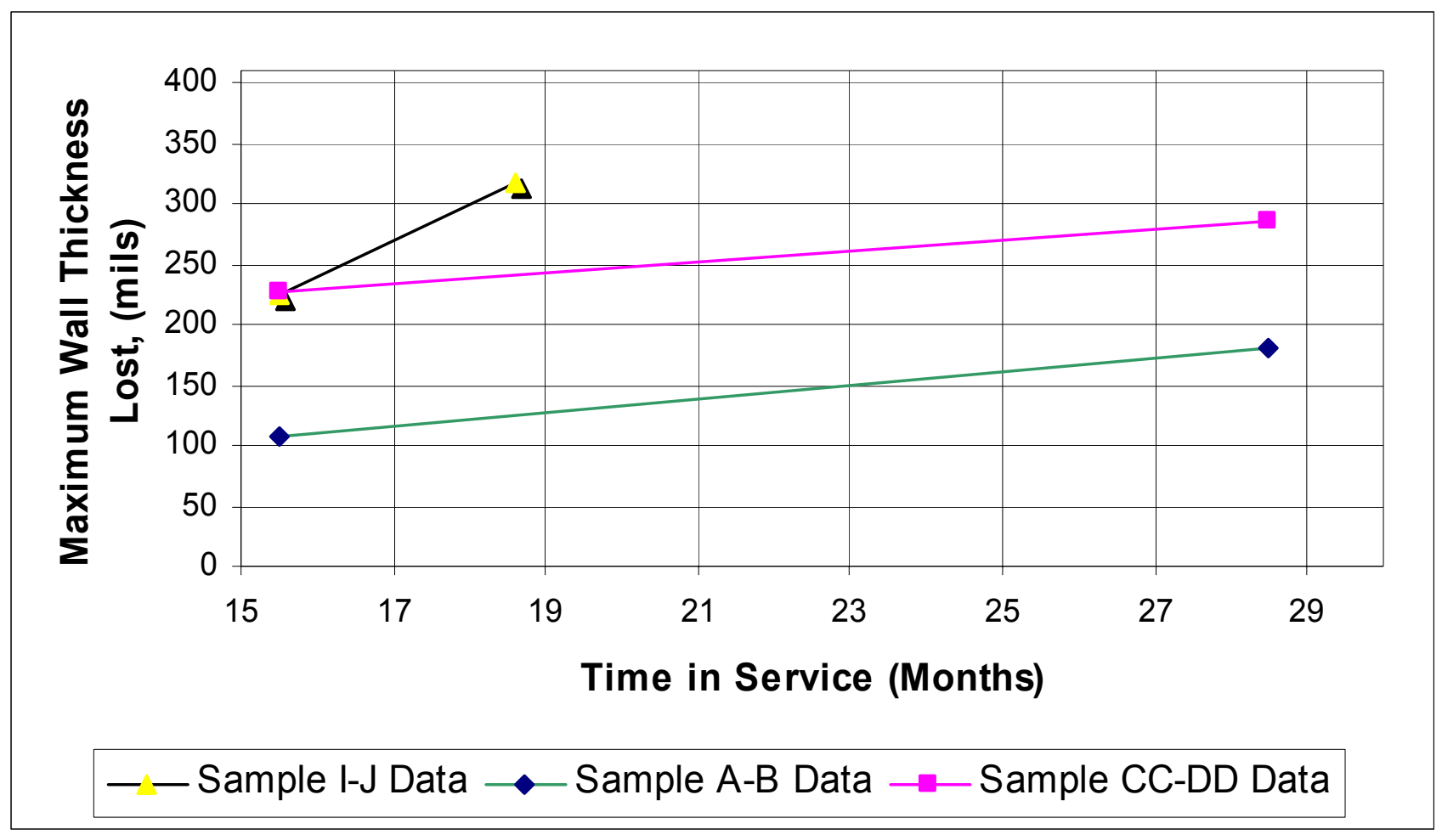

Figure C-14: Metal Loss as a Function of Time

Note: From the plot above it can be seen that, for Section C, Sample I-J was removed from service due to excessive metal losses at about the same time that Section A was removed for evaluation. Samples CC-DD and A-B both experienced the full exposure life for both Sections A and C. 


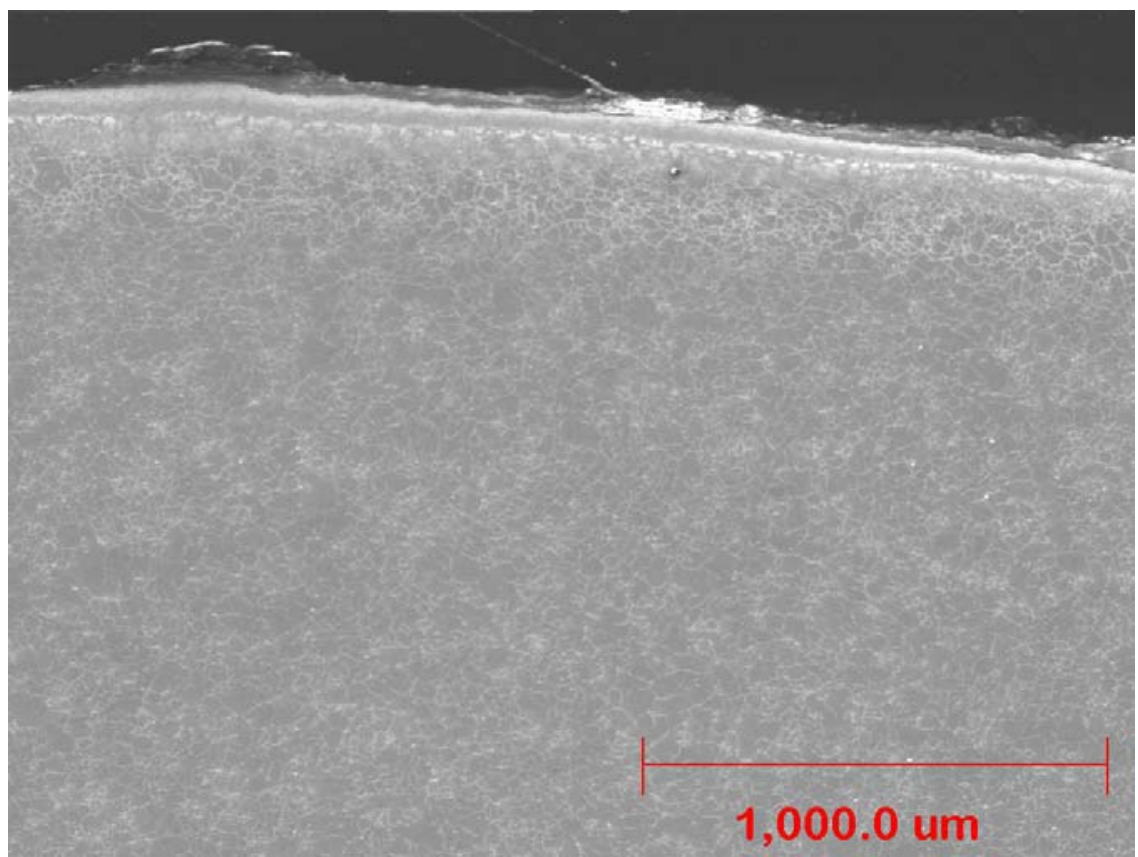

(a) External Scale/Metal Interface (SEI - Low Magnification)

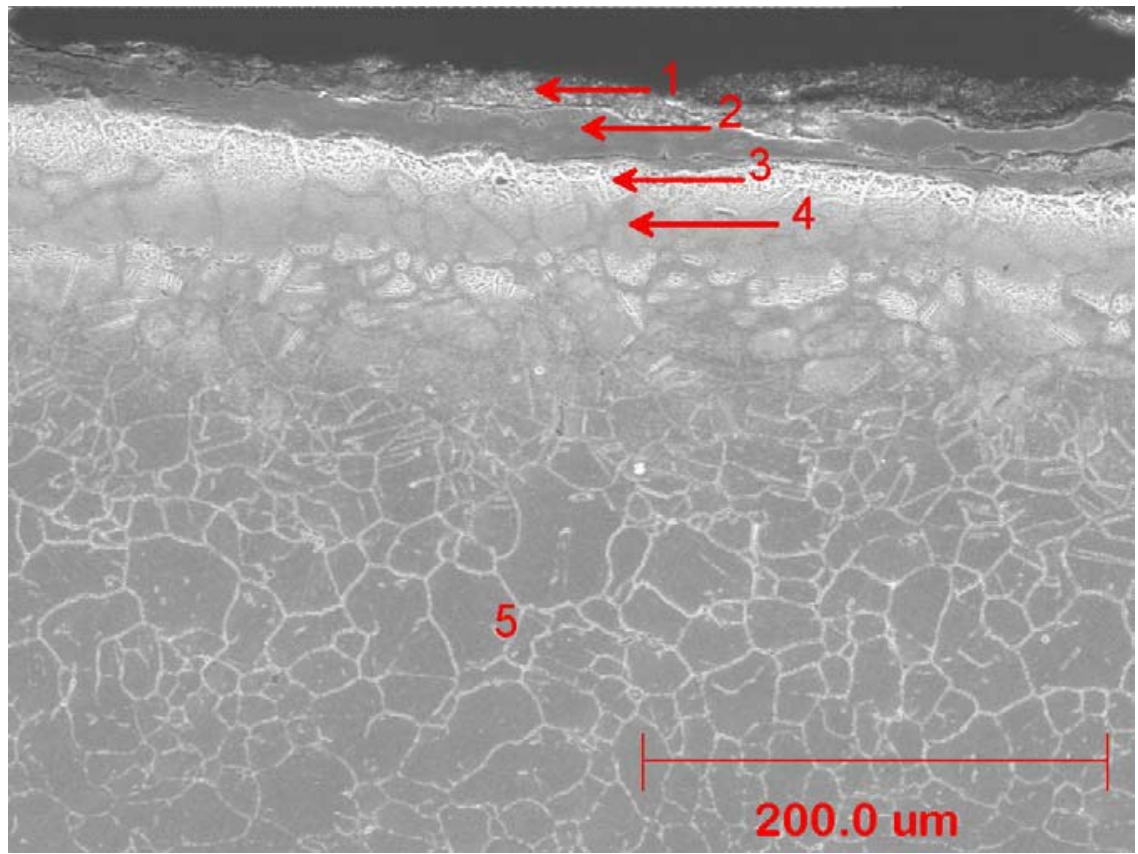

(b) External Scale/Metal Interface (SEI - Intermediate Magnification)

\section{C-15: Microstructure: 347 HFG}

Note: 1) Numbers on photos indicate distinct areas characterized by the visual assessment and EDS analyses that follow. 


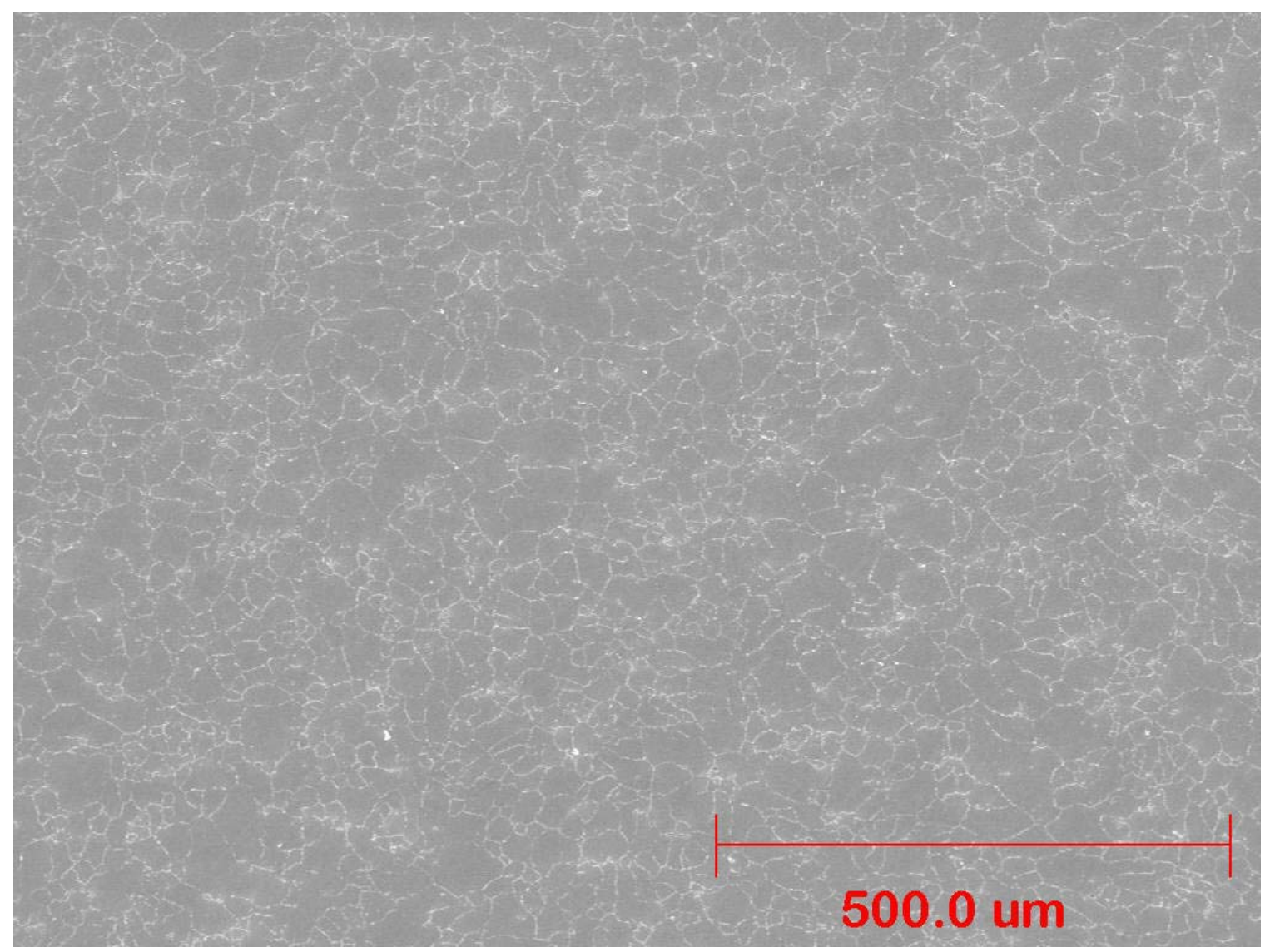

(SEI - Intermediate Magnification)

\section{C-16: Mid-Wall Microstructure: 347 HFG}

Note: Etchant - Electrolytic 10\% oxalic acid etch, 9V OCP 


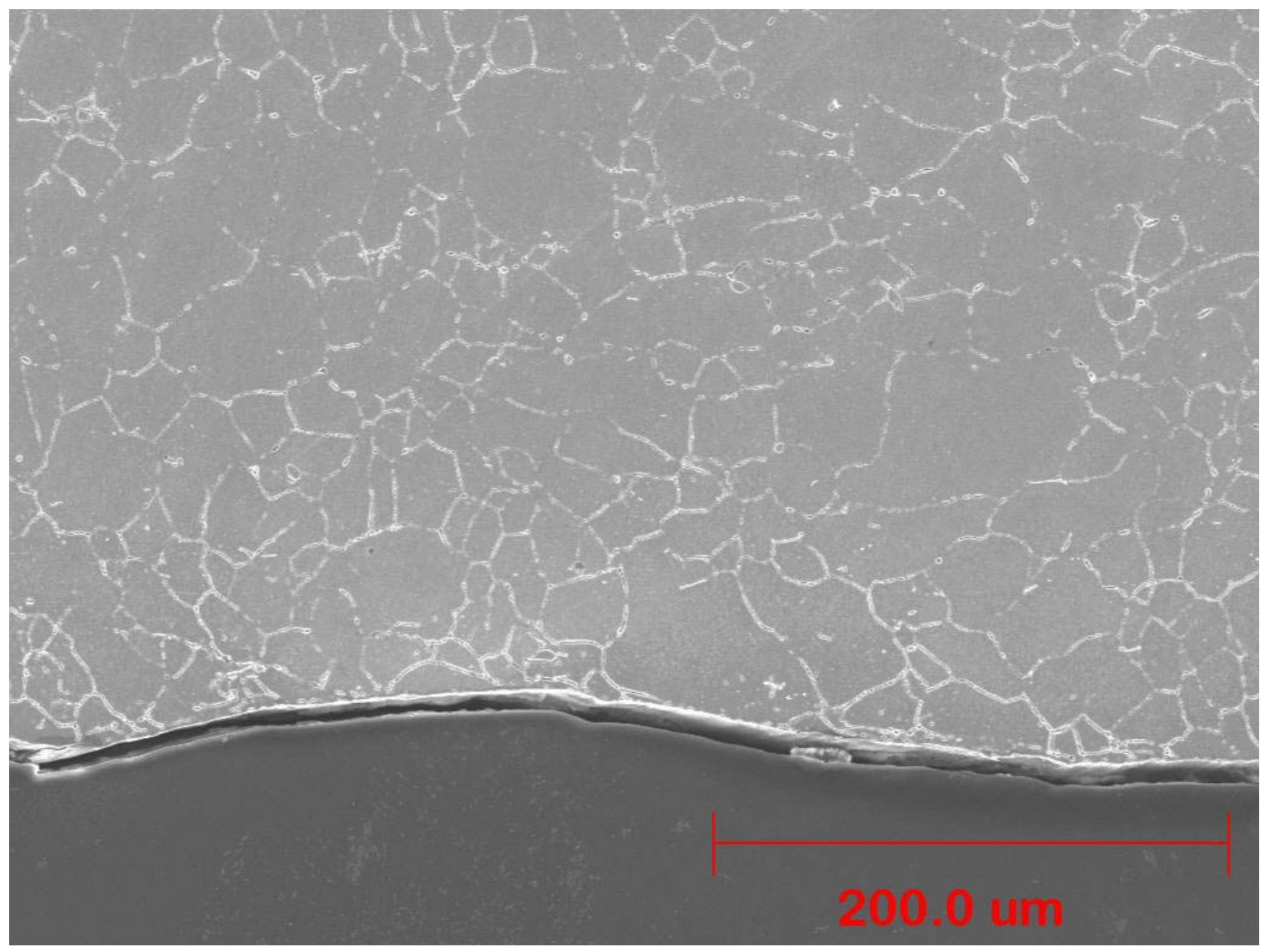

SEI - Internal Surface Appearance

Figure C-17: Microstructure: 347 HFG 


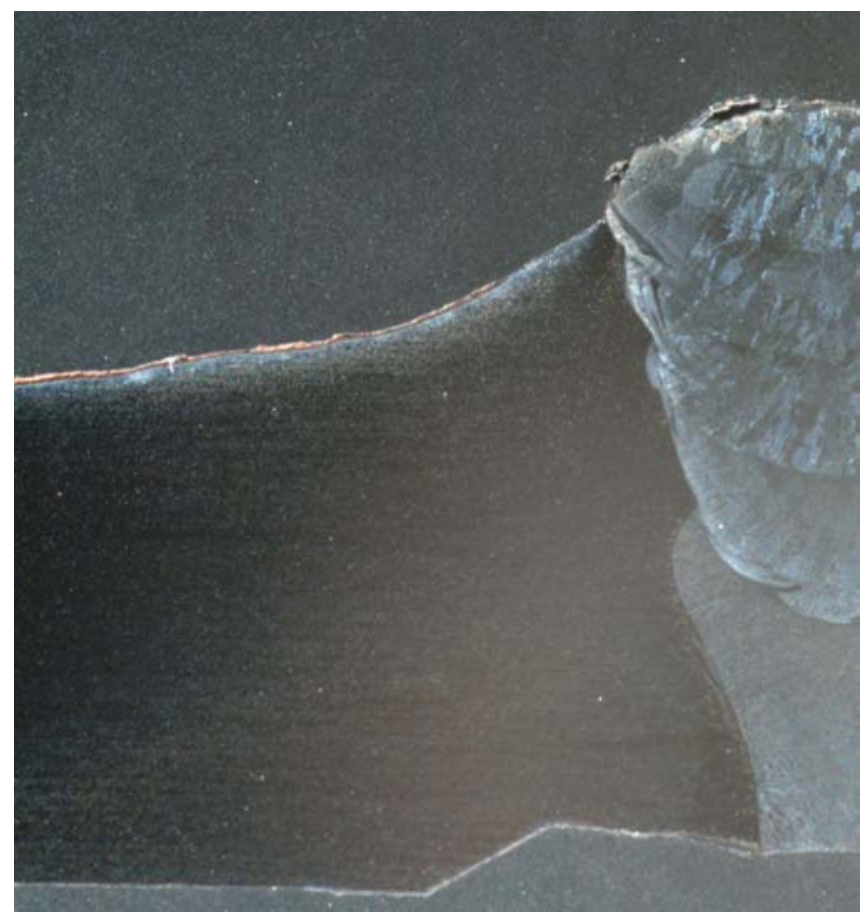

(a) 347 HFG Adjacent to Weld "B"

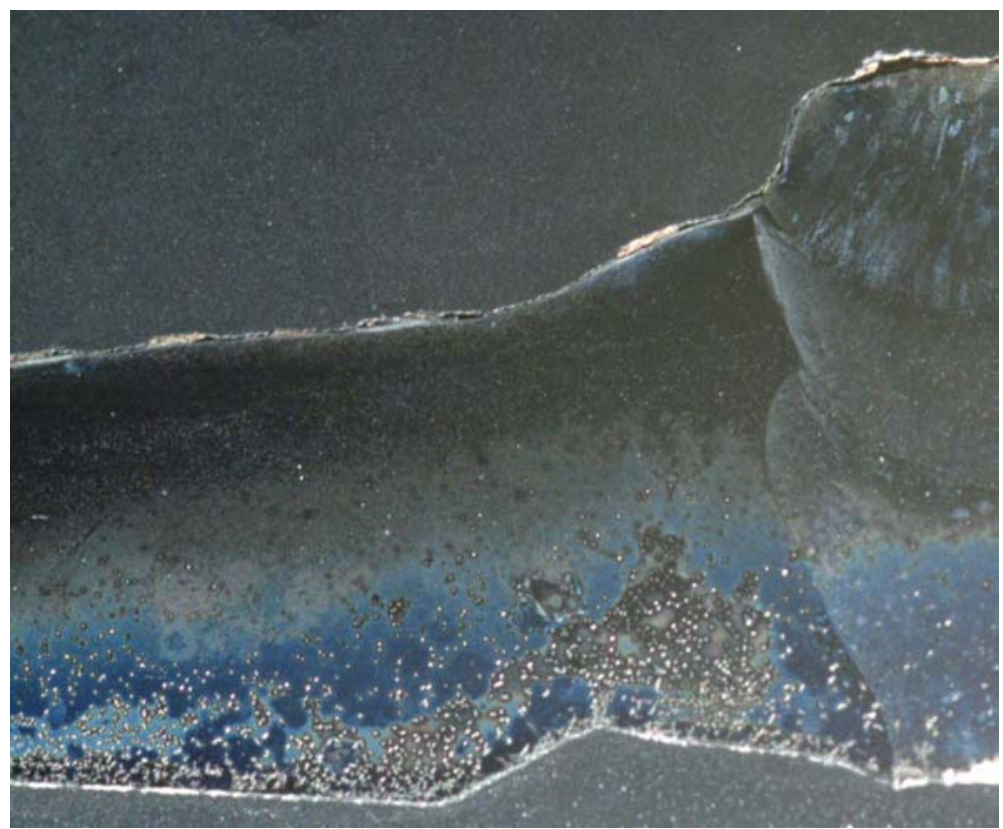

(b) 347 HFG Adjacent to Weld "DD"

Figure F-18: Longitudinal Cross-Sections: 347 HFG Adjacent to Welds 
Figure C-19: EDS Analysis of Deposit/Scale/Metal Interface for 347 HFG (Sample I-J)

\begin{tabular}{|c|c|c|c|c|c|c|c|c|c|c|c|c|c|c|c|c|c|c|}
\hline Area & Description & $\mathbf{N i}$ & $\mathrm{Cr}$ & $\mathbf{F e}$ & $\mathrm{Cu}$ & Mn & $\mathbf{N b}$ & Mo & Al & $\mathbf{S i}$ & $\mathbf{C}$ & $\mathbf{O}$ & $\mathbf{C l}$ & $\mathbf{K}$ & Zn & As & $\mathbf{P}$ & $\mathbf{S}$ \\
\hline 1 & $\begin{array}{l}\text { Bulk deposit } \\
\text { analysis }\end{array}$ & 0.52 & 2.15 & 49.6 & -- & -- & -- & -- & 1.21 & 2.82 & 31.1 & 11.4 & 0.31 & 0.58 & 1.01 & -- & 0.13 & 0.13 \\
\hline 2 & $\begin{array}{l}\text { Adherent } \\
\text { chromium- } \\
\text { oxide scale }\end{array}$ & 3.12 & 43.3 & 30.6 & -- & - & -- & -- & -- & 1.10 & -- & 19.1 & -- & 0.28 & -- & -- & -- & 1.42 \\
\hline 3 & $\begin{array}{l}\text { Deep etching } \\
\text { diffusion } \\
\text { zone at } \\
\text { scale/metal } \\
\text { interface. }\end{array}$ & 15.7 & 5.02 & 78.3 & -- & 0.67 & -- & - & -- & 0.26 & -- & -- & -- & -- & -- & -- & -- & -- \\
\hline 4 & $\begin{array}{l}\text { Gray etching } \\
\text { diffusion } \\
\text { zone near } \\
\text { interface. }\end{array}$ & 12.6 & 12.6 & 73.0 & -- & 1.06 & -- & -- & 0.39 & 0.30 & -- & -- & -- & -- & -- & -- & -- & -- \\
\hline \multirow[t]{2}{*}{5} & $\begin{array}{l}\text { Base metal } \\
\text { analysis. }\end{array}$ & 12.3 & 18.0 & 68.1 & & 1.05 & -- & -- & -- & 0.57 & -- & -- & -- & -- & -- & -- & -- & $\begin{array}{ll}- \\
\end{array}$ \\
\hline & $\begin{array}{l}\text { Reference } \\
\text { bulk } \\
\text { composition }\end{array}$ & 11.9 & 18.6 & 64.8 & 0.20 & 1.48 & 0.91 & 0.24 & 0.014 & 0.39 & 0.10 & -- & -- & -- & -- & -- & -- & -- \\
\hline
\end{tabular}




\section{Appendix D}

\section{Analysis of Performance}

\section{Test Section C}

NF 709 


\section{Overview of Results: NF 709}

NF 709 tube samples continued to evidence a high rate of metal loss due to service exposure. For samples in Section C the rate increased continuously with temperature, and ranged from 100 to 181 mils per year*. The fact that the starting wall thickness for these tubes was slightly less than 400 mils provides the reason why the sample with the highest corrosion rate (Sample J-K) had to be removed early to prolong the life of the section.

It should be noted that the metal loss rates determined to date for both Section A and Section C all were based on a ring sample that was extracted from mid-length of each tube sample. In this analysis additional attention was focused on metal losses in the vicinity of welds. Representative samples were taken and it was determined that, for the NF 709 samples evaluated, the Inconel 625 weld filler metal is reasonably resistant to coal ash corrosion and that the tube material immediately adjacent to the weld (i.e. the weld heat affected zone) was somewhat less affected by corrosion than was the remainder of the tube. Indeed the tube wall appeared to be progressively thinner toward the mid-point of the tube sample.

Analysis of deposit on the surface revealed that it had the known aggressive combination of species, namely iron, oxygen, the alkali metals, and sulfur. The presence of significant amounts of arsenic was unexpected, and its' significance in unclear. Examination and analysis of the scale adjacent to the metal surface revealed the chalky-white appearance associated with alkali-ironsulfates. 
Material: NF 709

\section{Summary of Findings}

\begin{tabular}{|c|c|}
\hline Issue & Discussion \\
\hline $\begin{array}{l}\text { Sample } \\
\text { Location and } \\
\text { Orientation. }\end{array}$ & $\begin{array}{l}\text { - In assessing the data for Section C, it is important to understand that } \\
\text { some tube samples had to be removed from the section early due to } \\
\text { excessive thinning. The schematic in Figure D-1 shows the relative } \\
\text { positions of the NF } 709 \text { samples in all three sections. Also, shown are } \\
\text { the positions of all of the excessively thinned tubes that were removed } \\
\text { and replaced in order to extend the life of Section C. } \\
\text { - One NF } 709 \text { sample was removed early. Sample J-K was removed in } \\
\text { November } 2001 \text {, (i.e. shortly after Section A was removed from } \\
\text { service for evaluation). The other two NF } 709 \text { samples survived for the } \\
\text { full Section C exposure period. } \\
\text { The sample that was removed early was set aside for later analysis, } \\
\text { and has been included in the current evaluation along with the other } \\
\text { tube samples that made up Section C. } \\
\text { Also shown in this figure is the calculated average surface metal } \\
\text { temperature at each location. }\end{array}$ \\
\hline $\begin{array}{l}\text { Composition } \\
\text { Verification. }\end{array}$ & $\begin{array}{l}\text { - Tubes from one heat of NF } 709 \text { (Heat 58733) were provided by } \\
\text { Nippon Steel Corporation (NSC) for testing in this program. NSC also } \\
\text { provided the composition for Heat 58733, and a reference target } \\
\text { composition that provided the tolerances for the alloying elements in } \\
\text { this material. These are shown in the table in Figure D-2 along with } \\
\text { the results of a semi-quantitative analysis that was used to verify the } \\
\text { composition of one of the NF } 709 \text { tubes in Section C. } \\
\text { During the evaluation of Section A, detailed composition verification } \\
\text { was undertaken for every tube sample that was removed from service. } \\
\text { This served, in part, to verify that the each tube material was located at } \\
\text { its' proper position within the test section. } \\
\text { Based on the good results for Section A, it was decided that it would } \\
\text { be unnecessary to analyze each and every tube sample for composition } \\
\text { verification. Rather, it was decided to select a representative sample } \\
\text { from each tube material type for confirmatory analysis. } \\
\text { During the Section A evaluation, the energy dispersive spectrographic } \\
\text { (EDS) capabilities of the scanning electron microscope (SEM) were } \\
\text { used to perform semi-quantitative analyses of each of the twelve } \\
\text { different candidate tube materials. Comparison of these EDS results } \\
\text { showed reasonably good correlation with results obtained using } \\
\text { quantitative analytical techniques, including optical emission } \\
\text { spectrometry. } \\
\text { Based on this, it was decided that the accuracy provided by EDS } \\
\text { would suffice for composition verification, but that more detailed } \\
\text { analyses would be undertaken if questions arose. } \\
\text { The results of the EDS analysis of tube sample C-D confirmed that the } \\
\text { correct material was used at this location. }\end{array}$ \\
\hline
\end{tabular}


Material: NF 709

\section{Summary of Findings, (continued)}

\begin{tabular}{|c|c|}
\hline Issue & Discussion \\
\hline $\begin{array}{l}\text { As-Received } \\
\text { Appearance }\end{array}$ & $\begin{array}{l}\text { - Figure D-3 shows the typical appearance of this tube material as it } \\
\text { came from NSC. } \\
\text { Figure D-4 through D- } 6 \text { document the as-received appearance of the } \\
\text { external surface after service in Section C. Three views are provided } \\
\text { for each of the three tube samples: } 12 \text { o'clock (the view that faces on- } \\
\text { coming gas flow), } 3 \text { o'clock (the view where the gas flow is tangent to } \\
\text { the surface), and } 6 \text { o'clock (the view that shows the back-side of the } \\
\text { tube relative to the gas flow direction. } \\
\text { - The reader should be alerted to the fact that, within the boiler, the gas } \\
\text { flows upward over these tubes. This implies that for the "o'clock" } \\
\text { conventions described above, (with the } 12 \text { o'clock orientation facing } \\
\text { the gas flow direction), the actual physical orientation of these tubes is } \\
\text { such that the } 12 \text { o'clock side of the tube faces downward, and the } 6 \\
\text { o'clock orientation faces upward within the boiler. } \\
\text { Wastage is most clearly visible at the } 3 \text { and } 12 \text { o'clock orientations for } \\
\text { all three samples. The raised surface of the adjoining Inconel } 625 \text { weld } \\
\text { metal provides a good reference for this. } \\
\text { Evidence of the gray/white scale associated with molten alkali attack } \\
\text { can best be seen at the } 3 \text { o'clock orientations for Samples C-D and } \\
\text { BB-CC. }\end{array}$ \\
\hline $\begin{array}{l}\text { Grit-Blasted } \\
\text { Surface } \\
\text { Appearance }\end{array}$ & $\begin{array}{l}\text { Figures D-7 through D-9 show the appearance of the external surface } \\
\text { of the NF } 709 \text { tube samples after grit-blasting to remove deposit and } \\
\text { scale. The } 12 \text { o'clock and } 3 \text { o'clock positions for all three samples } \\
\text { show the appearance of general wastage due to fireside corrosion. } \\
\text { The } 6 \text { o'clock position for Samples J-K and C-D show the pitting that } \\
\text { occurred on the back-side of these samples. Only minimal pitting was } \\
\text { noted at the } 6 \text { o'clock position for Sample BB-CC. }\end{array}$ \\
\hline $\begin{array}{l}\text { Wall } \\
\text { Thickness } \\
\text { Loss } \\
\text { Determined }\end{array}$ & $\begin{array}{l}\text { - Tube cross-sections were prepared to allow for measurement of wall } \\
\text { thickness to determine metal loss due to exposure. These are shown in } \\
\text { silhouette in Figures D-10 through D-12. } \\
\text { The same relative "o'clock" positions were maintained as discussed } \\
\text { earlier. Measurements were taken at eight evenly spaced locations } \\
\text { about the tube circumference. These were then compared with } \\
\text { measurements taken at these same locations prior to exposure. } \\
\text { - The before and after measurements were documented in the table that } \\
\text { accompanies each figure. These form the basis for the "delta" } \\
\text { calculation to determine the amount of wall thickness lost. } \\
\text { For all three samples, the minimum wall thickness in the cross section } \\
\text { was not at one of the eight predetermined locations. In these cases, } \\
\text { there were no "before" measurement for comparison to determine the } \\
\text { "delta". So, the average "before" wall thickness was used to calculate } \\
\text { the wall thickness lost at these off-locations. }\end{array}$ \\
\hline
\end{tabular}


Material: NF 709

\section{Summary of Findings, (continued)}

\begin{tabular}{|c|c|}
\hline Issue & Discussion \\
\hline $\begin{array}{l}\text { Rate of Wall } \\
\text { Thickness } \\
\text { Loss Versus } \\
\text { Average Metal } \\
\text { Temperature }\end{array}$ & $\begin{array}{l}\text { - The chart in Figure D-13 plots the maximum wall thickness lost } \\
\text { against the calculated average metal temperature for the three NF } 709 \\
\text { samples. Similar data for Section A also are plotted on this chart. } \\
\text { - For both sections, it was noted that there appears to be a "knee" in the } \\
\text { plotted curve that comes about due to a significant increase in the rate } \\
\text { of metal loss above an exposure temperature of about } 1160 \mathrm{~F} \text {. } \\
\text { - As was mentioned earlier, Sample J-K was removed early due to } \\
\text { excessive metal losses. }\end{array}$ \\
\hline $\begin{array}{l}\text { Metal Loss as } \\
\text { a Function of } \\
\text { Time at Each } \\
\text { Location }\end{array}$ & $\begin{array}{l}\text { - The chart in Figure D-14 plots metal loss as a function of time for each } \\
\text { sample location for both Sections A and C, with samples from Section } \\
\text { C having experienced generally longer exposure times. } \\
\text { - Shortly after Section A was removed for analysis, the tube Sample J-K } \\
\text { was also removed and replaced in Section C. } \\
\text { The chart shows a straight line fit to the maximum metal loss data } \\
\text { points for Samples C-D and BB-CC for both Sections A and C. } \\
\text { Evaluation of the rate of metal loss data depicted in Figure D-13 } \\
\text { suggests that a straight line fit might well be appropriate for these data } \\
\text { in that the rate of metal loss was similar for both locations for both } \\
\text { Section A and Section C. }\end{array}$ \\
\hline $\begin{array}{l}\text { Metallurgical } \\
\text { Analysis - } \\
\text { SEM }\end{array}$ & $\begin{array}{l}\text { - Sample C-D was selected for a detailed SEM/EDAX assessment of the } \\
\text { constituents and structure in the vicinity of the external and internal } \\
\text { surfaces. This sample was selected based on the fact that it had lost the } \\
\text { most wall thickness of the two NF } 709 \text { samples that survived the full } \\
\text { exposure period for Section C. The focus of this investigation was in } \\
\text { the region of the tube where the maximum amount of material was } \\
\text { lost. } \\
\text { - Figures D-15a, and b show secondary electron images that document } \\
\text { the appearance of the scale/metal interface for Sample C-D at } \\
\text { increasing magnifications. Six distinct regions are in evidence. These } \\
\text { regions are, starting within the metal and working out: } \\
\text { Base metal - oxalic acid etch delineates grain boundaries and } \\
\text { carbides. } \\
\text { Light etching base metal - this region is obvious as a light- } \\
\text { appearing band that is parallel to the metal surface, perhaps } \\
\text { see most clearly in Figure D-15b. This figure shows that this } \\
\text { band is about one grain wide, and affects only those grains } \\
\text { that are within a grain or two of the interface. This likely is } \\
\text { due to composition differences related to diffusion in this }\end{array}$ \\
\hline
\end{tabular}




\section{Summary of Findings, (continued)}

\section{Material: $\quad$ NF 709}

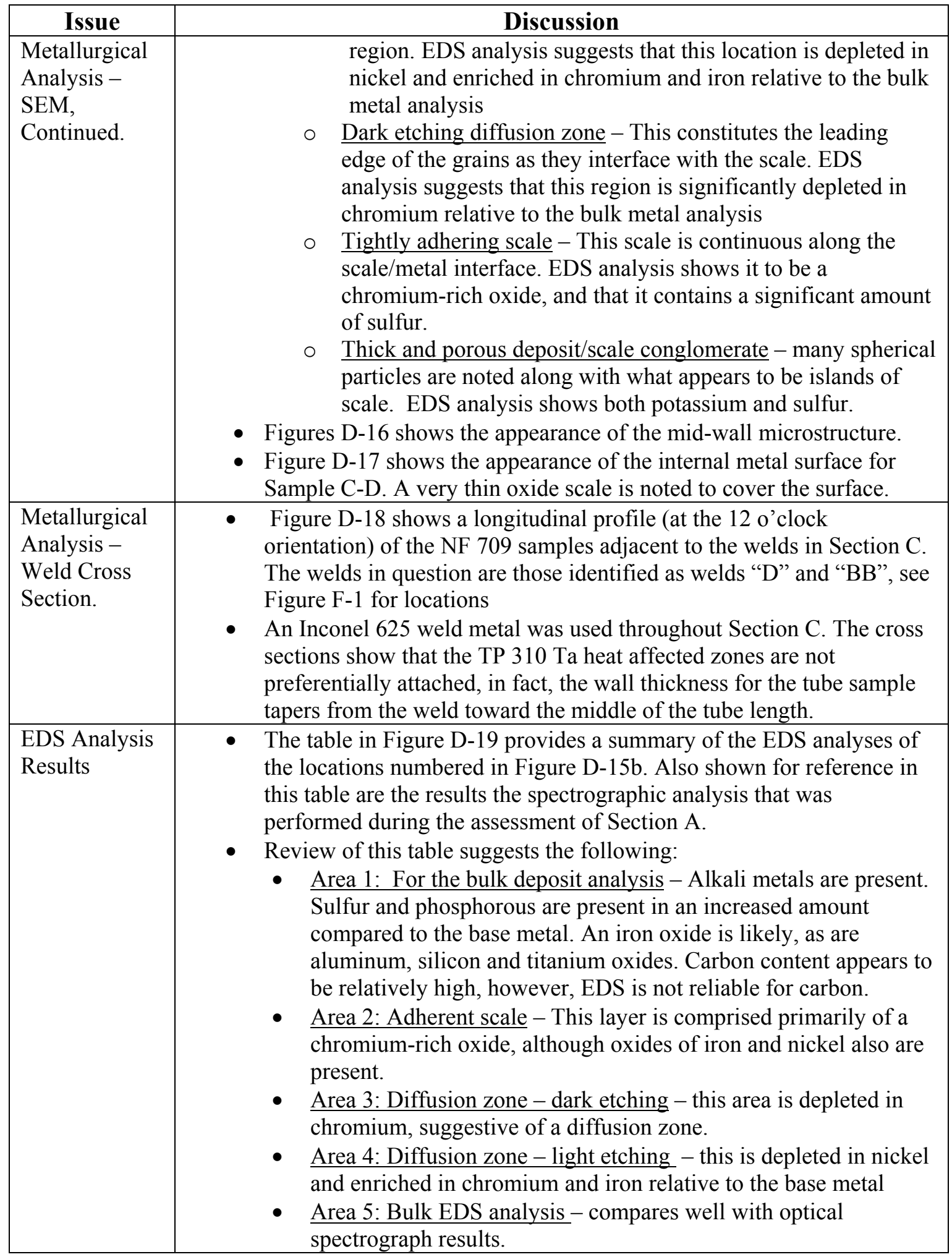




\section{Gas Flow}

Direction

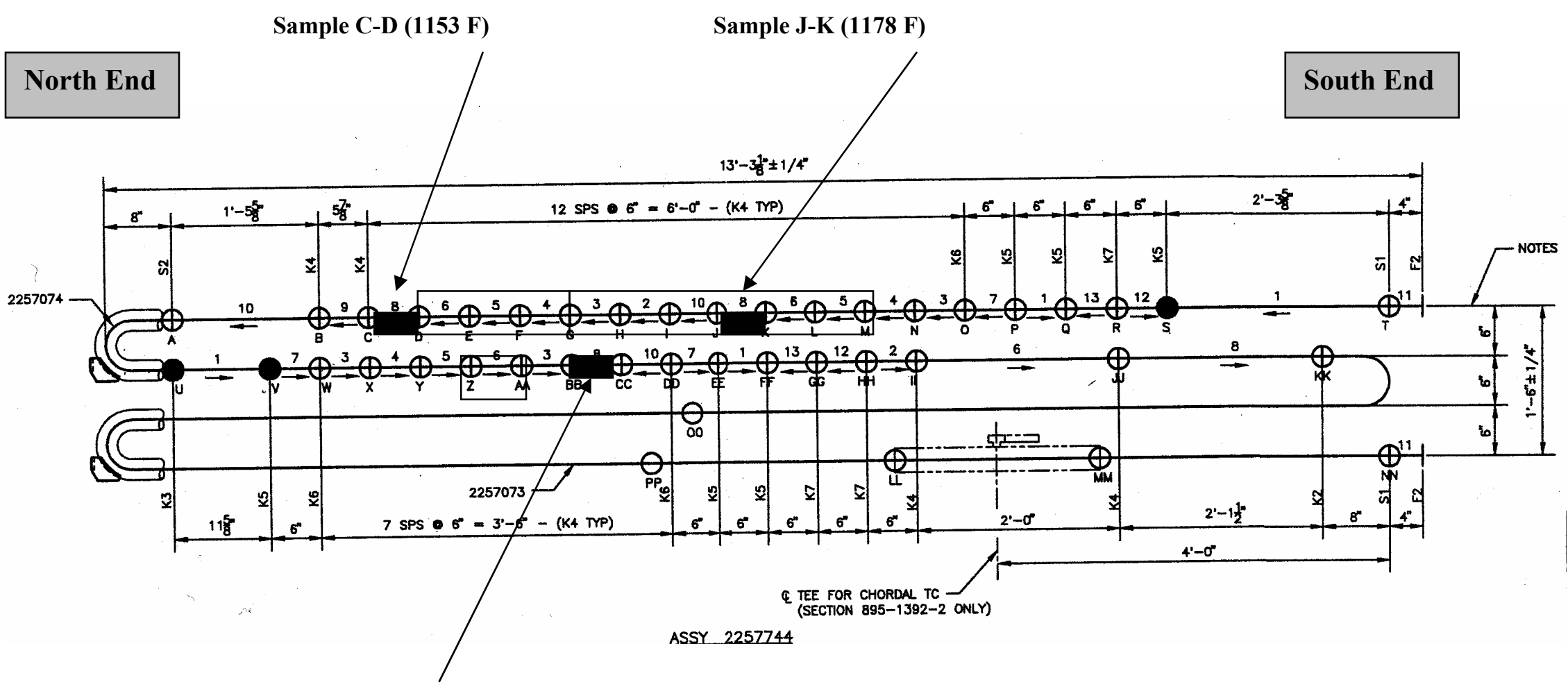

Sample BB-CC (1088 F)

Figure D-1: Schematic Drawing Showing Position of NF 709 Tube Samples Within Section C

1. Drawing shown in same relative orientation as per the actual installation.

2. This group of tube samples was removed from service in November 2001 - highlighted in red.

3. This group of tube samples was removed from service in June 2002 - highlighted in yellow. 
Figure D-2 Semi_Quantitative Composition Verification for NF709

Description:

Source:

Reference Composition: delivered.
Nominal $20 \mathrm{Cr} / 25 \mathrm{Ni}$ steel with $\mathrm{Nb}$ and $\mathrm{Ti}$ added to provide creep strength.

Nippon Steel Corporation (NSC)

"Specified" chemistry - per NSC inspection certificate for heat

\begin{tabular}{|c|c|c|c|c|c|}
\hline Element & $\begin{array}{c}\text { Reference } \\
\text { Composition }\end{array}$ & $\begin{array}{l}\text { Composition } \\
\text { Heat E58733 }\end{array}$ & $\begin{array}{c}\text { Tube Sample } \\
\text { C-D }\end{array}$ & $\begin{array}{c}\text { Tube Sample } \\
\text { J-K }\end{array}$ & $\begin{array}{c}\text { Tube Sample } \\
\text { BB-CC }\end{array}$ \\
\hline $\mathrm{Ni}$ & $22.00-28.00$ & 24.87 & 25.0 & $\mathrm{~N} / \mathrm{A}$ & $\mathrm{N} / \mathrm{A}$ \\
\hline $\mathrm{Cr}$ & $19.00-23.00$ & 22.29 & 21.9 & $\mathrm{~N} / \mathrm{A}$ & $\mathrm{N} / \mathrm{A}$ \\
\hline $\mathrm{Fe}$ & - & - & 50.4 & $\mathrm{~N} / \mathrm{A}$ & $\mathrm{N} / \mathrm{A}$ \\
\hline Mo & $1.00-2.00$ & 1.42 & 1.5 & N/A & N/A \\
\hline $\mathrm{Co}$ & - & - & - & $\mathrm{N} / \mathrm{A}$ & $\mathrm{N} / \mathrm{A}$ \\
\hline $\mathrm{C}$ & $0.1 \max$ & 0.02 & - & $\mathrm{N} / \mathrm{A}$ & $\mathrm{N} / \mathrm{A}$ \\
\hline $\mathrm{N}$ & $0.100-0.250$ & 0.176 & - & $\mathrm{N} / \mathrm{A}$ & $\mathrm{N} / \mathrm{A}$ \\
\hline B & $0.0020-0.0100$ & 0.0044 & - & $\mathrm{N} / \mathrm{A}$ & $\mathrm{N} / \mathrm{A}$ \\
\hline $\mathrm{Mn}$ & $1.50 \max$ & 0.97 & 0.73 & $\mathrm{~N} / \mathrm{A}$ & $\mathrm{N} / \mathrm{A}$ \\
\hline $\mathrm{Si}$ & $1.00 \mathrm{max}$ & 0.40 & 0.41 & $\mathrm{~N} / \mathrm{A}$ & $\mathrm{N} / \mathrm{A}$ \\
\hline $\mathrm{Al}$ & - & - & - & N/A & $\mathrm{N} / \mathrm{A}$ \\
\hline $\mathrm{Ti}$ & $0.02-0.20$ & 0.05 & - & $\mathrm{N} / \mathrm{A}$ & $\mathrm{N} / \mathrm{A}$ \\
\hline $\mathrm{Al}+\mathrm{Ti}$ & - & - & - & $\mathrm{N} / \mathrm{A}$ & $\mathrm{N} / \mathrm{A}$ \\
\hline $\mathrm{Nb}$ & $0.10-0.40$ & 0.23 & N/D & $\mathrm{N} / \mathrm{A}$ & N/A \\
\hline $\mathrm{Ta}$ & - & - & - & $\mathrm{N} / \mathrm{A}$ & $\mathrm{N} / \mathrm{A}$ \\
\hline $\mathrm{Nb}+\mathrm{Ta}$ & - & - & - & $\mathrm{N} / \mathrm{A}$ & $\mathrm{N} / \mathrm{A}$ \\
\hline $\mathrm{V}$ & - & - & - & $\mathrm{N} / \mathrm{A}$ & $\mathrm{N} / \mathrm{A}$ \\
\hline $\mathrm{W}$ & - & - & - & $\mathrm{N} / \mathrm{A}$ & N/A \\
\hline $\mathrm{Cu}$ & - & - & - & $\mathrm{N} / \mathrm{A}$ & $\mathrm{N} / \mathrm{A}$ \\
\hline $\mathrm{Sn}$ & - & - & - & $\mathrm{N} / \mathrm{A}$ & $\mathrm{N} / \mathrm{A}$ \\
\hline $\mathrm{P}$ & $0.030 \max$ & 0.010 & - & $\mathrm{N} / \mathrm{A}$ & $\mathrm{N} / \mathrm{A}$ \\
\hline $\mathrm{S}$ & $0.010 \max$ & 0.001 & - & $\mathrm{N} / \mathrm{A}$ & $\mathrm{N} / \mathrm{A}$ \\
\hline $\mathrm{O} 2$ & - & - & - & $\mathrm{N} / \mathrm{A}$ & $\mathrm{N} / \mathrm{A}$ \\
\hline
\end{tabular}

$\mathrm{N} / \mathrm{D}=\mathrm{EDS}$ technique (gold coating) precluded an accurate niobium determination.

$\mathrm{N} / \mathrm{A}=$ Tube sample not analyzed. 


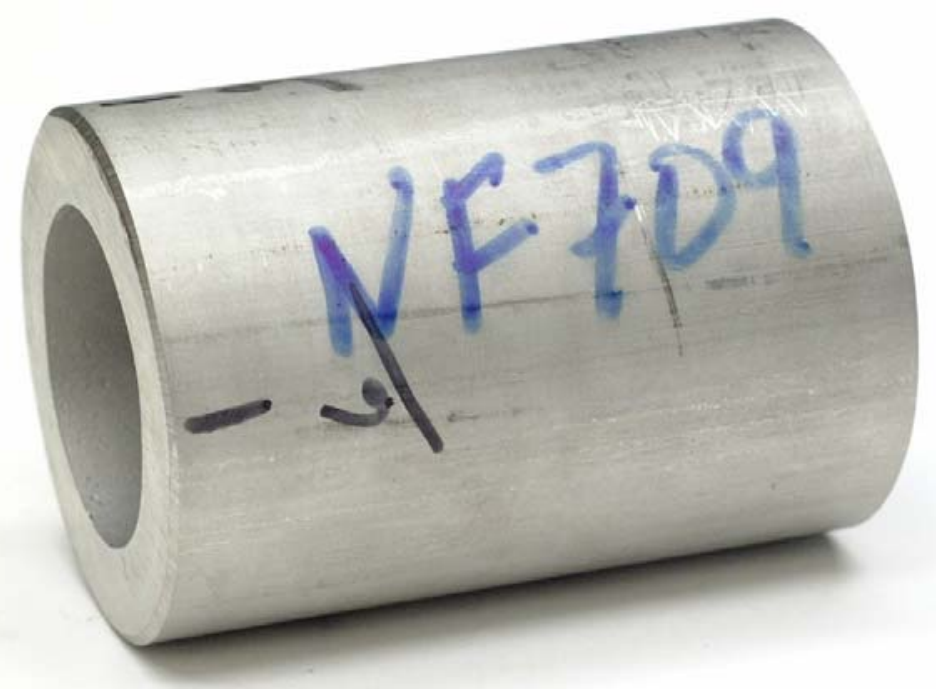

Figure D-3: Appearance of NF 709 Tube Sample Prior to Service 


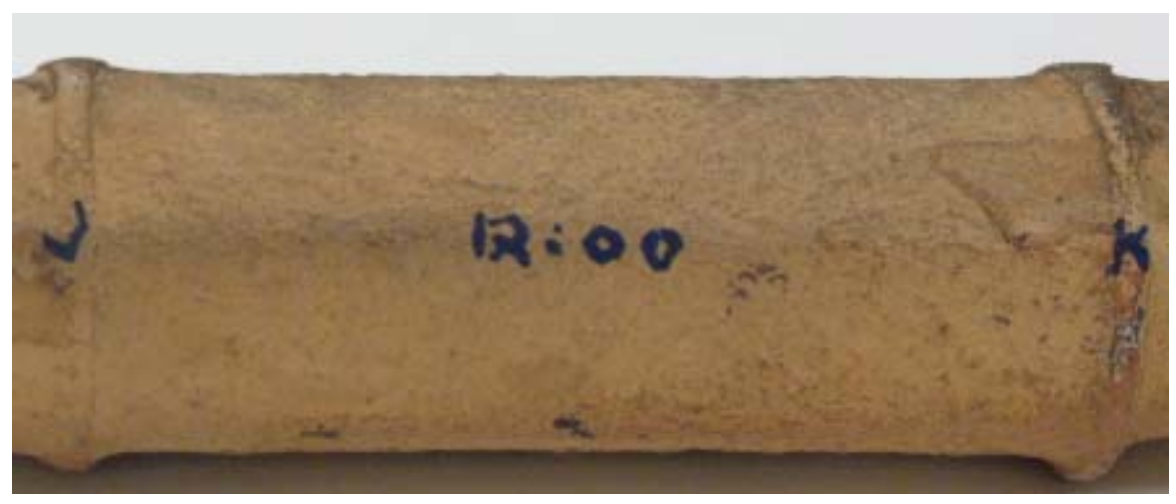

12 O'Clock - Facing Gas Flow

(Note: 3 o'clock is up in this image)

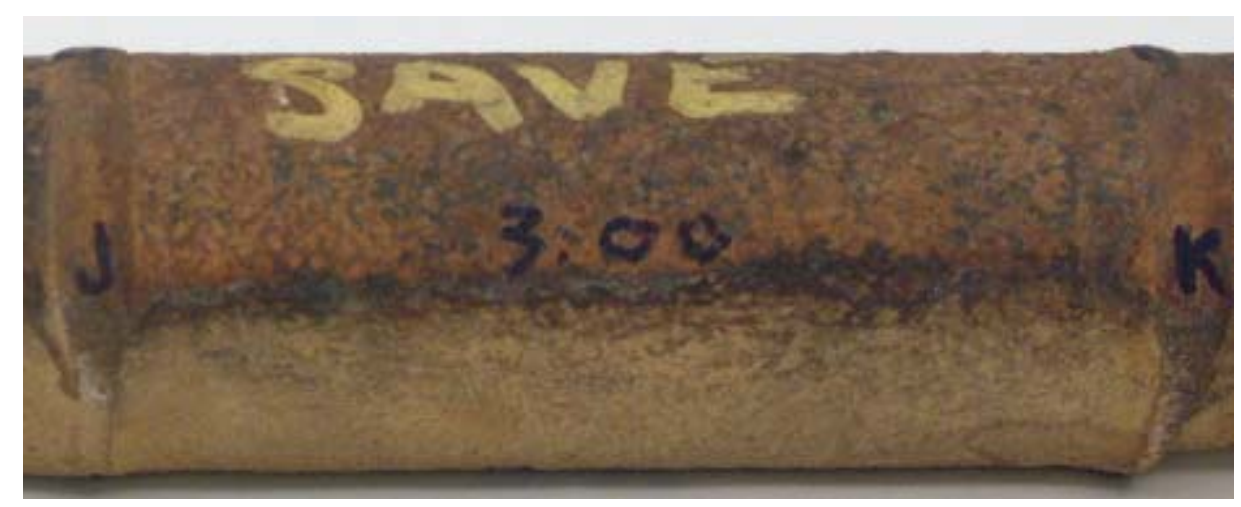

3 O'Clock

(Note: 6 o'clock is up in this image)

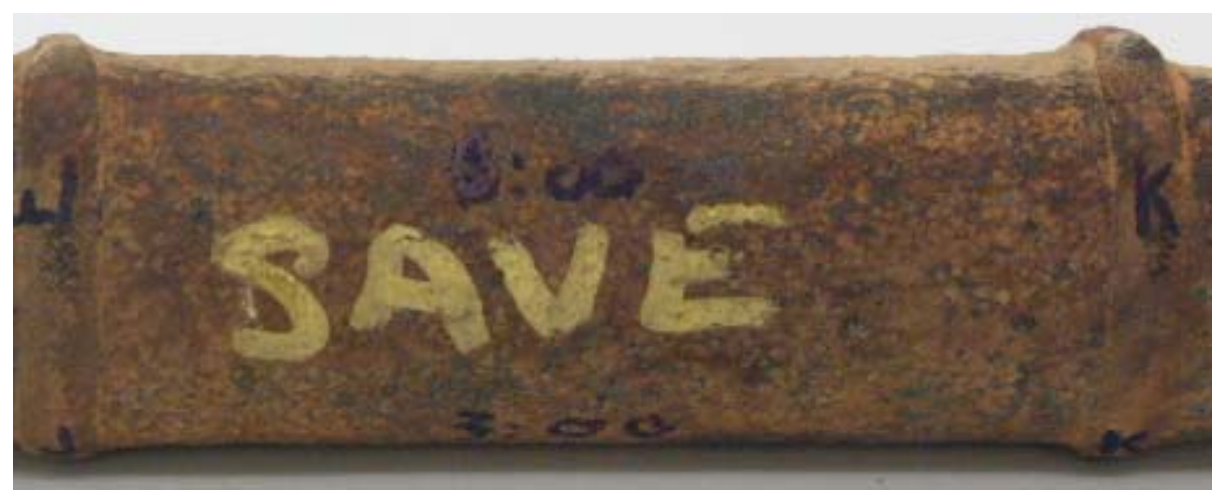

6 O'Clock - Opposite Gas Flow

Figure D-4 As-Received Appearance of Sample J-K (NF 709)

\section{(Average metal temperature: $1178 \mathrm{~F}$}

Note: this tube was removed from service on 11/6/01. 


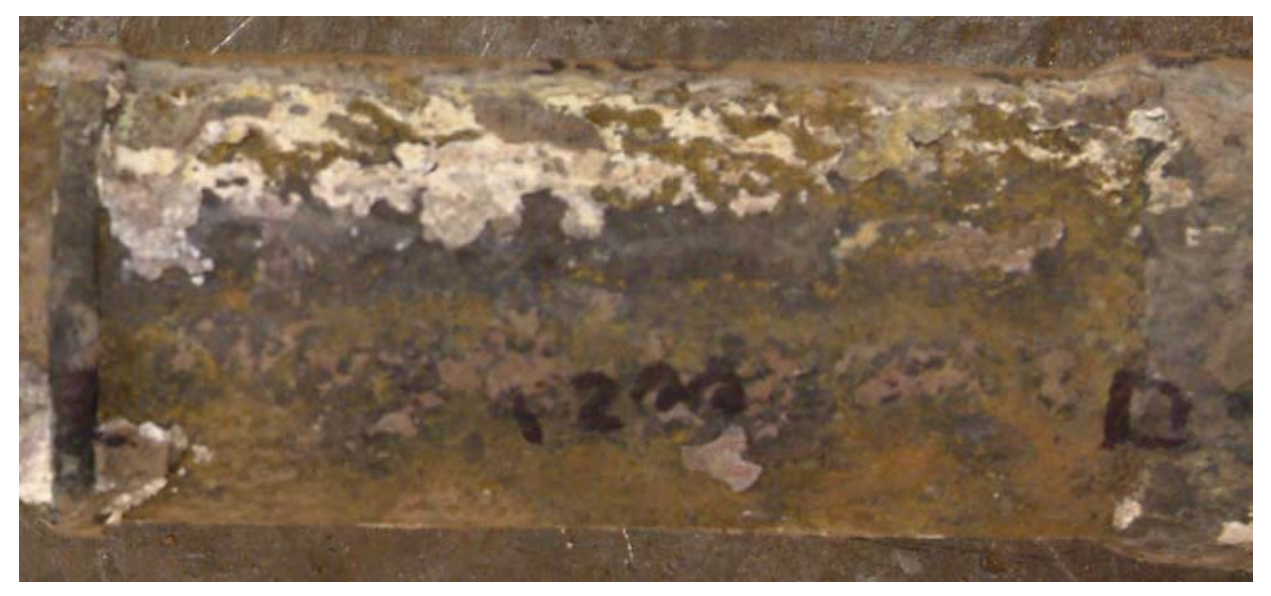

12 O'Clock - Facing Gas Flow

(Note: 3 o'clock is up in this image)

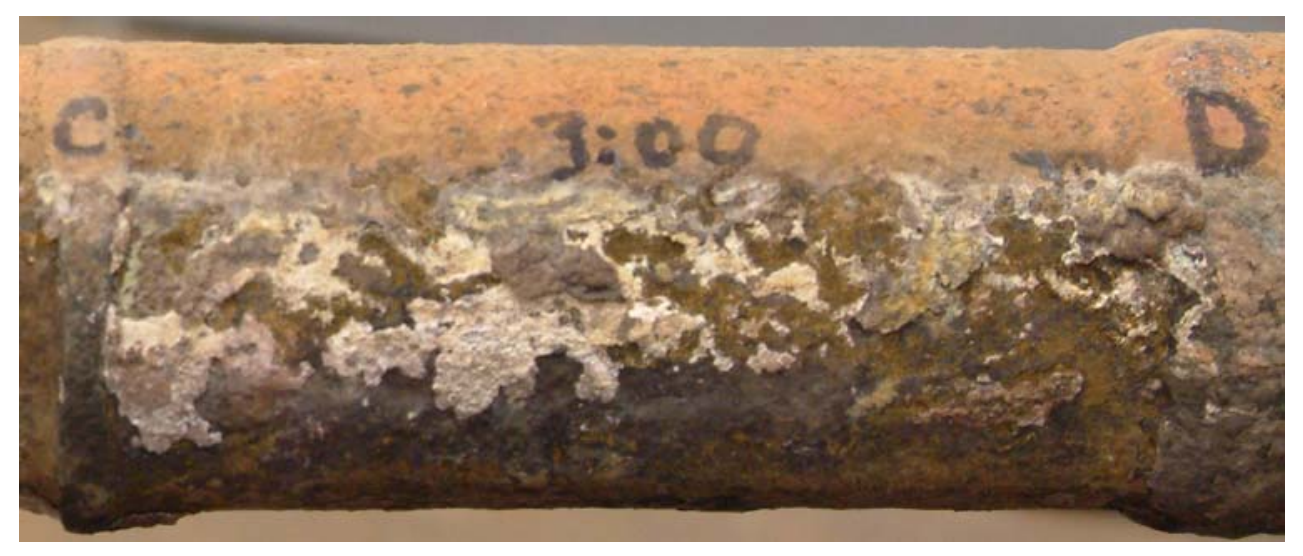

3 O'Clock

(Note: 6 o'clock is up in this image)

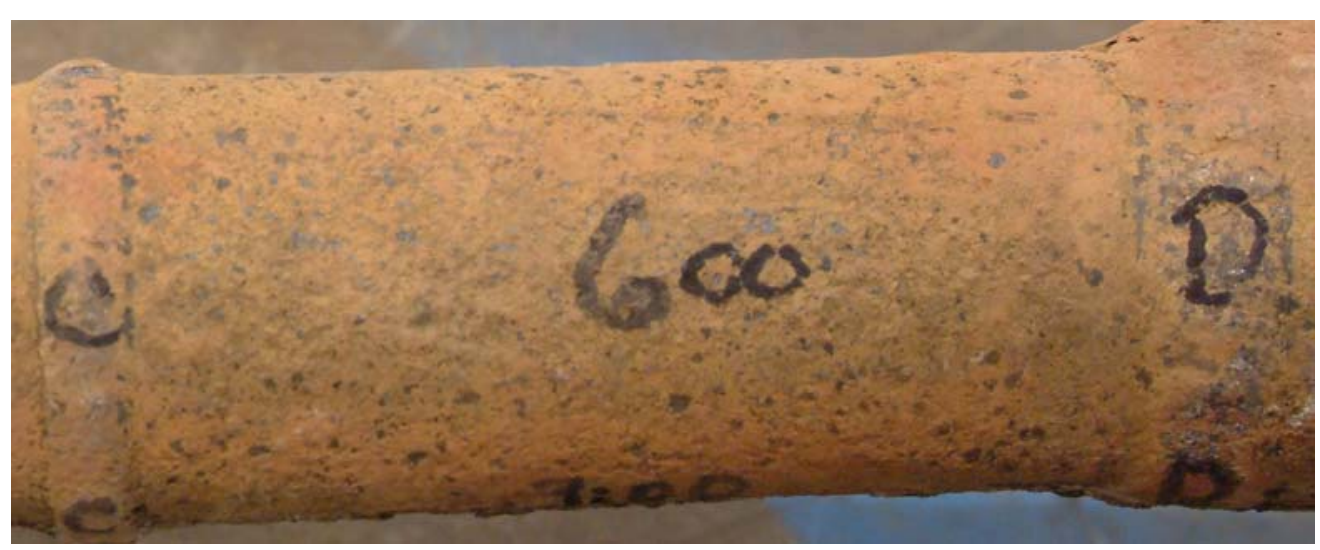

6 O'Clock - Opposite Gas Flow

Figure D-5 As-Received Appearance of Sample C-D (NF 709)

(Average metal temperature: $1153 \mathrm{~F}$ ) 
12 O'Clock - Facing Gas Flow

(Note: 3 o'clock is up in this image)

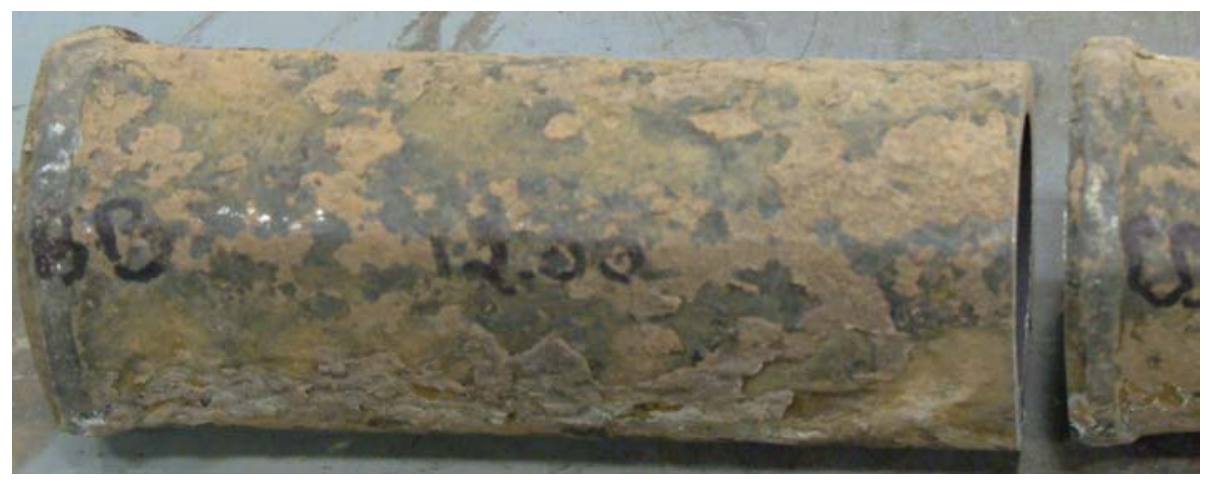

3 O'Clock

(Note: 6 o'clock is up in this image)

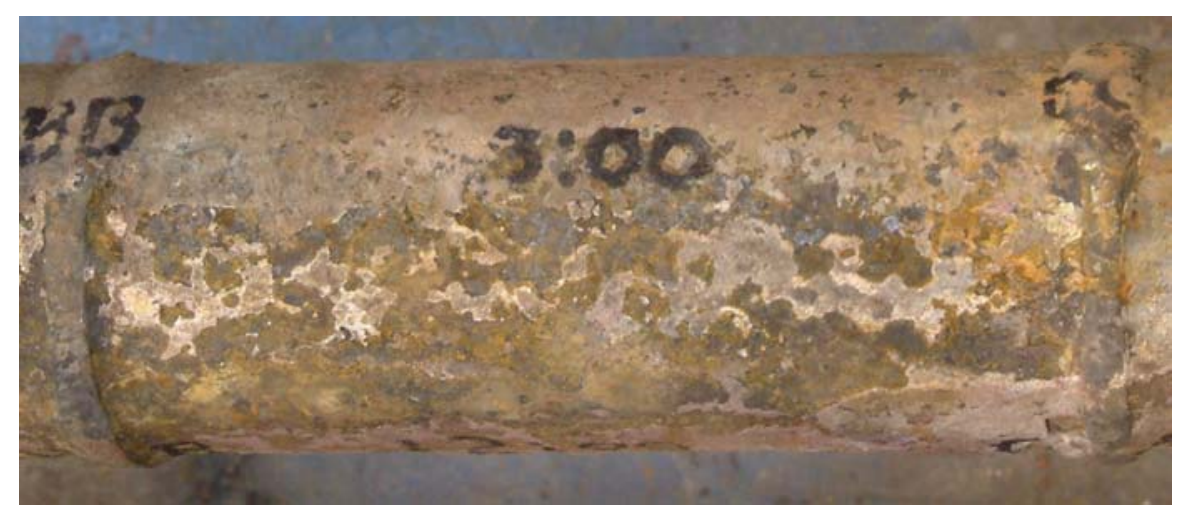

6 O’Clock - Opposite Gas Flow

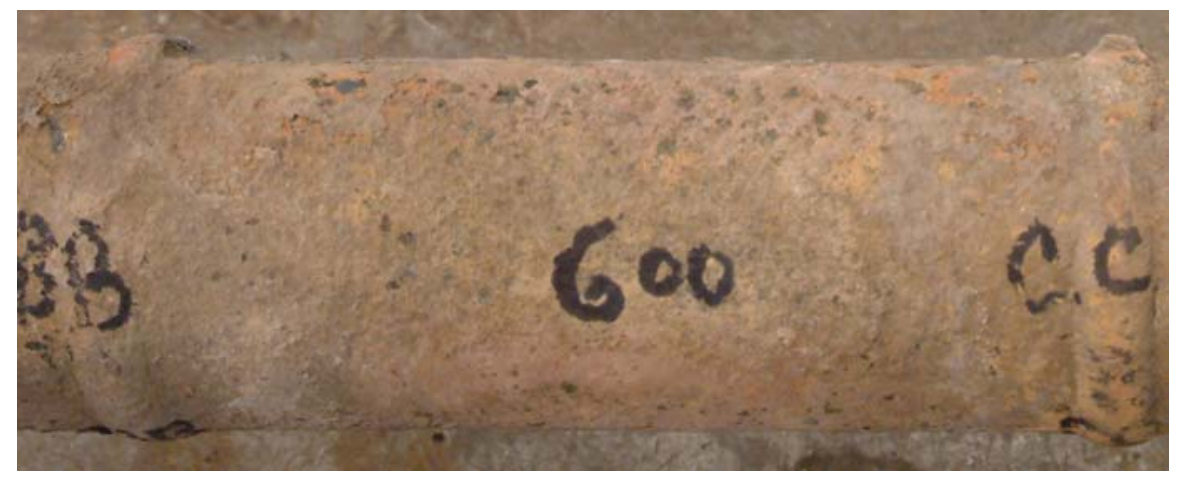

Figure D-6 As-Received Appearance of Sample BB-CC (NF 709)

(Average metal temperature: 1088 F) 
12 O'Clock

(201 mils lost)

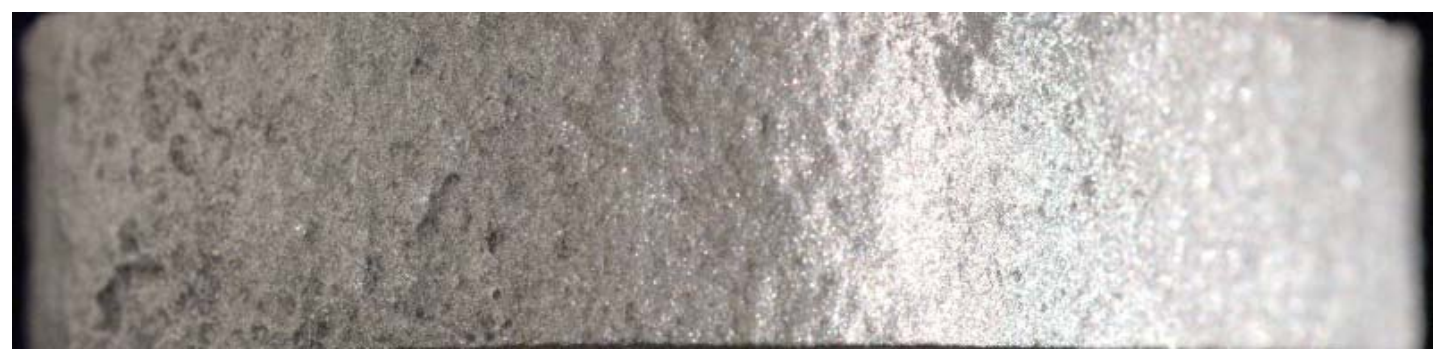

3 O'Clock

(224 mils lost)

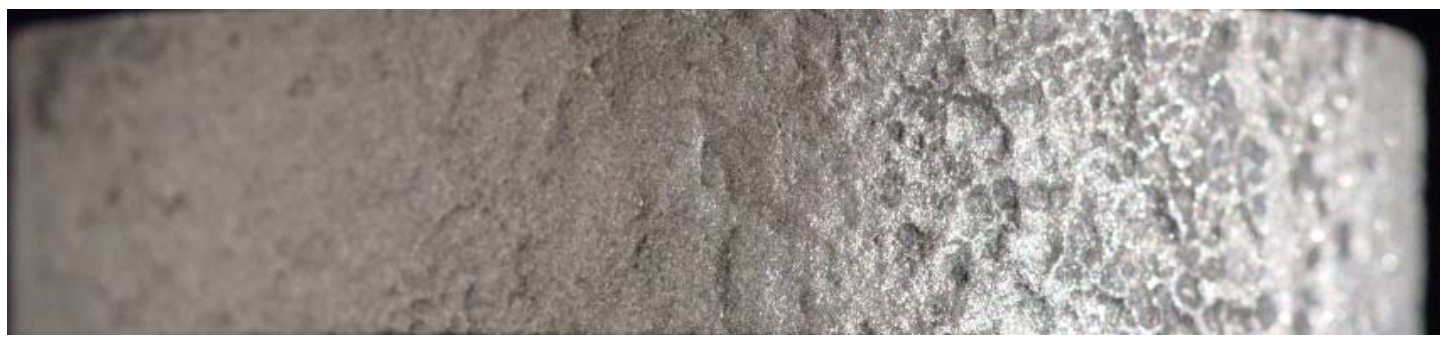

6 O'Clock

(1 mil lost)

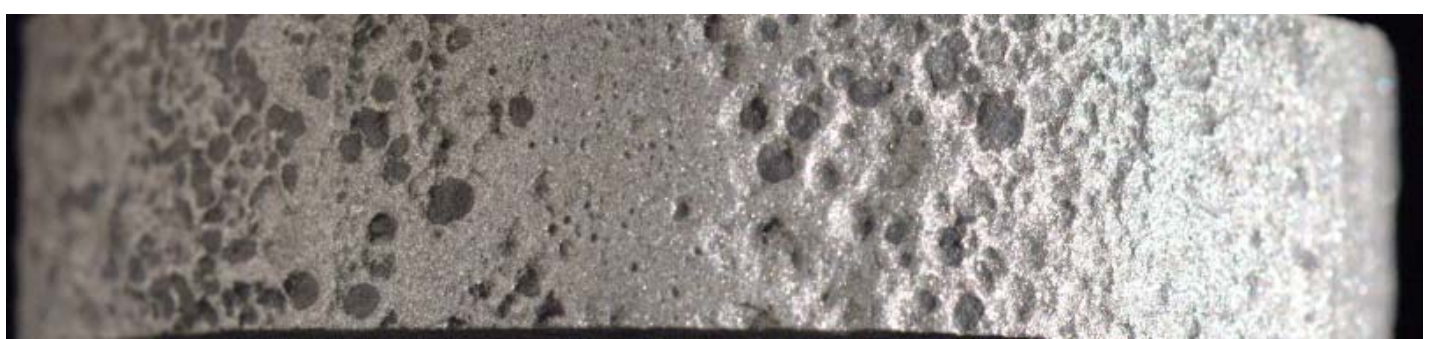

Figure D-7: OD Surface Appearance After Grit Blast

Material: $\quad$ NF 709

Sample: J-K 
12 O'Clock

(208 mils lost)

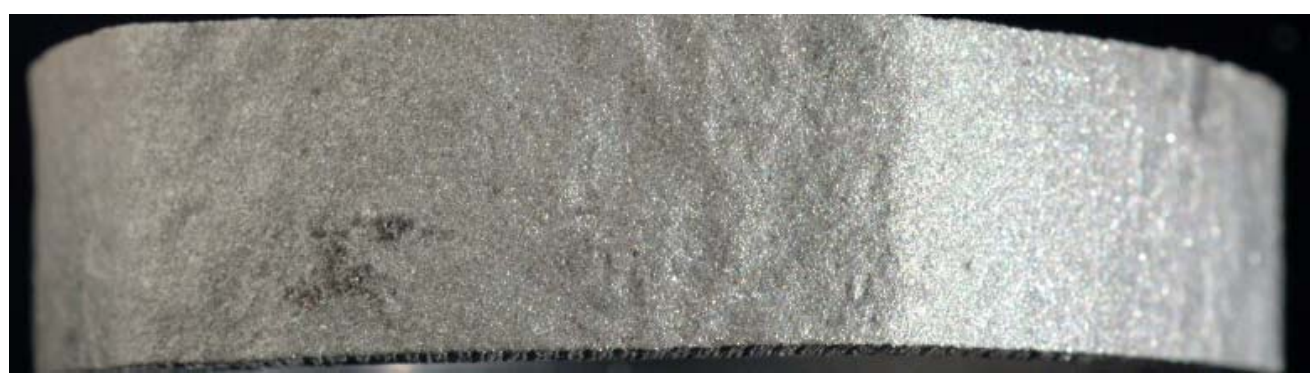

3 O'Clock

(204 mils lost)

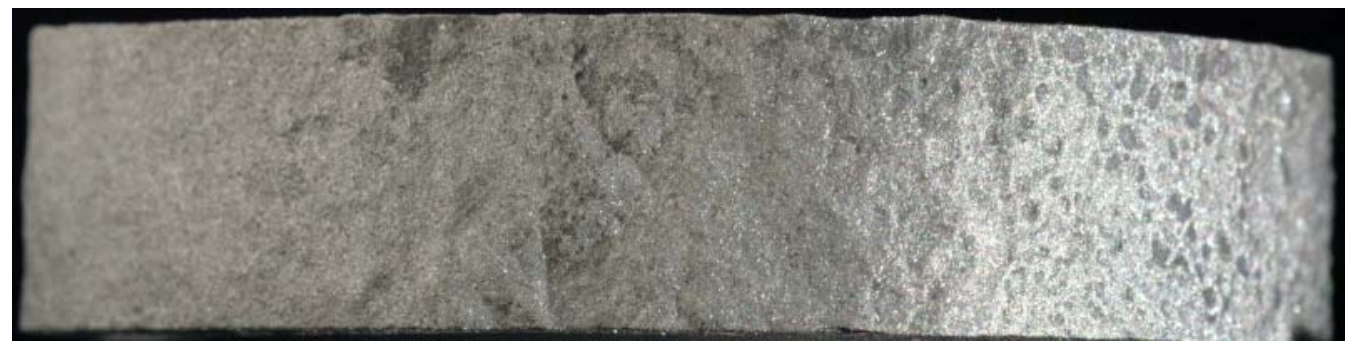

6 O'Clock

(13 mils lost)

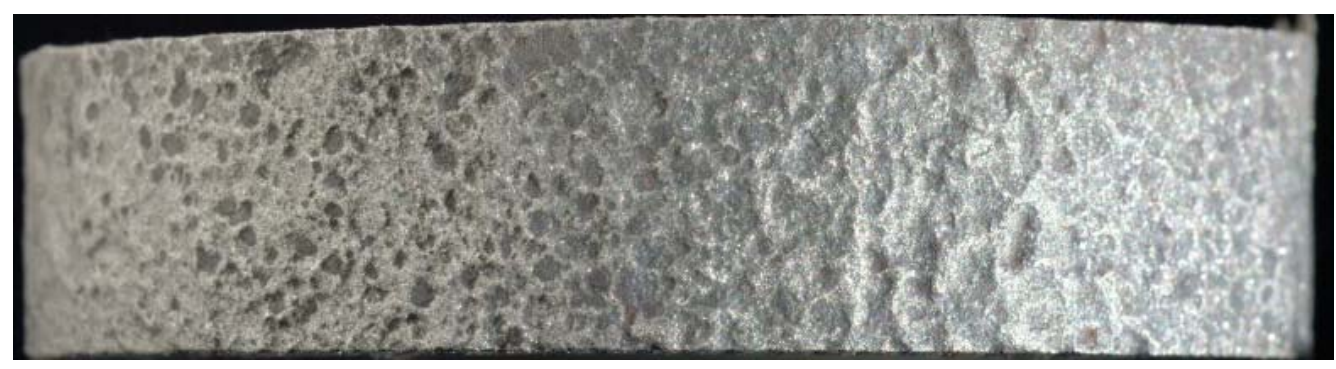

Figure D-8: OD Surface Appearance After Grit Blast

Material: $\quad$ NF 709

Sample: C-D 
12 O'Clock

(188 mils lost)

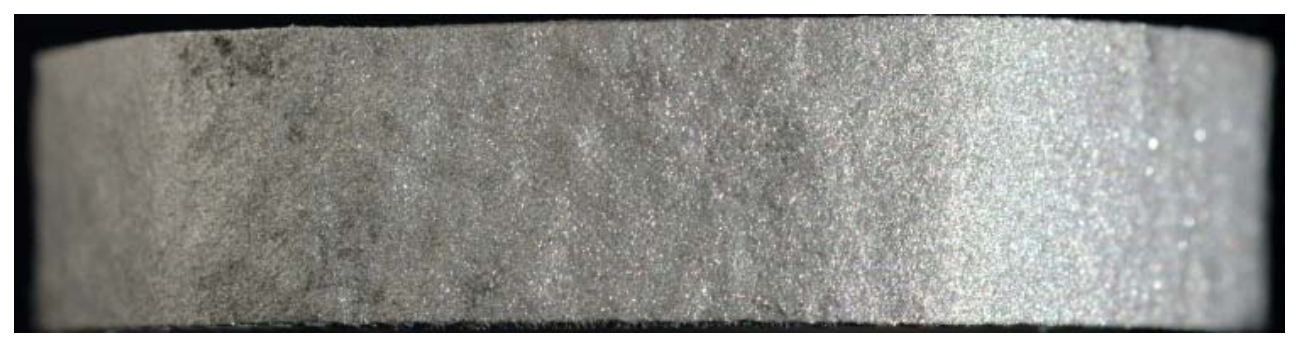

3 O'Clock

(207 mils lost)

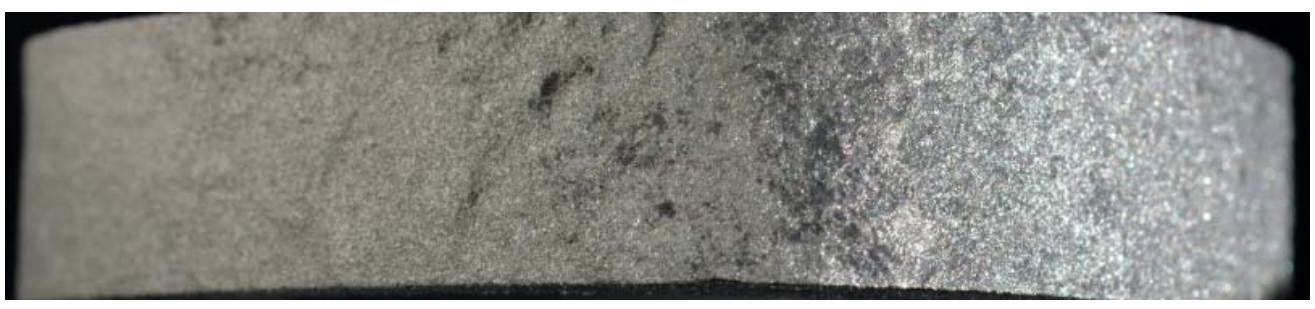

6 O'Clock

(2 mils lost)

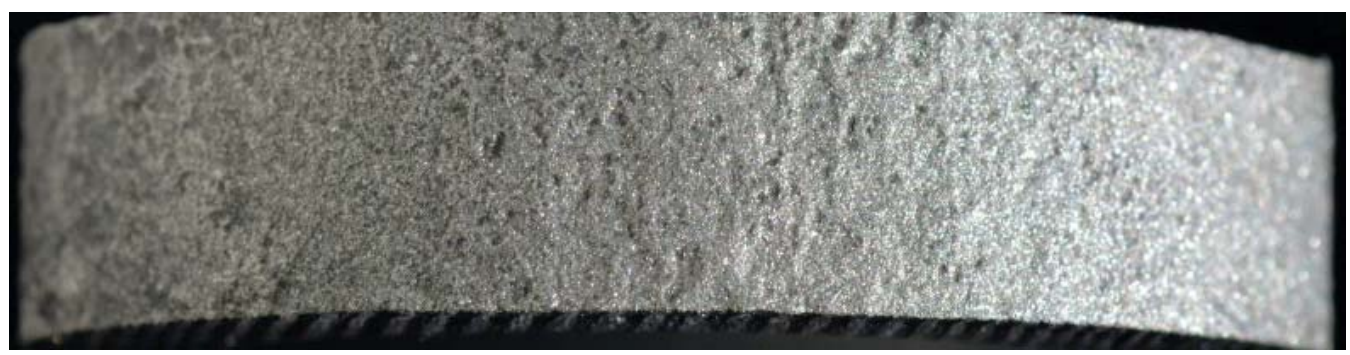

Figure D-9: OD Surface Appearance After Grit Blast

Material: $\quad$ NF 709

Sample: $\quad$ BB-CC 
Material:

Sample Location:

Maximum Rate:

Maximum Loss Orientation:

Average metal Temperature:
NF 709

J-K

$181 \mathrm{mils} / \mathrm{yr}$

Approx. 9:30 o'clock

$1178 \mathrm{~F}$

12 O'Clock

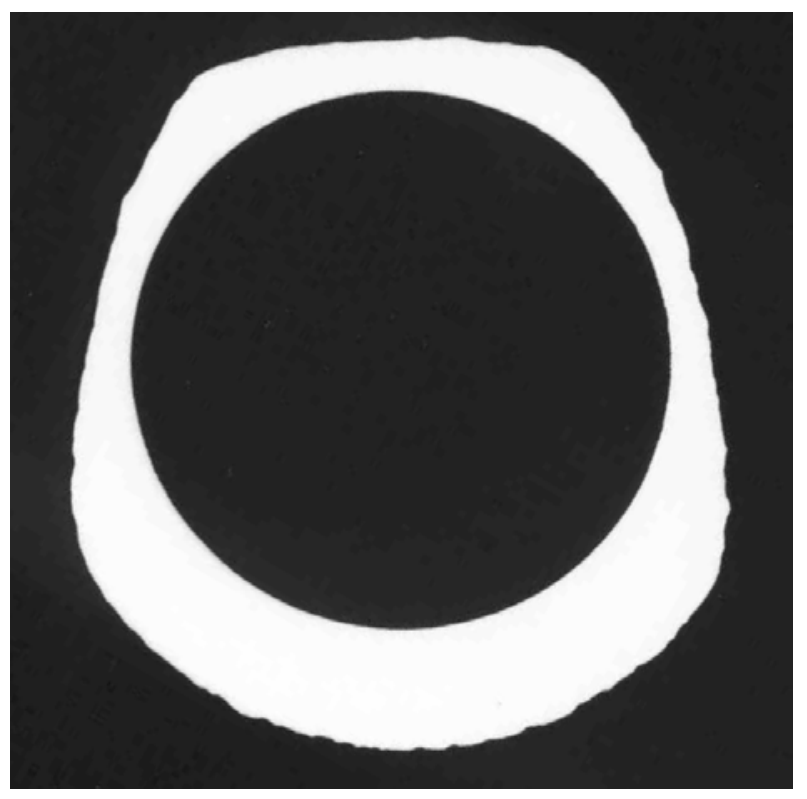

3 O'Clock

Approx. 1.2X

Wall Reduction by Position (in.)

\begin{tabular}{|l|c|c|c|c|c|c|c|c|c|c|}
\hline & $\begin{array}{c}\mathbf{1 2} \\
\text { o'clock }\end{array}$ & $\mathbf{1 : 3 0}$ & $\mathbf{3 : 0 0}$ & $\mathbf{4 : 3 0}$ & $\mathbf{6 : 0 0}$ & $\mathbf{7 : 3 0}$ & $\mathbf{9 : 0 0}$ & $\mathbf{1 0 : 3 0}$ & AVG & $\begin{array}{c}\text { Max } \\
\text { Loss* }\end{array}$ \\
\hline Before & 0.400 & 0.398 & 0.398 & 0.396 & 0.395 & 0.395 & 0.395 & 0.399 & 0.397 & 0.397 \\
\hline After & 0.199 & 0.160 & 0.174 & 0.378 & 0.394 & 0.365 & 0.146 & 0.254 & & 0.116 \\
\hline Delta & 0.201 & 0.238 & 0.224 & 0.018 & 0.001 & 0.030 & 0.249 & 0.145 & & 0.281 \\
\hline
\end{tabular}

Figure D-10: Silhouette and Wall Thickness Measurements for Sample J-K

Note: this tube was removed from service on 11/6/01.

* Measurement taken at "maximum loss orientation" noted above. The average

"before" wall thickness was used to calculate the "delta". 
Material:

Sample Location:

Maximum Rate:

Maximum Loss Orientation:

Average metal Temperature:
NF 709

C-D

114 mils/yr

Approx. 2:30 o'clock

$1153 \mathrm{~F}$

12 O'Clock

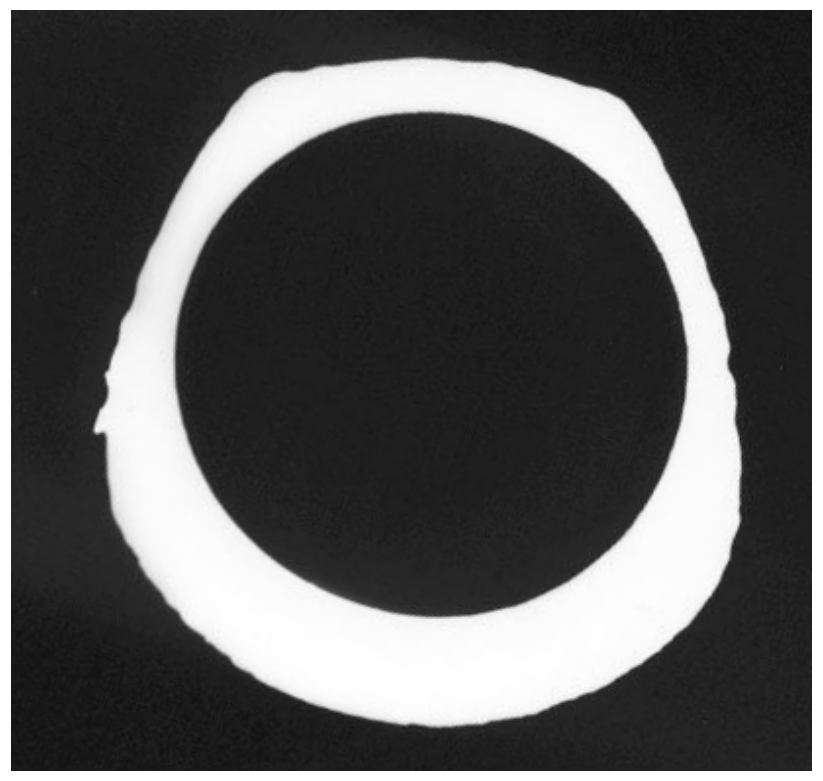

3 O’Clock

Approx. 1.2X

Wall Reduction by Position (in.)

\begin{tabular}{|l|c|c|c|c|c|c|c|c|c|c|}
\hline & $\begin{array}{c}\mathbf{1 2} \\
\text { o'clock }\end{array}$ & $\mathbf{1 : 3 0}$ & $\mathbf{3 : 0 0}$ & $\mathbf{4 : 3 0}$ & $\mathbf{6 : 0 0}$ & $\mathbf{7 : 3 0}$ & $\mathbf{9 : 0 0}$ & $\mathbf{1 0 : 3 0}$ & AVG $^{\text {Max }}$ & $\begin{array}{c}\text { Loss } \\
\text { (0) }\end{array}$ \\
\hline Before & 0.399 & 0.399 & 0.398 & 0.399 & 0.401 & 0.400 & 0.400 & 0.402 & 0.400 & 0.400 \\
\hline After & 0.191 & 0.163 & 0.194 & 0.386 & 0.388 & 0.394 & 0.219 & 0.297 & & 0.128 \\
\hline Delta & 0.208 & 0.236 & 0.204 & 0.013 & 0.013 & 0.006 & 0.181 & 0.105 & & 0.272 \\
\hline
\end{tabular}

Figure D-11: Silhouette and Wall Thickness Measurements for Sample C-D

* Measurement taken at "maximum loss orientation" noted above. The average

"before" wall thickness was used to calculate the "delta". 
Material:

Sample Location:

Maximum Rate:

Maximum Loss Orientation:

Average metal Temperature:
NF 709

BB-CC

$100 \mathrm{mils} / \mathrm{yr}$

Approx. 9:30 o'clock

$1088 \mathrm{~F}$

12 O’Clock

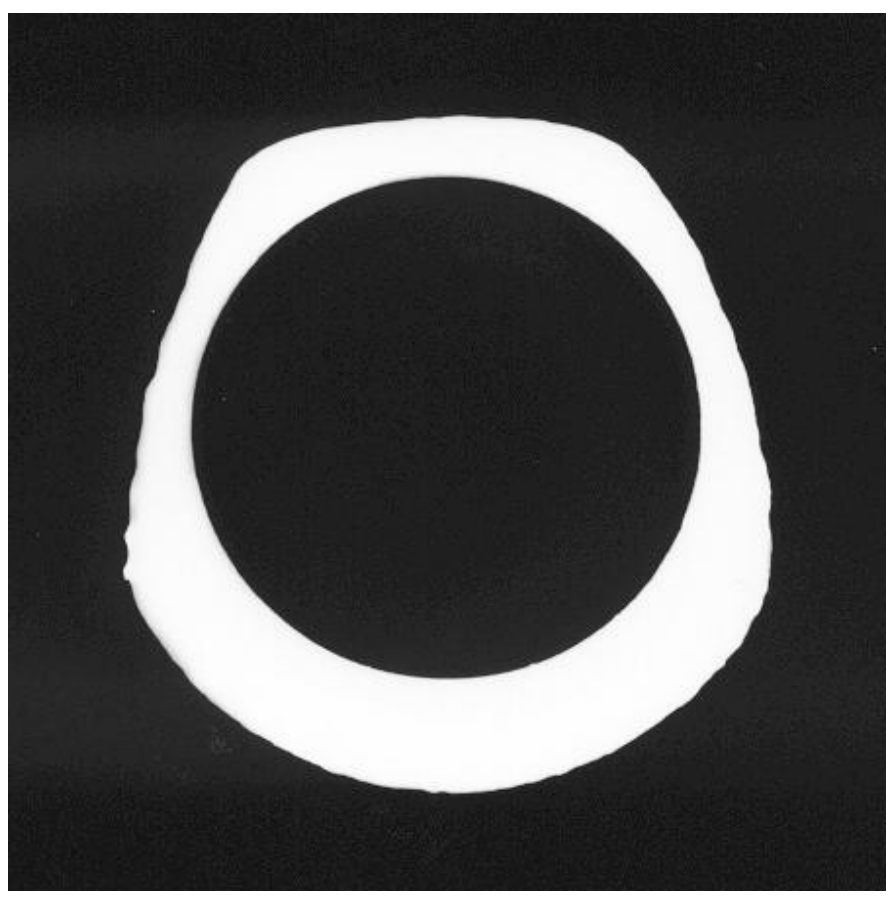

3 O'Clock

Approx. 1.2X

Wall Reduction by Position (in.)

\begin{tabular}{|l|c|c|c|c|c|c|c|c|c|c|}
\hline & $\begin{array}{c}\mathbf{1 2} \\
\text { o'clock }\end{array}$ & $\mathbf{1 : 3 0}$ & $\mathbf{3 : 0 0}$ & $\mathbf{4 : 3 0}$ & $\mathbf{6 : 0 0}$ & $\mathbf{7 : 3 0}$ & $\mathbf{9 : 0 0}$ & $\mathbf{1 0 : 3 0}$ & AVG & $\begin{array}{c}\text { Max } \\
\text { Loss }\end{array}$ \\
\hline Before & 0.398 & 0.397 & 0.399 & 0.397 & 0.396 & 0.400 & 0.399 & 0.398 & 0.398 & 0.398 \\
\hline After & 0.210 & 0.237 & 0.192 & 0.385 & 0.398 & 0.384 & 0.190 & 0.238 & & 0.159 \\
\hline Delta & 0.188 & 0.160 & 0.207 & 0.012 & 0.002 & 0.016 & 0.209 & 0.160 & & 0.239 \\
\hline
\end{tabular}

Figure D-12: Silhouette and Wall Thickness Measurements for Sample BB-CC

* Measurement taken at "maximum loss orientation" noted above. The average

"before" wall thickness was used to calculate the "delta". 


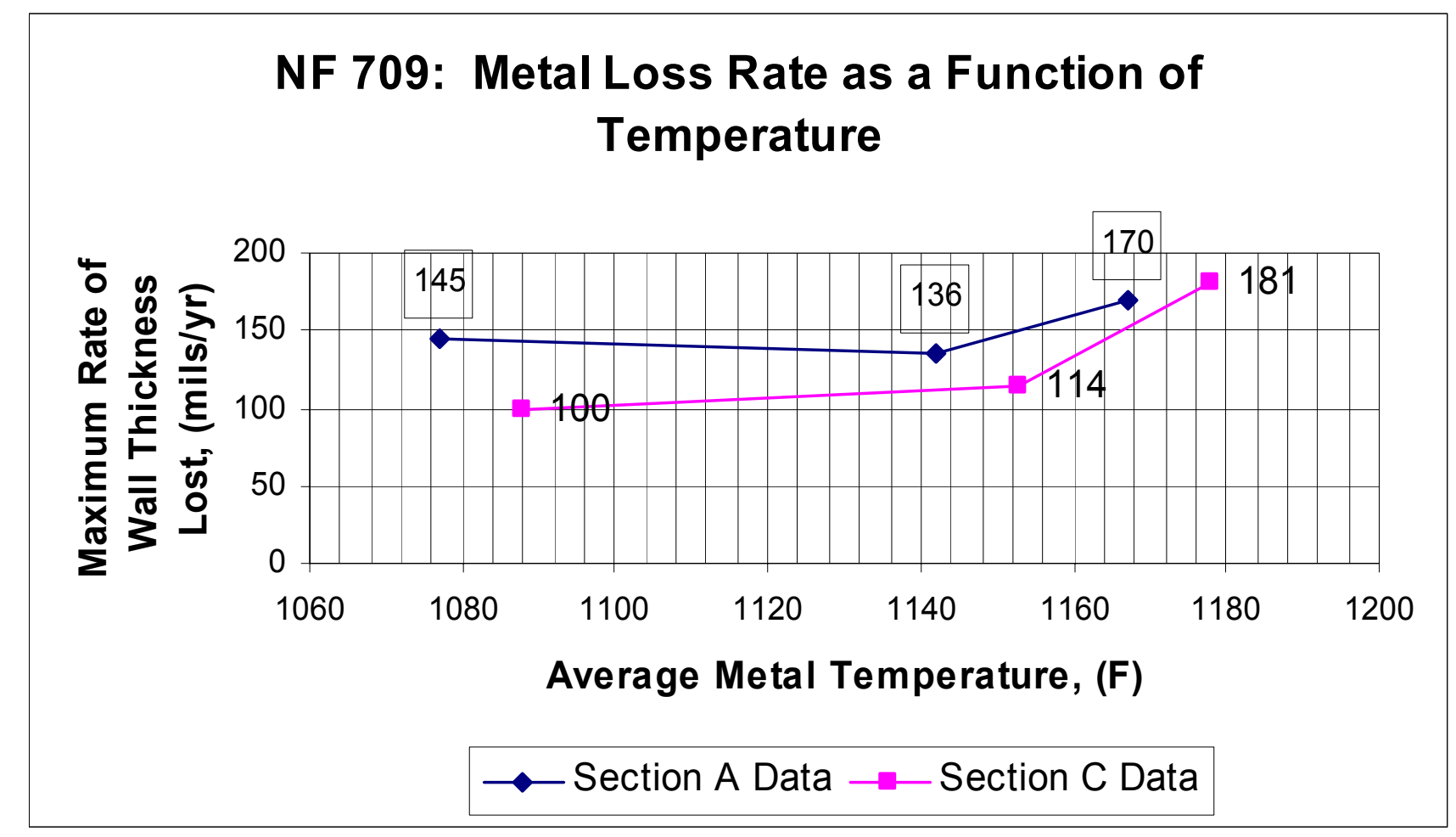

Figure D-13: Metal Loss Rate as a Function of Temperature

\section{Material: NF 709}

Note: In analyzing these data it is important to understand that the one of the three Section C samples was removed early due to excessive corrosion losses. Sample J-K (1178 F) was removed in November 2001, shortly after Section A was removed for analysis. Sample C-D (1153 F) and Sample BB-CC (1088 F) were removed with the remaining Section C samples in May 2003. 


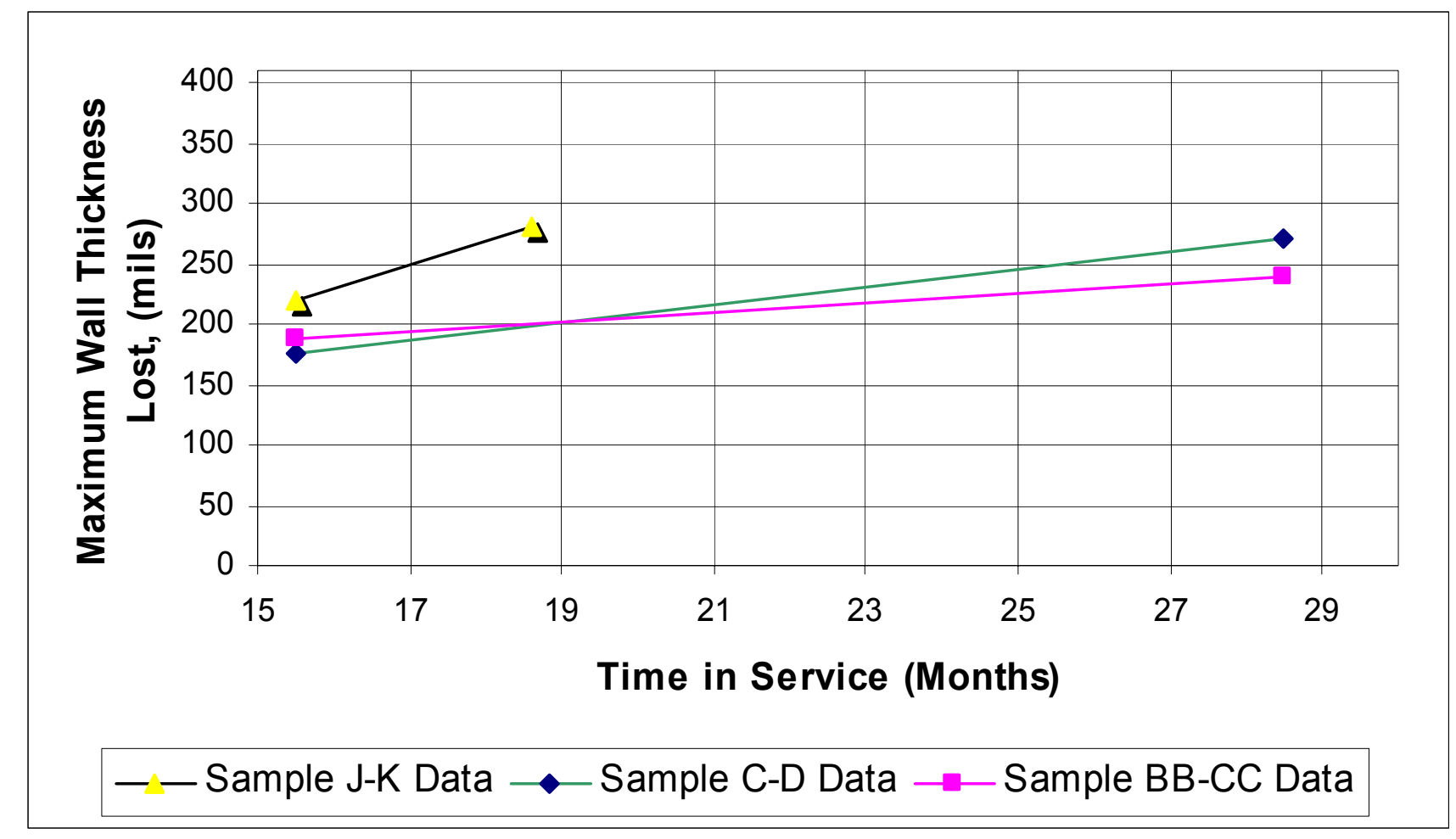

Figure B-14: Metal Loss as a Function of Time

\section{Material: Modified NF 709}

Note: From the plot above, Section C Sample J-K was removed shortly after Section A was removed for evaluation. Samples C-D and BB-CC experienced the full exposure life for both Sections A and C. 


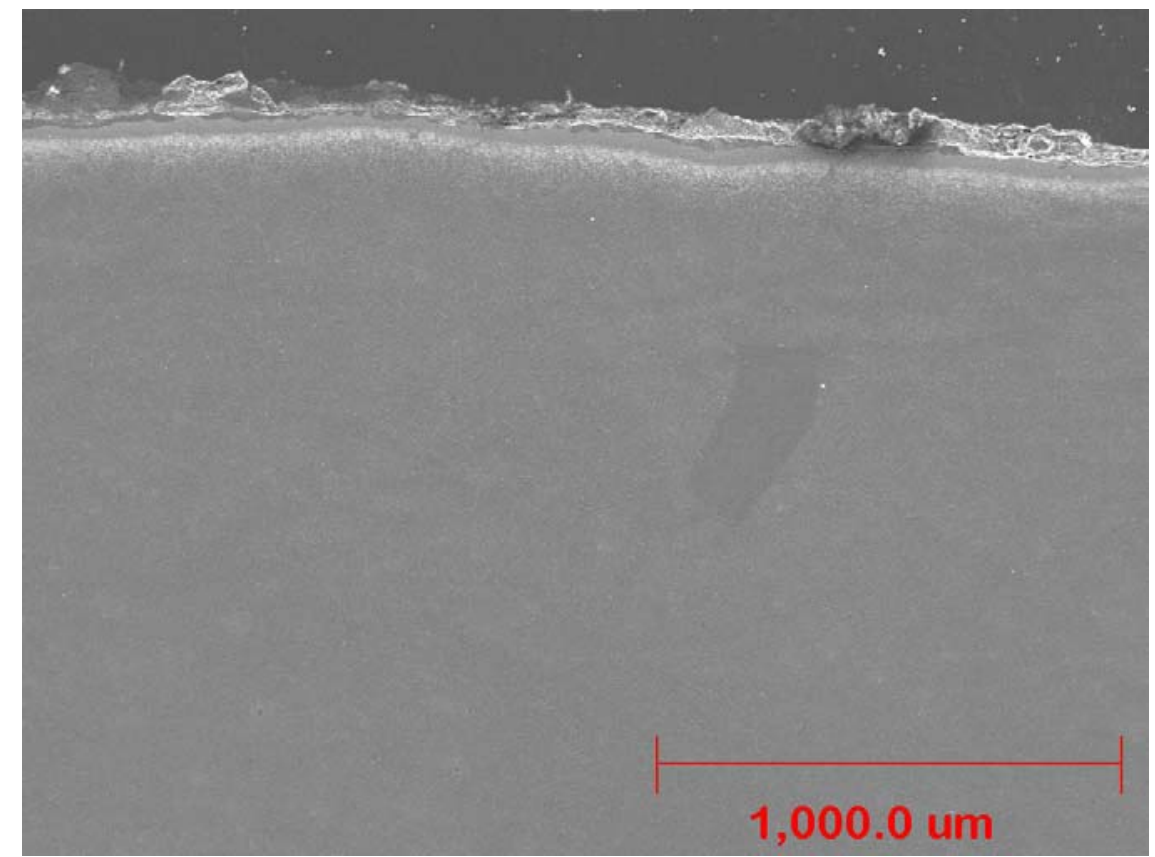

(a) External Scale/Metal Interface (SEI - Low Magnification)

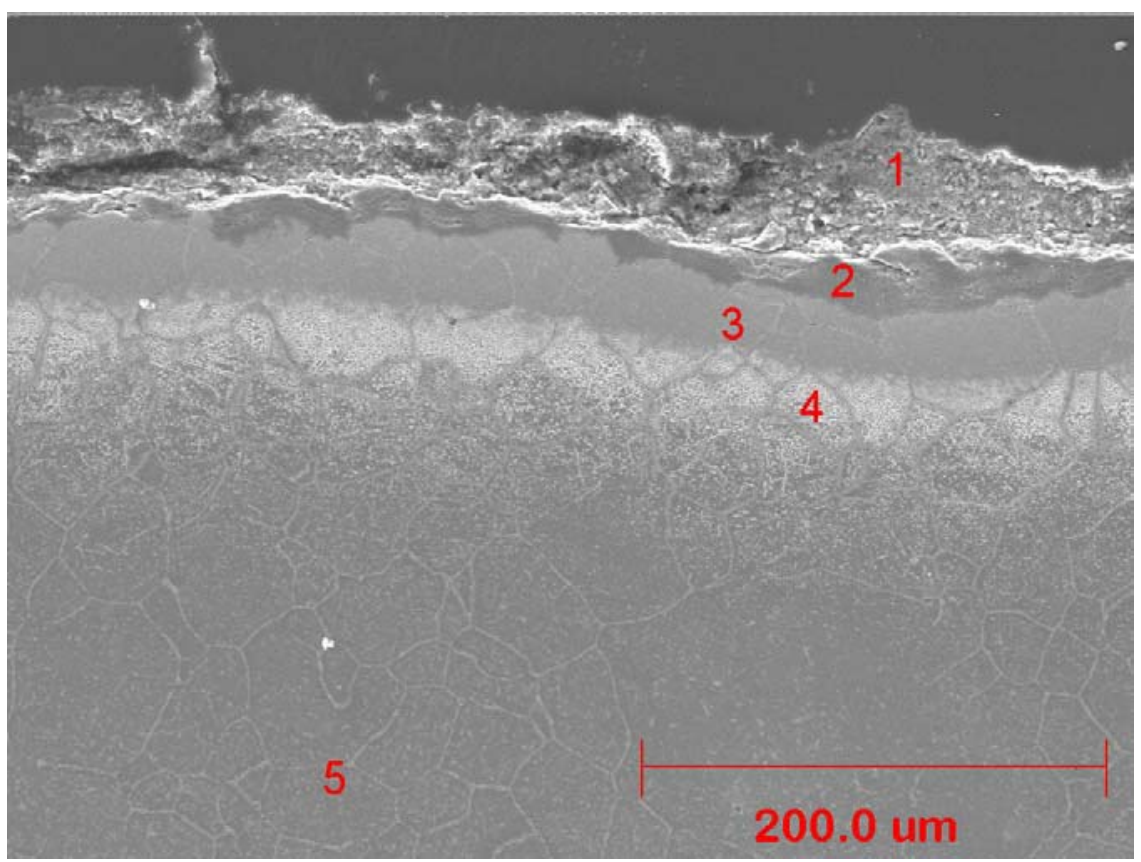

(b) External Scale/Metal Interface (SEI - Intermediate Magnification)

Figure D-15: Microstructure: NF 709

Note: 1) Numbers on photos indicate distinct areas characterized by the visual assessment and EDAX analyses that follow. 


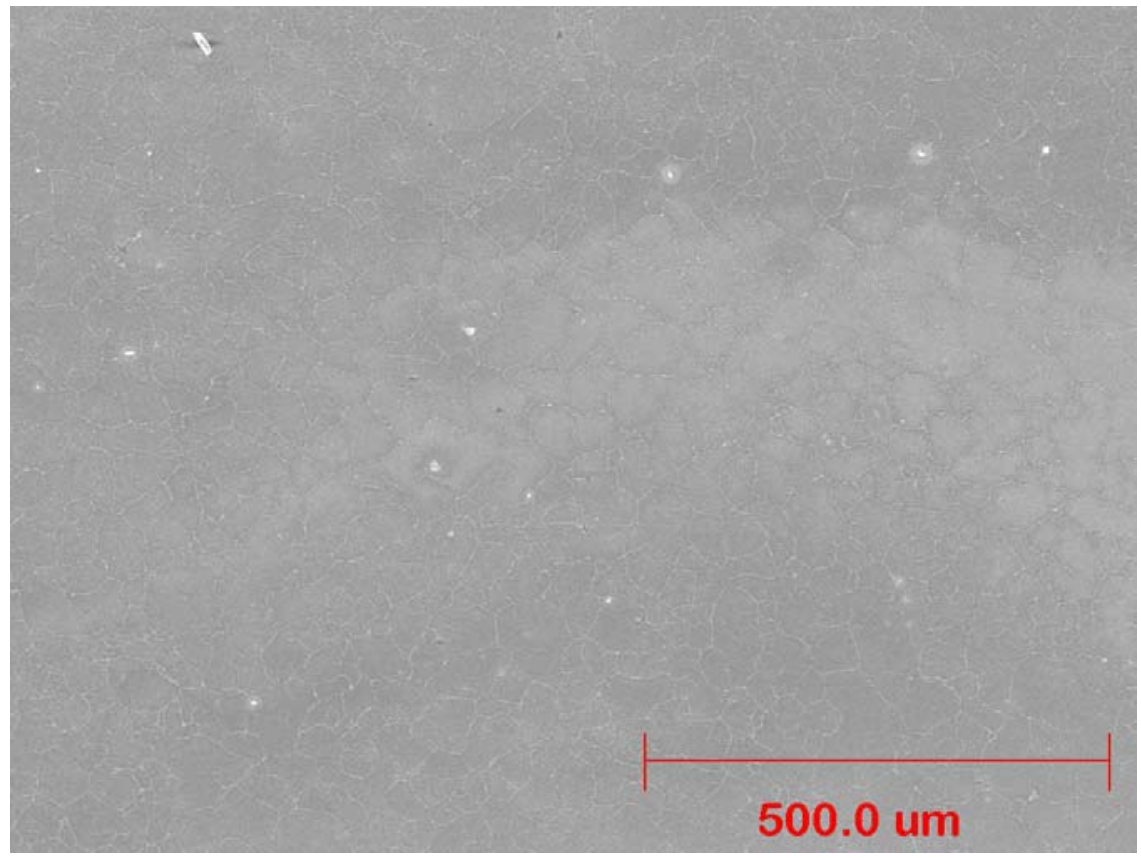

Mid-Wall Microstructure (SEI - Low Magnification)

Figure D-16: Microstructure: NF 709

Note: Photomicrograph at approximately 200X. Electrolytic 10\% oxalic acid etch, 9V OCP 


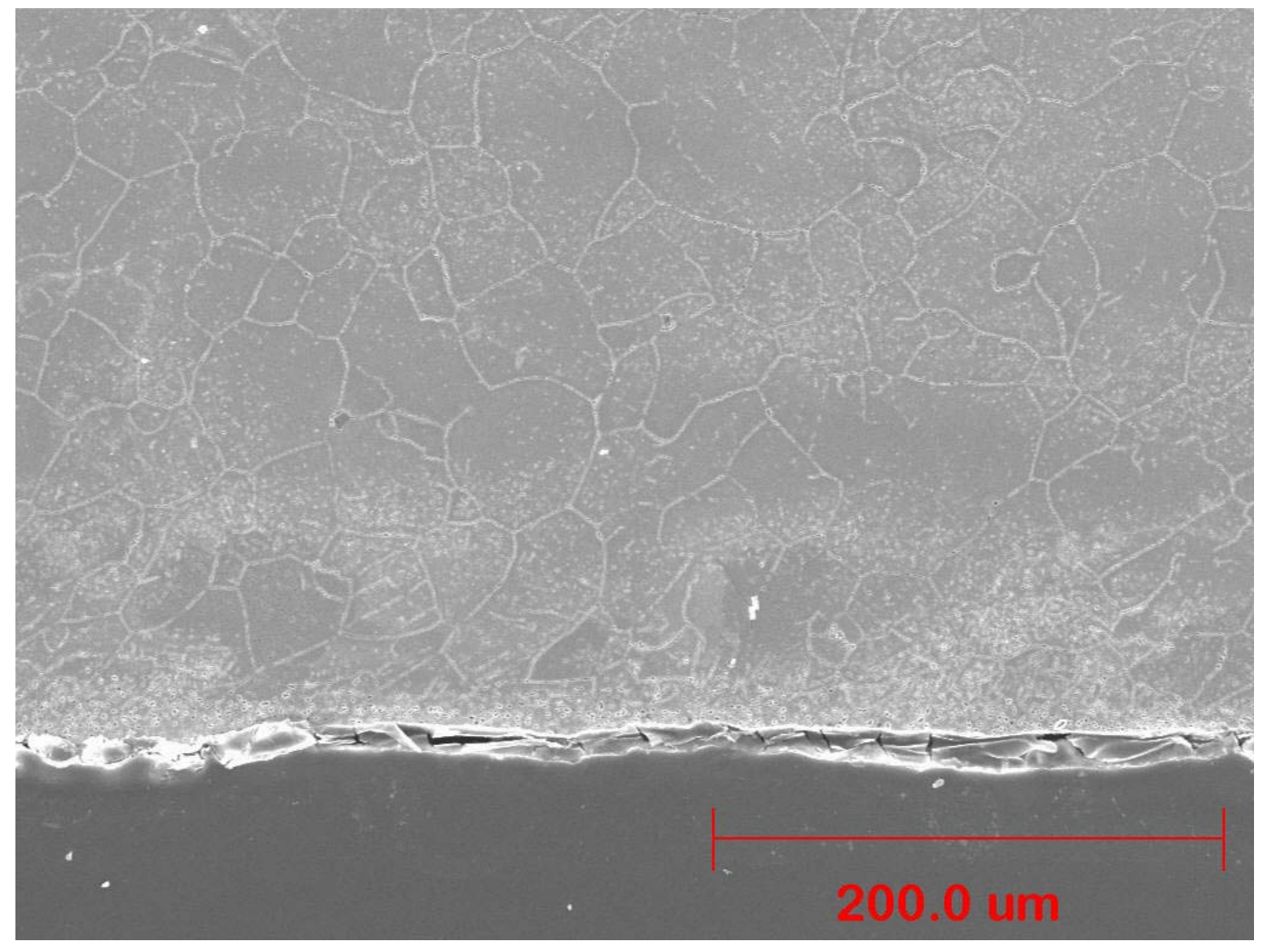

Internal Surface Appearance

Figure D-17: Microstructure: NF 709 


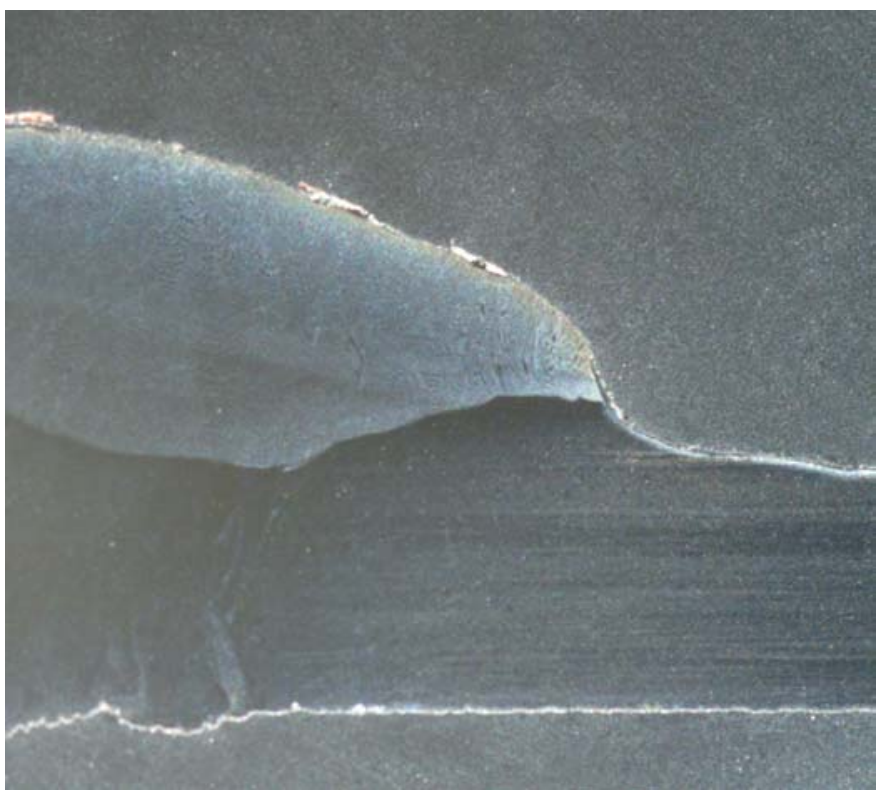

(a) NF 709 Adjacent to Weld "D"

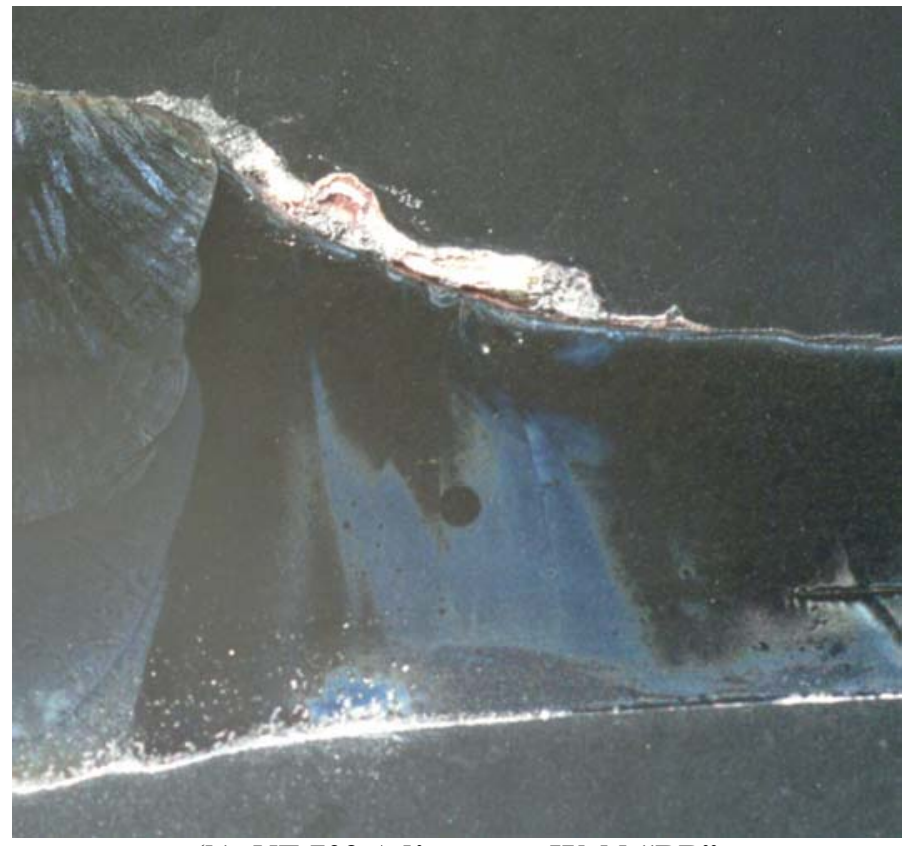

(b) NF 709 Adjacent to Weld "BB"

Figure D-18: Longitudinal Cross-Section: NF 709 Adjacent to Welds

Note: The weld shown in figure D-18a is not original with Section C. It was used in inserting a "dutchman" between welds D and G on June 10, 2002. 
Figure D-19: EDS Analysis of Deposit/Scale/Metal Interface for NF 709 (Sample C-D)

\begin{tabular}{|c|c|c|c|c|c|c|c|c|c|c|c|c|c|c|c|c|c|c|}
\hline Area & Description & $\mathbf{N i}$ & $\mathrm{Cr}$ & $\mathbf{F e}$ & $\mathrm{Cu}$ & Mn & $\mathbf{N b}$ & $\mathbf{T i}$ & Al & $\mathbf{S i}$ & $\mathrm{C}$ & $\mathbf{O}$ & Mo & $\mathbf{K}$ & Cl & As & $\mathbf{P}$ & $\mathbf{S}$ \\
\hline 1 & $\begin{array}{l}\text { Bulk deposit } \\
\text { analysis }\end{array}$ & 2.01 & 1.74 & 36.3 & -- & -- & -- & 0.64 & 6.43 & 9.55 & 14.4 & 24.0 & -- & 1.45 & -- & 1.15 & 1.72 & 0.62 \\
\hline 2 & $\begin{array}{l}\text { Adherent } \\
\text { scale near } \\
\text { metal } \\
\text { interface. }\end{array}$ & 6.21 & 50.6 & 13.2 & - & 0.51 & -- & -- & -- & 1.39 & -- & 17.9 & -- & -- & -- & -- & -- & 10.2 \\
\hline 3 & $\begin{array}{l}\text { Diffusion } \\
\text { zone-dark } \\
\text { etch }\end{array}$ & 30.9 & 12.2 & 56.0 & -- & 0.61 & -- & -- & -- & 0.34 & -- & -- & -- & -- & -- & -- & -- & -- \\
\hline 4 & $\begin{array}{l}\text { Diffusion } \\
\text { zone-light } \\
\text { etch }\end{array}$ & 13.5 & 24.3 & 60.7 & -- & 0.90 & -- & -- & -- & 0.64 & -- & -- & -- & -- & -- & -- & -- & -- \\
\hline \multirow[t]{2}{*}{5} & $\begin{array}{l}\text { Bulk base } \\
\text { metal per } \\
\text { EDAX }\end{array}$ & 25.0 & 21.9 & 50.4 & -- & 0.73 & -- & -- & -- & 0.40 & -- & -- & 1.51 & -- & -- & -- & -- & -- \\
\hline & $\begin{array}{l}\text { Reference } \\
\text { Bulk } \\
\text { Composition } \\
\text { Per optical } \\
\text { spectrograph. }\end{array}$ & 24.9 & 22.7 & 48.8 & 0.04 & 1.00 & 1.40 & 0.036 & 0.032 & 0.37 & 0.03 & -- & 1.39 & -- & -- & -- & 0.010 & 0.002 \\
\hline
\end{tabular}


Appendix E

Analysis of Performance

Test Section C

$\mathrm{Fe}_{3} \mathrm{Al} / \mathrm{TP} 304 \mathrm{H}$

E-1 


\section{Overview of Results: $\mathrm{Fe}_{3} \mathrm{Al} / \mathrm{TP} 304 \mathrm{H}$}

The rate of metal loss for the $\mathrm{Fe}_{3} \mathrm{Al} / \mathrm{TP} 304 \mathrm{H}$ tube sample (B-C) in Section C was somewhat less than that for the same material in Section A, (i.e. 100 mils per year versus 145 mils per year*). The reason for this reduced wastage rate is not clear. For both samples, the protective $\mathrm{Fe}_{3} \mathrm{Al}$ coating was not in evidence anywhere on the tube surface at the time of the analysis, and based on performance in Section A, it likely disappeared early during the life of the tube sample, (i.e. in less than 1.55 years). In any case, the limited number of $\mathrm{Fe}_{3} \mathrm{Al} / \mathrm{TP} 304 \mathrm{H}$ samples in this program, makes it difficult to draw substantive conclusions.

It should be noted that the metal loss rates determined to date for both Section A and Section C all were based on a ring specimen that was extracted from mid-length of each tube sample. In this analysis additional attention was focused on metal losses in the vicinity of welds. Representative samples were taken and it was determined that, for the $\mathrm{Fe}_{3} \mathrm{Al} / \mathrm{TP} 304 \mathrm{H}$ samples evaluated, the Inconel 625 weld filler metal is reasonably resistant to coal ash corrosion and that the tube material immediately adjacent to the weld (i.e. the weld heat affected zone) was somewhat less affected by corrosion than was the remainder of the tube. Indeed the tube wall appeared to be progressively thinner toward the mid-point of the tube sample. 


\section{Material: $\mathrm{Fe}_{3} \mathrm{Al} / \mathrm{TP}$ 304H}

\section{Summary of Findings}

\begin{tabular}{|c|c|}
\hline Issue & Discussion \\
\hline $\begin{array}{l}\text { Sample } \\
\text { Location and } \\
\text { Orientation. }\end{array}$ & $\begin{array}{l}\text { - The schematic in Figure E-1 shows the position of the one } \mathrm{Fe}_{3} \mathrm{Al} / \mathrm{TP} \\
\text { 304H sample in Section C. Also, shown is the calculated average } \\
\text { metal temperature at this location. }\end{array}$ \\
\hline $\begin{array}{l}\text { Composition } \\
\text { Verification. }\end{array}$ & $\begin{array}{l}\text { - The table in Figure E- } 2 \text { provides the composition of tube Sample B-C } \\
\text { after exposure. An attempt was made to find and analyze the } \\
\text { composition of the iron-aluminide coating on the "after service" } \\
\text { samples, but none could be found. It is assumed that the coating either } \\
\text { spalled or was corroded away from the surface at some point during } \\
\text { the } 2.38 \text { years of exposure in Section C. This is consistent with similar } \\
\text { results for Section A, after } 1.55 \text { years of exposure. } \\
\text { The table in Figure E-2 provides EDS analyses of the Fe } \mathrm{Al} \text { coating } \\
\text { prior to service. Numerous samplings were made and found to be } \\
\text { highly variable. The reported results are for material at the extreme } \\
\text { outer surface. A bulk analysis was also attempted and reported. The } \\
\text { significance of deviations from the target chemistry is unclear. } \\
\text { EDS semi-quantitative analysis at mid-wall for Sample B-C confirms } \\
\text { that this material is consistent with TP } 304 \mathrm{H} \text {, thus if verifies that the } \\
\text { correct sample was located at position B-C. }\end{array}$ \\
\hline $\begin{array}{l}\text { As-Received } \\
\text { Appearance }\end{array}$ & $\begin{array}{l}\text { - Figures E-3 shows the appearance of the surface of the } \mathrm{Fe}_{3} \mathrm{Al} \text { coated } \\
\text { tube prior to service. } \\
\text { - Figure E-4 documents the appearance of the external surface of } \\
\text { Sample B-C after service. Three views are provided for this tube: } 12 \\
\text { o'clock (the view that faces on-coming gas flow), } 3 \text { o'clock (the view } \\
\text { where the gas flow is tangent to the surface), and } 6 \text { o'clock (the view } \\
\text { that shows the back-side of the tube relative to the gas flow direction. } \\
\text { - The reader should be alerted to the fact that, within the boiler, the gas } \\
\text { flows upward over these tubes. This implies that for the "o'clock" } \\
\text { conventions described above, (with the } 12 \text { o'clock orientation facing } \\
\text { the gas flow direction), the actual physical orientation of these tubes is } \\
\text { such that the } 12 \text { o'clock side of the tube faces downward, and the } 6 \\
\text { o'clock orientation faces upward within the boiler. } \\
\text { - Wastage is obscured by the heavy deposit and scale at the } 3 \text { and } 12 \\
\text { o'clock orientations for Sample B-C. } \\
\text { A relatively thin red-brown deposit is seen at the } 6 \text { o'clock orientation. } \\
\text { The tube profile in this view also gives a sense of the extent of } \\
\text { wastage. } \\
\text { The gray/white scale associated with molten alkali-iron sulfate attack } \\
\text { is clearly visible at the } 3 \text { and } 12 \text { o'clock orientations.. }\end{array}$ \\
\hline $\begin{array}{l}\text { Grit-Blasted } \\
\text { Surface } \\
\text { Appearance }\end{array}$ & $\begin{array}{l}\text { - Figure E-5 shows the appearance of the external surface of the } \mathrm{Fe}_{3} \mathrm{Al} / \\
\text { TP } 304 \mathrm{H} \text { tube samples after grit-blasting to remove deposit and scale. } \\
\text { General wastage is most clearly shown at the } 12 \text { o'clock orientation. } \\
\text { - The } 6 \text { o'clock position shows very little evidence of attack. }\end{array}$ \\
\hline
\end{tabular}




\section{Material: $\quad \mathrm{Fe}_{3} \mathrm{Al} / \mathrm{TP}$ 304H}

\section{Summary of Findings, (continued)}

\begin{tabular}{|c|c|}
\hline Issue & Discussion \\
\hline $\begin{array}{l}\text { Wall } \\
\text { Thickness } \\
\text { Loss } \\
\text { Determined }\end{array}$ & $\begin{array}{l}\text { - Tube cross-sections were prepared to allow for measurement of wall } \\
\text { thickness to determine metal loss due to exposure. This is shown in } \\
\text { silhouette in Figure E-6. } \\
\text { The same relative "o'clock" positions were maintained as discussed } \\
\text { earlier. Measurements were taken at eight evenly spaced locations } \\
\text { about the tube circumference. These were then compared with } \\
\text { measurements taken at these same locations prior to exposure. } \\
\text { - The before and after measurements were documented in the table that } \\
\text { accompanies each figure. These form the basis for the "delta" } \\
\text { calculation to determine the amount of wall thickness lost. } \\
\text { - For the sample in question, the minimum wall thickness in the cross } \\
\text { section was not at one of the eight predetermined locations. So, there } \\
\text { were no "before" measurement for comparison to determine the } \\
\text { "delta". To address this, the average "before" wall thickness was used } \\
\text { to calculate the wall thickness lost at this off-location. }\end{array}$ \\
\hline $\begin{array}{l}\text { Wall } \\
\text { Thickness } \\
\text { Loss Versus } \\
\text { Average Metal } \\
\text { Temperature }\end{array}$ & $\begin{array}{l}\text { - The chart in Figure E-7 plots the maximum wall thickness lost against } \\
\text { the calculated average metal temperature for Sample B-C in both } \\
\text { Sections A and C. } \\
\text { I was noted that the rate metal loss was somewhat greater for Section } \\
\text { A than for Section C. It's difficult to draw conclusions from this } \\
\text { particularly given the fact that the iron-aluminide coating was likely } \\
\text { gone from the Section C sample very early in its life. }\end{array}$ \\
\hline $\begin{array}{l}\text { Metal Loss as } \\
\text { a Function of } \\
\text { Time }\end{array}$ & $\begin{array}{l}\text { - The chart in Figure E-8 plots metal loss as a function of time. } \\
\text { - Given the limited data, it's difficult to justify the straight line fit } \\
\text { shown, particularly in the light of the evidence of a decreasing metal } \\
\text { loss rate as a function of time, per the data shown in Figure E-7. }\end{array}$ \\
\hline $\begin{array}{l}\text { Metallurgical } \\
\text { Analysis - } \\
\text { SEM }\end{array}$ & $\begin{array}{l}\text { - Sample B-C was subjected to a detailed SEM/EDS assessment of the } \\
\text { constituents and structure in the vicinity of the external and internal } \\
\text { surfaces. The focus of this investigation was in the region of the tube } \\
\text { where the maximum amount of material was lost. } \\
\text { - Figures E-9a and b show secondary electron images (SEI) of the } \\
\text { external surface. A continuous scale was observed at the interface } \\
\text { along with a relatively thin deposit. } \\
\text { - The numbered locations in Figure E-9b show the locations of EDS } \\
\text { analyses that follow. Location } 1 \text { was taken to represent the bulk TP } \\
\text { 304H composition. Location } 4 \text { represented the continuous scale, and } \\
\text { Location } 5 \text { represented the diffusion zone along the external surface. } \\
\text { - Figure E-10 represents the microstructure at mid-wall in the tube. } \\
\text { - Figure E-11 shows the appearance of the internal metal surface for } \\
\text { Sample B-C. Local penetrations into the surface are observed. The } \\
\text { numbered locations show the locations of additional EDS analyses that } \\
\text { follow. Location } 2 \text { represents the composition of a corrosion } \\
\text { penetration into the surface. Location } 3 \text { represents the ID scale. }\end{array}$ \\
\hline
\end{tabular}


Material: $\mathrm{Fe}_{3} \mathrm{Al} / \mathrm{TP}$ 304H

\section{Summary of Findings, (continued)}

\begin{tabular}{|c|c|}
\hline Issue & Discussion \\
\hline $\begin{array}{l}\text { Metallurgical } \\
\text { Analysis - } \\
\text { Weld Cross } \\
\text { Section. }\end{array}$ & $\begin{array}{l}\text { - Figure E-12 shows a longitudinal profile (at the } 12 \text { o'clock } \\
\text { orientation) of the } \mathrm{Fe}_{3} \mathrm{Al} / \mathrm{TP} 304 \mathrm{H} \text { sample adjacent to weld "B" in } \\
\text { Section } \mathrm{C} \text {, see Figure E-1 for location. } \\
\text { - An Inconel } 625 \text { weld metal was used throughout Section C. The cross } \\
\text { sections show that the } \mathrm{Fe}_{3} \mathrm{Al} / \mathrm{TP} 304 \mathrm{H} \text { heat affected zone is not } \\
\text { preferentially attacked, in fact, the wall thickness for the tube sample } \\
\text { tapers from the weld toward the middle of the tube length. }\end{array}$ \\
\hline $\begin{array}{l}\text { EDS Analysis } \\
\text { Results }\end{array}$ & $\begin{array}{l}\text { - The table in Figure E-13 provides a summary of the EDS analyses of } \\
\text { the locations numbered in Figures E-9 and E-11. Also shown in this } \\
\text { table is a summary of the spectrographic analysis reported earlier. } \\
\text { Review of this table suggests the following: } \\
\text { - Area 1: Bulk metal analysis for TP } 304 \mathrm{H} \text { - These results are } \\
\text { taken as verification that the correct tube material was at position } \\
\text { B-C } \\
\text { - Area 2: Scale penetration at ID surface - The analysis suggests } \\
\text { - } \text { Area 3: Scale adhering along ID surface }- \text { This analysis also } \\
\text { suggests a nickel-rich oxide.. } \\
\text { - Area 4: OD surface deposit/scale - The analysis suggests that this } \\
\text { is primarily an iron-rich oxide. Sulfur also is noted. The reason } \\
\text { for the presence of copper is unclear. } \\
\text { - Area 5: OD diffusion zone - This zone is depleted in chromium } \\
\text { and thus enriched in nickel and iron. }\end{array}$ \\
\hline
\end{tabular}




\section{Gas Flow}

Direction

Sample B-C (1149 F)

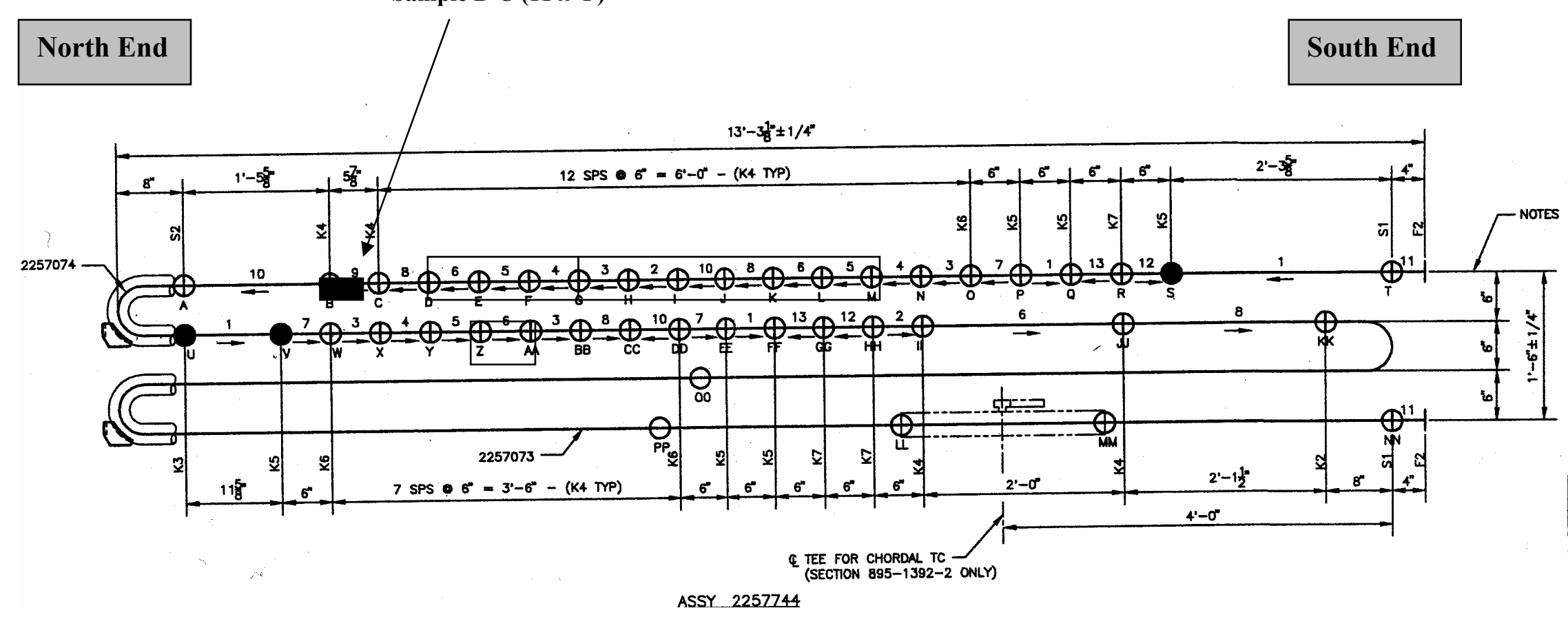

Figure E-1: Schematic Drawing Showing Position of Fe $\mathrm{F}_{3} \mathrm{Al}$ / TP 304HTube Sample Within Section C

1. Drawing shown in same relative orientation as per the actual installation.

2. This group of tube samples was removed from service in November 2001 - highlighted in red.

3. This group of tube samples was removed from service in June 2002 - highlighted in yellow. 


\section{Figure E-2 Semi-Quantitative Composition Verification for $\mathrm{Fe}_{3} \mathrm{Al}$ / TP 304H}

\section{Description:}

Source:

Reference Composition:
Commercially available iron Aluminide alloy (FAS) electrospark discharge coated onto Type 304H stainless steel (roughly 30 to 60 mils thick)..

FAS developed by Oak Ridge National Laboratory (ORNL), applied by

Pacific Northwest National Laboratory (PNNL)

"Target" chemistry for coating - per Reference 1 .

\begin{tabular}{|c|c|c|c|c|c|c|c|}
\hline Element & $\begin{array}{c}\text { Reference } \\
\text { Composition of } \\
\text { Coating }\end{array}$ & $\begin{array}{c}\text { Composition (a) } \\
\text { Heat } 17433\end{array}$ & $\begin{array}{c}\text { EDS of } \\
\text { Coating at } \\
\text { Outer Surface }\end{array}$ & $\begin{array}{l}\text { EDS of Bulk } \\
\text { Coating }\end{array}$ & Element & $\begin{array}{c}\text { Reference } \\
\text { Composition } \\
\text { SA } 213 \text { TP 304H }\end{array}$ & $\begin{array}{c}\text { Tube Sample } \\
\text { B-C }\end{array}$ \\
\hline $\mathrm{Ni}$ & - & $<0.01$ & 1.22 & 3.99 & $\mathrm{Ni}$ & $8.00-11.0$ & 8.23 \\
\hline $\mathrm{Cr}$ & 2.19 & 1.59 & 3.92 & 10.8 & $\mathrm{Cr}$ & $18.0-20.0$ & 16.5 \\
\hline $\mathrm{Fe}$ & Balance & - & 79.5 & 67.8 & $\mathrm{Fe}$ & - & Balance \\
\hline Mo & - & 0.01 & - & - & Mo & - & - \\
\hline $\mathrm{Co}$ & - & $<0.01$ & - & - & $\mathrm{Co}$ & - & - \\
\hline $\mathrm{C}$ & - & 0.008 & - & - & $\mathrm{C}$ & $0.04-0.10$ & - \\
\hline $\mathrm{N}_{2}$ & - & 0.001 & - & - & $\mathrm{N}_{2}$ & - & - \\
\hline $\mathrm{B}$ & 0.011 & 0.010 & - & - & $\mathrm{B}$ & - & - \\
\hline $\mathrm{Mn}$ & - & $<0.01$ & - & 1.34 & $\mathrm{Mn}$ & $2.00 \max$ & 1.19 \\
\hline $\mathrm{Si}$ & - & $<0.01$ & - & - & $\mathrm{Si}$ & $0.75 \max$ & 0.50 \\
\hline $\mathrm{Al}$ & 15.93 & 15.79 & 11.7 & 5.22 & $\mathrm{Al}$ & - & - \\
\hline $\mathrm{Ti}$ & - & - & - & - & $\mathrm{Ti}$ & - & - \\
\hline $\mathrm{Al}+\mathrm{Ti}$ & - & - & - & - & $\mathrm{Al}+\mathrm{Ti}$ & - & - \\
\hline $\mathrm{Nb}$ & - & $<0.01$ & 3.65 & 10.8 & $\mathrm{Nb}$ & - & - \\
\hline $\mathrm{Ta}$ & - & - & - & - & $\mathrm{Ta}$ & - & - \\
\hline Mo & - & - & - & - & $\mathrm{Nb}+\mathrm{Ta}$ & - & - \\
\hline $\mathrm{V}$ & - & $<0.01$ & - & - & $\mathrm{V}$ & - & - \\
\hline $\mathrm{W}$ & - & - & - & - & $\mathrm{W}$ & - & - \\
\hline $\mathrm{Cu}$ & - & - & - & - & $\mathrm{Cu}$ & - & - \\
\hline $\mathrm{Sn}$ & - & - & - & - & $\mathrm{Sn}$ & - & - \\
\hline $\mathrm{P}$ & - & - & - & - & $\mathrm{P}$ & $0.040 \max$ & - \\
\hline $\mathrm{S}$ & - & 0.002 & - & - & $\mathrm{S}$ & $0.030 \max$ & - \\
\hline
\end{tabular}

a - Reported analysis of wire used in deposition process by PNNL.

b- By difference. 


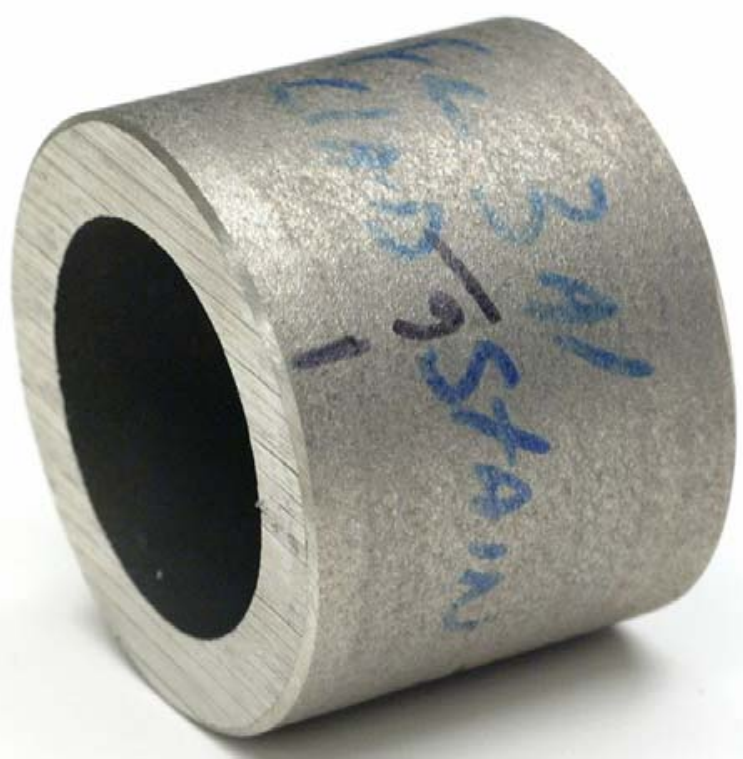

Figure E-3: Appearance of $\mathrm{Fe}_{3} \mathrm{Al}$ Coated TP 304H Tube Sample Prior to Service 


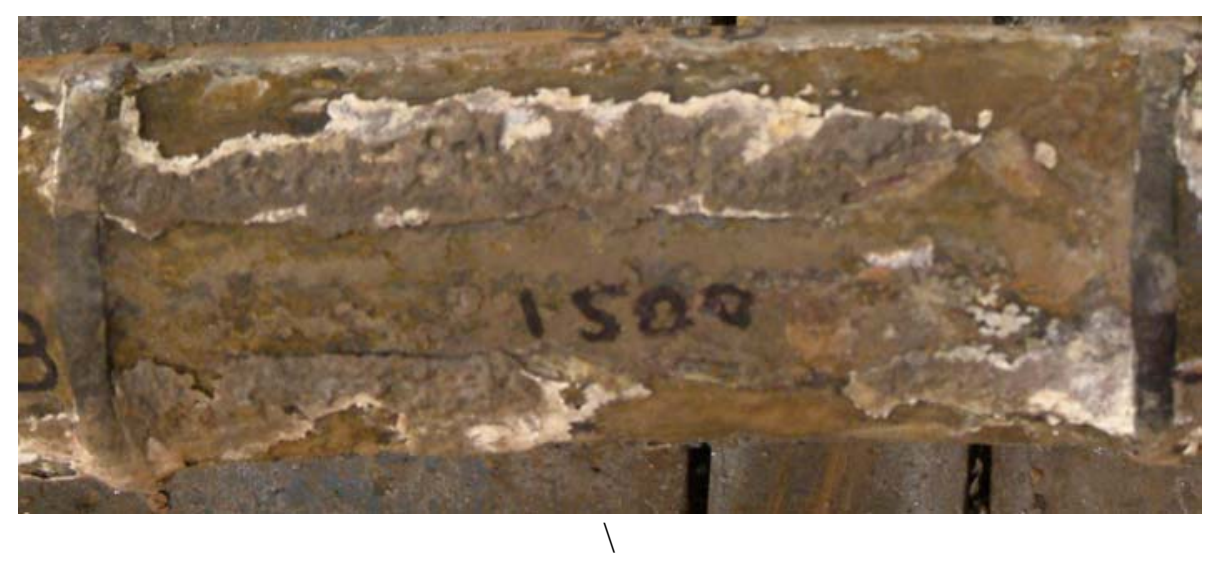

12 O'Clock - Facing Gas Flow

(Note: 3 o'clock is up in this image)

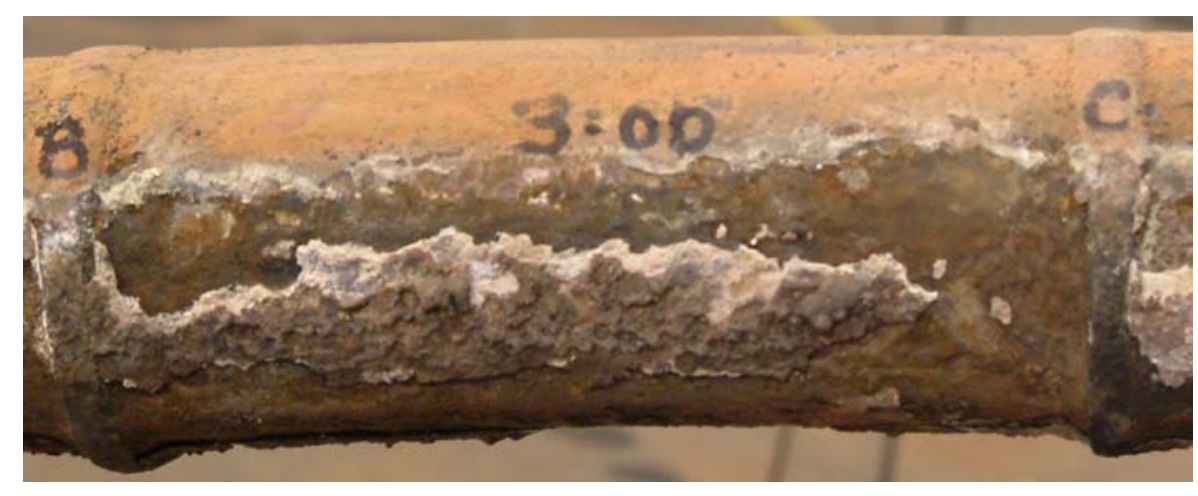

3 O'Clock

(Note: 6 o'clock is up in this image)

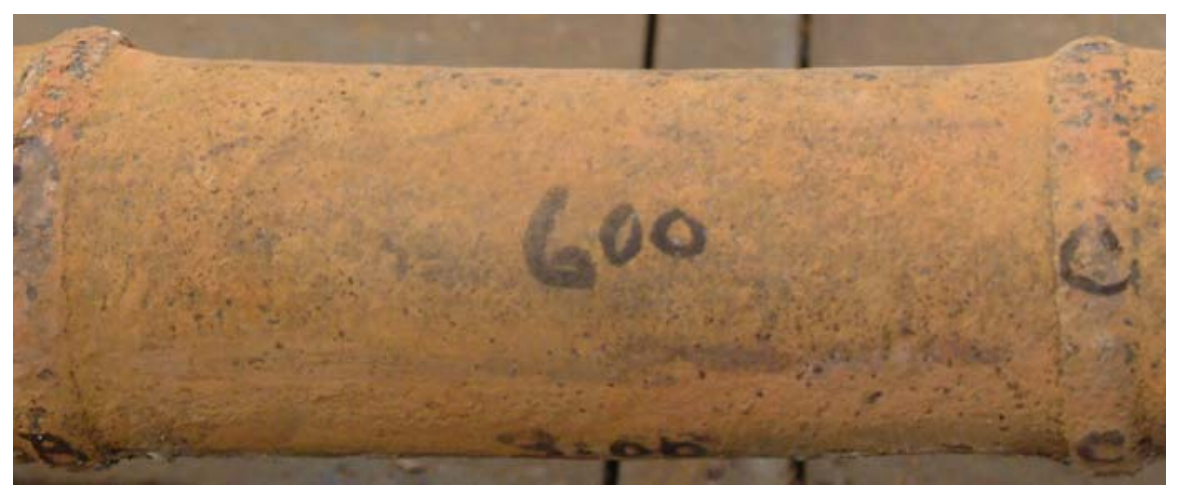

6 O'Clock - Opposite Gas Flow

Figure E-4 As-Received Appearance of Sample B-C ( $\left.\mathrm{Fe}_{3} \mathrm{Al} / \mathrm{TP} 304 \mathrm{H}\right)$

(Average metal temperature: 1149 F) 
12 O'Clock

(227 mils lost)

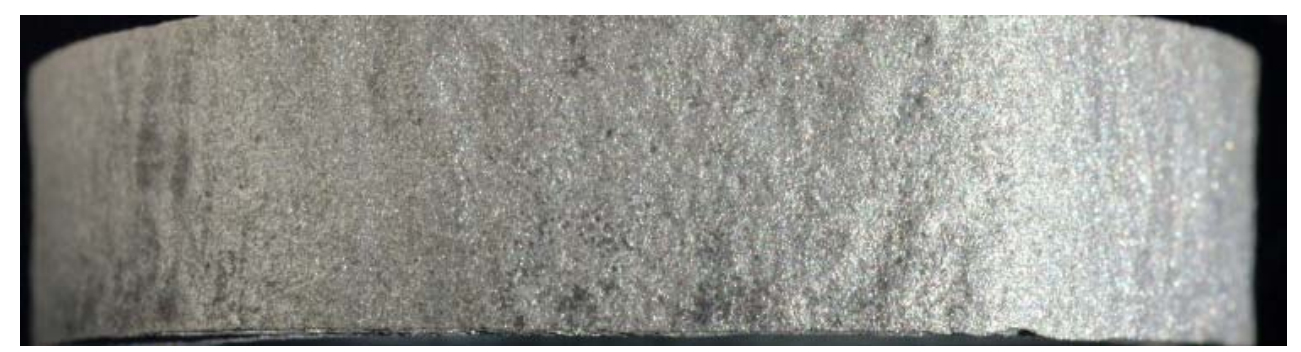

3 O'Clock

(194 mils lost)

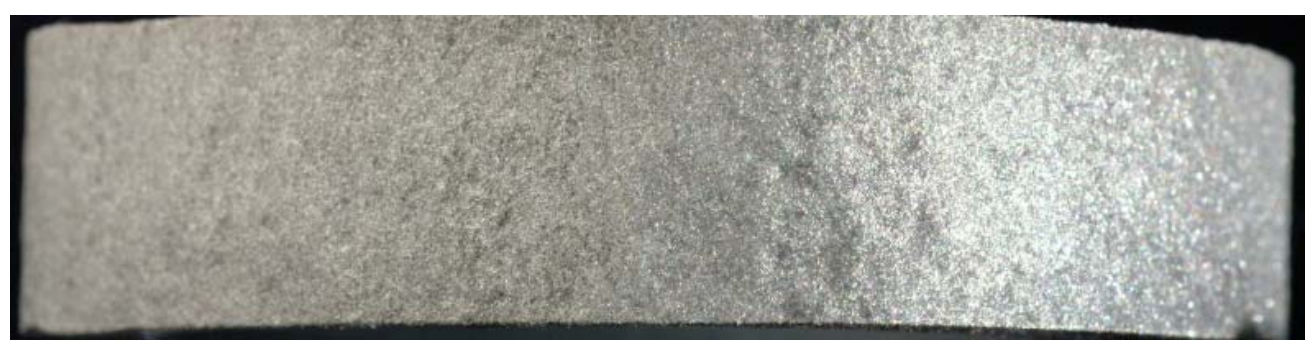

6 O'Clock

(27 mils lost)

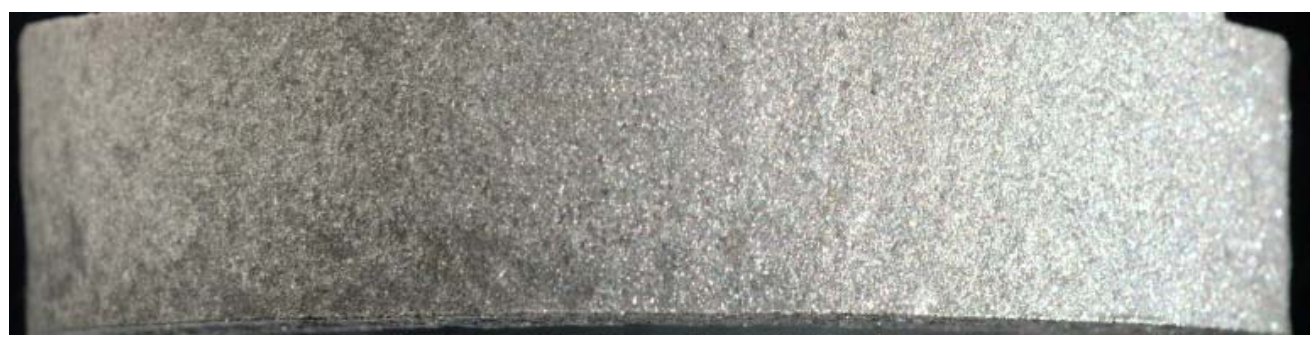

Figure E-5: OD Surface Appearance After Grit Blast

Material: $\quad \mathrm{Fe}_{3} \mathrm{Al} / \mathrm{TP} 304 \mathrm{H}$

Sample: B-C 


\section{Material:}

Sample Location:

Maximum Rate:

Maximum Loss Orientation:

Average metal Temperature:
$\mathrm{Fe}_{3} \mathrm{Al} / \mathrm{TP} 304 \mathrm{H}$

B-C

$100 \mathrm{mils} / \mathrm{yr}$

Approx. 2:30 o'clock 1149 F

12 O'Clock

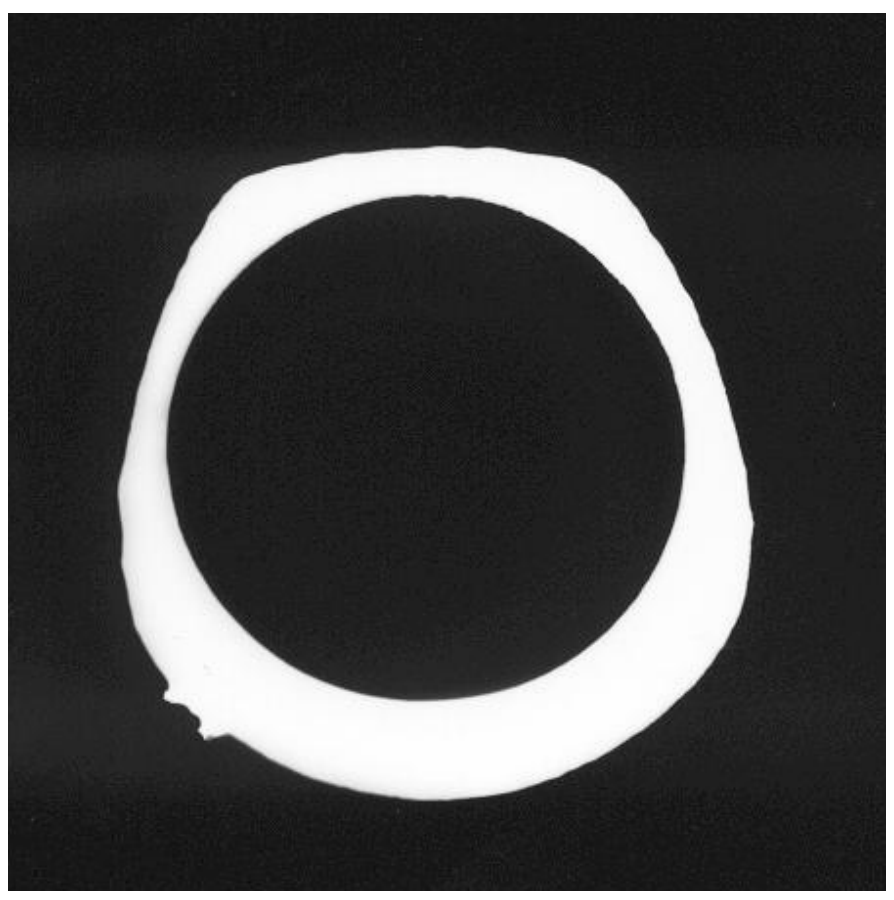

3 O'Clock

Approx. 1.2X

Wall Reduction by Position (in.)

\begin{tabular}{|l|c|c|c|c|c|c|c|c|c|c|}
\hline & $\begin{array}{c}\mathbf{1 2} \\
\text { o'clock }\end{array}$ & $\mathbf{1 : 3 0}$ & $\mathbf{3 : 0 0}$ & $\mathbf{4 : 3 0}$ & $\mathbf{6 : 0 0}$ & $\mathbf{7 : 3 0}$ & $\mathbf{9 : 0 0}$ & $\mathbf{1 0 : 3 0}$ & AVG & $\begin{array}{c}\text { Max }^{*} \\
\text { Loss }\end{array}$ \\
\hline Before & 0.392 & 0.391 & 0.392 & 0.389 & 0.388 & 0.389 & 0.391 & 0.392 & 0.390 & 0.390 \\
\hline After & 0.165 & 0.194 & 0.198 & 0.356 & 0.361 & 0.364 & 0.190 & 0.236 & & 0.151 \\
\hline Delta & 0.227 & 0.197 & 0.194 & 0.033 & 0.027 & 0.025 & 0.201 & 0.156 & & 0.239 \\
\hline
\end{tabular}

Figure E-6: Silhouette and Wall Thickness Measurements for Sample B-C 


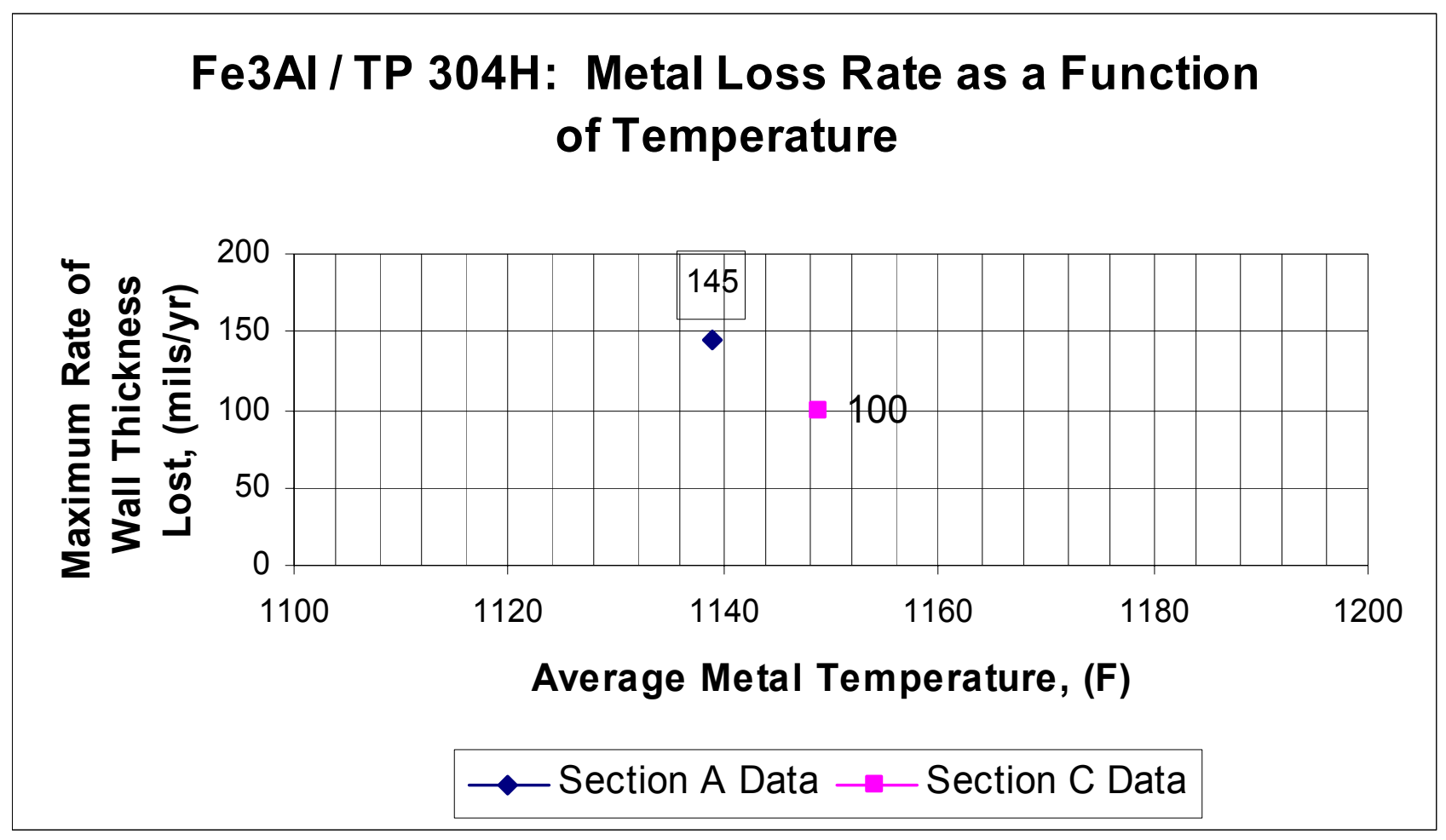

Figure E-7: Metal Loss Rate as a Function of Temperature

Material: $\quad \mathrm{Fe}_{3} \mathrm{Al} / \mathrm{TP} 304 \mathrm{H}$ 


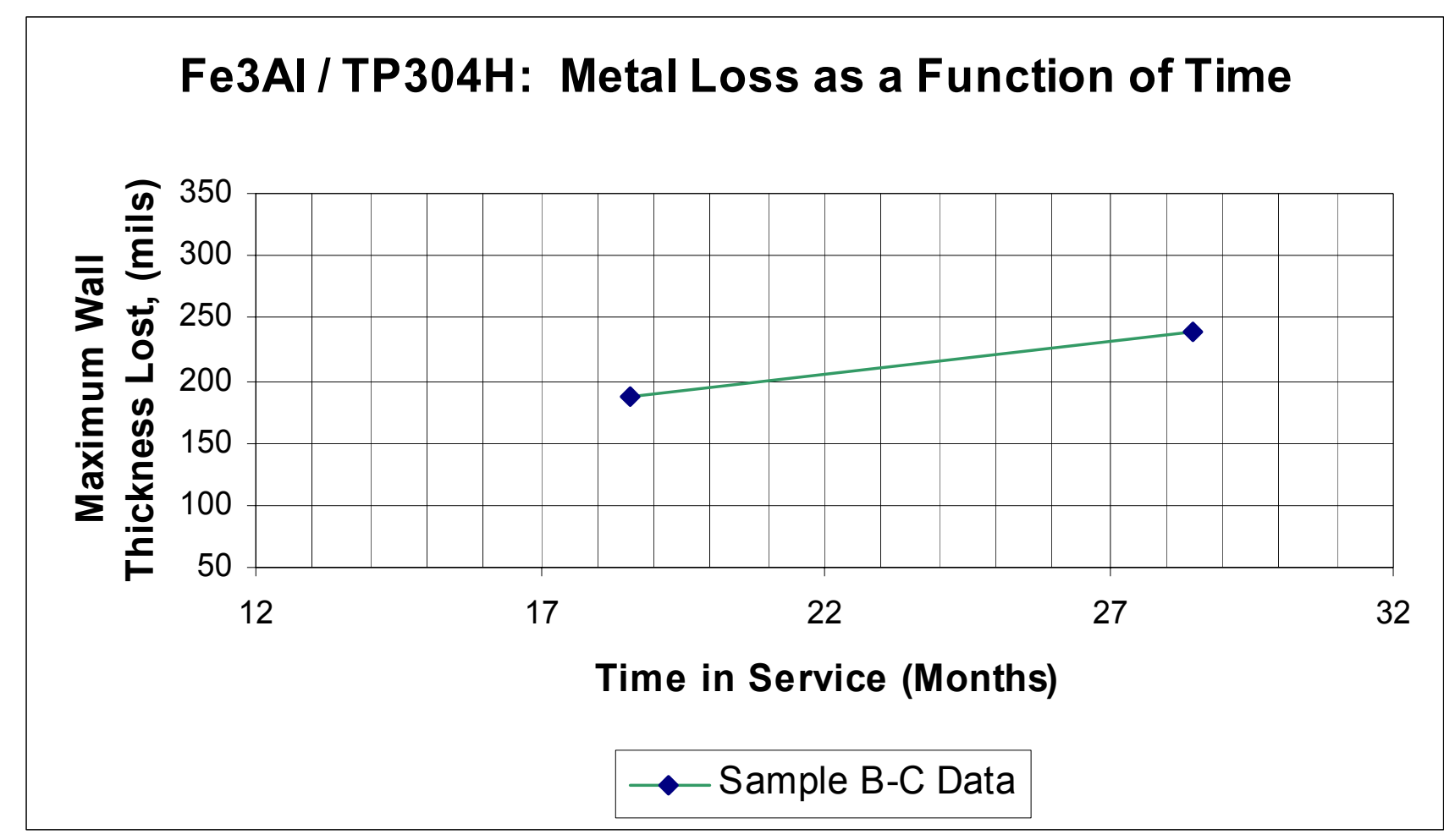

Figure E-7: Metal Loss as a Function of Time

Material: $\quad \mathrm{Fe}_{3} \mathrm{Al} / \mathrm{TP} 304$ 


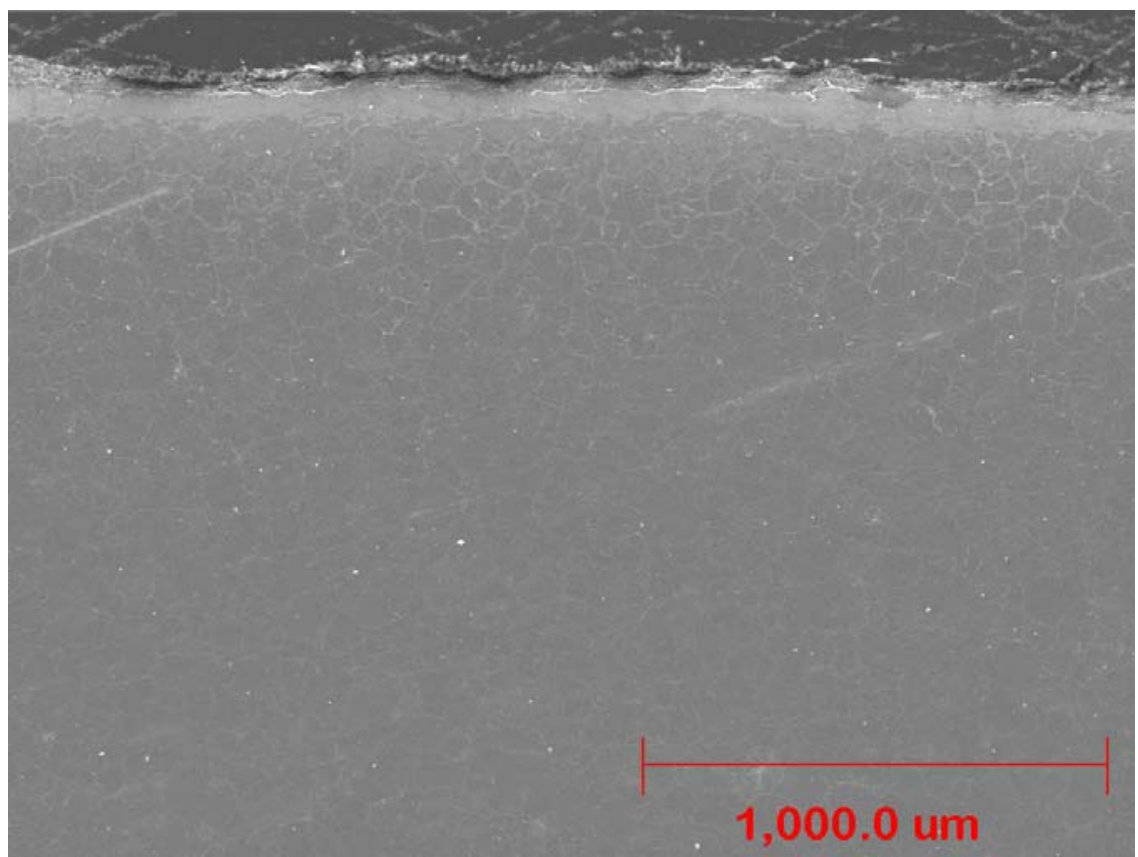

(a) External Scale/Metal Interface (SEI - Low Magnification)

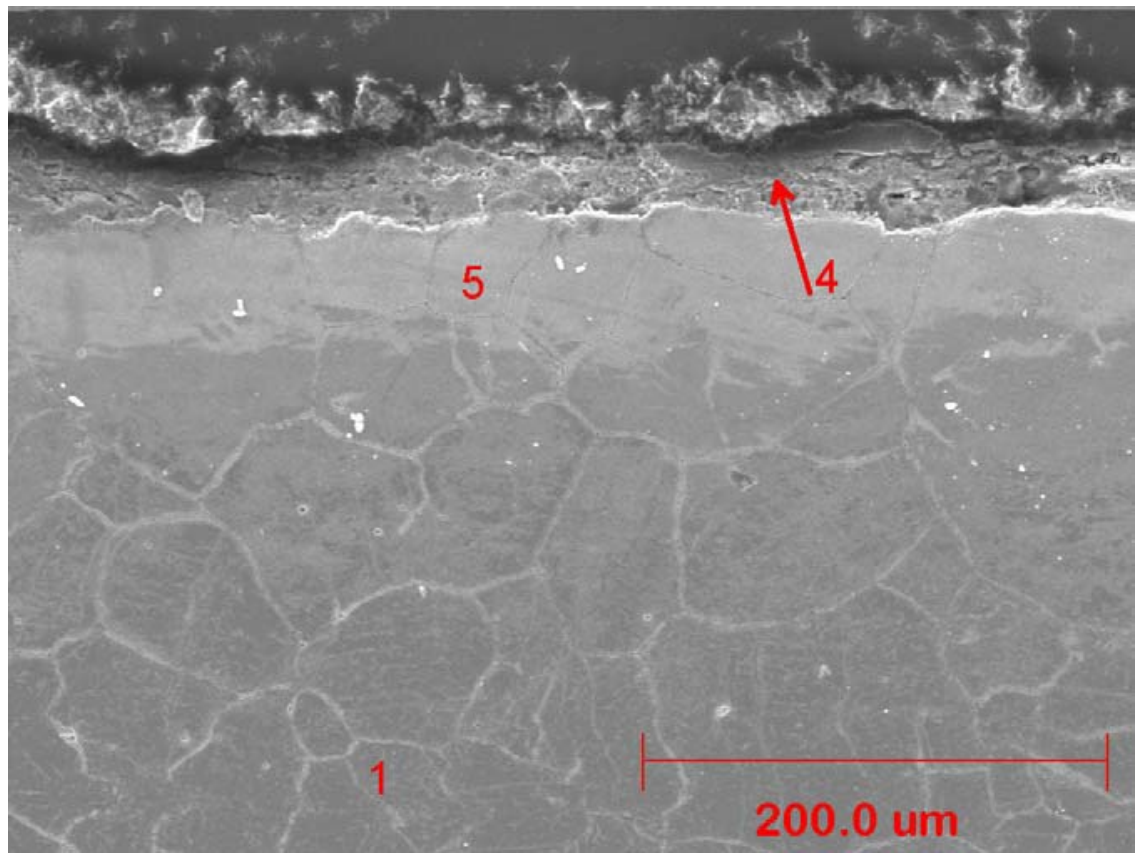

(b) External Scale/Metal Interface (SEI - Intermediate Magnification)

Note: Numbers indicate distinct areas characterized by the visual assessment and EDS analyses that follow.

Figure E-9: Microstructure: $\mathrm{Fe}_{3} \mathrm{Al} / \mathrm{TP}$ 304H 


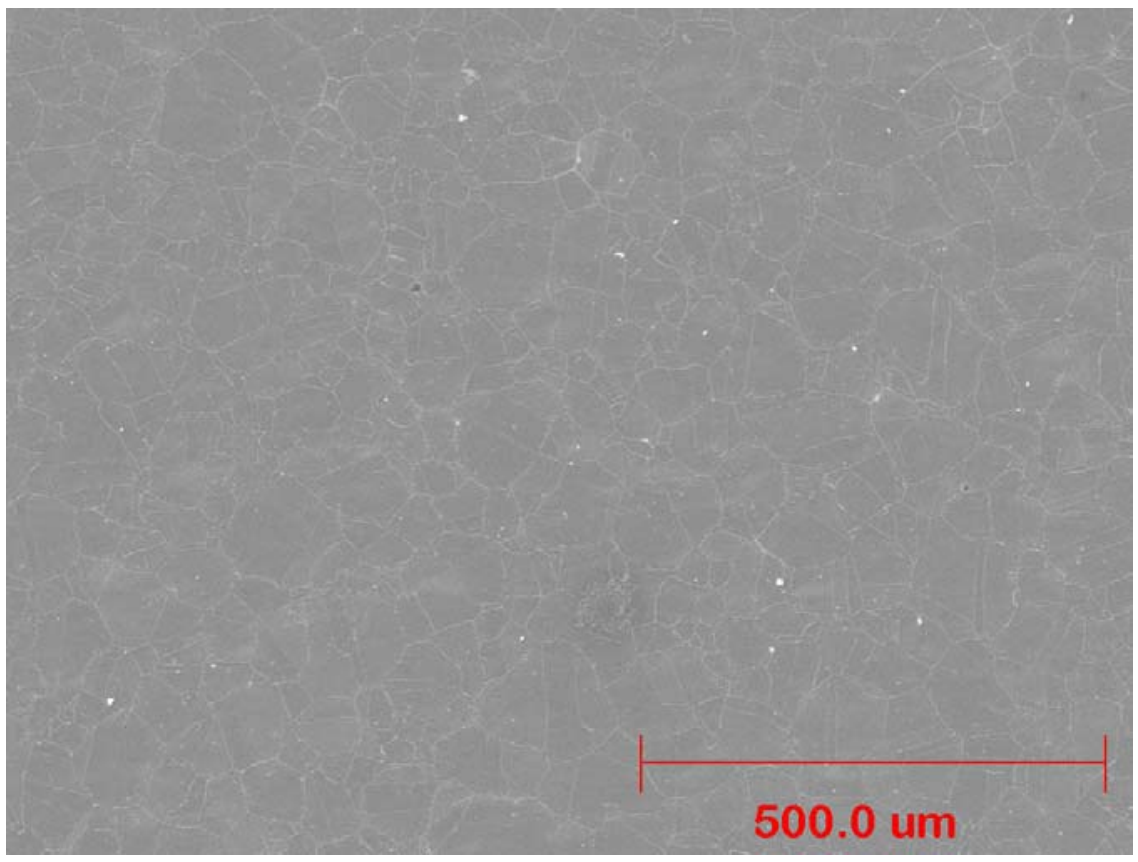

(SEI - Low Magnification)

Figure E-10: Mid-wall Microstructure: $\mathrm{Fe}_{3} \mathrm{Al} / \mathrm{TP} 304 \mathrm{H}$

Note: Photomicrograph at approximately 200X. Electrolytic 10\% oxalic acid etch, 9V OCP 


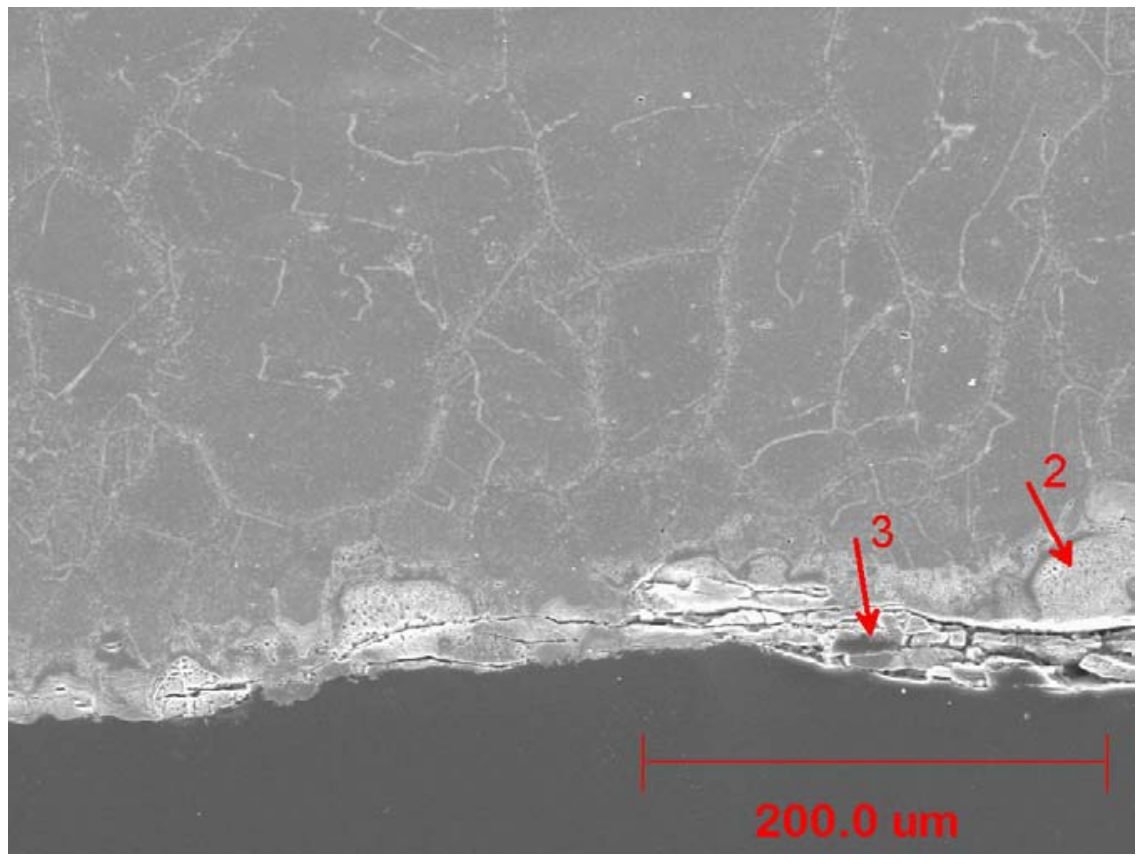

(SEI - Intermediate Magnification)

Figure E-11: Microstructure: Internal Scale/Metal Interface : $\mathrm{Fe}_{3} \mathrm{Al}$ / TP 304H

Note: Numbers indicate distinct areas characterized by the visual assessment and EDS analyses that follow. 


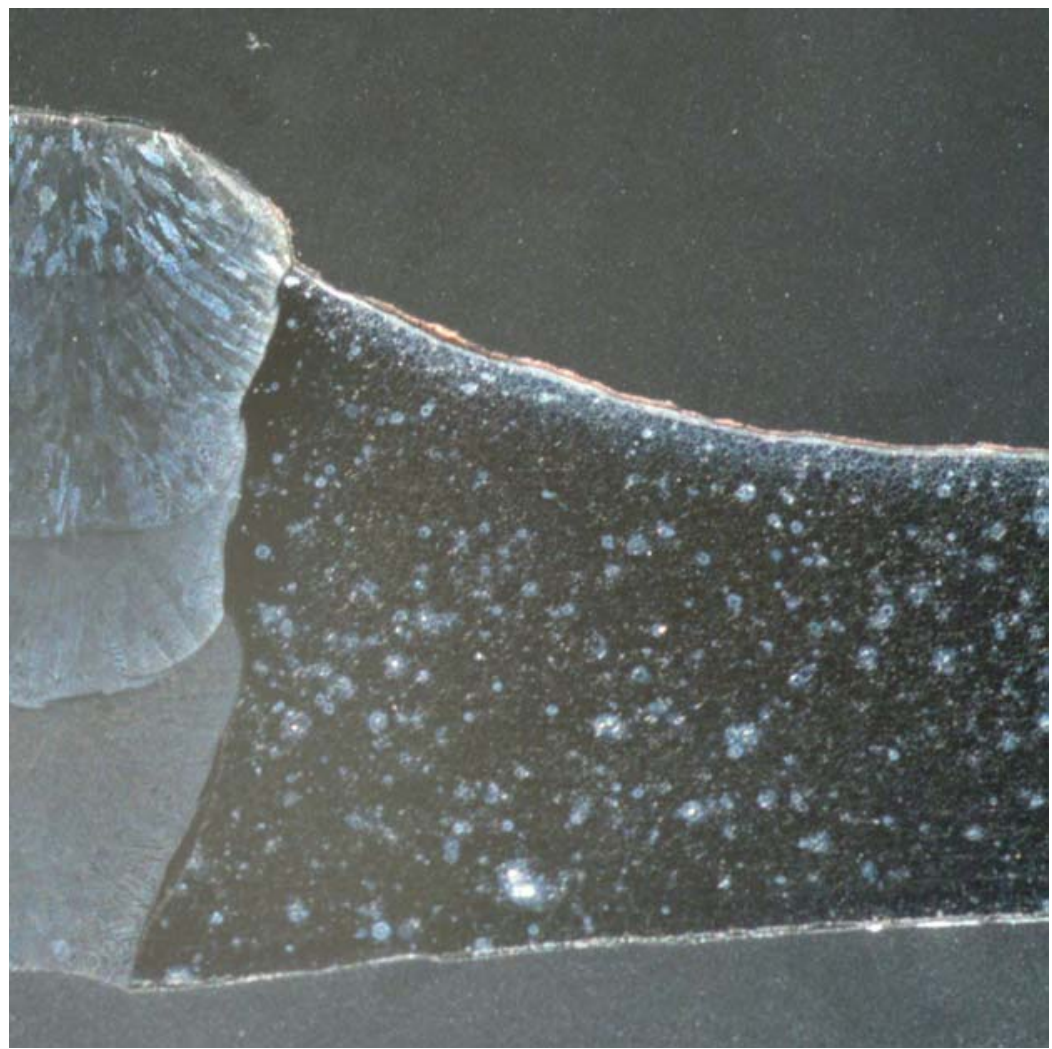

$\mathrm{Fe}_{3} \mathrm{Al}$ / TP 304H Adjacent to Weld "B"

Figure E-12: Longitudinal Cross-Section: $\mathrm{Fe}_{3} \mathrm{Al}$ / TP 304H Adjacent to Weld 
Figure E-13: EDS Analysis of Deposit/Scale/Metal Interface for $\mathrm{Fe}_{3} \mathrm{Al} / \mathrm{TP304H}$ (Sample B-C)

\begin{tabular}{|c|c|c|c|c|c|c|c|c|c|c|c|c|c|c|c|c|c|c|}
\hline Area & Description & $\mathbf{N i}$ & $\mathrm{Cr}$ & $\mathbf{F e}$ & $\mathrm{Cu}$ & Mn & $\mathbf{W}$ & $\mathbf{T i}$ & Al & Si & $\mathrm{C}$ & $\mathbf{O}$ & $\mathrm{Cu}$ & $\mathbf{K}$ & $\mathbf{N a}$ & As & $\mathbf{P}$ & $\mathbf{S}$ \\
\hline 1 & $\begin{array}{l}\text { Mid-wall } \\
\text { bulk analysis } \\
\text { TP 304H }\end{array}$ & 8.23 & 16.5 & 73.6 & -- & 1.19 & -- & -- & -- & 0.50 & -- & -- & -- & -- & -- & -- & -- & -- \\
\hline 2 & $\begin{array}{l}\text { ID surface } \\
\text { scale. }\end{array}$ & 47.2 & 8.58 & 33.7 & -- & 1.04 & -- & -- & 1.59 & -- & -- & 7.86 & -- & -- & -- & -- & -- & -- \\
\hline 3 & $\begin{array}{l}\text { ID surface } \\
\text { scale. }\end{array}$ & 20.4 & 16.8 & 44.9 & -- & 2.14 & -- & -- & -- & 0.79 & -- & 15.0 & -- & -- & -- & -- & -- & -- \\
\hline 4 & $\begin{array}{l}\text { OD surface } \\
\text { deposit/scale. }\end{array}$ & -- & 0.81 & 69.7 & -- & -- & -- & -- & 4.89 & 5.16 & -- & 13.6 & 4.02 & -- & -- & 1.37 & -- & 0.50 \\
\hline 5 & $\begin{array}{l}\text { OD diffusion } \\
\text { zone. }\end{array}$ & 10.2 & 8.47 & 79.5 & -- & 1.19 & -- & -- & -- & 0.59 & -- & -- & -- & -- & -- & -- & -- & -- \\
\hline & $\begin{array}{l}\text { Reference } \\
\text { analysis }\end{array}$ & 8.51 & 18.8 & 69.4 & 0.17 & 1.77 & 0.028 & 0.002 & 0.007 & 0.37 & 0.05 & -- & -- & -- & -- & -- & 0.029 & 0.001 \\
\hline
\end{tabular}


Appendix F

Analysis of Performance

Test Section C

310 Ta

F-1 


\section{Overview of Results: 310 Ta}

Type 310 Ta experienced a high rate of metal loss at two of the three locations in Section C. The metal loss rates for Samples M-N and F-G were 131 and 127 mils per year* respectively. These rates were very similar to those determined for the samples from Section A which had metal loss rates that ranged from 120 mils per year to 136 mils per year. This suggests a linear rate of metal loss for samples at these locations. It was noted that Sample F-G needed to be removed early from Section C due to concerns about excessive metal loss. This poor performance of TP $310 \mathrm{Ta}$ is consistent with that of those other alloys having approximately $20 \%$ to $25 \%$ chromium.

The sample at the lowest temperature location, Sample X-Y, had a metal loss rate of 120 mils per year for Section A and 64 mils per year for Section C. The reason for this reduced rate is not clear, particularly given the fact that the average surface metal temperature for tube Sample X-Y is somewhat higher (calculated to be $11^{\circ} \mathrm{F}$ ) in Section $\mathrm{C}$ than in Section A, and otherwise the corrosion rate appears to increase with temperature over the temperature range in question.

It should be noted that the metal loss rates determined to date for both Section A and Section C all were based on a ring sample that was extracted from mid-length of each tube sample. In this analysis additional attention was focused on metal losses in the vicinity of welds. Representative samples were taken and it was determined that, for the TP 310 Ta samples evaluated, the Inconel 625 weld filler metal is reasonably resistant to coal ash corrosion and that the tube material immediately adjacent to the weld (i.e. the weld heat affected zone) was somewhat less affected by corrosion than was the remainder of the tube. Indeed the tube wall appeared to be progressively thinner toward the mid-point of the tube sample.

Analysis of deposit on the surface revealed that it had the known aggressive combination of species, namely iron, oxygen, the alkali metals, and sulfur. The presence of significant amounts of arsenic was consistent with the analysis of samples from Section A, and further is consistent with the other samples from Section C. Examination and analysis of the scale adjacent to the metal surface revealed the chalky-white appearance associated with Alkali-iron-sulfates.

Furthermore high sulfur was consistently indicated in this area.

Finally, the analysis of the deposit and the metal surface immediately adjacent to the interface revealed the presence of carbon. While carbon determinations using EDS are unreliable, the implications of carbon in the deposit are worthy of additional consideration, as is the potential for carbon ingress from the deposit into the abutting metal.

* $\quad$ Note throughout this appendix, the reported metal loss rate was calculated based on time at temperature rather than total time within the boiler. 
Material: 310 Ta

Summary of Findings

\begin{tabular}{|c|c|}
\hline Issue & Discussion \\
\hline $\begin{array}{l}\text { Sample } \\
\text { Location and } \\
\text { Orientation. }\end{array}$ & $\begin{array}{l}\text { - In assessing the data for Section C, it is important to understand that } \\
\text { some tube samples had to be removed from the section early due to } \\
\text { excessive thinning. The schematic in Figure F-1 shows the relative } \\
\text { positions of the TP } 310 \text { Ta samples in all three sections. Also, shown } \\
\text { are the positions of all of the excessively thinned tubes that were } \\
\text { removed and replaced in order to extend the life of Section C. } \\
\text { - One TP } 310 \text { Ta sample was removed early. Sample F-G was removed } \\
\text { in June } 2002 \text {. Based on this, Sample F-G experienced approximately } \\
\text { three additional months at service temperature than the Section A } \\
\text { samples. It saw approximately seven less months at service } \\
\text { temperature than full exposure period for samples in Section C. } \\
\text { - Samples M-N and X-Y survived for the full Section C exposure } \\
\text { period. } \\
\text { The Sample F-G that was removed early was set aside for later } \\
\text { analysis, and has been included in the current evaluation along with } \\
\text { the other tube samples that made up Section C. } \\
\text { Also shown in Figure F-1 is the calculated average metal temperature } \\
\text { at each location. }\end{array}$ \\
\hline $\begin{array}{l}\text { Composition } \\
\text { Verification. }\end{array}$ & $\begin{array}{l}\text { - During the Section A evaluation, detailed composition verification was } \\
\text { undertaken for each tube sample that was removed from service. This } \\
\text { served, in part, to verify that the each tube material was located at its' } \\
\text { proper position within the test section. } \\
\text { - Based on the good results for Section A, it was decided that it would } \\
\text { be unnecessary to analyze each and every tube sample for composition } \\
\text { verification. Rather, it was decided to select a representative sample } \\
\text { from each tube material type for confirmatory analysis. } \\
\text { - During the Section A evaluation, the energy dispersive spectrographic } \\
\text { (EDS) capabilities of the scanning electron microscope (SEM) were } \\
\text { used to perform semi-quantitative analyses of each of the twelve } \\
\text { different candidate tube materials. Comparison of these EDS results } \\
\text { showed reasonably good correlation with results obtained using } \\
\text { quantitative analytical techniques, including optical emission } \\
\text { spectrometry. } \\
\text { Based on this, it was decided that the accuracy provided by EDS } \\
\text { would suffice for composition verification, but that more detailed } \\
\text { analyses would be undertaken if questions arose. } \\
\text { The results of the EDS analysis of tube sample M-N confirmed that } \\
\text { the correct material was used at this location. } \\
\text { For reference it should be noted Sample M-N was taken from Heat } \\
\text { 17425. Sample X-Y was taken from Heat } 17426 \text {. } \\
\text { The table in Figure F-2 also shows the "target" composition as } \\
\text { reported by ORNL. }\end{array}$ \\
\hline
\end{tabular}


Material: 310 Ta

\section{Summary of Findings, (continued)}

\begin{tabular}{|c|c|}
\hline Issue & Discussion \\
\hline $\begin{array}{l}\text { As-Received } \\
\text { Appearance }\end{array}$ & $\begin{array}{l}\text { - Figure F-3 shows the typical appearance of the } 310 \text { Ta samples as } \\
\text { delivered by ORNL. } \\
\text { - Figures F-4 through F- } 6 \text { document the appearance of the external } \\
\text { surface for all three samples after service exposure. Three views are } \\
\text { provided for each tube: } 12 \text { o'clock (the view that faces on-coming gas } \\
\text { flow), } 3 \text { o'clock (the view where the gas flow is tangent to the } \\
\text { surface), and } 6 \text { o'clock (the view that shows the back-side of the tube } \\
\text { relative to the gas flow direction. } \\
\text { The reader should be alerted to the fact that, within the boiler, the gas } \\
\text { flows upward over these tubes. This implies that for the "o'clock" } \\
\text { conventions described above, (with the } 12 \text { o'clock orientation facing } \\
\text { the gas flow direction), the actual physical orientation of these tubes is } \\
\text { such that the } 12 \text { o'clock side of the tube faces downward, and the } 6 \\
\text { o'clock orientation faces upward within the boiler. } \\
\text { - Wastage is visible at the } 12 \text { o'clock and } 3 \text { o'clock positions for all } \\
\text { three samples, as was a relatively thick red-brown deposit/scale. } \\
\text { The gray-white deposit typically associated with coal ash corrosion } \\
\text { can clearly been seen at the } 3 \text { o'clock position for Sample X-Y. EDS } \\
\text { analysis will show that the aggressive species implied by this } \\
\text { corrosion mechanism are also found in the scale of Sample M-N. }\end{array}$ \\
\hline $\begin{array}{l}\text { Grit-Blasted } \\
\text { Surface } \\
\text { Appearance }\end{array}$ & $\begin{array}{l}\text { - Figures F-7 through F-9 show the appearance of the external surface of } \\
\text { the } 301 \text { Ta tube samples after grit-blasting to remove deposit and } \\
\text { scale. The } 12 \text { o'clock and } 3 \text { o'clock positions for all three samples } \\
\text { show the appearance of general wastage due to fireside corrosion. } \\
\text { - The } 6 \text { o'clock position for Samples M-N shows that significant pitting } \\
\text { occurred on the back-side (i.e. the side of the tube opposite the gas } \\
\text { flow direction). }\end{array}$ \\
\hline $\begin{array}{l}\text { Wall } \\
\text { Thickness } \\
\text { Loss } \\
\text { Determined }\end{array}$ & $\begin{array}{l}\text { - Tube cross-sections were prepared to allow for measurement of wall } \\
\text { thickness to determine metal loss due to exposure. These are shown in } \\
\text { silhouette in Figures F-10 through F-12. } \\
\text { The same relative "o'clock" positions were maintained as discussed } \\
\text { earlier. Measurements were taken at eight evenly spaced locations } \\
\text { about the tube circumference. These were then compared with } \\
\text { measurements taken at these same locations prior to exposure. } \\
\text { - The before and after measurements were documented in the table that } \\
\text { accompanies each figure. These form the basis for the "delta" } \\
\text { calculation to determine the amount of wall thickness lost. } \\
\text { For all three tube samples, the minimum wall thickness in the cross } \\
\text { section was not at one of the eight predetermined locations. In these } \\
\text { cases, there were no "before" measurements for comparison. So, the } \\
\text { average "before" wall thickness was used to calculate the wall } \\
\text { thickness lost at these off-locations. }\end{array}$ \\
\hline
\end{tabular}




\section{Material: 310 Ta}

\section{Summary of Findings, (continued)}

\begin{tabular}{|c|c|}
\hline Issue & Discussion \\
\hline $\begin{array}{l}\text { Wall } \\
\text { Thickness } \\
\text { Loss Rate } \\
\text { Versus } \\
\text { Average } \\
\text { Surface Metal } \\
\text { Temperature }\end{array}$ & $\begin{array}{l}\text { - The chart in Figure F-13 plots the maximum wall thickness lost } \\
\text { against the calculated average surface metal temperature for the three } \\
310 \text { Ta samples in Section C. These same data also are plotted for the } \\
\text { tube samples from Section A. } \\
\text { It should be noted that, with one exception (Sample X-Y from Section } \\
\text { C) the remaining samples exhibited a consistent rate of metal loss from } \\
\text { section to section. These results typically would be suggestive of a } \\
\text { linear rate of metal loss. } \\
\text { It is difficult to account for the lower rate of metal loss ( } 64 \text { mils per } \\
\text { year) for Sample X-Y other than to note that it was situated at a } \\
\text { relatively low temperature position. }\end{array}$ \\
\hline $\begin{array}{l}\text { Wall } \\
\text { Thickness } \\
\text { Loss as a } \\
\text { Function of } \\
\text { Time and } \\
\text { Location }\end{array}$ & $\begin{array}{l}\text { - The chart in Figure F-13 plots metal loss as a function. Consistent with } \\
\text { the rates above Samples M-N and F-G demonstrated a nearly linear } \\
\text { rate of metal loss. } \\
\text { Also, apparent from this chart is that, while all three sample locations } \\
\text { exhibited approximately the same rate of metal loss for Section A (i.e. } \\
\text { after } 18.6 \text { months of service), Sample X-Y in Section C exhibited a } \\
\text { reduced rate of metal loss. }\end{array}$ \\
\hline $\begin{array}{l}\text { Metallurgical } \\
\text { Analysis - } \\
\text { SEM }\end{array}$ & 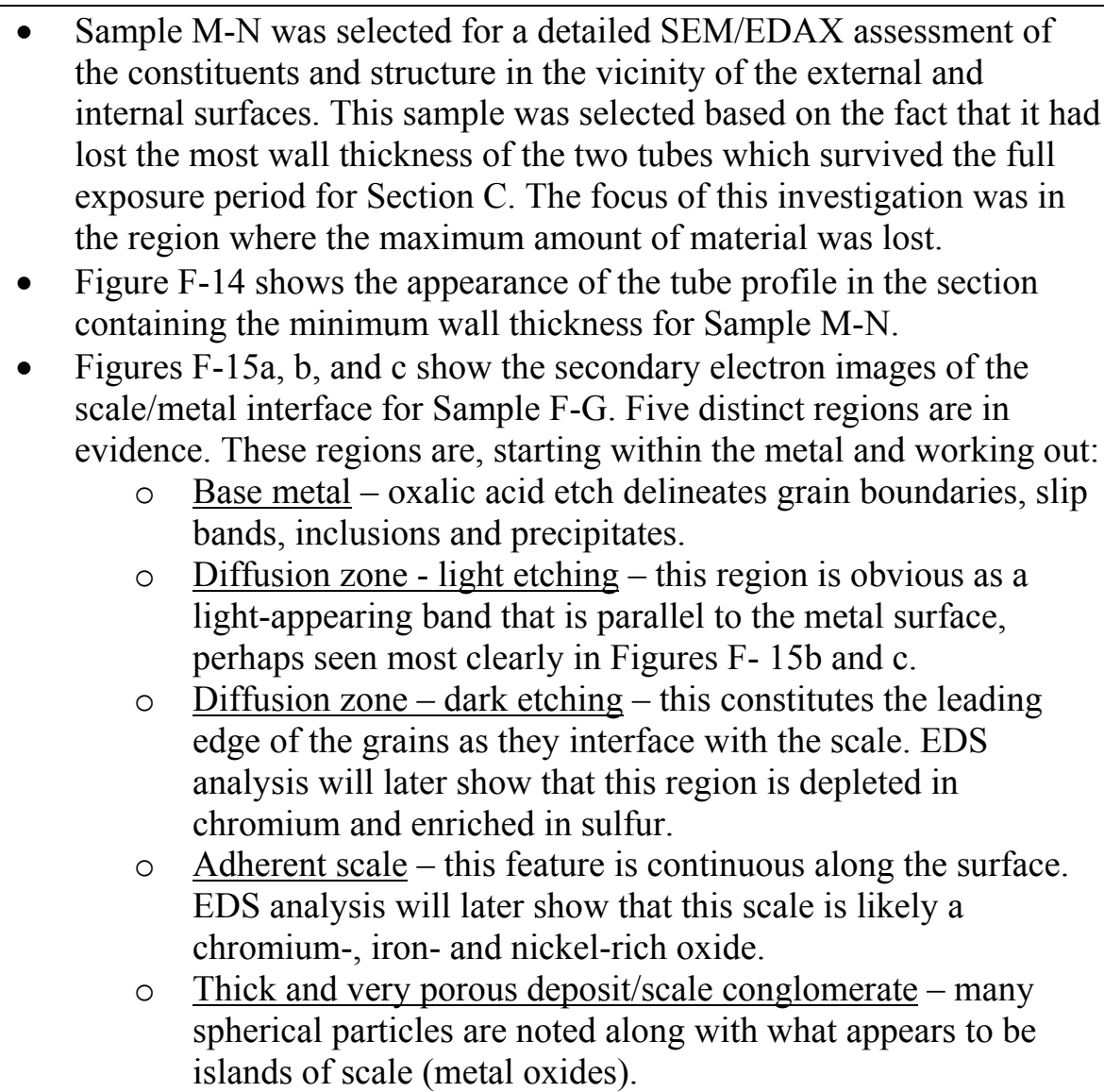 \\
\hline
\end{tabular}




\section{Summary of Findings, (continued)}

\section{Material: $\quad 310$ Ta}

\begin{tabular}{|c|c|}
\hline Issue & Discussion \\
\hline $\begin{array}{l}\text { Metallurgical } \\
\text { Analysis - } \\
\text { SEM, } \\
\text { Continued. }\end{array}$ & $\begin{array}{l}\text { - Figure F-15b shows numbered positions where EDS analyses were } \\
\text { performed to characterize the materials in the vicinity of the } \\
\text { scale/metal interface. } \\
\text { - Figure F-16 shows the appearance of the microstructure at mid-wall in } \\
\text { the specimen. } \\
\text { - Figure F-17 internal metal surface for Sample M-N. An extremely thin } \\
\text { oxide scale is noted to cover the surface. }\end{array}$ \\
\hline $\begin{array}{l}\text { Metallurgical } \\
\text { Analysis - } \\
\text { Weld Cross } \\
\text { Section. }\end{array}$ & $\begin{array}{l}\text { - Figure F-18 shows a longitudinal profile (at the } 12 \text { o'clock } \\
\text { orientation) of the TP } 310 \text { Ta samples adjacent to the welds in Section } \\
\text { C. The welds in question are those identified as welds "N" and "X", } \\
\text { see Figure F-1 for locations } \\
\text { - An Inconel } 625 \text { weld metal was used throughout Section C. The cross } \\
\text { sections show that the TP } 310 \text { Ta heat affected zones are not } \\
\text { preferentially attacked, in fact, the wall thickness for the tube sample } \\
\text { tapers from the weld toward the middle of the tube length. }\end{array}$ \\
\hline $\begin{array}{l}\text { EDS Analysis } \\
\text { Results }\end{array}$ & $\begin{array}{l}\text { - The table in Figure F-19 provides a summary of the EDS analyses of } \\
\text { the locations numbered in Figure F-15b. Also shown in this table is a } \\
\text { summary of the spectrographic analysis reported earlier. } \\
\text { Review of this table suggests the following: } \\
\text { - Area 1: For the bulk deposit analysis - Alkali metals are present. } \\
\text { Sulfur and phosphorous are present in an increased amount } \\
\text { compared to the base metal. An iron oxide is likely, as are } \\
\text { aluminum, silicon and titanium oxides. Carbon content is } \\
\text { relatively high, however, EDS is not reliable quantitatively for } \\
\text { low atomic number elements. } \\
\text { - Area 2: Tightly adhering scale - This scale immediately adjacent } \\
\text { to the metal/scale is comprised predominantly of a chromium-rich } \\
\text { oxide. Also, of significance is the relatively high level of sulfur in } \\
\text { this region. } \\
\text { Area 3: Diffusion zone - dark etching - This region, adjacent to } \\
\text { the scale/metal interface, is less than one grain wide. The results } \\
\text { of the analysis suggest that this is a diffusion zone that is depleted } \\
\text { in chromium and enriched in iron and nickel. This is consistent } \\
\text { with the chromium-rich oxide scale that adheres to the metal } \\
\text { surface. } \\
\text { Area } 4 \text { : Diffusion zone - light etching - This region is } \\
\text { approximately one grain wide. It appears to be slightly depleted } \\
\text { in chromium. } \\
\text { Area 5: Bulk EDS analysis - The analysis at this location served } \\
\text { as the reference composition for verification of the sample } \\
\text { identity. }\end{array}$ \\
\hline
\end{tabular}




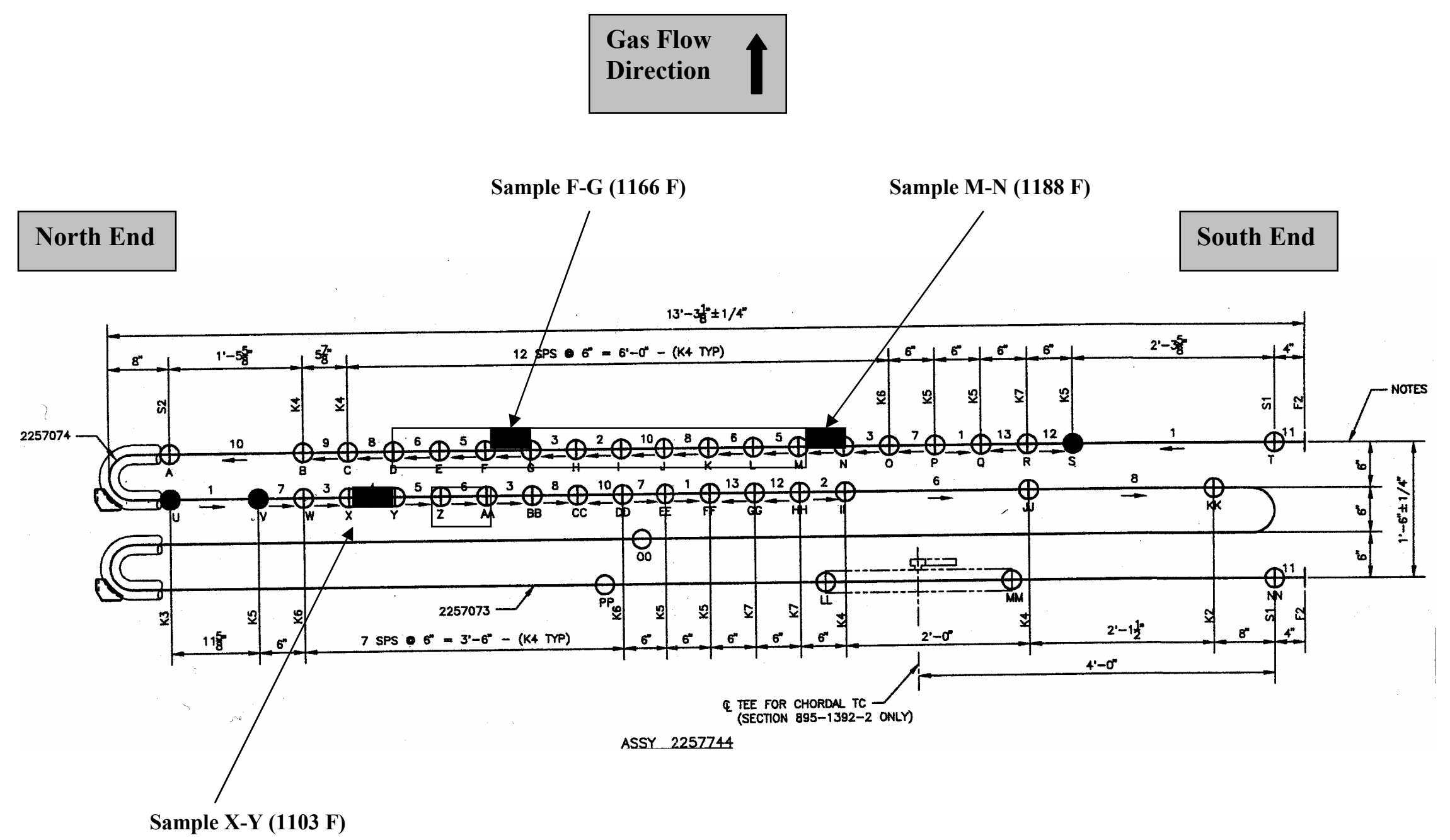

Figure F-1: Schematic Drawing Showing Position of 310 Ta Tube Samples in Section C

1. Drawing shown in same relative orientation as per the actual installation.

2. This group of tube samples was removed from service in November 2001 - highlighted in red.

3. This group of tube samples was removed from service in June 2002 - highlighted in yellow. 


\section{Figure F-2 Semi-Quantitative Composition Verification for Type 310 Ta}

Description:

20-25 Cr steel with improved creep strength due to addition of tantalum and nitrogen

Oak Ridge National Laboratory (ORNL)

Source:
Reference Composition:

"Target" Chemistry per ORNL (Reference 1).

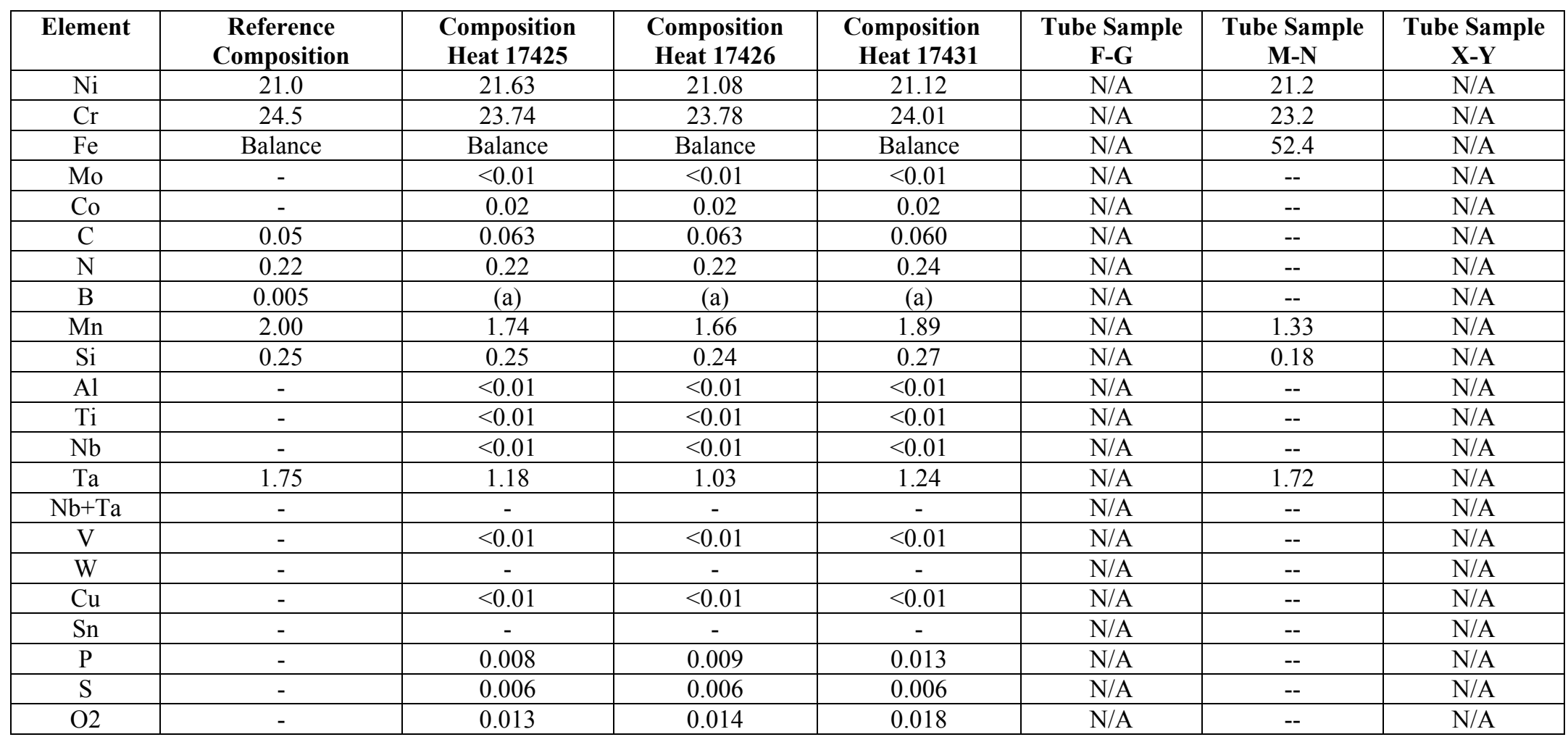

a- Not analyzed.

b- By difference.
Reference 1:

"Fabrication of Test Tubes for Coal Ash Corrosion Testing”, Sikka, V.K., Swindeman, R.W.,

Wright, I.G., Judkins, R.R., Johnson, R., Proc, 13 ${ }^{\text {th }}$ Annual Conf. on Fossil Energy Mat'ls, May 1999.

Sample M-N was from Heat $17425 \quad$ N/A = Tube sample not analyzed. 


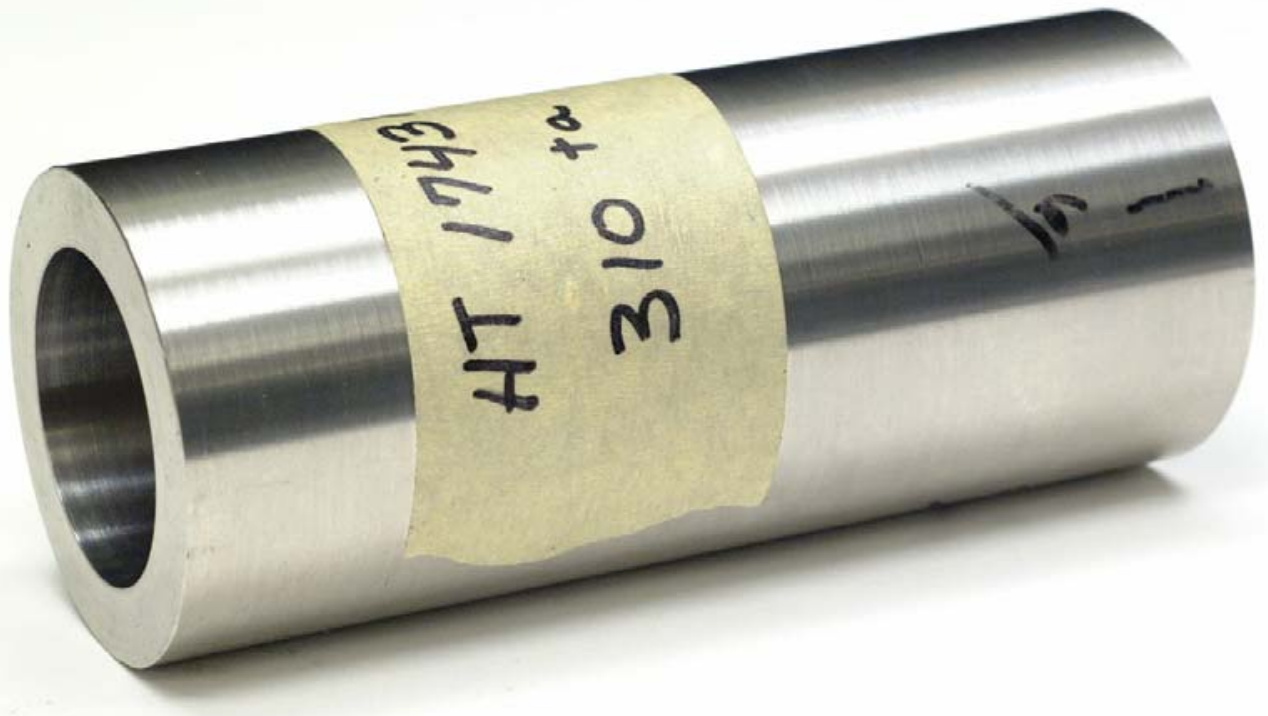

Figure F-3: Appearance of 310 Ta Tube Sample Prior to Service 


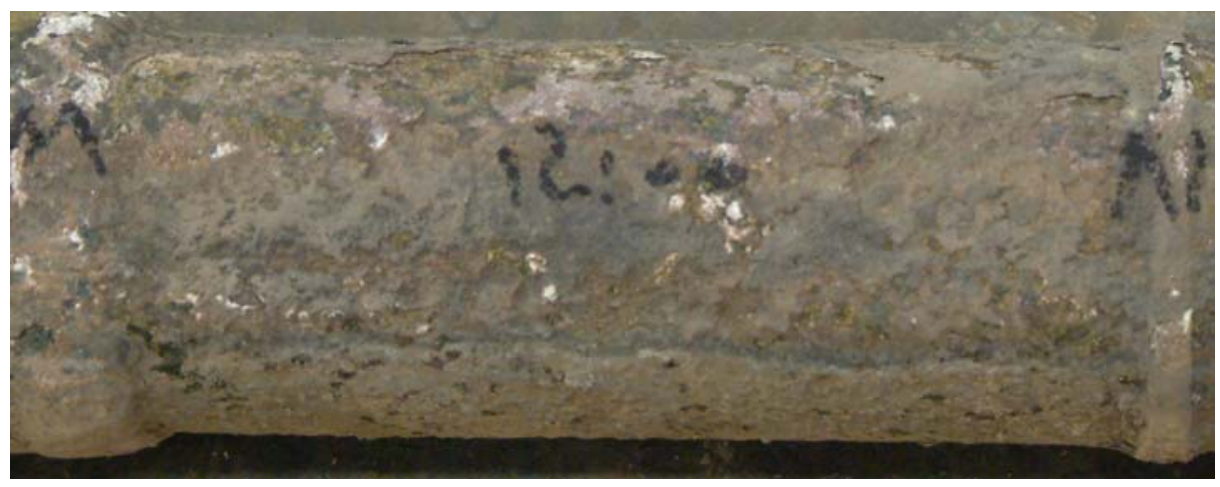

12 O'Clock - Facing Gas Flow

(Note: 3 o'clock is up in this image)

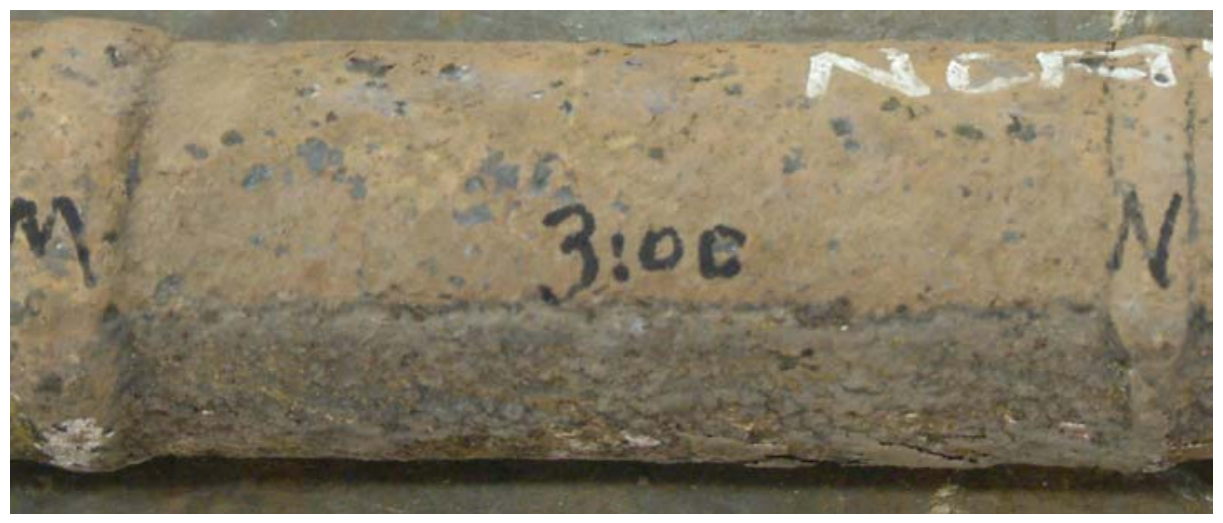

3 O'Clock

(Note: 6 o'clock is up in this image)

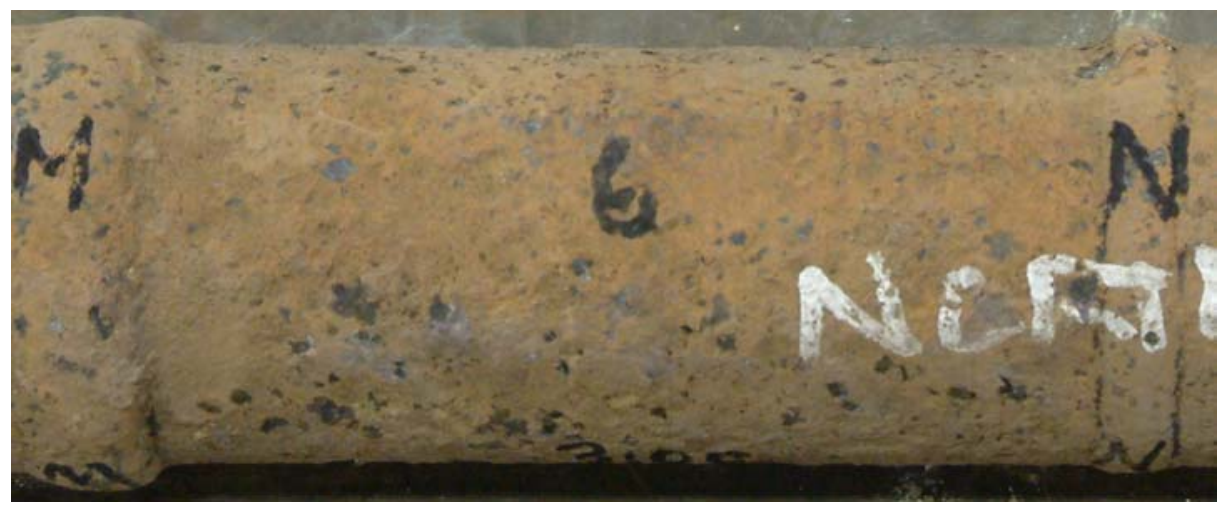

6 O'Clock - Opposite Gas Flow

Figure F-4 As-Received Appearance of Sample M-N (310 Ta)

(Average metal temperature: 1188 F) 


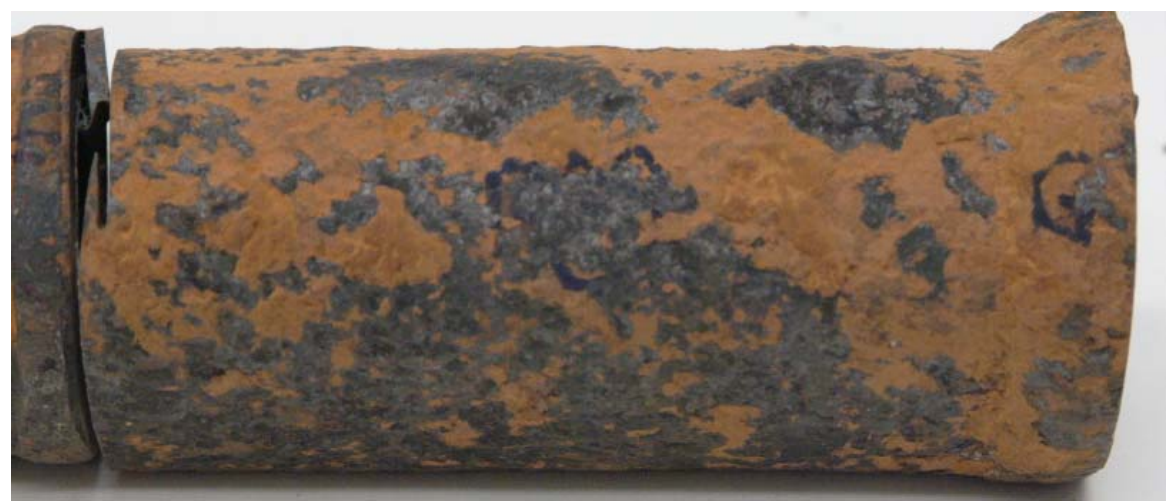

12 O'Clock - Facing Gas Flow

(Note: 3 o'clock is up in this image)

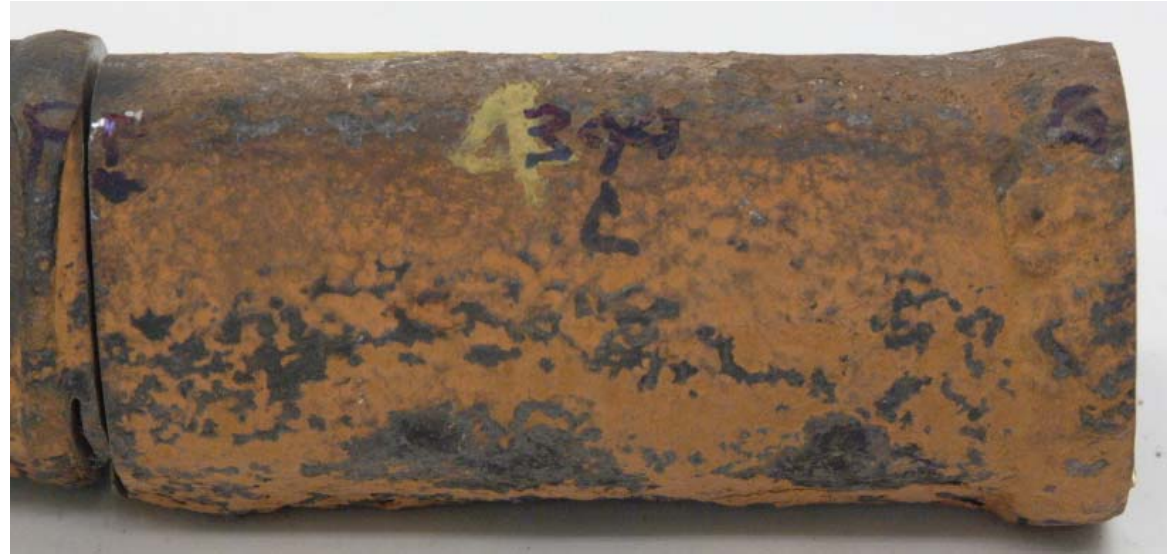

3 O'Clock

(Note: 6 o'clock is up in this image)

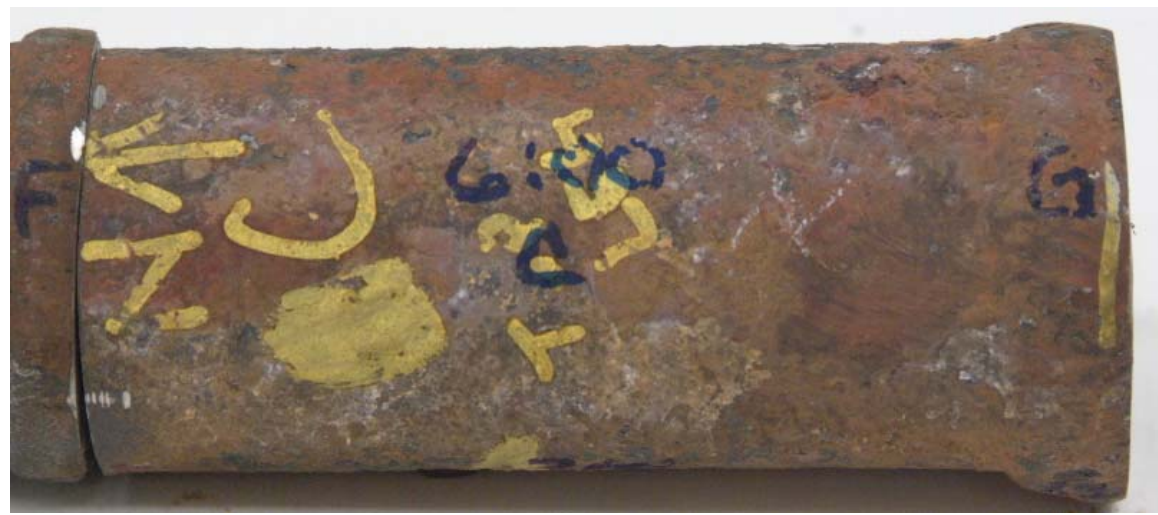

6 O’Clock - Opposite Gas Flow

Figure F-5 As-Received Appearance of Sample F-G (310 Ta)

(Average metal temperature: $1166 \mathrm{~F}$ ) 


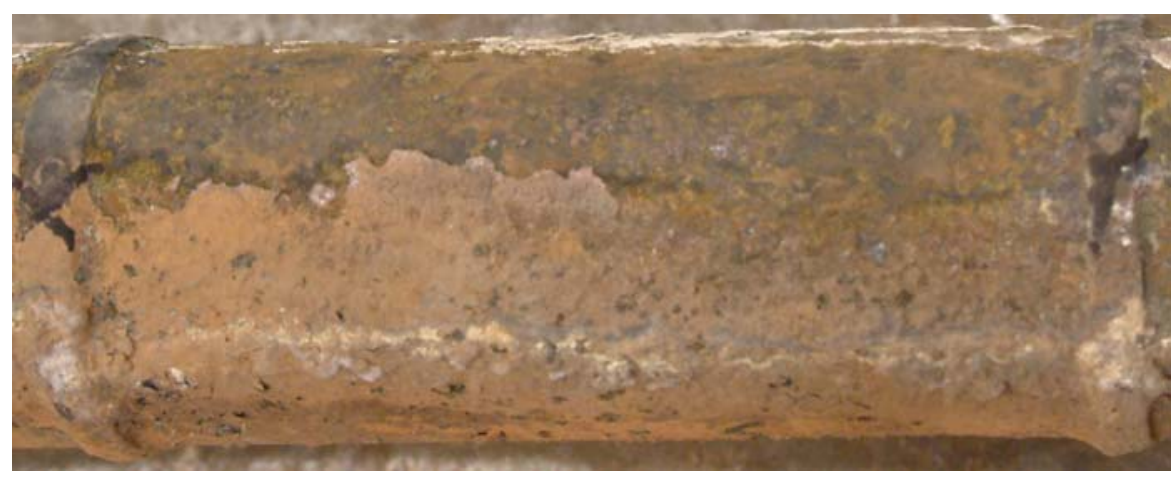

12 O'Clock - Facing Gas Flow

(Note: 3 o'clock is up in this image)

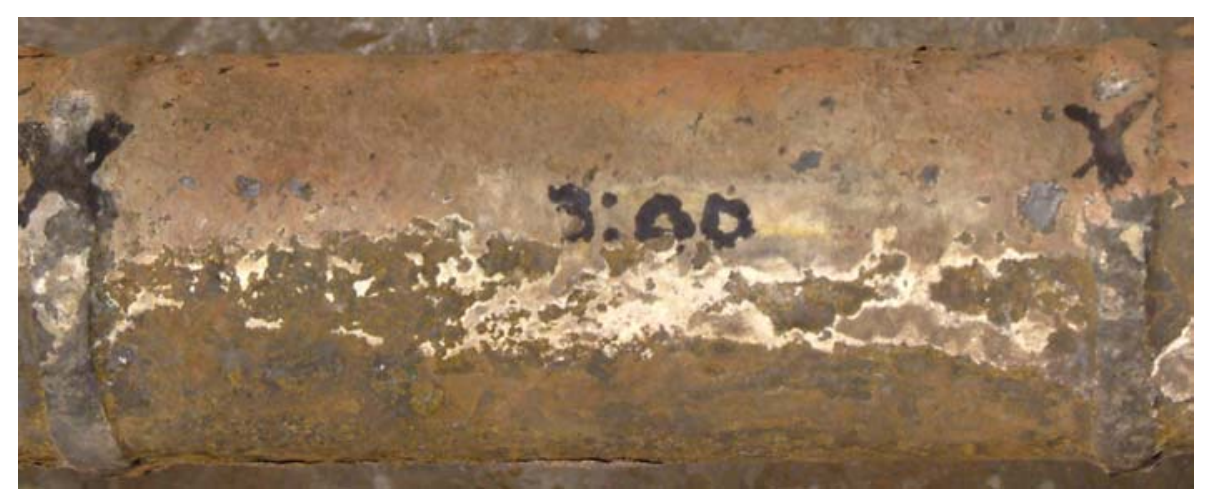

3 O'Clock

(Note: 6 o'clock is up in this image)

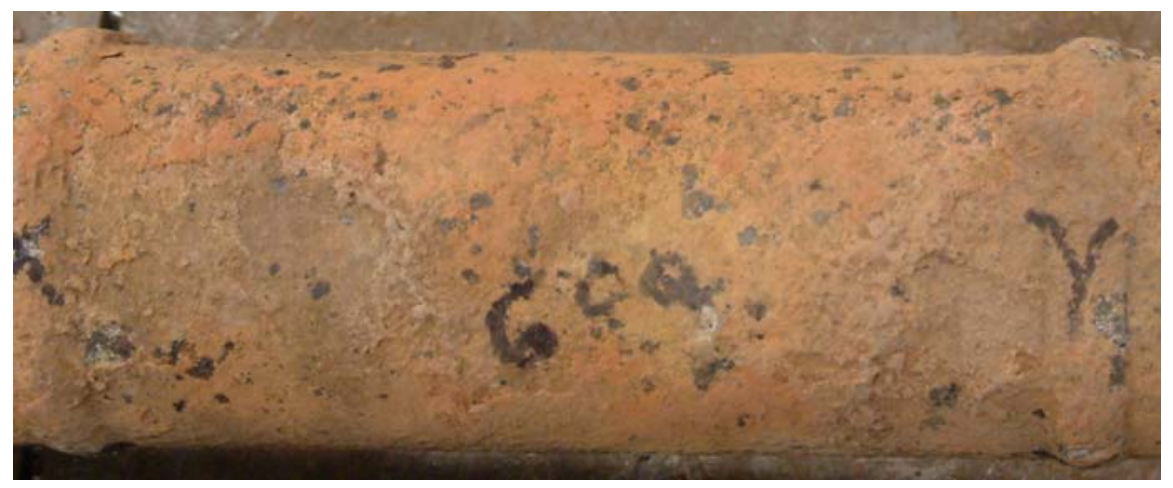

6 O'Clock - Opposite Gas Flow

Figure F-6 As-Received Appearance of Sample X-Y (310 Ta)

(Average metal temperature: $1103 \mathrm{~F}$ ) 
12 O'Clock

(212 mils lost)

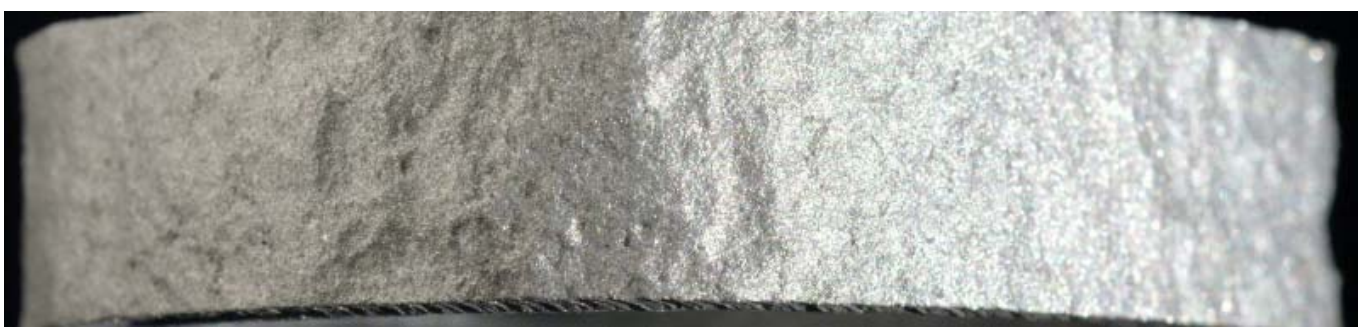

3 O'Clock

(255 mils lost)

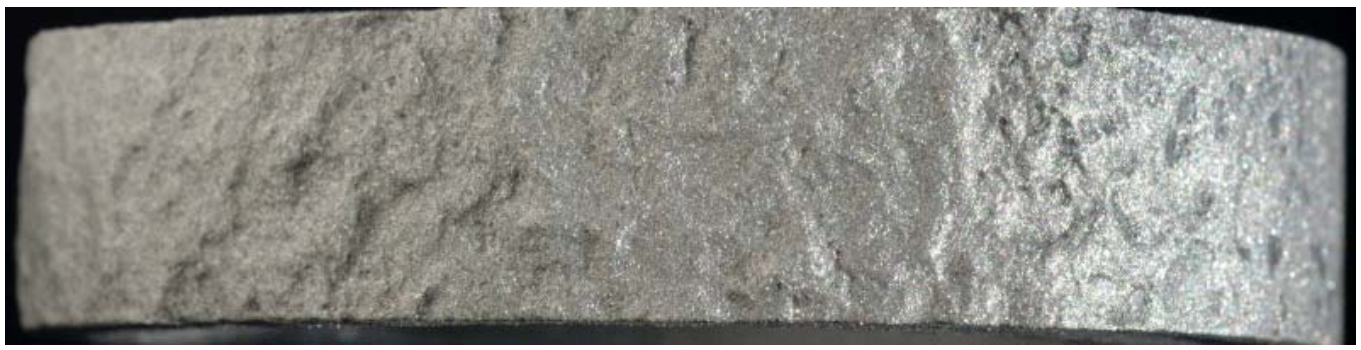

6 O'Clock

(0 mils lost)

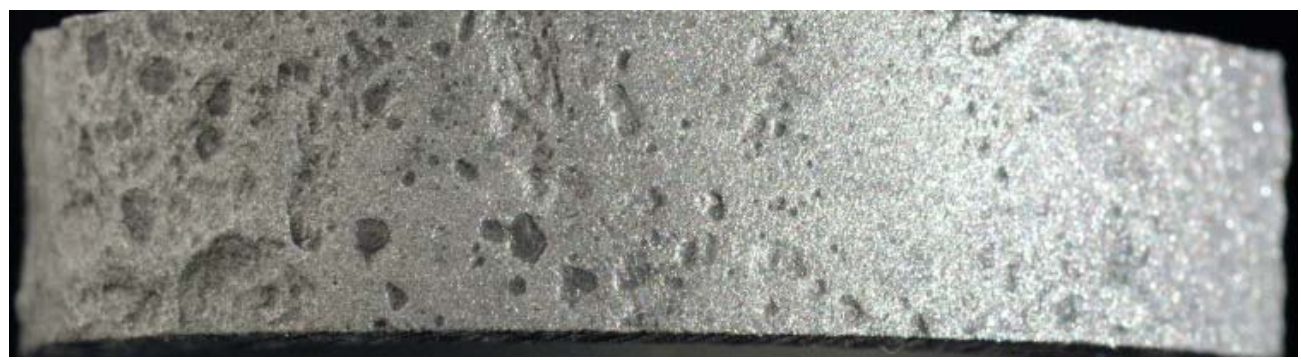

Figure F-7: OD Surface Appearance After Grit Blast

Material: $\quad 310$ Ta

Sample: M-N 
12 O'Clock

(119 mils lost)

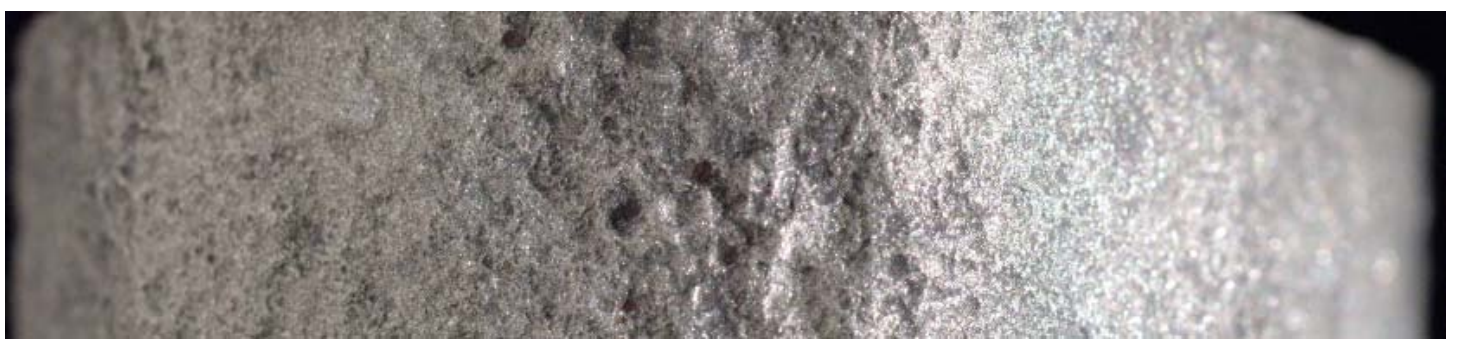

3 O'Clock

(188 mils lost)

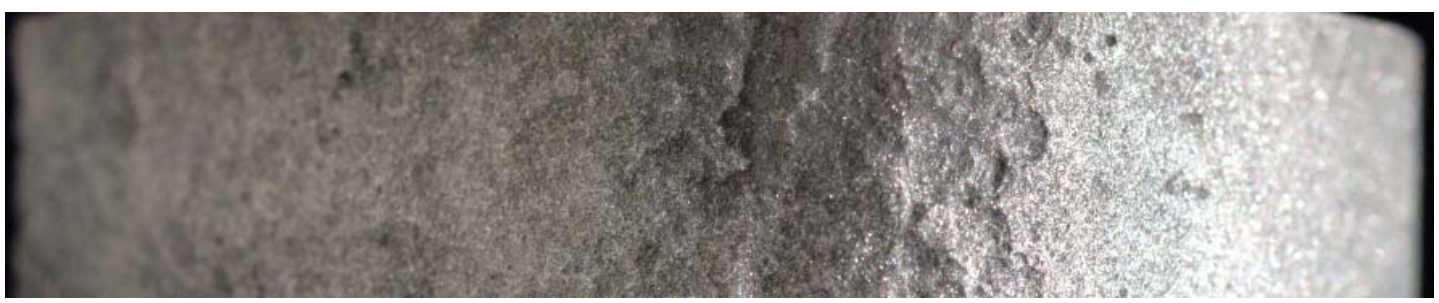

6 O'Clock

(2 mils lost)

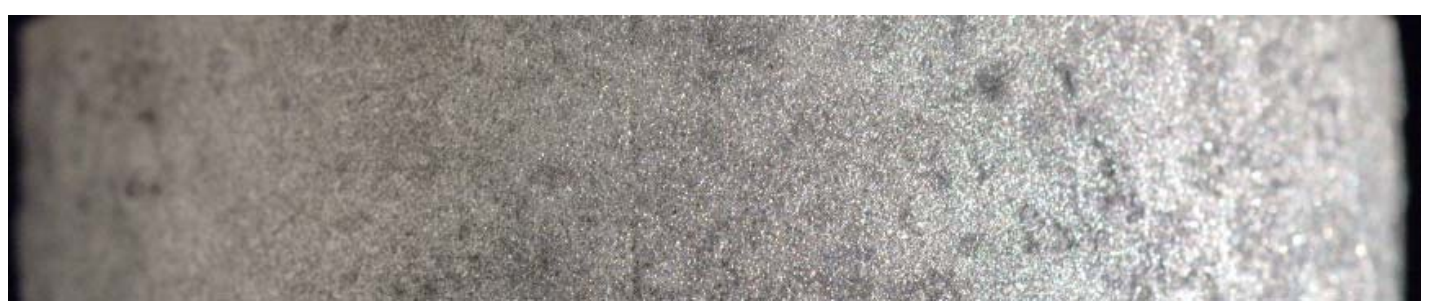

Figure F-8: OD Surface Appearance After Grit Blast

Material: $\quad 310$ Ta

Sample: $\quad$ F-G 
12 O'Clock

(105 mils lost)

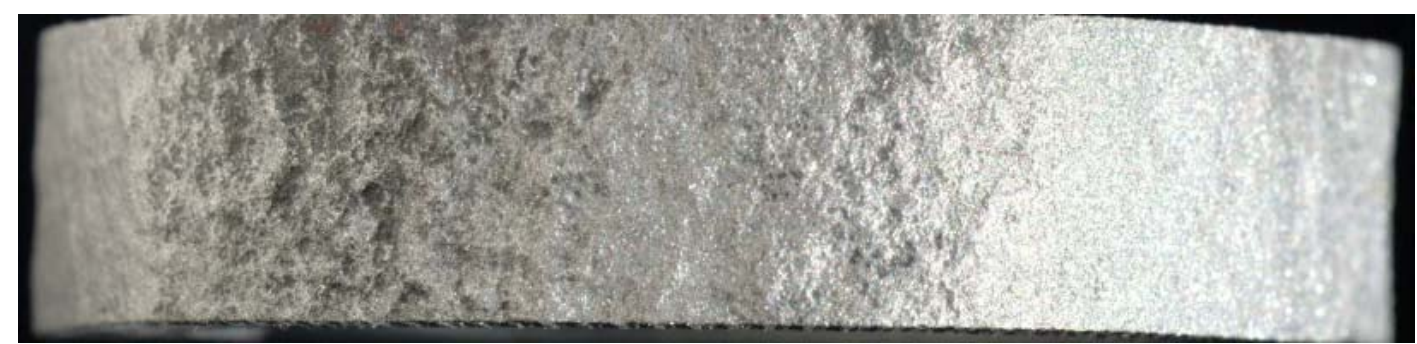

3 O'Clock

(61 mils lost)

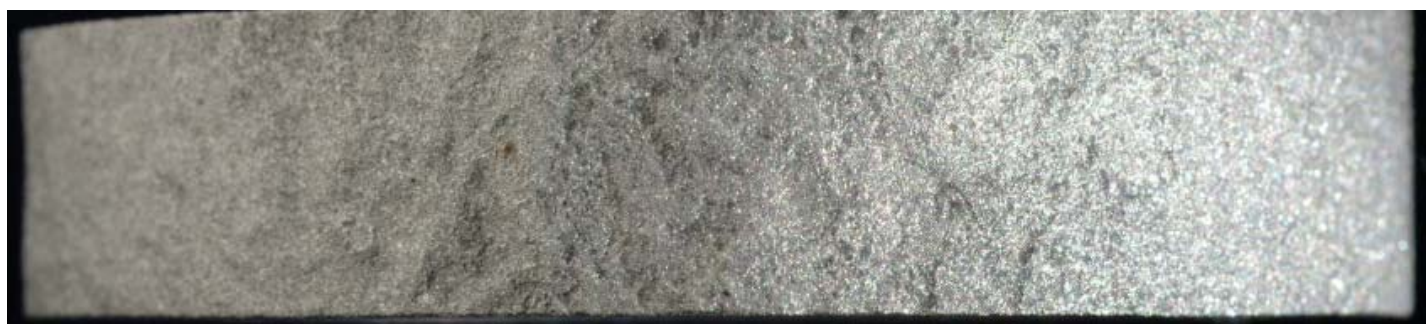

6 O'Clock

(0 mils lost)

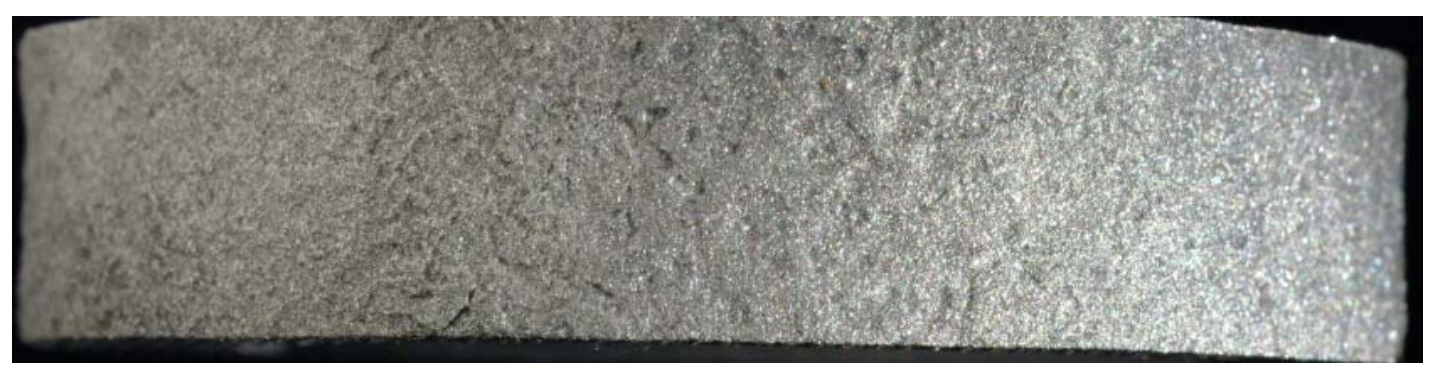

Figure F-9: OD Surface Appearance After Grit Blast

Material: $\quad 310$ Ta

Sample: $\quad \mathrm{X}-\mathrm{Y}$ 


\section{Material:}

Sample Location:

Maximum Rate:

Maximum Loss Orientation:

Average metal Temperature:

\section{Ta}

M-N

131 mils/yr

Approx. 2:30 o'clock

$1188 \mathrm{~F}$

\section{O’Clock}

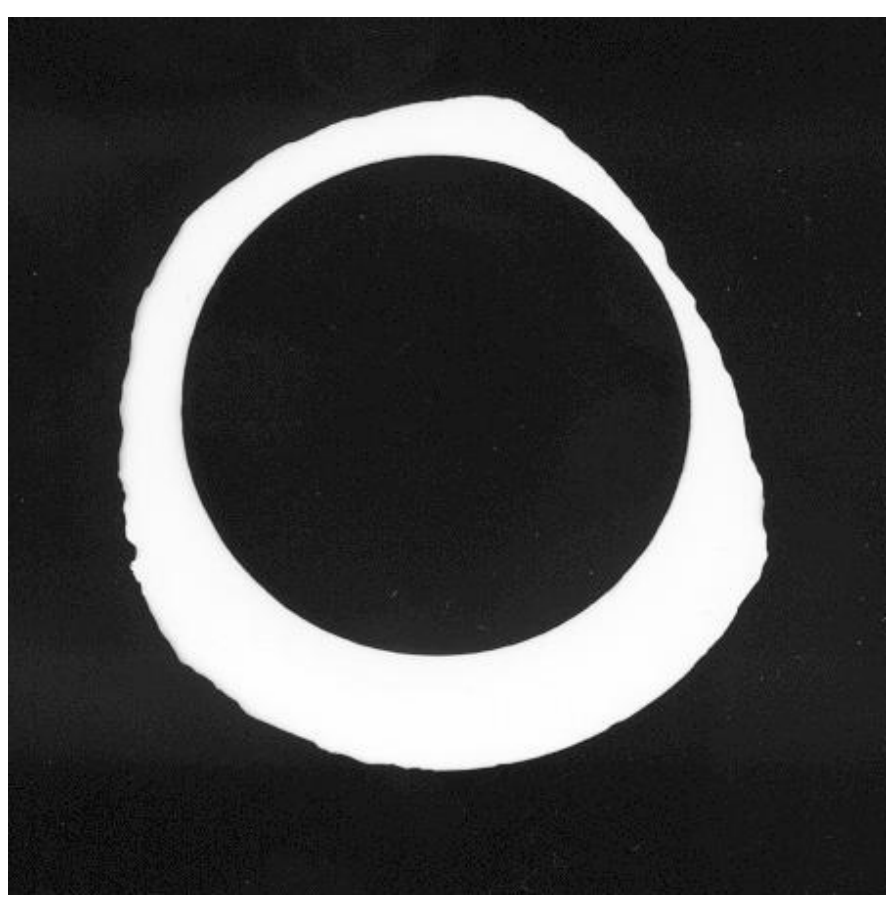

3 O’Clock

Approx. 1.2X

\section{Wall Reduction by Position (in.)}

\begin{tabular}{|l|c|c|c|c|c|c|c|c|c|c|}
\hline & $\begin{array}{c}\mathbf{1 2} \\
\text { o'clock }\end{array}$ & $\mathbf{1 : 3 0}$ & $\mathbf{3 : 0 0}$ & $\mathbf{4 : 3 0}$ & $\mathbf{6 : 0 0}$ & $\mathbf{7 : 3 0}$ & $\mathbf{9 : 0 0}$ & $\mathbf{1 0 : 3 0}$ & AVG & $\begin{array}{c}\text { Max }^{*} \\
\text { Loss }\end{array}$ \\
\hline Before & 0.400 & 0.401 & 0.401 & 0.401 & 0.401 & 0.400 & 0.400 & 0.400 & 0.400 & 0.400 \\
\hline After & 0.188 & 0.118 & 0.146 & 0.381 & 0.401 & 0.381 & 0.231 & 0.203 & & 0.089 \\
\hline Delta & 0.212 & 0.283 & 0.255 & 0.020 & 0.000 & 0.019 & 0.169 & 0.197 & & 0.311 \\
\hline
\end{tabular}

Figure F-10: Silhouette and Wall Thickness Measurements for Sample M-N

* Measurement taken at "maximum loss orientation" noted above. The average

"before" wall thickness was used to calculate the "delta". 


\section{Material:}

Sample Location:

Maximum Rate:

Maximum Loss Orientation:

Average metal Temperature:

\section{Ta}

F-G

$127 \mathrm{mils} / \mathrm{yr}$

Approx. 2:30 o'clock $1166 \mathrm{~F}$

\section{O’Clock}

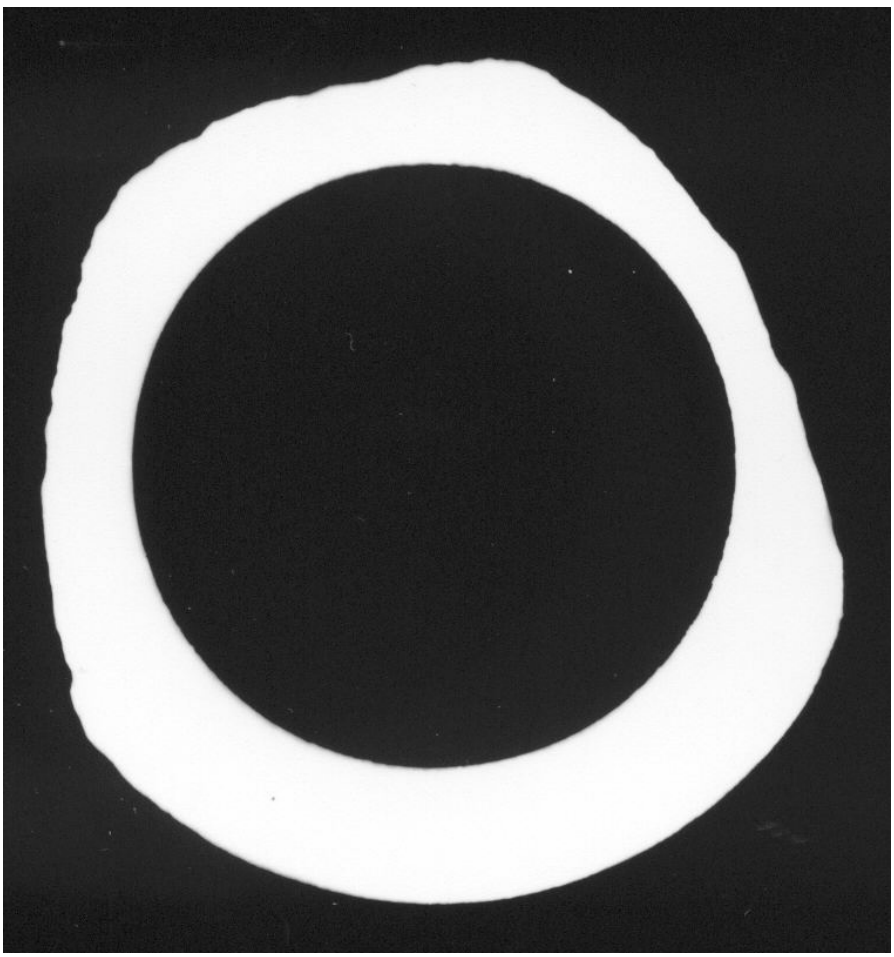

3 O’Clock

Approx. 1.2X

Wall Reduction by Position (in.)

\begin{tabular}{|l|c|c|c|c|c|c|c|c|c|c|}
\hline & $\begin{array}{c}\mathbf{1 2} \\
\text { o'clock }\end{array}$ & $\mathbf{1 : 3 0}$ & $\mathbf{3 : 0 0}$ & $\mathbf{4 : 3 0}$ & $\mathbf{6 : 0 0}$ & $\mathbf{7 : 3 0}$ & $\mathbf{9 : 0 0}$ & $\mathbf{1 0 : 3 0}$ & AVG & $\begin{array}{c}\text { Max } \\
\text { Loss }\end{array}$ \\
\hline Before & 0.402 & 0.402 & 0.402 & 0.401 & 0.402 & 0.399 & 0.398 & 0.399 & 0.401 & 0.401 \\
\hline After & 0.283 & 0.207 & 0.214 & 0.399 & 0.399 & 0.395 & 0.270 & 0.333 & & 0.173 \\
\hline Delta & 0.119 & 0.195 & 0.188 & 0.002 & 0.003 & 0.004 & 0.128 & 0.066 & & 0.228 \\
\hline
\end{tabular}

Figure F-11: Silhouette and Wall Thickness Measurements for Sample F-G

Note: this tube was removed from service on $6 / 10 / 02$.

* Measurement taken at "maximum loss orientation" noted above. The average

"before" wall thickness was used to calculate the "delta". 


\section{Material:}

Sample Location:

Maximum Rate:

Maximum Loss Orientation:

Average metal Temperature:

\section{Ta}

X-Y

$64 \mathrm{mils} / \mathrm{yr}$

Approx. 9:30 o'clock $1103 \mathrm{~F}$

12 O’Clock

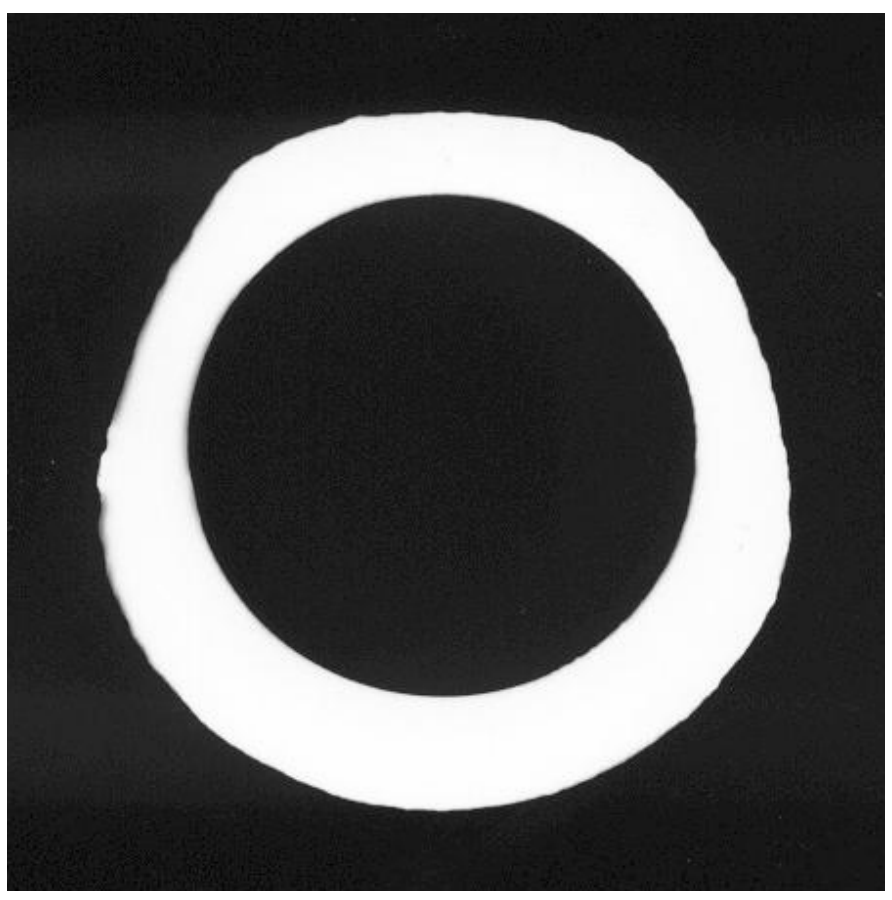

3 O'Clock

Approx. 1.2X

Wall Reduction by Position (in.)

\begin{tabular}{|l|c|c|c|c|c|c|c|c|c|c|}
\hline & $\begin{array}{c}\mathbf{1 2} \\
\text { o'clock }\end{array}$ & $\mathbf{1 : 3 0}$ & $\mathbf{3 : 0 0}$ & $\mathbf{4 : 3 0}$ & $\mathbf{6 : 0 0}$ & $\mathbf{7 : 3 0}$ & $\mathbf{9 : 0 0}$ & $\mathbf{1 0 : 3 0}$ & AVG & $\begin{array}{c}\text { Max }^{*} \\
\text { Loss }\end{array}$ \\
\hline Before & 0.400 & 0.400 & 0.399 & 0.400 & 0.399 & 0.401 & 0.401 & 0.400 & 0.400 & 0.400 \\
\hline After & 0.295 & 0.291 & 0.338 & 0.391 & 0.399 & 0.394 & 0.285 & 0.334 & & 0.248 \\
\hline Delta & 0.105 & 0.109 & 0.061 & 0.009 & 0.000 & 0.006 & 0.116 & 0.166 & & 0.152 \\
\hline
\end{tabular}

Figure F-12: Silhouette and Wall Thickness Measurements for Sample X-Y

* Measurement taken at "maximum loss orientation" noted above. The average

"before" wall thickness was used to calculate the "delta". 


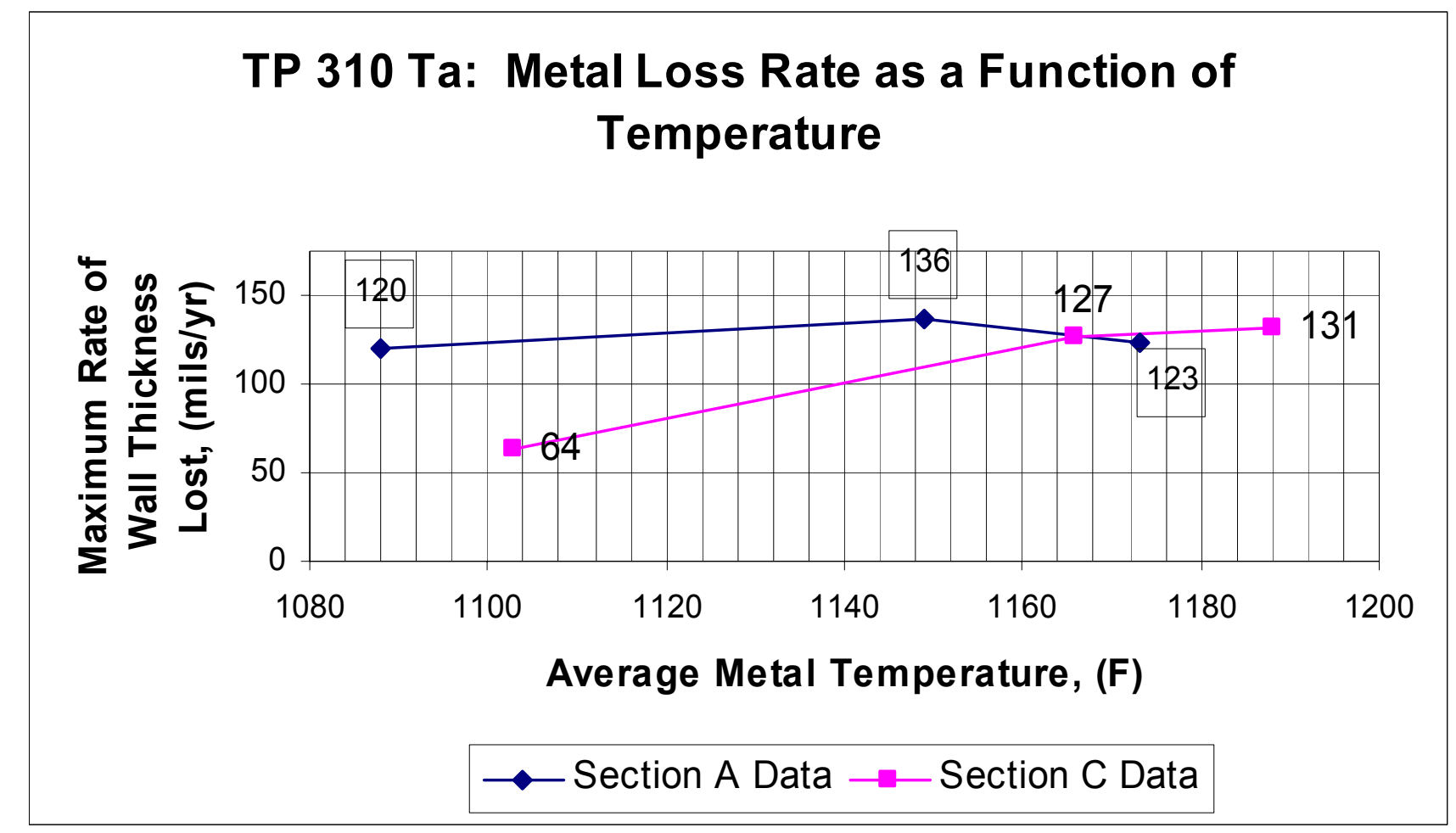

Figure F-13: Metal Loss Rate as a Function of Temperature

Material: 310 Ta

Note: In analyzing these data it is important to understand that the one of the three Section C samples was removed early due to excessive corrosion losses. Sample F-G (1166 F) was removed in June 2002. Sample M-N (1188 F) and Sample X-Y (1103 F) were removed with the remaining Section C samples in May 2003. 


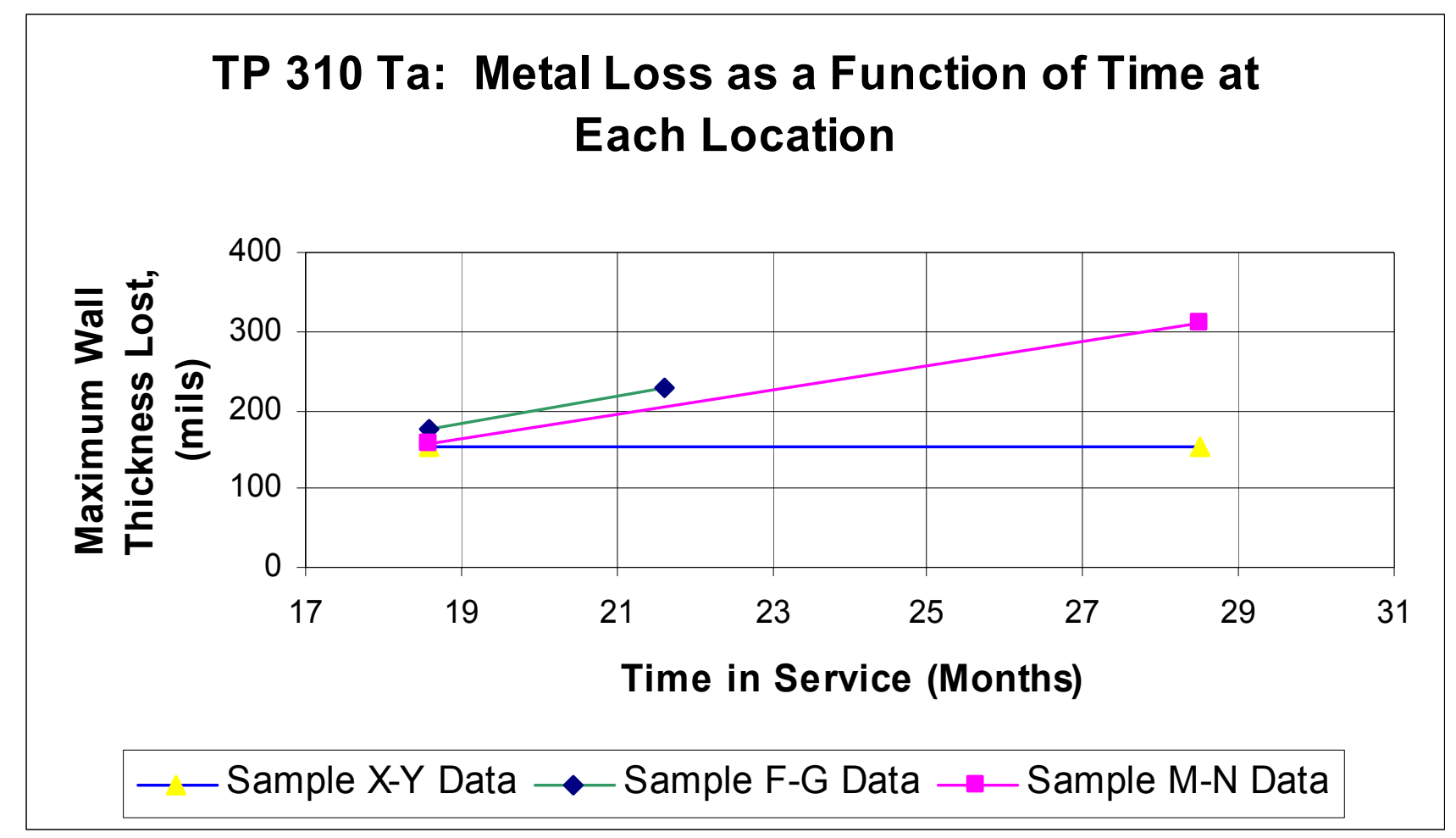

Figure F-13: Metal Loss as a Function of Time

Note: From the plot above it can be seen that, for Section C, Sample F-G was removed early (in June 2002) due to concerns about excessive metal loss. Samples $\mathrm{X}-\mathrm{Y}$ and $\mathrm{M}-\mathrm{N}$ experienced the full exposure life for both Sections A and C. 


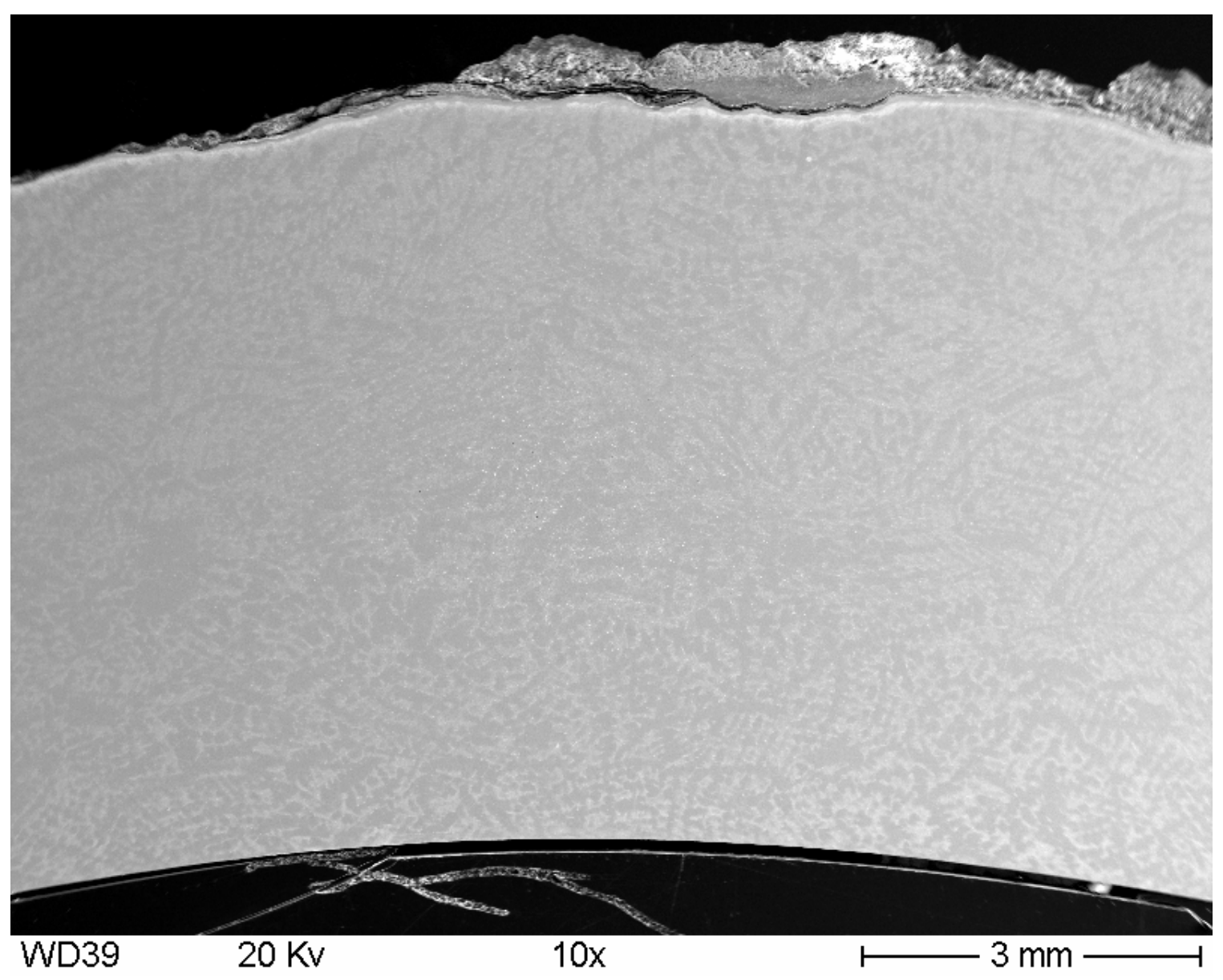

Figure F-14: Low Magnification Image Showing Cross-Section Through Thinnest Region of Sample F-G (310 Ta) 


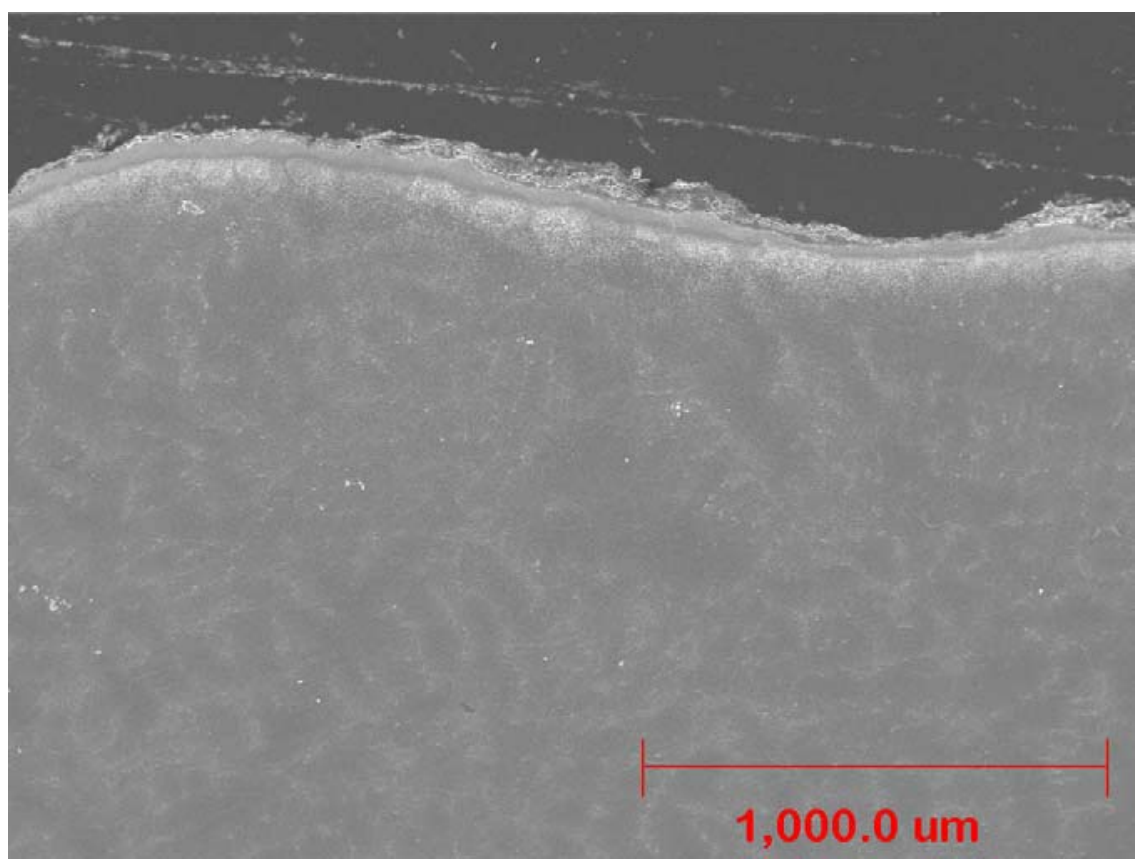

(a) External Scale/Metal Interface (SEI - Low Magnification)

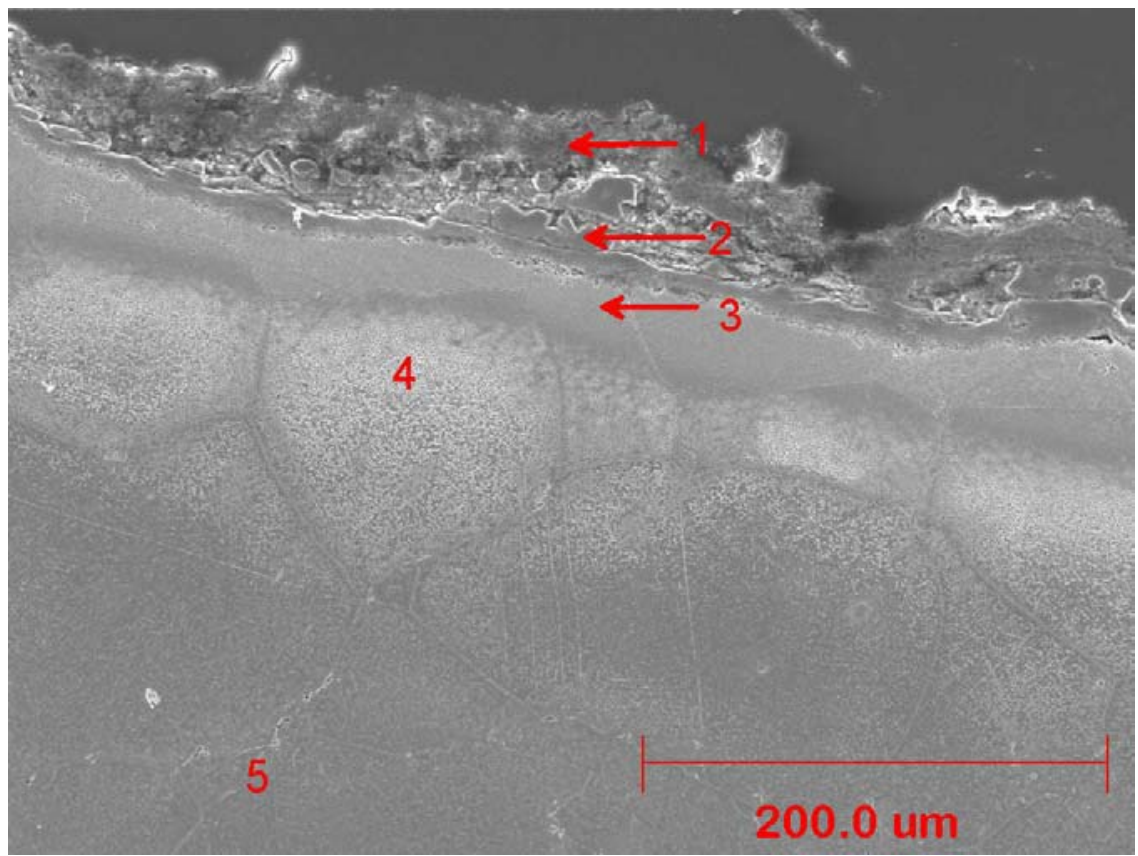

(b) External Scale/Metal Interface (SEI - Intermediate Magnification)

Figure F-15: Microstructure: 310 Ta

Note: Numbers on photos indicate distinct areas characterized by the visual assessment and EDS analyses that follow. 


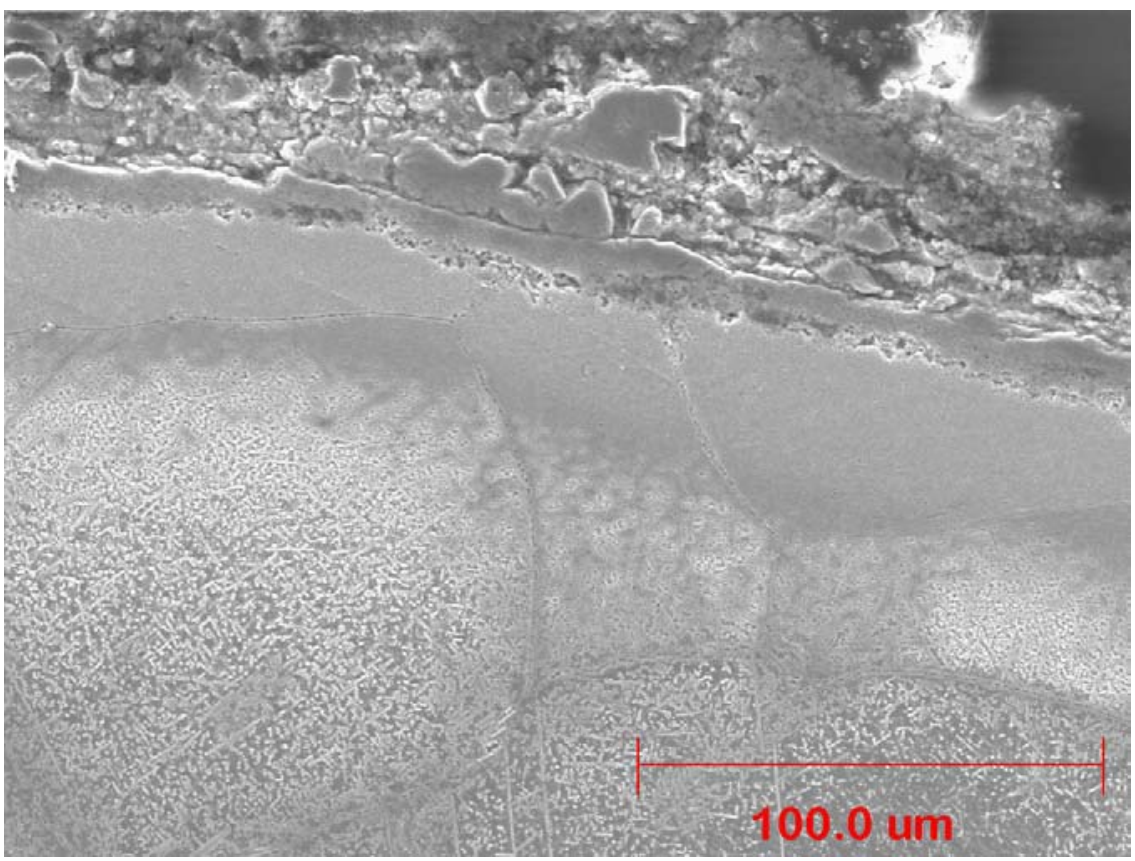

(c) External Scale/Metal Interface (SEI - High Magnification)

Figure F-15 (Cont.): Microstructure: 310 Ta 


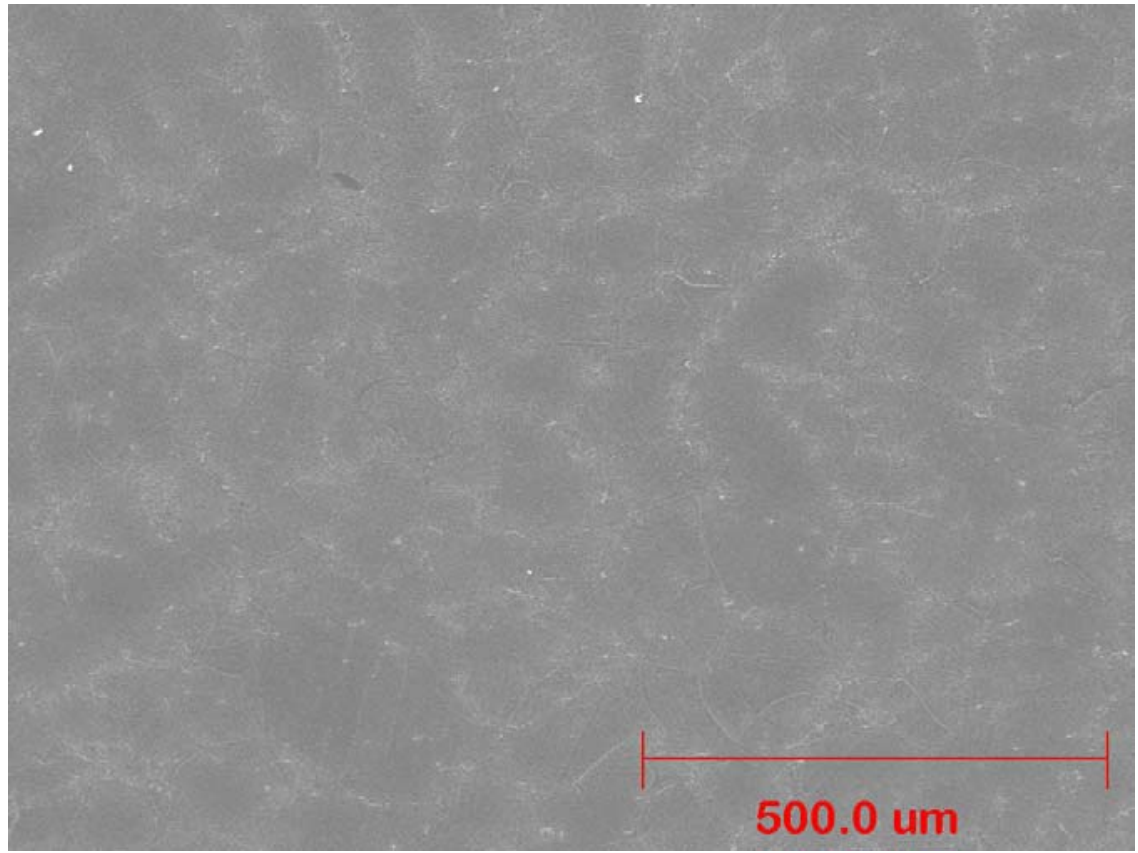

(SEI - Low Magnification)

Figure F-16: Mid-Wall Microstructure: 310 Ta

Note: Photomicrograph at approximately 200X. Electrolytic 10\% oxalic acid etch, 9V OCP 


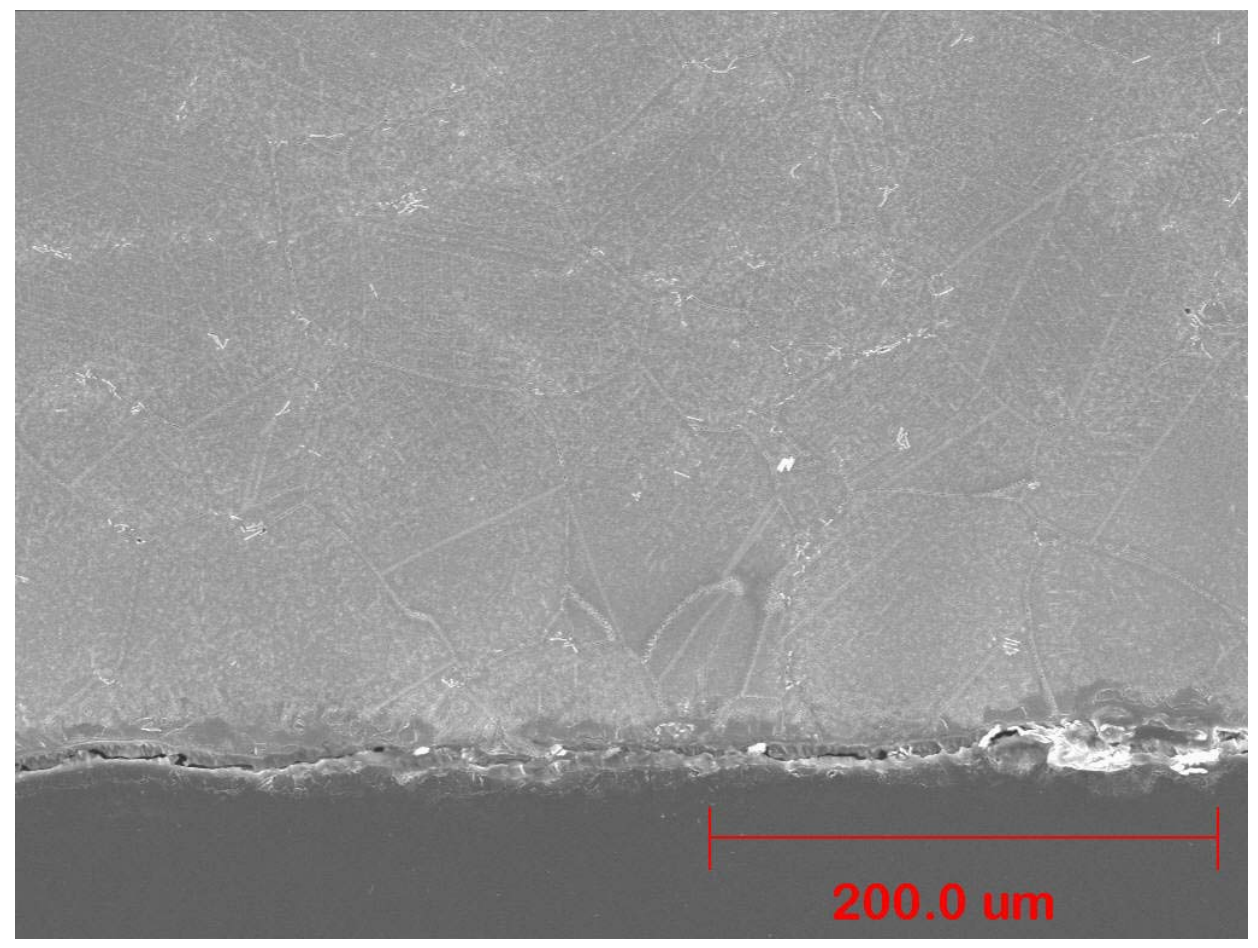

Internal Surface Appearance

Figure F-17: Microstructure: 310 Ta 


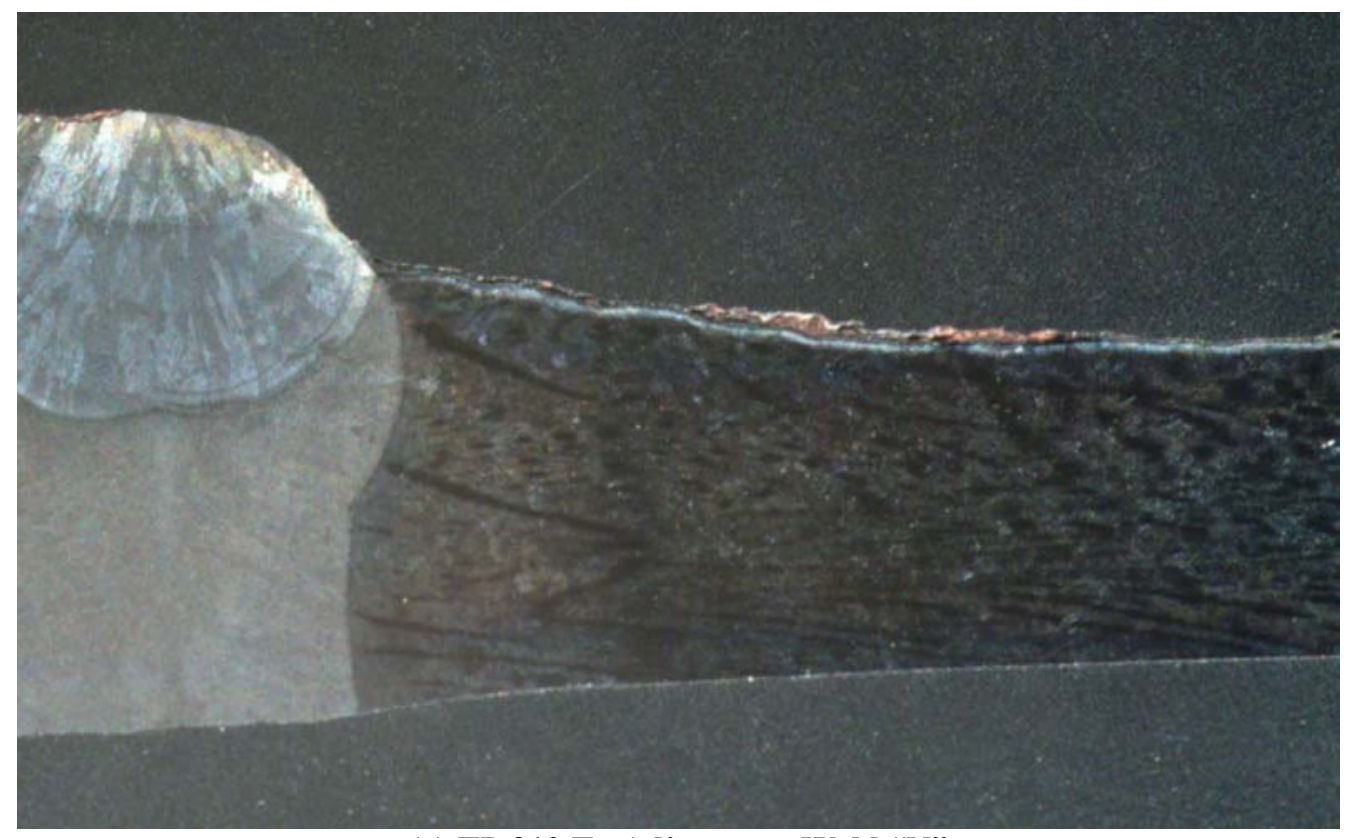

(a) TP 310 Ta Adjacent to Weld "N"

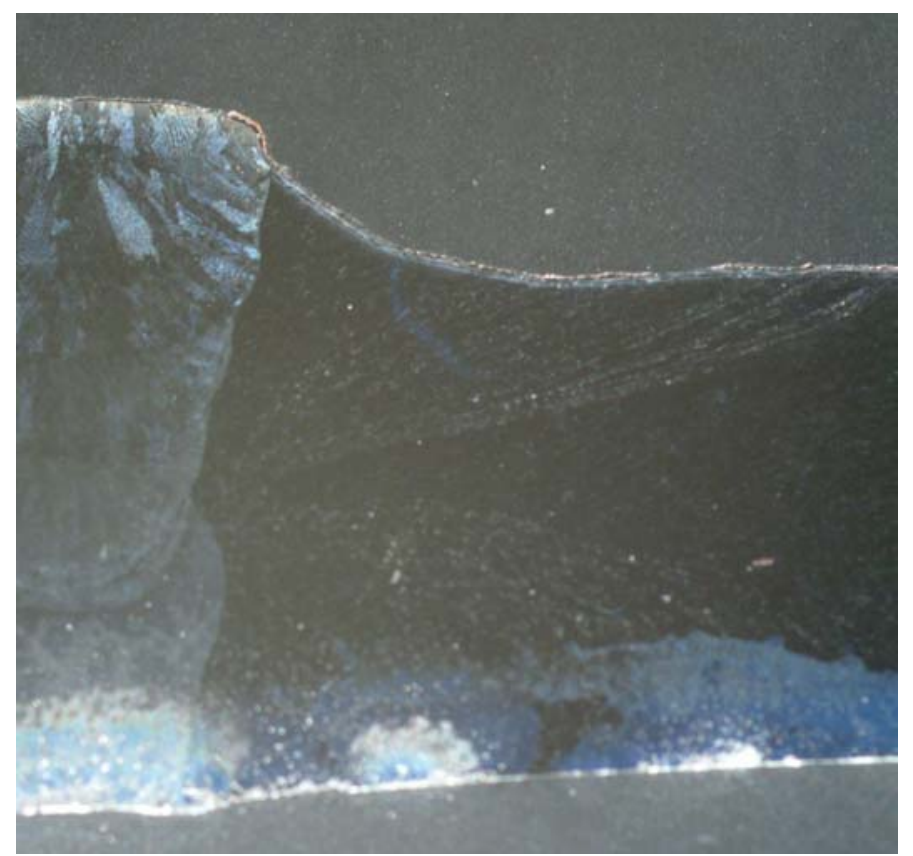

(b) TP 310 Ta Adjacent to Weld "X"

Figure F-18: Longitudinal Cross-Section: 310 Ta Adjacent to Welds 
Figure F-19: EDS Analysis of Deposit/Scale/Metal Interface for 310 Ta (Sample M-N)

\begin{tabular}{|c|c|c|c|c|c|c|c|c|c|c|c|c|c|c|c|c|c|c|}
\hline Area & Description & $\mathbf{N i}$ & $\mathrm{Cr}$ & $\mathbf{F e}$ & $\mathrm{Cu}$ & Mn & Ta & $\mathbf{T i}$ & Al & $\mathbf{S i}$ & $\mathrm{C}$ & $\mathbf{O}$ & $\mathrm{Ca}$ & $\mathbf{K}$ & $\mathbf{N a}$ & As & $\mathbf{P}$ & $\mathbf{S}$ \\
\hline 1 & $\begin{array}{l}\text { Bulk deposit } \\
\text { analysis }\end{array}$ & 1.96 & 0.90 & 26.1 & & & & 0.56 & 4.78 & 10.8 & 18.4 & 27.3 & & 1.28 & & 3.94 & 1.62 & 2.31 \\
\hline 2 & $\begin{array}{l}\text { Tightly } \\
\text { adhering } \\
\text { scale.. }\end{array}$ & 3.48 & 54.5 & 18.2 & & 1.98 & & & 0.26 & 1.39 & & 15.2 & & & & & & 4.98 \\
\hline 3 & $\begin{array}{l}\text { Diffusion } \\
\text { zone - dark } \\
\text { etching } \\
\text { adjacent to } \\
\text { interface. }\end{array}$ & 25.2 & 12.2 & 60.0 & & 1.09 & 0.91 & & & 0.58 & & & & & & & & \\
\hline 4 & $\begin{array}{l}\text { Diffusion } \\
\text { zone - light } \\
\text { etching. }\end{array}$ & 21.4 & 21.1 & 54.7 & & 1.01 & 1.34 & & & 0.45 & & & & & & & & \\
\hline 5 & $\begin{array}{l}\text { Bulk EDS } \\
\text { analysis }\end{array}$ & 21.2 & 23.2 & 52.4 & & 1.33 & 1.72 & & & 0.18 & & & & & & & & \\
\hline & $\begin{array}{l}\text { Reference } \\
\text { bulk analysis. }\end{array}$ & 21.0 & 24.7 & 51.2 & -- & 1.72 & 0.73 & - & 0.002 & 0.24 & 0.07 & -- & -- & -- & -- & -- & 0.003 & 0.007 \\
\hline
\end{tabular}


Appendix G

Analysis of Performance

Test Section C

310 HCbN (HR3C) 


\section{Overview of Results: $310 \mathrm{HCbN}$ (HR3C)}

One $310 \mathrm{HCbN}$ sample in Section C, Sample G-H experienced a significantly high rate of metal loss (141 mils per year*) that it was removed and replaced after 18.6 months of exposure, i.e. shortly after Section A was removed for examination. Sample N-O remained in Section C for continued testing and experienced a high rate of metal loss to the point where, less than $25 \%$ of the wall remained at the time that Section $\mathrm{C}$ was removed from service. The other two 310 $\mathrm{HCbN}$ samples, which experienced lower exposure temperatures, experienced little if any additional corrosion relative to the samples from Section A. The reason for this is not clear.

It should be noted that the metal loss rates determined to date for both Section A and Section C all were based on a ring specimen that was extracted from mid-length of each tube sample. In this analysis additional attention was focused on metal losses in the vicinity of welds. Representative samples were taken and it was determined that, for the $310 \mathrm{HCbN}$ samples evaluated, the Inconel 625 weld filler metal is reasonably resistant to coal ash corrosion and that the tube material immediately adjacent to the weld (i.e. the weld heat affected zone) was somewhat less affected by corrosion than was the remainder of the tube. Indeed the tube wall appeared to be progressively thinner toward the mid-point of the tube sample.

Analysis of deposit on the surface revealed that it had the known aggressive combination of species, namely iron, oxygen, the alkali metals, and sulfur. Examination and analysis of the scale adjacent to the metal surface revealed the chalky-white appearance associated with alkali-ironsulfates. Thus, the required corrosive constituent was available to test the performance limits of this alloy. 
Material: 310 HCbN (HR3C)

\section{Summary of Findings}

\begin{tabular}{|c|c|}
\hline Issue & Discussion \\
\hline $\begin{array}{l}\text { Sample } \\
\text { Location and } \\
\text { Orientation. }\end{array}$ & $\begin{array}{l}\text { - In assessing the data for Section C, it is important to understand that } \\
\text { some tube samples had to be removed from the section early due to } \\
\text { excessive thinning. The schematic in Figure G-1 shows the relative } \\
\text { positions of the } 310 \mathrm{HCbN} \text { samples in all three sections. Also, shown } \\
\text { are the positions of all of the excessively thinned tubes that were } \\
\text { removed and replaced in order to extend the life of Section C. } \\
\text { - One } 310 \mathrm{HCbN} \text { sample was removed early. Sample G-H was removed } \\
\text { in November 2001, (i.e. shortly after Section A was removed from } \\
\text { service for evaluation). Samples N-O, W-X and AA-BB survived for } \\
\text { the full Section C exposure period. } \\
\text { The sample that was removed early was set aside for later analysis, } \\
\text { and has been included in the current evaluation along with the other } \\
\text { tube samples that made up Section C. } \\
\text { Also shown in this figure is the calculated average surface metal } \\
\text { temperature at each location. }\end{array}$ \\
\hline $\begin{array}{l}\text { Composition } \\
\text { Verification. }\end{array}$ & $\begin{array}{l}\text { - The table in Figure G-1 provides the ASTM specified composition for } \\
\text { this grade of material. } \\
\text { - Sumitomo Metal Industries supplied tube from one heat of material for } \\
\text { use in this program, Heat D8A0809. The composition for this heat also } \\
\text { is provided in Figure G-1. } \\
\text { During the Section A evaluation, detailed composition verification was } \\
\text { undertaken for each tube sample that was removed from service. This } \\
\text { served, in part, to verify that the each tube material was located at its' } \\
\text { proper position within the test section. } \\
\text { Based on the good results for Section A, it was decided that it would } \\
\text { be unnecessary to analyze each and every tube sample for composition } \\
\text { verification. Rather, it was decided to select a representative sample } \\
\text { from each tube material type for confirmatory analysis. } \\
\text { During the Section A evaluation, the energy dispersive spectrographic } \\
\text { (EDS) capabilities of the scanning electron microscope (SEM) were } \\
\text { used to perform semi-quantitative analyses of each of the twelve } \\
\text { different candidate tube materials. Comparison of these EDS results } \\
\text { showed reasonably good correlation with results obtained using } \\
\text { quantitative analytical techniques, including optical emission } \\
\text { spectrometry. } \\
\text { Based on this, it was decided that the accuracy provided by EDS }\end{array}$ \\
\hline
\end{tabular}




\begin{tabular}{|l|l|}
\hline & $\begin{array}{l}\text { would suffice for composition verification, but that more detailed } \\
\text { analyses would be undertaken if questions arose. } \\
\text { The results of the EDS analysis of tube sample N-O confirmed that the } \\
\text { correct material was used at this location. }\end{array}$ \\
\hline
\end{tabular}

\section{Material: 310 HCbN (HR3C)}

\section{Summary of Findings, (continued)}

\begin{tabular}{|c|c|}
\hline Issue & Discussion \\
\hline $\begin{array}{l}\text { As-Received } \\
\text { Appearance }\end{array}$ & $\begin{array}{l}\text { - Figure G-3 shows the typical appearance of } 310 \mathrm{HCbN} \text { sample on } \\
\text { receipt from Sumitomo. } \\
\text { - Figures G-4 through G-7 document the as-received appearance of the } \\
\text { external surface of the Save } 25 \text { tube samples. Three views are } \\
\text { provided for each tube: } 12 \text { o'clock (the view that faces on-coming gas } \\
\text { flow), } 3 \text { o'clock (the view where the gas flow is tangent to the } \\
\text { surface), and } 6 \text { o'clock (the view that shows the back-side of the tube } \\
\text { relative to the gas flow direction. } \\
\text { - The reader should be alerted to the fact that, within the boiler, the gas } \\
\text { flows upward over these tubes. This implies that for the "o'clock" } \\
\text { conventions described above, (with the } 12 \text { o'clock orientation facing } \\
\text { the gas flow direction), the actual physical orientation of these tubes is } \\
\text { such that the } 12 \text { o'clock side of the tube faces downward, and the } 6 \\
\text { o'clock orientation faces upward within the boiler. } \\
\text { Wastage was visible at the } 12 \text { o'clock for all four samples; however, it } \\
\text { was obscured to some extent by deposit on those surfaces.. } \\
\text { A chalky gray/white deposit is clearly seen at the } 3 \text { o'clock position } \\
\text { for Samples W-X and AA-BB. This of course is consistent with } \\
\text { molten alkali-iron-sulfate accelerated attack. }\end{array}$ \\
\hline $\begin{array}{l}\text { Grit-Blasted } \\
\text { Surface } \\
\text { Appearance }\end{array}$ & $\begin{array}{l}\text { - Figures G- } 8 \text { through G-11 show the appearance of the external surface } \\
\text { of the } 310 \text { HCbN tube samples after grit-blasting to remove deposit } \\
\text { and scale. The } 12 \text { o'clock and } 3 \text { o'clock positions for all four samples } \\
\text { show the appearance of general wastage due to fireside corrosion. } \\
\text { - The } 6 \text { o'clock position shows some evidence of pitting for all four } \\
\text { samples; otherwise, is was relatively unaffected, and exhibited little or } \\
\text { no wastage at this surface. }\end{array}$ \\
\hline $\begin{array}{l}\text { Wall } \\
\text { Thickness } \\
\text { Loss } \\
\text { Determined }\end{array}$ & $\begin{array}{l}\text { - Tube cross-sections were prepared to allow for measurement of wall } \\
\text { thickness to determine metal loss due to exposure. These are shown in } \\
\text { silhouette in Figures G-12 through G-15. } \\
\text { - The same relative "o'clock" positions were maintained as discussed } \\
\text { earlier. Measurements were taken at eight evenly spaced locations } \\
\text { about the tube circumference. These were then compared with } \\
\text { measurements taken at these same locations prior to exposure. } \\
\text { - The before and after measurements were documented in the table that } \\
\text { accompanies each figure. These form the basis for the "delta" } \\
\text { calculation to determine the amount of wall thickness lost. }\end{array}$ \\
\hline
\end{tabular}




\begin{tabular}{|l|l|}
\hline$\bullet$ & $\begin{array}{l}\text { For all four tube samples, the minimum wall thickness in the cross } \\
\text { section was not at one of the eight predetermined locations. In these } \\
\text { cases, there was no "before" measurement for comparison. So, the } \\
\text { average "before" wall thickness was used to calculate the wall } \\
\text { thickness lost at these off locations. }\end{array}$ \\
\hline
\end{tabular}

\section{Material: 310 HCbN (HR3C)}

\section{Summary of Findings, (continued)}

\begin{tabular}{|c|c|}
\hline Issue & Discussion \\
\hline $\begin{array}{l}\text { Rate of Wall } \\
\text { Thickness } \\
\text { Loss Versus } \\
\text { Average } \\
\text { Surface Metal } \\
\text { Temperature }\end{array}$ & $\begin{array}{l}\text { - The chart in Figure G-16 plots the maximum rate of wall thickness } \\
\text { loss against the calculated average surface metal temperature for each } \\
\text { of the four } 310 \mathrm{HCbN} \text { samples in Section C. The Section A data also is } \\
\text { plotted. } \\
\text { - Review of the data shows that the lower temperature samples in } \\
\text { Section C experienced a lower than expected rate of metal loss. } \\
\text { Otherwise, the rates are fairly consistent, and moderately high. }\end{array}$ \\
\hline $\begin{array}{l}\text { Wall } \\
\text { Thickness } \\
\text { Loss as a } \\
\text { Function of } \\
\text { Time and } \\
\text { Location }\end{array}$ & $\begin{array}{l}\text { - The chart in Figure G- } 17 \text { plots metal loss as a function of time for each } \\
\text { sample location for Sections A and C. with samples from Section C } \\
\text { having experienced generally longer exposure times. } \\
\text { - Shortly after Section A was removed for analysis, the tube Sample G- } \\
\text { H was also removed and replaced in Section C. It was noted that the } \\
\text { rate of metal loss appears to be greater for the Sample G-H from } \\
\text { Section C. This may have been due to the somewhat higher } \\
\text { temperatures for the G-H position in Section C (1167 F vs } 1157 \mathrm{~F}) \text {, but } \\
\text { due to limited sampling it is difficult to draw this conclusion this with } \\
\text { certainty. } \\
\text { It was noted that the tube sample at position AA-BB experienced only } \\
\text { somewhat more metal loss, relative to the sample at the same position } \\
\text { in Section A, after the full exposure period for Section C (i.e. } 189 \\
\text { versus } 166 \text { mils total). } \\
\text { The Sample W-X in Section C actually experienced less metal loss } \\
\text { than the sample at the same position in Section A even though it saw } \\
\text { approximately ten additional months at the service temperature. }\end{array}$ \\
\hline $\begin{array}{l}\text { Metallurgical } \\
\text { Analysis - } \\
\text { SEM }\end{array}$ & $\begin{array}{l}\text { - Sample N-O was selected for a detailed SEM/EDS assessment of the } \\
\text { constituents and structure in the vicinity of the external and internal } \\
\text { surfaces. This sample was selected based on the fact that it had lost the } \\
\text { most wall thickness among the three } 310 \mathrm{HCbN} \text { samples that survived } \\
\text { the full exposure period for Section C. The focus of this investigation } \\
\text { was in the region of the tube where the maximum amount of material } \\
\text { was lost. } \\
\text { - Figures G-18a, b, c and d, show the secondary electron images of the } \\
\text { scale/metal interface for Sample G-H. Six distinct regions are in } \\
\text { evidence. These regions are, starting within the metal and working out: } \\
\text { Base metal - oxalic acid etch delineates grain boundaries and } \\
\text { carbides. } \\
\text { Diffusion zone - light etch - this region is obvious as a light- }\end{array}$ \\
\hline
\end{tabular}




\begin{tabular}{|l|l|}
\hline & $\begin{array}{l}\text { appearing band that is parallel to the metal surface, perhaps } \\
\text { see most clearly in Figure G-18b. }\end{array}$ \\
$\circ \begin{array}{l}\text { Featureless grain surface }- \text { This constitutes the leading edge of } \\
\text { the grains as they interface with the scale. As EDS analysis } \\
\text { later shows that this zone seems related to diffusion of } \\
\text { alloying elements both into and out of the metal surface as } \\
\text { scale formation progresses. }\end{array}$ \\
\hline
\end{tabular}

\section{Material: $\quad 310 \mathrm{HCbN}$ (HR3C)}

\section{Summary of Findings, (continued)}

\begin{tabular}{|c|c|}
\hline Issue & Discussion \\
\hline $\begin{array}{l}\text { Metallurgical } \\
\text { Analysis - } \\
\text { SEM, } \\
\text { Continued. }\end{array}$ & $\begin{array}{l}\circ \begin{array}{l}\text { Tightly adhering scale }- \text { this is seen continuous along the } \\
\text { surface. EDS analysis will later show that this is a chromium-, } \\
\text { nickel-, and iron-rich -oxide. }\end{array} \\
\circ \begin{array}{l}\text { Thick and very porous deposit/scale conglomerate - many } \\
\text { spherical particles are noted along with what appears to be } \\
\text { islands of scale (metal oxides). }\end{array} \\
\text { - Figures G-19 shows the appearance of the tube microstructure at mid- } \\
\text { wall. } \\
\text { - Figure G-20 shows the appearance of the internal metal surface for } \\
\text { Sample N-O. Little if any oxide scale is visible at this surface. }\end{array}$ \\
\hline $\begin{array}{l}\text { Metallurgical } \\
\text { Analysis - } \\
\text { Weld Cross } \\
\text { Section. }\end{array}$ & $\begin{array}{l}\text { - Figure G-21 shows a longitudinal profile (at the } 12 \text { o'clock } \\
\text { orientation) of the } 310 \mathrm{HCbN} \text { samples adjacent to the welds in Section } \\
\text { C. Three welds were cross sectioned. These were identified as "N", } \\
\text { "X" and "BB" in the schematic shown in Figure G-1. } \\
\text { - An Inconel } 625 \text { weld metal was used throughout Section C. The cross } \\
\text { sections show that the } 310 \mathrm{HCbN} \text { heat affected zones are not } \\
\text { preferentially attached, in fact, the wall thickness for the tube sample } \\
\text { tapers from the weld toward the middle of the tube length. }\end{array}$ \\
\hline $\begin{array}{l}\text { EDS Analysis } \\
\text { Results }\end{array}$ & $\begin{array}{l}\text { - The table in Figure G-22 provides a summary of the EDAX analyses } \\
\text { of the locations numbered in Figure G-18b. Also shown in this table is } \\
\text { a summary of the spectrographic analysis reported earlier, see Figure } \\
\text { G-2. } \\
\text { Review of this table suggests the following: } \\
\text { - } \text { Area 1: For the bulk deposit analysis - Alkali metals are present. } \\
\text { Sulfur and phosphorous are present in an increased amount } \\
\text { compared to the base metal. An iron oxide is likely, as are } \\
\text { aluminum, silicon and titanium oxides. Carbon content is } \\
\text { relatively high, however, EDS is not reliable quantitatively. } \\
\text { - } \text { Area 2: Tightly adhering scale - This appears to be an iron-, } \\
\text { chromium-, nickel-rich oxide. Also significant is the presence of } \\
\text { sulfur in this layer. } \\
\text { - Area 3:- Diffusion zone - dark etching - This region of the tube } \\
\text { metal is depleted in chromium relative to the bulk composition } \\
\text { (roughly } 9 \% \text { versus } 22 \% \text { per EDS). } \\
\text { Area 4: - Diffusion zone - light etching - This region of is only } \\
\text { somewhat depleted in chromium (roughly } 20 \% \text { versus } 22 \% \text { per } \\
\text { EDS). }\end{array}$ \\
\hline
\end{tabular}




\begin{tabular}{|l|l|}
\hline & $\bullet \frac{\text { Area 5: Bulk metal analysis }}{\text { composition verification. }}-$ This analysis was used for \\
\hline
\end{tabular}




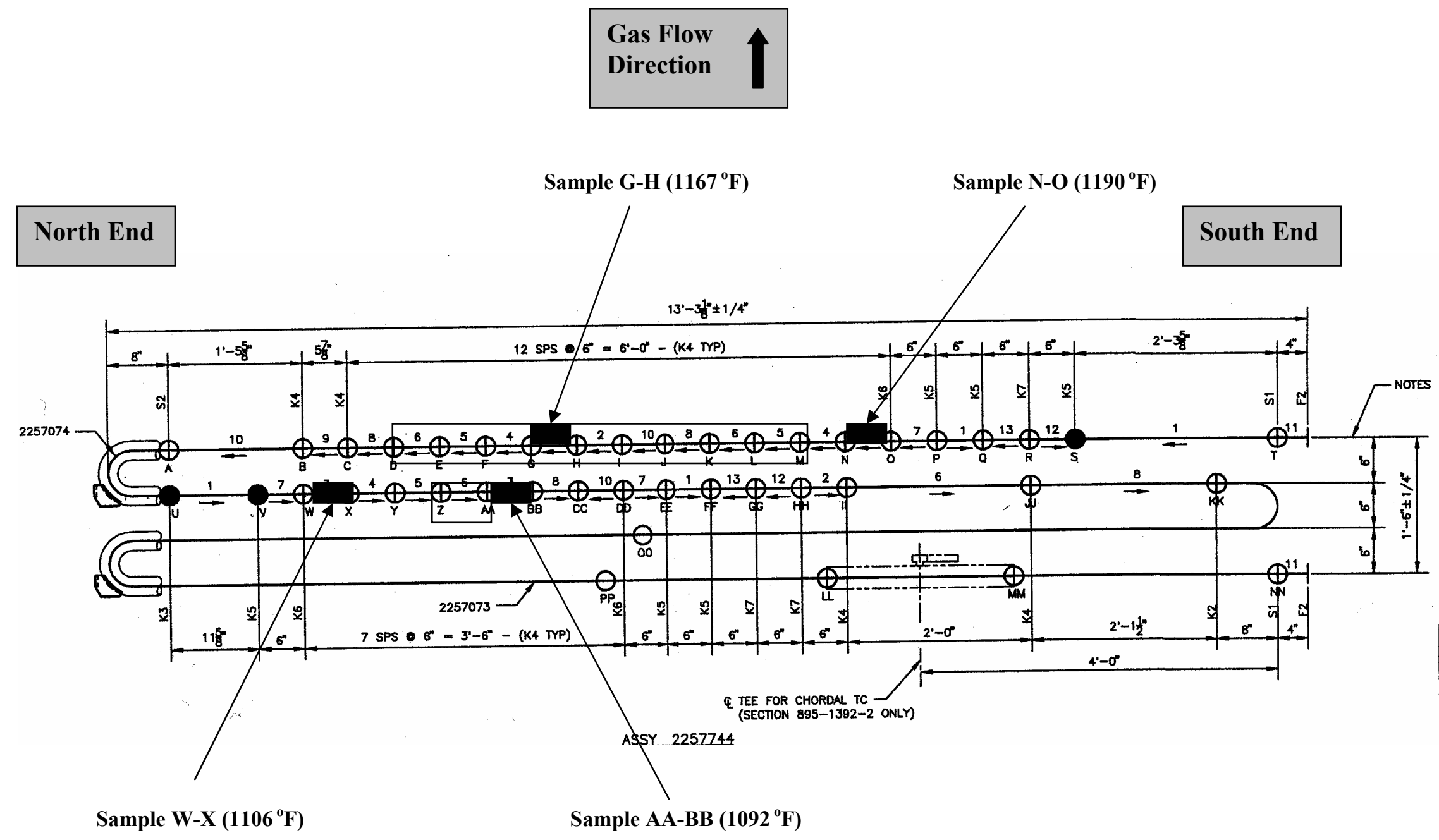

Figure G-1: Schematic Drawing Showing Position of 310 HCbN (HR3C) Tube Samples Within Test Section C

1. Drawing shown in same relative orientation as per the actual installation.

2. This group of tube samples was removed from service in November 2001 - highlighted in red.

3. This group of tube samples was removed from service in June 2002 - highlighted in yellow. 
Figure G-2 Semi-Quantitative Composition Verification for 310 HCbN (HR 3C)

Description: $\quad$ Nominal $25 \mathrm{Cr} / 20 \mathrm{Ni}$ steel with $\mathrm{Nb}$, Ta, and $\mathrm{N}$ added to provide creep strength.

Source:

Reference Composition:

"Specified" chemistry - ASTM A213 (TP310 HCbN).

\begin{tabular}{|c|c|c|c|c|c|c|}
\hline Element & $\begin{array}{c}\text { Reference } \\
\text { Composition }\end{array}$ & $\begin{array}{c}\text { Composition (a) } \\
\text { Heat D8A0809 }\end{array}$ & $\begin{array}{c}\text { Tube Sample } \\
\text { G-H }\end{array}$ & $\begin{array}{c}\text { Tube Sample } \\
\text { N-O }\end{array}$ & $\begin{array}{c}\text { Tube Sample } \\
\text { W-X }\end{array}$ & $\begin{array}{c}\text { Tube Sample } \\
\text { AA-BB }\end{array}$ \\
\hline $\mathrm{Ni}$ & $17.00-23.00$ & 19.97 & N/A & 21.1 & N/A & $\mathrm{N} / \mathrm{A}$ \\
\hline $\mathrm{Cr}$ & $24.00-26.00$ & 25.61 & N/A & 22.1 & N/A & N/A \\
\hline $\mathrm{Fe}$ & Balance & Balance & N/A & 55.6 & N/A & $\mathrm{N} / \mathrm{A}$ \\
\hline Mo & - & - & $\mathrm{N} / \mathrm{A}$ & - & $\mathrm{N} / \mathrm{A}$ & $\mathrm{N} / \mathrm{A}$ \\
\hline $\mathrm{Co}$ & - & - & $\mathrm{N} / \mathrm{A}$ & - & N/A & N/A \\
\hline $\mathrm{C}$ & $0.04-0.10$ & 0.05 & N/A & - & N/A & N/A \\
\hline $\mathrm{N}$ & $0.15-0.35$ & 0.24 & N/A & - & N/A & N/A \\
\hline $\mathrm{B}$ & - & - & N/A & - & N/A & $\mathrm{N} / \mathrm{A}$ \\
\hline $\mathrm{Mn}$ & $2.00 \max$ & 1.18 & N/A & 0.86 & N/A & N/A \\
\hline $\mathrm{Si}$ & $0.75 \max$ & 0.36 & N/A & 0.28 & N/A & $\mathrm{N} / \mathrm{A}$ \\
\hline $\mathrm{Al}$ & - & - & N/A & - & N/A & N/A \\
\hline $\mathrm{Ti}$ & - & - & N/A & - & N/A & N/A \\
\hline $\mathrm{Al}+\mathrm{Ti}$ & - & - & N/A & - & N/A & $\mathrm{N} / \mathrm{A}$ \\
\hline $\mathrm{Nb}$ & - & 0.47 & N/A & - & N/A & $\mathrm{N} / \mathrm{A}$ \\
\hline $\mathrm{Ta}$ & - & (c) & N/A & - & N/A & N/A \\
\hline $\mathrm{Nb}+\mathrm{Ta}$ & $0.20-0.60$ & 0.47 & $\mathrm{~N} / \mathrm{A}$ & - & $\mathrm{N} / \mathrm{A}$ & $\mathrm{N} / \mathrm{A}$ \\
\hline $\mathrm{V}$ & - & - & N/A & - & N/A & N/A \\
\hline W & - & - & N/A & - & N/A & N/A \\
\hline $\mathrm{Cu}$ & - & - & N/A & - & N/A & N/A \\
\hline $\mathrm{Sn}$ & - & - & N/A & - & N/A & N/A \\
\hline $\mathrm{P}$ & 0.030 max & 0.015 & N/A & - & N/A & N/A \\
\hline $\mathrm{S}$ & $0.030 \mathrm{max}$ & (c) & $\mathrm{N} / \mathrm{A}$ & - & N/A & $\mathrm{N} / \mathrm{A}$ \\
\hline
\end{tabular}
a- Ladle analysis.
N/A = Sample not analyzed
b- By difference.
c- Not detected. 


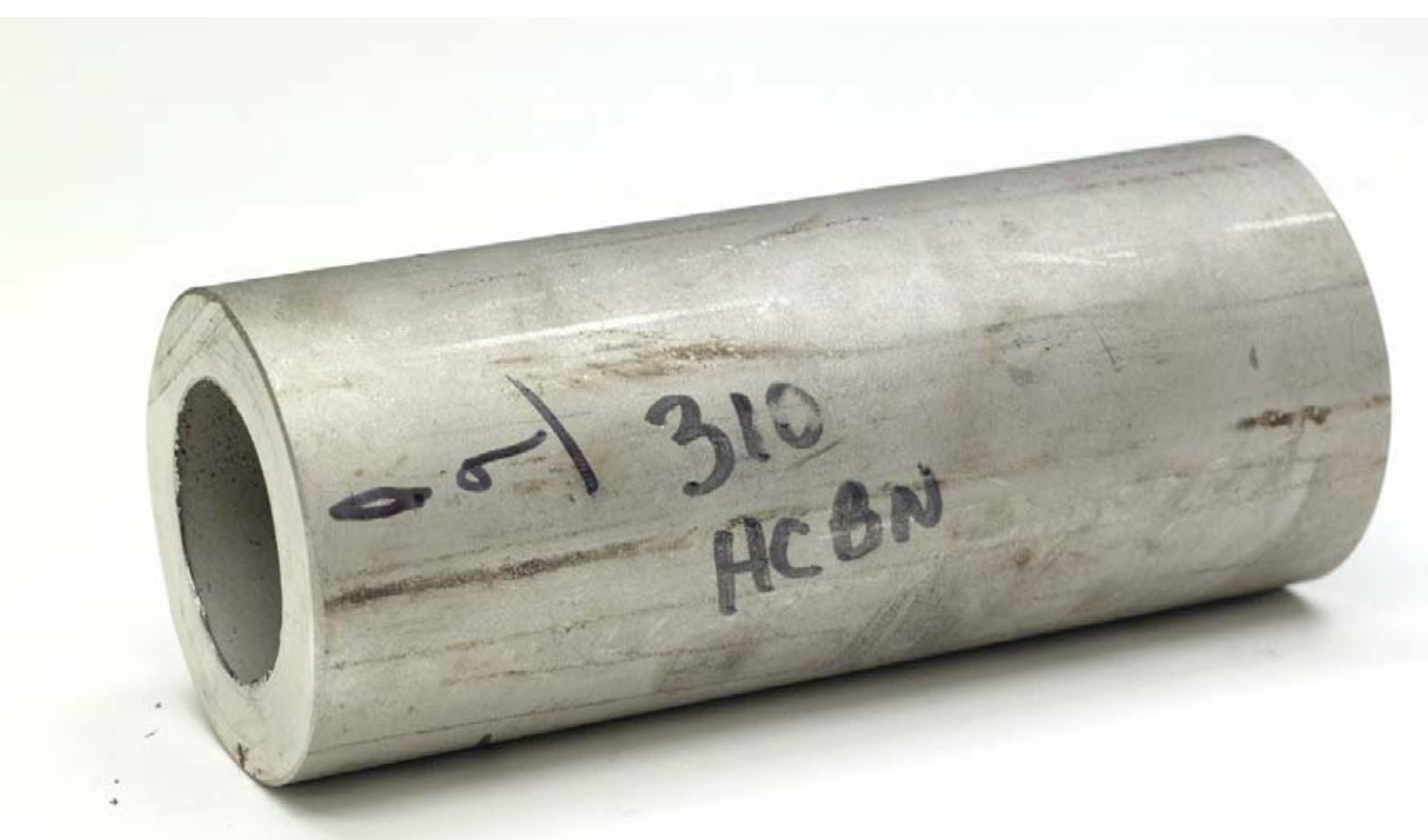

Figure G-3: Appearance of 310 HCbN (HR3C) Tube Sample Prior to Service 


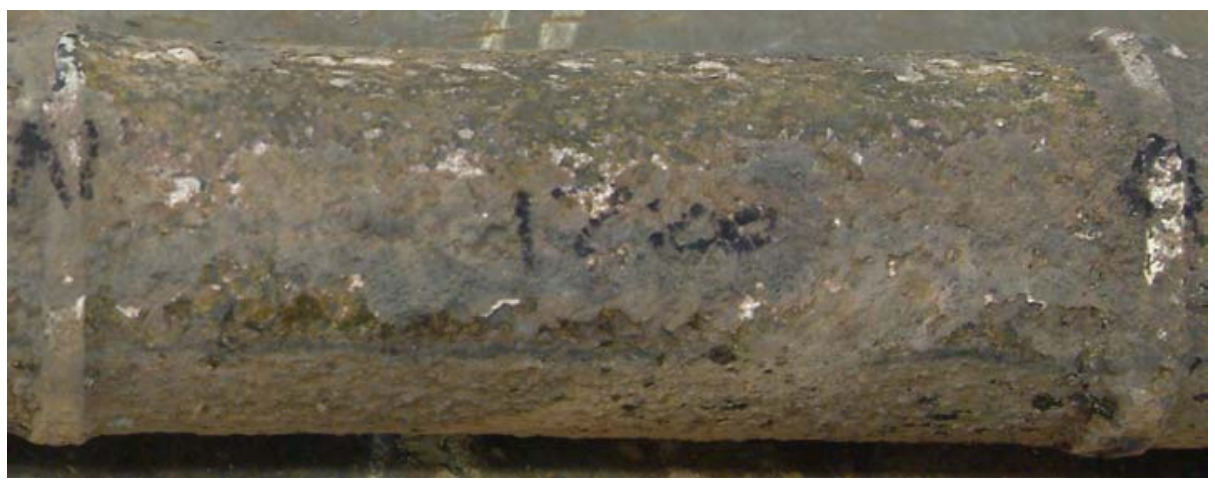

12 O'Clock - Facing Gas Flow

(Note: 3 o'clock is up in this image)

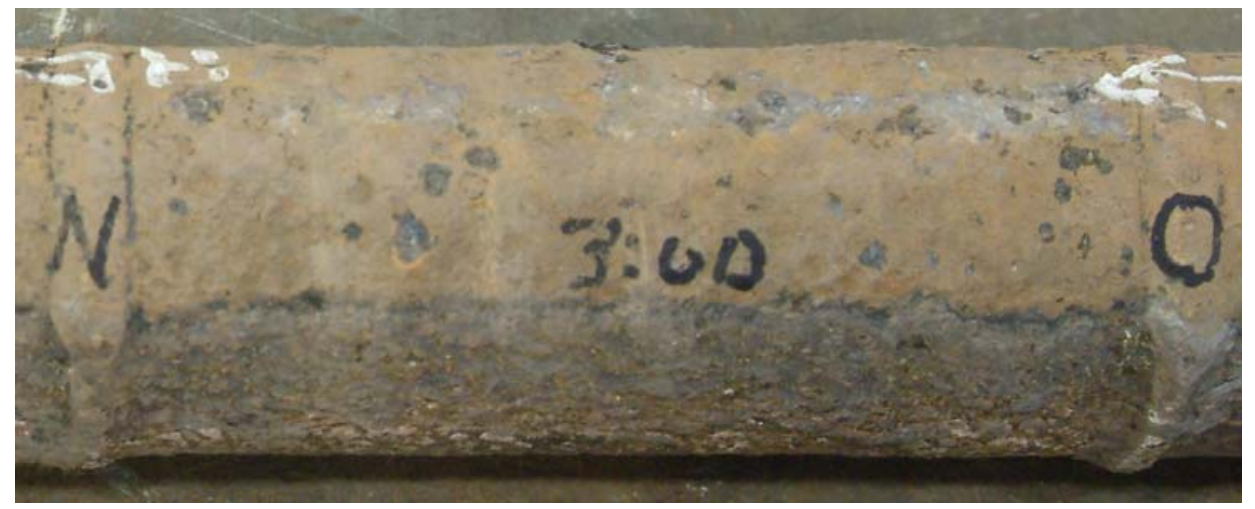

3 O'Clock

(Note: 6 o'clock is up in this image)

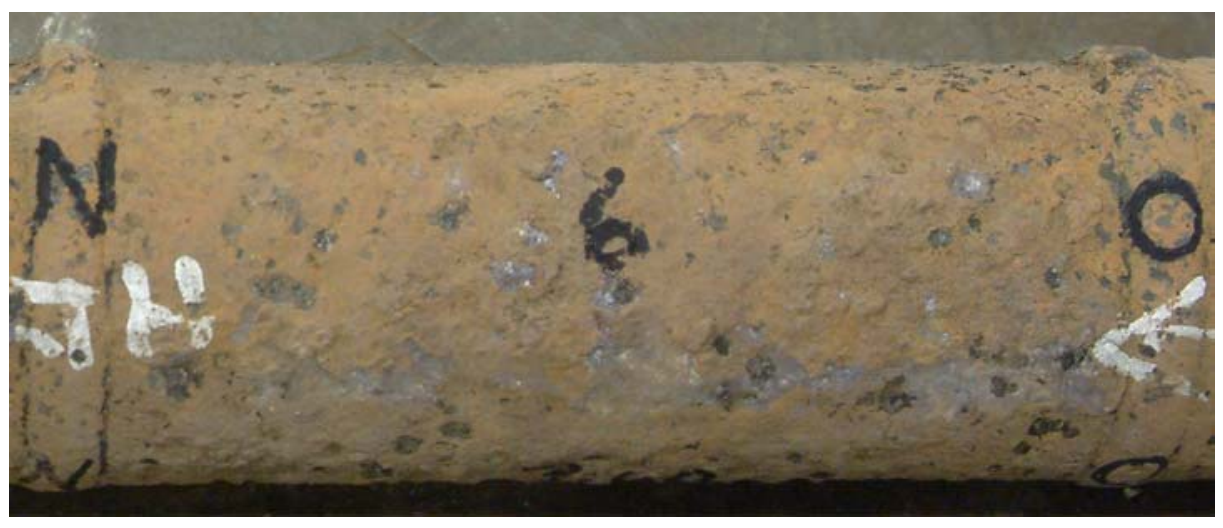

6 O'Clock - Opposite Gas Flow

Figure G-4 As-Received Appearance of Sample N-O (310 HCbN)

(Average surface metal temperature: $1190{ }^{\circ} \mathrm{F}$ ) 


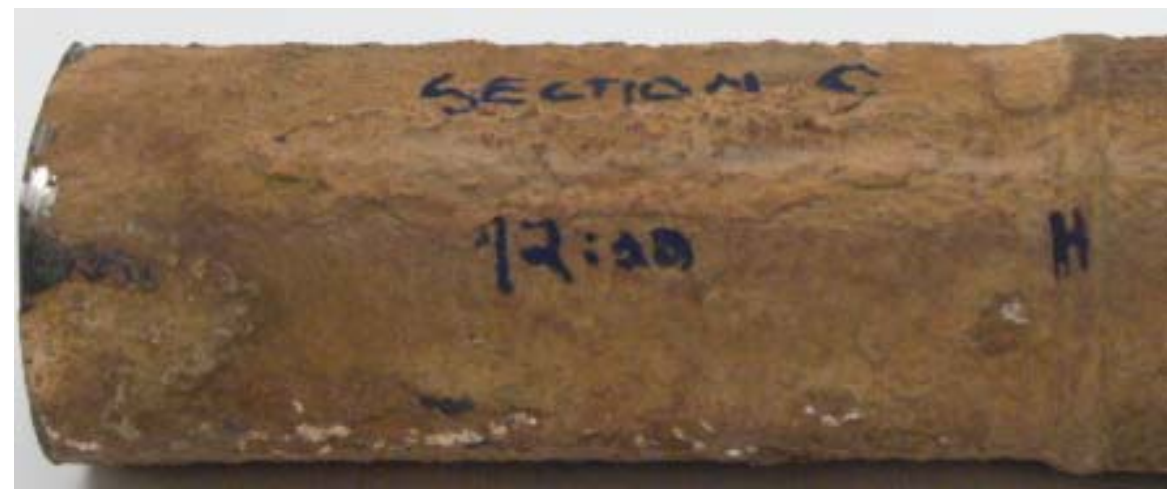

12 O'Clock - Facing Gas Flow

(Note: 3 o'clock is up in this image)

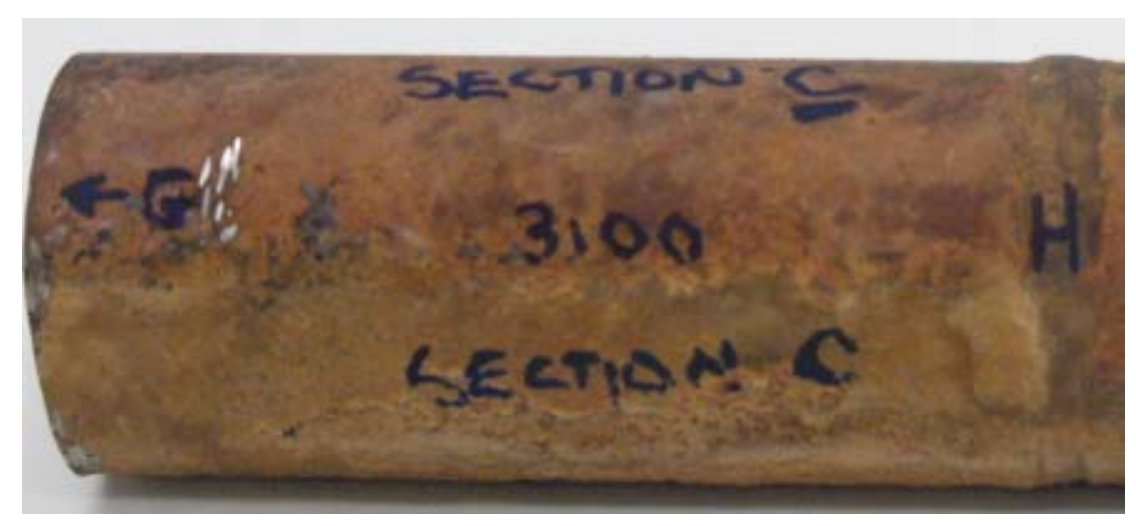

3 O'Clock

(Note: 6 o'clock is up in this image)

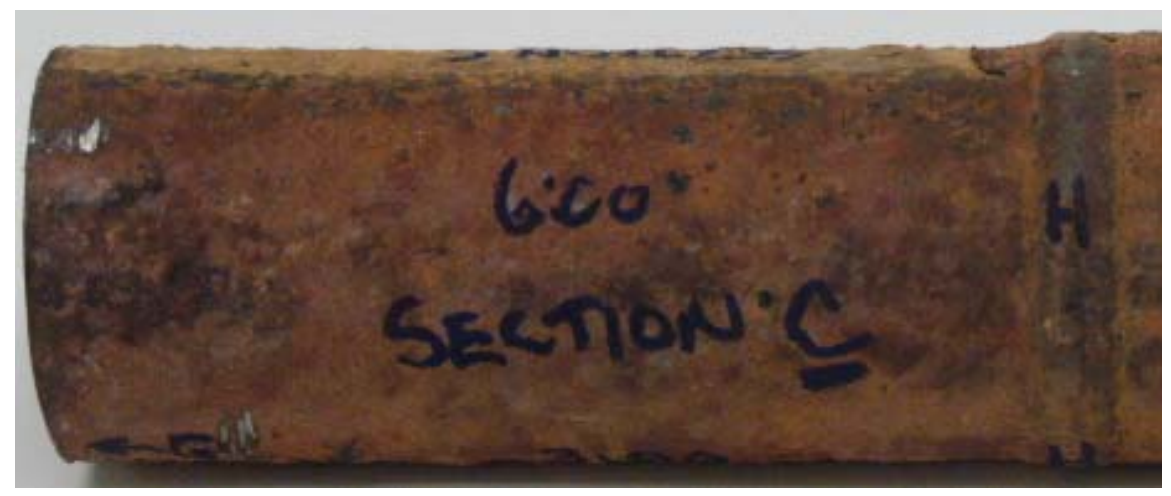

6 O'Clock - Opposite Gas Flow

Figure G-5 As-Received Appearance of Sample G-H (310 HCbN)

(Average surface metal temperature: $1167^{\circ} \mathrm{F}$ )

Note: this tube was removed from service on 11/6/01. 


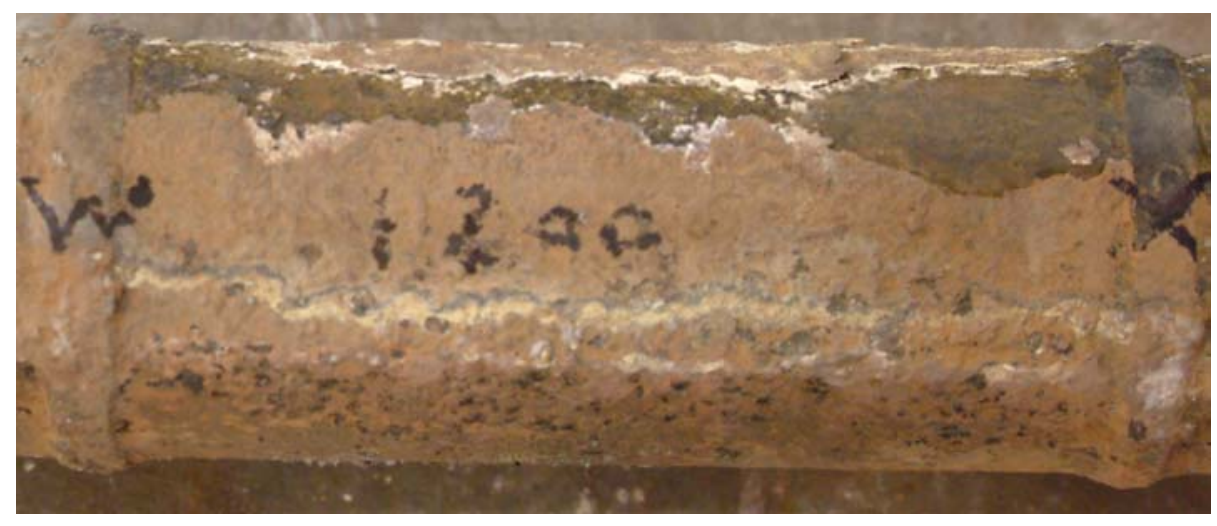

12 O'Clock - Facing Gas Flow

(Note: 3 o'clock is up in this image)

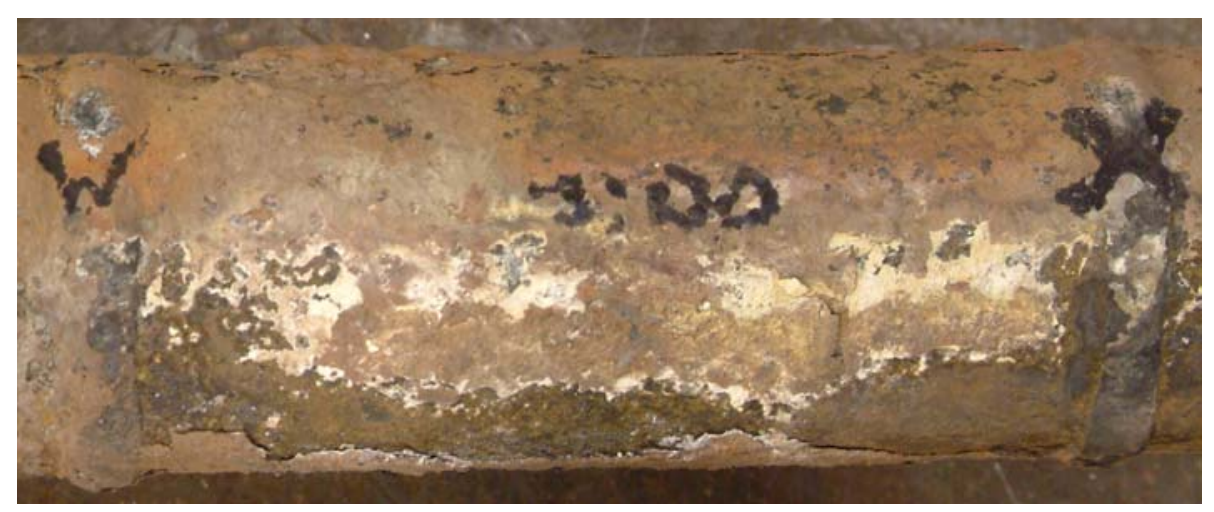

3 O'Clock

(Note: 6 o'clock is up in this image)

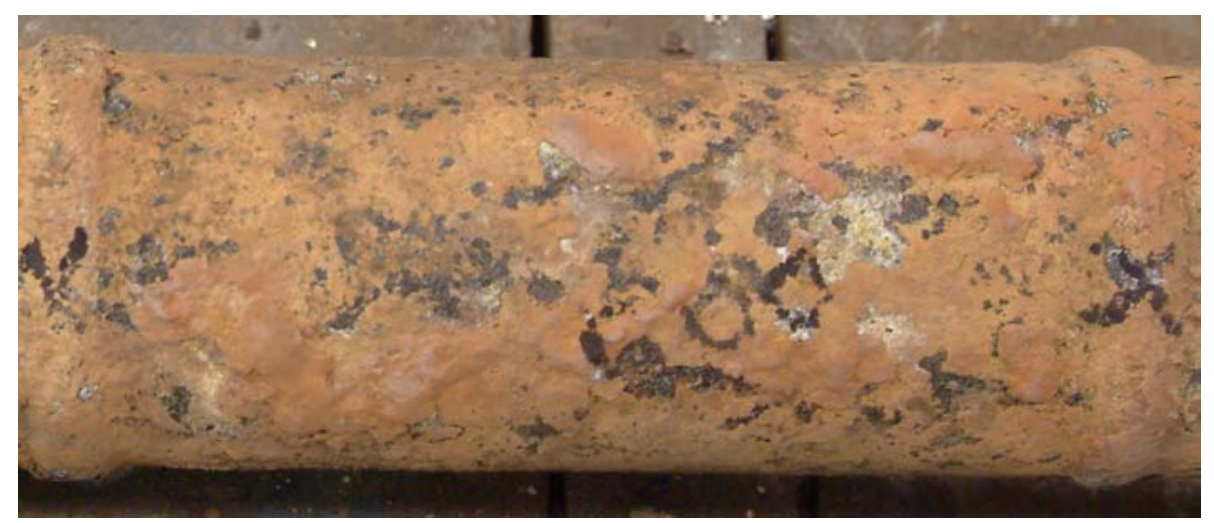

6 O'Clock - Opposite Gas Flow

Figure G-6 As-Received Appearance of Sample W-X (310 HCbN)

(Average surface metal temperature: $1106^{\circ} \mathrm{F}$ ) 


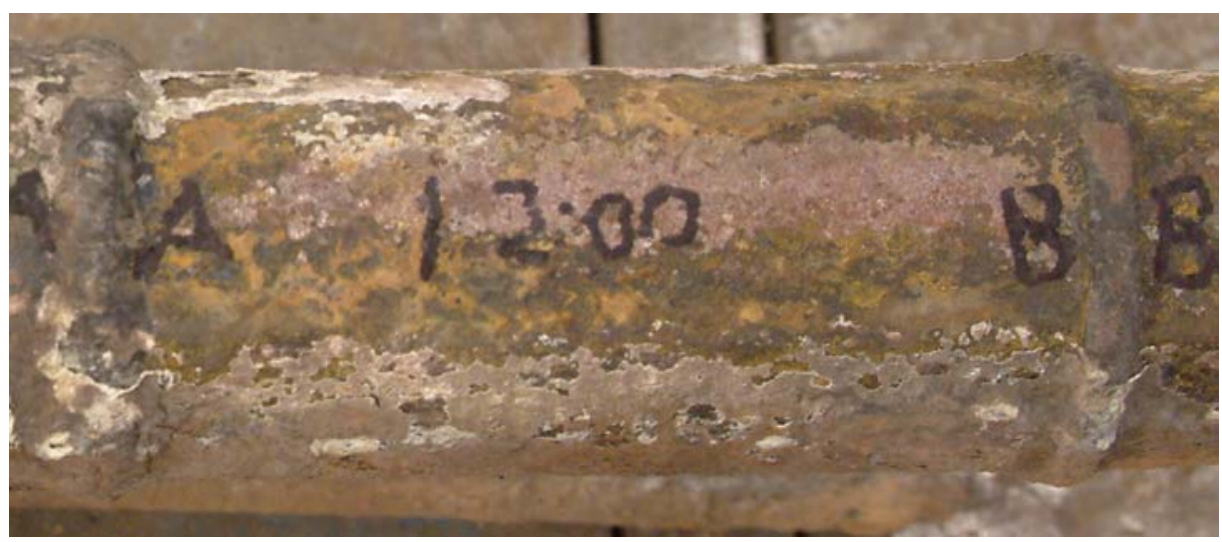

12 O'Clock - Facing Gas Flow

(Note: 3 o'clock is up in this image)

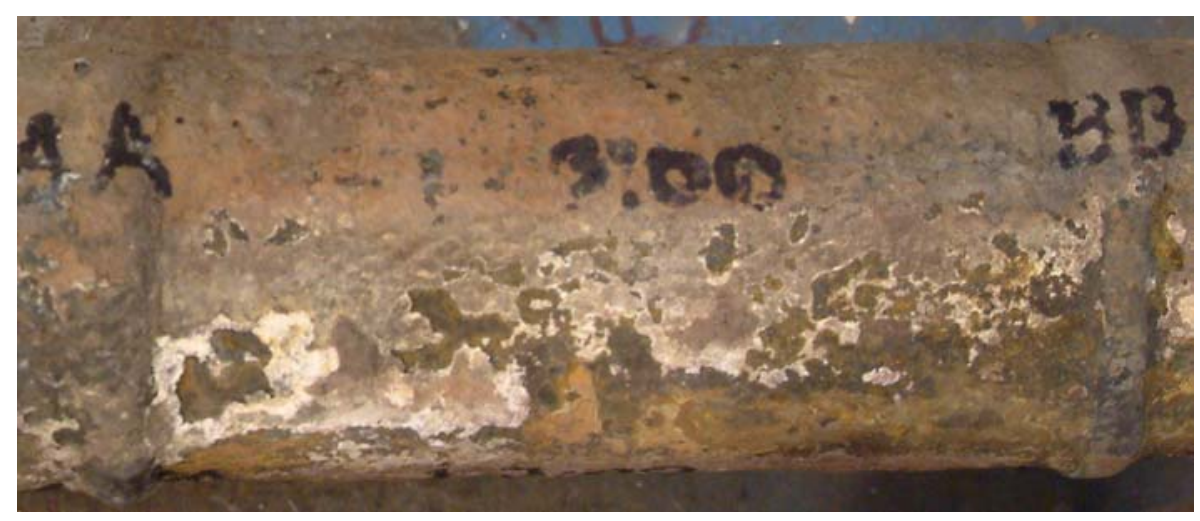

3 O'Clock

(Note: 6 o'clock is up in this image)

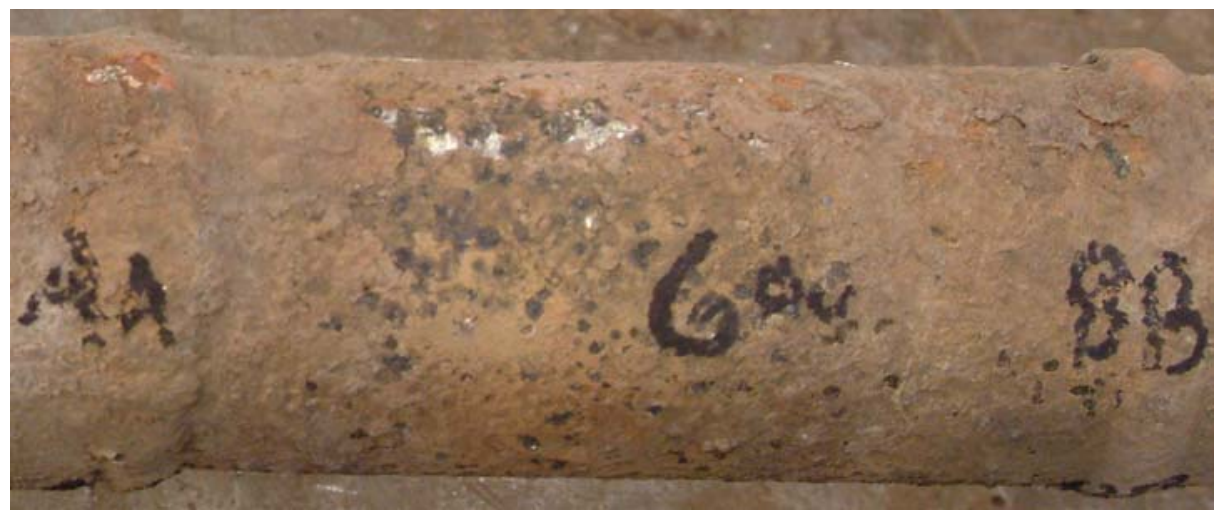

6 O'Clock - Opposite Gas Flow

Figure G-7 As-Received Appearance of Sample AA-BB (310 HCbN)

(Average surface metal temperature: $1092{ }^{\circ} \mathrm{F}$ ) 
12 O'Clock

(201 mils lost)

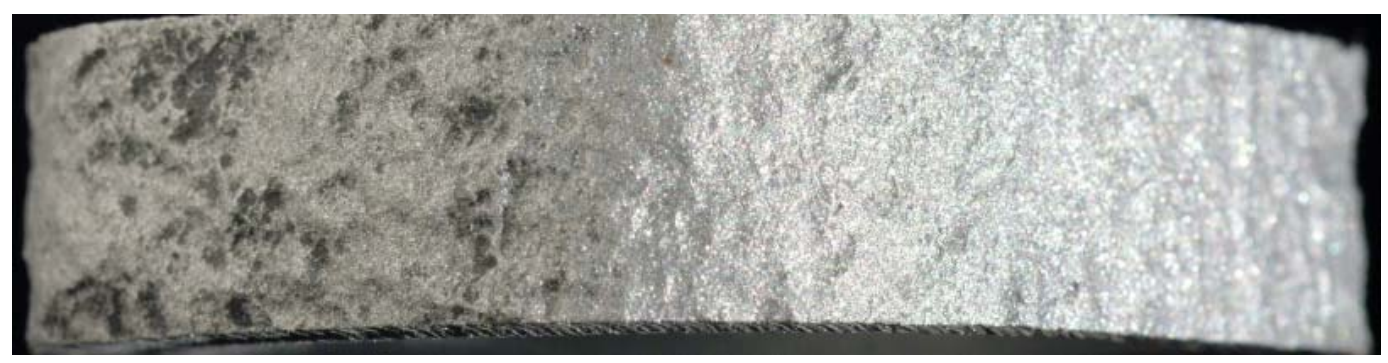

3 O'Clock

(205 mils lost)

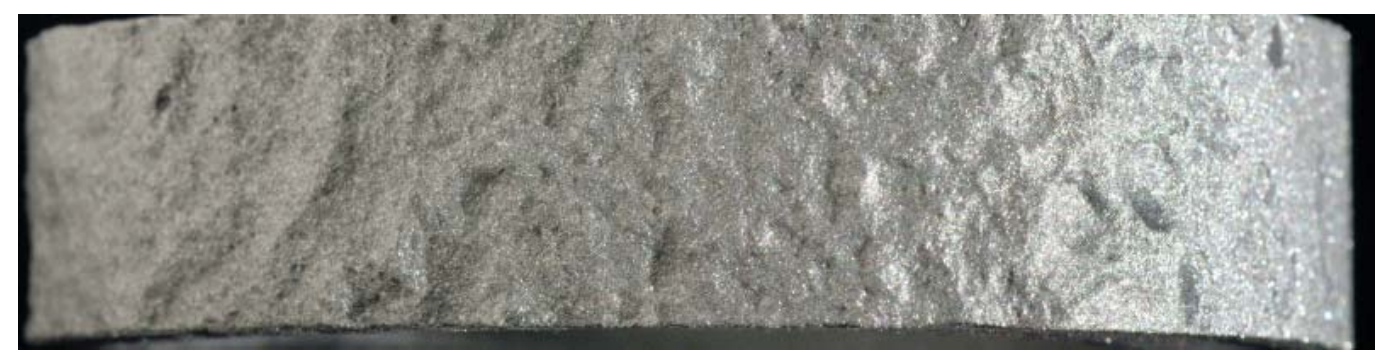

6 O'Clock

(6 mils lost)

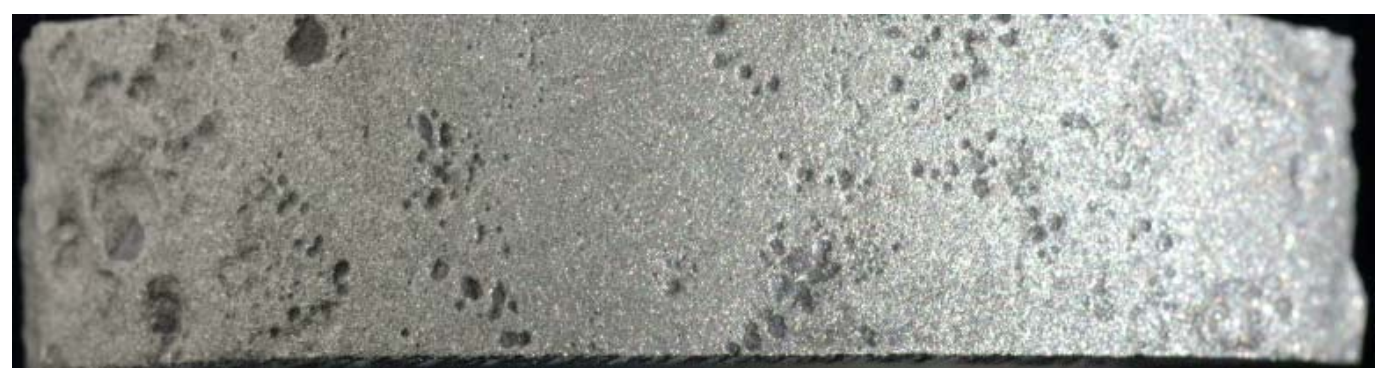

Figure G-8: OD Surface Appearance After Grit Blast

Material: $310 \mathrm{HCbN}$ (HR3C)

Sample: N-O 
12 O'Clock

(146 mils lost)

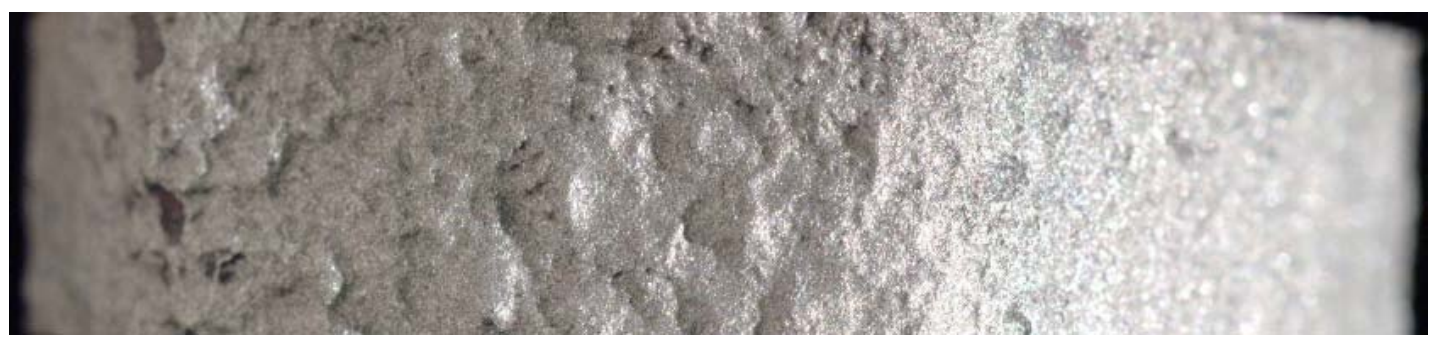

3 O'Clock

(173 mils lost)

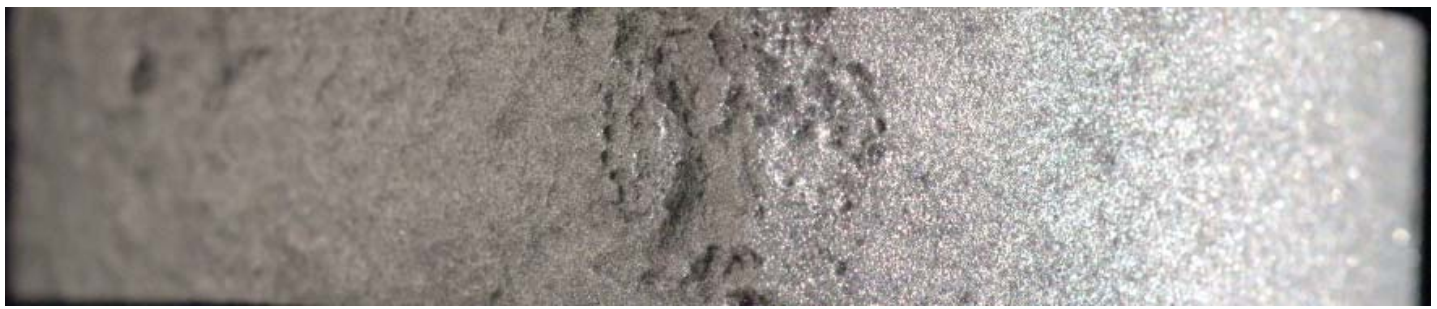

6 O'Clock

(2 mils lost)

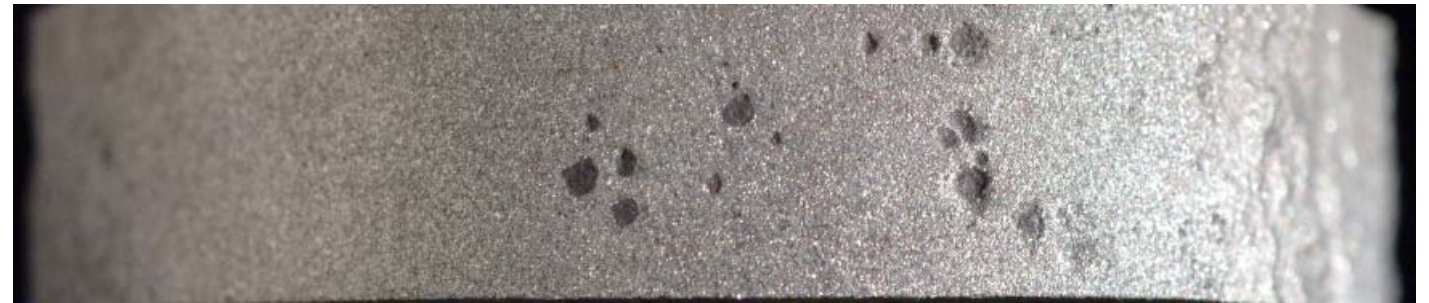

Figure G-9: OD Surface Appearance After Grit Blast

Material: $310 \mathrm{HCbN}(\mathrm{HR3C})$

Sample: G-H 
12 O'Clock

(61 mils lost)

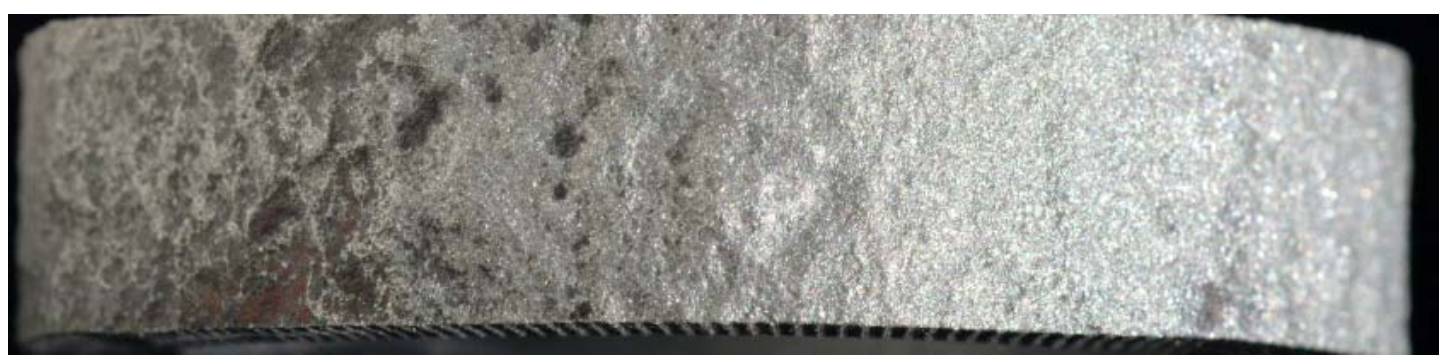

3 O'Clock

(45 mils lost)

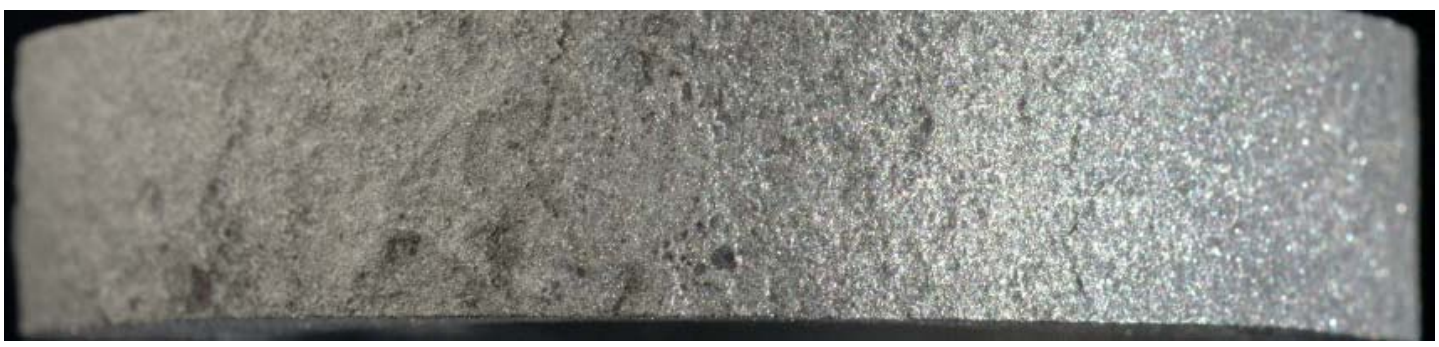

6 O'Clock

(7 mils lost)

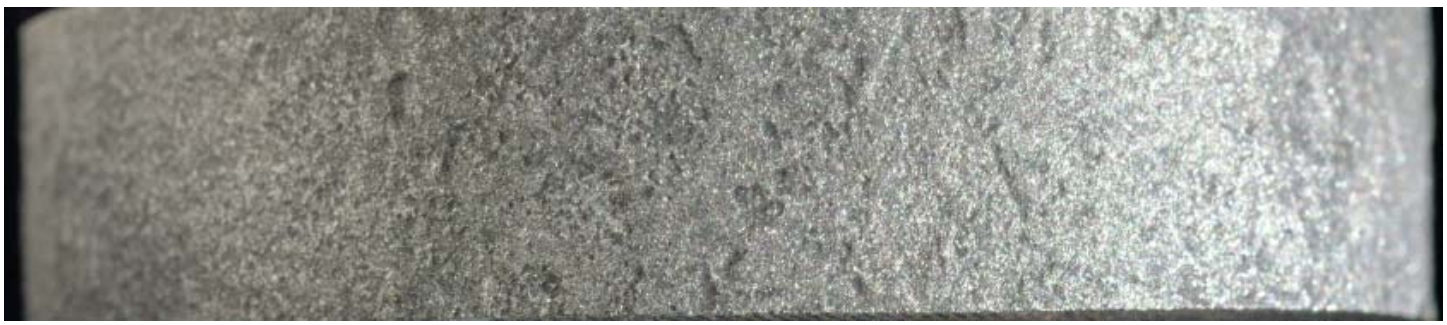

Figure G-10: OD Surface Appearance After Grit Blast

Material: $310 \mathrm{HCbN}$ (HR3C)

Sample: W-X 
12 O'Clock

(146 mils lost)

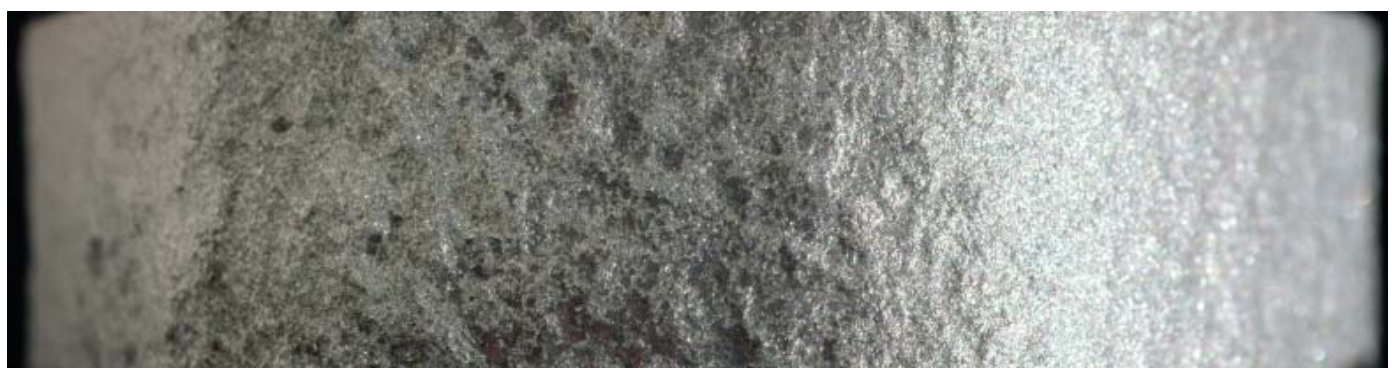

3 O'Clock

(148 mils lost)

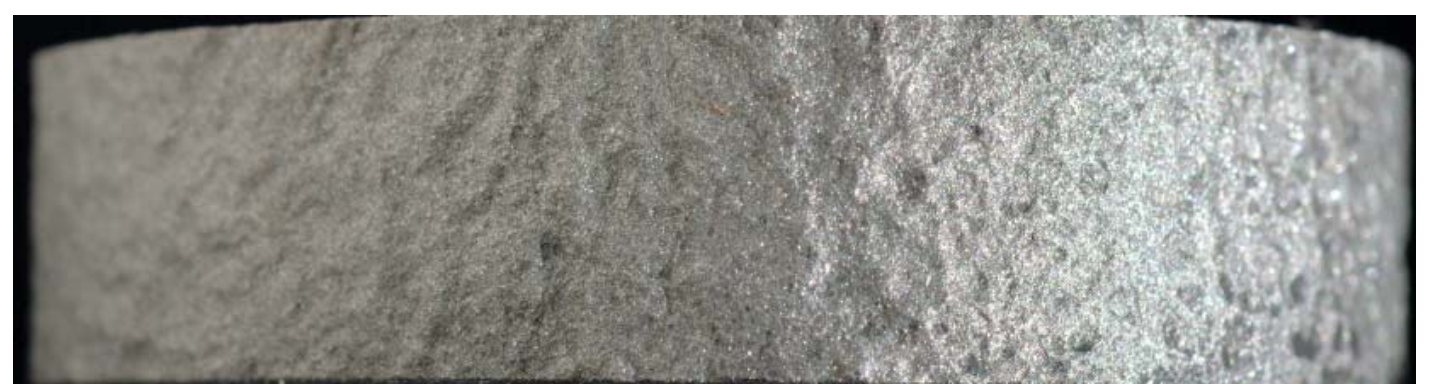

6 O'Clock

(7 mils lost)

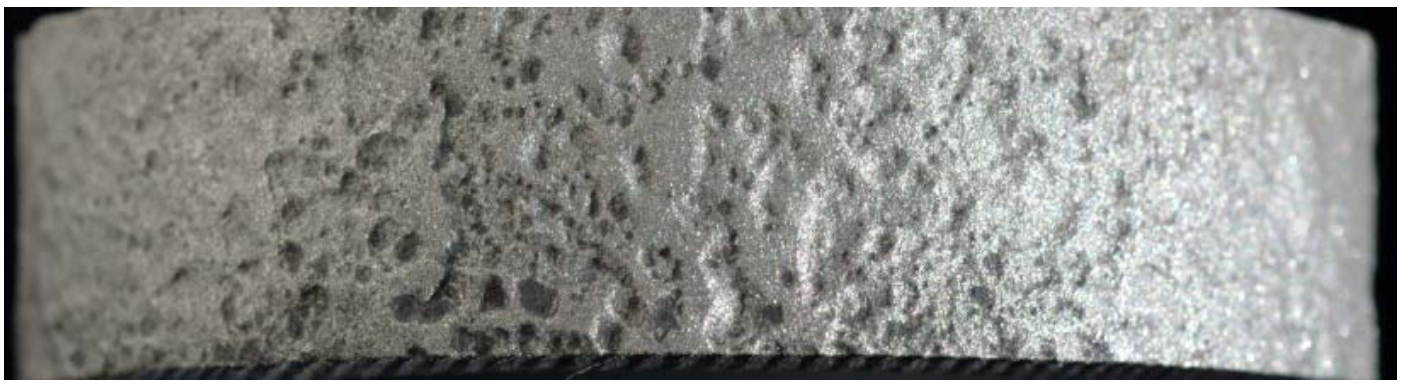

Figure G-11: OD Surface Appearance After Grit Blast

Material: $310 \mathrm{HCbN}$ (HR3C)

Sample: AA-BB 


\section{Material:}

Sample Location:

Maximum Rate:

Maximum Loss Orientation:

Average Surface Metal Temperature:
310 HCbN (HR3C)

N-O

$133 \mathrm{mils} / \mathrm{yr}$

Approx. 2 o'clock

$1190{ }^{\circ} \mathrm{F}$

\section{O'Clock}

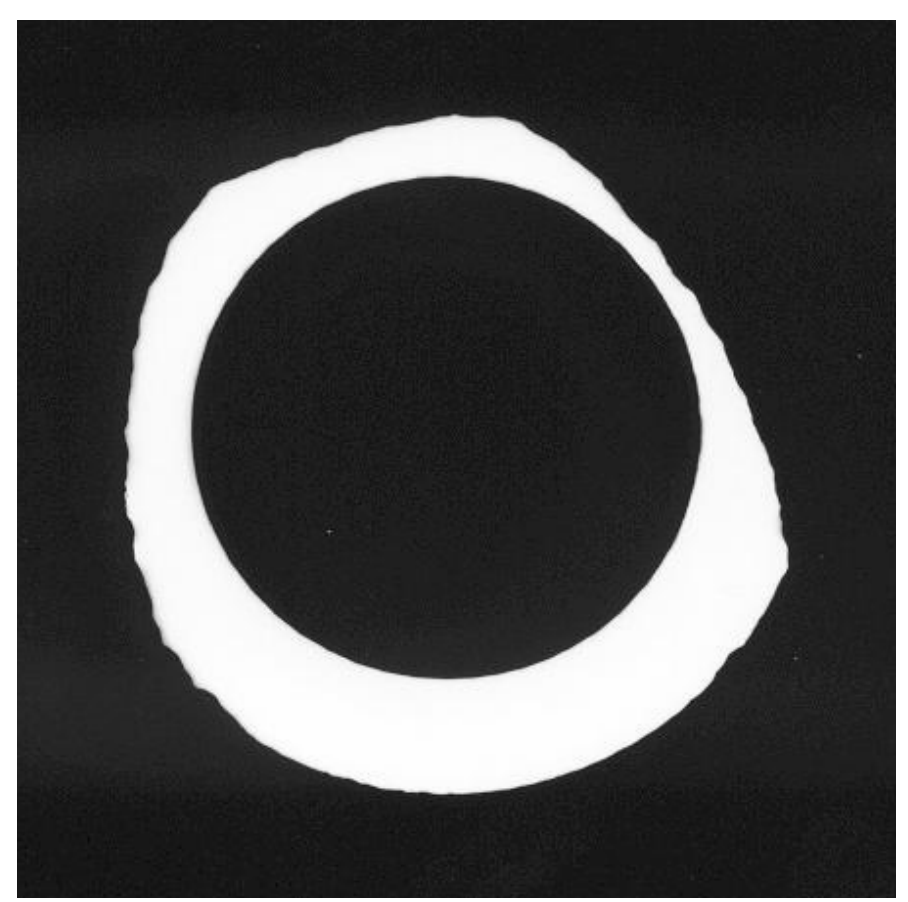

3 O'Clock

Approx. 1.2X

Wall Reduction by Position (in.)

\begin{tabular}{|l|c|c|c|c|c|c|c|c|c|c|}
\hline & $\begin{array}{c}\mathbf{1 2} \\
\text { o'clock }\end{array}$ & $\mathbf{1 : 3 0}$ & $\mathbf{3 : 0 0}$ & $\mathbf{4 : 3 0}$ & $\mathbf{6 : 0 0}$ & $\mathbf{7 : 3 0}$ & $\mathbf{9 : 0 0}$ & $\mathbf{1 0 : 3 0}$ & AVG & $\begin{array}{c}\text { Max* } \\
\text { Loss }\end{array}$ \\
\hline Before & 0.396 & 0.396 & 0.398 & 0.401 & 0.400 & 0.398 & 0.401 & 0.399 & 0.399 & 0.399 \\
\hline After & 0.195 & 0.093 & 0.193 & 0.391 & 0.394 & 0.399 & 0.252 & 0.293 & & 0.083 \\
\hline Delta & 0.201 & 0.303 & 0.205 & 0.010 & 0.006 & -0.001 & 0.149 & 0.106 & & 0.316 \\
\hline
\end{tabular}

Figure G-12: Silhouette and Wall Thickness Measurements for Sample N-O

* Measurement taken at "maximum loss orientation" noted above. The average

"before" wall thickness was used to calculate the "delta". 


\section{Material:}

Sample Location:

Maximum Rate:

Maximum Loss Orientation:

Average Surface Metal Temperature:
310 HCbN (HR3C)

G-H

$141 \mathrm{mils} / \mathrm{yr}$

Approx. 2:30 o'clock

\section{O’Clock}

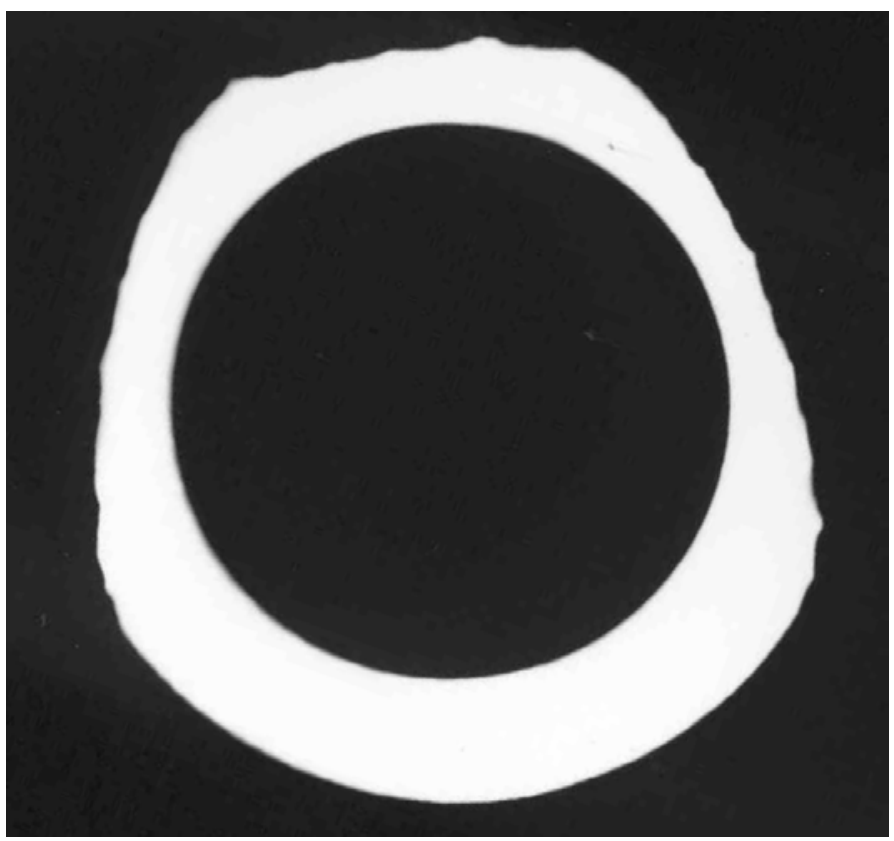

3 O’Clock

Approx. 1.2X

Wall Reduction by Position (in.)

\begin{tabular}{|l|c|c|c|c|c|c|c|c|c|c|}
\hline & $\begin{array}{c}\mathbf{1 2} \\
\text { o'clock }\end{array}$ & $\mathbf{1 : 3 0}$ & $\mathbf{3 : 0 0}$ & $\mathbf{4 : 3 0}$ & $\mathbf{6 : 0 0}$ & $\mathbf{7 : 3 0}$ & $\mathbf{9 : 0 0}$ & $\mathbf{1 0 : 3 0}$ & AVG & $\begin{array}{c}\text { Max } \\
\text { Loss }\end{array}$ \\
\hline Before & 0.404 & 0.401 & 0.399 & 0.402 & 0.400 & 0.399 & 0.400 & 0.402 & 0.401 & 0.401 \\
\hline After & 0.258 & 0.221 & 0.226 & 0.401 & 0.398 & 0.399 & 0.232 & 0.311 & & 0.182 \\
\hline Delta & 0.146 & 0.180 & 0.173 & 0.001 & 0.002 & 0.000 & 0.168 & 0.091 & & 0.219 \\
\hline
\end{tabular}

Figure G-13: Silhouette and Wall Thickness Measurements for Sample G-H

Note: this tube was removed from service on 11/6/01.

* Measurement taken at "maximum loss orientation" noted above. The average

"before" wall thickness was used to calculate the "delta". 


\section{Material:}

Sample Location:

Maximum Rate:

Maximum Loss Orientation:

Average Surface Metal Temperature:
$310 \mathrm{HCbN}(\mathrm{HR} 3 \mathrm{C})$

W-X

52 mils/yr

Approx. 9:30 o'clock

$1106^{\circ} \mathrm{F}$

\section{O’Clock}

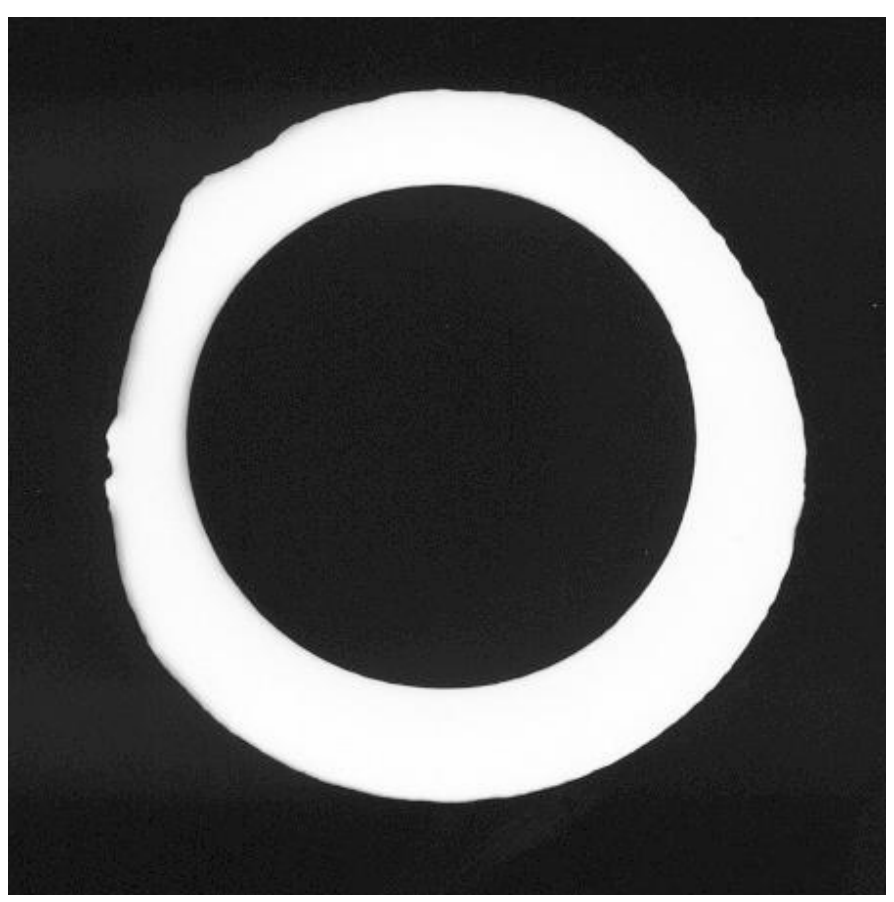

3 O'Clock

Approx. 1.2X

Wall Reduction by Position (in.)

\begin{tabular}{|l|c|c|c|c|c|c|c|c|c|c|}
\hline & $\begin{array}{c}\mathbf{1 2} \\
\text { o'clock }\end{array}$ & $\mathbf{1 : 3 0}$ & $\mathbf{3 : 0 0}$ & $\mathbf{4 : 3 0}$ & $\mathbf{6 : 0 0}$ & $\mathbf{7 : 3 0}$ & $\mathbf{9 : 0 0}$ & $\mathbf{1 0 : 3 0}$ & AVG & $\begin{array}{c}\text { Max* } \\
\text { Loss }\end{array}$ \\
\hline Before & 0.398 & 0.398 & 0.398 & 0.401 & 0.399 & 0.397 & 0.400 & 0.397 & 0.398 & 0.398 \\
\hline After & 0.337 & 0.322 & 0.353 & 0.398 & 0.392 & 0.396 & 0.301 & 0.346 & & 0.273 \\
\hline Delta & 0.061 & 0.076 & 0.045 & 0.003 & 0.007 & 0.001 & 0.099 & 0.051 & & 0.125 \\
\hline
\end{tabular}

Figure G-14: Silhouette and Wall Thickness Measurements for Sample W-X

* Measurement taken at "maximum loss orientation" noted above. The average

"before" wall thickness was used to calculate the "delta". 


\section{Material:}

Sample Location:

Maximum Rate:

Maximum Loss Orientation:

Average Surface Metal Temperature:

\section{HCbN (HR3C)}

\author{
AA-BB \\ 79 mils/yr \\ Approx. 9:30 o'clock \\ $1092{ }^{\circ} \mathrm{F}$
}

\section{O'Clock}

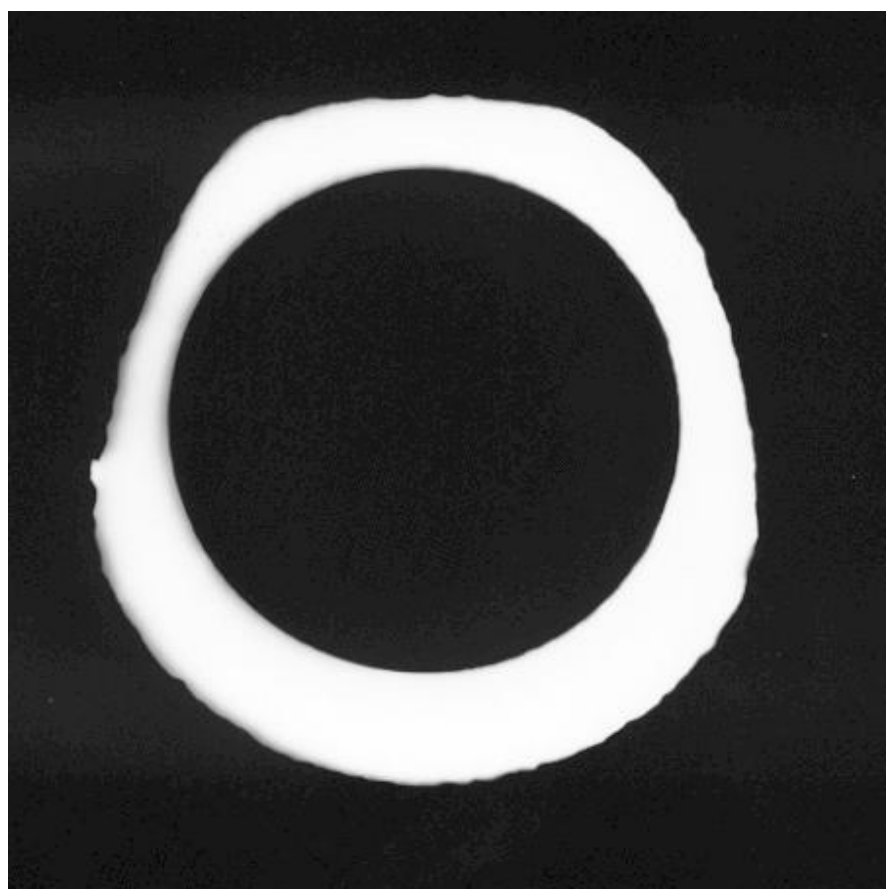

3 O'Clock

Approx. 1.2X

Wall Reduction by Position (in.)

\begin{tabular}{|l|c|c|c|c|c|c|c|c|c|c|}
\hline & $\begin{array}{c}\mathbf{1 2} \\
\text { o'clock }\end{array}$ & $\mathbf{1 : 3 0}$ & $\mathbf{3 : 0 0}$ & $\mathbf{4 : 3 0}$ & $\mathbf{6 : 0 0}$ & $\mathbf{7 : 3 0}$ & $\mathbf{9 : 0 0}$ & $\mathbf{1 0 : 3 0}$ & AVG & $\begin{array}{c}\text { Max } \\
\text { Loss }\end{array}$ \\
\hline Before & 0.399 & 0.400 & 0.400 & 0.397 & 0.394 & 0.399 & 0.399 & 0.399 & 0.398 & 0.398 \\
\hline After & 0.253 & 0.278 & 0.252 & 0.380 & 0.387 & 0.387 & 0.256 & 0.269 & & 0.209 \\
\hline Delta & 0.146 & 0.122 & 0.148 & 0.017 & 0.007 & 0.012 & 0.143 & 0.130 & & 0.189 \\
\hline
\end{tabular}

Figure G-15: Silhouette and Wall Thickness Measurements for Sample AA-BB

\footnotetext{
* Measurement taken at "maximum loss orientation" noted above. The average
} "before" wall thickness was used to calculate the "delta". 


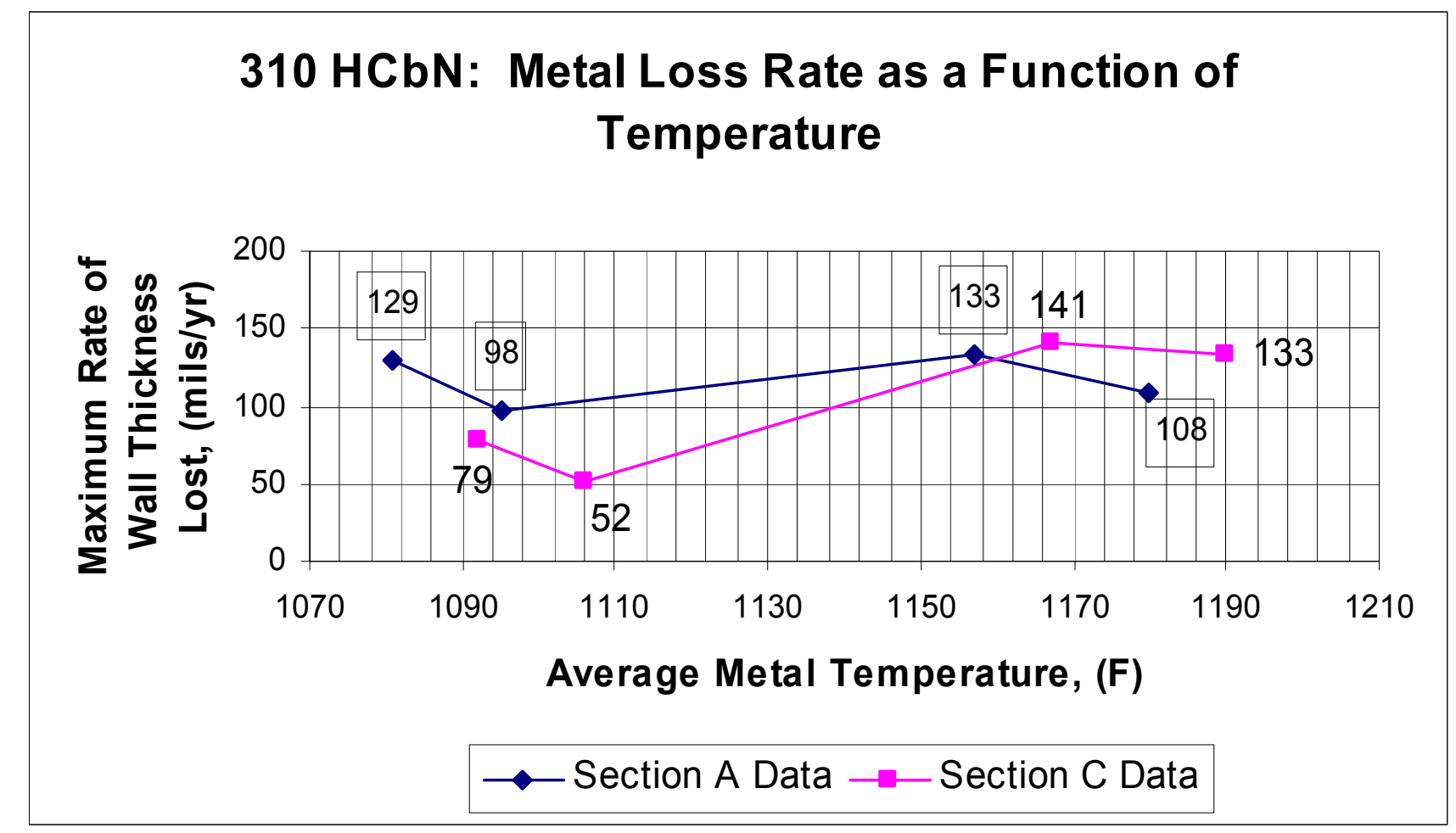

Figure G-16: Metal Loss Rate as a Function of Average Surface Metal Temperature

\section{Material: $310 \mathrm{HCbN}(\mathrm{HR3C})$}

Note: In analyzing these data it is important to understand that the one of the three Section C samples was removed early due to excessive corrosion losses. Sample G-H (1167 F) was removed in November 2001, shortly after Section A was removed for evaluation. Sample N-O (1190 F), Sample W-X (1106 F), and Sample AA-BB (1092 F) were removed with the remaining Section C samples in May 2003. 


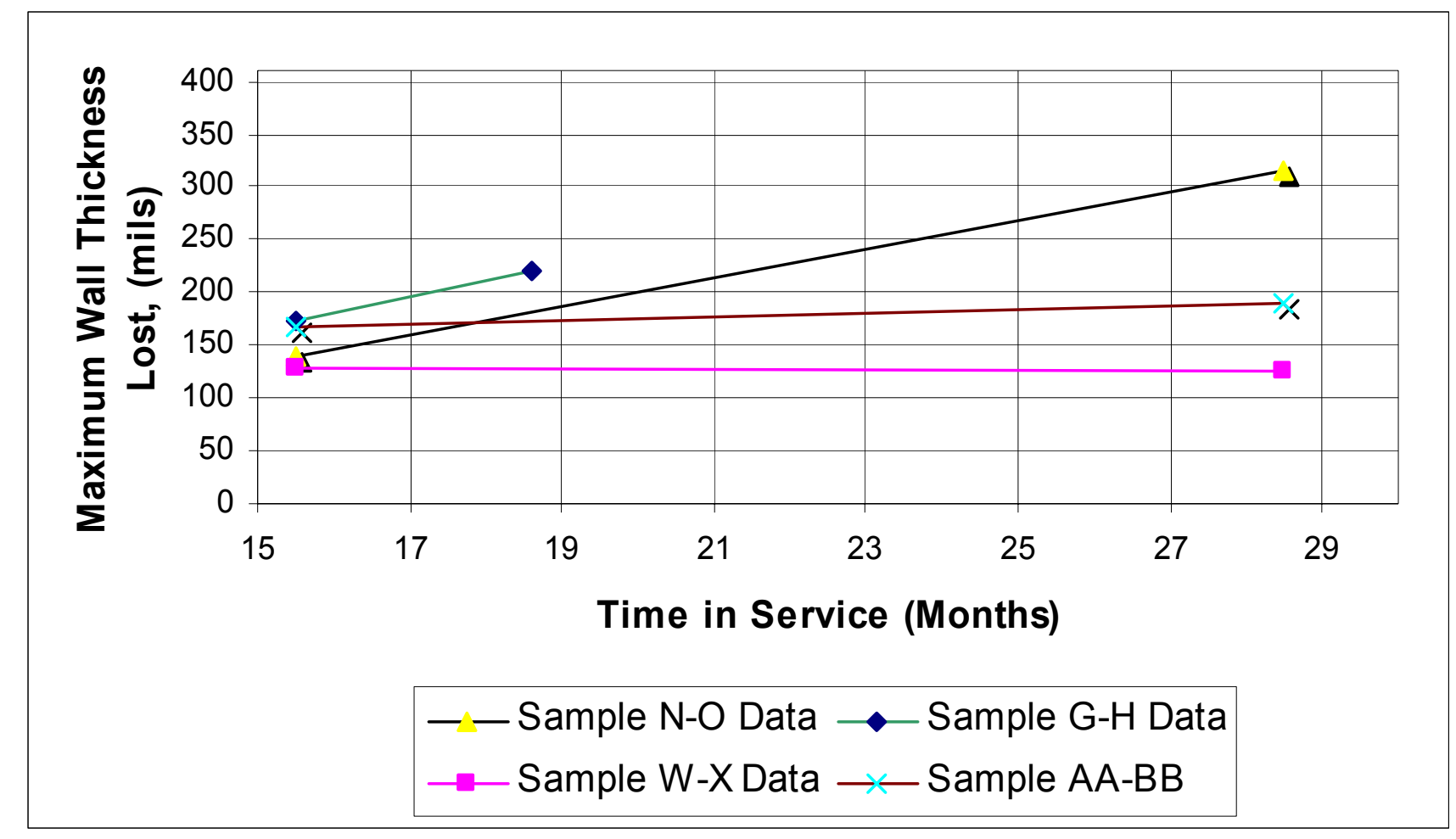

Figure G-17: Metal Loss as a Function of Time

\section{Material: $310 \mathrm{HCbN}(\mathrm{HR3C})$}

Note: From the plot above it can be seen that, for Section C, the Sample G-H was removed shortly after Section A was removed for evaluation. The other three sample locations experienced the full exposure life for both Sections A and C. 


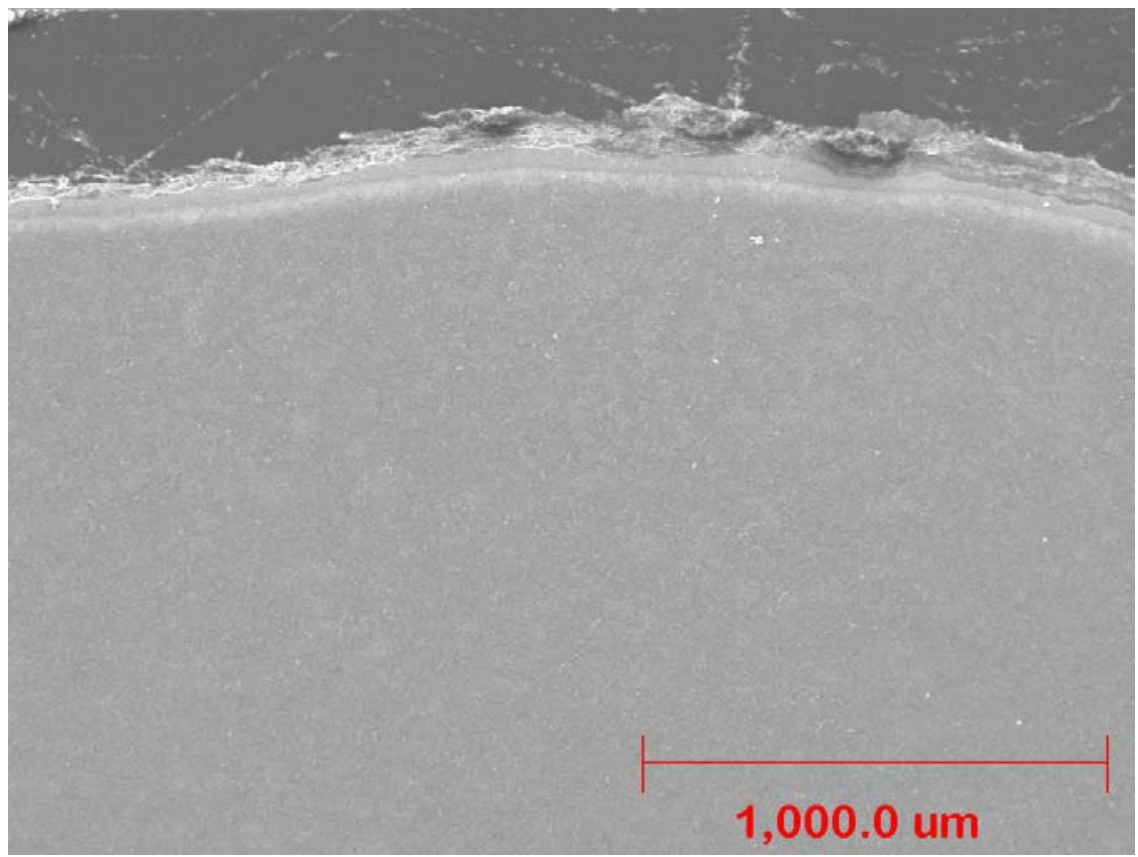

(a) External Scale/Metal Interface (SEI - Low Magnification)

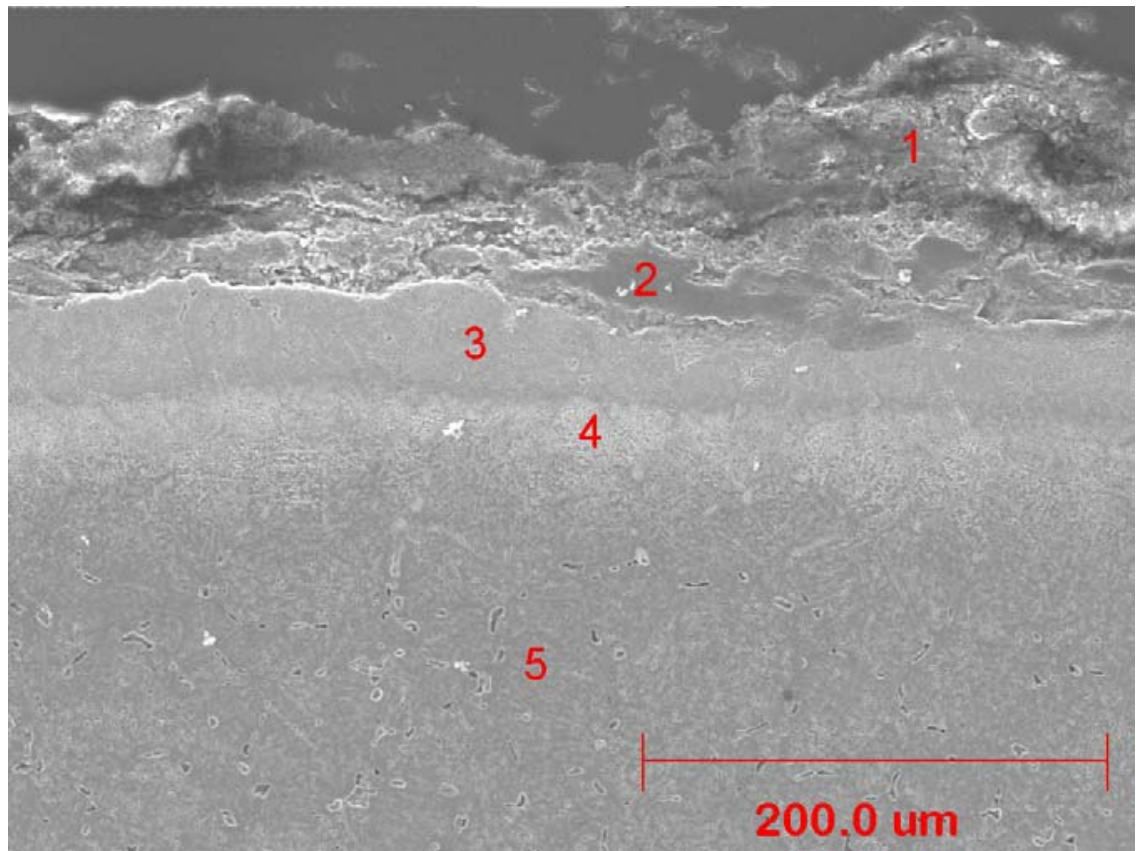

(b) External Scale/Metal Interface (SEI - Intermediate Magnification)

\section{Figure G-18: Microstructure: $310 \mathrm{HCbN}$}

Note: Numbers on photo indicate distinct areas characterized by the visual assessment and EDS analyses that follow. 


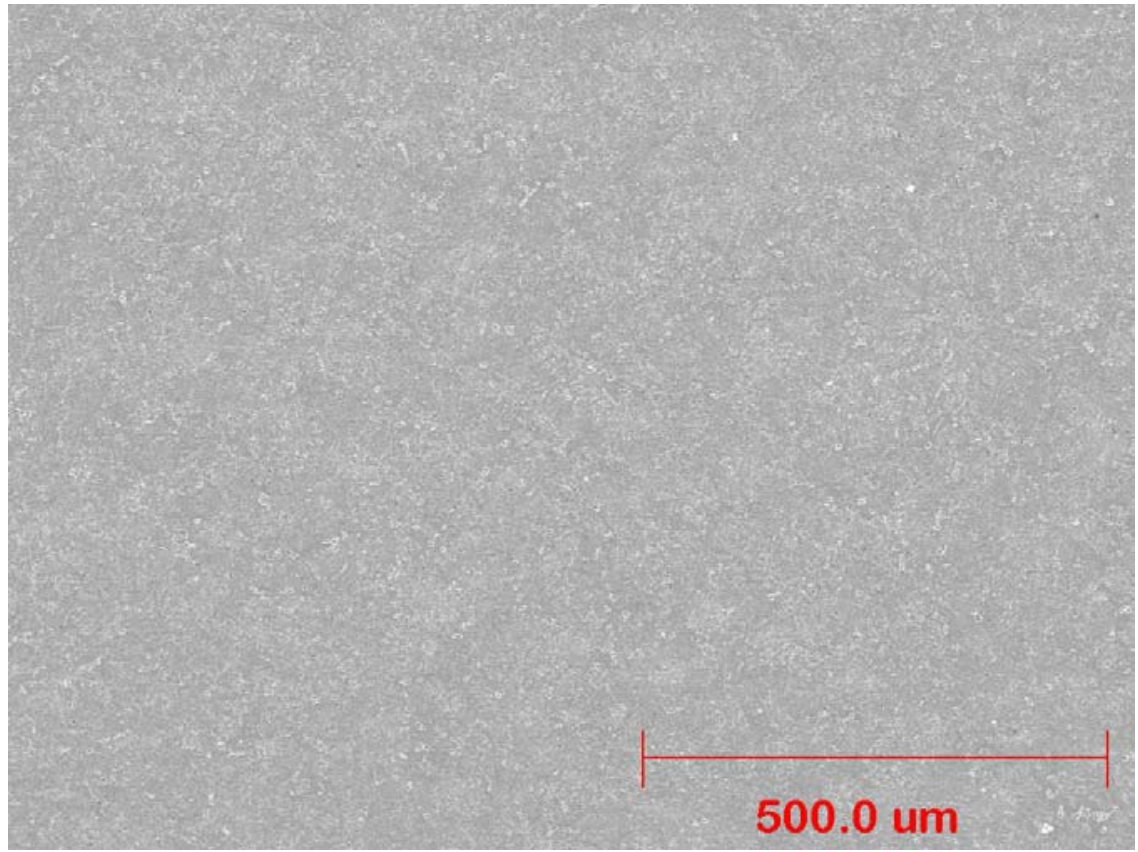

(SEI - Low Magnification)

\section{Figure G-19: Mid-Wall Microstructure: 310 HCbN}

Note: Photomicrograph at approximately 200X. Electrolytic 10\% oxalic acid etch, 9V OCP 


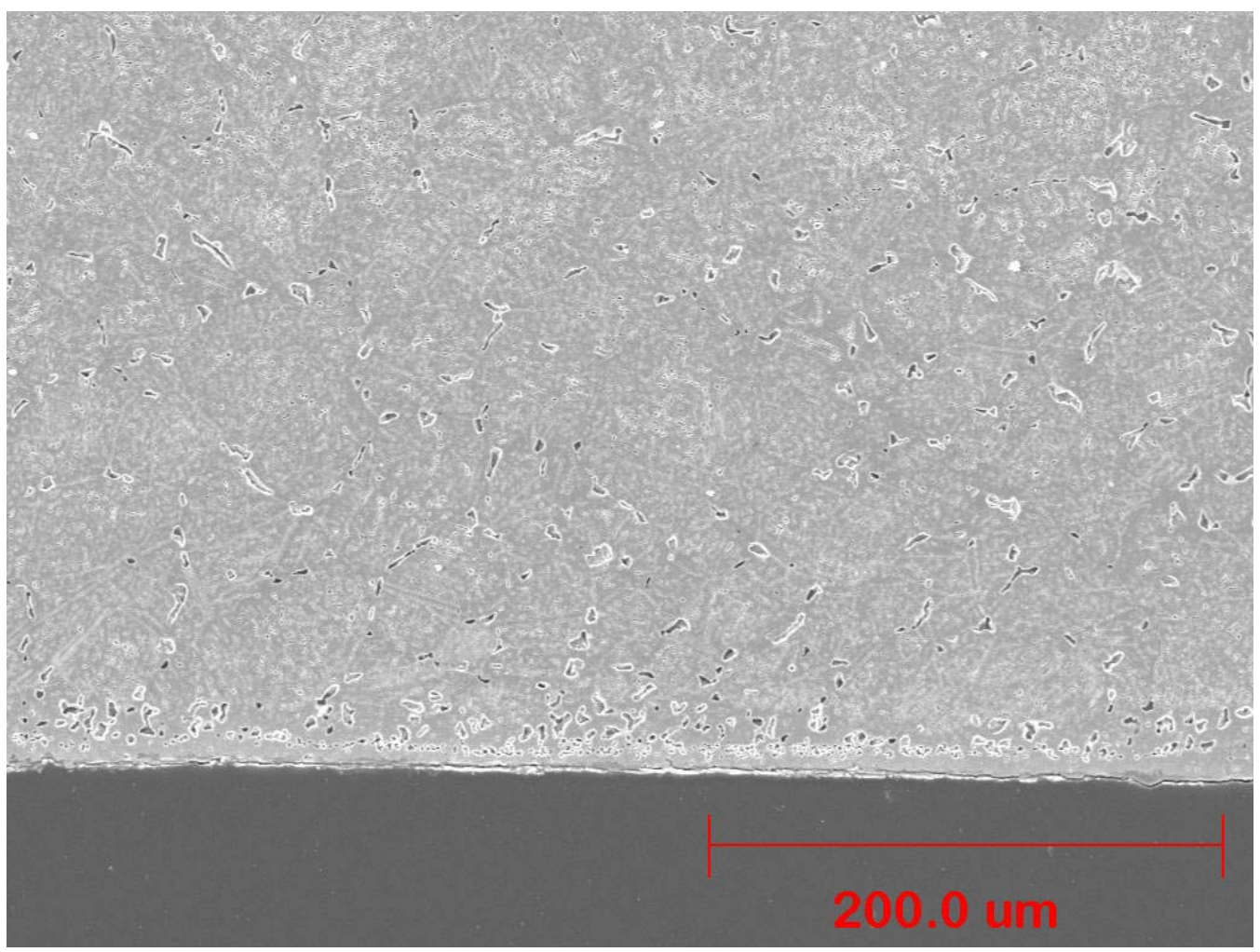

Internal Surface Appearance (SEI - Intermediate Magnification)

Figure G-20: Microstructure: $310 \mathrm{HCbN}$ 


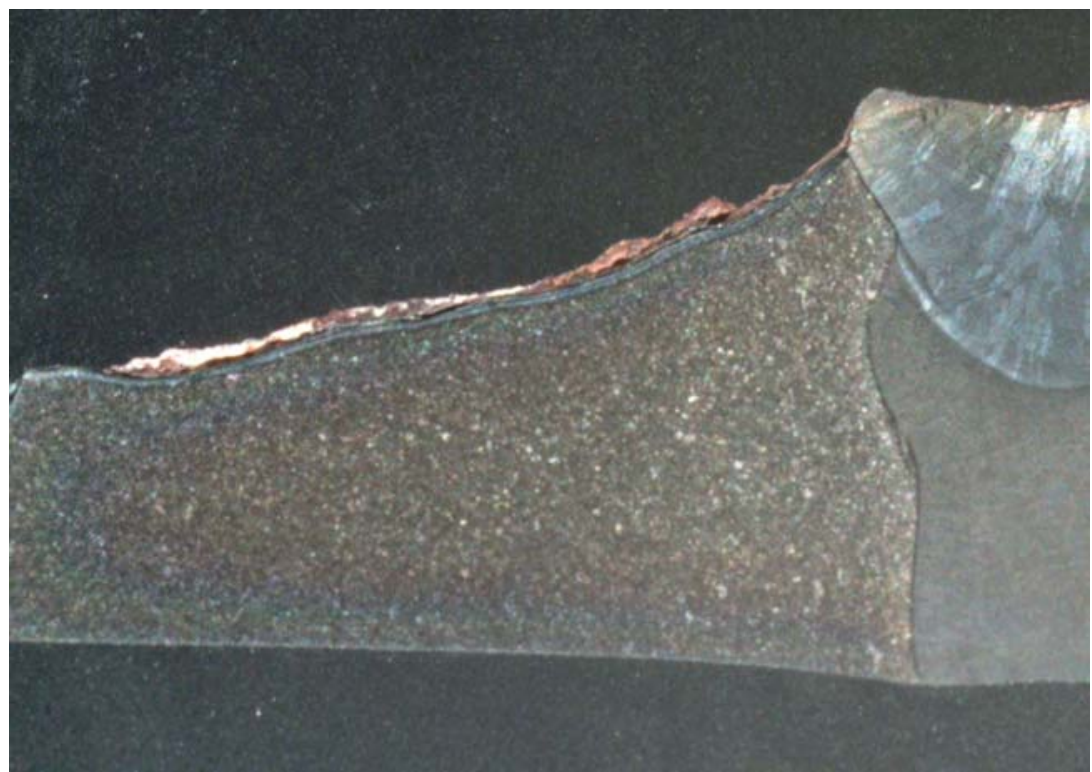

(a) 310 HCbN Adjacent to Weld "N"

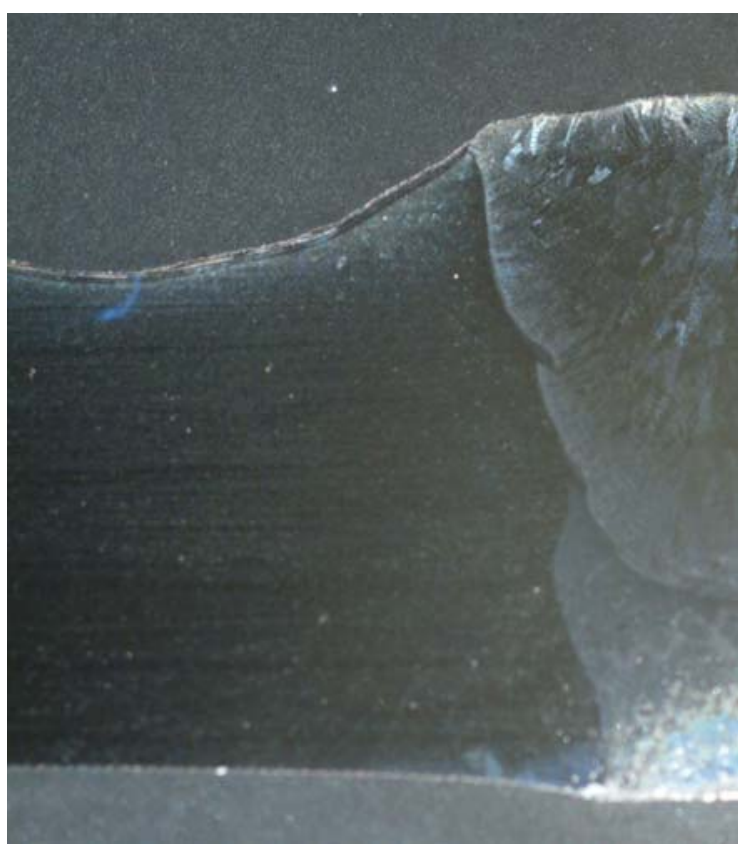

(b) 310 HCbN Adjacent to Weld "X"

Figure G-21: Longitudinal Cross-Sections: $310 \mathrm{HCbN}$ Adjacent to Welds 


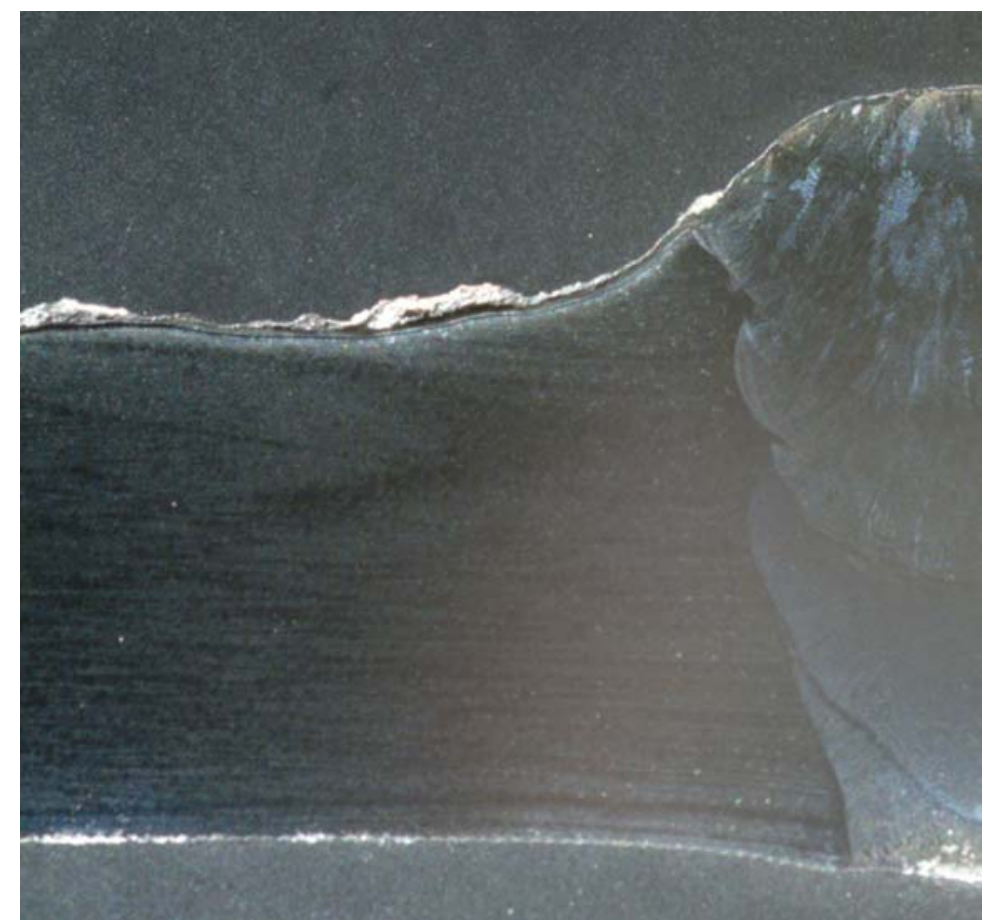

(c) $310 \mathrm{HCbN}$ Adjacent to Weld "BB"

Figure G-21, Continued: Longitudinal Cross-Sections: $310 \mathrm{HCbN}$ Adjacent to Welds 
Figure G-22: EDS Analysis of Deposit/Scale/Metal Interface for 310 HCbN (HR3C) (Sample G-H)

\begin{tabular}{|c|c|c|c|c|c|c|c|c|c|c|c|c|c|c|c|c|c|c|}
\hline Area & Description & $\mathbf{N i}$ & $\mathrm{Cr}$ & $\mathbf{F e}$ & $\mathrm{Cu}$ & Mn & $\mathbf{N b}$ & $\mathbf{T i}$ & Al & $\mathbf{S i}$ & $\mathrm{C}$ & $\mathbf{O}$ & $\mathrm{Ca}$ & $\mathbf{K}$ & $\mathbf{N a}$ & As & $\mathbf{P}$ & $\mathbf{S}$ \\
\hline 1 & $\begin{array}{l}\text { Bulk deposit } \\
\text { analysis }\end{array}$ & 1.03 & 0.57 & 22.5 & -- & 0.14 & -- & 0.36 & 6.77 & 12.5 & 25.3 & 21.6 & 0.16 & 1.08 & -- & 4.96 & 2.00 & 1.07 \\
\hline 2 & $\begin{array}{l}\text { Tightly } \\
\text { adhering } \\
\text { scale.. }\end{array}$ & 18.9 & 20.3 & 42.1 & -- & -- & -- & -- & 0.17 & 0.51 & -- & 13.7 & 0.54 & -- & -- & -- & -- & 3.74 \\
\hline 3 & $\begin{array}{l}\text { Diffusion } \\
\text { zone - dark } \\
\text { etching } \\
\text { adjacent to } \\
\text { interface. }\end{array}$ & 27.3 & 8.68 & 62.7 & -- & 0.71 & - & - & -- & 0.46 & - & -- & 0.22 & - & -- & -- & -- & - \\
\hline 4 & $\begin{array}{l}\text { Diffusion } \\
\text { zone - light } \\
\text { etching. }\end{array}$ & 21.5 & 19.9 & 57.3 & -- & 0.96 & -- & - & -- & 0.31 & -- & -- & -- & -- & -- & -- & -- & -- \\
\hline 5 & $\begin{array}{l}\text { Bulk tube } \\
\text { metal } \\
\text { analysis }\end{array}$ & 21.1 & 22.1 & 55.6 & -- & 0.86 & -- & -- & -- & 0.28 & - & -- & -- & -- & -- & -- & -- & -- \\
\hline & $\begin{array}{l}\text { Reference } \\
\text { bulk analysis. }\end{array}$ & 19.9 & 26.0 & 50.5 & -- & 1.20 & 0.47 & 0.008 & 0.017 & 0.35 & 0.07 & -- & -- & -- & -- & -- & 0.014 & 0.001 \\
\hline
\end{tabular}




\section{Appendix H}

\section{Analysis of Performance}

\section{Test Section C}

HR 120 


\section{Overview of Results: HR 120}

Of the three HR 120 samples in Section C, Sample O-P experienced the highest rate of metal loss (124 mils per year). Given a total exposure period of 2.38 years, this metal loss rate resulted in a total wall loss of 295 mils at one location, i.e. $79 \%$ of the initial wall thickness was lost. This problem was exacerbated in that when the section was being manufactured, the wall thickness was reduced by machining to provide for a weld transition to an adjacent tube that had a thinner wall. The end result was that only a very thin ligament remained. Accordingly, this tube section was removed and replaced for Section B which is continuing in service to gain data beyond the 2.38 years associated with Section $\mathrm{C}$.

It was also noted that, for both Section A and Section C, the sample that saw the lowest exposure temperature, Sample DD-EE had a higher metal loss rate than Sample V-W which saw an intermediate exposure temperature. While limited data is available, it might be postulated that somewhat different mechanisms are active at the low and high temperature locations.

It should be noted that the metal loss rates determined to date for both Section A and Section C all were based on a ring specimen that was extracted from mid-length of each tube sample. In this analysis additional attention was focused on metal losses in the vicinity of welds. Representative samples were taken and it was determined that, for the HR 120 samples evaluated, the Inconel 625 weld filler metal is reasonably resistant to coal ash corrosion. Two cases were noted wherein the wall thickness of the HR 120 was machined thinner to facilitate welding during construction of the test section. These areas must be taken into consideration during future in situ measurements of similarly designed samples.

Analysis of deposit on the surface revealed that it had the known aggressive combination of species, namely iron, oxygen, potassium, and sulfur. Examination and analysis of the scale adjacent to the metal surface revealed the chalky-white appearance associated with alkali-ironsulfates. Thus the required corrosive constituent was available to test the performance limits of this alloy. time at temperature rather than total time within the boiler.
} 
Material: HR 120

Summary of Findings

\begin{tabular}{|c|c|}
\hline Issue & Discussion \\
\hline $\begin{array}{l}\text { Sample } \\
\text { Location and } \\
\text { Orientation. }\end{array}$ & $\begin{array}{l}\text { - The schematic in Figure H-1 shows the relative positions of the } \\
\text { three HR } 120 \text { samples in Test Section C. Note that none of the HR } 120 \\
\text { samples were included in the tube lengths that were removed early and } \\
\text { replaced, i.e. in November } 2001 \text { or in June } 2002 \text {. } \\
\text { - Also, shown is the calculated average surface metal temperature at } \\
\text { each location. }\end{array}$ \\
\hline $\begin{array}{l}\text { Composition } \\
\text { Verification. }\end{array}$ & $\begin{array}{l}\text { - The HR } 120 \text { tubing for this program came from one heat of material } \\
\text { - } \\
\text { Theat } 854487538 \text { ) supplied from Haynes International, Inc. } \\
\text { Haynes literature. It also provides the reported heat analysis. } \\
\text { O It is interesting to note that while the heat supplied met the } \\
\text { constraints of the nominal composition, it was somewhat lean } \\
\text { in alloying element additions: molybdenum (actual } 0.23 \% \\
\text { versus } 2.5 \% \text { max nomimal), cobalt ( } 0.088 \% \text { versus } 3 \% \text { max), } \\
\text { and tungsten (not detectable versus } 2.5 \% \text { max). } \\
\text { - } \\
\text { uuring the Section A evaluation, detailed composition verification was } \\
\text { undertaken for each tube sample that was removed from service. This } \\
\text { served, in part, to verify that the each tube material was located at its' } \\
\text { proper position within the test section. } \\
\text { Based on the good results for Section A, it was decided that it would } \\
\text { be unnecessary to analyze each and every tube sample for composition } \\
\text { verification. Rather, it was decided to select a representative sample } \\
\text { from each tube material type for confirmatory analysis. } \\
\text { During the Section A evaluation, the energy dispersive spectrographic } \\
\text { (EDS) capabilities of the scanning electron microscope (SEM) were } \\
\text { used to perform semi-quantitative analyses of each of the twelve } \\
\text { different candidate tube materials. Comparison of these EDS results } \\
\text { showed reasonably good correlation with results obtained using } \\
\text { quantitative analytical techniques, including optical emission } \\
\text { spectrometry. } \\
\text { Based on this, it was decided that the accuracy provided by EDS } \\
\text { would suffice for composition verification, but that more detailed } \\
\text { analyses would be undertaken if questions arose. } \\
\text { The results of the EDS analysis of tube sample O-P confirmed that the } \\
\text { correct material was used at this location. }\end{array}$ \\
\hline $\begin{array}{l}\text { As-Received } \\
\text { Appearance }\end{array}$ & $\begin{array}{l}\text { - Figure H-3 shows the typical appearance of an HR } 120 \text { tube sample } \\
\text { prior to service. } \\
\text { - Figures H-4 through H-6 document the as-received appearance of the } \\
\text { external surface of the HR } 120 \text { tube samples. Three views are provided } \\
\text { for each tube: } 12 \text { o'clock (the view that faces on-coming gas flow), } 3 \\
\text { o'clock (the view where the gas flow is tangent to the surface), and } 6 \\
\text { o'clock (the view opposite to the gas flow direction). }\end{array}$ \\
\hline
\end{tabular}




\section{Material: HR 120}

\section{Summary of Findings, (continued)}

\begin{tabular}{|c|c|}
\hline Issue & Discussion \\
\hline $\begin{array}{l}\text { As-Received } \\
\text { Appearance, } \\
\text { Continued. }\end{array}$ & $\begin{array}{l}\text { - The reader should be alerted to the fact that, within the boiler, the gas } \\
\text { flows upward over these tubes. This implies that for the "o'clock" } \\
\text { conventions described above, (with the } 12 \text { o'clock orientation facing } \\
\text { the gas flow direction), the actual physical orientation of these tubes is } \\
\text { such that the } 12 \text { o'clock side of the tube faces downward, and the } 6 \\
\text { o'clock orientation faces upward within the boiler. } \\
\text { - Wastage on these tubes is, for the most part, obscured by deposit on } \\
\text { the } 12 \text { o'clock and } 3 \text { o'clock positions. } \\
\text { - The gray/white scale commonly associated with molten alkali-iron } \\
\text { sulfate attack is seen most readily at the } 3 \text { o'clock position in Sample } \\
\text { DD-EE. }\end{array}$ \\
\hline $\begin{array}{l}\text { Grit-Blasted } \\
\text { Surface } \\
\text { Appearance }\end{array}$ & $\begin{array}{l}\text { - Figures H-7 through H-9 show the appearance of the external surface } \\
\text { of the HR } 120 \text { tube samples after grit-blasting to remove deposit and } \\
\text { scale. The } 12 \text { o'clock and } 3 \text { o'clock positions for all three samples } \\
\text { show the appearance of general wastage due to fireside corrosion. } \\
\text { - Pitting was noted on at the } 6 \text { o'clock position for Sample O-P. }\end{array}$ \\
\hline $\begin{array}{l}\text { Wall } \\
\text { Thickness } \\
\text { Loss } \\
\text { Determined }\end{array}$ & $\begin{array}{l}\text { - Tube cross-sections were prepared to allow for measurement of wall } \\
\text { thickness to determine metal loss due to exposure. These are shown in } \\
\text { silhouette in Figures H-10 through H-12. } \\
\text { The same relative "o'clock" positions were maintained as discussed } \\
\text { earlier. Measurements were taken at eight evenly spaced locations } \\
\text { about the tube circumference. These were then compared with } \\
\text { measurements taken at these same locations prior to exposure. } \\
\text { The before and after measurements were documented in the table that } \\
\text { accompanies each figure. These form the basis for the "delta" } \\
\text { calculation to determine the amount of wall thickness lost. Note that } \\
\text { the delta is negative for the } 6 \text { o'clock position for Sample O-P. This is } \\
\text { believed due to wall thickness variability and difficulty in locating the } \\
\text { exact location of the "before" measurement. }\end{array}$ \\
\hline $\begin{array}{l}\text { Wall } \\
\text { Thickness } \\
\text { Loss Versus } \\
\text { Average Metal } \\
\text { Temperature }\end{array}$ & $\begin{array}{l}\text { - The chart in Figure H-13 plots the maximum wall thickness lost } \\
\text { against the calculated average metal temperature for each of the three } \\
\text { HR } 120 \text { samples in Section C. Section A data also are plotted. } \\
\text { Review of these data suggests that the highest temperature sample, } \\
\text { Sample O-P, experienced the greatest rate of metal loss for both } \\
\text { sections. } \\
\text { It also was noted that the rate of metal loss appears to drop off for } \\
\text { Sample V-W which experienced an intermediate exposure } \\
\text { temperature. The reason for this has not been resolved but it suggests } \\
\text { that different corrosion mechanisms are active at the higher and lower } \\
\text { exposure temperatures. }\end{array}$ \\
\hline
\end{tabular}




\section{Material: HR 120}

\section{Summary of Findings, (continued)}

\begin{tabular}{|c|c|}
\hline Issue & Discussion \\
\hline $\begin{array}{l}\text { Wall } \\
\text { Thickness } \\
\text { Loss as a } \\
\text { Function of } \\
\text { Time and } \\
\text { Location }\end{array}$ & $\begin{array}{l}\text { - The chart in Figure H-14 plots metal loss as a function of time for each } \\
\text { sample location for Sections A and C. with samples from Section C } \\
\text { having experienced generally longer exposure times. } \\
\text { - It was noted that metal loss rate increased as a function of time for } \\
\text { Sample O-P at the highest exposure temperature. Likewise it was } \\
\text { noted that the rate decreased with time for Samples V-W and DD-EE. } \\
\text { This suggests that the straight line fit shown in Figure H-14 may } \\
\text { pertain. }\end{array}$ \\
\hline $\begin{array}{l}\text { Metallurgical } \\
\text { Analysis - } \\
\text { SEM }\end{array}$ & $\begin{array}{l}\text { - Sample O-P was selected for a detailed SEM/EDAX assessment of the } \\
\text { constituents and structure in the vicinity of the external and internal } \\
\text { surfaces. This sample was selected based on the fact that it was } \\
\text { experiencing metal wastage at about the maximum rate. The focus of } \\
\text { this investigation was in the region of the tube where the maximum } \\
\text { amount of material was lost. } \\
\text { - Figures H-15a and b show the secondary electron images of the } \\
\text { scale/metal interface for Sample O-P. Six distinct regions are in } \\
\text { evidence. These regions are, starting within the metal and working out: } \\
\text { O Base metal - oxalic acid etch delineates grain boundaries, } \\
\text { carbides and inclusions. } \\
\text { Diffusion zone - light etching - this region is obvious as a } \\
\text { light-appearing band that is parallel to the metal surface, } \\
\text { perhaps see most clearly in Figure H-15b. This band is } \\
\text { approximately one-half grain wide, and affects only those } \\
\text { grains contiguous with the surface. Later EDS analysis will } \\
\text { show that this region is very similar in composition to the bulk } \\
\text { tube, with the possible exception that the sulfur content may } \\
\text { be somewhat increased in this area. } \\
\text { Diffusion zone - dark etching - This constitutes the leading } \\
\text { edge of the grains as they interface with the scale. EDS } \\
\text { analysis later shows that this zone is depleted in chromium } \\
\text { (11.2\% versus roughly 24\%). } \\
\text { - Tightly adherent scale - this is seen continuous along the } \\
\text { surface. EDS analysis will later show that this is } \\
\text { predominantly a chromium-rich oxide with a significant } \\
\text { amount of sulfur present. } \\
\text { Fulk Deposit - The deposit was comprised of oxides of iron, } \\
\text { Figure H-16 shows the appearance of the mid-wall microstructure for } \\
\text { silicon, and aluminum. Arsenic was noted, as were sulfur and } \\
\text { possibly carbon. } \\
\text { appears to be coated with a } 1 \text { mil thick oxide. }\end{array}$ \\
\hline
\end{tabular}




\section{Summary of Findings, (continued)}

\section{Material: $\quad$ HR 120}

\begin{tabular}{|c|c|}
\hline Issue & Discussion \\
\hline $\begin{array}{l}\text { Metallurgical } \\
\text { Analysis - } \\
\text { Weld Cross } \\
\text { Section. }\end{array}$ & $\begin{array}{l}\text { - Figure H-18 shows a longitudinal profile (at the } 12 \text { o'clock } \\
\text { orientation) of the HR } 120 \text { samples adjacent to the welds in Section C. } \\
\text { Three welds were cross sectioned. These were identified as "P", "V" } \\
\text { and "DD" in the schematic shown in Figure H-1. } \\
\text { An Inconel } 625 \text { weld metal was used throughout Section C. The cross } \\
\text { sections show that the HR } 120 \text { heat affected zones are not } \\
\text { preferentially attached, in fact, the wall thickness for the tube sample } \\
\text { tapers from the weld toward the middle of the tube length. } \\
\text { An important point to notice is that welds "P" and "V" both joint HR } \\
120 \text { to Incoclad } 671 \text { which had a thinner wall thickness (nominal } 260 \\
\text { mils vs nominal } 375 \text { mils). In order to complete this joint successfully } \\
\text { the wall thickness of the HR } 120 \text { tube had to be reduced considerably. } \\
\text { This accounts for the weld cross section appearance in Figures H-18a } \\
\text { and b. It is important to note from this that only a thin ligament } \\
\text { remained for Sample O-P at the time that it was removed from service. } \\
\text { This fact should be kept in mind during in situ measurements to } \\
\text { determine the remaining wall thickness of similarly constructed test } \\
\text { sections. }\end{array}$ \\
\hline $\begin{array}{l}\text { EDS Analysis } \\
\text { Results }\end{array}$ & $\begin{array}{l}\text { - The table in Figure H-19 provides a summary of the EDAX analyses } \\
\text { of the numbered in Figure H-15b. Also shown in this table is a } \\
\text { summary of the spectrographic analysis reported earlier, Figure H-2. } \\
\text { Review of this table suggests the following: } \\
\text { - Area 1: Bulk deposit/scale analysis - Potassium is present, as are } \\
\text { sulfur and phosphorous. An iron oxide is likely, as are aluminum, } \\
\text { silicon and titanium oxides. The analysis suggests that the carbon } \\
\text { content is relatively high, however, these results need to be } \\
\text { considered carefully given the inherent inaccuracies in EDS } \\
\text { carbon analysis. } \\
\text { - Area 2: Loosely adhering deposit/scale - This appears to be } \\
\text { chromium-, iron-, and nickel-rich oxide. Sulfur is present. } \\
\text { - Area 3: Tightly adhering scale - This is the chromium-rich scale } \\
\text { that is immediately adjacent to the metal surface. Again high } \\
\text { sulfur is indicated. } \\
\text { - Area 4: Diffusion zone - dark etching - This region that abuts } \\
\text { the tightly adhering scale is depleted in chromium. } \\
\text { Area 5: Diffusion zone - light etching - The presence of a } \\
\text { significant amount of sulfur is the only thing that distinguishes } \\
\text { this region from the bulk tube metal.. } \\
\text { Bulk EDS analysis - This analysis compares well with the } \\
\text { reference spectrographic analysis. }\end{array}$ \\
\hline
\end{tabular}




\section{Gas Flow}

Direction

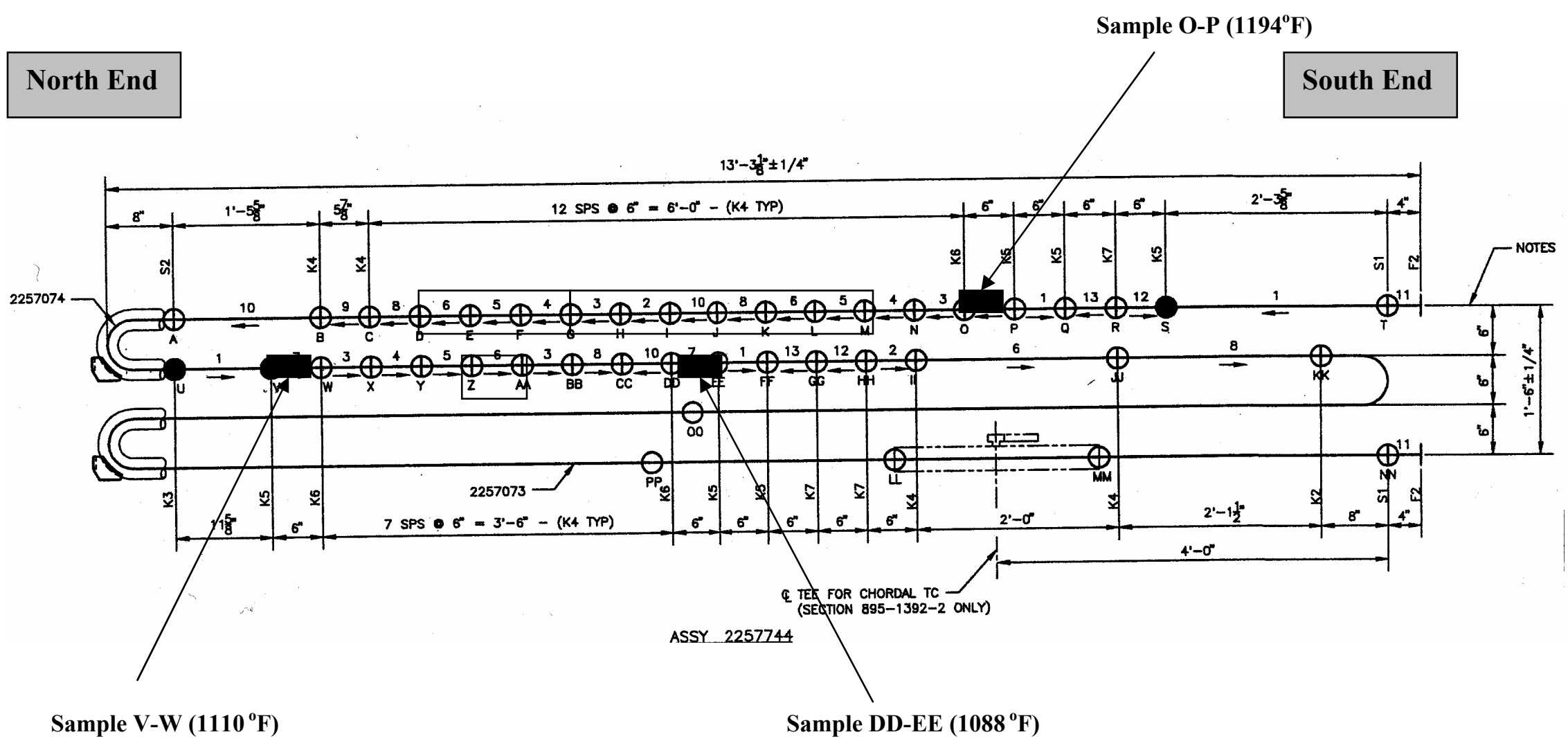

Figure H-1: Schematic Drawing Showing Position of HR 120 Tube Samples Within Test Section C

1. Drawing shown in same relative orientation as per the actual installation.

2. This group of tube samples was removed from service in November 2001 - highlighted in red.

3. This group of tube samples was removed from service in June 2002 - highlighted in yellow. 
Figure H-2 Semi-Quantitative Composition Verification for HR 120

Description:

Source:

Reference Composition:
Nominal $33 \mathrm{Fe} / 25 \mathrm{Cr} / 37 \mathrm{Ni}$ alloy with $\mathrm{Co}, \mathrm{W}$, and $\mathrm{Nb}$ added to provide creep strength.

Haynes International, Inc.

"Nominal" chemistry - per Haynes literature.

\begin{tabular}{|c|c|c|c|c|c|}
\hline Element & $\begin{array}{c}\text { Reference } \\
\text { Composition }\end{array}$ & $\begin{array}{c}\text { Composition } \\
\text { Heat } 854487538\end{array}$ & $\begin{array}{c}\text { Tube Sample } \\
\text { O-P }\end{array}$ & $\begin{array}{c}\text { Tube Sample } \\
\text { V-W }\end{array}$ & $\begin{array}{c}\text { Tube Sample } \\
\text { DD-EE }\end{array}$ \\
\hline $\mathrm{Ni}$ & 37 & 37.61 & 38.2 & $\mathrm{~N} / \mathrm{A}$ & $\mathrm{N} / \mathrm{A}$ \\
\hline $\mathrm{Cr}$ & 25 & 25.28 & 24.1 & N/A & N/A \\
\hline $\mathrm{Fe}$ & Balance & Balance & 36.5 & N/A & N/A \\
\hline Mo & $2.5 \max$ & 0.33 & - & $\mathrm{N} / \mathrm{A}$ & N/A \\
\hline $\mathrm{Co}$ & $3 \max$ & 0.09 & - & N/A & N/A \\
\hline $\mathrm{C}$ & 0.05 & 0.06 & - & $\mathrm{N} / \mathrm{A}$ & N/A \\
\hline $\mathrm{N}$ & 0.2 & 0.20 & - & N/A & N/A \\
\hline $\mathrm{B}$ & 0.004 & $<0.002$ & - & N/A & N/A \\
\hline $\mathrm{Mn}$ & 0.7 & 0.68 & 0.46 & $\mathrm{~N} / \mathrm{A}$ & $\mathrm{N} / \mathrm{A}$ \\
\hline $\mathrm{Si}$ & 0.6 & 0.52 & 0.65 & $\mathrm{~N} / \mathrm{A}$ & $\mathrm{N} / \mathrm{A}$ \\
\hline $\mathrm{Al}$ & 0.1 & 0.06 & - & N/A & N/A \\
\hline $\mathrm{Ti}$ & - & 0.02 & - & N/A & N/A \\
\hline $\mathrm{Al}+\mathrm{Ti}$ & - & - & - & N/A & N/A \\
\hline $\mathrm{Nb}$ & 0.7 & 0.62 & - & N/A & N/A \\
\hline $\mathrm{Ta}$ & - & - & - & N/A & N/A \\
\hline $\mathrm{Nb}+\mathrm{Ta}$ & - & - & - & $\mathrm{N} / \mathrm{A}$ & $\mathrm{N} / \mathrm{A}$ \\
\hline $\mathrm{V}$ & - & - & - & N/A & N/A \\
\hline $\mathrm{W}$ & $2.5 \max$ & $<0.10$ & - & $\mathrm{N} / \mathrm{A}$ & N/A \\
\hline $\mathrm{Cu}$ & - & 0.10 & - & $\mathrm{N} / \mathrm{A}$ & N/A \\
\hline $\mathrm{Sn}$ & - & - & - & N/A & N/A \\
\hline $\mathrm{P}$ & - & 0.016 & - & N/A & N/A \\
\hline $\mathrm{S}$ & - & $<0.002$ & - & N/A & N/A \\
\hline $\mathrm{O} 2$ & - & - & - & N/A & N/A \\
\hline
\end{tabular}

N/A = Sample not analyzed. 


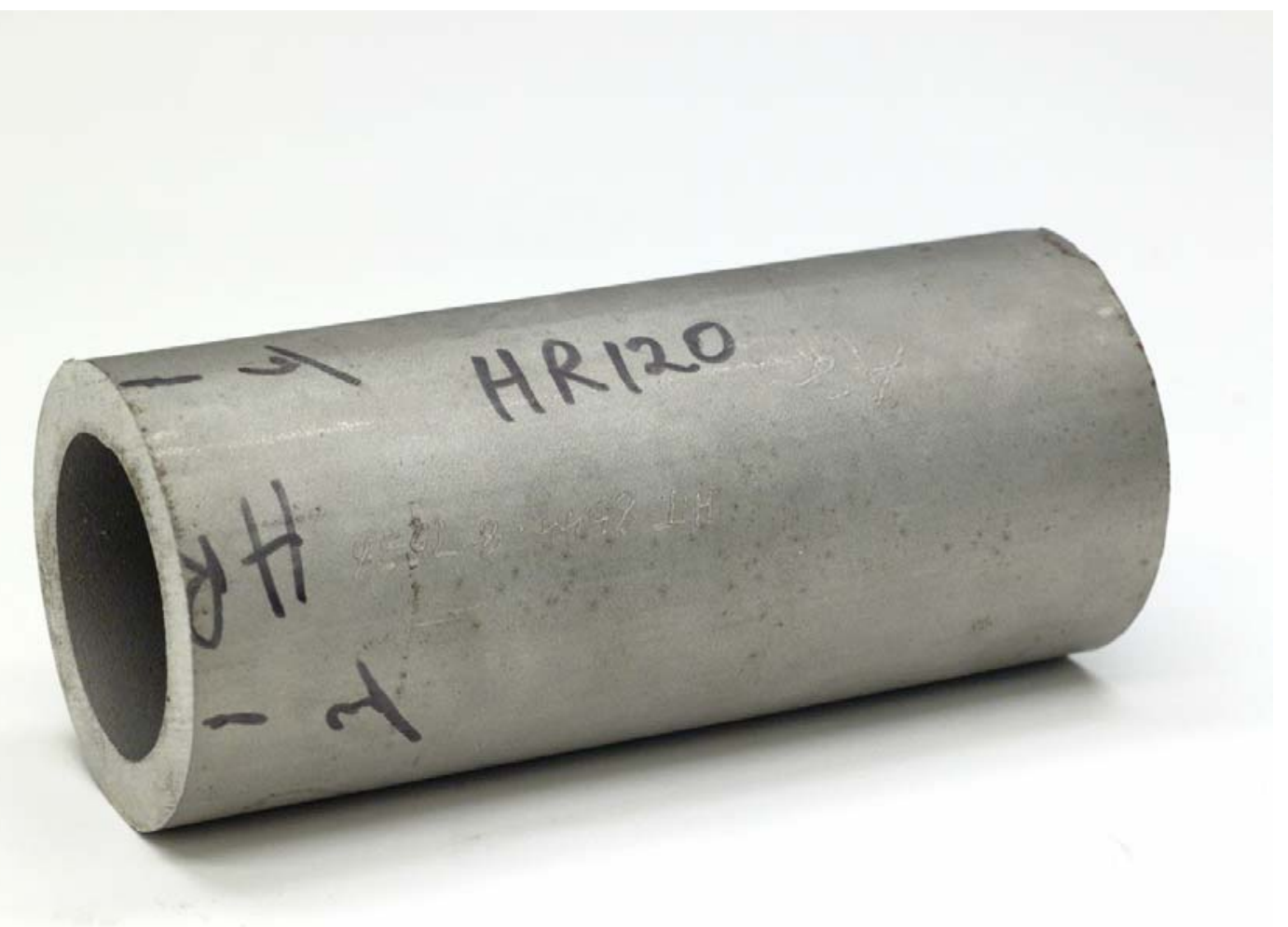

Figure H-3: Appearance of HR 120 Tube Sample Prior to Service 


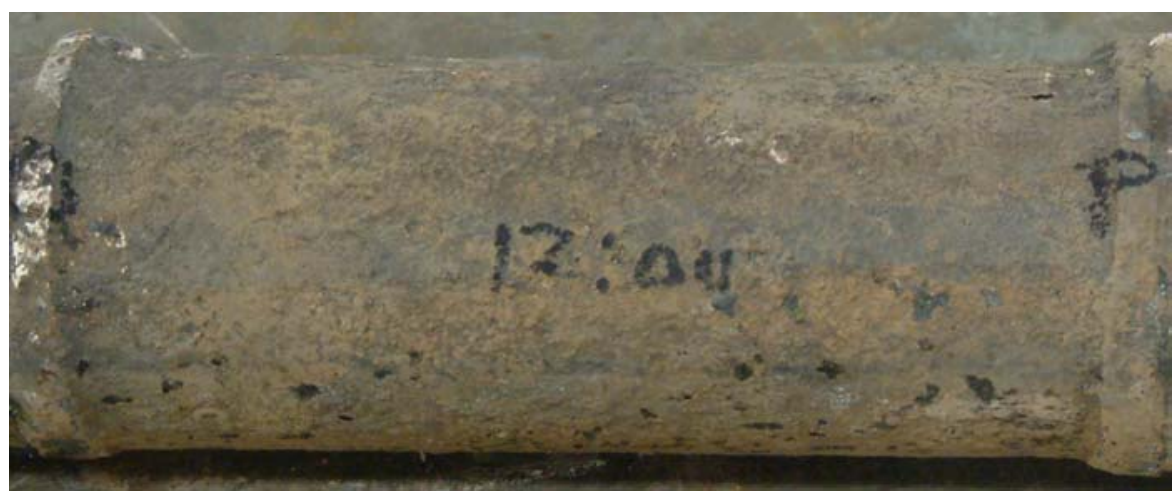

12 O'Clock - Facing Gas Flow

(Note: 3 o'clock is up in this image)

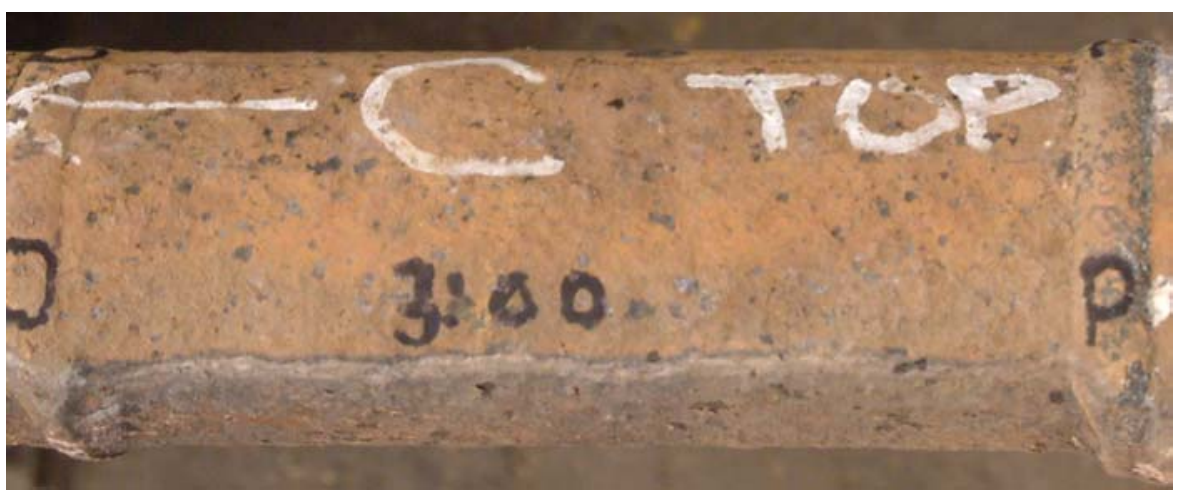

3 O'Clock

(Note: 6 o'clock is up in this image)

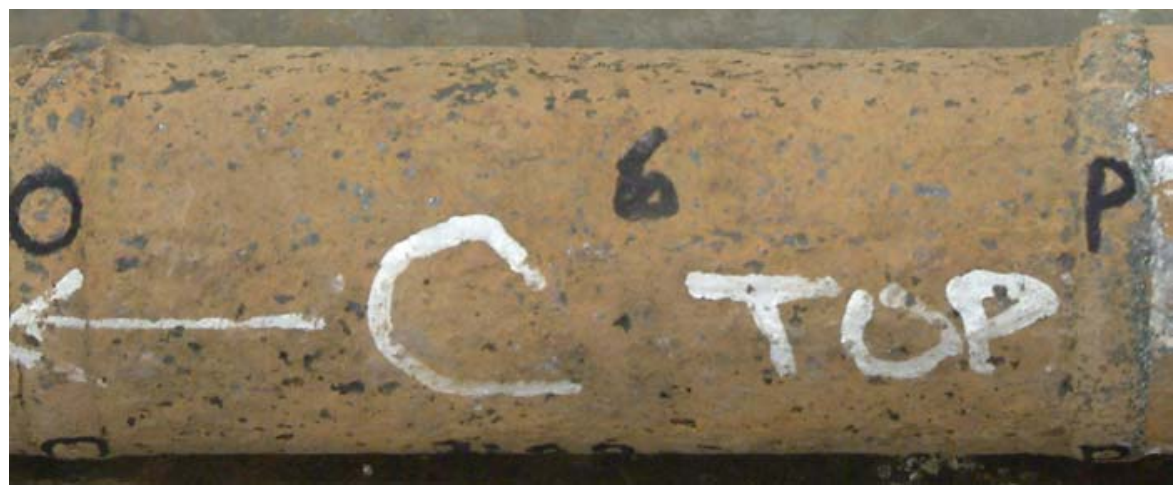

6 O'Clock - Opposite Gas Flow

Figure H-4 As-Received Appearance of Sample O-P (HR 120)

(Average surface metal temperature: $1194^{\circ} \mathrm{F}$ ) 


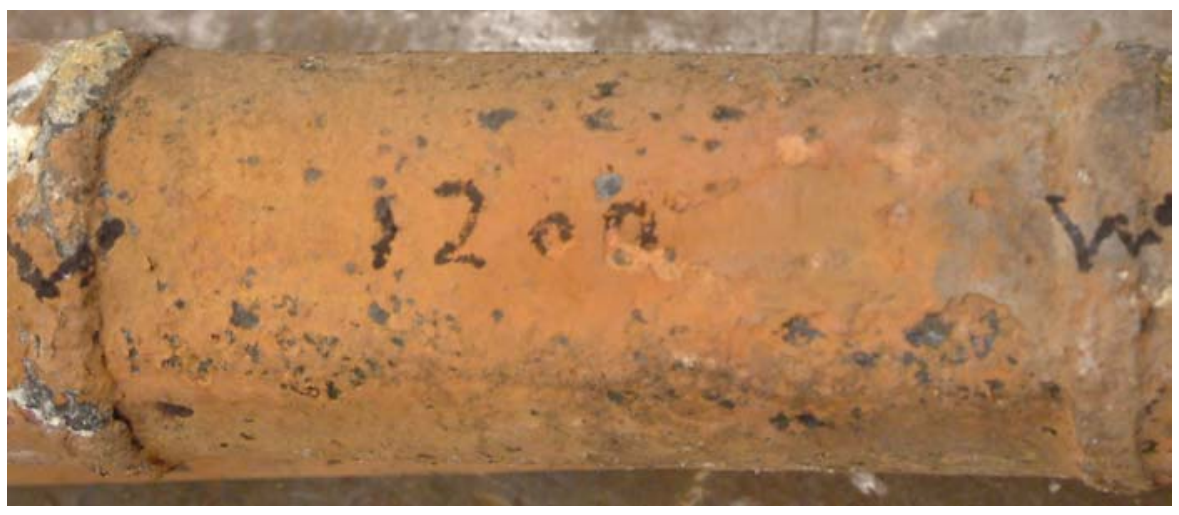

12 O'Clock - Facing Gas Flow

(Note: 3 o'clock is up in this image)

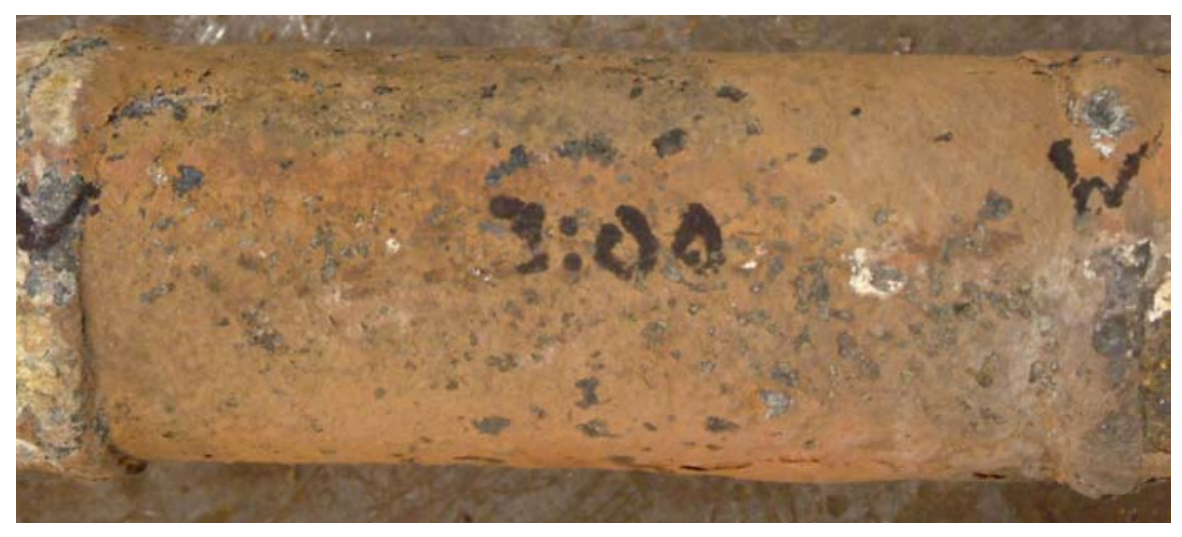

3 O'Clock

(Note: 6 o'clock is up in this image)

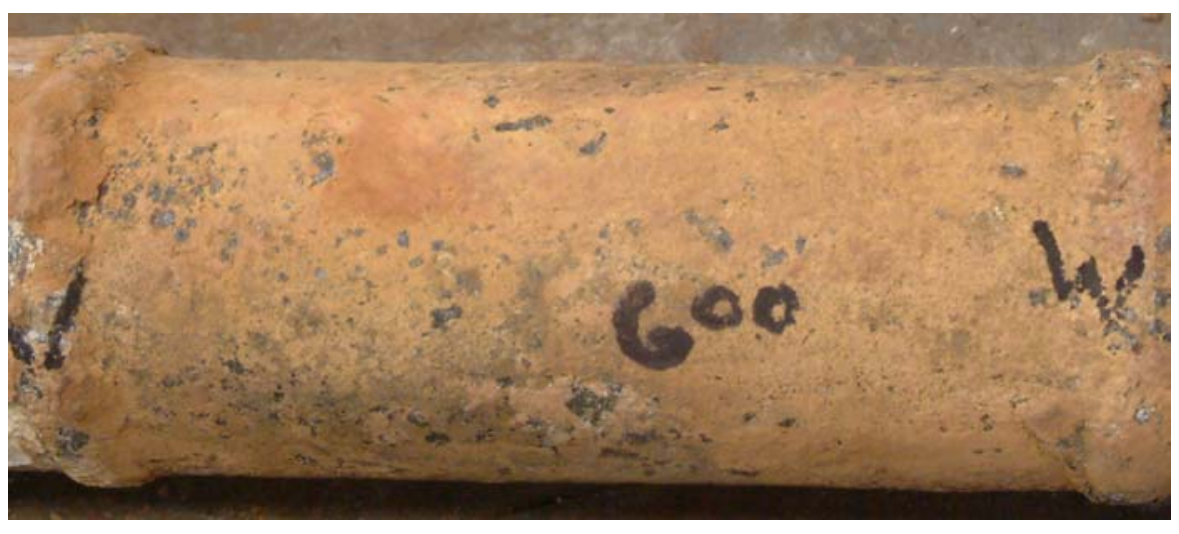

6 O'Clock - Opposite Gas Flow

Figure H-5 As-Received Appearance of Sample V-W (HR 120)

(Average surface metal temperature: $1110^{\circ} \mathrm{F}$ ) 


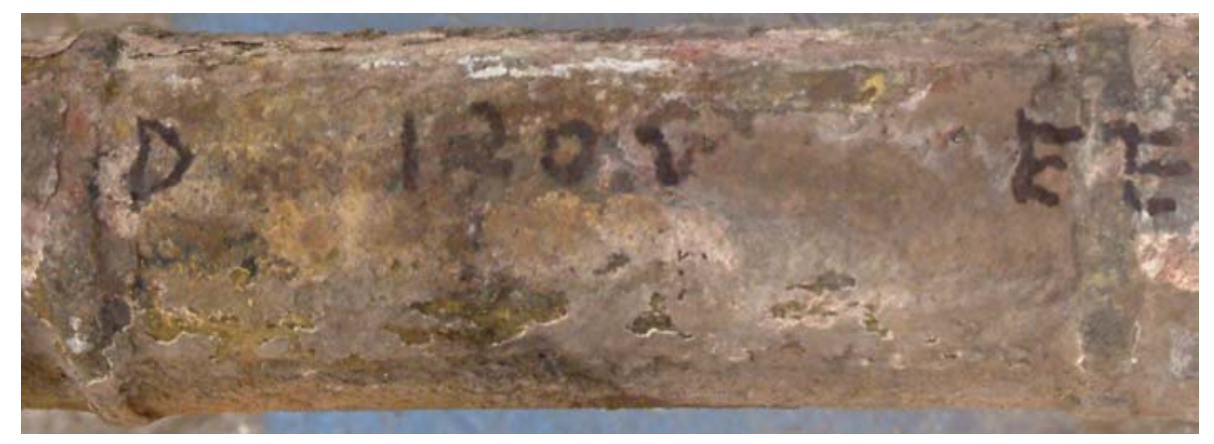

12 O'Clock - Facing Gas Flow

(Note: 3 o'clock is up in this image)

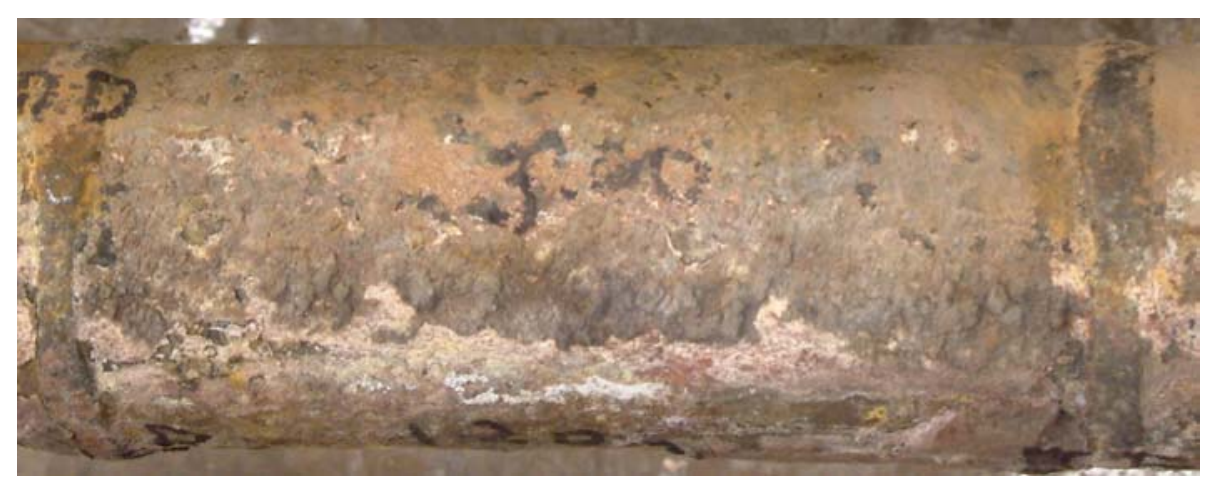

3 O'Clock

(Note: 6 o'clock is up in this image)

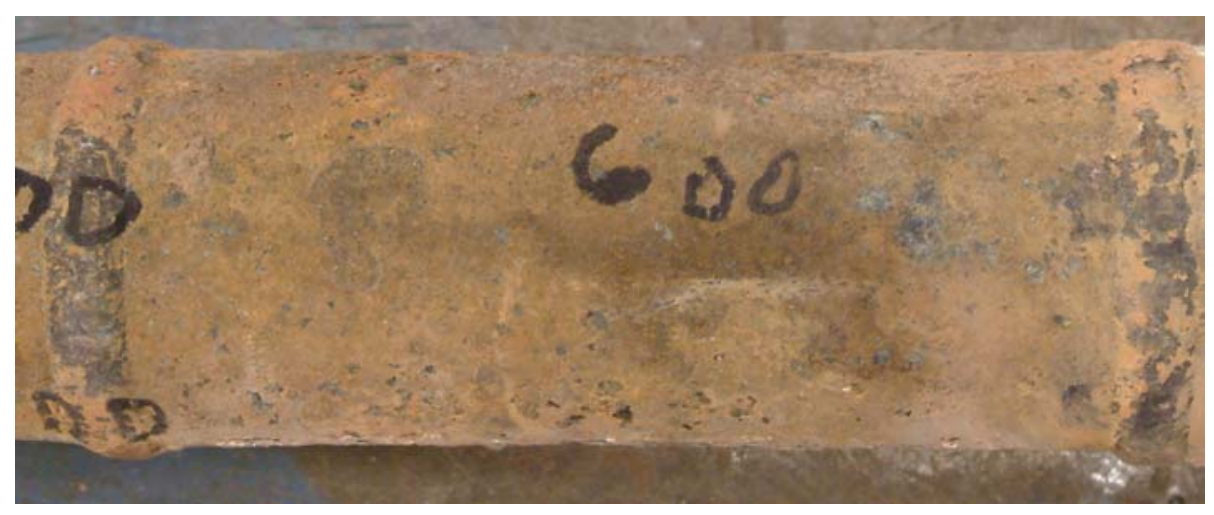

6 O'Clock - Opposite Gas Flow

Figure H-6 As-Received Appearance of Sample DD-EE (HR 120)

(Average surface metal temperature: $1088^{\circ} \mathrm{F}$ ) 
12 O'Clock

(204 mils lost)

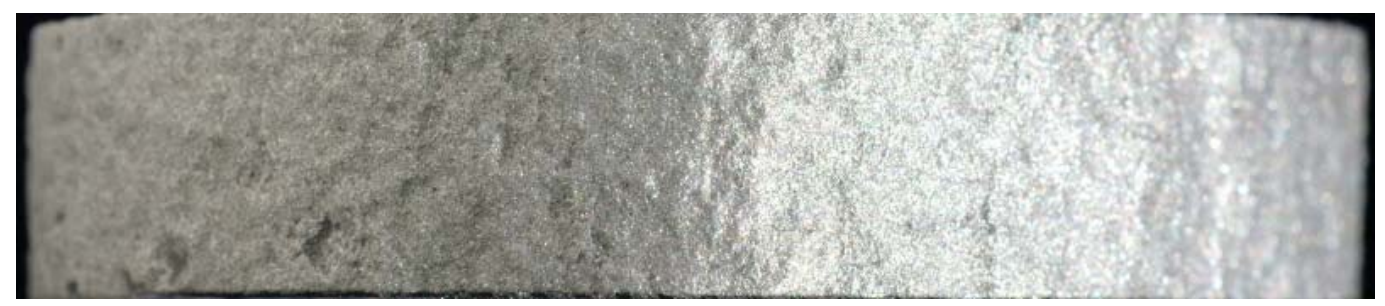

3 O'Clock

(208 mils lost)

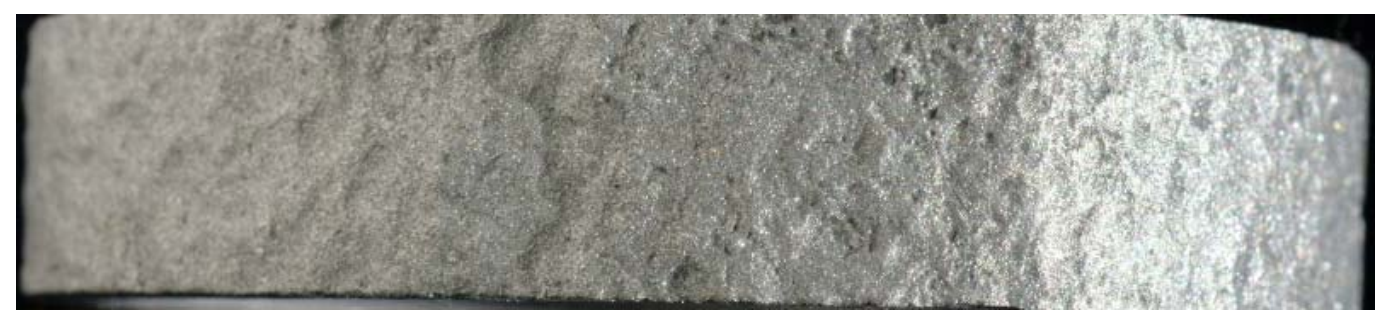

6 O'Clock

(0 mils lost)

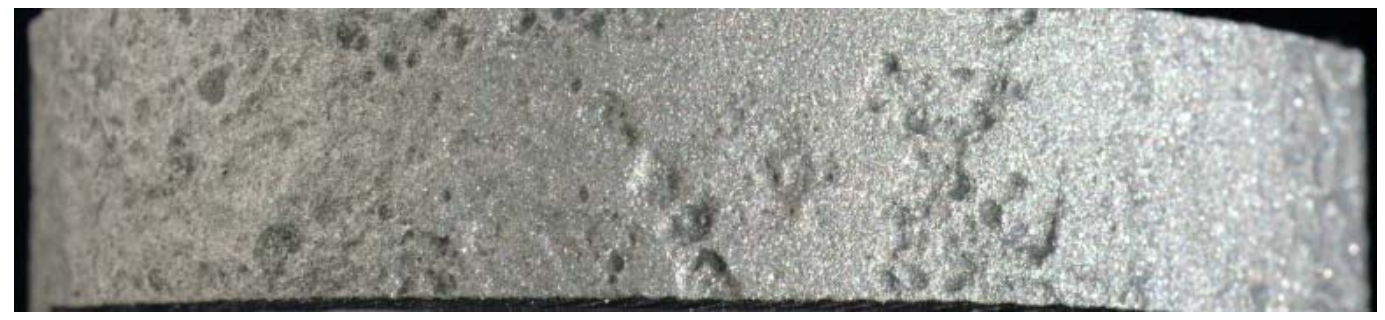

Figure H-7: OD Surface Appearance After Grit Blast

Material: HR 120

Sample: O-P 
12 O'Clock

(76 mils lost)

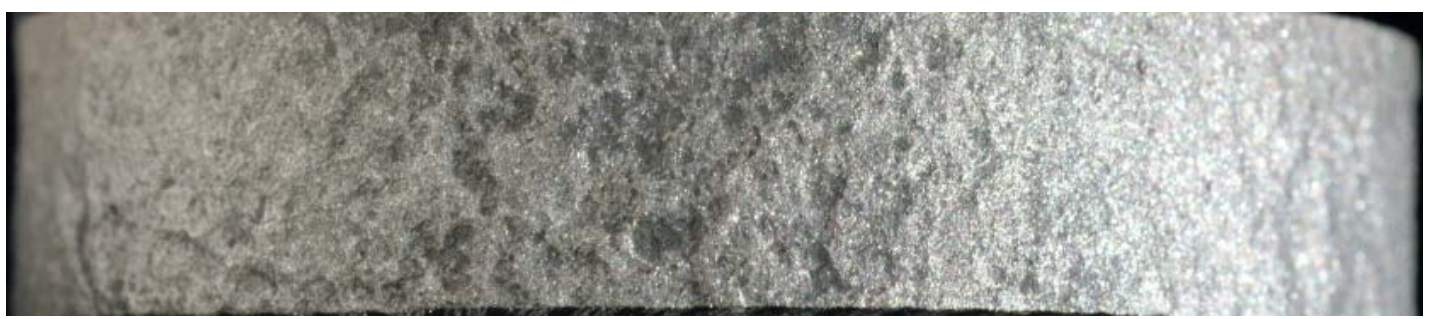

3 O'Clock

(43 mils lost)

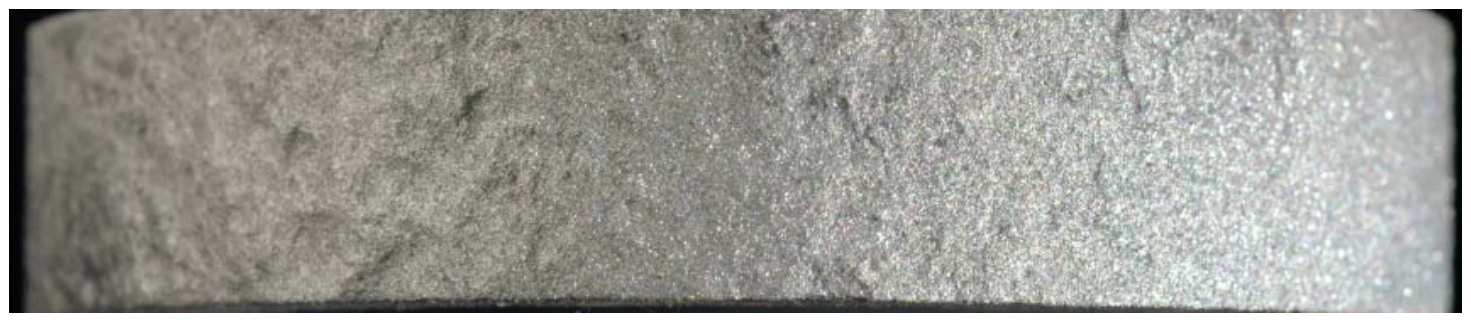

6 O'Clock

(2 mils lost)

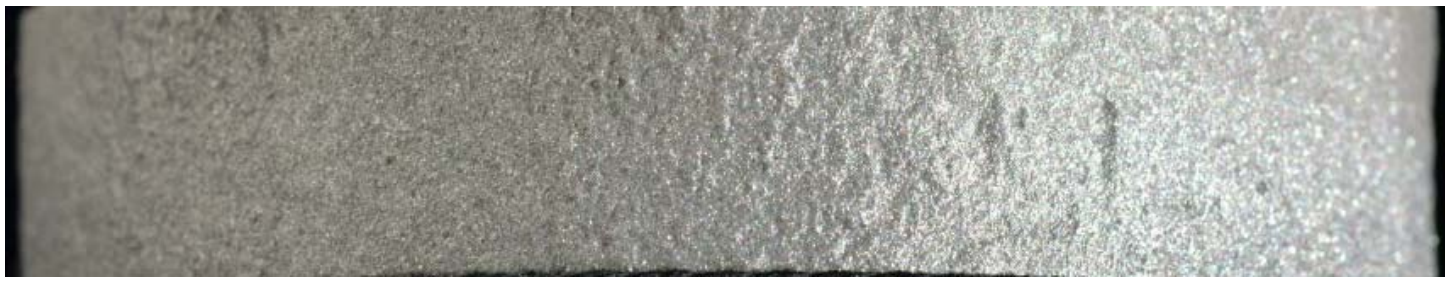

Figure H-8: OD Surface Appearance After Grit Blast

Material: HR 120

Sample: V-W 
12 O'Clock

(131 mils lost)

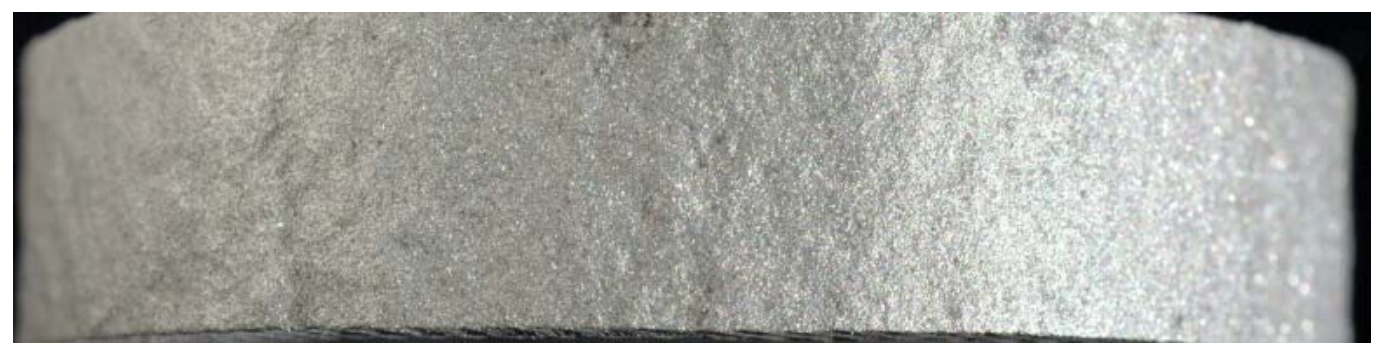

3 O'Clock

(177 mils lost)

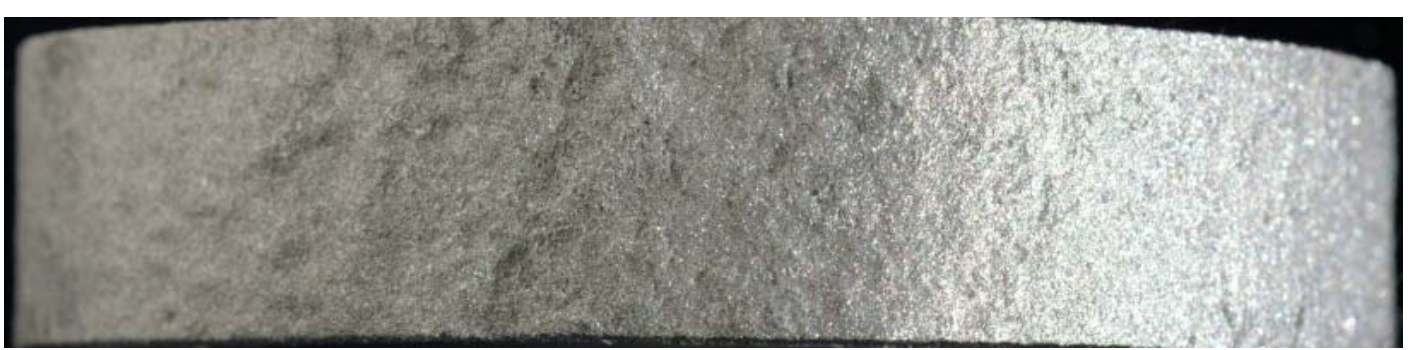

6 O'Clock

(11 mils lost)

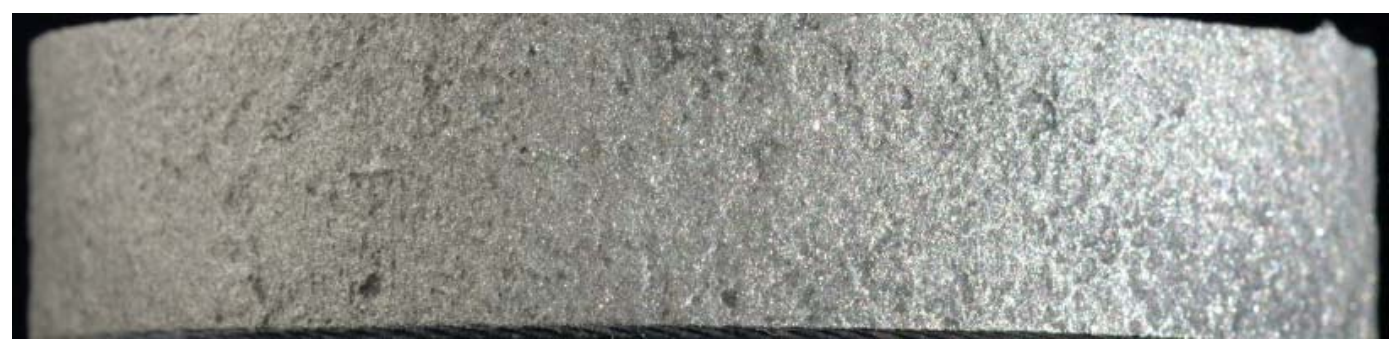

Figure H-9: OD Surface Appearance After Grit Blast

\author{
Material: HR 120 \\ Sample: DD-EE
}




\section{Material:}

Sample Location:

Maximum Rate:

Maximum Loss Orientation:

Average Surface Metal Temperature:
HR 120

O-P

$124 \mathrm{mils} / \mathrm{yr}$

Approx. 1:30 o'clock

$1194{ }^{\circ} \mathrm{F}$

\section{O’Clock}

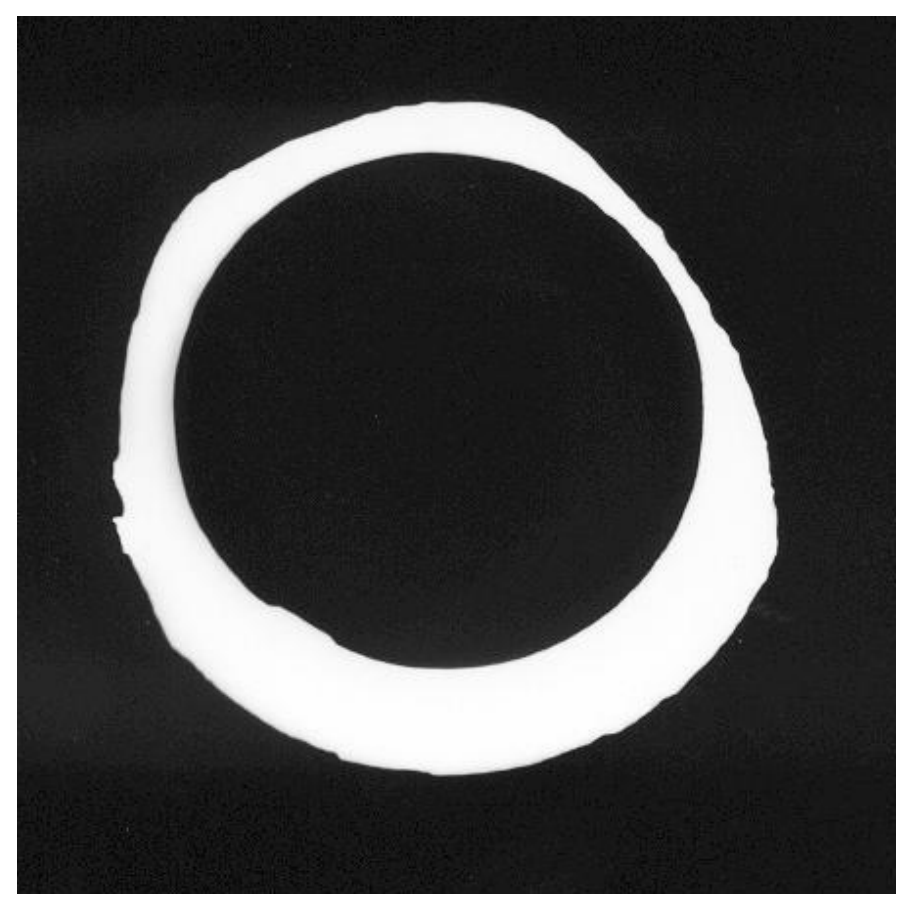

3 O'Clock

Approx. 1.2X

Wall Reduction by Position (in.)

\begin{tabular}{|l|c|c|c|c|c|c|c|c|c|c|}
\hline & $\begin{array}{c}\mathbf{1 2} \\
\text { o'clock }\end{array}$ & $\mathbf{1 : 3 0}$ & $\mathbf{3 : 0 0}$ & $\mathbf{4 : 3 0}$ & $\mathbf{6 : 0 0}$ & $\mathbf{7 : 3 0}$ & $\mathbf{9 : 0 0}$ & $\mathbf{1 0 : 3 0}$ & AVG & $\begin{array}{c}\text { Max } \\
\text { Loss }\end{array}$ \\
\hline Before & 0.374 & 0.376 & 0.378 & 0.377 & 0.374 & 0.375 & 0.378 & 0.376 & 0.376 & 0.376 \\
\hline After & 0.170 & 0.081 & 0.170 & 0.363 & 0.376 & 0.362 & 0.249 & 0.229 & & 0.081 \\
\hline Delta & 0.204 & 0.295 & 0.208 & 0.014 & -0.002 & 0.013 & 0.129 & 0.147 & & 0.295 \\
\hline
\end{tabular}

Figure H-10: Silhouette and Wall Thickness Measurements for Sample O-P 


\section{Material:}

Sample Location:

Maximum Rate:

Maximum Loss Orientation:

Average Surface Metal Temperature:
HR 120

V-W

46 mils/yr

Approx. 9:30 o'clock

$1110^{\circ} \mathrm{F}$

\section{O’Clock}

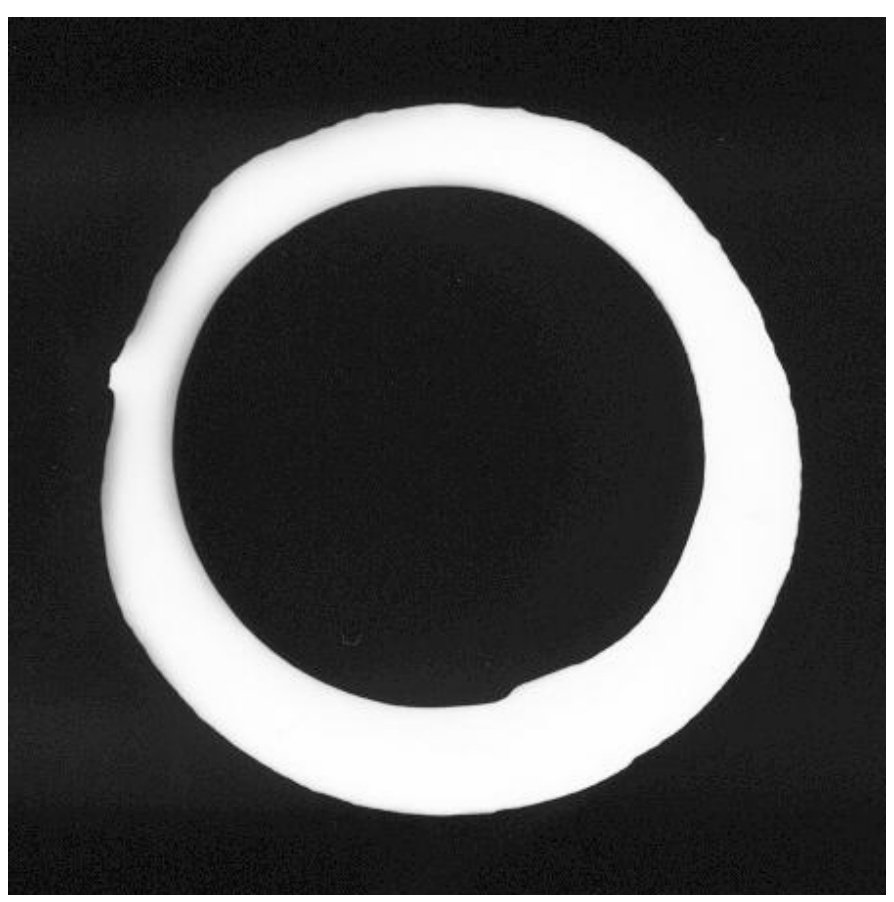

3 O'Clock

Approx. 1.2X

\section{Wall Reduction by Position (in.)}

\begin{tabular}{|l|c|c|c|c|c|c|c|c|c|c|}
\hline & $\begin{array}{c}\mathbf{1 2} \\
\text { o'clock }\end{array}$ & $\mathbf{1 : 3 0}$ & $\mathbf{3 : 0 0}$ & $\mathbf{4 : 3 0}$ & $\mathbf{6 : 0 0}$ & $\mathbf{7 : 3 0}$ & $\mathbf{9 : 0 0}$ & $\mathbf{1 0 : 3 0}$ & AVG & $\begin{array}{c}\text { Max }^{*} \\
\text { Loss }\end{array}$ \\
\hline Before & 0.375 & 0.376 & 0.371 & 0.372 & 0.379 & 0.379 & 0.377 & 0.375 & 0.376 & 0.376 \\
\hline After & 0.299 & 0.297 & 0.328 & 0.367 & 0.377 & 0.372 & 0.299 & 0.314 & & 0.267 \\
\hline Delta & 0.076 & 0.079 & 0.043 & 0.005 & 0.002 & 0.007 & 0.078 & 0.061 & & 0.109 \\
\hline
\end{tabular}

Figure H-11: Silhouette and Wall Thickness Measurements for Sample V-W

* Measurement taken at "maximum loss orientation" noted above. The average

"before" wall thickness was used to calculate the "delta". 


\section{Material:}

Sample Location:

Maximum Rate:

Maximum Loss Orientation:

Average Surface Metal Temperature:
HR 120

DD-EE

79 mils/yr

Approx. 10:00 o'clock

$1088^{\circ} \mathrm{F}$

\section{O’Clock}

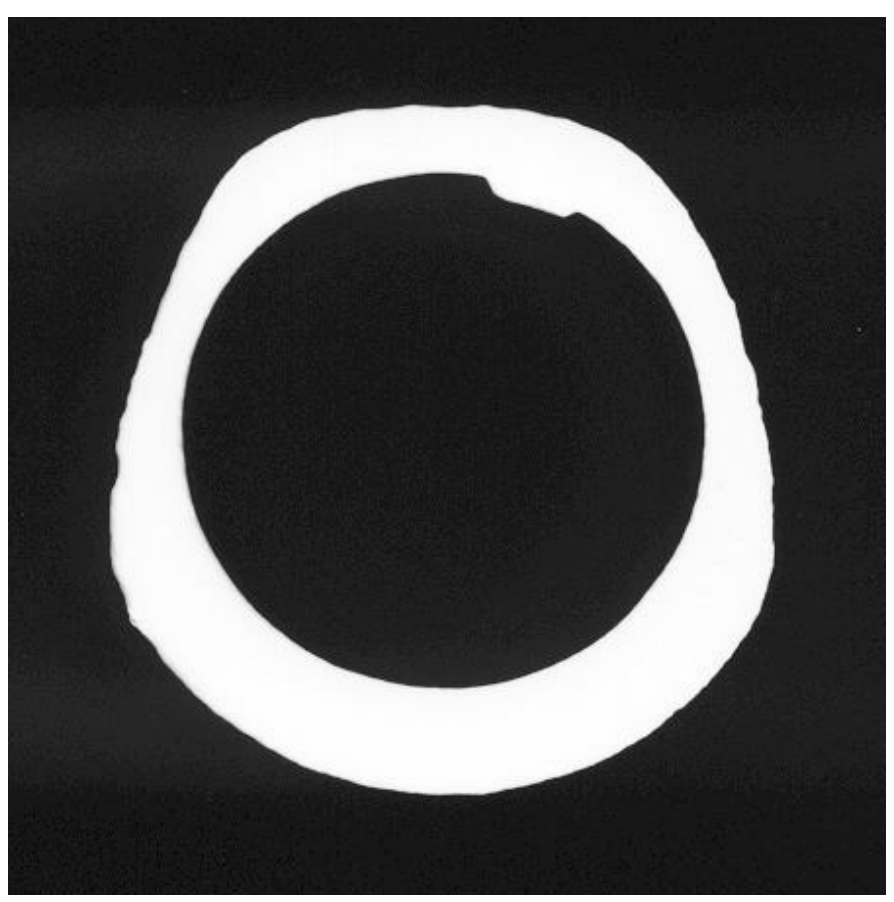

3 O'Clock

Approx. 1.2X

\section{Wall Reduction by Position (in.)}

\begin{tabular}{|l|c|c|c|c|c|c|c|c|c|c|}
\hline & $\begin{array}{c}\mathbf{1 2} \\
\text { o'clock }\end{array}$ & $\mathbf{1 : 3 0}$ & $\mathbf{3 : 0 0}$ & $\mathbf{4 : 3 0}$ & $\mathbf{6 : 0 0}$ & $\mathbf{7 : 3 0}$ & $\mathbf{9 : 0 0}$ & $\mathbf{1 0 : 3 0}$ & AVG & $\begin{array}{c}\text { Max } \\
\text { Loss }\end{array}$ \\
\hline Before & 0.372 & 0.375 & 0.374 & 0.379 & 0.375 & 0.374 & 0.371 & 0.374 & 0.374 & 0.374 \\
\hline After & 0.241 & 0.271 & 0.197 & 0.366 & 0.364 & 0.364 & 0.249 & 0.225 & & 0.186 \\
\hline Delta & 0.131 & 0.104 & 0.177 & 0.013 & 0.011 & 0.010 & 0.122 & 0.149 & & 0.188 \\
\hline
\end{tabular}

Figure H-12: Silhouette and Wall Thickness Measurements for Sample DD-EE

* Measurement taken at "maximum loss orientation" noted above. The average

"before" wall thickness was used to calculate the "delta". 


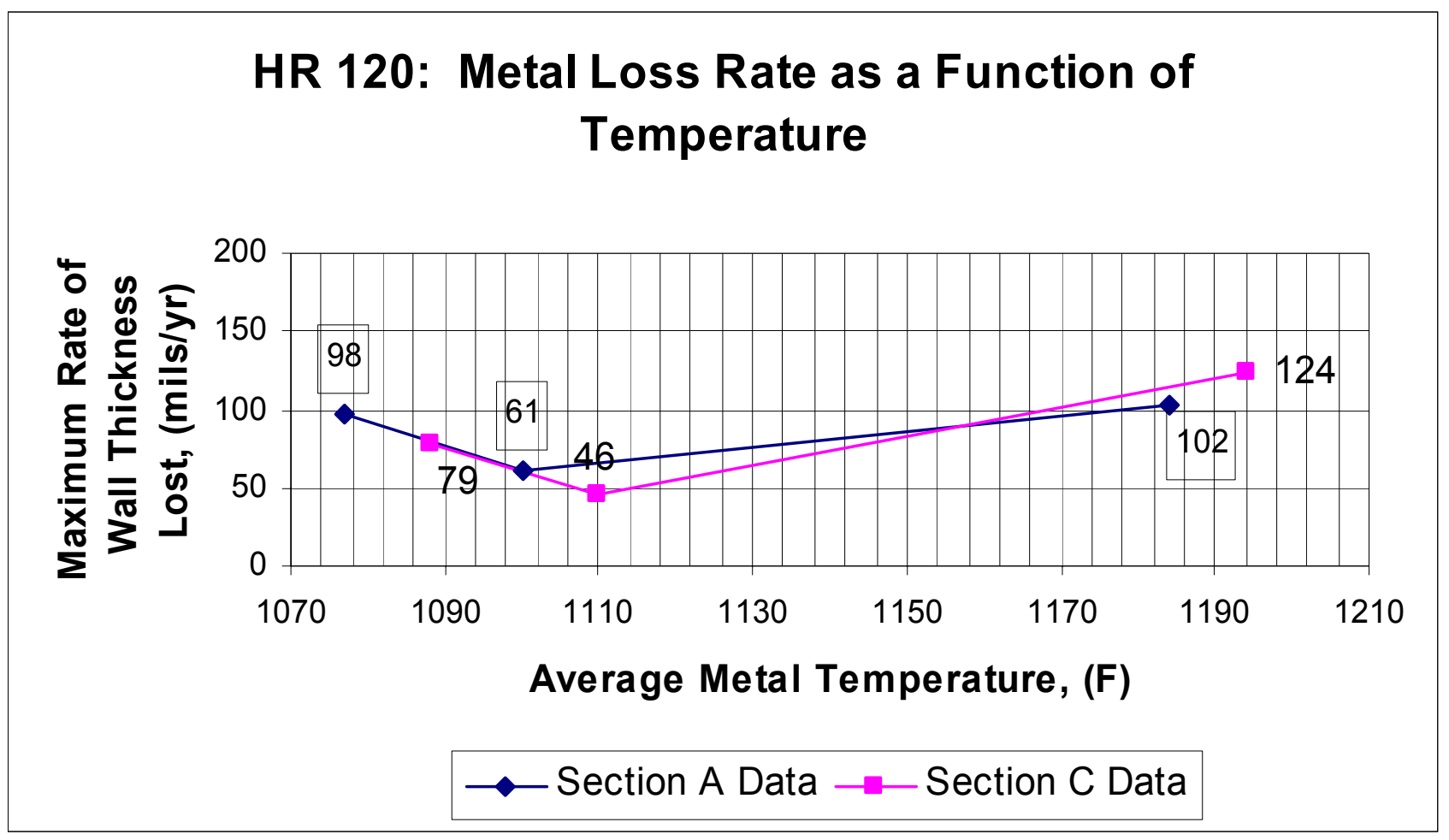

Figure H-13: Metal Loss Rate as a Function of Average Surface Metal Temperature

Material: HR 120 


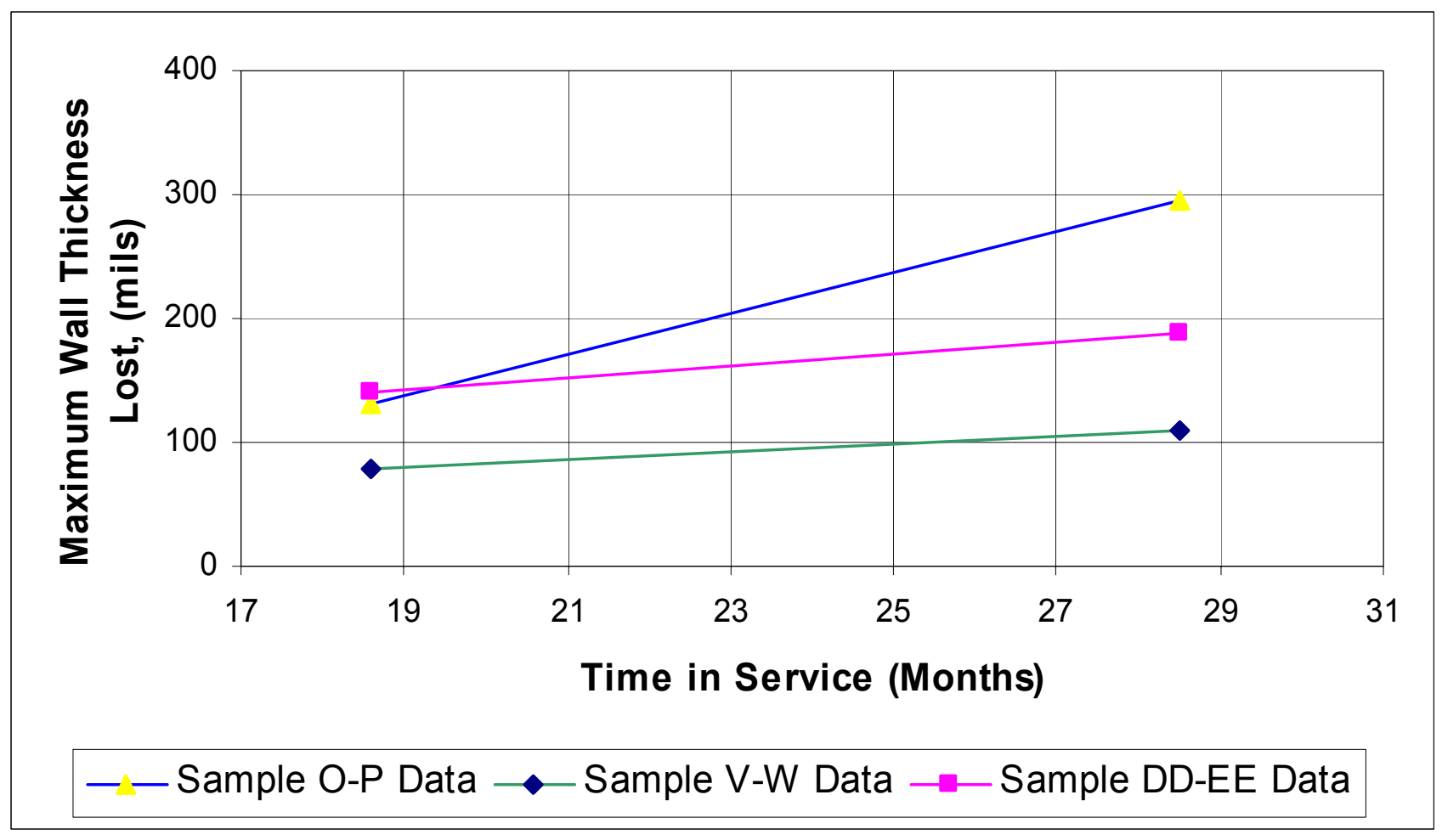

Figure H-14: Metal Loss as a Function of Time

Material: HR 120 


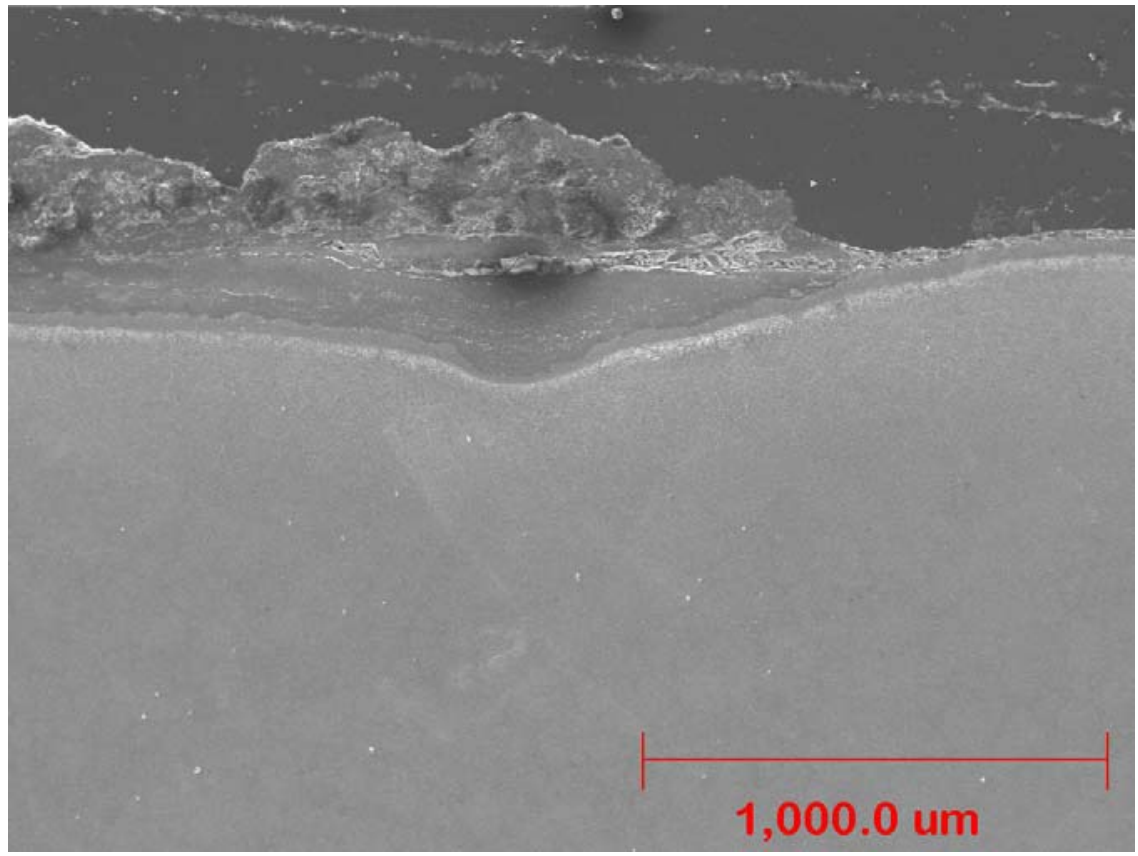

(a) External Scale/Metal Interface (SEI- Low Magnification)

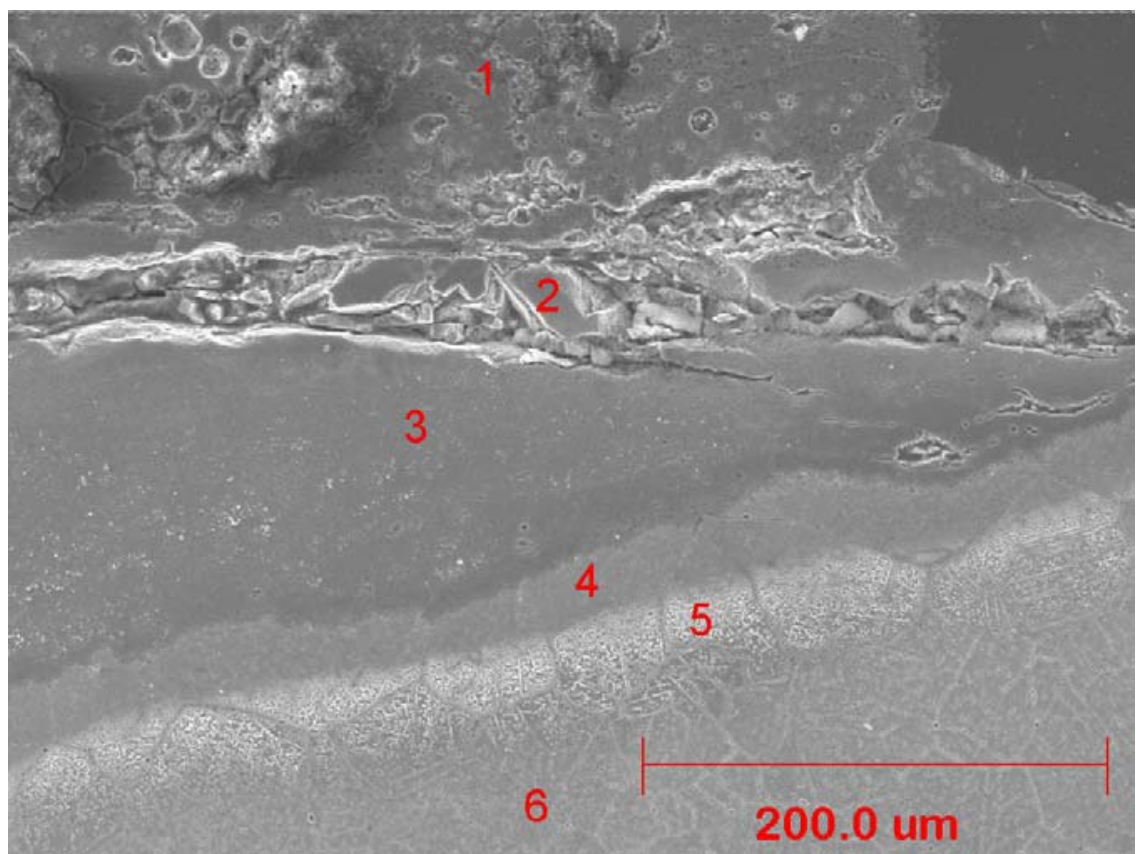

(b) External Scale/Metal Interface (SEI - Intermediate Magnification)

\section{Figure H-15: Microstructure: HR 120}

Note: Numbers on photo indicate distinct areas characterized by the visual assessment and EDS analyses that follow. 


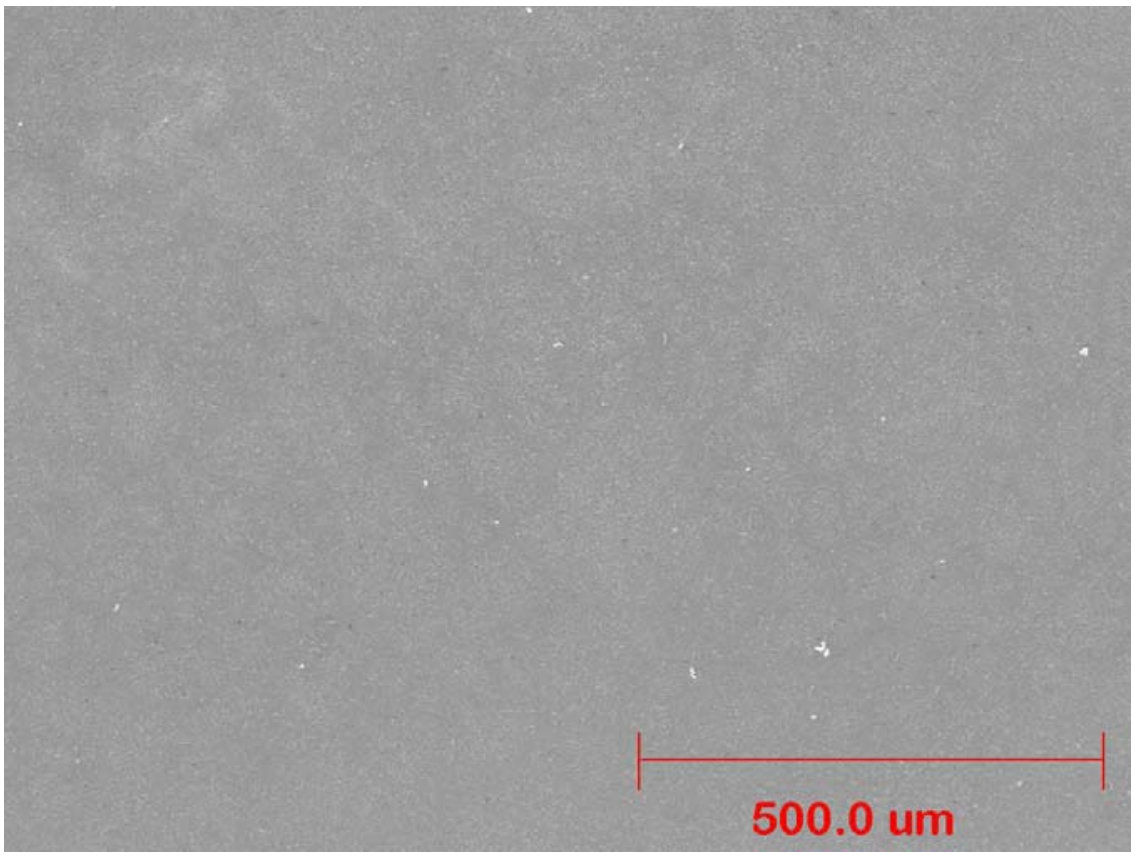

Internal Surface Appearance (SEI- Low Magnification)

Figure H-16: Mid-Wall Microstructure: HR 120

Note: Photomicrograph at approximately 200X. Electrolytic 10\% oxalic acid etch, 9V OCP 


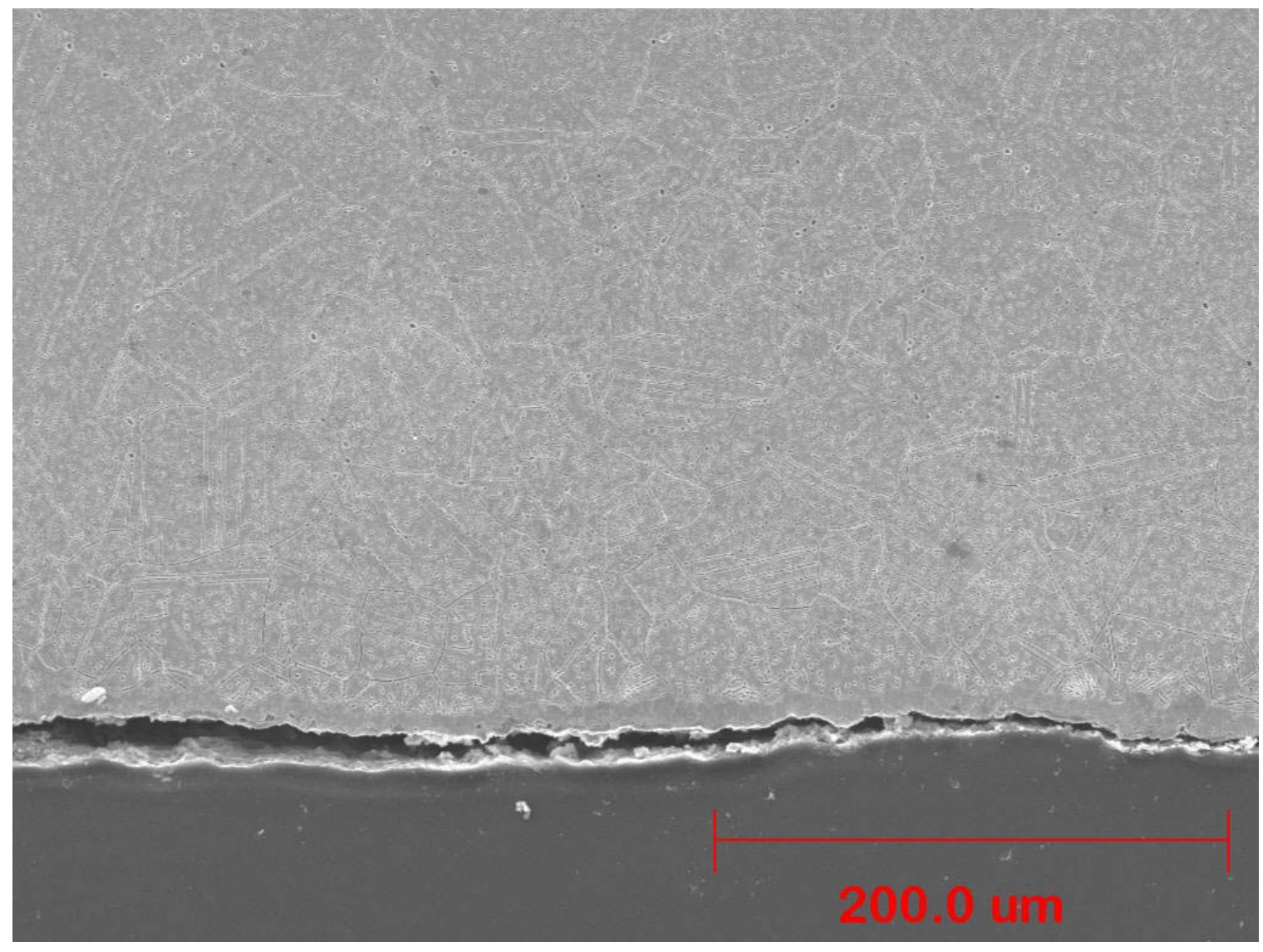

Internal Surface Appearance

Figure H-17: Microstructure: HR 120 


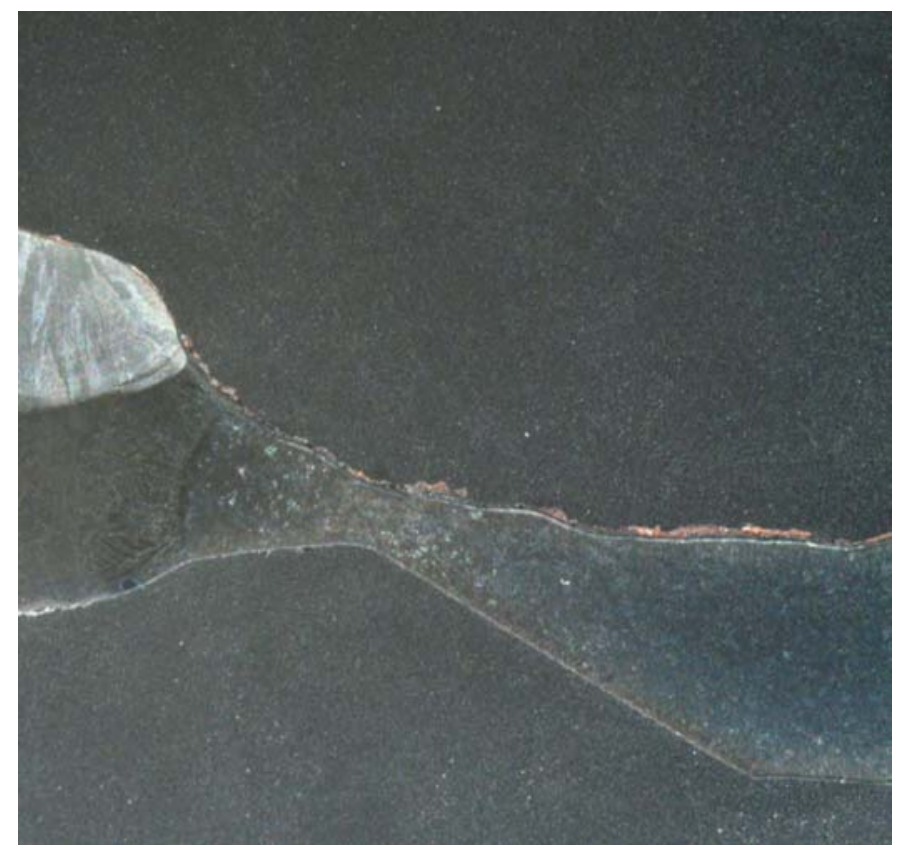

(a) HR 120 Adjacent to Weld "P"

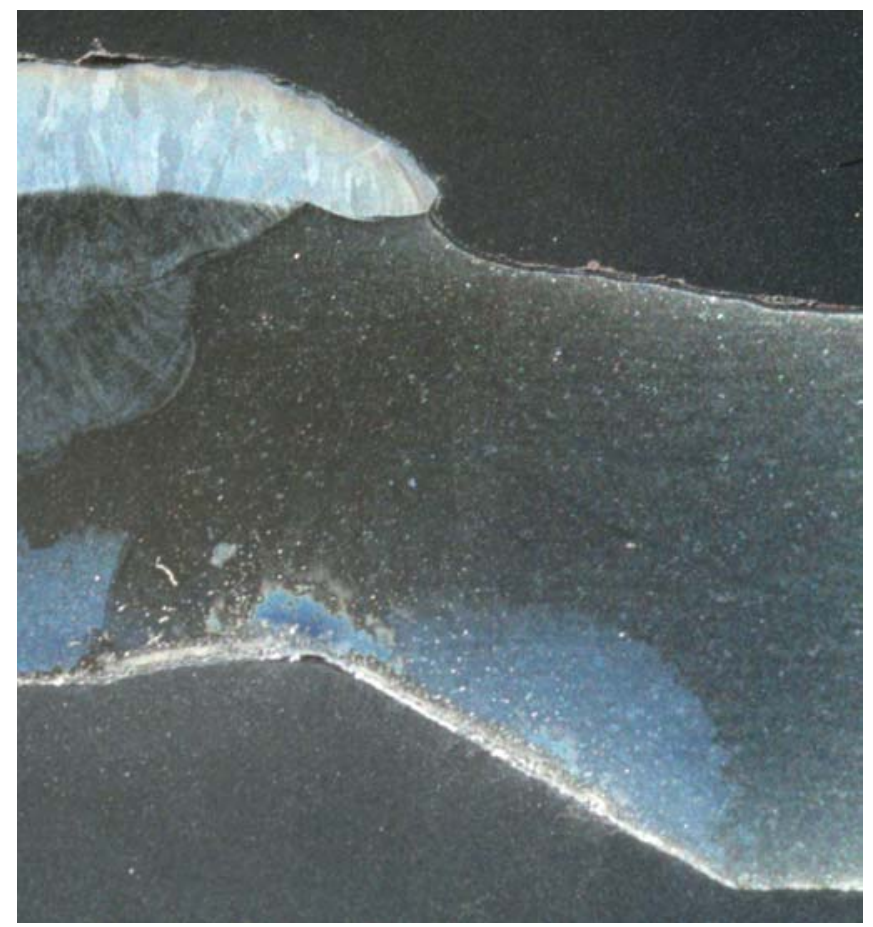

(b) HR 120 Adjacent to Weld "V"

Figure H-18: Longitudinal Cross-Sections: HR 120 Adjacent to Welds 


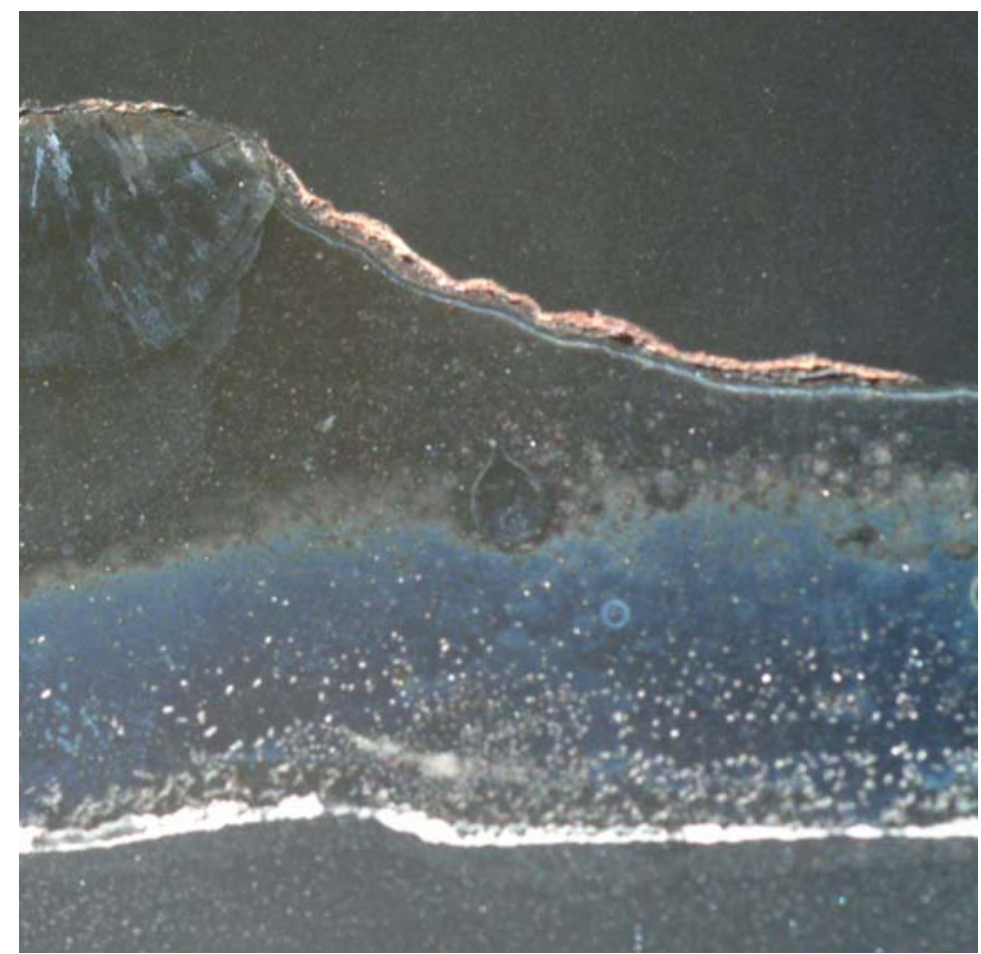

(c) HR 120 Adjacent to Weld "DD"

Figure H-18, Continued: Longitudinal Cross-Sections: HR 120 Adjacent to Welds 
Figure H-19: EDS Analysis of Deposit/Scale/Metal Interface for HR 120 (Sample O-P)

\begin{tabular}{|c|c|c|c|c|c|c|c|c|c|c|c|c|c|c|c|c|c|c|}
\hline Area & Description & $\mathbf{N i}$ & $\mathrm{Cr}$ & $\mathbf{F e}$ & Mo & Mn & $\mathbf{N b}$ & $\mathbf{T i}$ & $\mathbf{A l}$ & Si & $\mathbf{C}$ & $\mathbf{O}$ & $\mathbf{C a}$ & $\mathbf{K}$ & $\mathbf{N a}$ & As & $\mathbf{P}$ & $\mathbf{S}$ \\
\hline 1 & $\begin{array}{l}\text { Bulk deposit } \\
\text { analysis }\end{array}$ & 6.74 & 0.25 & 26.0 & -- & -- & -- & 0.39 & 8.17 & 11.9 & 20.9 & 19.1 & -- & 2.30 & -- & 2.96 & 0.85 & 0.48 \\
\hline 2 & $\begin{array}{l}\text { Loosely } \\
\text { adhering } \\
\text { scale/deposit }\end{array}$ & 12.7 & 47.7 & 19.1 & -- & -- & -- & -- & -- & 3.74 & -- & 15.8 & -- & -- & -- & -- & -- & 0.87 \\
\hline 3 & $\begin{array}{l}\text { Tightly } \\
\text { adhering } \\
\text { scale. }\end{array}$ & 8.58 & 57.4 & 9.28 & -- & - & -- & -- & -- & 1.42 & - & 17.6 & - & - & -- & -- & -- & 5.76 \\
\hline 4 & $\begin{array}{l}\text { Diffusion } \\
\text { zone - dark } \\
\text { etching near } \\
\text { scale/metal } \\
\text { interface }\end{array}$ & 47.1 & 11.2 & 40.6 & -- & 0.64 & -- & -- & - & 0.54 & -- & -- & -- & -- & -- & -- & - & -- \\
\hline 5 & $\begin{array}{l}\text { Diffusion } \\
\text { zone - light } \\
\text { etching } \\
\end{array}$ & 37.3 & 25.6 & 35.5 & -- & 0.48 & - & -- & 0.13 & 0.68 & - & -- & - & -- & -- & -- & - & 0.31 \\
\hline 6 & $\begin{array}{l}\text { Bulk EDS } \\
\text { Analysis } \\
\end{array}$ & 38.2 & 24.1 & 36.5 & -- & 0.46 & -- & -- & 0.65 & -- & -- & -- & -- & -- & -- & -- & -- & -- \\
\hline & $\begin{array}{l}\text { Reference } \\
\text { bulk analysis. }\end{array}$ & 37.9 & 25.3 & 34.3 & 0.23 & 0.69 & 0.56 & 0.01 & 0.064 & 0.51 & 0.06 & -- & -- & -- & -- & -- & -- & 0.001 \\
\hline
\end{tabular}


Appendix I

Analysis of Performance

Test Section C

Thermie 


\section{Overview of Results: Thermie}

Thermie experienced an intermediate rate of metal loss of 76 and 34 mils per year* at positions H-I and HH-II respectively. It was noted that Sample H-I was removed early, but given the metal loss rate above, it would have been able to survive for the 2.38 years required for the full Section $\mathrm{C}$ exposure period.

Metallographic examination of a Sample HH-II in Section C revealed evidence of significant grain boundary penetration at the external surface of the tube. It should also be noted that, for Section A, the metallographic examination focused on Sample H-I, instead of Sample HH-II, and that Sample HH-II has a much larger grain size that Sample H-I. It is not clear whether this difference in microstructure gave rise to the observed difference in mechanism.

It should be noted that the metal loss rates determined to date for both Section A and Section C all were based on a ring specimens that were extracted from mid-length of each tube sample. In this analysis additional attention was focused on metal losses in the vicinity of welds. Representative samples were taken and it was determined that, for the Thermie samples evaluated, the Inconel 625 weld filler metal is reasonably resistant to coal ash corrosion and that the tube material immediately adjacent to the weld (i.e. the weld heat affected zone) was somewhat less affected by corrosion than was the remainder of the tube. Indeed the tube wall appeared to be progressively thinner toward the mid-point of the tube sample.

Visual examination of the scale adjacent to the metal surface revealed the chalky-white appearance associated with alkali-iron-sulfates. Thus, the required corrosive elements likely were available to test the performance limits of this alloy. 


\section{Material: Thermie}

\section{Summary of Findings}

\begin{tabular}{|c|c|}
\hline Issue & Discussion \\
\hline $\begin{array}{l}\text { Sample } \\
\text { Location and } \\
\text { Orientation. }\end{array}$ & $\begin{array}{l}\text { - In assessing the data for Section C, it is important to understand that } \\
\text { some tube samples had to be removed from the section early due to } \\
\text { excessive thinning. The schematic in Figure I-1 shows the relative } \\
\text { positions of the Thermie samples in all three sections. Also, shown are } \\
\text { the positions of all of the excessively thinned tubes that were removed } \\
\text { and replaced in order to extend the life of Section C. } \\
\text { - One Thermie sample was removed early. Sample H-I was removed in } \\
\text { November 2001, (i.e. at the same time that Section A was removed } \\
\text { from service for evaluation). Sample HH-II survived for the full } \\
\text { Section C exposure period. } \\
\text { The Sample HH-II, that was removed early, was set aside for later } \\
\text { analysis, and has been included in the current evaluation along with } \\
\text { the other tube samples that made up Section C. } \\
\text { Also shown in this figure is the calculated average surface metal } \\
\text { temperature at both locations. }\end{array}$ \\
\hline $\begin{array}{l}\text { Composition } \\
\text { Verification. }\end{array}$ & $\begin{array}{l}\text { - Oak Ridge National Laboratory (ORNL) provided the Thermie } \\
\text { samples that were used in this program. Samples H-I and HH-II were } \\
\text { reported to be from different heats. } \\
\text { - The table in Figure I-1 shows the ORNL "target" chemistry for these } \\
\text { alloys along with the ORNL-provided analysis for both heats. } \\
\text { - During the Section A evaluation, detailed composition verification was } \\
\text { undertaken for each tube sample that was removed from service. This } \\
\text { served, in part, to verify that the each tube material was located at its' } \\
\text { proper position within the test section. } \\
\text { - Based on the good results for Section A, it was decided that it would } \\
\text { be unnecessary to analyze each and every tube sample for composition } \\
\text { verification. Rather, it was decided to select a representative sample } \\
\text { from each tube material type for confirmatory analysis. } \\
\text { During the Section A evaluation, the energy dispersive spectrographic } \\
\text { (EDS) capabilities of the scanning electron microscope (SEM) were } \\
\text { used to perform semi-quantitative analyses of each of the twelve } \\
\text { different candidate tube materials. Comparison of these EDS results } \\
\text { showed reasonably good correlation with results obtained using } \\
\text { quantitative analytical techniques, including optical emission } \\
\text { spectrometry. } \\
\text { Based on this, it was decided that the accuracy provided by EDS } \\
\text { would suffice for composition verification, but that more detailed } \\
\text { analyses would be undertaken if questions arose. } \\
\text { The results of the EDS analysis of Sample HH-II confirmed that the } \\
\text { correct material was used at this location. }\end{array}$ \\
\hline
\end{tabular}




\section{Material: Thermie}

\section{Summary of Findings, (continued)}

\begin{tabular}{|c|c|}
\hline Issue & Discussion \\
\hline $\begin{array}{l}\text { As-Received } \\
\text { Appearance }\end{array}$ & $\begin{array}{l}\text { - Figure I-3 shows the typical appearance of a Thermie tube sample } \\
\text { prior to service } \\
\text { - Figures I-4 and I-5 document the appearance of the external surface of } \\
\text { the Thermie tube samples after service in Section C. Three views are } \\
\text { provided for each tube: } 12 \text { o'clock (the view that faces on-coming gas } \\
\text { flow), } 3 \text { o'clock (the view where the gas flow is tangent to the } \\
\text { surface), and } 6 \text { o'clock (the view that shows the back-side of the tube } \\
\text { relative to the gas flow direction. } \\
\text { - The reader should be alerted to the fact that, within the boiler, the gas } \\
\text { flows upward over these tubes. This implies that for the "o'clock" } \\
\text { conventions described above, (with the } 12 \text { o'clock orientation facing } \\
\text { the gas flow direction), the actual physical orientation of these tubes is } \\
\text { such that the } 12 \text { o'clock side of the tube faces downward, and the } 6 \\
\text { o'clock orientation faces upward within the boiler. } \\
\text { Evidence of the gray/white scale associated with molten alkali-iron } \\
\text { sulfate attack clearly in evidence for Sample HH-II, Figure I-5. }\end{array}$ \\
\hline $\begin{array}{l}\text { Grit-Blasted } \\
\text { Surface } \\
\text { Appearance }\end{array}$ & $\begin{array}{l}\text { - Figures I- } 6 \text { and I-7 show the appearance of the external surface of the } \\
\text { Thermie tube samples after grit-blasting to remove deposit and scale. } \\
\text { - The } 12 \text { o'clock and } 3 \text { o'clock positions for Samples H-I clearly show } \\
\text { the appearance of general wastage due to fireside corrosion. Some } \\
\text { level of pitting also is suggested at the } 6 \text { o'clock position for this } \\
\text { sample. } \\
\text { - Wastage and pitting are much is less evident for Sample HH-II. }\end{array}$ \\
\hline $\begin{array}{l}\text { Wall } \\
\text { Thickness } \\
\text { Loss } \\
\text { Determined }\end{array}$ & $\begin{array}{l}\text { - Tube cross-sections were prepared to allow for measurement of wall } \\
\text { thickness to determine metal loss due to exposure. These are shown in } \\
\text { silhouette in Figures I-8 and I-9. } \\
\text { The same relative "o'clock" positions were maintained as discussed } \\
\text { earlier. Measurements were taken at eight evenly spaced locations } \\
\text { about the tube circumference. These were then compared with } \\
\text { measurements taken at these same locations prior to exposure. } \\
\text { - The before and after measurements were documented in the table that } \\
\text { accompanies each figure. These form the basis for the "delta" } \\
\text { calculation to determine the amount of wall thickness lost. } \\
\text { Note that for some positions in Sample H-I, the delta is negative. This } \\
\text { is believed due to wall thickness variability and difficulty in locating } \\
\text { the exact location of the "before" measurement. }\end{array}$ \\
\hline $\begin{array}{l}\text { Wall } \\
\text { Thickness } \\
\text { Loss Versus } \\
\text { Average Metal } \\
\text { Temperature }\end{array}$ & $\begin{array}{l}\text { - The chart in Figure I-10 plots the maximum wall thickness lost against } \\
\text { the calculated average metal temperature for each of the two Thermie } \\
\text { samples. Data for the Thermie samples in Section A also are included } \\
\text { in this plot. } \\
\text { - From these limited data it appears as if the rate of metal loss increases } \\
\text { with increasing temperature. }\end{array}$ \\
\hline
\end{tabular}




\section{Material: Thermie}

\section{Summary of Findings, (continued)}

\begin{tabular}{|c|c|}
\hline Issue & Discussion \\
\hline $\begin{array}{l}\text { Wall } \\
\text { Thickness } \\
\text { Loss as a } \\
\text { Function of } \\
\text { Time and } \\
\text { Location }\end{array}$ & $\begin{array}{l}\text { - The chart in Figure I- } 11 \text { plots metal loss as a function of time for each } \\
\text { sample location for Sections A and C. with samples from Section C } \\
\text { having experienced generally longer exposure times. } \\
\text { About the time that Section A was removed for analysis, the tube } \\
\text { Sample H-I was also removed and replaced in Section C. Given the } \\
\text { fact that these samples had an initial wall thickness of } 400 \text { mils, the } \\
\text { metal loss rate for Sample H-I would have predicted that this sample } \\
\text { should have been able to survive the } 2.38 \text { years the comprise the full } \\
\text { exposure period for Section C. Nonetheless, this sample was located } \\
\text { between a series of samples that were experiencing a much higher } \\
\text { corrosion rate, and the decision was made to extract and replace } \\
\text { Sample H-I along with these other samples. } \\
\text { Sample HH-II demonstrated approximately the same rate of metal loss } \\
\text { for Section C as it did in Section A (34 mils per year vs } 31 \text { mils per } \\
\text { year). Based on this, the linear data fit may well be appropriate. } \\
\text { Additional data would be required to confirm this. }\end{array}$ \\
\hline $\begin{array}{l}\text { Metallurgical } \\
\text { Analysis - } \\
\text { SEM }\end{array}$ & $\begin{array}{l}\text { - Sample HH-II was selected for a detailed SEM/EDS assessment of the } \\
\text { constituents and structure in the vicinity of the external and internal } \\
\text { surfaces. This sample was selected based on the fact that it had seen } \\
\text { the full exposure period for Section C. The focus of this investigation } \\
\text { was in the region of the tube where the maximum amount of material } \\
\text { was lost. } \\
\text { - Figures I-12a and b show backscattered electron images of the } \\
\text { scale/metal interface for Sample HH-II. The multiple zones evident } \\
\text { for this material during the Section A analysis were not seen in this } \\
\text { sample from Section C. A very thin scale was noted in isolated patches } \\
\text { on the surface of the tube. Scale-filled cracks were noted along the } \\
\text { surface of the tube. No diffusion zone was in evidence. } \\
\text { - Figure I- } 13 \text { provides a view of the microstructure at mid-wall. } \\
\text { - Figure I-14 shows a cross section at the internal surface of Sample } \\
\text { HH-II. Little or no scale is noted on this surface. }\end{array}$ \\
\hline $\begin{array}{l}\text { Metallurgical } \\
\text { Analysis - } \\
\text { Weld Cross } \\
\text { Section. }\end{array}$ & $\begin{array}{l}\text { - Figure I-15 shows a longitudinal profile (at the } 12 \text { o'clock orientation) } \\
\text { of Sample HH-II adjacent to the weld "HH" in Section C. } \\
\text { - An Inconel } 625 \text { weld metal was used throughout Section C. The cross } \\
\text { sections show that the Thermie weld heat affected zones are not } \\
\text { preferentially attacked, in fact, the wall thickness for the tube sample } \\
\text { tapers from the weld toward the middle of the tube length. } \\
\text { - Of note in this cross section is the fact that, when Section C was being } \\
\text { built, the wall thickness of the Thermie sample had to be reduced so } \\
\text { that it could be attached to the thinner-walled IN } 52 \text { Weld Overlay } \\
\text { specimen that was adjacent to it. It is important to remember this as we } \\
\text { are examine the Thermie samples that remain is service in Section B. } \\
\text { In situ diameter measurements to estimate remaining wall thickness } \\
\text { need to take into account this locally thinner wall adjacent to the weld. }\end{array}$ \\
\hline
\end{tabular}


Summary of Findings, (continued)

\section{Material: Thermie}

\begin{tabular}{|c|c|}
\hline Issue & Discussion \\
\hline $\begin{array}{l}\text { EDS Analysis } \\
\text { Results }\end{array}$ & $\begin{array}{l}\text { - The table in Figure I-16 provides a summary of the EDS analyses of } \\
\text { the locations numbered in Figures I-12b. Also shown in this table is a } \\
\text { summary of the spectrographic analysis reported earlier, see Figure } \\
\text { I-2. } \\
\text { Review of this table suggests the following: } \\
\text { - Area 1: Scale/metal interface - Analysis of this region suggests } \\
\text { the formation of a chromium-rich oxide; however, given the small } \\
\text { volume of scale at this location, it seems likely that, to a great } \\
\text { extent, this analysis reflects the composition of the tube metal } \\
\text { adjacent to the interface. } \\
\text { - Areas 2: Corrosion product in crack near the surface - This } \\
\text { analysis suggests that the crack was lined with a chromium-rich } \\
\text { oxide. A significant amount of sulfur also was found at this } \\
\text { location. } \\
\text { Area 3: Corrosion product in crack near the surface - This } \\
\text { analysis suggests the presence of a nickel-rich corrosion product. } \\
\text { Area 4: Bulk metal analysis - Given its limitations, this EDS } \\
\text { analysis compares favorably with that of the reference } \\
\text { spectrographic analysis below it. }\end{array}$ \\
\hline
\end{tabular}




\section{Gas Flow}

Direction

\section{Sample H-I $\left(1171^{\circ} \mathrm{F}\right)$}

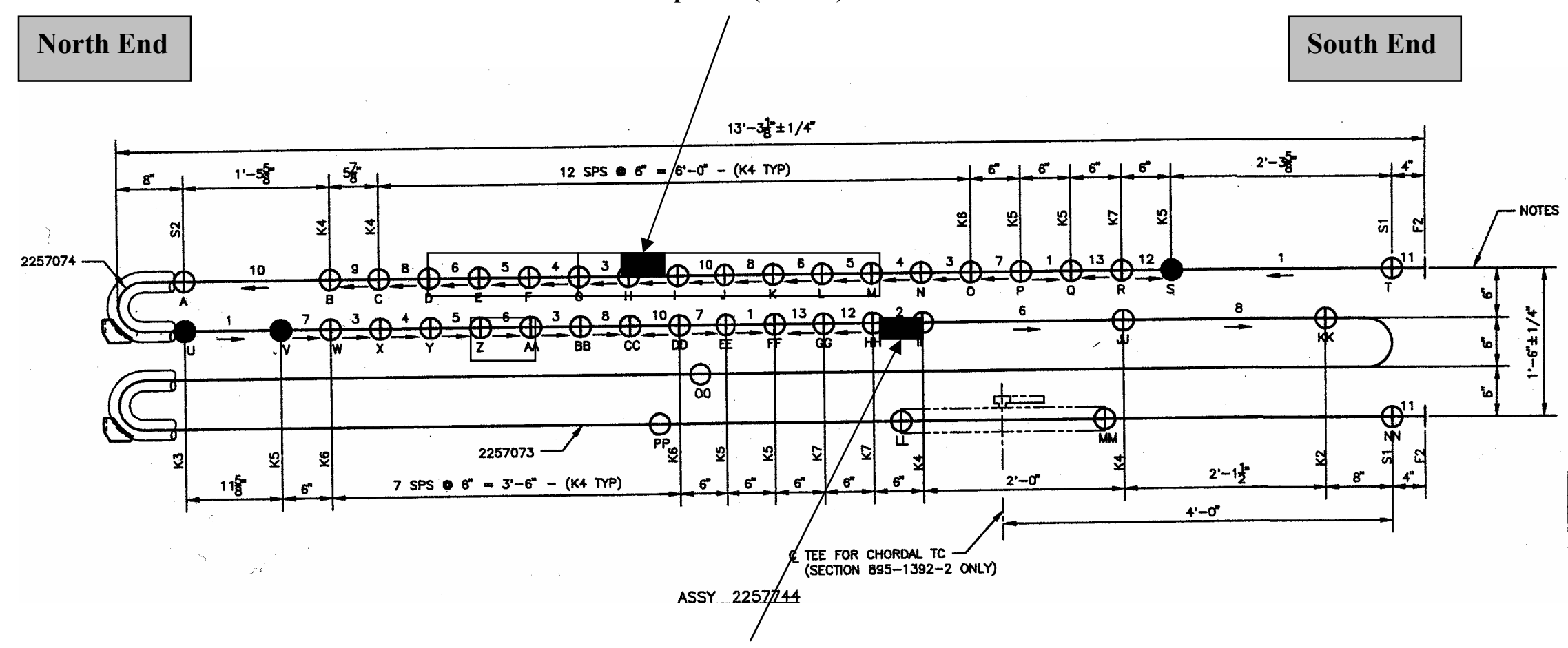

Sample HH-II $\left(1067^{\circ} \mathrm{F}\right)$

Figure I-1: Schematic Drawing Showing Position of Thermie Tube Samples Within Test Section C

1. Drawing shown in same relative orientation as per the actual installation.

2. This group of tube samples was removed from service in November 2001 - highlighted in red.

3. This group of tube samples was removed from service in June 2002 - highlighted in yellow. 


\section{Figure I-2 Semi-Quantitative Composition Verification for Thermie}

Description:

Source:

Reference Composition:
Nominal $25 \mathrm{Cr} / 20 \mathrm{Co}$ nickel alloy with additions of $\mathrm{Nb}$ and $\mathrm{Ti}$ to provide creep strength.

Oak Ridge National Laboratory (ORNL)

"Target” Chemistry per ORNL (Reference 1).

\begin{tabular}{|c|c|c|c|c|c|}
\hline Element & $\begin{array}{c}\text { Reference } \\
\text { Composition }\end{array}$ & $\begin{array}{c}\text { Composition } \\
\text { Heat } 17422\end{array}$ & $\begin{array}{r}\text { Composition } \\
\text { Heat } 17454\end{array}$ & $\begin{array}{c}\text { Tube Sample } \\
\text { H-I }\end{array}$ & $\begin{array}{c}\text { Tube Sample } \\
\text { HH-II }\end{array}$ \\
\hline $\mathrm{Ni}$ & 48.0 & 49.06 & 48.92 & $\mathrm{~N} / \mathrm{A}$ & 51.3 \\
\hline $\mathrm{Cr}$ & 24.0 & 23.08 & 23.63 & N/A & 24.8 \\
\hline $\mathrm{Fe}$ & 2.0 & 2.06 & 2.11 & N/A & 1.99 \\
\hline Mo & 0.5 & 0.43 & 0.42 & N/A & - \\
\hline $\mathrm{Co}$ & 19.8 & 19.39 & 19.42 & $\mathrm{~N} / \mathrm{A}$ & 18.4 \\
\hline $\mathrm{C}$ & 0.07 & 0.088 & 0.094 & N/A & - \\
\hline $\mathrm{N}$ & - & 0.013 & 0.022 & N/A & - \\
\hline $\mathrm{B}$ & $0.002 \max$ & (a) & (a) & N/A & - \\
\hline $\mathrm{Mn}$ & 0.3 & 0.32 & 0.32 & N/A & - \\
\hline $\mathrm{Si}$ & 0.5 & 0.51 & 0.49 & $\mathrm{~N} / \mathrm{A}$ & 0.53 \\
\hline $\mathrm{Al}$ & 0.8 & 0.74 & 0.60 & N/A & 0.66 \\
\hline $\mathrm{Ti}$ & 2.0 & 2.10 & 2.01 & N/A & 2.22 \\
\hline $\mathrm{Al}+\mathrm{Ti}$ & - & - & - & $\mathrm{N} / \mathrm{A}$ & - \\
\hline $\mathrm{Nb}$ & 2.0 & 2.20 & 1.95 & $\mathrm{~N} / \mathrm{A}$ & - \\
\hline $\mathrm{Cu}$ & - & $<0.01$ & $<0.01$ & N/A & - \\
\hline $\mathrm{Sn}$ & - & - & - & N/A & - \\
\hline $\mathrm{P}$ & - & $<0.002$ & $<0.002$ & $\mathrm{~N} / \mathrm{A}$ & - \\
\hline$S$ & - & 0.003 & 0.009 & N/A & - \\
\hline $\mathrm{O} 2$ & - & 0.003 & 0.004 & N/A & - \\
\hline $\mathrm{Zr}$ & $0.015 \max$ & (a) & (a) & N/A & - \\
\hline $\mathrm{Mg}$ & - & - & - & N/A & - \\
\hline
\end{tabular}




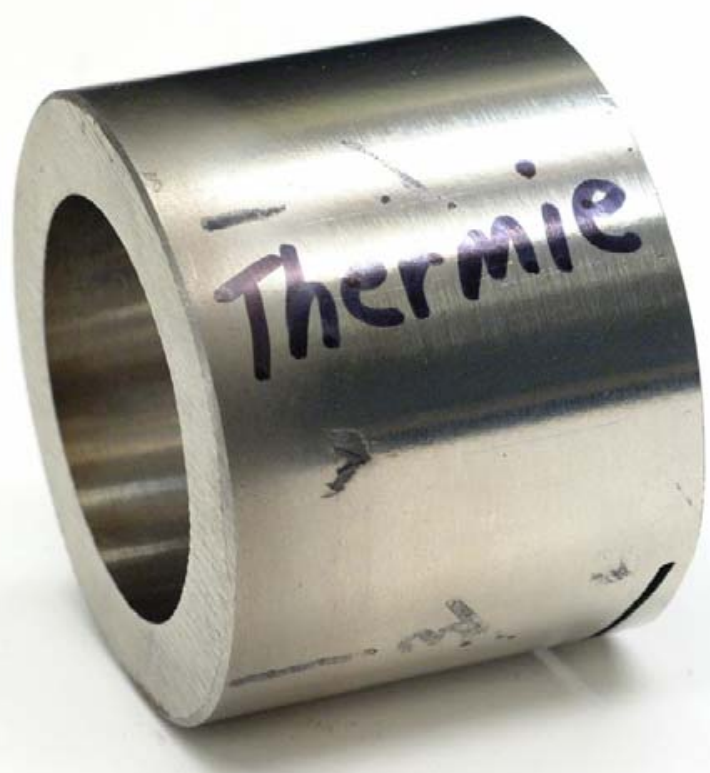

Figure I-3: Appearance of Thermie Tube Sample Prior to Service 


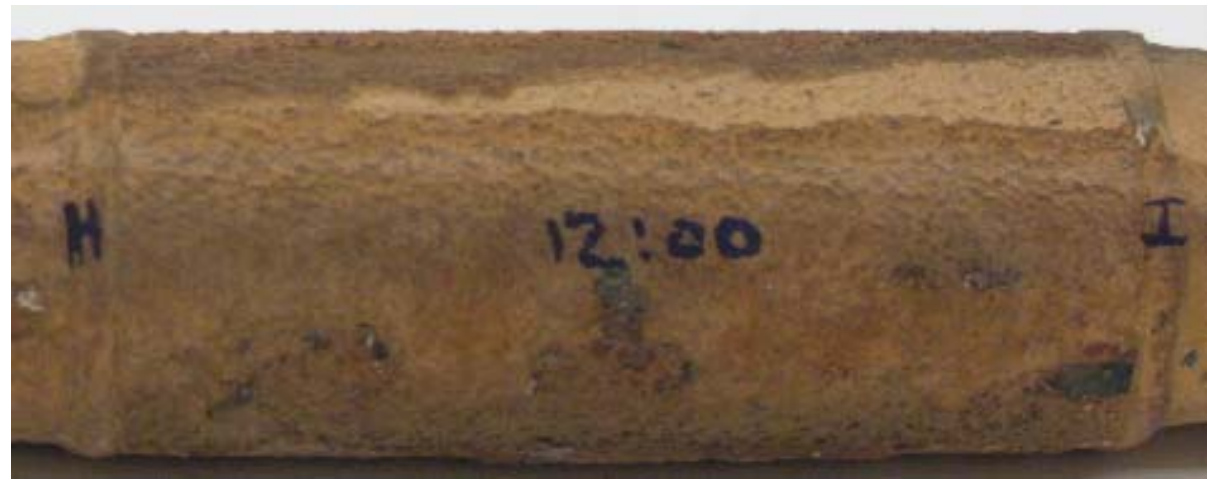

12 O'Clock - Facing Gas Flow

(Note: 3 o'clock is up in this image)

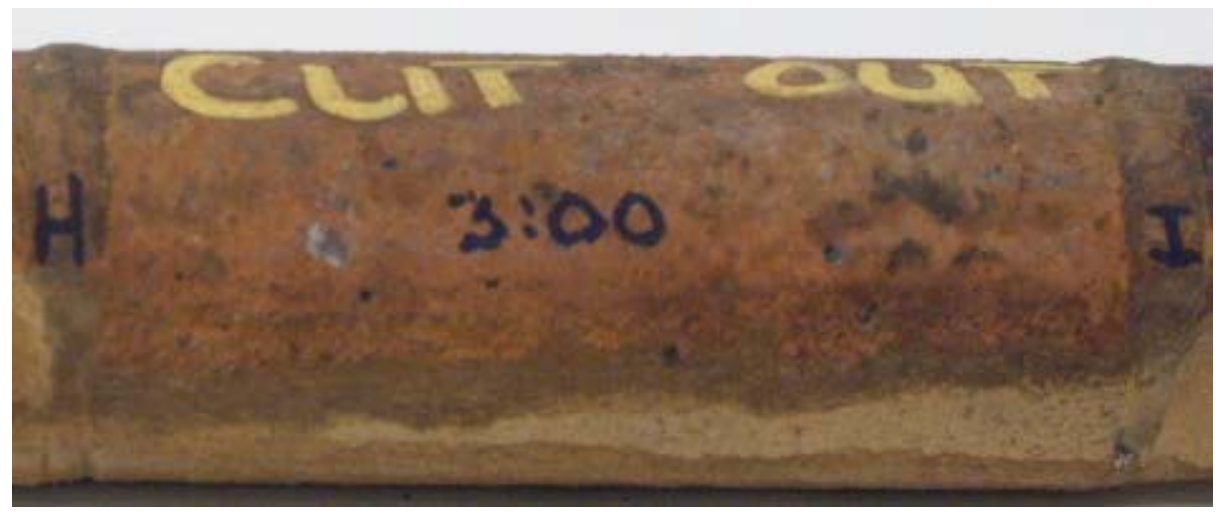

3 O'Clock

(Note: 6 o'clock is up in this image)

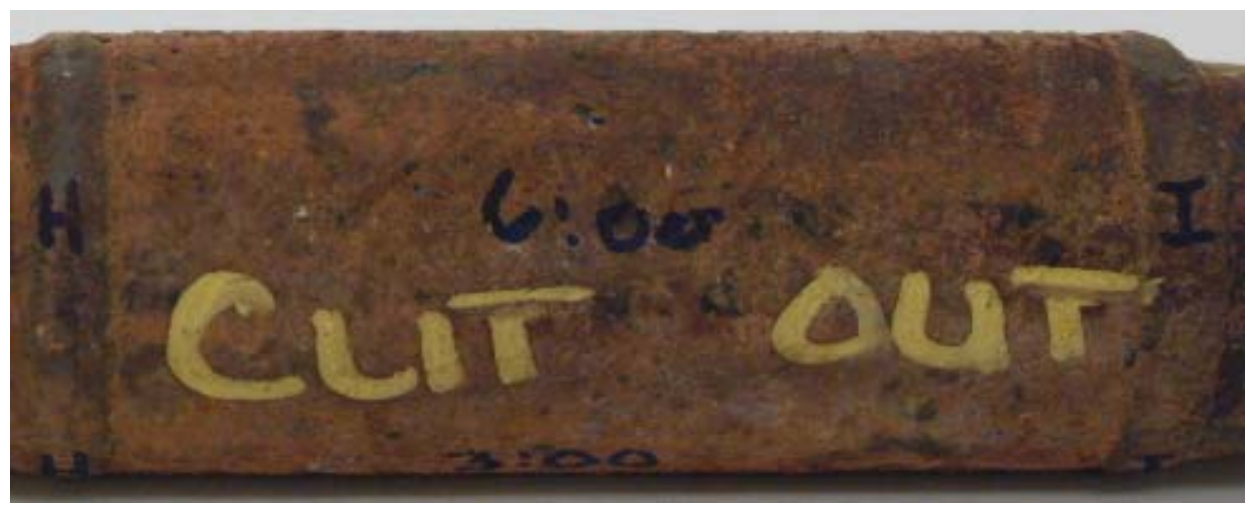

6 O'Clock - Opposite Gas Flow

Figure I-4 As-Received Appearance of Sample H-I (Thermie)

(Average surface metal temperature: $1171^{\circ} \mathrm{F}$ )

Note: this tube was removed from service on 11/6/01. 


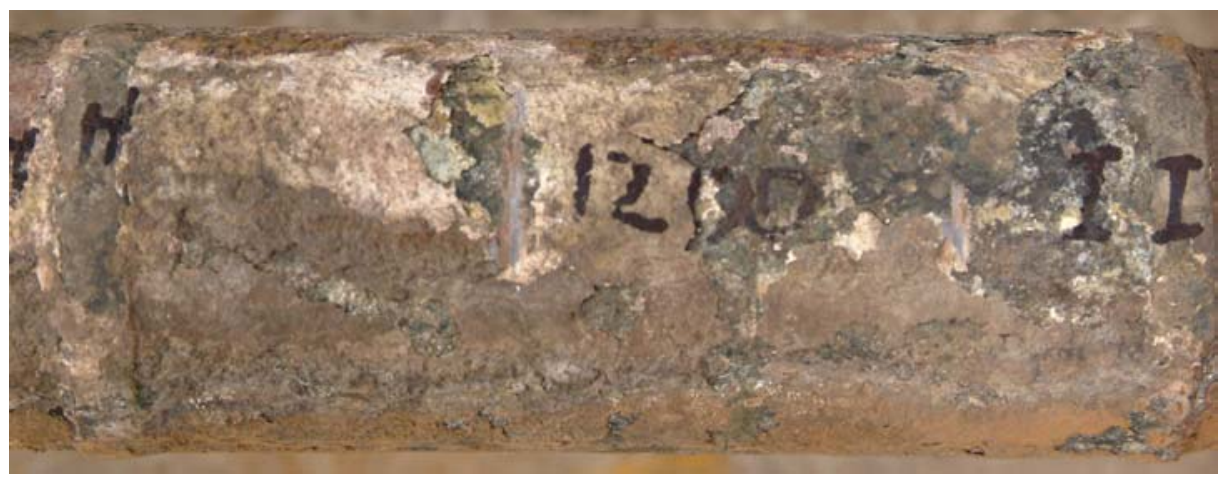

12 O'Clock - Facing Gas Flow

(Note: 3 o'clock is up in this image)

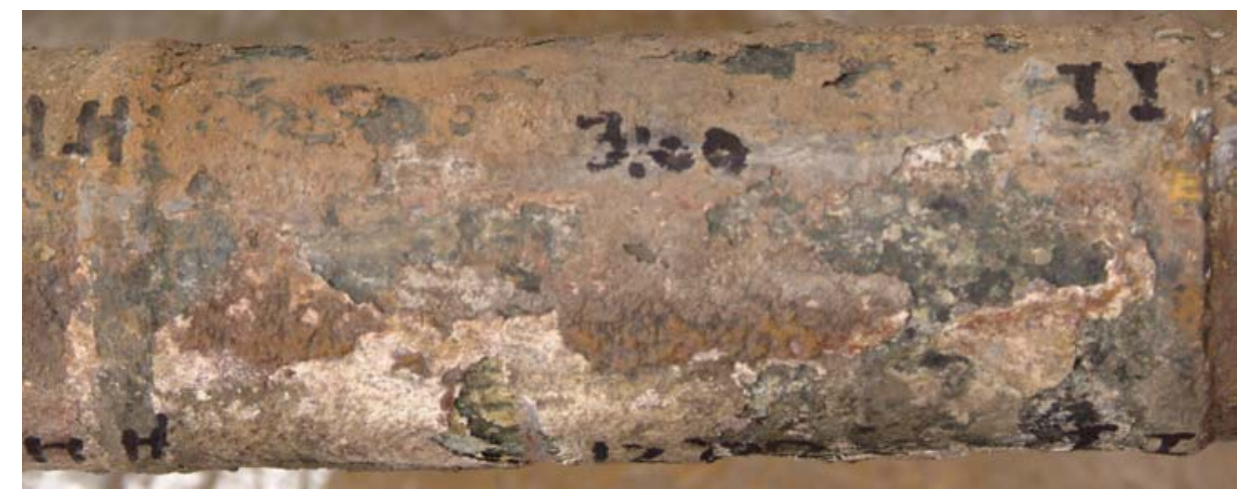

3 O'Clock

(Note: 6 o'clock is up in this image)

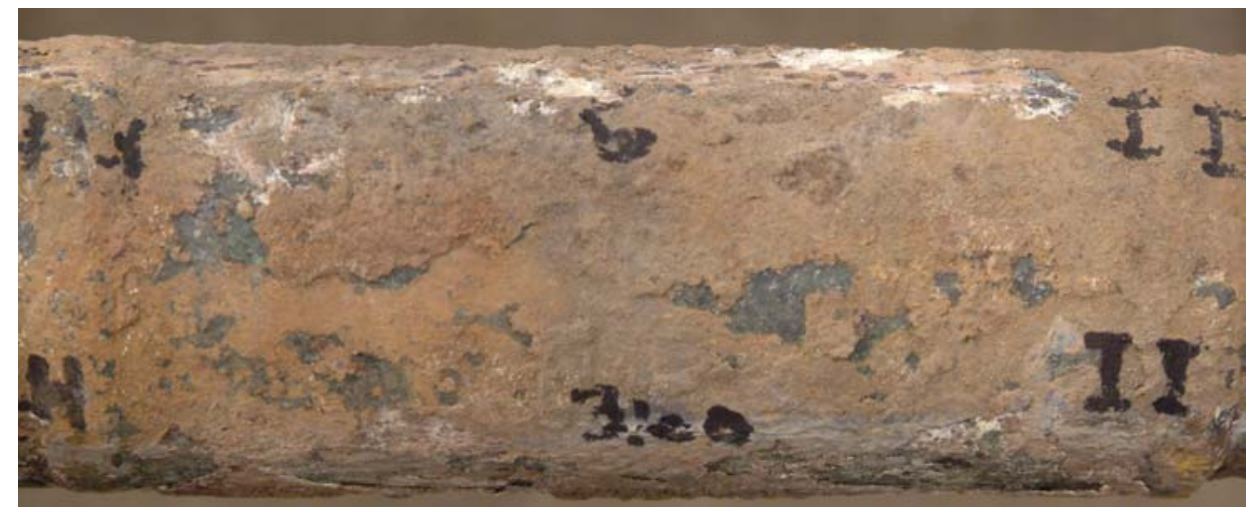

6 O'Clock - Opposite Gas Flow

Figure I-5 As-Received Appearance of Sample HH-II (Thermie)

(Average surface metal temperature: $1067^{\circ} \mathrm{F}$ ) 
12 O'Clock

(86 mils lost)

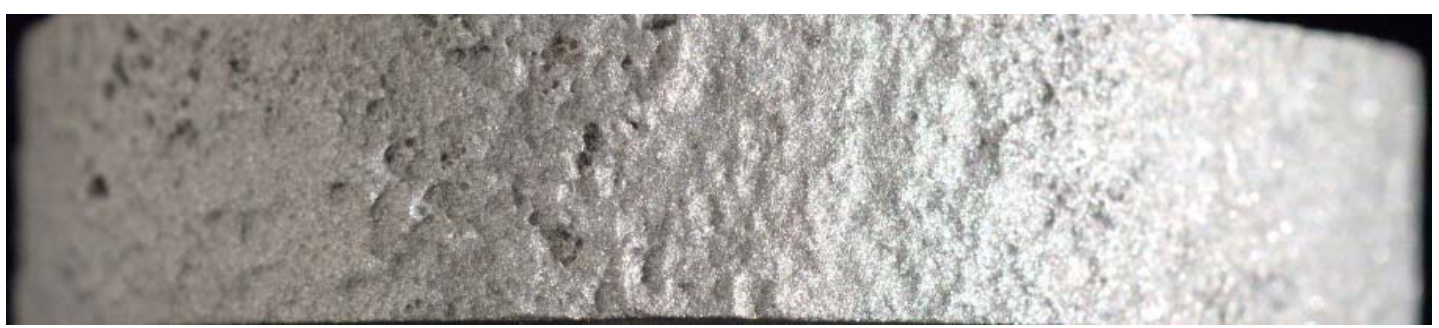

3 O'Clock

(0 mils lost)

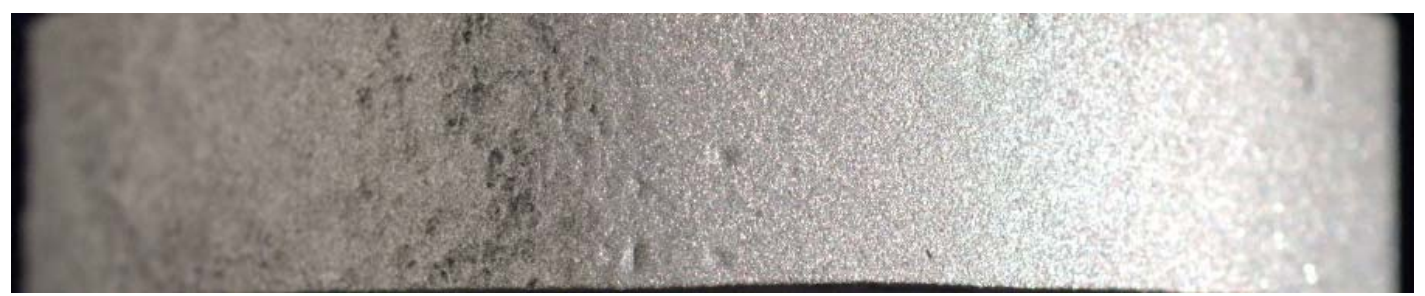

6 O'Clock

(6 mils lost)

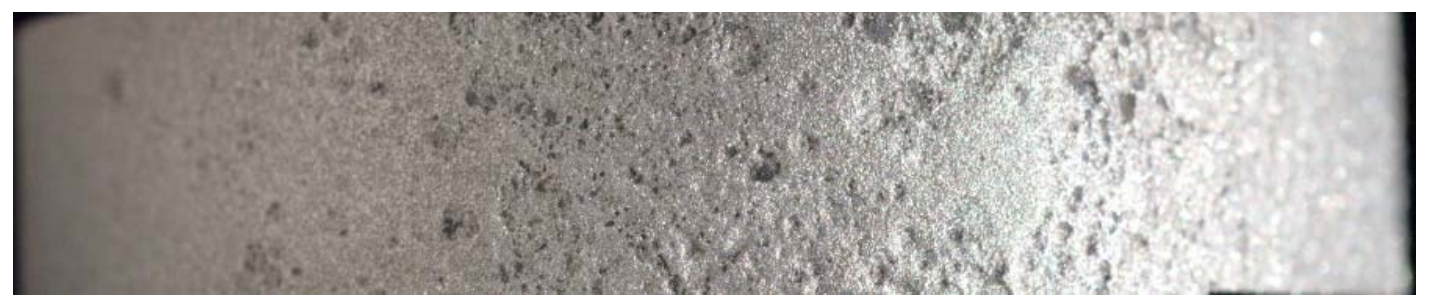

Figure I-6: OD Surface Appearance After Grit Blast

Material: Thermie

Sample: H-I 
12 O'Clock

(39 mils lost)

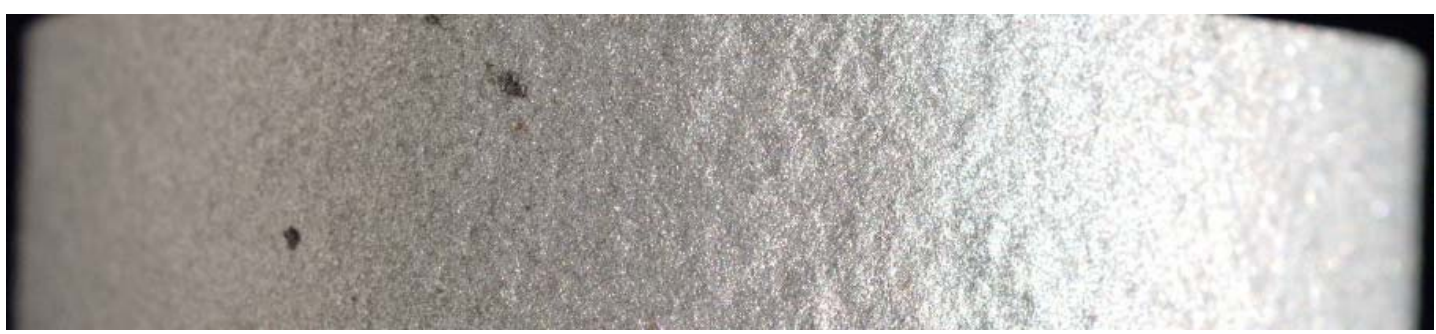

3 O'Clock

(55 mils lost)

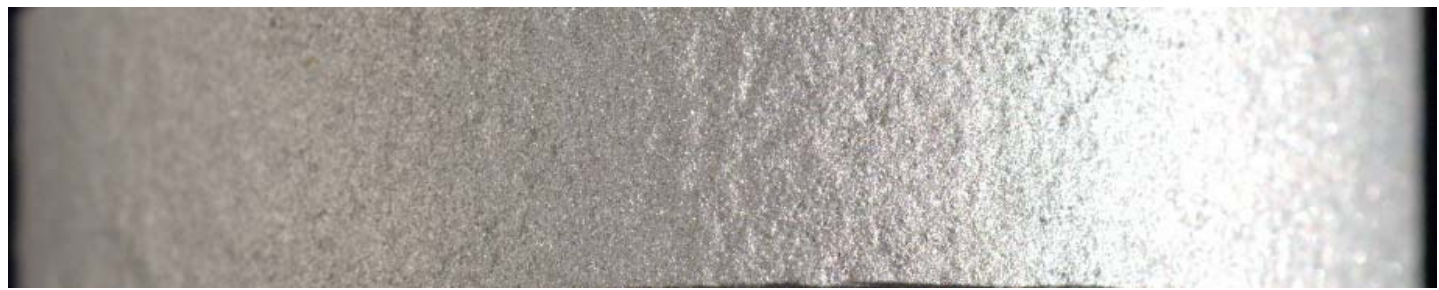

6 O'Clock

(6 mils lost)

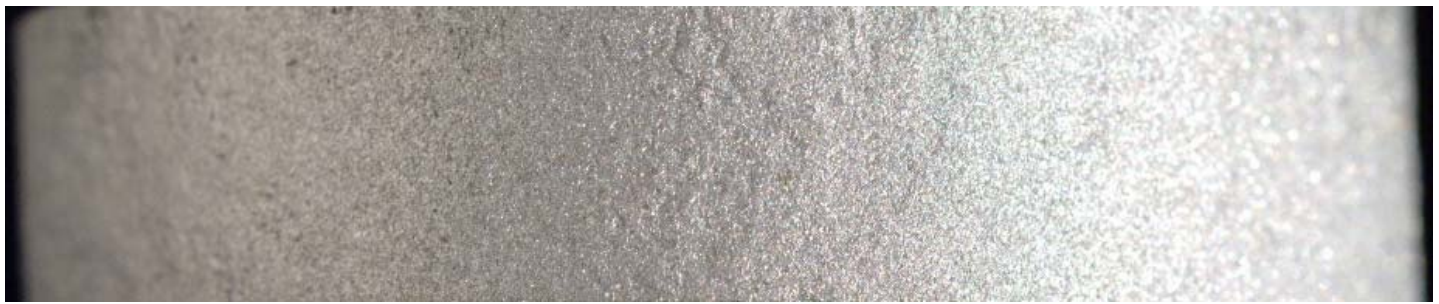

Figure I-7: OD Surface Appearance After Grit Blast

Material: Thermie

Sample: HH-II 


\section{Material:}

Sample Location:

Maximum Rate:

Maximum Loss Orientation:

Average Surface Metal Temperature:

\section{Thermie}

H-I

77 mils/yr

Approx. 1:30 o'clock

\section{O’Clock}

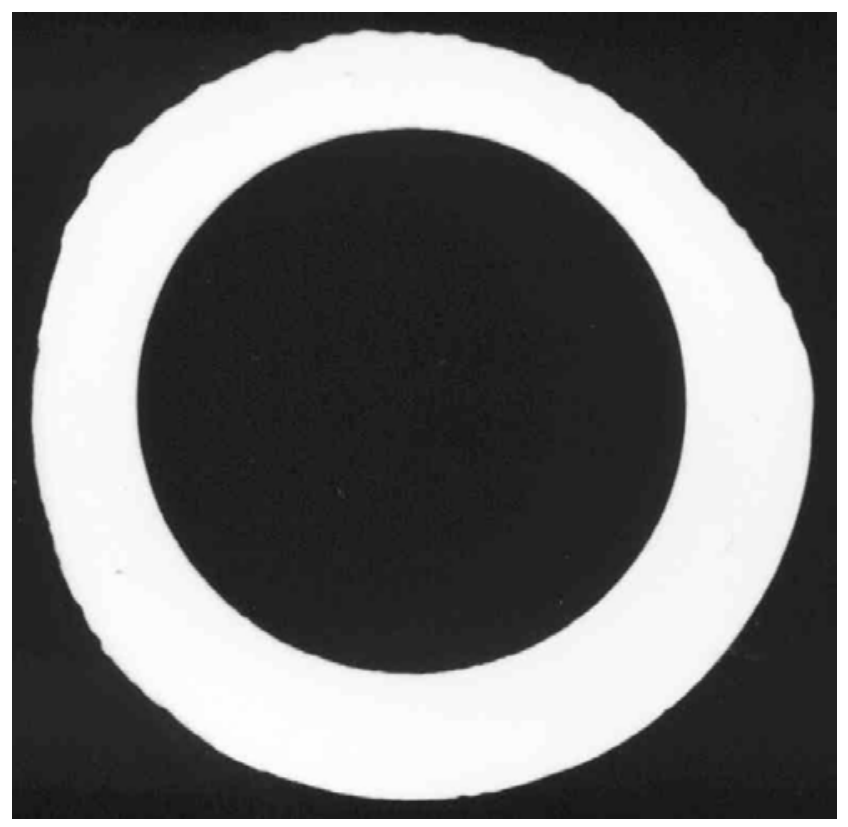

3 O’Clock

Approx. 1.2X

Wall Reduction by Position (in.)

\begin{tabular}{|l|c|c|c|c|c|c|c|c|c|c|}
\hline & $\begin{array}{c}\mathbf{1 2} \\
\text { o'clock }\end{array}$ & $\mathbf{1 : 3 0}$ & $\mathbf{3 : 0 0}$ & $\mathbf{4 : 3 0}$ & $\mathbf{6 : 0 0}$ & $\mathbf{7 : 3 0}$ & $\mathbf{9 : 0 0}$ & $\mathbf{1 0 : 3 0}$ & AVG & $\begin{array}{c}\text { Max } \\
\text { Loss }\end{array}$ \\
\hline Before & 0.399 & 0.399 & 0.400 & 0.400 & 0.400 & 0.400 & 0.399 & 0.398 & 0.399 & 0.399 \\
\hline After & 0.313 & 0.280 & 0.401 & 0.401 & 0.394 & 0.397 & 0.330 & 0.343 & & 0.280 \\
\hline Delta & 0.086 & 0.119 & -0.001 & -0.001 & 0.006 & 0.003 & 0.069 & 0.055 & & 0.119 \\
\hline
\end{tabular}

Figure I-8: Silhouette and Wall Thickness Measurements for Sample H-I

Note: this tube was removed from service on 11/6/01. 


\section{Material:}

Sample Location:

Maximum Rate:

Maximum Loss Orientation:

Average Surface Metal Temperature:

\section{Thermie}

HH-II

$34 \mathrm{mils} / \mathrm{yr}$

Approx. 2:30 o'clock

$1067^{\circ} \mathrm{F}$

\section{O’Clock}

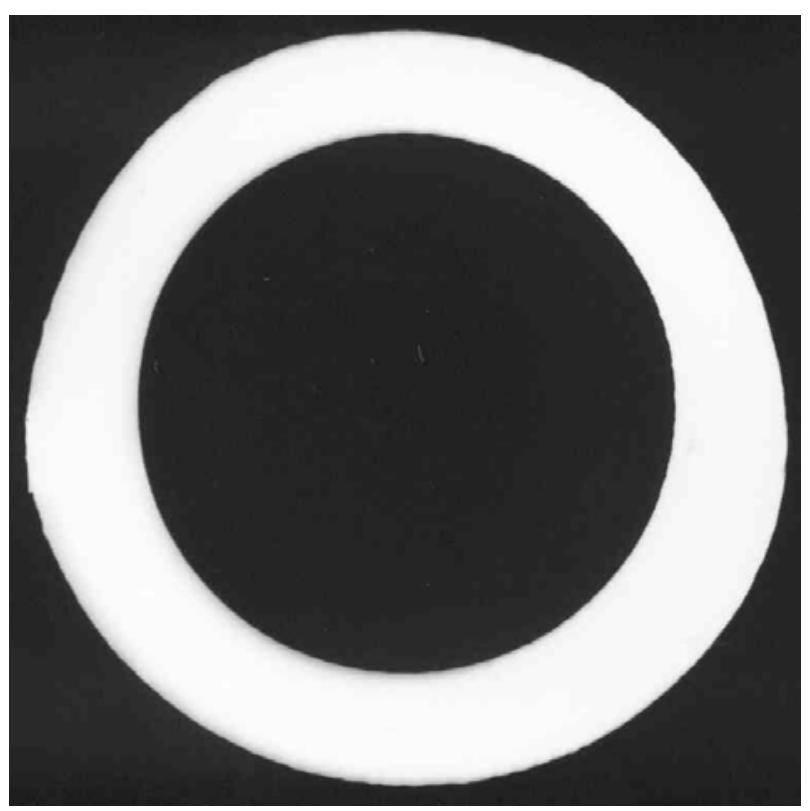

3 O’Clock

Approx. 1.2X

Wall Reduction by Position (in.)

\begin{tabular}{|l|c|c|c|c|c|c|c|c|c|c|}
\hline & $\begin{array}{c}\mathbf{1 2} \\
\text { o'clock }\end{array}$ & $\mathbf{1 : 3 0}$ & $\mathbf{3 : 0 0}$ & $\mathbf{4 : 3 0}$ & $\mathbf{6 : 0 0}$ & $\mathbf{7 : 3 0}$ & $\mathbf{9 : 0 0}$ & $\mathbf{1 0 : 3 0}$ & AVG & $\begin{array}{c}\text { Max } \\
\text { Loss }\end{array}$ \\
\hline Before & 0.399 & 0.399 & 0.398 & 0.399 & 0.399 & 0.399 & 0.399 & 0.399 & 0.399 & 0.399 \\
\hline After & 0.360 & 0.344 & 0.343 & 0.395 & 0.394 & 0.399 & 0.382 & 0.340 & & 0.318 \\
\hline Delta & 0.039 & 0.055 & 0.055 & 0.005 & 0.006 & 0.000 & 0.017 & 0.059 & & 0.081 \\
\hline
\end{tabular}

Figure I-9: Silhouette and Wall Thickness Measurements for Sample HH-II

* Measurement taken at "maximum loss orientation" noted above. The average

"before" wall thickness was used to calculate the "delta". 


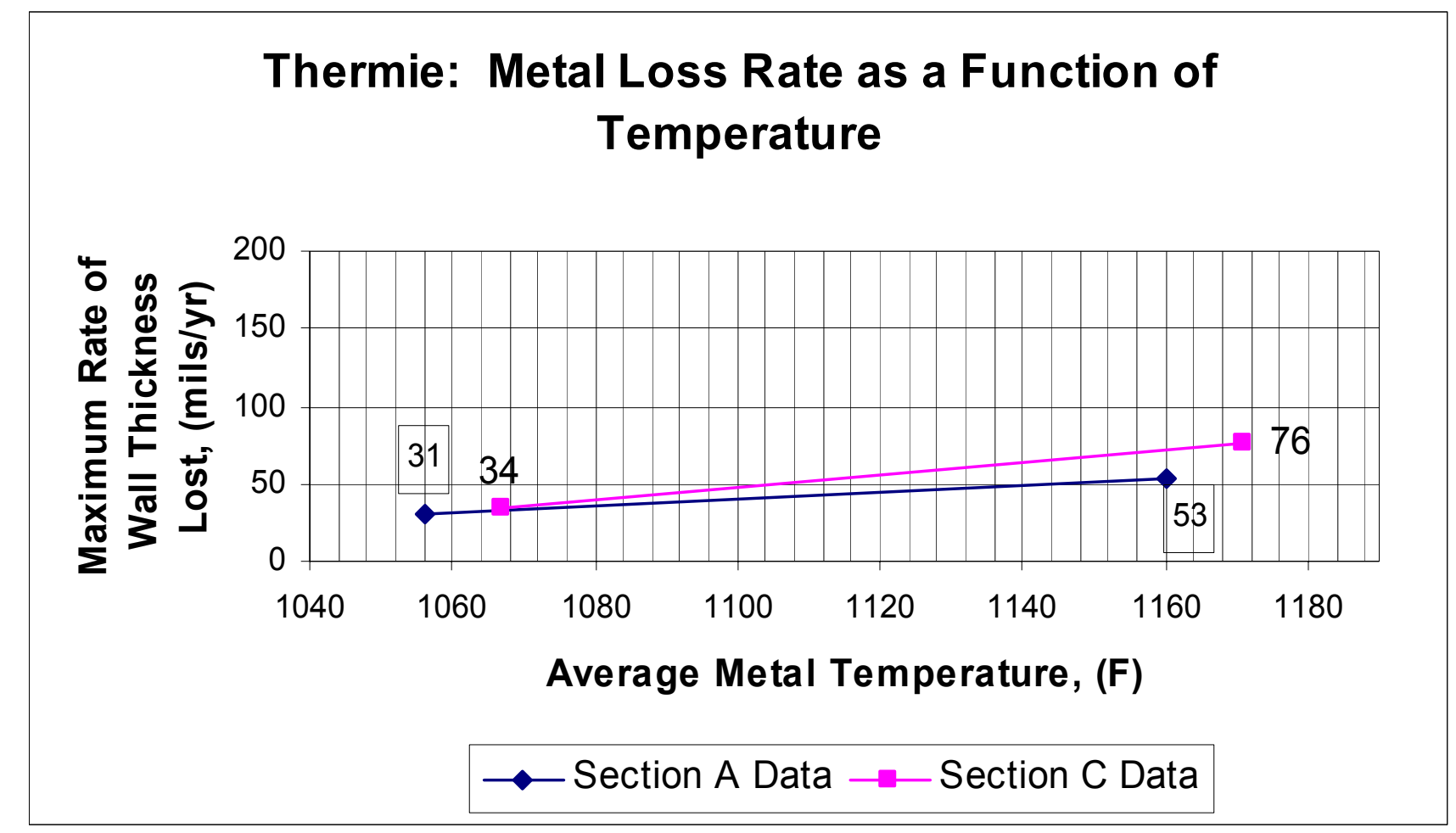

Figure I-10: Metal Loss Rate as a Function of Average Surface Metal Temperature

\section{Material: Thermie}

Note: In analyzing these data it is important to understand that the one of the two Section C samples was removed early due to excessive corrosion losses. Sample H-I (1171 F) was removed in November 2001. Sample HH-II was removed with the remaining Section C samples in May 2003. 


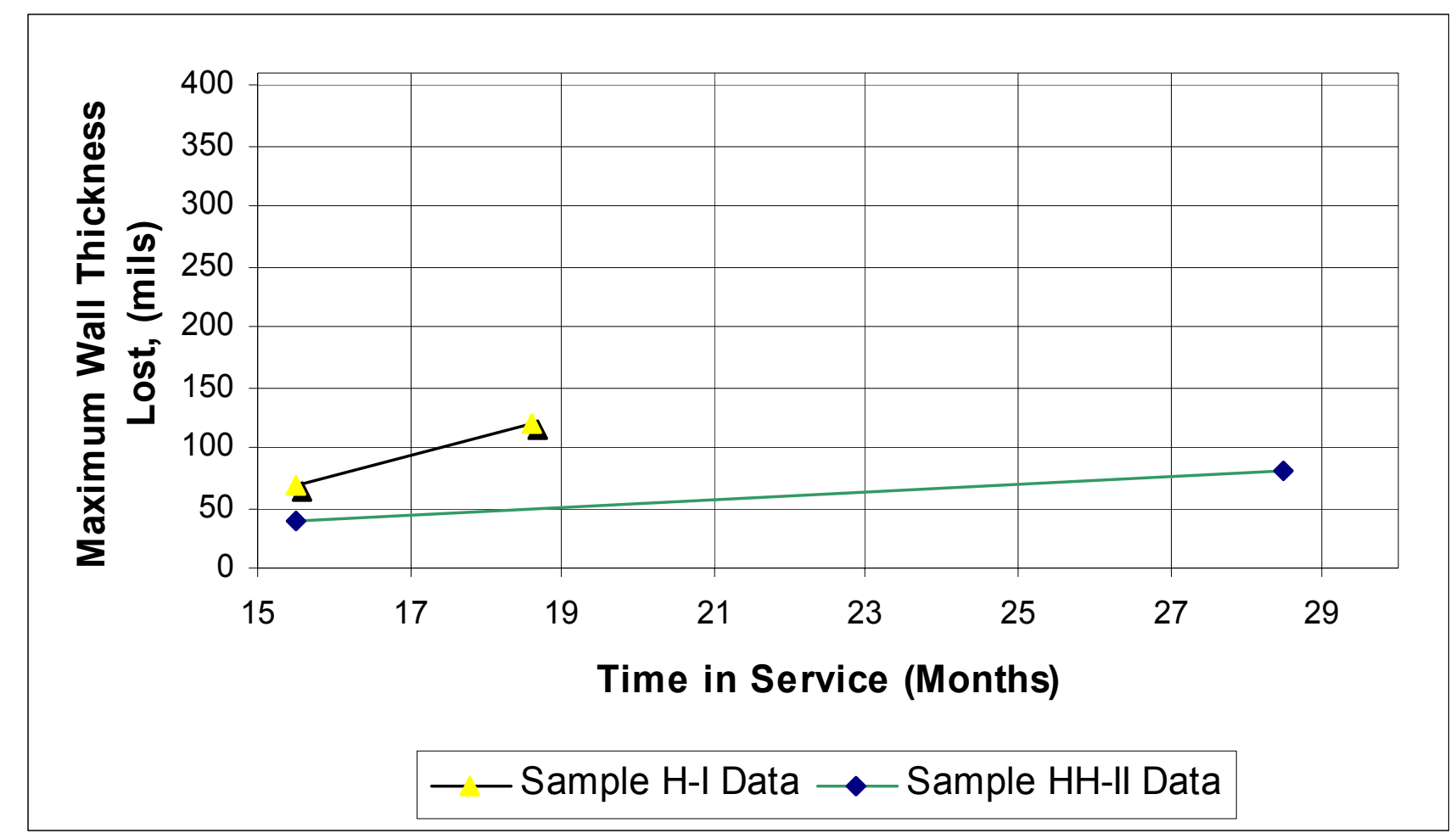

Figure I-10: Metal Loss as a Function of Time

\section{Material: Thermie}

Note: From the plot above it can be seen that Section C Sample H-I was removed early. Sample HH-II experienced the full exposure life for both Sections A and $\mathrm{C}$. 


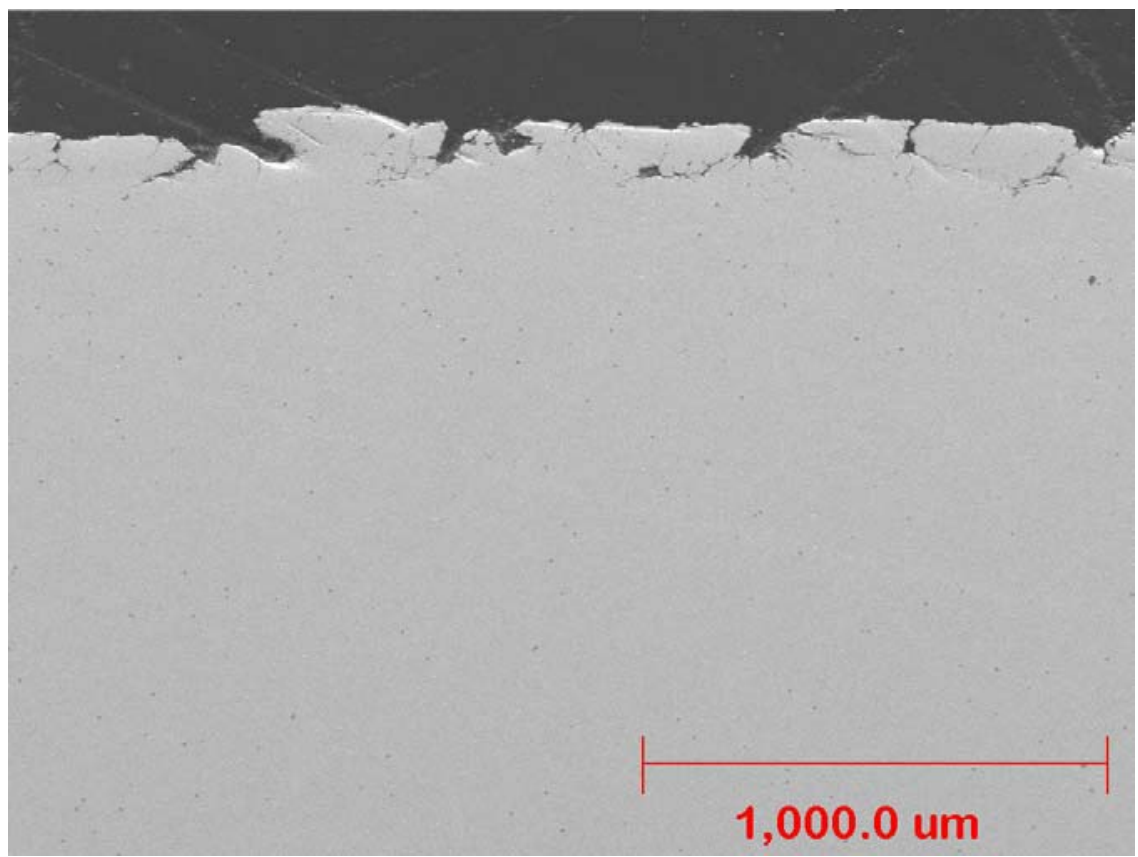

(a) External Scale/Metal Interface (BEI - Low Magnification)

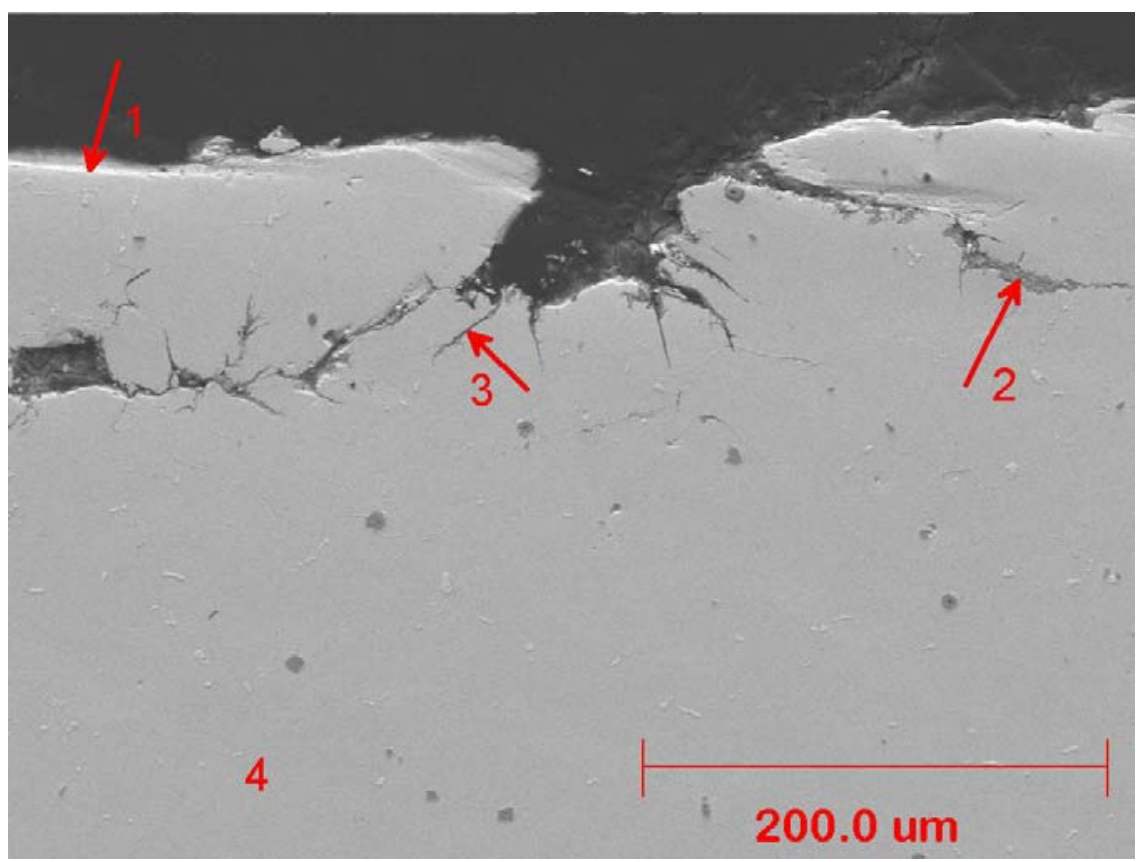

(b) External Scale/Metal Interface (BEI - Intermediate Magnification)

\section{Figure I-12: Microstructure: Thermie}

Note: Numbers on photo indicate distinct areas characterized by the visual assessment and EDS analyses that follow. 


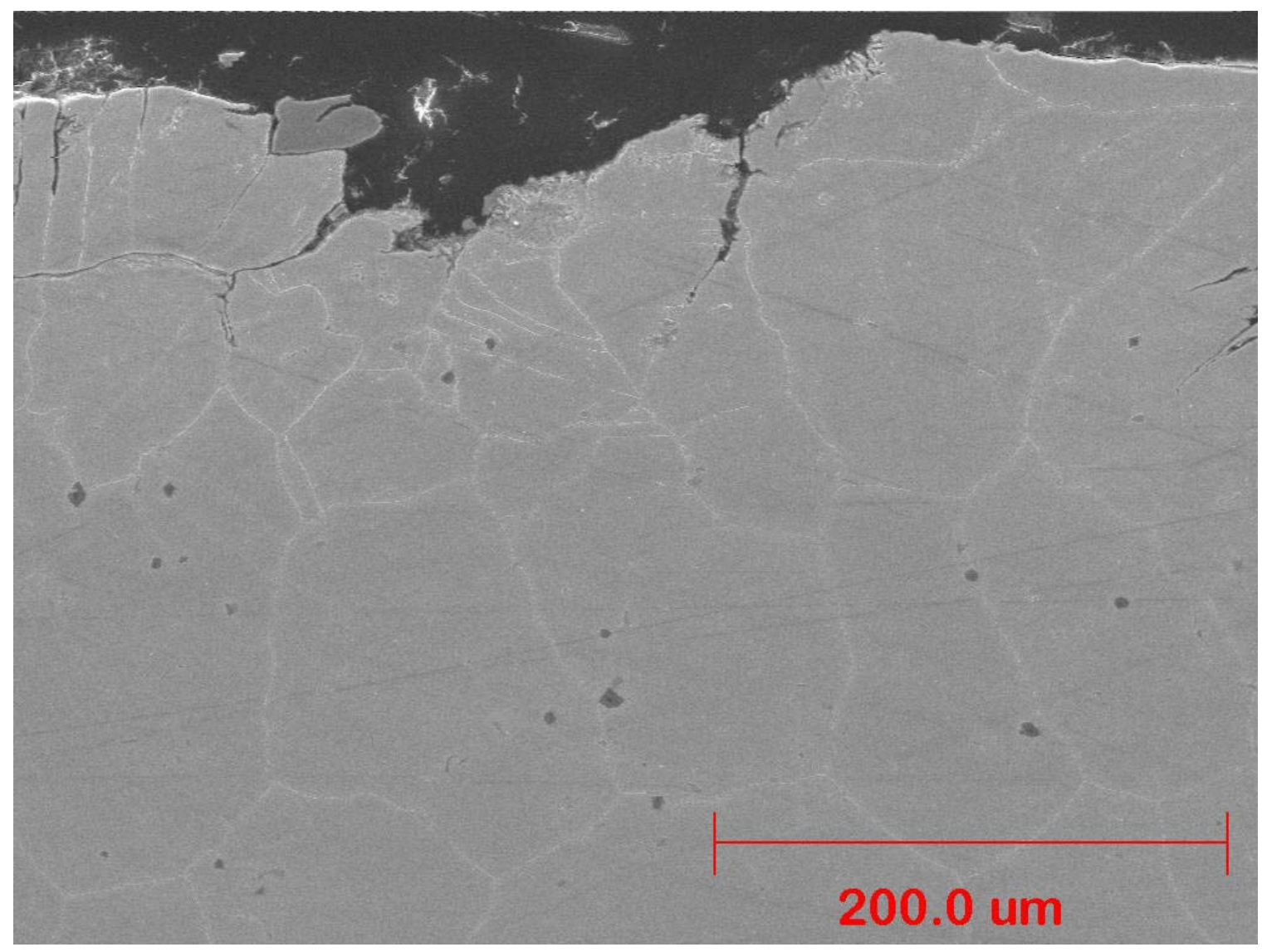

(SEI - Intermediate Magnification)

Figure I-13: Microstructure at OD Surface: Thermie

Note: Electrolytic 10\% oxalic acid etch, 9V OCP 


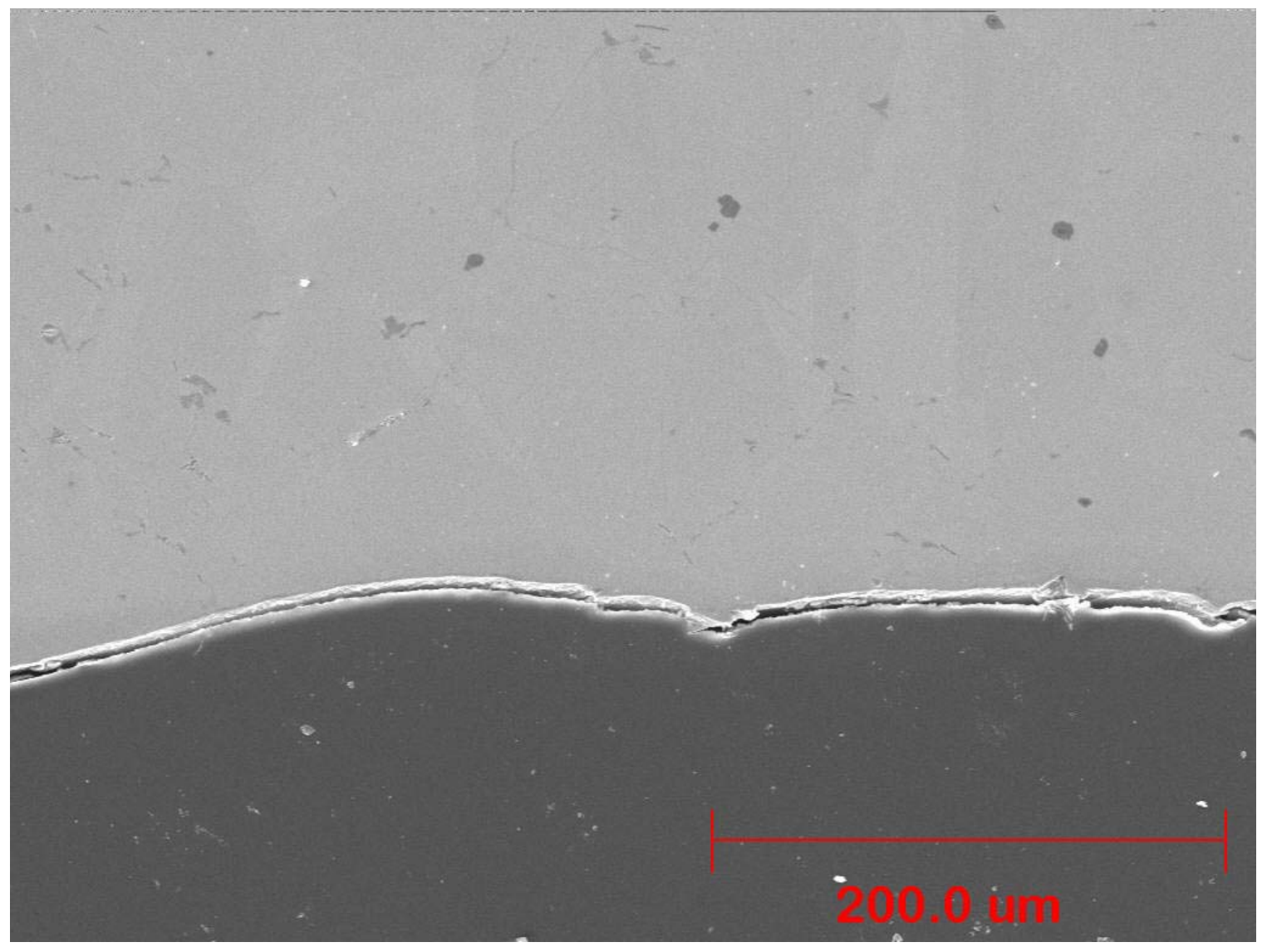

Internal Surface Appearance (SEI - Intermediate Magnification)

Figure I-14: Microstructure: Thermie 


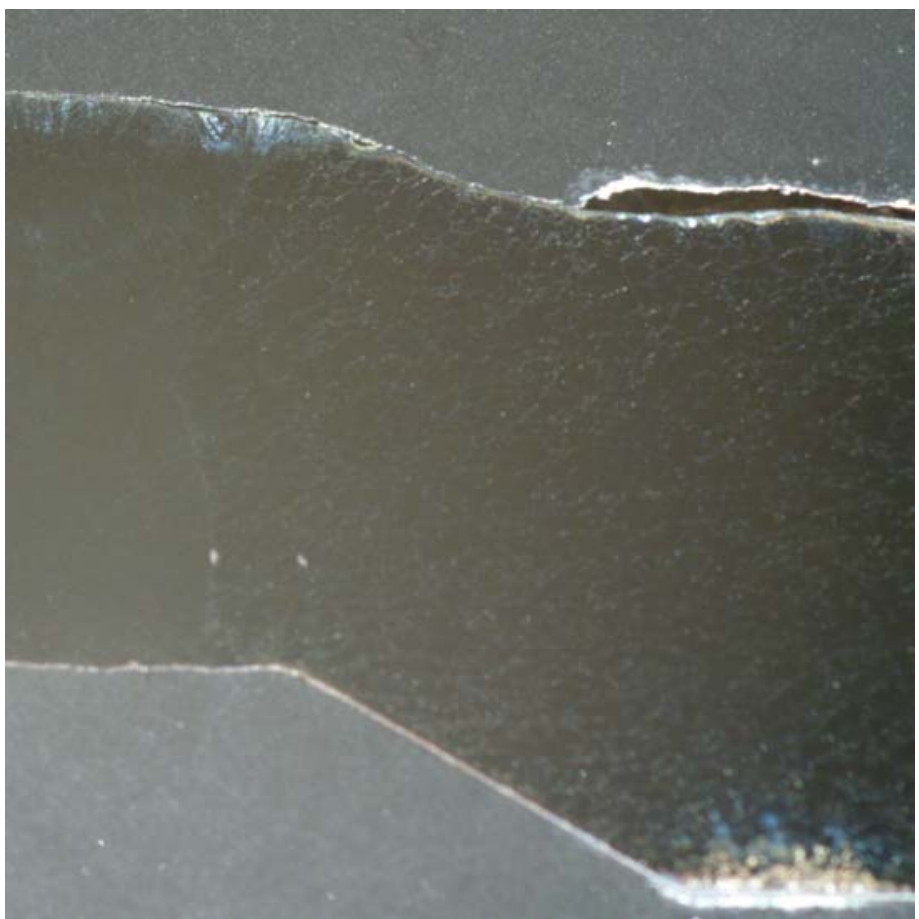

Thermie Adjacent to Weld "HH"

Figure I-15: Longitudinal Cross-Sections: Thermie Adjacent to Weld 
Figure I-16: EDS Analysis of Deposit/Scale/Metal Interface for Thermie (Sample HH-II)

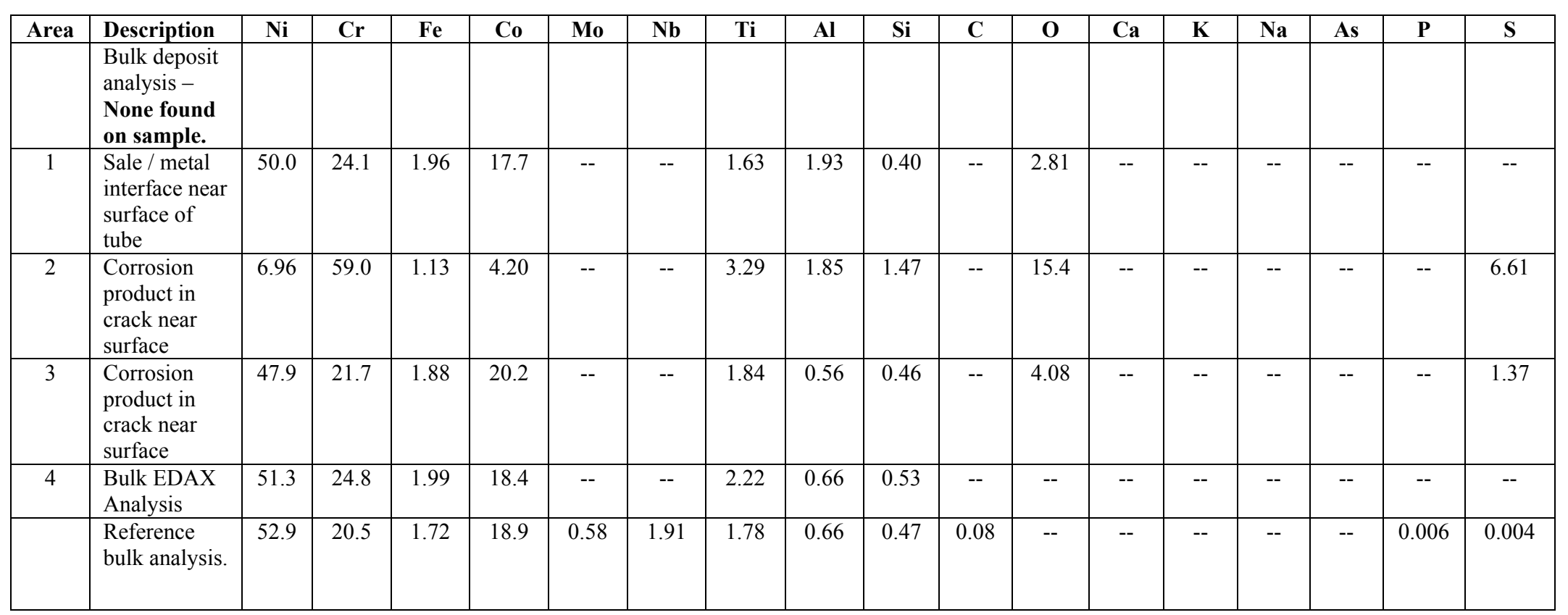


Appendix J

Analysis of Performance

Test Section C

IN52 Weld Overlay 


\section{Overview of Results: IN52 Weld Overlay}

The IN52 Weld Overlay experienced an intermediate rate of metal loss (48 mils per year to 63 mils per year*) in the service environment of Test Section C. The 80 mil thick IN 52 weld overlay was found to have been completely breached during their 2.38 years of exposure. Now that the overlay has been breached, the performance of this material will be based on the scaling resistance of the underlying $800 \mathrm{H}$ base metal. As this point, slightly more than half of the original wall thickness remains for Sample GG-HH which experienced a lower corrosion rate than Sample $\mathrm{R}-\mathrm{S}$ which experienced a higher average surface metal temperature (1208 F vs $1073 \mathrm{~F})$. This increased rate of metal loss at lower temperatures is consistent with the results found for the other high-nickel, high-chromium alloys provided by Special Metals Corporation.

Comparison of the IN 52 data with that for the more highly alloyed IN 72 clad overlay suggests that IN 72, having a maximum rate of wall loss of 14 mils per year, is significantly more resistant to corrosion in the environment of the Niles boiler. However, this result is somewhat mitigated by the fact that IN 72 was found to have suffered an internal cracking problem within the cladding that may make it a somewhat less desirable candidate cladding material. While the IN 52 cladding showed evidence of lack of fusion -type defects, no evidence could be found of the grain boundary cracking found in the IN 72 weld overlay material. This IN 72 cracking may take on additional importance as this program draws to an end in that examination of archive material along with material from Sections A and C suggest that the extent of internal cracking is increasing with increased exposure time.

It should be noted that the metal loss rates determined to date for both Section A and Section C all were based on a ring specimen that was extracted from mid-length of each tube sample. In the current analysis, additional attention was focused on metal losses in the vicinity of welds. Indeed examination revealed that, for the IN 52 samples, the IN 52 cladding material wasted away more rapidly than the Inconel 625 weld metal that was used to join the tube samples together to construct the section.

No significant amount of deposit was found on these samples. Analysis of the scale showed a high level of sulfur confirming the presence of a potentially aggressive environment. The loss of wall thickness in these samples further bears this out.

* Note throughout this appendix, the reported metal loss rate was calculated based on time at temperature rather than total time within the boiler. 


\section{Material: IN52 Weld Overlay}

\section{Summary of Findings}

\begin{tabular}{|c|c|}
\hline Issue & Discussion \\
\hline $\begin{array}{l}\text { Sample } \\
\text { Location and } \\
\text { Orientation. }\end{array}$ & $\begin{array}{l}\text { - The schematic in Figure J-1 shows the relative positions of IN52 Weld } \\
\text { Overlay samples in Test Section A. Note that none of the IN52 overlay } \\
\text { samples was within the tube lengths that were removed early, i.e. in } \\
\text { November } 2001 \text { or in June } 2002 \text {. } \\
\text { - Also, shown is the calculated average metal temperature at both } \\
\text { locations. }\end{array}$ \\
\hline $\begin{array}{l}\text { Composition } \\
\text { Verification. }\end{array}$ & $\begin{array}{l}\text { - The table in Figure J-2 provides the reference composition for the } \\
\text { filler metal as per Special Metals Company (SMC) literature. } \\
\text { - The table also shows the SMC heat analysis for the heat of filler wire } \\
\text { used to deposit the cladding on these samples, Heat NX 9090JK. } \\
\text { During the Section A evaluation, detailed composition verification was } \\
\text { undertaken for every tube sample that was removed from service. This } \\
\text { served, in part, to verify that the each tube material was located at its' } \\
\text { proper position within the test section. } \\
\text { Based on the good results for Section A, it was decided that it would } \\
\text { be unnecessary to analyze each and every tube sample for composition } \\
\text { verification. Rather, it was decided to select a representative sample } \\
\text { from each tube material type for confirmatory analysis. } \\
\text { During the Section A evaluation, the energy dispersive spectrographic } \\
\text { (EDS) capabilities of the scanning electron microscope (SEM) were } \\
\text { used to perform semi-quantitative analyses of each of the twelve } \\
\text { different candidate tube materials. Comparison of these EDS results } \\
\text { showed reasonably good correlation with results obtained using } \\
\text { quantitative analytical techniques, including optical emission } \\
\text { spectrometry. } \\
\text { Based on this, it was decided that the accuracy provided by EDS } \\
\text { would suffice for composition verification, but that more detailed } \\
\text { analyses would be undertaken if questions arose. } \\
\text { The results of the EDS analysis of tube sample GG-HH confirmed that } \\
\text { the correct material was used at this location. }\end{array}$ \\
\hline $\begin{array}{l}\text { As-Received } \\
\text { Appearance }\end{array}$ & $\begin{array}{l}\text { Figure J-3 shows the appearance of the IN52 Weld Overlay tube as } \\
\text { delivered from SMC. Note the distinctive appearance of the weld } \\
\text { cladding. The total wall thickness for this tube is approximately } 290 \\
\text { mils. The clad layer is about } 80 \text { mils thick. An inner layer of } 800 \mathrm{H} \\
\text { provides support. } \\
\text { Figures J-4 and J-5 document the appearance of the external surface of } \\
\text { the IN52 Weld Overlay tube samples after service in Test Section C. } \\
\text { Three views are provided for each tube: } 12 \text { o'clock (the view that } \\
\text { faces on-coming gas flow), } 3 \text { o'clock (the view where the gas flow is } \\
\text { tangent to the surface), and } 6 \text { o'clock (the view that shows the back- } \\
\text { side of the tube relative to the gas flow direction. }\end{array}$ \\
\hline
\end{tabular}




\section{Material: IN52 Weld Overlay}

\section{Summary of Findings, (continued)}

\begin{tabular}{|c|c|}
\hline Issue & Discussion \\
\hline $\begin{array}{l}\text { As-Received } \\
\text { Appearance, } \\
\text { Continued }\end{array}$ & $\begin{array}{l}\text { - The reader should be alerted to the fact that, within the boiler, the gas } \\
\text { flows upward over these tubes. This implies that for the "o'clock" } \\
\text { conventions described above, (with the } 12 \text { o'clock orientation facing } \\
\text { the gas flow direction), the actual physical orientation of these tubes is } \\
\text { such that the } 12 \text { o'clock side of the tube faces downward, and the } 6 \\
\text { o'clock orientation faces upward within the boiler. } \\
\text { - No wastage is visible on either sample, but this likely is obscured by } \\
\text { the deposit/scale at the } 12 \text { o'clock and } 3 \text { o'clock positions on both } \\
\text { samples. } \\
\text { Evidence of the gray/white scale associated with molten alkali-iron } \\
\text { sulfate attack was found on both samples. }\end{array}$ \\
\hline $\begin{array}{l}\text { Grit-Blasted } \\
\text { Surface } \\
\text { Appearance }\end{array}$ & $\begin{array}{l}\text { - Figures J-6 through J-7 show the appearance of the external surface of } \\
\text { the IN52 Weld Overlay tube samples after grit-blasting to remove } \\
\text { deposit and scale. The } 12 \text { o'clock and } 3 \text { o'clock positions for both } \\
\text { samples show the appearance of general wastage due to fireside } \\
\text { corrosion. Some pitting is also suggested at these locations. } \\
\text { - The } 6 \text { o'clock position, for tube Sample GG-HH, shows evidence of } \\
\text { pitting attach. Very little attack is found at the } 6 \text { o'clock position for } \\
\text { Sample R-S. }\end{array}$ \\
\hline $\begin{array}{l}\text { Wall } \\
\text { Thickness } \\
\text { Loss } \\
\text { Determined }\end{array}$ & $\begin{array}{l}\text { - Tube cross-sections were prepared to allow for measurement of wall } \\
\text { thickness to determine metal loss due to exposure. These are shown in } \\
\text { silhouette in Figures J-8 and J-9. } \\
\text { The same relative "o'clock" positions were maintained as discussed } \\
\text { earlier. Measurements were taken prior to service at eight evenly } \\
\text { spaced locations about the tube circumference. To prepare for this, the } \\
\text { external surface of the cladding was ground flush to minimize } \\
\text { thickness variability due to surface contour. After-service wall } \\
\text { thickness measurements were taken at these same locations then } \\
\text { compared with the initial thickness. } \\
\text { The before and after measurements were documented in the table that } \\
\text { accompanies each figure. These form the basis for the "delta" } \\
\text { calculation to determine the amount of wall thickness lost. Note that in } \\
\text { some cases, the delta is negative (e.g at the } 6 \text { o'clock position for } \\
\text { Sample GG-HH). This is believed due to wall thickness variability and } \\
\text { difficulty in locating the exact location of the "before" measurement. }\end{array}$ \\
\hline $\begin{array}{l}\text { Wall } \\
\text { Thickness } \\
\text { Loss Versus } \\
\text { Average Metal } \\
\text { Temperature }\end{array}$ & $\begin{array}{l}\text { - The chart in Figure J-10 plots the maximum wall thickness lost against } \\
\text { the calculated average metal temperature for each of the two IN52 } \\
\text { Weld Overlay samples. The data for Section A also are plotted. } \\
\text { The data in the chart suggest that the metal loss rate decreases with } \\
\text { increased temperature for the IN52 Weld Overlay samples. } \\
\text { A significant metal loss rate (of } 63 \text { mils per year for Sample GG-HH, } \\
\text { and } 48 \text { mils per year for Sample R-S in Section C). This rate was } \\
\text { adequate to breach the cladding thickness ( } 80 \text { mils) for both samples, } \\
\text { exposing the underlying Incoloy } 800 \mathrm{H} \text {. }\end{array}$ \\
\hline
\end{tabular}




\section{Material: IN52 Weld Overlay}

\section{Summary of Findings, (continued)}

\begin{tabular}{|c|c|}
\hline Issue & Discussion \\
\hline $\begin{array}{l}\text { Wall } \\
\text { Thickness } \\
\text { Loss as a } \\
\text { Function of } \\
\text { Time and } \\
\text { Location }\end{array}$ & $\begin{array}{l}\text { - The chart in Figure J-11 plots metal loss as a function of time for each } \\
\text { sample location for both Sections A and C, with samples from Section } \\
\text { C having experienced generally longer exposure times. } \\
\text { Both tube samples experienced increased metal loss with time; } \\
\text { however, the data above suggest the rate decreased as a function of } \\
\text { time, so, a straight-line fit likely is not appropriate. }\end{array}$ \\
\hline $\begin{array}{l}\text { Metallurgical } \\
\text { Analysis - } \\
\text { SEM }\end{array}$ & 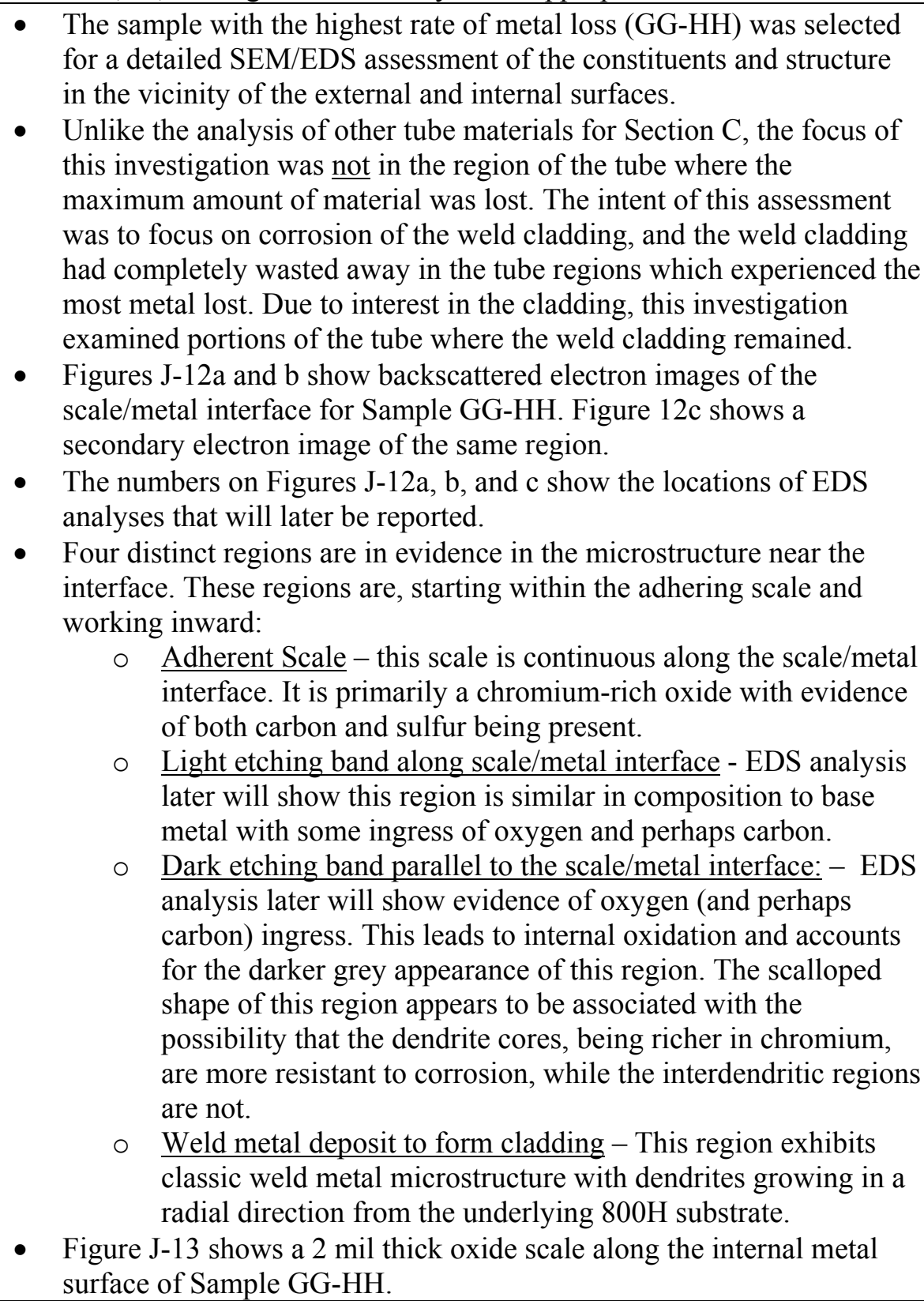 \\
\hline
\end{tabular}




\section{Summary of Findings, (continued)}

\section{Material: IN52 Weld Overlay}

\begin{tabular}{|c|c|}
\hline Issue & Discussion \\
\hline $\begin{array}{l}\text { Metallurgical } \\
\text { Analysis - } \\
\text { Weld Cross } \\
\text { Section. }\end{array}$ & $\begin{array}{l}\text { - Figure J-14 shows a longitudinal profile (at the } 12 \text { o'clock orientation) } \\
\text { of the IN52 Weld Overlay samples adjacent to the welds in Section C. } \\
\text { The welds in question are those identified as welds "R" and "HH", see } \\
\text { Figure J-1 for locations } \\
\text { - Examination of Figure J-14 reveals that in both cases the IN } 52 \text { weld } \\
\text { cladding has been completely wasted away. Also note that the Inconel } \\
625 \text { weld metal appears to have wasted to a lesser extent. In any case, } \\
\text { the wall thickness adjacent to the weld is somewhat thicker and it } \\
\text { tapers toward the middle of the tube length. This seems to lend validity } \\
\text { to the dimensions taken from a ring that was extracted from the middle } \\
\text { of this tube. }\end{array}$ \\
\hline $\begin{array}{l}\text { EDAX } \\
\text { Analysis } \\
\text { Results }\end{array}$ & $\begin{array}{l}\text { - The table in Figure J-15 provides a summary of the EDS analyses of } \\
\text { the numbered locations in Figure J-12. Also shown in this table is a } \\
\text { summary of the spectrographic analysis reported earlier, see Figure } \\
\text { J-2. } \\
\text { - Review of this table suggests the following: } \\
\text { - } \text { Area 1: Adherent scale - This is primarily a chromium-rich } \\
\text { oxide. The data also suggest a high concentration of sulfur and } \\
\text { perhaps carbon. } \\
\text { - Area 2: Light etching band along scale interface - The } \\
\text { composition of this band is similar to that of the base metal with } \\
\text { some ingress of oxygen and perhaps carbon. } \\
\text { Area 3: Dendrite core - This analysis suggests that the dendrite } \\
\text { core has a composition very similar to that of the bulk metal } \\
\text { analysis. } \\
\text { - Area 4: Inderdendritic region - corrosion possible - This analysis } \\
\text { suggests that the region between dendrites has a reduced level of } \\
\text { nickel relative to the bulk metal analysis. Evidence of oxygen and } \\
\text { perhaps carbon suggest that corrosion has affected this region that } \\
\text { is located near to the scale/metal interface. } \\
\text { Area 5: Interdendritic region - not corroded - This interdendritic } \\
\text { region is further away from the scale/metal interface and shows } \\
\text { no evidence of corrosion. } \\
\text { Area 6: Grain boundary that crosses corroded zone - This region } \\
\text { was chosen because it did not evidence corrosion while the } \\
\text { adjacent grains did. Analysis shows that this boundary was } \\
\text { enriched in chromium. } \\
\text { Area 7: Bulk metal analysis - This analysis was used to verify } \\
\text { that the weld cladding had a composition consistent with IN 52. }\end{array}$ \\
\hline
\end{tabular}




\section{Gas Flow}

Direction

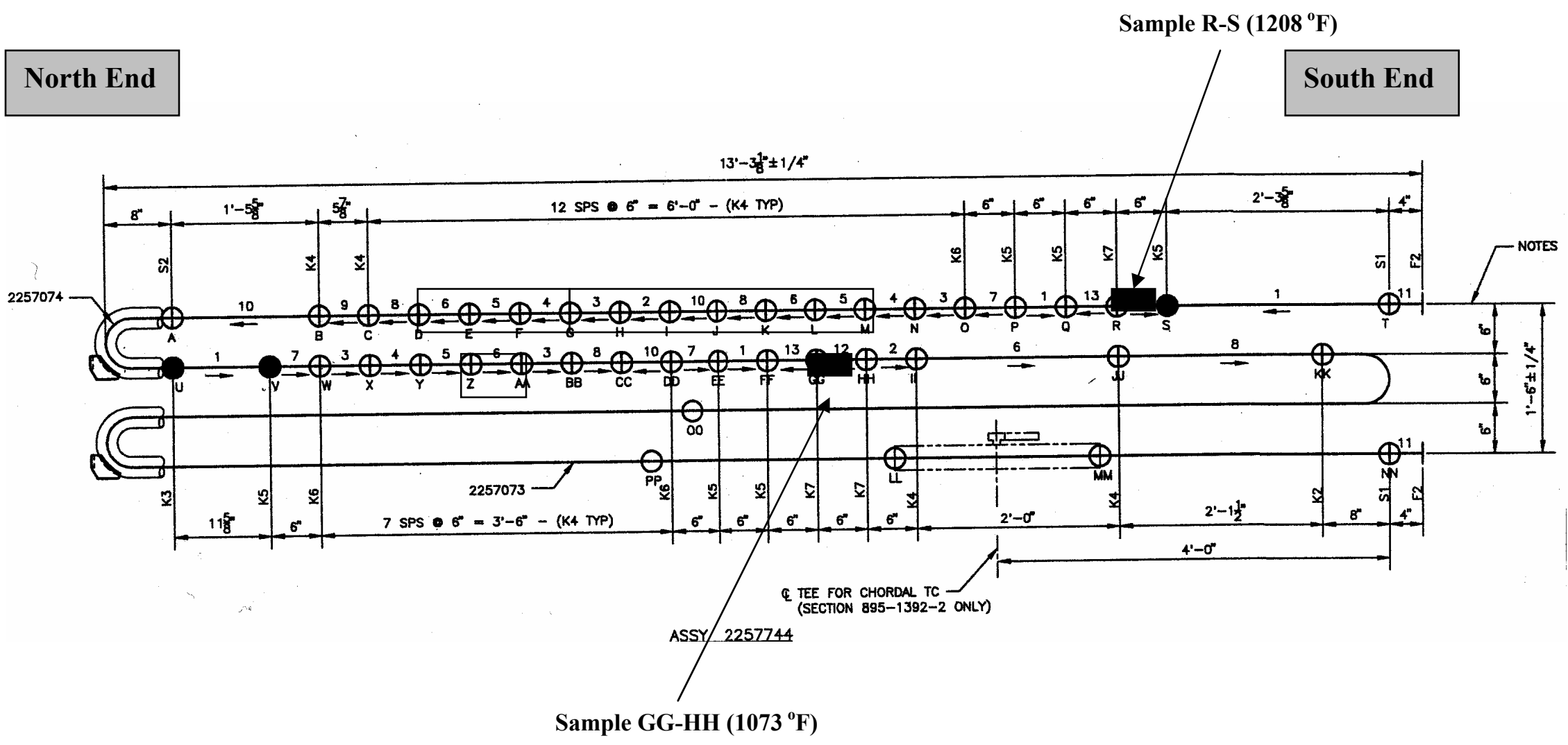

Figure J-1: Schematic Drawing Showing Position of IN52 Weld Overlay Tube Samples Within Test Section C

1. Drawing shown in same relative orientation as per the actual installation.

2. This group of tube samples was removed from service in November 2001 - highlighted in red.

3. This group of tube samples was removed from service in June 2002 - highlighted in yellow. 
Figure J-2 Semi-Quantitative Composition Verification for Inco 52 / 800 HT (Weld clad duplex tubing)

Description:

Source:

Reference Composition:
Nominal $30 \mathrm{Cr}$ nickel alloy cladding for oxidation/corrosion protection. Incoloy 800HT substrate provides creep strength. Special Metals Corporation (SMC)

Filler wire "limiting chemical composition" - Per SMC literature. (ANSI AWS 5.14M-97 Class ERNiCrFe-7)

\begin{tabular}{|c|c|c|c|c|}
\hline Element & $\begin{array}{c}\text { Reference } \\
\text { Composition for } \\
\text { Weld Cladding }\end{array}$ & $\begin{array}{c}\text { Composition } \\
\text { Heat NX 9090JK }\end{array}$ & $\begin{array}{c}\text { Tube Sample } \\
\text { R-S }\end{array}$ & $\begin{array}{c}\text { Tube Sample } \\
\text { GG-HH }\end{array}$ \\
\hline $\mathrm{Ni}$ & Balance & 60.48 & $\mathrm{~N} / \mathrm{A}$ & 59.9 \\
\hline $\mathrm{Cr}$ & $28.0-31.5$ & 28.97 & $\mathrm{~N} / \mathrm{A}$ & 29.2 \\
\hline $\mathrm{Fe}$ & $7.0-11.0$ & 8.86 & $\mathrm{~N} / \mathrm{A}$ & 9.25 \\
\hline Mo & $0.50 \max$ & 0.01 & N/A & - \\
\hline $\mathrm{Co}$ & - & 0.01 & $\mathrm{~N} / \mathrm{A}$ & - \\
\hline $\mathrm{C}$ & $0.04 \max$ & 0.02 & $\mathrm{~N} / \mathrm{A}$ & - \\
\hline $\mathrm{N}$ & - & - & $\mathrm{N} / \mathrm{A}$ & - \\
\hline $\mathrm{B}$ & - & - & $\mathrm{N} / \mathrm{A}$ & - \\
\hline $\mathrm{Mn}$ & $1.0 \max$ & 0.24 & $\mathrm{~N} / \mathrm{A}$ & - \\
\hline $\mathrm{Si}$ & $0.50 \max$ & 0.14 & $\mathrm{~N} / \mathrm{A}$ & 0.36 \\
\hline $\mathrm{Al}$ & $1.10 \max$ & 0.69 & $\mathrm{~N} / \mathrm{A}$ & 0.68 \\
\hline $\mathrm{Ti}$ & $1.0 \max$ & 0.58 & N/A & 0.61 \\
\hline $\mathrm{Al}+\mathrm{Ti}$ & $1.5 \max$ & 1.27 & N/A & 1.29 \\
\hline $\mathrm{Nb}$ & $0.10 \max$ & 0.01 & $\mathrm{~N} / \mathrm{A}$ & - \\
\hline $\mathrm{Ta}$ & - & $<0.01$ & N/A & - \\
\hline $\mathrm{Nb}+\mathrm{Ta}$ & - & 0.02 & $\mathrm{~N} / \mathrm{A}$ & - \\
\hline V & - & - & N/A & - \\
\hline $\mathrm{W}$ & - & - & $\mathrm{N} / \mathrm{A}$ & - \\
\hline $\mathrm{Cu}$ & $0.30 \max$ & 0.01 & $\mathrm{~N} / \mathrm{A}$ & - \\
\hline $\mathrm{Sn}$ & - & - & N/A & - \\
\hline $\mathrm{P}$ & $0.020 \max$ & 0.004 & $\mathrm{~N} / \mathrm{A}$ & - \\
\hline $\mathrm{S}$ & $0.015 \max$ & $<0.001$ & $\mathrm{~N} / \mathrm{A}$ & - \\
\hline $\mathrm{O} 2$ & - & - & $\mathrm{N} / \mathrm{A}$ & - \\
\hline Others & $0.50 \max$ & $<0.50$ & $\mathrm{~N} / \mathrm{A}$ & - \\
\hline
\end{tabular}

$\mathrm{N} / \mathrm{A}=$ Sample not analyzed

Note: Reference composition is for filler wire, not as-deposited. 


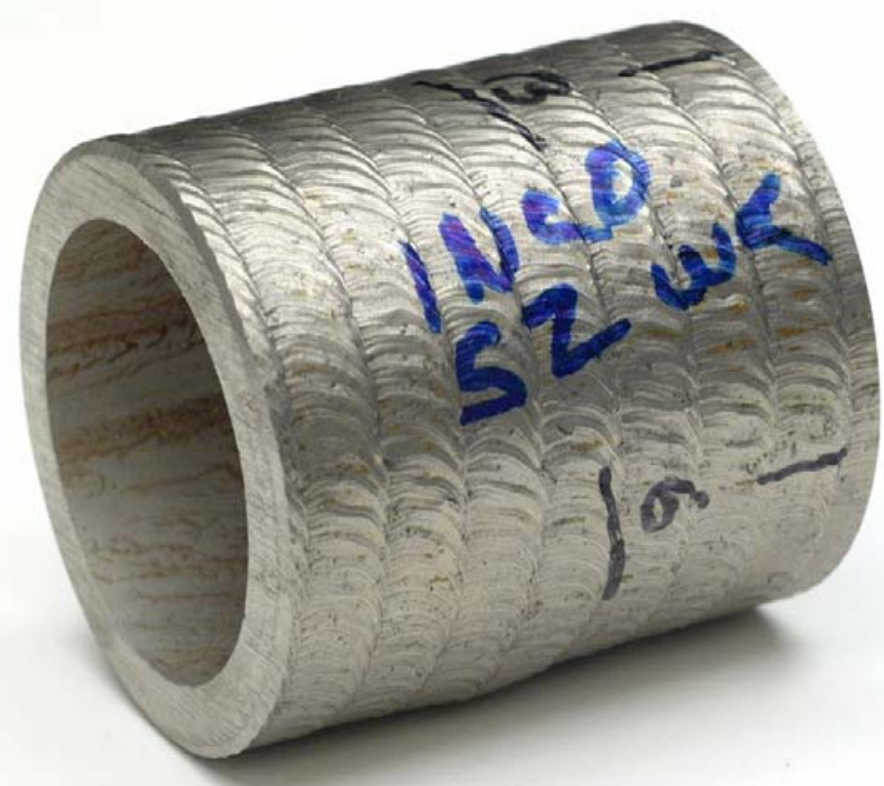

Figure J-3: Appearance of IN52 Weld Overlay Tube Sample Prior to Service 


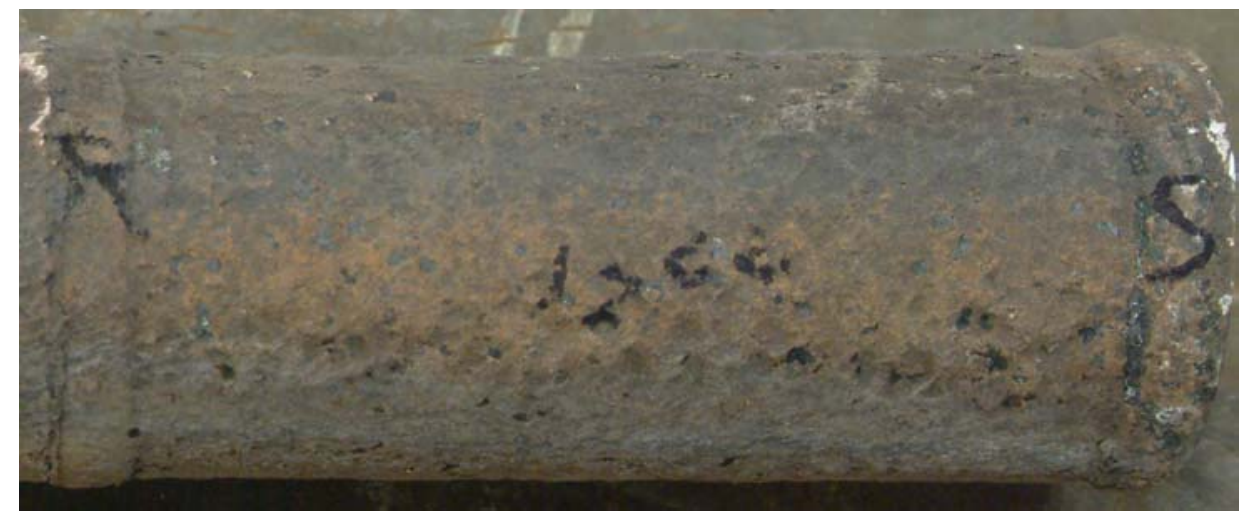

12 O'Clock - Facing Gas Flow

(Note: 3 o'clock is up in this image)

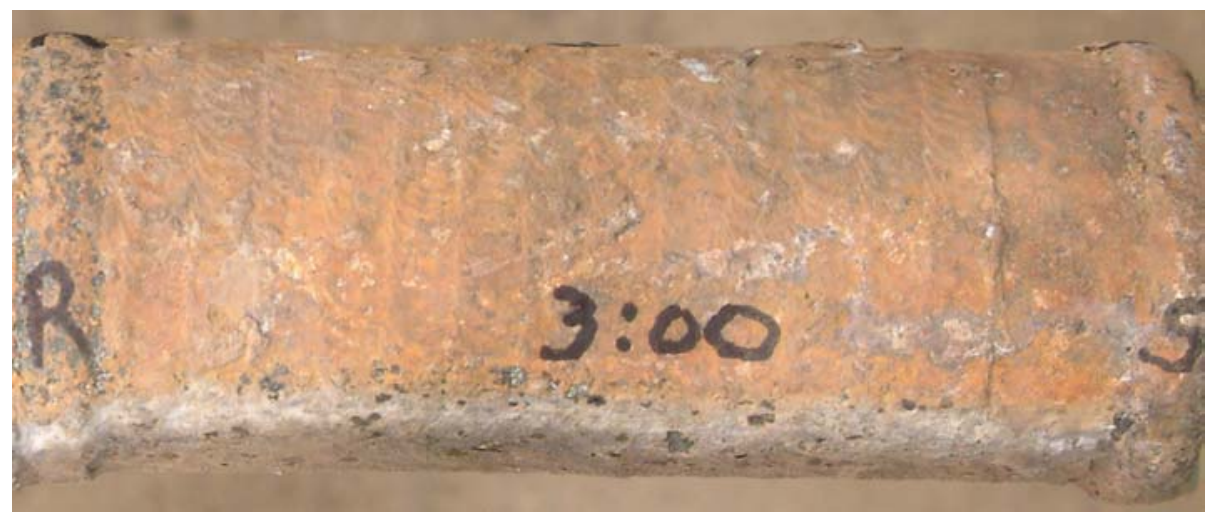

3 O'Clock

(Note: 6 o'clock is up in this image)

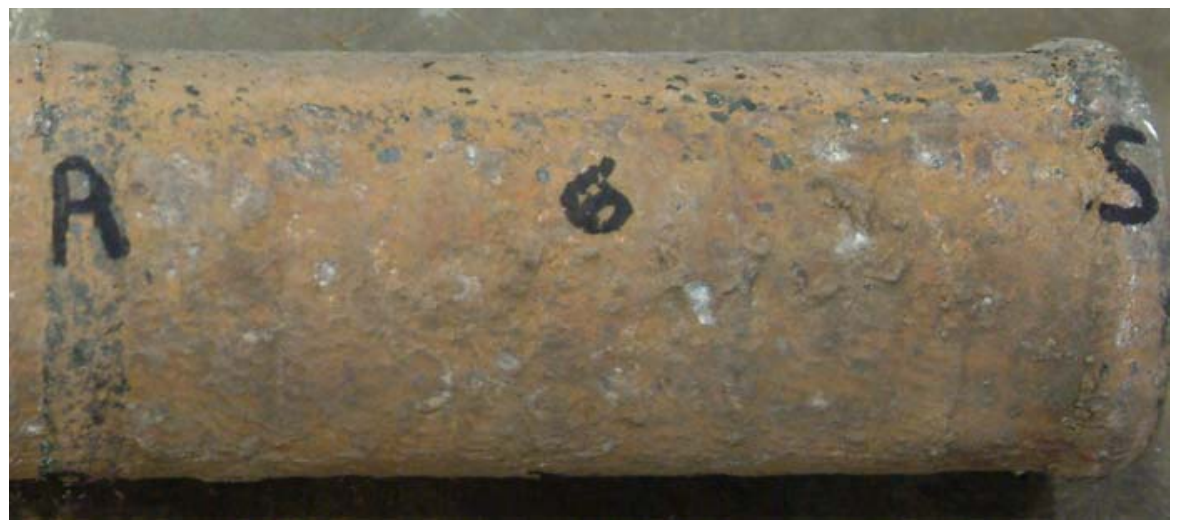

6 O'Clock - Opposite Gas Flow

Figure J-4: As-Received Appearance of Sample R-S (IN52 Weld Overlay)

(Average surface metal temperature: $1208^{\circ} \mathrm{F}$ ) 


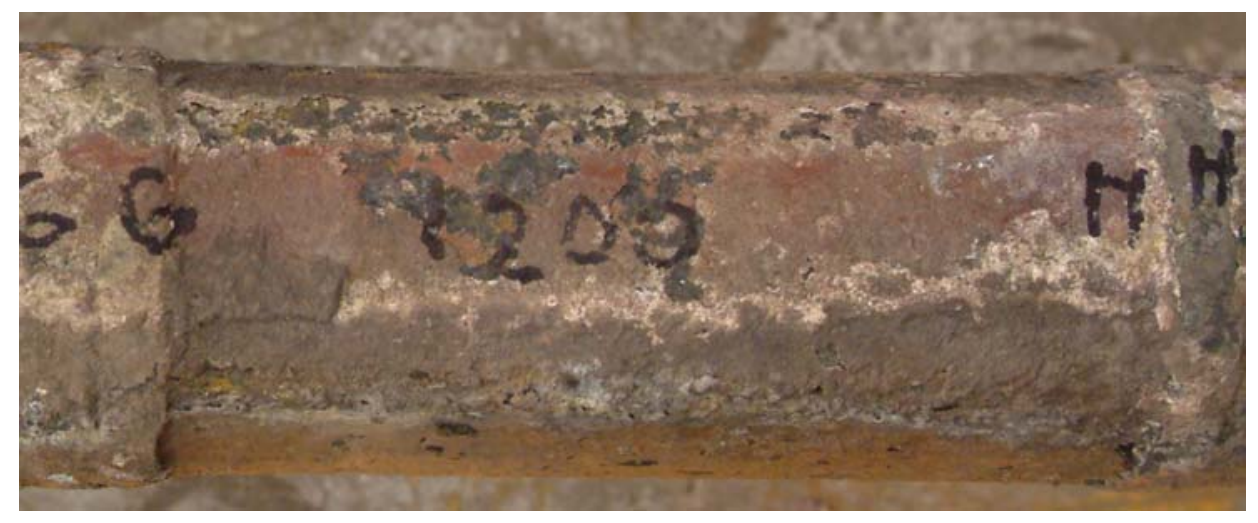

12 O'Clock - Facing Gas Flow

(Note: 3 o'clock is up in this image)

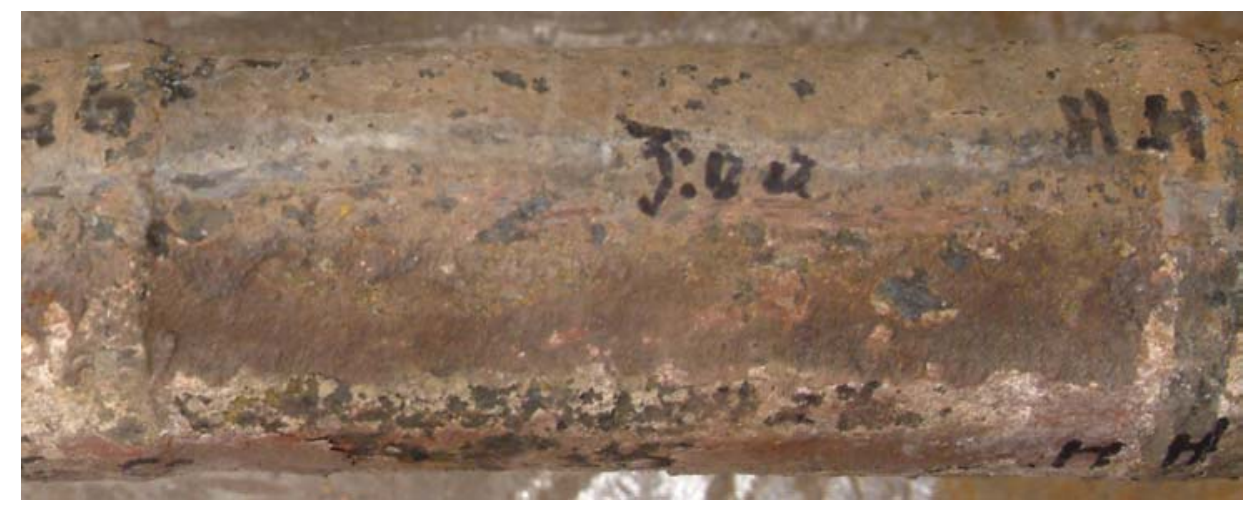

3 O'Clock

(Note: 6 o'clock is up in this image)

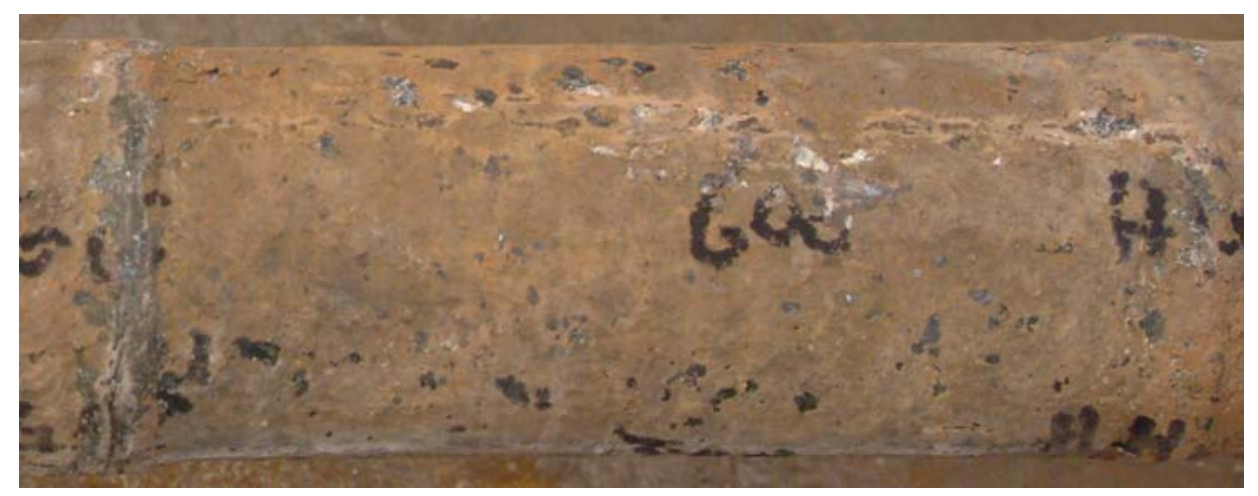

6 O'Clock - Opposite Gas Flow

Figure J-5: As-Received Appearance of Sample GG-HH (IN52 Weld Overlay)

(Average surface metal temperature: $1073^{\circ} \mathrm{F}$ ) 
12 O'Clock

(N/A mils lost)

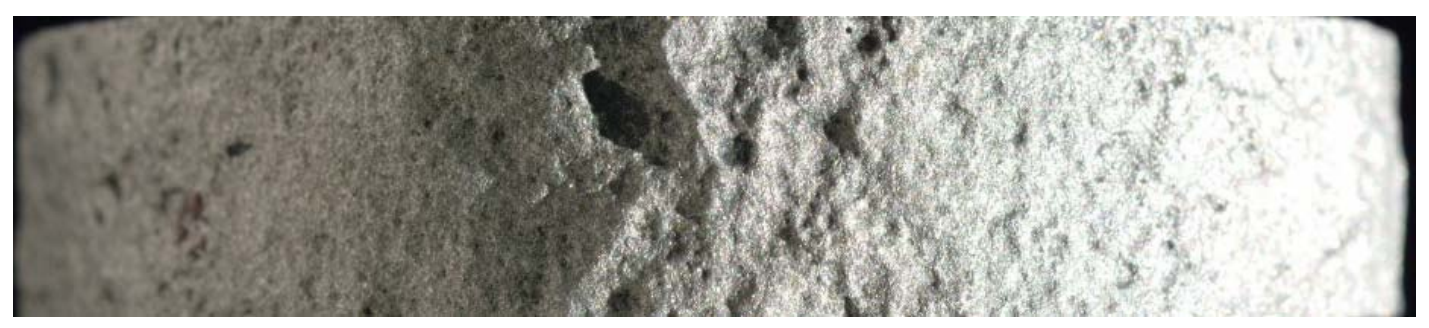

3 O'Clock

(91 mils lost)

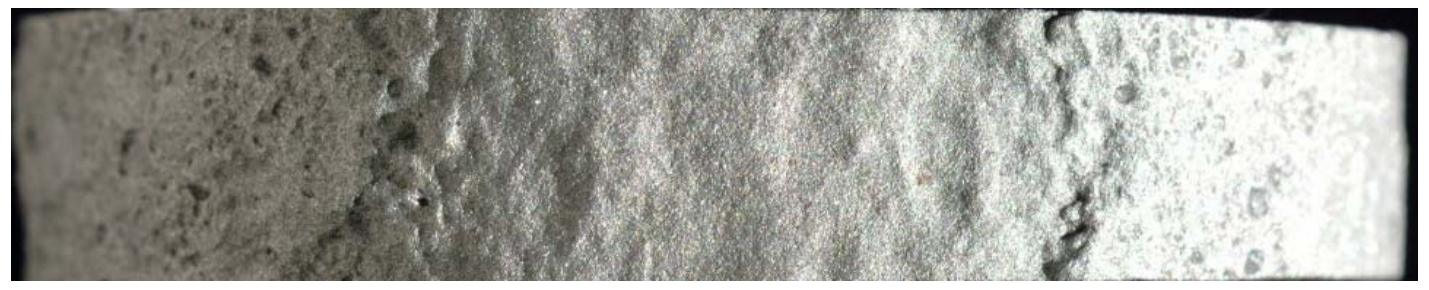

6 O'Clock

(3 mils lost)

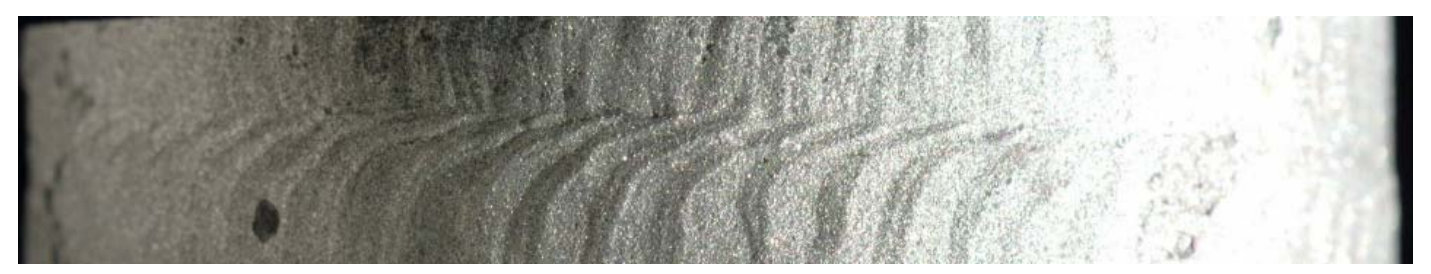

Figure J-6: OD Surface Appearance After Grit Blast

Material: IN52 Weld Overlay

Sample: R-S 
12 O'Clock

(N/A mils lost)

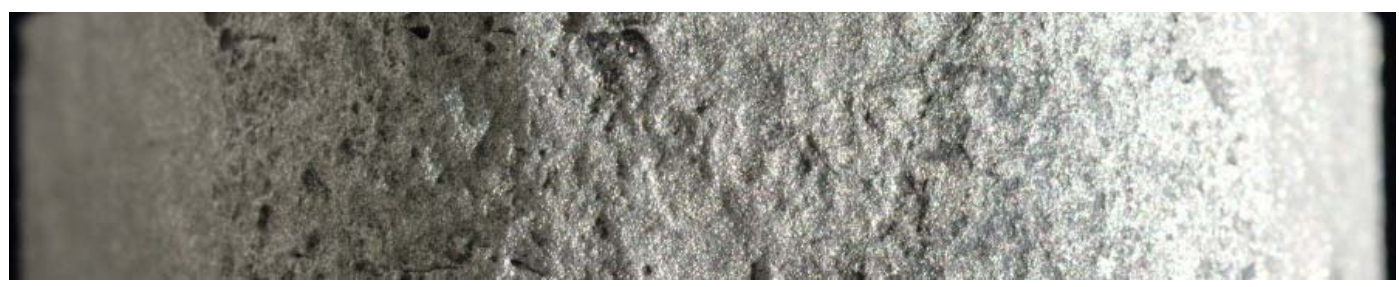

3 O'Clock

(106 mils lost)

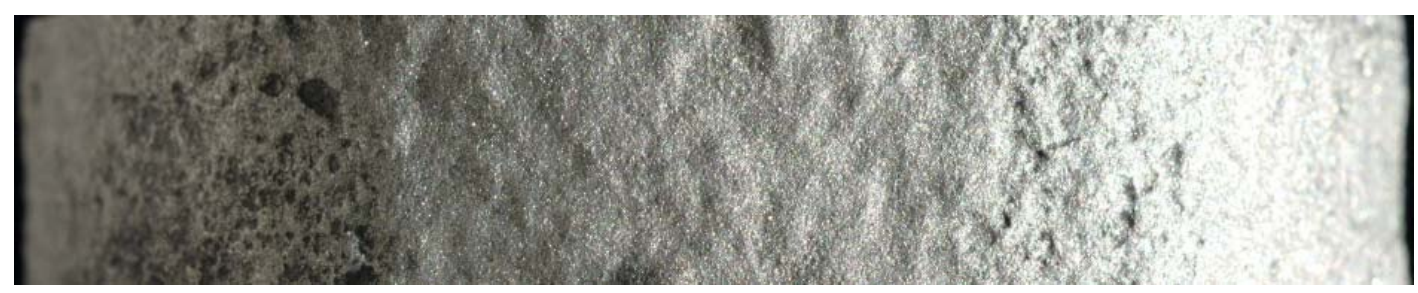

6 O'Clock

(0 mils lost)

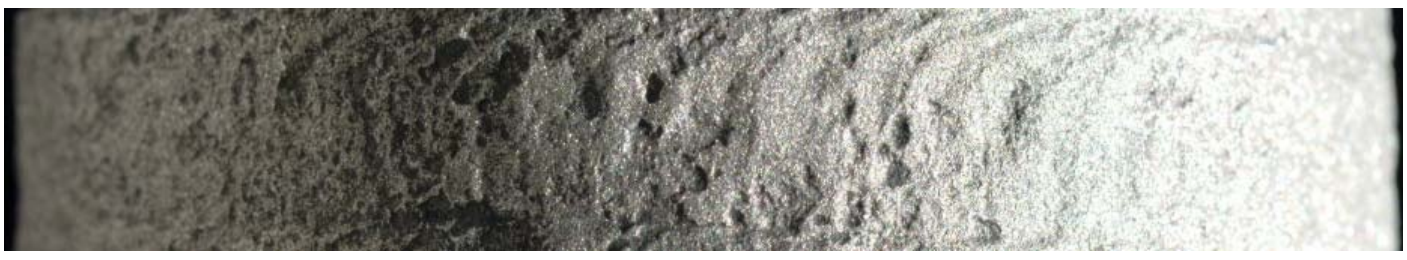

Figure J-7: OD Surface Appearance After Grit Blast

Material: IN52 Weld Overlay

Sample: GG-HH 


\section{Material:}

Sample Location:

Maximum Rate:

Maximum Loss Orientation:

Average Surface Metal Temperature:

\section{IN52 Weld Overlay}

R-S

$48 \mathrm{mils} / \mathrm{yr}$

Approx. 2:30 o'clock

\section{O’Clock}

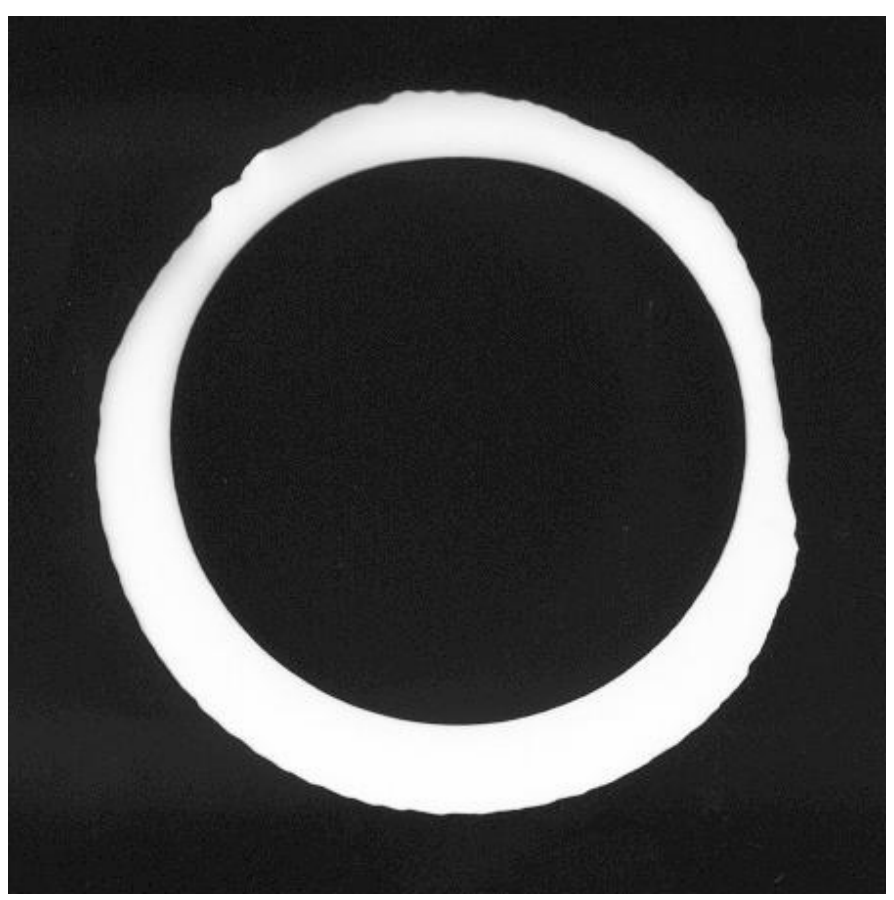

3 O'Clock

Approx. 1.2X

\section{Wall Reduction by Position (in.)}

\begin{tabular}{|l|c|c|c|c|c|c|c|c|c|c|}
\hline & $\begin{array}{c}\mathbf{1 2} \\
\text { o'clock }\end{array}$ & $\mathbf{1 : 3 0}$ & $\mathbf{3 : 0 0}$ & $\mathbf{4 : 3 0}$ & $\mathbf{6 : 0 0}$ & $\mathbf{7 : 3 0}$ & $\mathbf{9 : 0 0}$ & $\mathbf{1 0 : 3 0}$ & AVG & $\begin{array}{c}\text { Max }^{*} \\
\text { Loss }\end{array}$ \\
\hline Before & 0.287 & 0.285 & 0.290 & 0.295 & 0.302 & 0.301 & 0.293 & 0.286 & 0.292 & 0.292 \\
\hline After & N/A & 0.223 & 0.199 & 0.291 & 0.299 & 0.299 & 0.296 & 0.249 & & 0.178 \\
\hline Delta & N/A & 0.062 & 0.091 & 0.004 & 0.003 & 0.002 & -0.003 & 0.037 & & 0.114 \\
\hline
\end{tabular}

Figure J-8: Silhouette and Wall Thickness Measurements for Sample R-S

* Measurement taken at "maximum loss orientation" noted above. The average

"before" wall thickness was used to calculate the "delta". 


\section{Material:}

Sample Location:

Maximum Rate:

Maximum Loss Orientation:

Average Surface Metal Temperature:

\section{IN52 Weld Overlay}

GG-HH

$63 \mathrm{mils} / \mathrm{yr}$

Approx. 10 o'clock

$1073^{\circ} \mathrm{F}$

\section{O’Clock}

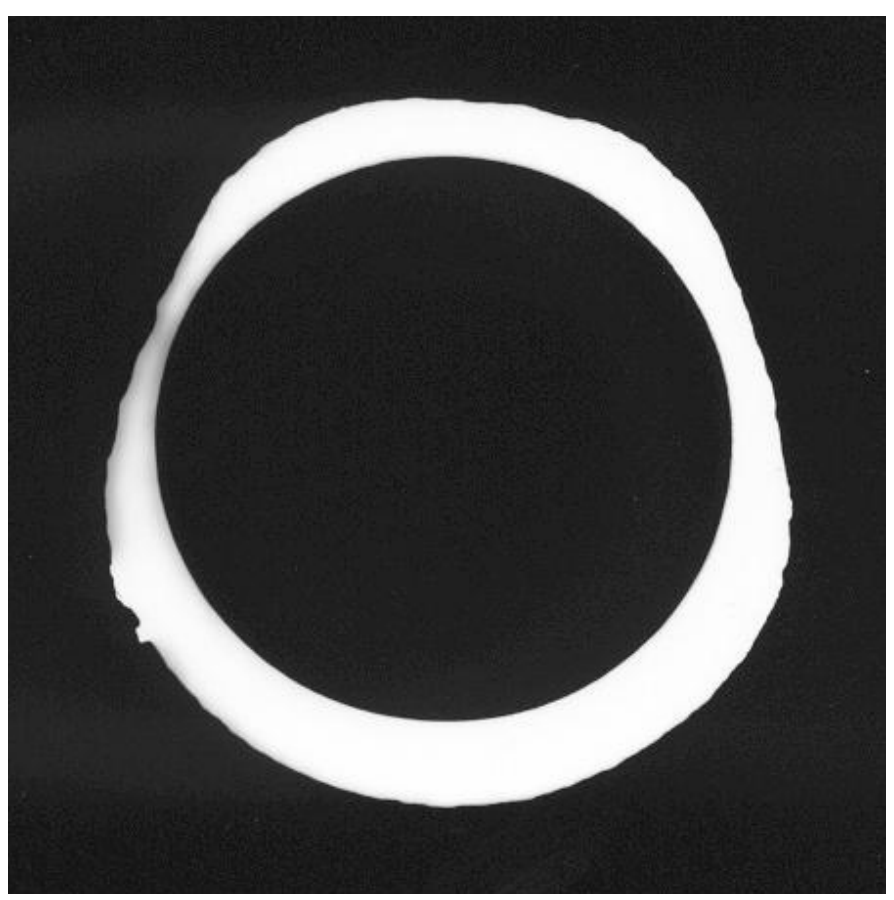

3 O'Clock

Approx. 1.2X

\section{Wall Reduction by Position (in.)}

\begin{tabular}{|l|c|c|c|c|c|c|c|c|c|c|}
\hline & $\begin{array}{c}\mathbf{1 2} \\
\text { o'clock }\end{array}$ & $\mathbf{1 : 3 0}$ & $\mathbf{3 : 0 0}$ & $\mathbf{4 : 3 0}$ & $\mathbf{6 : 0 0}$ & $\mathbf{7 : 3 0}$ & $\mathbf{9 : 0 0}$ & $\mathbf{1 0 : 3 0}$ & AVG & $\begin{array}{c}\text { Max }^{*} \\
\text { Loss }\end{array}$ \\
\hline Before & 0.284 & 0.282 & 0.282 & 0.287 & 0.293 & 0.294 & 0.293 & 0.289 & 0.288 & 0.288 \\
\hline After & N/A & 0.213 & 0.176 & 0.283 & 0.295 & 0.283 & 0.247 & 0.162 & & 0.138 \\
\hline Delta & N/A & 0.069 & 0.106 & 0.004 & -0.002 & 0.011 & 0.046 & 0.127 & & 0.150 \\
\hline
\end{tabular}

Figure J-9: Silhouette and Wall Thickness Measurements for Sample GG-HH

* Measurement taken at "maximum loss orientation" noted above. The average

"before" wall thickness was used to calculate the "delta". 


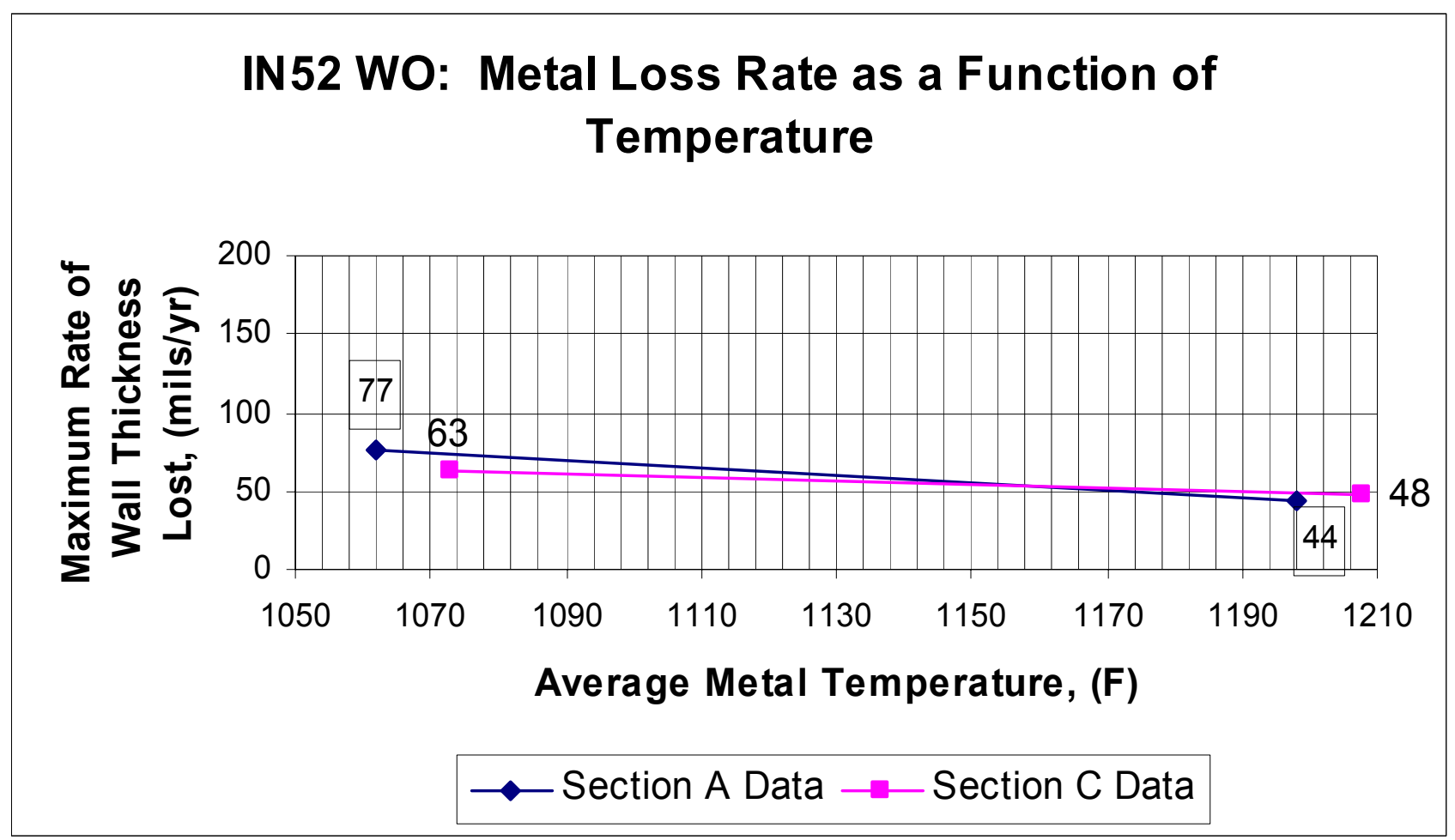

Figure J-10: Metal Loss Rate as a Function of Average Surface Metal Temperature

Material: IN52 Weld Overlay 


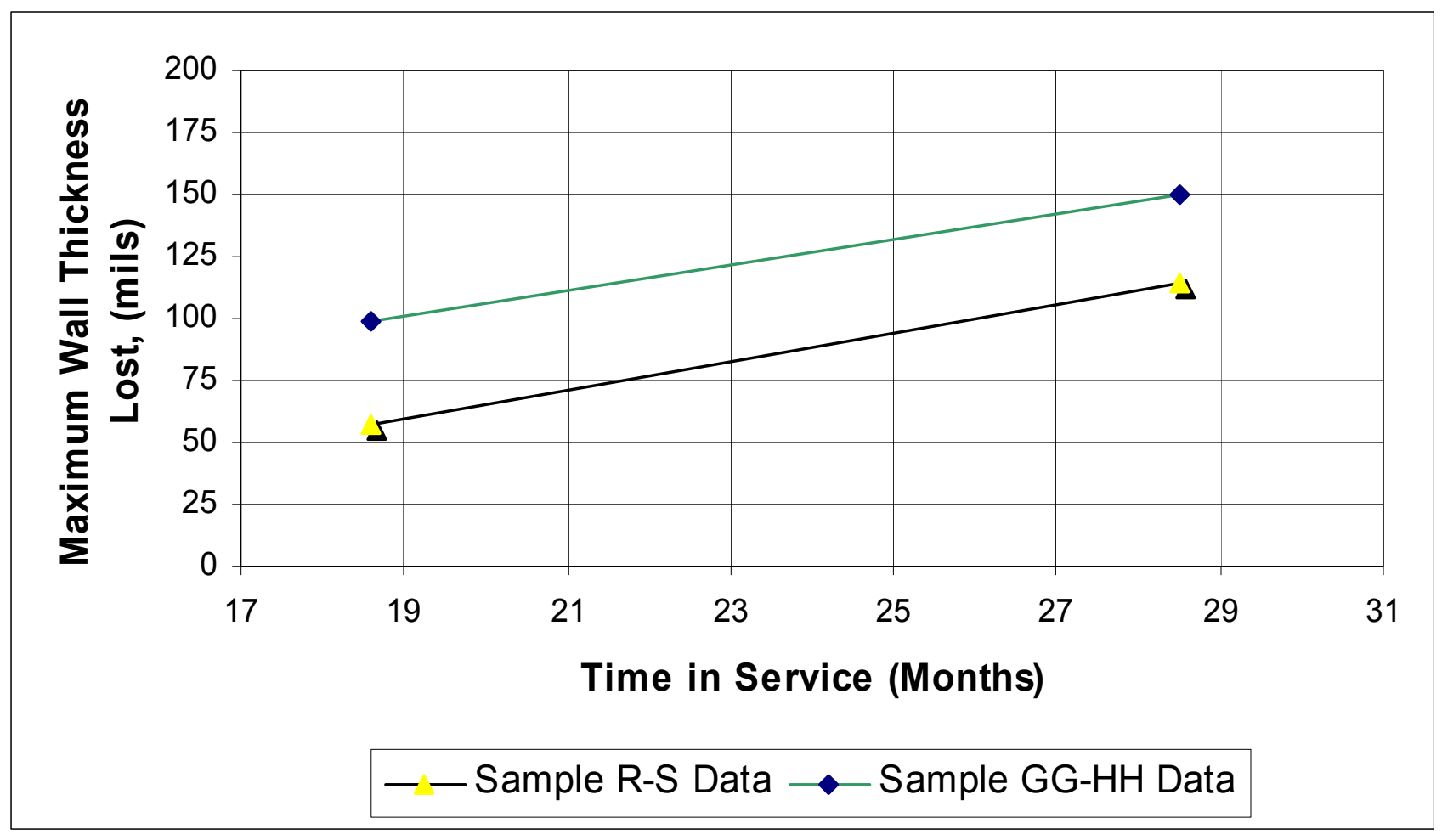

Figure J-11: Metal Loss as a Function of Time

Material: IN52 Weld Overlay 


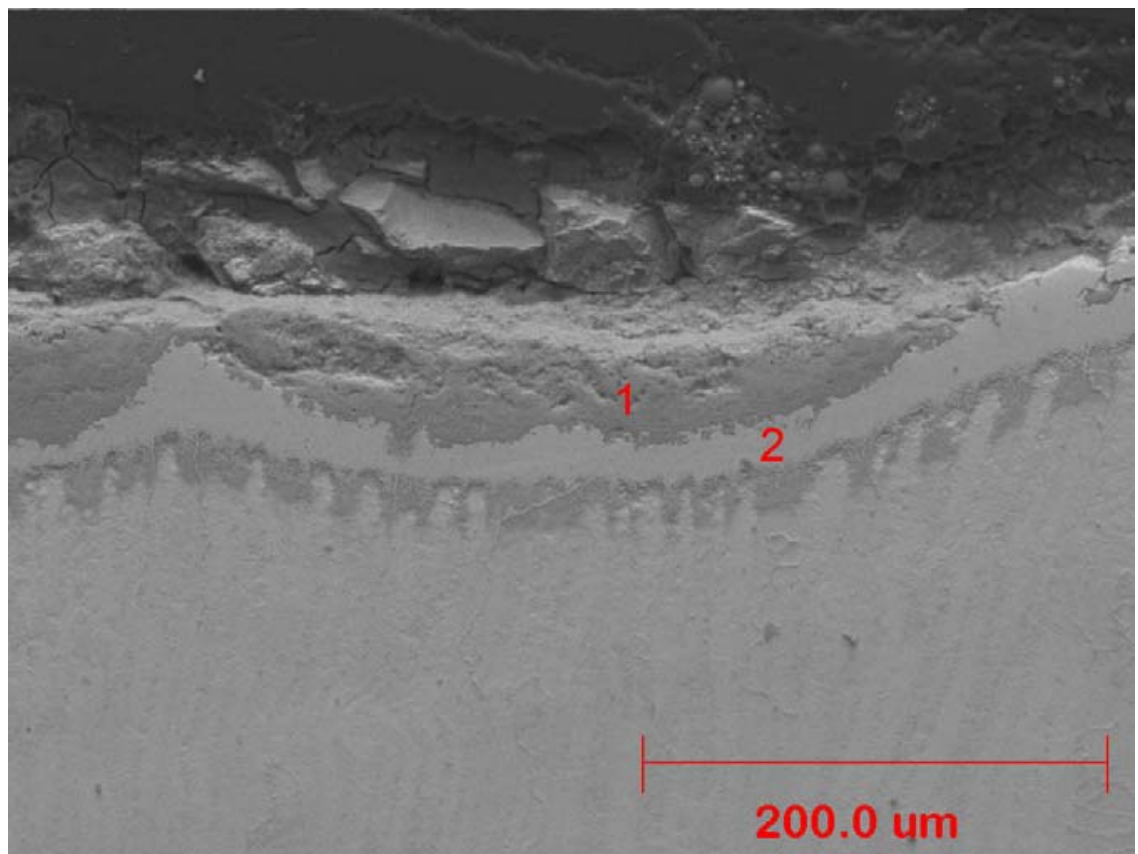

(a) External Scale/Metal Interface (BEI - Intermediate Magnification)

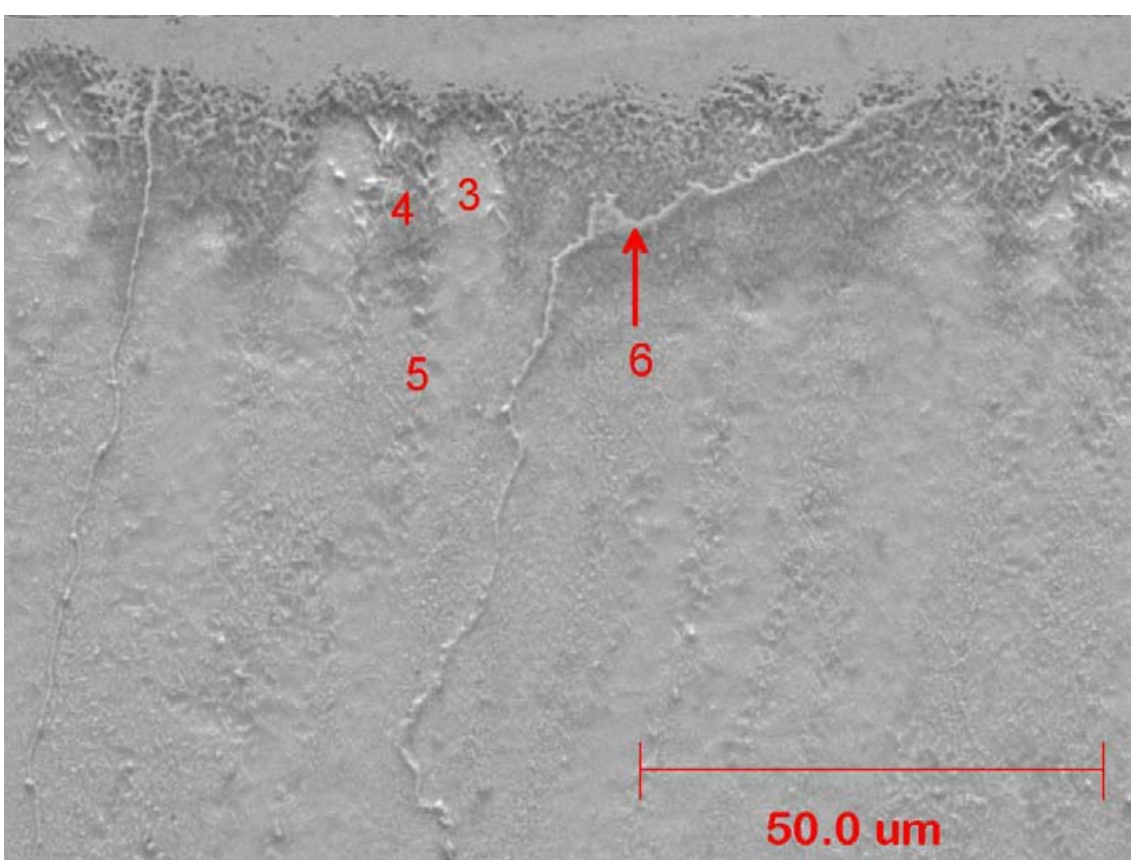

(b) External Scale/Metal Interface (BEI - High Magnification)

Figure J-12 Microstructure: IN52 Weld Overlay

Note: Numbers on photos indicate distinct areas characterized by the visual assessment and EDS analyses that follow. 


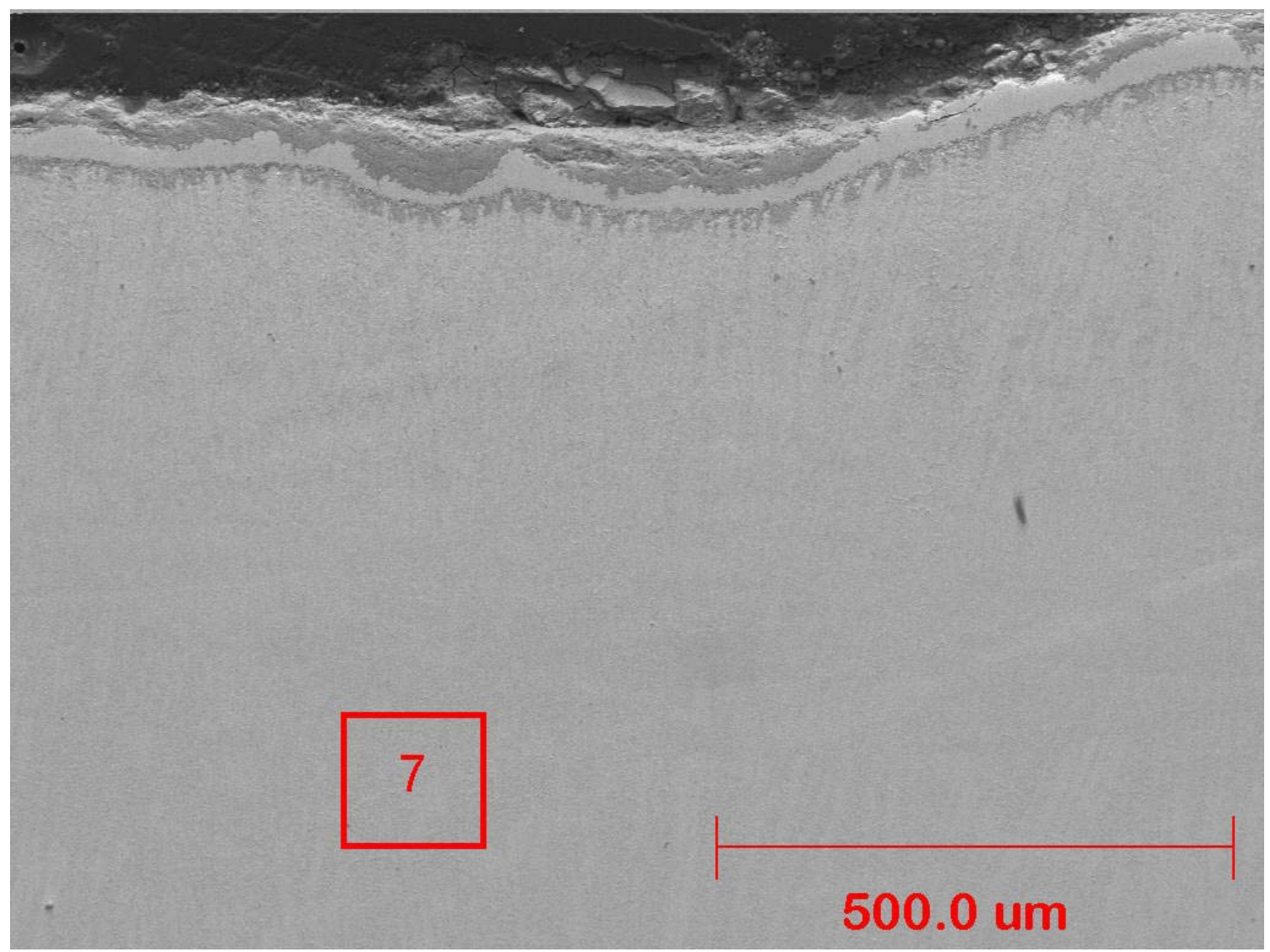

(c) External Scale/Metal Interface (SEI - Intermediate Magnification)

\section{Figure J-12(Cont.): Microstructure: IN52 Weld Overlay}

Note: Numbers on photos indicate distinct areas characterized by the visual assessment and EDS analyses that follow. 


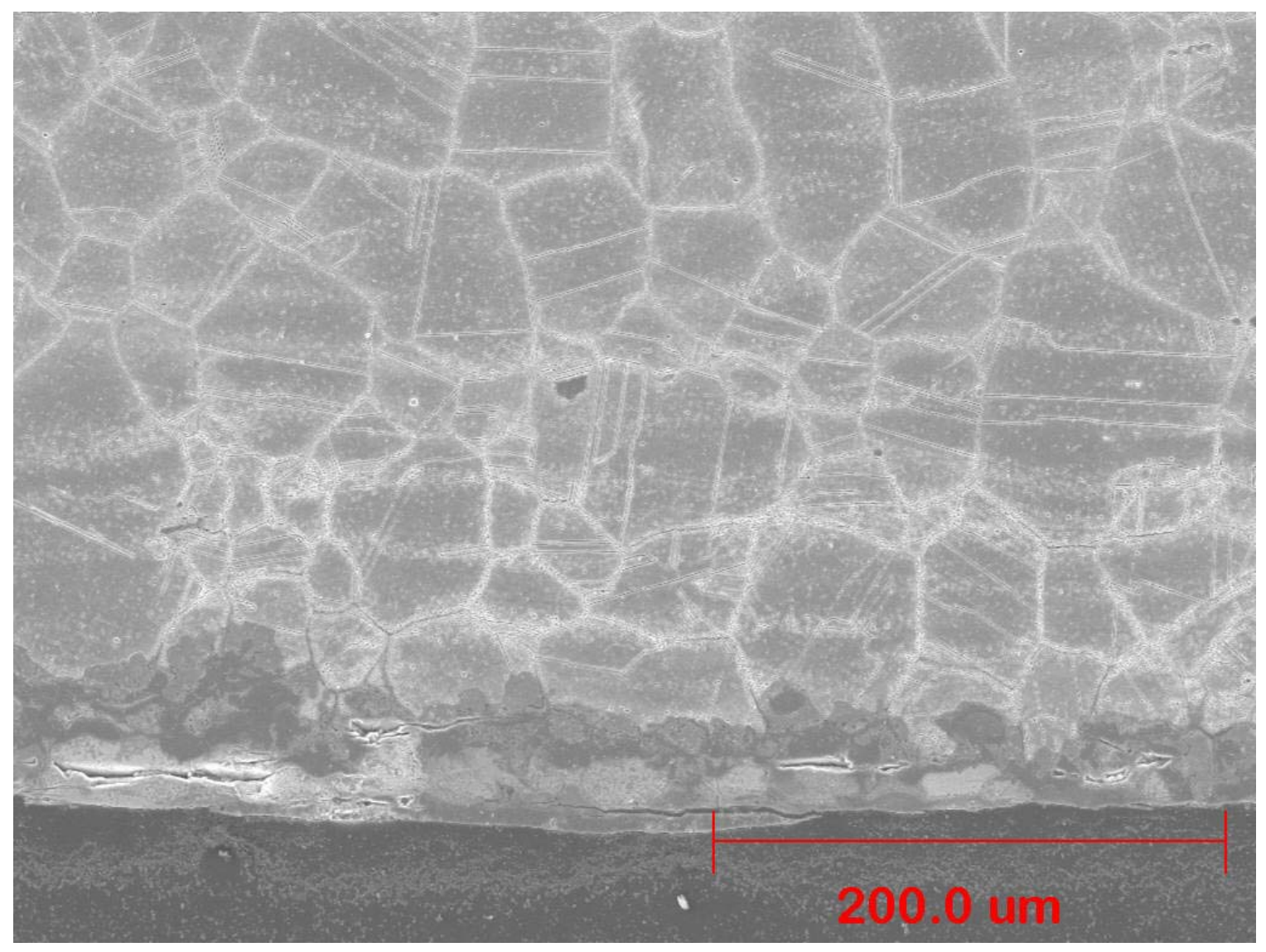

Internal Surface Appearance

Figure J-13 Microstructure: 800H Substrate Internal Surface of Tube with IN52 Weld Overlay

Note: Photomicrograph at approximately 200X. Electrolytic 10\% oxalic acid etch, $9 \mathrm{~V}$ OCP 


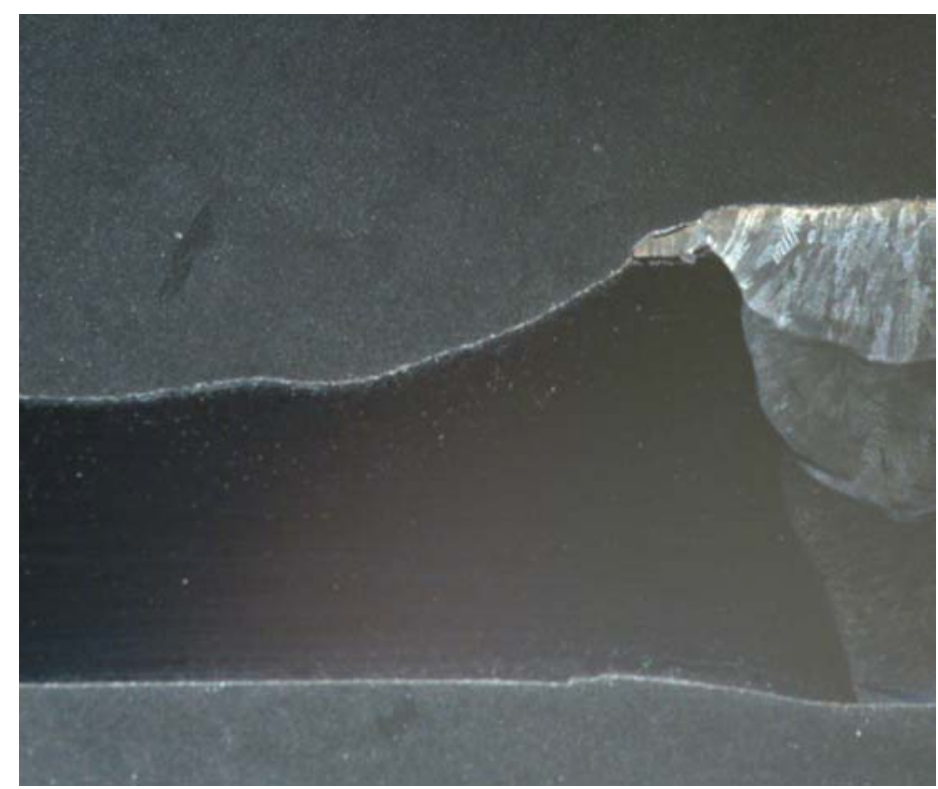

(a) IN52 Weld Overlay Adjacent to Weld "R"

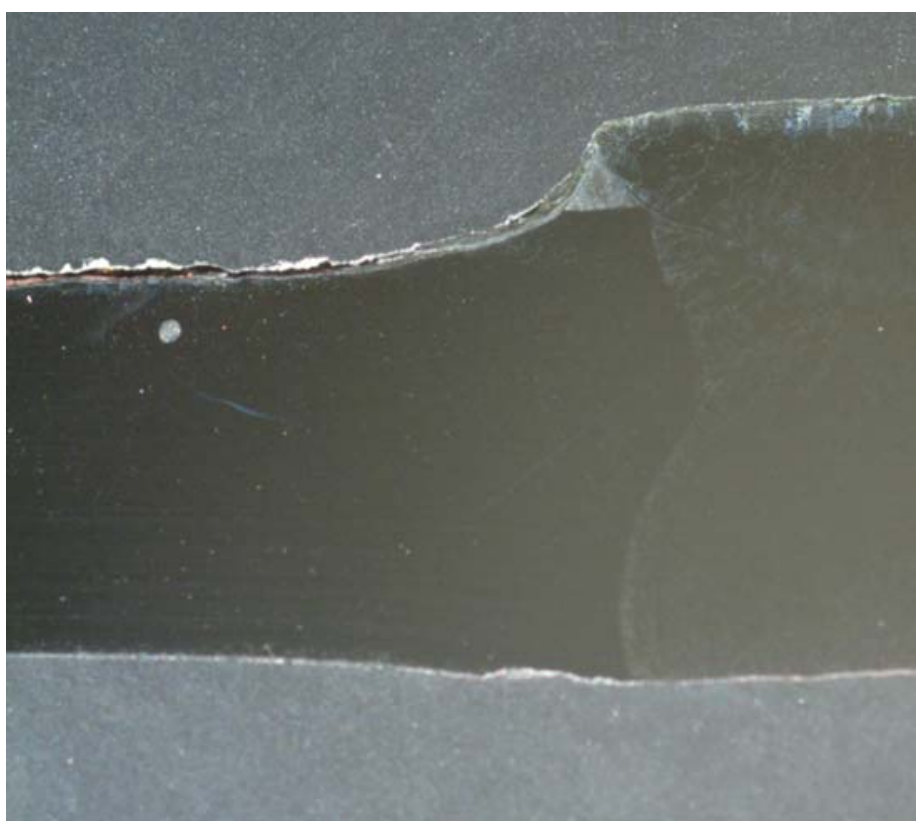

(b) IN52 Weld Overlay Adjacent to Weld "HH"

Figure J-14: Longitudinal Cross-Sections: IN52 Weld Overlay Adjacent to Welds

Note: None of the original weld overlay remains in this view, only $800 \mathrm{H}$ substrate. 
Figure J-15: EDS Analysis of Deposit/Scale/Metal Interface for IN52 Weld Overlay (Sample GG-HH)

\begin{tabular}{|c|c|c|c|c|c|c|c|c|c|c|c|c|c|c|c|c|c|c|}
\hline Area & Description & $\mathbf{N i}$ & $\mathrm{Cr}$ & $\mathbf{F e}$ & Co & Mn & $\mathbf{N b}$ & $\mathbf{T i}$ & Al & $\mathbf{S i}$ & $\mathrm{C}$ & $\mathbf{O}$ & $\mathrm{Ca}$ & $\mathbf{K}$ & $\mathbf{N a}$ & As & $\mathbf{P}$ & $\mathbf{S}$ \\
\hline 1 & $\begin{array}{l}\text { Adherent } \\
\text { scale }\end{array}$ & 7.76 & 55.1 & 3.80 & -- & -- & -- & 0.60 & 1.42 & 0.25 & 4.88 & 17.4 & -- & -- & -- & -- & -- & 8.78 \\
\hline 2 & $\begin{array}{l}\text { Light etching } \\
\text { band along } \\
\text { scale } \\
\text { interface }\end{array}$ & 57.2 & 25.3 & 9.16 & -- & -- & -- & 0.26 & 0.36 & -- & 5.15 & 2.53 & -- & -- & -- & -- & -- & -- \\
\hline 3 & Dendrite core & 61.9 & 27.9 & 9.73 & -- & - & -- & - & 0.39 & -- & -- & -- & -- & -- & -- & -- & -- & -- \\
\hline 4 & $\begin{array}{l}\text { Interdendritic } \\
\text { - corrosion } \\
\text { possible. }\end{array}$ & 49.8 & 28.0 & 7.13 & -- & -- & -- & 0.51 & 0.80 & 0.24 & 7.02 & 6.44 & -- & -- & -- & -- & -- & -- \\
\hline 5 & $\begin{array}{l}\text { Interdendritic } \\
- \text { not } \\
\text { corroded. }\end{array}$ & 59.0 & 30.1 & 8.95 & -- & -- & -- & 0.87 & 0.82 & 0.28 & -- & -- & -- & -- & -- & -- & -- & -- \\
\hline 6 & $\begin{array}{l}\text { Grain } \\
\text { boundary } \\
\text { possible } \\
\text { corroded } \\
\text { zone }\end{array}$ & 50.6 & 39.8 & 7.36 & -- & -- & -- & 1.08 & 1.17 & -- & -- & -- & -- & -- & -- & -- & -- & -- \\
\hline 7 & $\begin{array}{l}\text { Bulk } \\
\text { composition } \\
\text { mid-overlay. }\end{array}$ & 59.9 & 29.2 & 9.25 & -- & -- & -- & 0.61 & 0.68 & 0.36 & -- & -- & -- & -- & -- & -- & -- & -- \\
\hline & $\begin{array}{l}\text { Reference } \\
\text { bulk analysis. }\end{array}$ & 61.8 & 27.3 & 8.99 & -- & 0.28 & -- & 0.51 & 0.83 & 0.23 & 0.03 & -- & -- & -- & -- & -- & -- & -- \\
\hline
\end{tabular}




\section{Appendix K}

\section{Analysis of Performance}

\section{Test Section C}

Incoclad 671

K-1 


\section{Overview of Results: Incoclad 671}

Incoclad 671 experienced a very low rate of metal loss due to exposure in Test Section C. The maximum rate was determined to be 10 mils per year*. The chromium and overall alloy content of this material seem to account for its' good performance in this environment. This further would be assisted by the fine grain size of the cladding which likely would facilitate the diffusion that would aid in the formation of a protective chromium-rich scale.

Examination revealed that, while the corrosion rate for Incoclad 671 was very low at all of the exposure temperatures, it was highest at the lowest exposure. The Section C data and the Section A data are consistent in this regard. The reason for the somewhat increased corrosion rate at lower temperatures is not entirely clear; however, the evidence of high sulfur in the reaction zone may give some guidance in this regard. It was noted that physical examination of the as-received tube surfaces showed evidence on the surface of all three tube samples of the alkali sulfates that are known to be particularly aggressive.

It should be noted that the metal loss rates determined to date for both Section A and Section C all were based on a ring specimen that was extracted from mid-length of each tube sample. In the current analysis, additional attention was focused on metal losses in the vicinity of welds. Indeed examination revealed that, for the Incoclad samples, the welds were the only regions where any significant corrosion occurred. Analysis showed that the Inconel 625 weld filler metal, that was used for joining tubes to one another, while being reasonably resistant to coal ash corrosion, was less resistant to corrosion that the adjacent Incoclad 671 material. This was particularly obvious for the Sample P-Q which experienced the highest exposure temperature (1203 F) of the three Incoclad 671 specimens. It is believed that dissolution of the Inconel 625 filler metal accounts for the local loss of cross section at the weld at location "P". This suggests that perhaps an IN72 filler metal may have been a better choice at this location. 


\section{Material: Incoclad 671}

\section{Summary of Findings}

\begin{tabular}{|c|c|}
\hline Issue & Discussion \\
\hline $\begin{array}{l}\text { Sample } \\
\text { Location and } \\
\text { Orientation. }\end{array}$ & $\begin{array}{l}\text { - The schematic in Figure K-1 shows the relative positions of the three } \\
\text { Incoclad } 671 \text { samples in Test Section C. Note that none of the Incoclad } \\
671 \text { overlay samples was within the tube lengths that were removed } \\
\text { early, i.e. in November } 2001 \text { or in June } 2002 \text {. } \\
\text { - Also, shown is the calculated average metal temperature at each } \\
\text { location. }\end{array}$ \\
\hline $\begin{array}{l}\text { Composition } \\
\text { Verification. }\end{array}$ & $\begin{array}{l}\text { - The table in Figure K-2 shows a reference nominal composition that } \\
\text { was provided by the Special Metals Corporation (SMC). } \\
\text { No mill certificate or heat analysis was offered so it's not clear } \\
\text { whether these samples came from one or multiple heats of material. } \\
\text { - During the Section A evaluation, detailed composition verification was } \\
\text { undertaken for every tube sample that was removed from service. This } \\
\text { served, in part, to verify that the each tube material was located at its' } \\
\text { proper position within the test section. } \\
\text { Based on the good results for Section A, it was decided that it would } \\
\text { be unnecessary to analyze each and every tube sample for composition } \\
\text { verification. Rather, it was decided to select a representative sample } \\
\text { from each tube material type for confirmatory analysis. } \\
\text { During the Section A evaluation, the energy dispersive spectrographic } \\
\text { (EDS) capabilities of the scanning electron microscope (SEM) were } \\
\text { used to perform semi-quantitative analyses of each of the twelve } \\
\text { different candidate tube materials. Comparison of these EDS results } \\
\text { showed reasonably good correlation with results obtained using } \\
\text { quantitative analytical techniques, including optical emission } \\
\text { spectrometry. } \\
\text { Based on this, it was decided that the accuracy provided by EDS } \\
\text { would suffice for composition verification, but that more detailed } \\
\text { analyses would be undertaken if questions arose. } \\
\text { The results of the EDS analysis of tube sample EE-FF confirmed that } \\
\text { the correct material was used at this location. }\end{array}$ \\
\hline $\begin{array}{l}\text { As-Received } \\
\text { Appearance }\end{array}$ & $\begin{array}{l}\text { - Figure K-3 shows the typical appearance of the Incoclad } 671 \text { samples } \\
\text { as they were received from SMC. This tube has a total wall thickness } \\
\text { of about } 260 \text { mils, with a } 75 \text { mil thick outer layer of IN671 to provide } \\
\text { corrosion resistance, and an inner supporting layer of } 800 \mathrm{H} \text {. } \\
\text { - Figures K-4 through K-6 document the appearance of the external } \\
\text { surface of the Incoclad } 671 \text { tube samples after they had been removed } \\
\text { from service. Three views are provided for each tube: } 12 \text { o'clock (the } \\
\text { view that faces on-coming gas flow), } 3 \text { o'clock (the view where the } \\
\text { gas flow is tangent to the surface), and } 6 \text { o'clock (the view that shows } \\
\text { the back-side of the tube relative to the gas flow direction.. }\end{array}$ \\
\hline
\end{tabular}




\section{Material: Incoclad 671}

\section{Summary of Findings, (continued)}

\begin{tabular}{|c|c|}
\hline Issue & Discussion \\
\hline $\begin{array}{l}\text { As-Received } \\
\text { Appearance, } \\
\text { Continued }\end{array}$ & $\begin{array}{l}\text { - The reader should be alerted to the fact that, within the boiler, the gas } \\
\text { flows upward over these tubes. This implies that for the "o'clock" } \\
\text { conventions described above, (with the } 12 \text { o'clock orientation facing } \\
\text { the gas flow direction), the actual physical orientation of these tubes is } \\
\text { such that the } 12 \text { o'clock side of the tube faces downward, and the } 6 \\
\text { o'clock orientation faces upward within the boiler. } \\
\text { - Wastage is not visible in any of these three tubes in any orientation. } \\
\text { - The chalky white scale associated with molten alkali-iron sulfate } \\
\text { attack can be seen on all three tube samples.. }\end{array}$ \\
\hline $\begin{array}{l}\text { Grit-Blasted } \\
\text { Surface } \\
\text { Appearance }\end{array}$ & $\begin{array}{l}\text { - Figures K7 through K-9 show the appearance of the external surface of } \\
\text { the Incoclad } 671 \text { tube samples after grit-blasting to remove deposit and } \\
\text { scale. The } 3 \text { and } 12 \text { o'clock positions on Sample EE-FF show the } \\
\text { appearance of general wastage due to fireside corrosion. } \\
\text { - Wastage is not apparent in viewing the remaining surfaces. }\end{array}$ \\
\hline $\begin{array}{l}\text { Wall } \\
\text { Thickness } \\
\text { Loss } \\
\text { Determined }\end{array}$ & $\begin{array}{l}\text { - Tube cross-sections were prepared to allow for measurement of wall } \\
\text { thickness to determine metal loss due to exposure. These are shown in } \\
\text { silhouette in Figures K-10 through K-12. } \\
\text { - Measurements were taken at eight evenly spaced "o'clock" locations } \\
\text { about the tube circumference. These were then compared with } \\
\text { measurements taken at these same locations prior to exposure. } \\
\text { - The before and after measurements were documented in the table that } \\
\text { accompanies each figure. These form the basis for the "delta" } \\
\text { calculation to determine wall thickness losses. In some cases, the delta } \\
\text { is negative. This is believed due to wall thickness variability and } \\
\text { difficulty in locating the exact location of the "before" measurement. } \\
\text { For tube Sample EE-FF, the minimum wall thickness in the cross } \\
\text { section was not at one of the eight predetermined locations. In this } \\
\text { case, there was no "before" measurement for comparison. So, the } \\
\text { average "before" wall thickness was used to calculate the wall } \\
\text { thickness lost at these off-locations. }\end{array}$ \\
\hline $\begin{array}{l}\text { Wall } \\
\text { Thickness } \\
\text { Loss Versus } \\
\text { Average Metal } \\
\text { Temperature }\end{array}$ & $\begin{array}{l}\text { - The chart in Figure K-13 plots the maximum wall thickness lost } \\
\text { against the calculated average metal temperature for the three samples. } \\
\text { The data from Section A also are plotted. } \\
\text { The wastage rate was low for all three samples; however, note that the } \\
\text { sample having the lowest average exposure temperature }\left(1086^{\circ} \mathrm{F}\right) \text { had } \\
\text { the highest wastage rate. This is confirmed by the appearance of the } \\
\text { grit blasted surface of this sample, see Figure K-9. }\end{array}$ \\
\hline $\begin{array}{l}\text { Wall } \\
\text { Thickness } \\
\text { Loss as a } \\
\text { Function of } \\
\text { Time and } \\
\text { Location } \\
\end{array}$ & $\begin{array}{l}\text { - The chart in Figure K-14 plots metal loss as a function of time for each } \\
\text { sample location for both Sections A and C, with samples from Section } \\
\text { C having experienced generally longer exposure times. } \\
\text { - The chart emphasizes the point that the higher temperature samples } \\
\text { experienced little if any additional wall loss, while the low temperature } \\
\text { sample, Sample EE-FF experienced increased metal loss with time. }\end{array}$ \\
\hline
\end{tabular}




\section{Material: Incoclad 671}

\section{Summary of Findings, (continued)}

\begin{tabular}{|c|c|}
\hline Issue & Discussion \\
\hline $\begin{array}{l}\text { Metallurgical } \\
\text { Analysis - } \\
\text { SEM }\end{array}$ & $\begin{array}{l}\text { - Sample EE-FF was selected for a detailed SEM/EDS assessment of the } \\
\text { constituents and structure at the external and internal surfaces. } \\
\text { Figure K-15 shows the appearance of the tube profile for Sample EE- } \\
\text { FF. The scale/deposit on the external surface is approximately } 2 \text { mils } \\
\text { thick. No scale is evident on the internal surface at this magnification. } \\
\text { - Figures K-15a and b show secondary electron images of the } \\
\text { scale/metal interface for Sample EE-Ff. Figures } 15 \text { c and d show back- } \\
\text { scattered electron images is this same region to enhance the } \\
\text { appearance of local composition and topological differences. } \\
\text { - The numbers on Figures K-15b and d show the locations of EDS } \\
\text { analysis that will be reported later in this document. } \\
\text { Examination of the microstructure reveals four distinct regions: } \\
\text { Incoclad 671 Base metal - Oxalic acid etch delineates grain } \\
\text { boundaries and carbides. Of particular note is the extremely } \\
\text { fine grain size in the cladding material. Also note from, Figure } \\
\text { 15d, that two phases are apparent in the bulk tube metal. Later } \\
\text { EDS analysis will show that these phases contain nickel and } \\
\text { chromium in different proportions. The chromium-rich phase } \\
\text { is resistant to attack by the oxalic acid etchant, see point \#5, } \\
\text { Figure K-15d. The nickel-rich phase is less resistant, see point } \\
\text { \#6 in the same figure. } \\
\text { Metal at the Interface - A very narrow diffusion zone is noted } \\
\text { at the metal/scale interface. This is indicated at point \#3 in } \\
\text { Figure } 15 b, \text { but perhaps is shown more clearly in Figure } 15 \mathrm{~d} \text {. } \\
\text { Examination reveals that the scale is progressing into the } \\
\text { metal surface along this narrow zone. EDS analysis has shown } \\
\text { that the zone is depleted in chromium. } \\
\text { Adherent scale with embedded deposit - EDS analysis will } \\
\text { later show that this is predominantly a chromium-rich oxide } \\
\text { with a significant amount of sulfur present. } \\
\text { Thick and very porous deposit/scale conglomerate - many } \\
\text { spherical particles are noted along with what appears to be } \\
\text { islands of scale (metal oxides). } \\
\text { surface. N-16 shows the condition of the tube wall at its internal } \\
\text { - No evidence found of significant corrosion in this region. }\end{array}$ \\
\hline $\begin{array}{l}\text { Metallurgical } \\
\text { Analysis - } \\
\text { Weld Cross } \\
\text { Section. }\end{array}$ & $\begin{array}{l}\text { - Figure K-17 shows a longitudinal profile (at the } 12 \text { o'clock } \\
\text { orientation) of the Incoclad } 671 \text { samples adjacent to the welds in } \\
\text { Section C. The welds in question are those identified as welds "P", } \\
\text { "V", and "FF", see Figure L-1 for locations } \\
\text { - Examination of Figure K-17a suggests that the Inconel } 625 \text { weld filler } \\
\text { metal was less resistant to corrosion than the clad tube adjacent to the } \\
\text { weld at location "P". It is believed that dissolution of the Inconel } 625 \\
\text { filler metal is the principle reason for the local loss of cross section at } \\
\text { this weld. This suggests that a different filler metal may have been a } \\
\text { better choice at this location. }\end{array}$ \\
\hline
\end{tabular}




\section{Summary of Findings, (continued)}

\section{Material: $\quad$ Incoclad 671}

\begin{tabular}{|c|c|}
\hline Issue & Discussion \\
\hline \begin{tabular}{|l|} 
EDAX \\
Analysis \\
Results, \\
Continued
\end{tabular} & $\begin{array}{l}\text { - The table in Figure K-18 provides a summary of the EDS analyses of } \\
\text { the numbered locations in Figure K-15b and d. Also shown in this } \\
\text { table is a summary of the spectrographic analysis reported earlier, see } \\
\text { Figure K-2. } \\
\text { - Review of this table suggests the following: } \\
\text { - Areas 1: Bulk deposit/scale analysis - Alkali metals and sulfur } \\
\text { are present. The relatively high chromium content suggests that a } \\
\text { significant percentage of the chromium-oxide scale was included } \\
\text { in this analysis. The oxides of iron, aluminum, and titanium also } \\
\text { are indicated. An iron oxide is likely, as are aluminum, silicon } \\
\text { and titanium oxides. } \\
\text { - Area 2: Adherent scale- This scale is predominantly a } \\
\text { chromium-rich oxide with a significant amount of sulfur noted. } \\
\text { Area 3: Diffusion zone - adjacent to the scale/metal interface - } \\
\text { This zone is depleted in chromium (21.7\% vs roughly } 50 \% \text { in the } \\
\text { bulk tube). } \\
\text { Area 4: Bulk metal analysis - This confirms that the sample was } \\
\text { correctly identified as Incoclad } 671 \text {. } \\
\text { Area 5: Shallow etching region in cladding - This constituent of } \\
\text { the microstructure is rich in chromium (nominally } 85 \% \text { Cr / } 15 \% \\
\text { Ni). } \\
\text { Area 6: Deep etching region in cladding - This constituent of the } \\
\text { microstructure is rich in nickel (nominally } 70 \% \text { Ni / } 30 \% \text { Cr). }\end{array}$ \\
\hline
\end{tabular}




\section{Gas Flow}

Direction

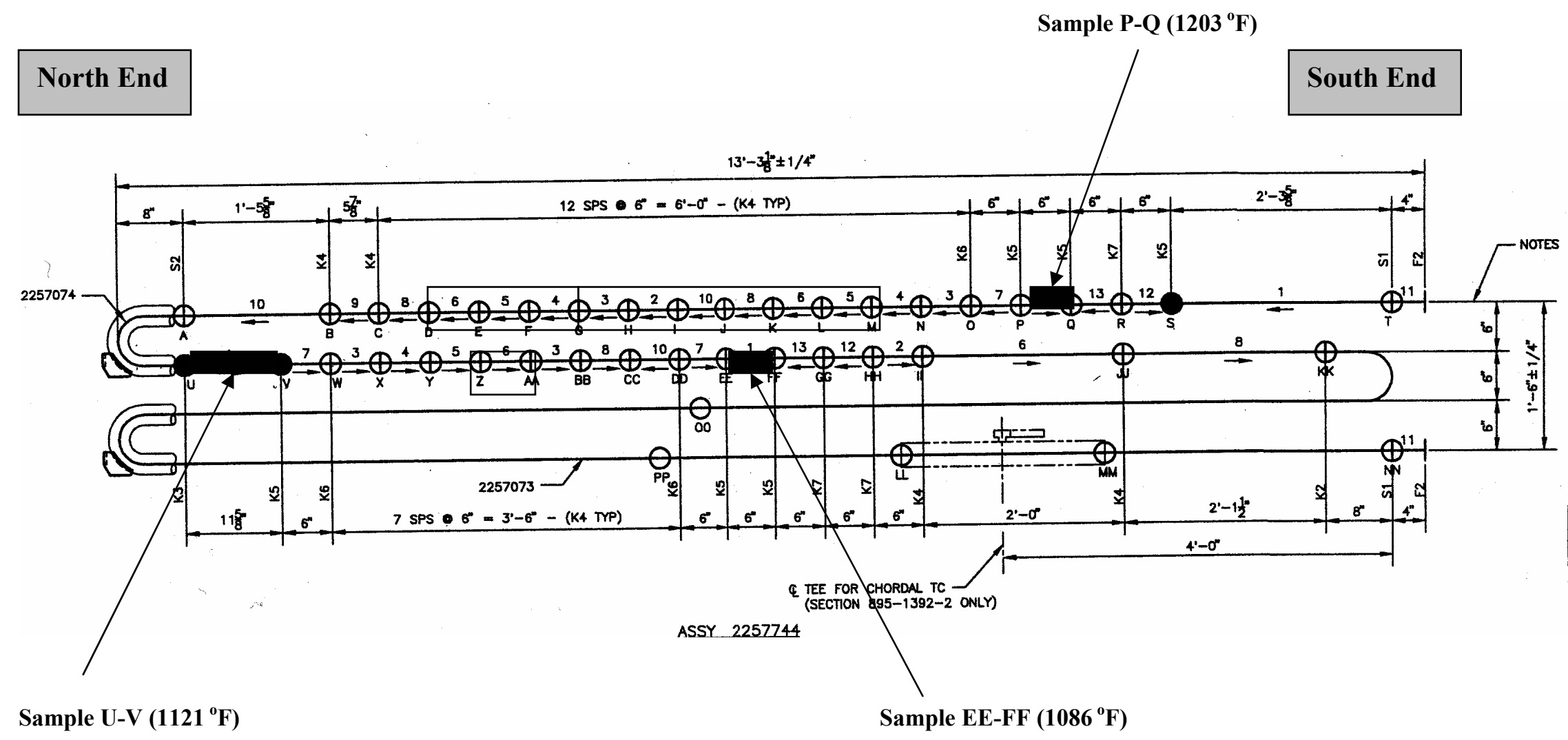

Figure K-1: Schematic Drawing Showing Position of Incoclad 671 Tube Samples Within Test Section C

1. Drawing shown in same relative orientation as per the actual installation.

2. This group of tube samples was removed from service in November 2001 - highlighted in red.

3. This group of tube samples was removed from service in June 2002 - highlighted in yellow. 
Figure K-2 Semi-Quantitative Composition Verification for Incoclad 671 / 800 HT (Co-extruded duplex tubing)

Description: $\quad$ Nominal $50 \mathrm{Cr} / 50 \mathrm{Ni}$ alloy cladding for oxidation/corrosion protection. Incoloy $800 \mathrm{HT}$ substrate to provide creep strength.

Source: Special Metals Corporation (SMC)

Reference Composition: Cladding "nominal" chemistry - per SMC literature.

\begin{tabular}{|c|c|c|c|c|c|}
\hline Element & $\begin{array}{c}\text { Reference } \\
\text { Composition }\end{array}$ & $\begin{array}{c}\text { Certified } \\
\text { Composition } \\
\text { (not provided) }\end{array}$ & $\begin{array}{c}\text { Tube Sample } \\
\text { P-Q }\end{array}$ & $\begin{array}{c}\text { Tube Sample } \\
\text { U-V }\end{array}$ & $\begin{array}{c}\text { Tube Sample } \\
\text { EE-FF }\end{array}$ \\
\hline $\mathrm{Ni}$ & 51.5 & & $\mathrm{~N} / \mathrm{A}$ & $\mathrm{N} / \mathrm{A}$ & 47.0 \\
\hline $\mathrm{Cr}$ & 48.0 & & $\mathrm{~N} / \mathrm{A}$ & N/A & 52.5 \\
\hline $\mathrm{Fe}$ & - & & $\mathrm{N} / \mathrm{A}$ & N/A & - \\
\hline Mo & - & & $\mathrm{N} / \mathrm{A}$ & N/A & - \\
\hline $\mathrm{Co}$ & - & & N/A & N/A & - \\
\hline $\mathrm{C}$ & 0.05 & & N/A & N/A & - \\
\hline $\mathrm{N}$ & - & & N/A & N/A & - \\
\hline $\mathrm{B}$ & - & & $\mathrm{N} / \mathrm{A}$ & N/A & - \\
\hline $\mathrm{Mn}$ & - & & N/A & $\mathrm{N} / \mathrm{A}$ & - \\
\hline $\mathrm{Si}$ & - & & N/A & $\mathrm{N} / \mathrm{A}$ & - \\
\hline $\mathrm{Al}$ & - & & $\mathrm{N} / \mathrm{A}$ & $\mathrm{N} / \mathrm{A}$ & 0.23 \\
\hline $\mathrm{Ti}$ & - & & N/A & N/A & 0.24 \\
\hline $\mathrm{Al}+\mathrm{Ti}$ & - & & N/A & N/A & - \\
\hline $\mathrm{Nb}$ & - & & N/A & N/A & - \\
\hline $\mathrm{Ta}$ & - & & $\mathrm{N} / \mathrm{A}$ & N/A & - \\
\hline $\mathrm{Nb}+\mathrm{Ta}$ & - & & N/A & N/A & - \\
\hline $\mathrm{V}$ & - & & N/A & $\mathrm{N} / \mathrm{A}$ & - \\
\hline $\mathrm{W}$ & - & & N/A & N/A & - \\
\hline $\mathrm{Cu}$ & - & & $\mathrm{N} / \mathrm{A}$ & N/A & - \\
\hline $\mathrm{Sn}$ & - & & N/A & N/A & - \\
\hline $\mathrm{P}$ & - & & N/A & N/A & - \\
\hline $\mathrm{S}$ & - & & N/A & N/A & - \\
\hline $\mathrm{O} 2$ & - & & N/A & N/A & - \\
\hline Others & - & & N/A & N/A & - \\
\hline
\end{tabular}

$\mathrm{N} / \mathrm{A}=$ Sample not analyzed. 


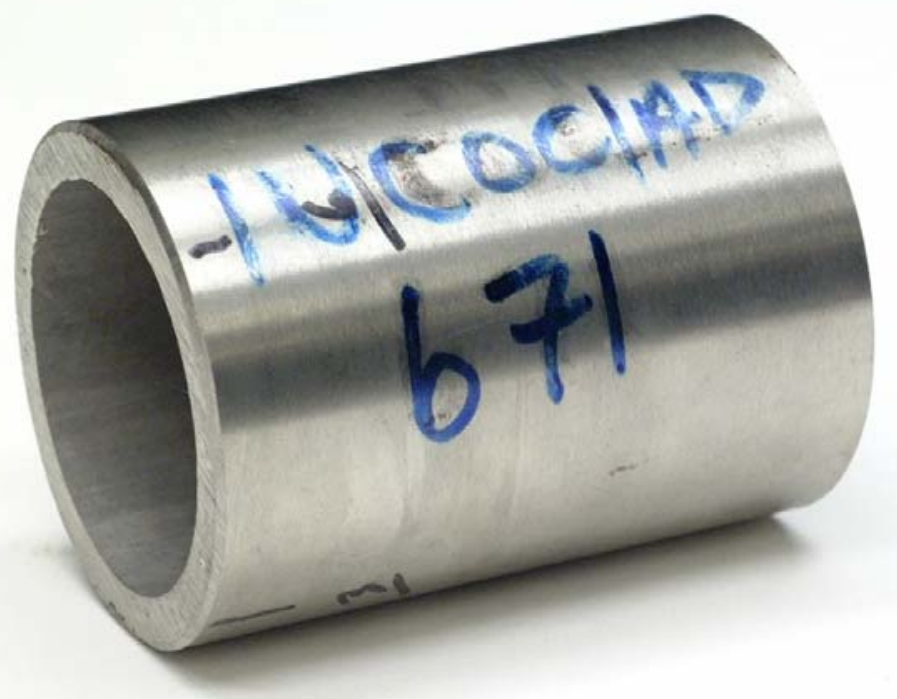

Figure K-3: Appearance of Incoclad 671 Tube Sample Prior to Service 


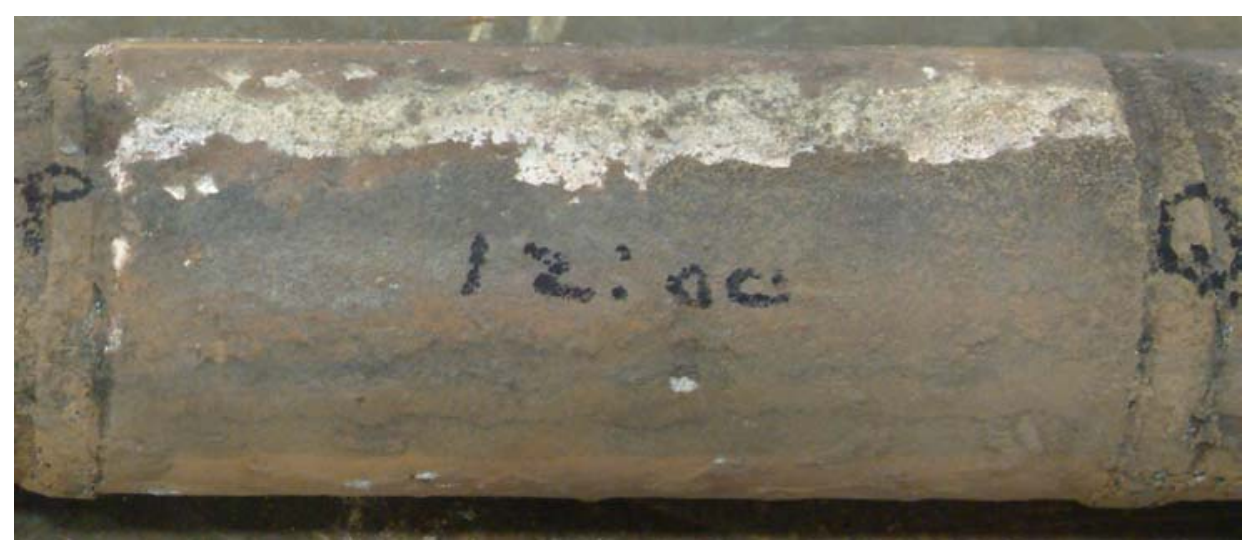

12 O'Clock - Facing Gas Flow

(Note: 3 o'clock is up in this image)

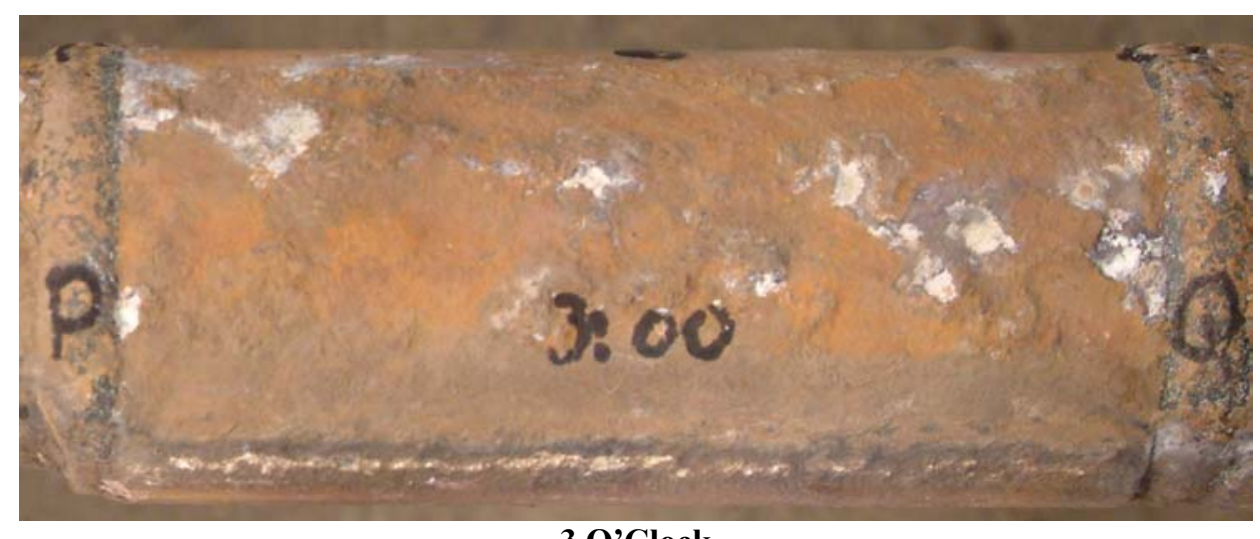

3 O'Clock

(Note: 6 o'clock is up in this image)

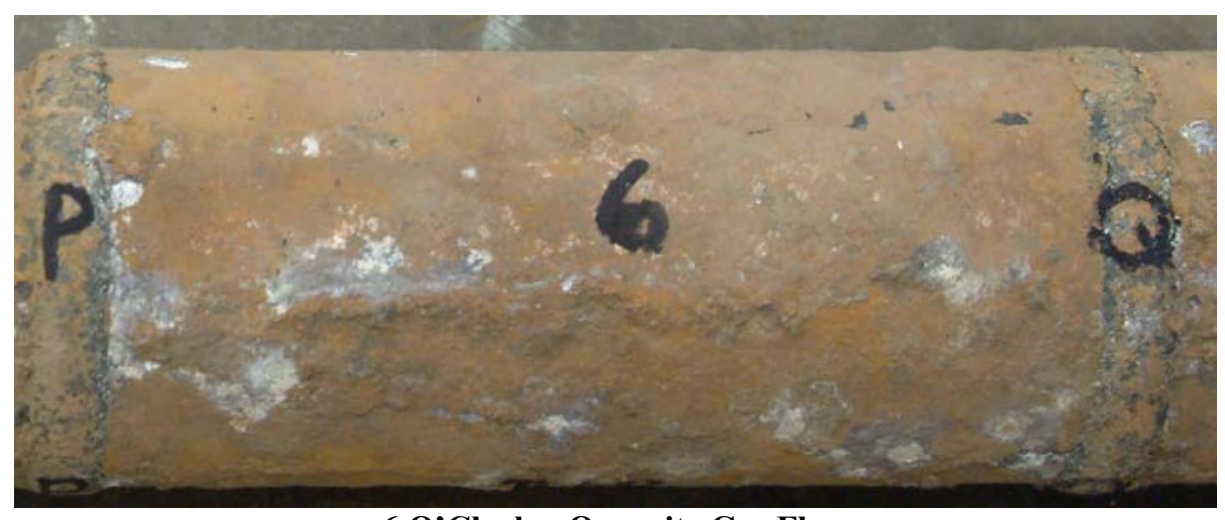

6 O'Clock - Opposite Gas Flow

Figure K-4: As-Received Appearance of Sample P-Q (Incoclad 671)

(Average surface metal temperature: $1203{ }^{\circ} \mathrm{F}$ ) 


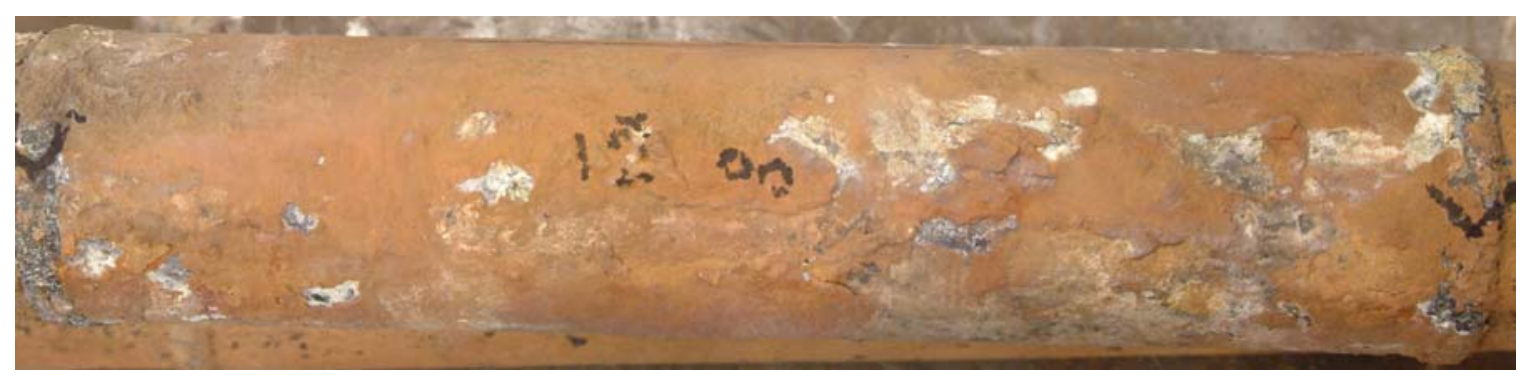

12 O'Clock - Facing Gas Flow

(Note: 3 o'clock is up in this image)

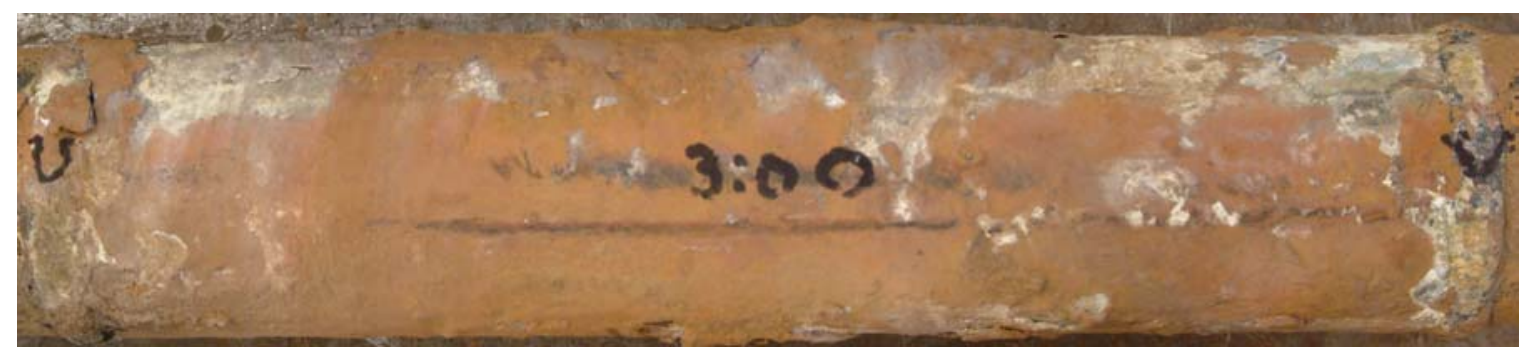

3 O'Clock

(Note: 6 o'clock is up in this image)

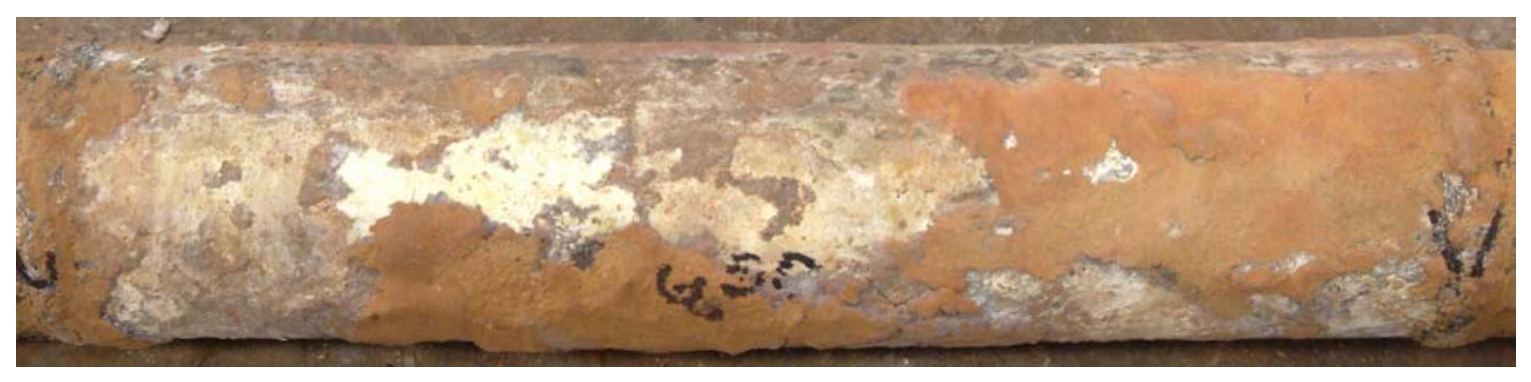

6 O'Clock - Opposite Gas Flow

Figure K-5 As-Received Appearance of Sample U-V (Incoclad 671)

(Average surface metal temperature: $1121{ }^{\circ} \mathrm{F}$ )

Note: this tube sample was 11-5/8-in. long. 


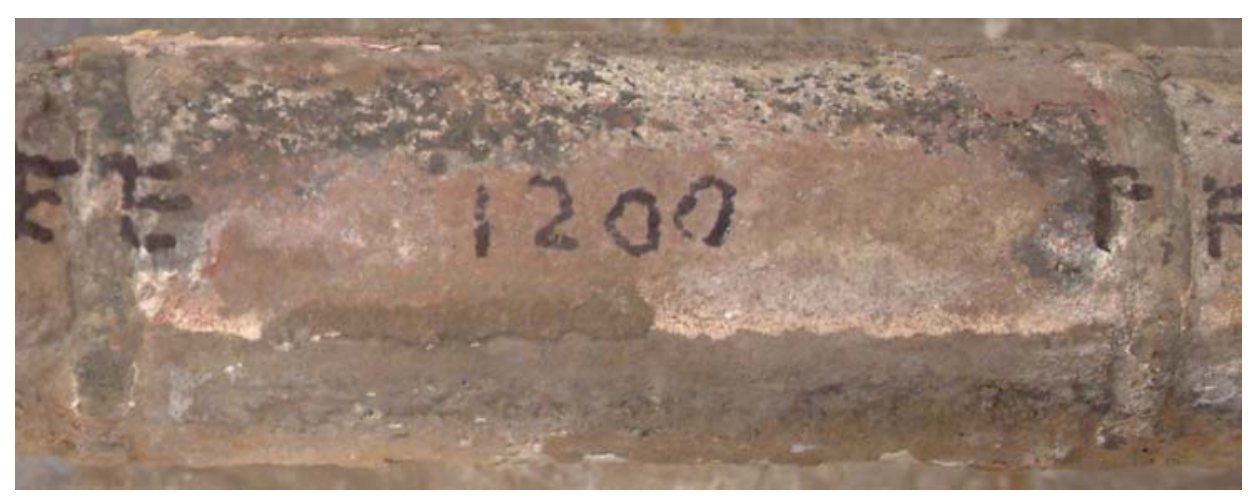

12 O'Clock - Facing Gas Flow

(Note: 3 o'clock is up in this image)

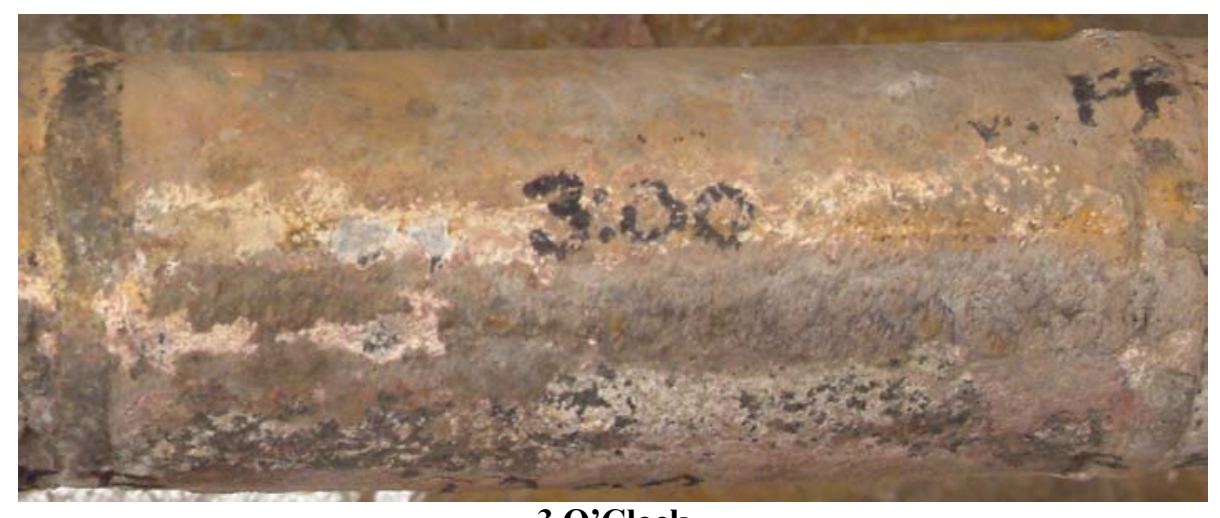

\section{O'Clock}

(Note: 6 o'clock is up in this image)

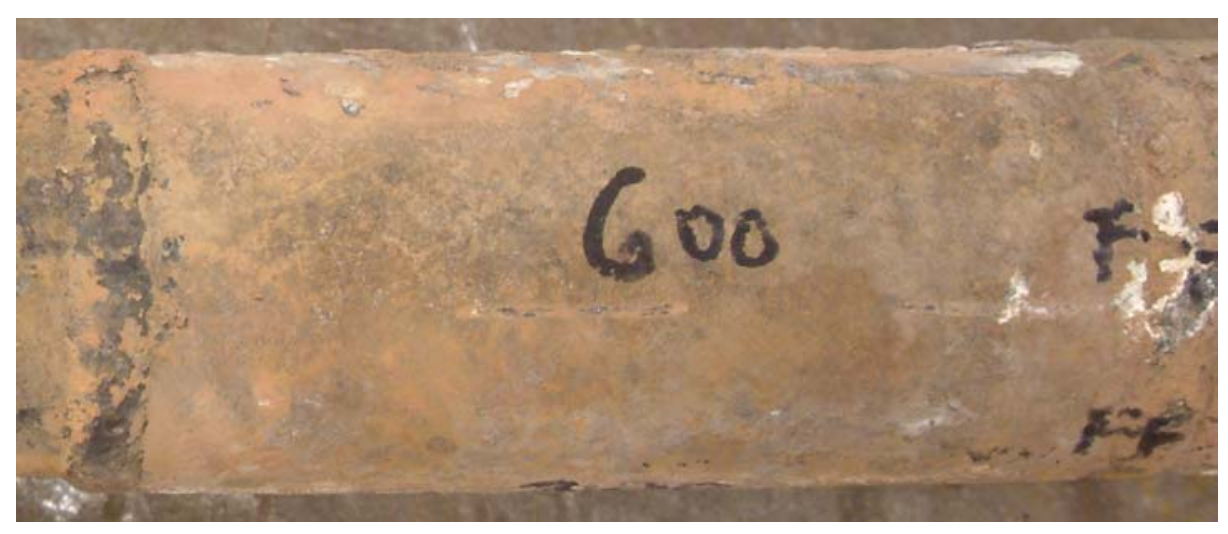

6 O'Clock - Opposite Gas Flow

Figure K-6: As-Received Appearance of Sample EE-FF (Incoclad 671)

(Average surface metal temperature: $1086^{\circ} \mathrm{F}$ ) 
12 O'Clock

(0 mils lost)

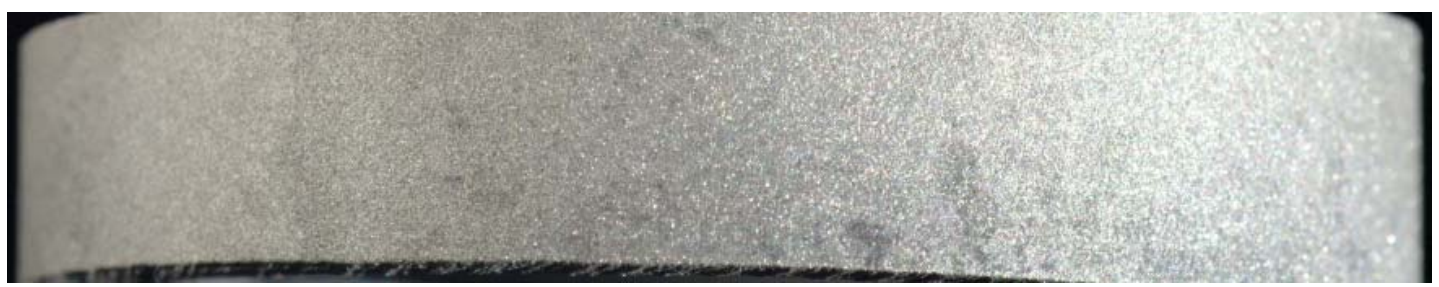

3 O'Clock

(0 mils lost)

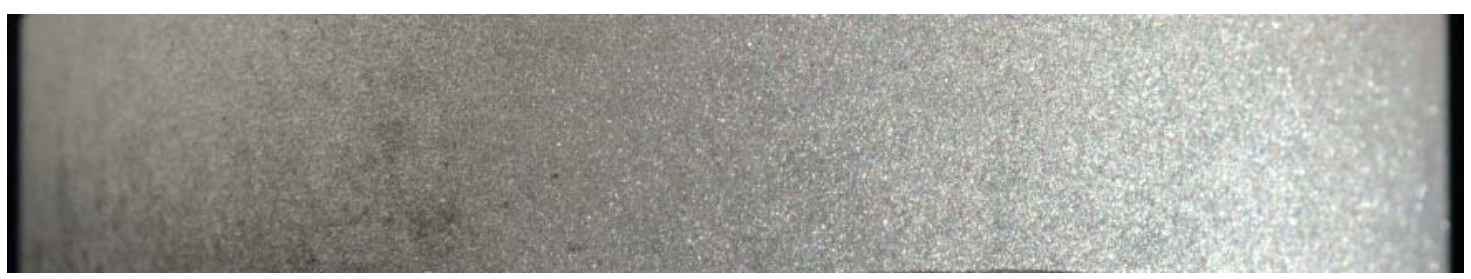

6 O'Clock

$(0$ mils lost $)$

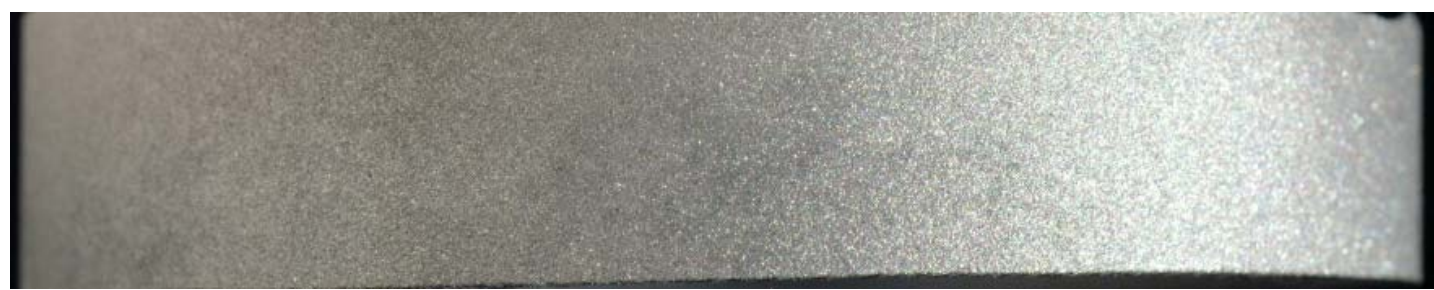

Figure K-7: OD Surface Appearance After Grit Blast

Material: Incoclad 671

Sample: P-Q 
12 O'Clock

(3 mils lost)

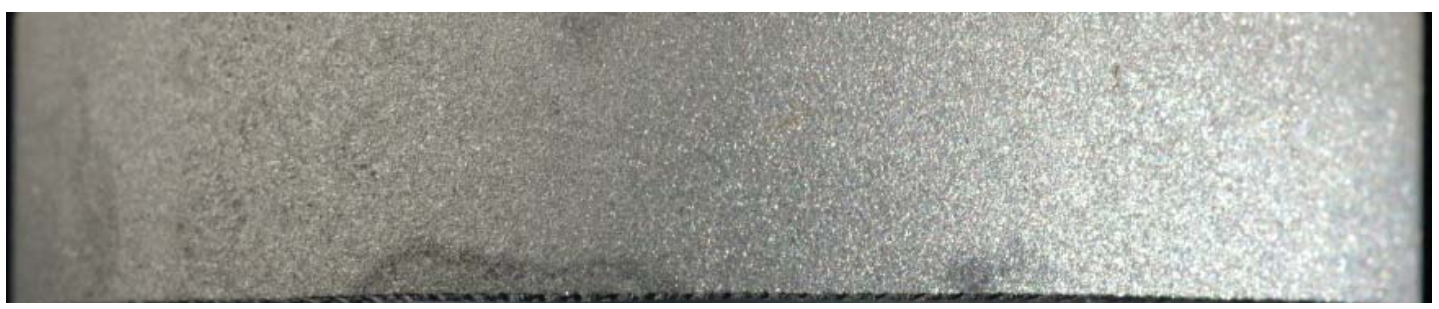

3 O'Clock

(1 mils lost)

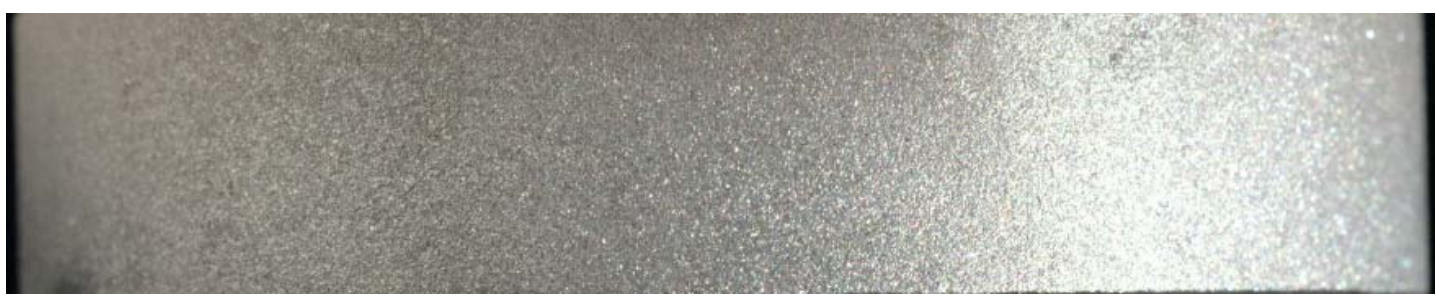

6 O'Clock

$(0$ mils lost $)$

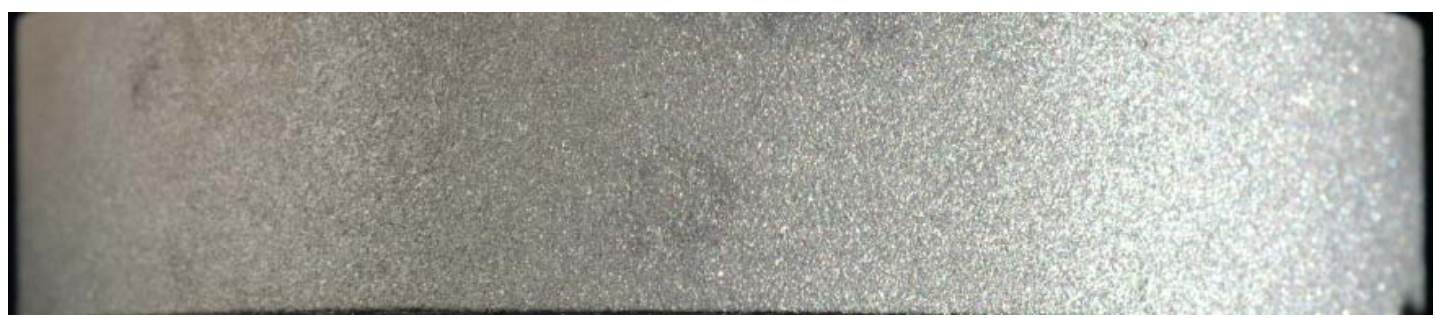

Figure K-8: OD Surface Appearance After Grit Blast

Material: Incoclad 671

Sample: U-V 
12 O'Clock

(4 mils lost)

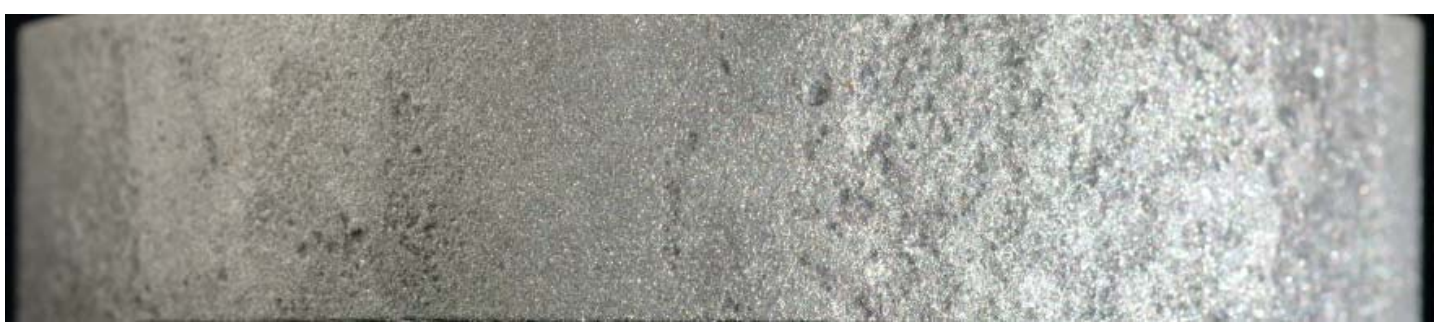

3 O'Clock

(0 mils lost)

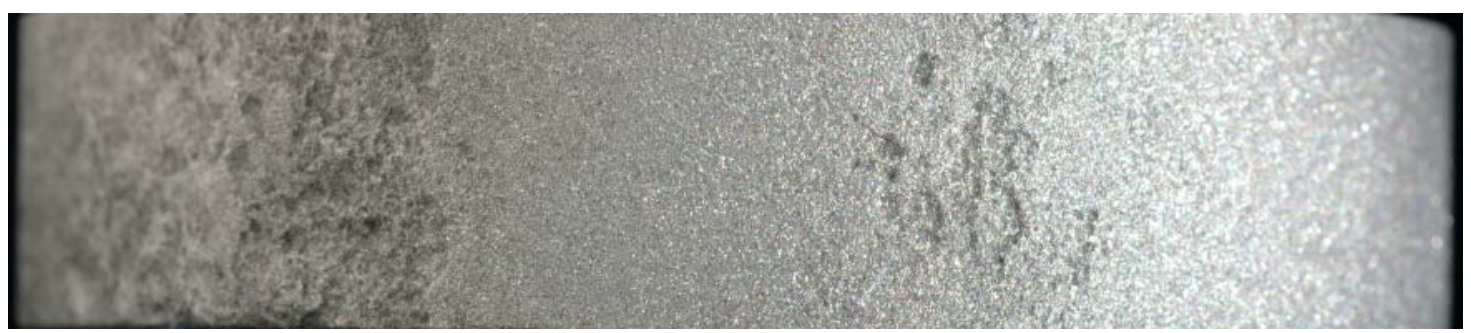

6 O'Clock

(0 mils lost)

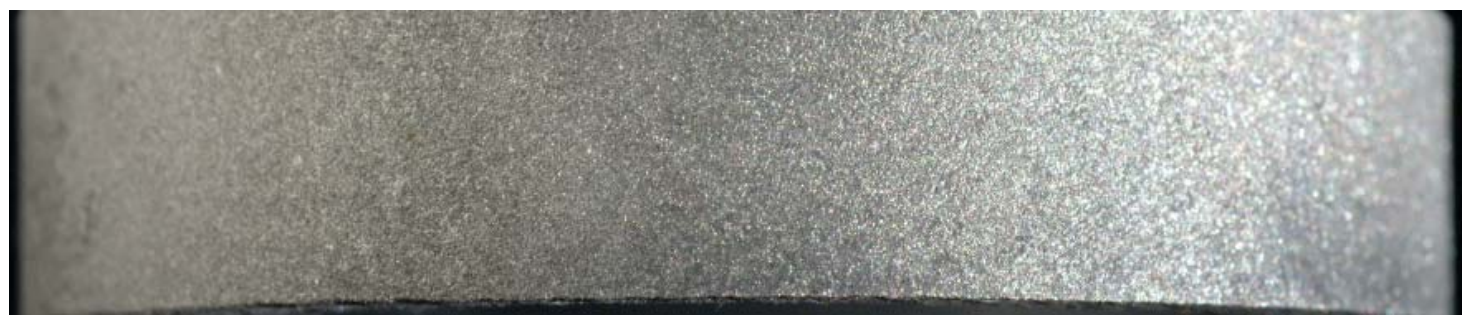

Figure K-9: OD Surface Appearance After Grit Blast

Material: Incoclad 671

Sample: EE-FF 


\section{Material:}

Sample Location:

Maximum Rate:

Maximum Loss Orientation:

Average Surface Metal Temperature:

\section{Incoclad 671}

P-Q

$2 \mathrm{mils} / \mathrm{yr}$

Approx. 10:30 o'clock

$1203^{\circ} \mathrm{F}$

12 O’Clock

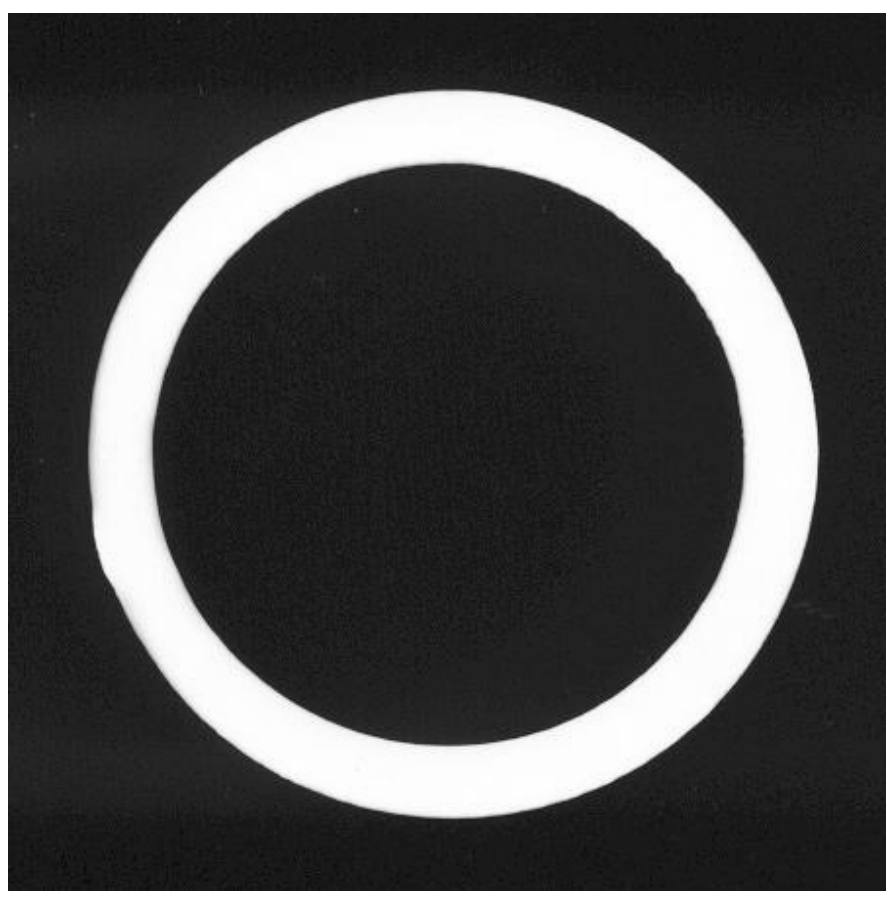

3 O'Clock

Approx. 1.2X

Wall Reduction by Position (in.)

\begin{tabular}{|l|c|c|c|c|c|c|c|c|c|c|}
\hline & $\begin{array}{c}\mathbf{1 2} \\
\text { o'clock }\end{array}$ & $\mathbf{1 : 3 0}$ & $\mathbf{3 : 0 0}$ & $\mathbf{4 : 3 0}$ & $\mathbf{6 : 0 0}$ & $\mathbf{7 : 3 0}$ & $\mathbf{9 : 0 0}$ & $\mathbf{1 0 : 3 0}$ & AVG & $\begin{array}{c}\text { Max } \\
\text { Loss }\end{array}$ \\
\hline Before & 0.259 & 0.264 & 0.262 & 0.258 & 0.256 & 0.255 & 0.256 & 0.259 & 0.259 & 0.259 \\
\hline After & 0.259 & 0.261 & 0.263 & 0.261 & 0.258 & 0.256 & 0.253 & 0.254 & & 0.254 \\
\hline Delta & 0.000 & 0.003 & -0.001 & -0.003 & -0.002 & -0.001 & 0.003 & 0.005 & & 0.005 \\
\hline
\end{tabular}

Figure K-10: Silhouette and Wall Thickness Measurements for Sample P-Q 


\section{Material:}

Sample Location:

Maximum Rate:

Maximum Loss Orientation:

Average Surface Metal Temperature:

\section{Incoclad 671}

U-V

$1 \mathrm{mils} / \mathrm{yr}$

Approx. 12 o'clock

12 O'Clock

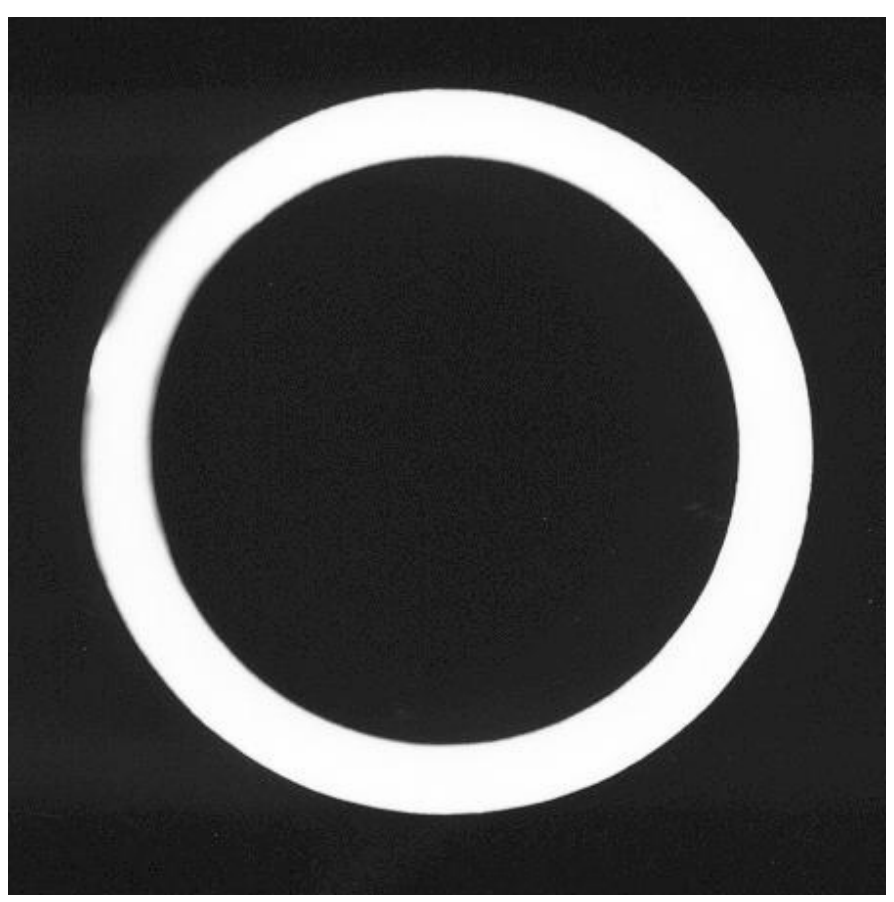

3 O'Clock

Approx. 1.2X

Wall Reduction by Position (in.)

\begin{tabular}{|l|c|c|c|c|c|c|c|c|c|c|}
\hline & $\begin{array}{c}\mathbf{1 2} \\
\text { o'clock }\end{array}$ & $\mathbf{1 : 3 0}$ & $\mathbf{3 : 0 0}$ & $\mathbf{4 : 3 0}$ & $\mathbf{6 : 0 0}$ & $\mathbf{7 : 3 0}$ & $\mathbf{9 : 0 0}$ & $\mathbf{1 0 : 3 0}$ & AVG & $\begin{array}{c}\text { Max } \\
\text { Loss }\end{array}$ \\
\hline Before & 0.258 & 0.253 & 0.257 & 0.260 & 0.262 & 0.262 & 0.262 & 0.258 & 0.259 & 0.258 \\
\hline After & 0255 & 0.256 & 0.256 & 0.260 & 0.263 & 0.261 & 0.259 & 0.257 & & 0.255 \\
\hline Delta & 0.003 & -0.003 & 0.001 & 0.000 & -0.001 & 0.001 & 0.003 & 0.001 & & 0.003 \\
\hline
\end{tabular}

Figure K-11: Silhouette and Wall Thickness Measurements for Sample U-V 


\section{Material:}

Sample Location:

Maximum Rate:

Maximum Loss Orientation:

Average Surface Metal Temperature:

\section{Incoclad 671}

EE-FF

$10 \mathrm{mils} / \mathrm{yr}$

Approx. 2 o'clock

$1086^{\circ} \mathrm{F}$

\section{O’Clock}

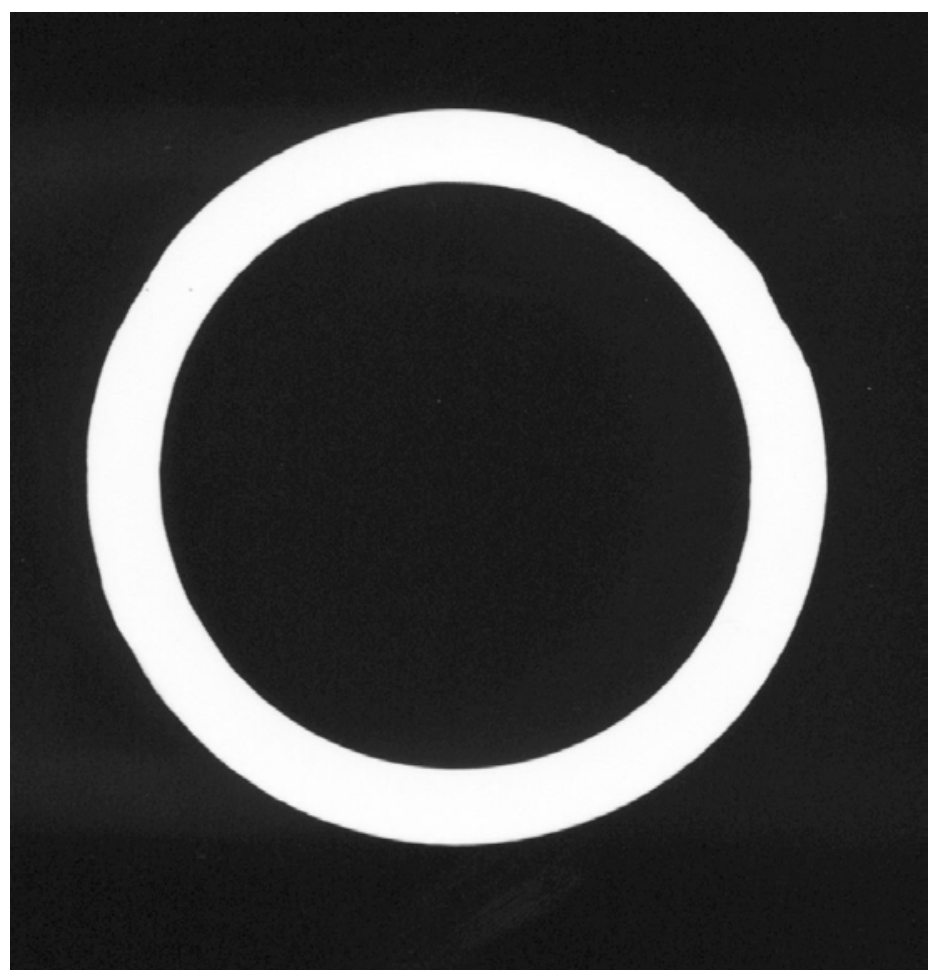

3 O'Clock

Approx. 1.2X

\section{Wall Reduction by Position (in.)}

\begin{tabular}{|l|c|c|c|c|c|c|c|c|c|c|}
\hline & $\begin{array}{c}\mathbf{1 2} \\
\text { o'clock }\end{array}$ & $\mathbf{1 : 3 0}$ & $\mathbf{3 : 0 0}$ & $\mathbf{4 : 3 0}$ & $\mathbf{6 : 0 0}$ & $\mathbf{7 : 3 0}$ & $\mathbf{9 : 0 0}$ & $\mathbf{1 0 : 3 0}$ & AVG & $\begin{array}{c}\text { Max } \\
\text { Loss }\end{array}$ \\
\hline Before & 0.256 & 0.258 & 0.260 & 0.263 & 0.261 & 0.259 & 0.257 & 0.255 & 0.259 & 0.259 \\
\hline After & 0.252 & 0.235 & 0.260 & 0.263 & 0.263 & 0.260 & 0.256 & 0.248 & & 0.234 \\
\hline Delta & 0.004 & 0.023 & 0.000 & 0.000 & -0.002 & -0.001 & 0.001 & 0.007 & & 0.025 \\
\hline
\end{tabular}

Figure K-12: Silhouette and Wall Thickness Measurements for Sample EE-FF

\footnotetext{
* Measurement taken at "maximum loss orientation" noted above. The average
} "before" wall thickness was used to calculate the "delta". 


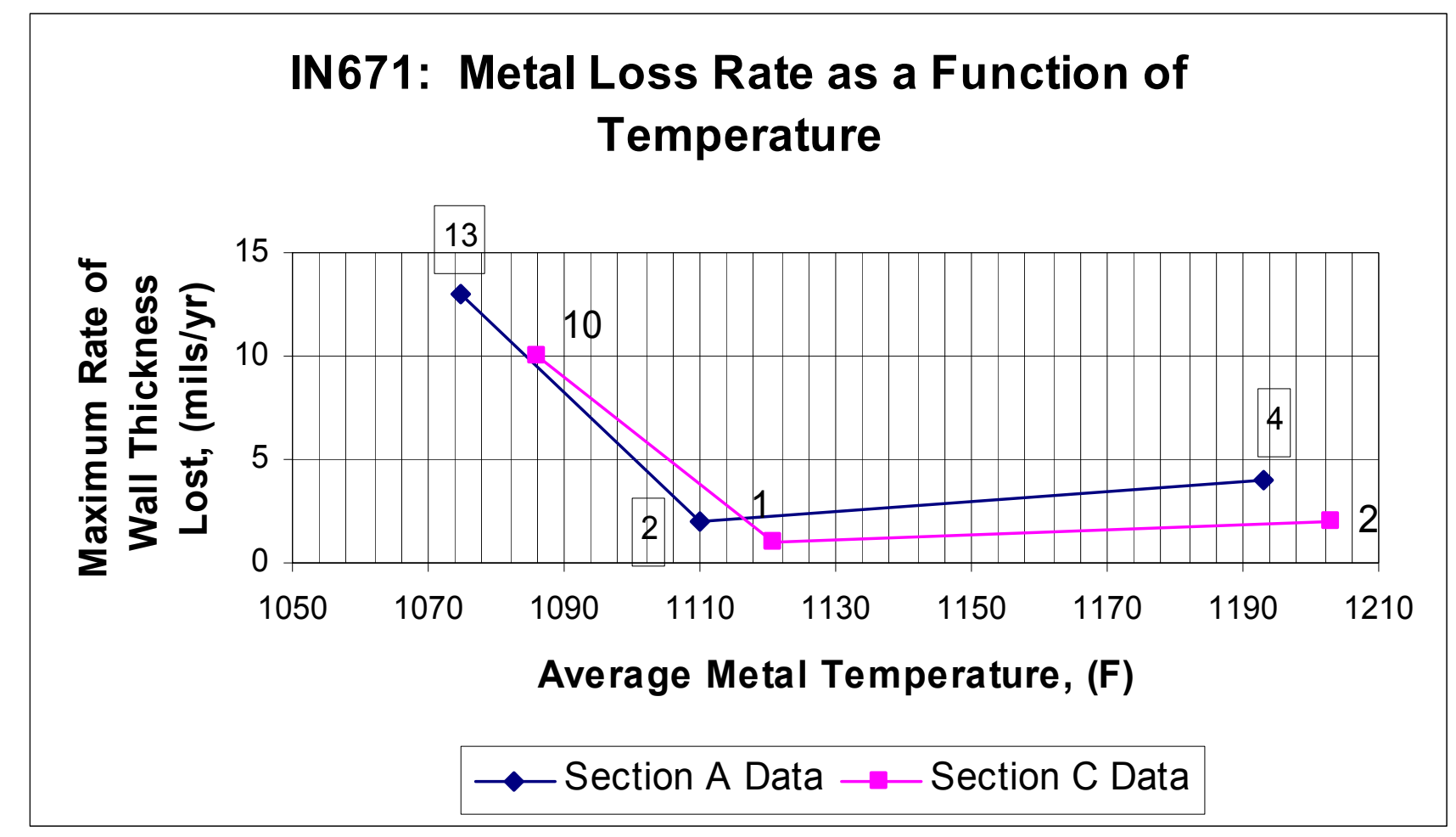

Figure K-13: Metal Loss Rate as a Function of Average Surface Metal Temperature

Material: Incoclad 671 


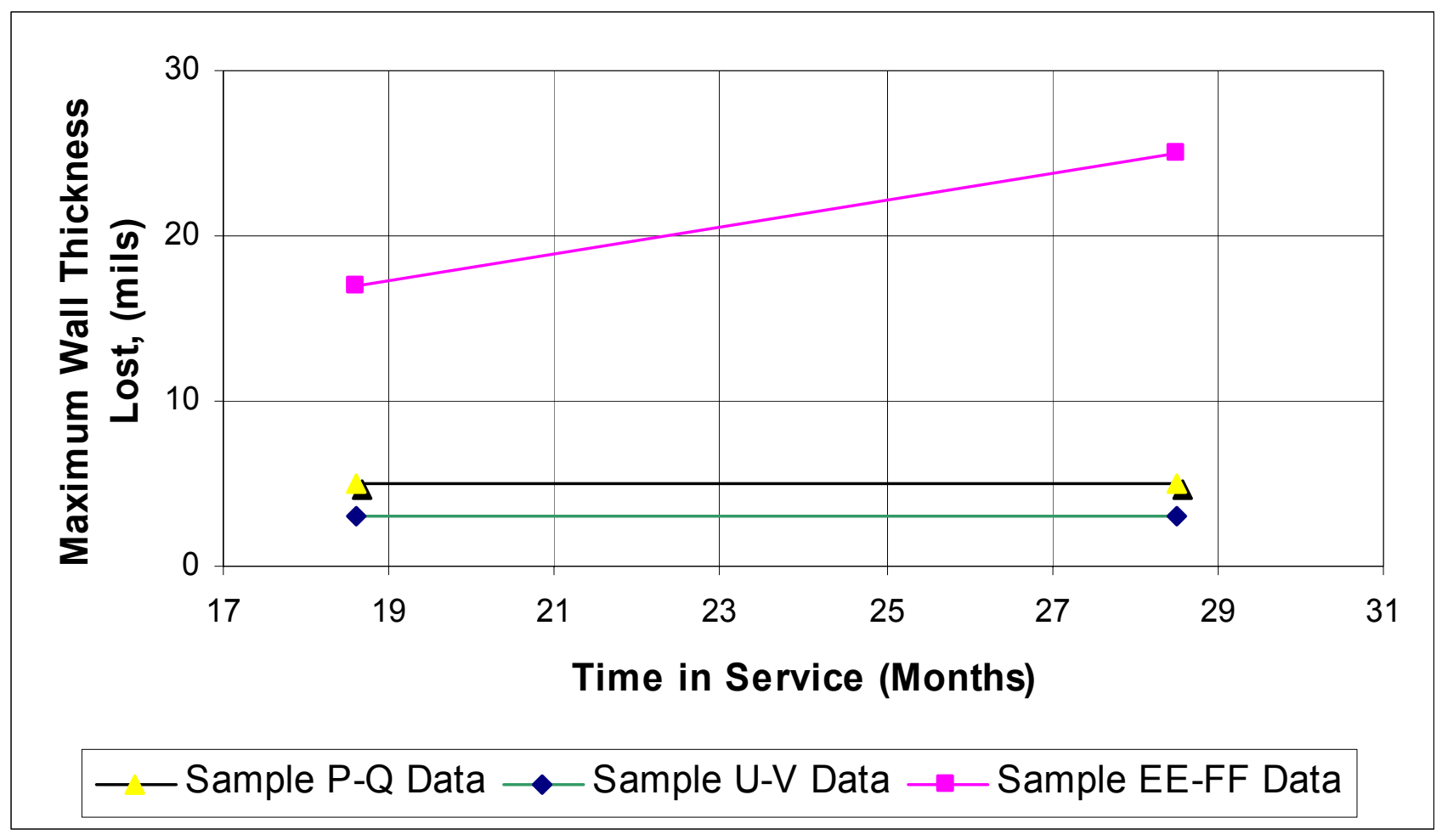

Figure K-14: Metal Loss as a Function of Time

Material: Incoclad 67 


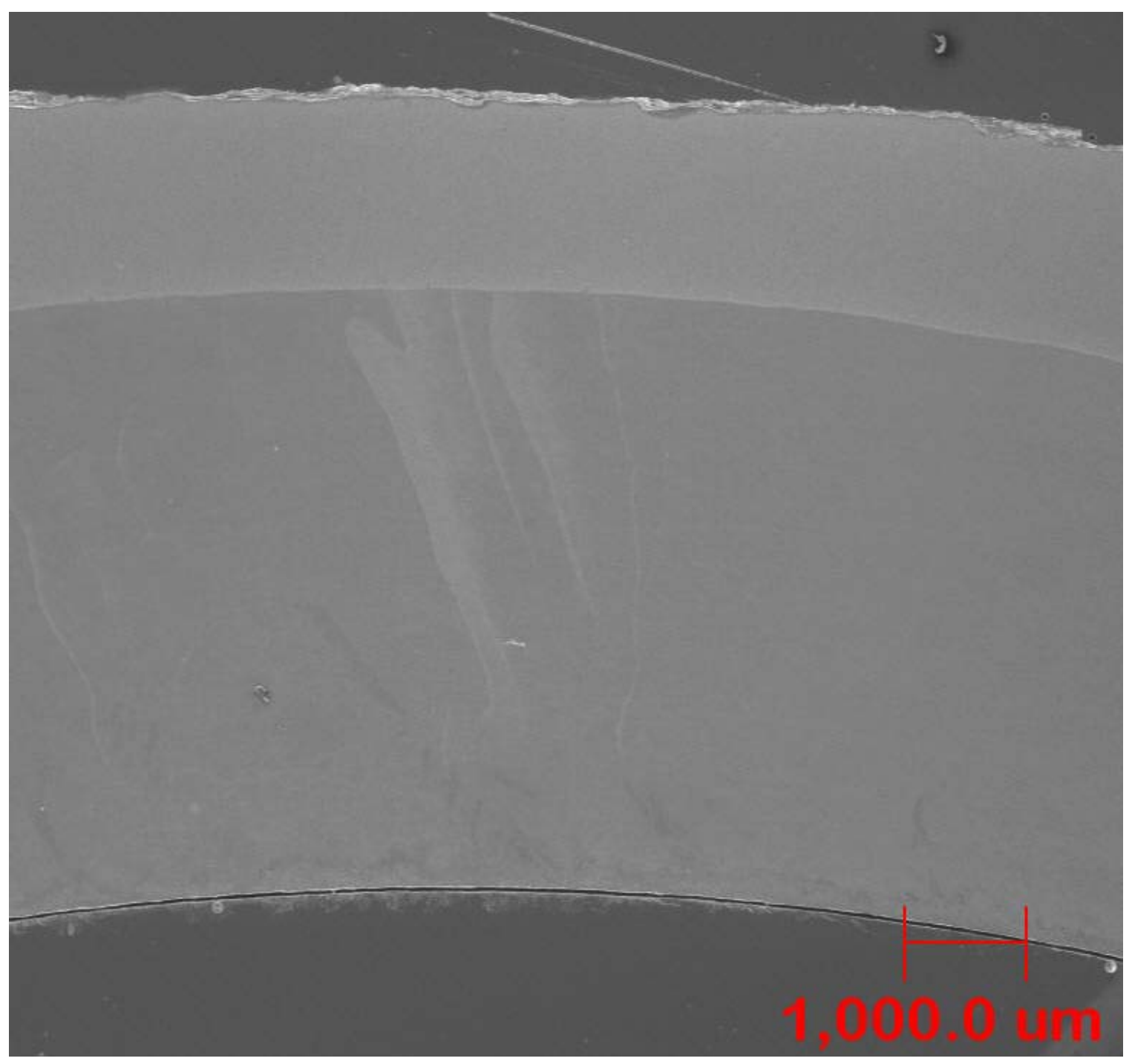

Figure K-15: Low Magnification Image Showing Cross-Section Through Thinnest Region of Sample EE-Ff (Incoclad 671) 


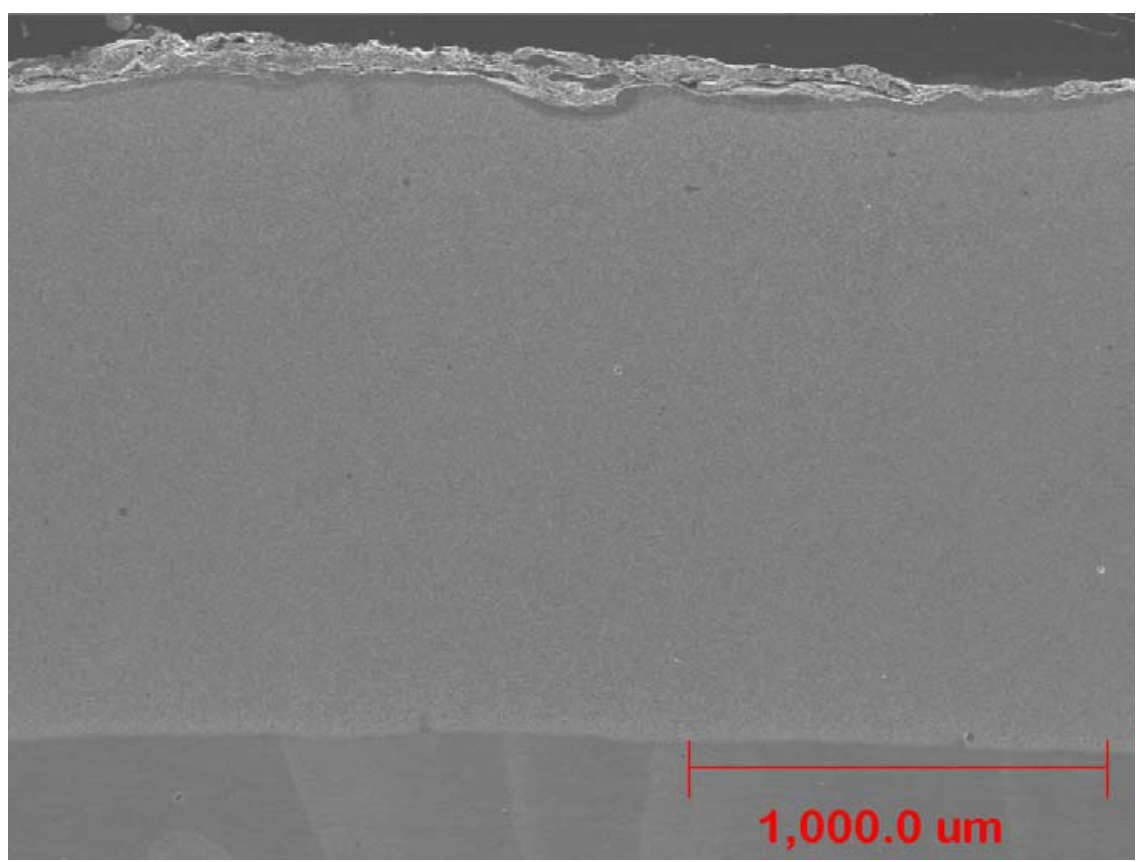

(a) External Scale/Metal Interface (SEI - Low Magnification)

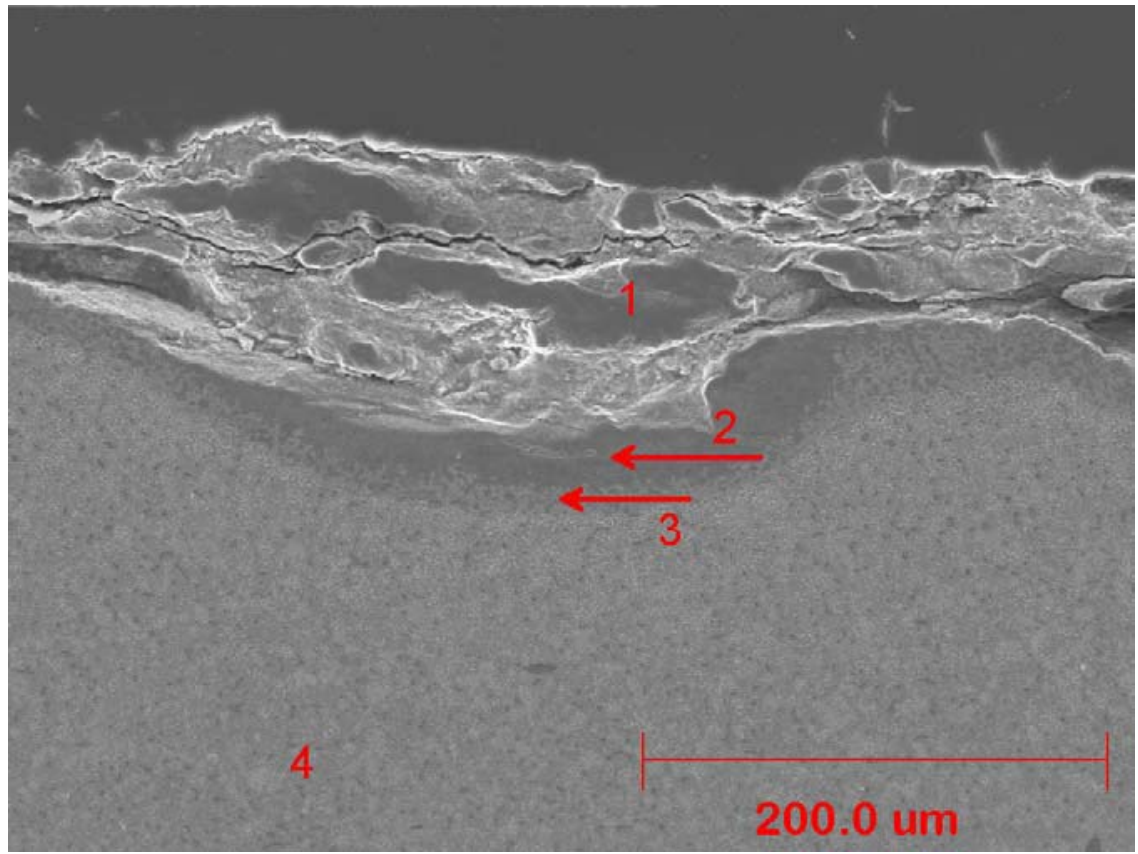

(b) External Scale/Metal Interface (SEI - Intermediate Magnification)

\section{Figure K-16: Microstructure: Incoclad 671}

Note: Numbers on photos indicate distinct areas characterized by the visual assessment and EDS analyses that follow. 


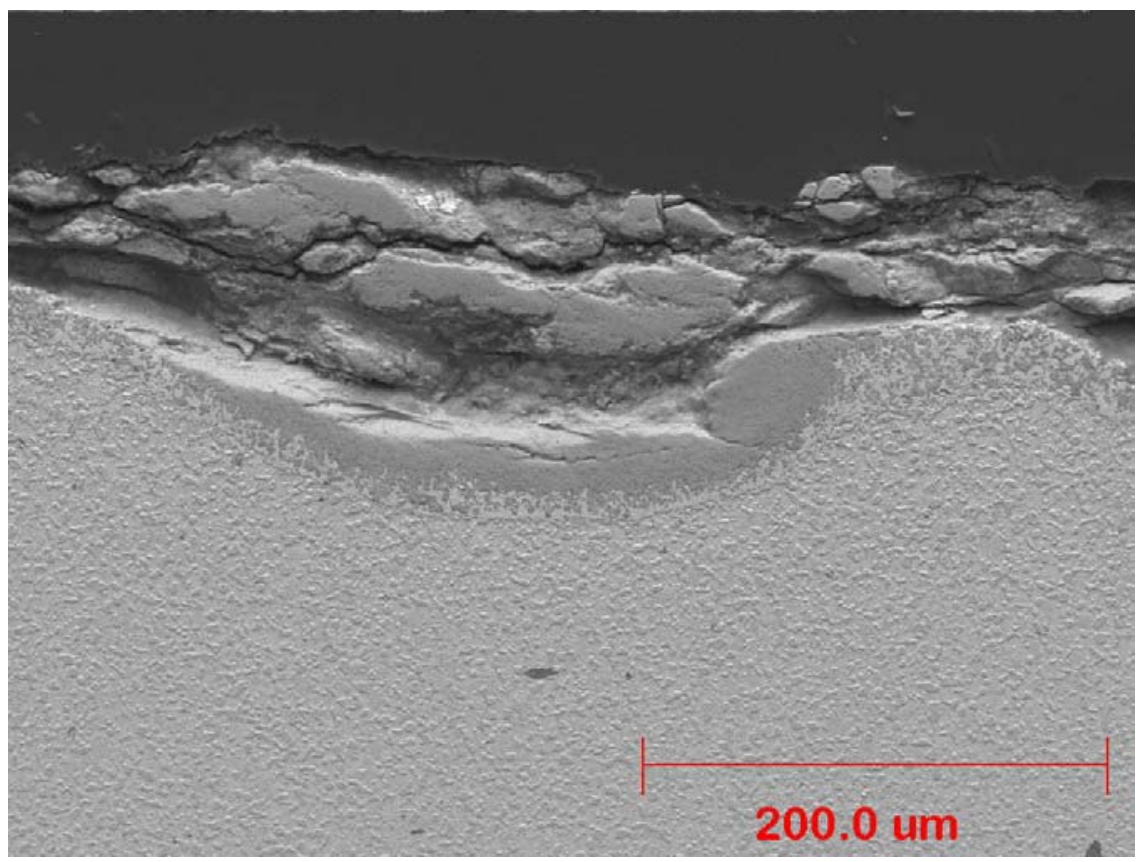

(c) External Scale/Metal Interface (BEI - High Magnification)

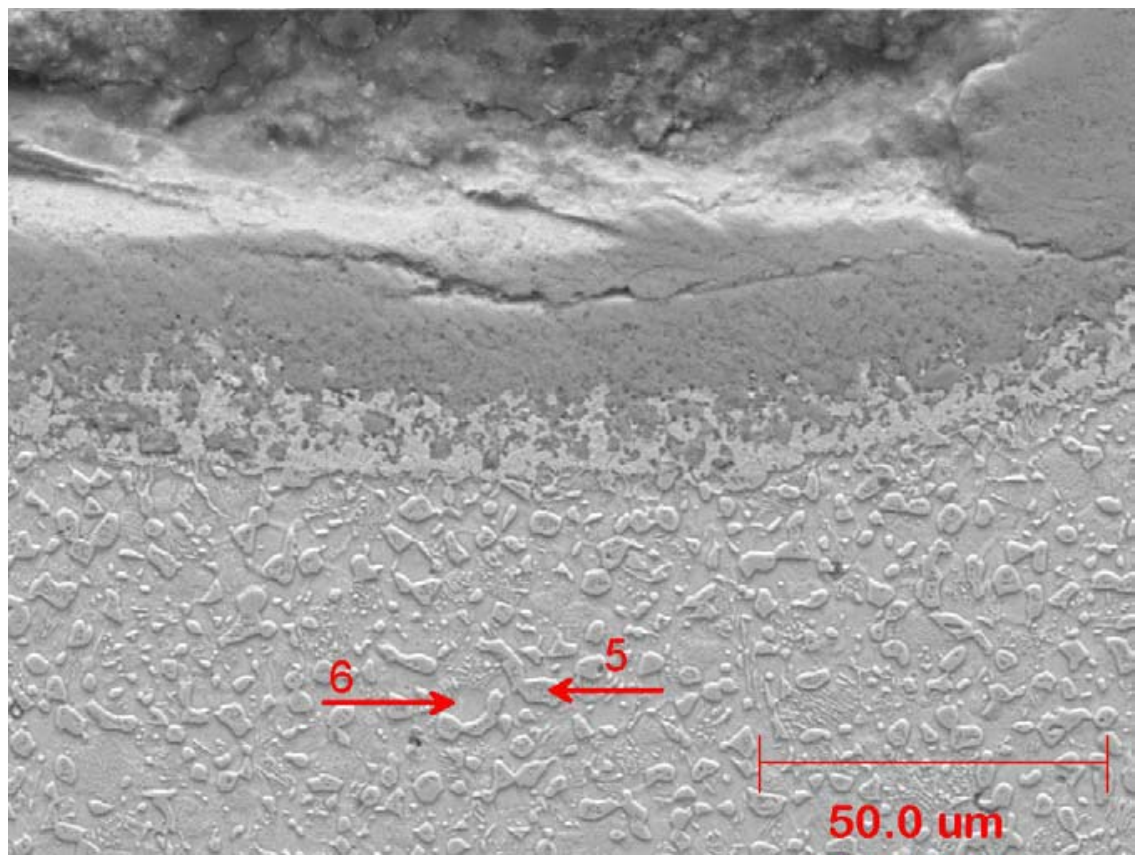

(d) External Scale/Metal Interface (SEI - High Magnification)

Figure K-15 (Cont.): Microstructure: Incoclad 671 


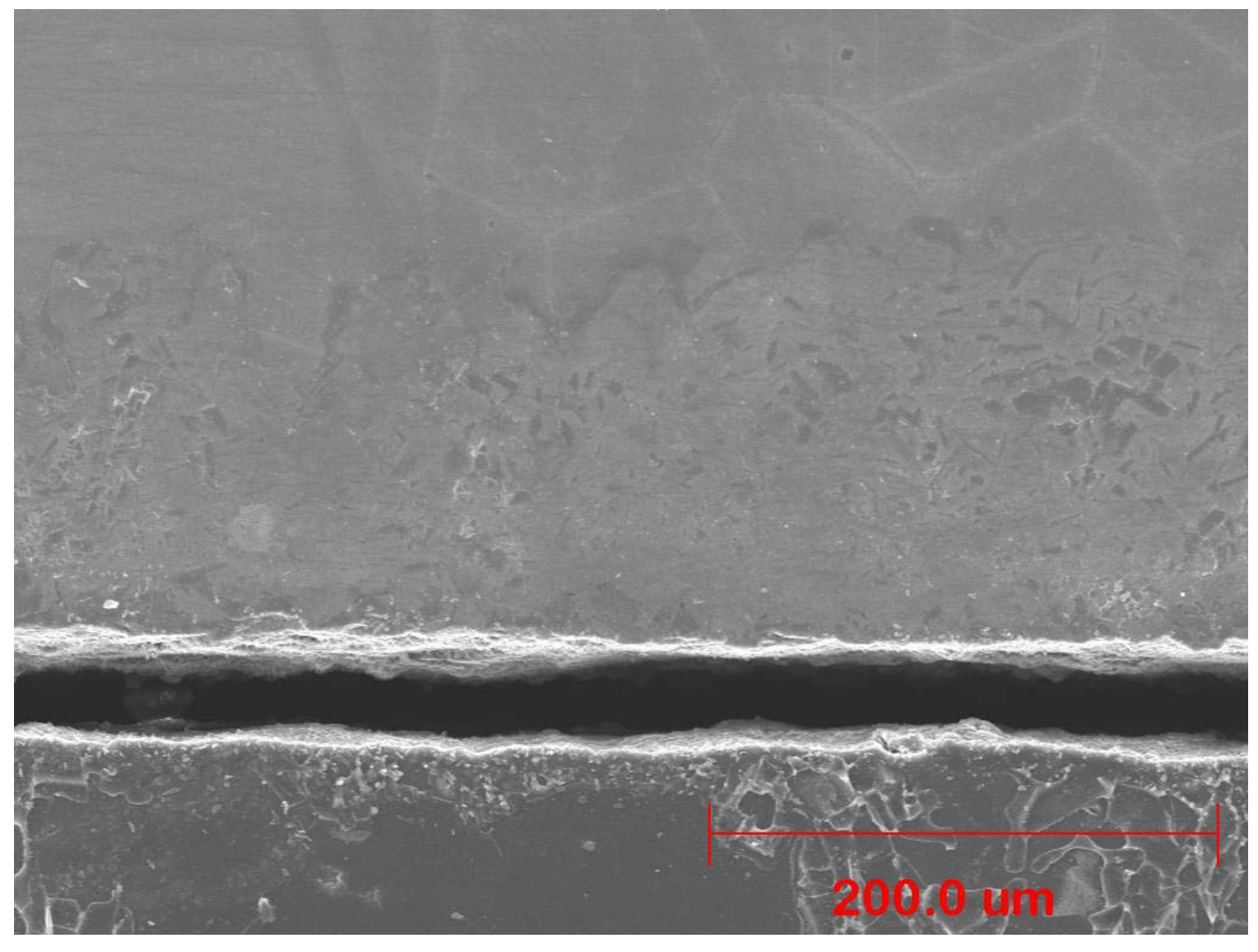

Internal Surface Appearance

Figure K-16: Microstructure: Incoclad 671 


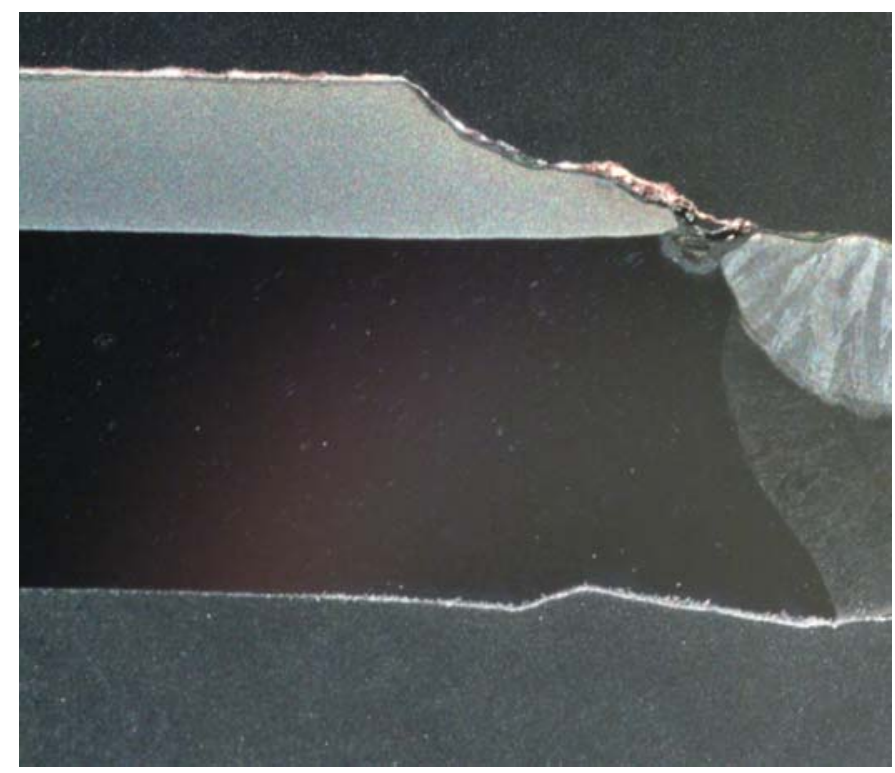

(a) Incoclad 671 Adjacent to Weld "P"

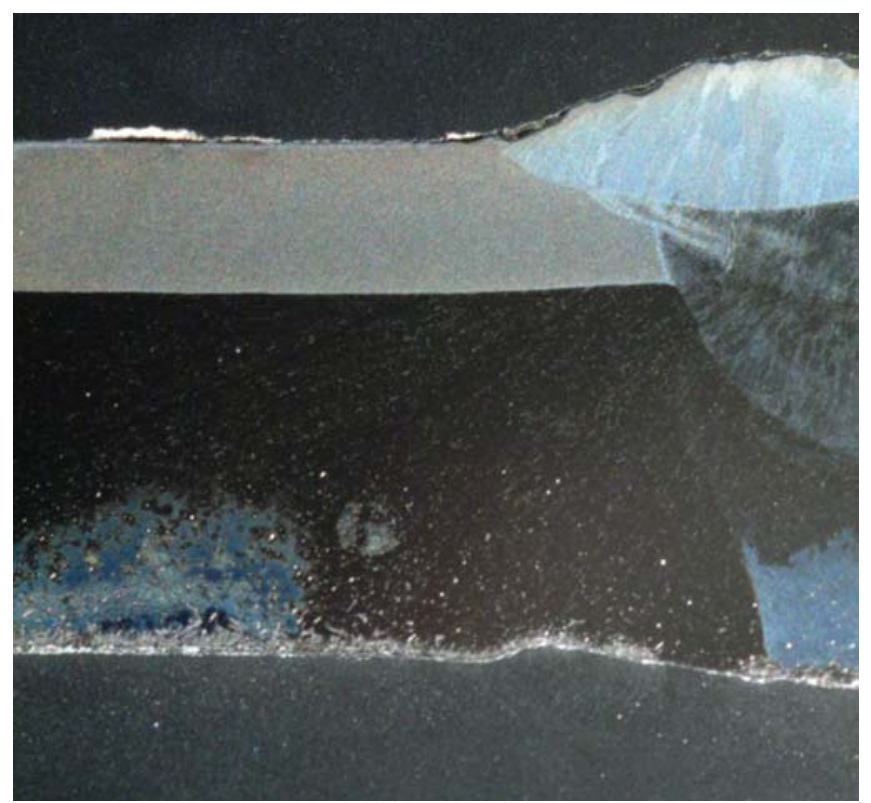

(b) Incoclad 671 Adjacent to Weld "V"

Figure K-17: Longitudinal Cross-Sections: Incoclad 671 Adjacent to Welds 


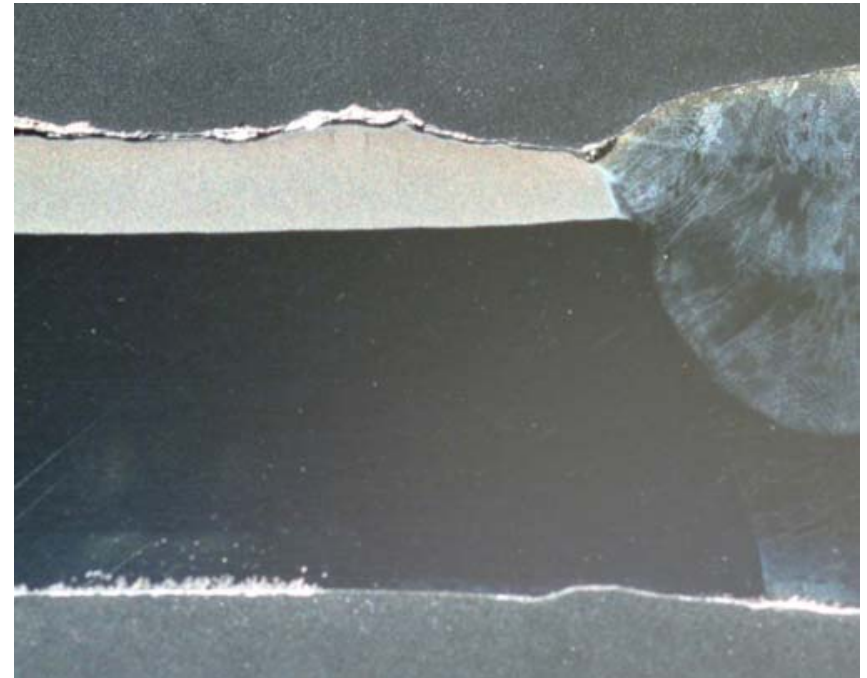

(c) Incoclad 671 Adjacent to Weld FF"

Figure K-17, Continued: Longitudinal Cross-Sections: Incoclad 671 Adjacent to Welds 
Figure K-18: EDS Analysis of Deposit/Scale/Metal Interface for Incoclad 671 (Sample EE-FF)

\begin{tabular}{|c|c|c|c|c|c|c|c|c|c|c|c|c|c|c|c|c|c|c|}
\hline Area & Description & $\mathbf{N i}$ & $\mathrm{Cr}$ & $\mathbf{F e}$ & Co & Mn & $\mathbf{V}$ & $\mathbf{T i}$ & Al & $\mathbf{S i}$ & $\mathbf{C}$ & $\mathbf{O}$ & $\mathbf{C a}$ & $\mathbf{K}$ & Cl & As & $\mathbf{P}$ & $\mathbf{S}$ \\
\hline 1 & $\begin{array}{l}\text { Bulk deposit } \\
\text { analysis }\end{array}$ & 1.25 & 75.6 & 3.26 & -- & -- & -- & 1.07 & 1.13 & -- & -- & 17.0 & 0.11 & 0.18 & -- & -- & -- & 0.56 \\
\hline 2 & $\begin{array}{l}\text { Tightly } \\
\text { adhering } \\
\text { scale. }\end{array}$ & 1.95 & 71.9 & -- & -- & -- & -- & 0.90 & 1.41 & -- & -- & 13.5 & -- & -- & -- & -- & -- & 10.2 \\
\hline 3 & $\begin{array}{l}\text { Diffusion } \\
\text { zone - dark } \\
\text { etching }\end{array}$ & 72.6 & 21.7 & -- & -- & - & -- & - & 0.45 & -- & -- & 2.24 & - & -- & -- & -- & -- & 3.01 \\
\hline 4 & $\begin{array}{l}\text { Bulk tube } \\
\text { analyais/ }\end{array}$ & 47.0 & 52.5 & -- & -- & - & -- & 0.24 & 0.23 & -- & - & -- & - & - & -- & -- & -- & -- \\
\hline 5 & $\begin{array}{l}\text { Shallow } \\
\text { etching } \\
\text { constituent in } \\
\text { tube. }\end{array}$ & 15.4 & 84.6 & -- & -- & -- & -- & -- & -- & -- & -- & -- & -- & -- & -- & -- & -- & -- \\
\hline 6 & $\begin{array}{l}\text { Deep etching } \\
\text { constituent in } \\
\text { tube. }\end{array}$ & 68.3 & 31.0 & -- & - & -- & -- & 0.30 & 0.36 & -- & -- & -- & -- & -- & -- & -- & -- & -- \\
\hline & $\begin{array}{l}\text { Reference } \\
\text { bulk analysis }\end{array}$ & 51.1 & 47.7 & .014 & 0.24 & 0.001 & 0.011 & 0.38 & 0.23 & 0.02 & 0.05 & -- & -- & -- & -- & -- & -- & 0.01 \\
\hline
\end{tabular}


Appendix L

Analysis of Performance

Test Section C

IN72 Weld Overlay 


\section{Overview of Results: IN72 Weld Overlay}

IN72 Weld Overlay continued to demonstrate a low rate of metal loss due to hot side corrosion. The maximum rate, based on diameter measurements, was determined to be 14 mils per year* (for Sample FF-GG). However, perhaps of equal importance is the fact that the overlay material appears to have suffered from internal cracking some grain boundaries that lie at roughly mid-point in the cladding. These internal cracks were shown to range from about 10 to 30 mils in length and seemed to be concentrated in one-half of the tube circumference and almost totally absent in the other.

The evidence associated with this internal cracking seems to suggest that hot corrosion is not the only active degradation mechanism for this material. The cause for the cracking has not been conclusively determined to date. However, evidence of cracking deep within the cladding, and lacking evidence of corrosion within the cracks, suggests that these, at least initially, are two separate phenomena. This investigation was able to identify a number of internal cracks in both Section C specimens. These internal cracks generally were not filled with corrosion product, thus they where apparently not connected to the external surface of the tube. However, in at least one instance (for Sample FF-GG) hot corrosion had removed sufficient surface material to expose an internal crack that was approximately 20 mils long. This exposed crack showed evidence of corrosion along its' length. If the additional 20 mils associated with this crack had been taken into account, then the calculated metal loss rate would have added 8 mils per year for Sample FF-GG. It should be noted that the cladding thickness was about 80 mils. So, for this case, the corrosion plus crack length breached about half of the cladding thickness.

To further explore the weld overlay cracking phenomenon, archive material was examined to verify determine whether the original material contained cracks. Dye penetrant and magnetic particle tests were performed on a piece of IN72 Weld Overlay archive material that had been set aside when the sections were originally constructed. Non-destructive testing of this six inch long sample showed no evidence of cracking. A ring section of the archive material was then cut and metallurgically examined. This showed evidence of a few small grain boundary cracks. When the Section A IN72 Weld Overlay samples were re-examined, it was found that these too had internal cracks that in only one case (noted in the Section A report) breached the surface. However, more importantly, more cracking was found in the Section A than in the archive material. Furthermore, Section C samples showed more cracking than the Section A samples. This suggests that service exposure may give rise to and grow additional cracks as a function of time.

Consideration of the evidence above suggests that IN72 Weld Overlay shows promise as to its ability to resist corrosion: however, serious consideration must be given to understanding the internal cracking phenomenon and its implications. If the internal cracking problem can be solved through processing or otherwise, then the IN72 Weld Overlay approach will offer a good practical solution toward addressing the problem of high temperature corrosion in similar applications. However, lacking an understanding of, and solution for, the observed internal cracking, and given the fact that cracking appears to be increasing with time at temperature, suggests that users would be well served in being cautious about employing this material. A major concern would be that the tubes may well show no visible evidence of underlying cracking during in situ inspection in the boiler.

Nonetheless, as was true for the other nickel-base alloys, the chromium and overall alloy content of this material likely account for the good corrosion resistance of the IN72 Weld Overlay in this environment. Also, like the other high-nickel, high-chromium materials, the corrosion rate 
appears to be higher at the lower exposure temperature. The reason for the somewhat increased corrosion rate at lower temperatures has not been determined.

It should be noted that the metal loss rates determined to date for both Section A and Section C all were based on a ring specimen that was extracted from mid-length of each tube sample. In this analysis additional attention was focused on metal losses in the vicinity of welds. Representative samples were taken and it was determined that the Inconel 625 weld filler metal, that was used for joining tubes to one another, while being reasonably resistant to coal ash corrosion, was less resistant to corrosion that the adjacent IN72 weld overlay. It is believed that dissolution of the Inconel 625 filler metal is the principle reason for the local loss of cross section at the weld at location "R". This suggests that IN72 filler metal may have been a better choice at this location.

Analysis of deposit on the surface revealed that it had the known aggressive combination of species, namely iron, oxygen, the alkali metals, and sulfur. Thus, the required corrosive elements were available to test the performance limits of this alloy.

* $\quad$ Note throughout this appendix, the reported metal loss rate was calculated based on time at temperature rather than total time within the boiler. 


\section{Material: IN72 Weld Overlay}

\section{Summary of Findings}

\begin{tabular}{|c|c|}
\hline Issue & Discussion \\
\hline $\begin{array}{l}\text { Sample } \\
\text { Location and } \\
\text { Orientation. }\end{array}$ & $\begin{array}{l}\text { - The schematic in Figure L-1 shows the relative positions of the two } \\
\text { IN72 Weld Overlay samples in Test Section C. Note that neither of the } \\
\text { IN72 overlay samples was within the tube lengths that were removed } \\
\text { early, i.e. in November } 2001 \text { or in June } 2002 \text {. } \\
\text { - Also, shown is the calculated average metal temperature at each } \\
\text { location. }\end{array}$ \\
\hline $\begin{array}{l}\text { Composition } \\
\text { Verification. }\end{array}$ & $\begin{array}{l}\text { - The weld wire for cladding the IN } 72 \text { Weld Overlay samples used in } \\
\text { this program was drawn from Special Metals Corporation (SMC) Heat } \\
\text { UKL8138. The composition for this heat is provided in the table in } \\
\text { Figure L-2 along with the target composition from SMC literature. } \\
\text { During the Section A evaluation, detailed composition verification was } \\
\text { undertaken for every tube sample that was removed from service. This } \\
\text { served, in part, to verify that the each tube material was located at its' } \\
\text { proper position within the test section. } \\
\text { Based on the good results for Section A, it was decided that it would } \\
\text { be unnecessary to analyze each and every tube sample for composition } \\
\text { verification. Rather, it was decided to select a representative sample } \\
\text { from each tube material type for confirmatory analysis. } \\
\text { During the Section A evaluation, the energy dispersive spectrographic } \\
\text { (EDS) capabilities of the scanning electron microscope (SEM) were } \\
\text { used to perform semi-quantitative analyses of each of the twelve } \\
\text { different candidate tube materials. Comparison of these EDS results } \\
\text { showed reasonably good correlation with results obtained using } \\
\text { quantitative analytical techniques, including optical emission } \\
\text { spectrometry. } \\
\text { Based on this, it was decided that the accuracy provided by EDS } \\
\text { would suffice for composition verification, but that more detailed } \\
\text { analyses would be undertaken if questions arose. } \\
\text { The results of the EDS analysis of tube sample FF-GG confirmed that } \\
\text { the correct material was used at this location. } \\
\text { It was noted that the actual tube sample composition was higher in } \\
\text { iron than the reported filler wire chemistry; however, is consistent } \\
\text { with what would be expected for a weld deposit, and compares } \\
\text { reasonable well the desired nominal chemistry in terms of chromium } \\
\text { content. }\end{array}$ \\
\hline $\begin{array}{l}\text { As-Received } \\
\text { Appearance }\end{array}$ & $\begin{array}{l}\text { - Figure L-3 shows the appearance of the archive sample for IN72 Weld } \\
\text { Overlay material. This photograph documents the condition of the tube } \\
\text { sample as it was delivered from SMC. The weld bead surface profile is } \\
\text { clearly visible in this view. The cladding thickness was measured to be } \\
\text { approximately } 80 \text { mils. Thus, it comprised about } 27 \% \text { of the total wall } \\
\text { thickness of } 292 \text { mils. }\end{array}$ \\
\hline
\end{tabular}




\section{Material: IN72 Weld Overlay}

\section{Summary of Findings, (continued)}

\begin{tabular}{|c|c|}
\hline Issue & Discussion \\
\hline $\begin{array}{l}\text { As-Received } \\
\text { Appearance, } \\
\text { Continued }\end{array}$ & $\begin{array}{l}\text { - Figures L-4 and L-5 document the appearance of the external surface } \\
\text { of the IN72 Weld Overlay tube samples after service. Three views are } \\
\text { provided for each tube: } 12 \text { o'clock (the view that faces on-coming gas } \\
\text { flow), } 3 \text { o'clock (the view where the gas flow is tangent to the } \\
\text { surface), and } 6 \text { o'clock (the view that shows the back-side of the tube } \\
\text { relative to the gas flow direction. } \\
\text { The reader should be alerted to the fact that, within the boiler, the gas } \\
\text { flows upward over these tubes. This implies that for the "o'clock" } \\
\text { conventions described above, (with the } 12 \text { o'clock orientation facing } \\
\text { the gas flow direction), the actual physical orientation of these tubes is } \\
\text { such that the } 12 \text { o'clock side of the tube faces downward, and the } 6 \\
\text { o'clock orientation faces upward within the boiler. } \\
\text { No wastage is visible in these views. The original weld bead profile is } \\
\text { somewhat obscured by deposit on Sample FF-GG (Figure L-5); } \\
\text { however, it is clearly visible on Sample Q-R (Figure L-4) which is } \\
\text { relatively free from scale. } \\
\text { All three views of Sample Q-R show evidence of the gray/white scale } \\
\text { associated with molten alkali-iron sulfate attack. This is also in } \\
\text { evidence in the } 6 \text { o'clock view for Sample FF-GG. }\end{array}$ \\
\hline $\begin{array}{l}\text { Grit-Blasted } \\
\text { Surface } \\
\text { Appearance }\end{array}$ & $\begin{array}{l}\text { - Figures L-6 and L-7 show the appearance of the external surface of the } \\
\text { IN } 72 \text { Weld Overlay tube samples after grit-blasting to remove deposit } \\
\text { and scale. The } 12 \text { o'clock and } 3 \text { o'clock positions for Sample FF-GG } \\
\text { show the appearance of general wastage due to fireside corrosion. } \\
\text { - Scattered pitting is in evidence at the } 12 \text { and } 3 \text { o'clock positions for } \\
\text { Sample Q-R. }\end{array}$ \\
\hline $\begin{array}{l}\text { Wall } \\
\text { Thickness } \\
\text { Loss } \\
\text { Determined }\end{array}$ & $\begin{array}{l}\text { - Tube cross-sections were prepared to allow for measurement of wall } \\
\text { thickness to determine metal loss due to exposure. These are shown in } \\
\text { silhouette in Figures L-8 and L-9. } \\
\text { The same relative "o'clock" positions were maintained as discussed } \\
\text { earlier. Measurements were taken at eight evenly spaced locations } \\
\text { about the tube circumference. These were then compared with } \\
\text { measurements taken at these same locations prior to exposure. } \\
\text { The before and after measurements were documented in the table that } \\
\text { accompanies each figure. These form the basis for the "delta" } \\
\text { calculation to determine the amount of wall thickness lost. Note that in } \\
\text { some cases, the delta is negative (particularly around the } 6 \text { o'clock } \\
\text { positon). This is believed due to wall thickness variability and } \\
\text { difficulty in locating the exact location of the "before" measurement. } \\
\text { Sample FF-GG experienced the maximum wall thickness reduction } \\
\text { due to corrosion of } 14 \text { mils per year. As was mentioned in the } \\
\text { overview section of this report, an additional } 8 \text { mils per year would } \\
\text { need to be included if one were to take into account weld cracking that } \\
\text { intersects the external surface. }\end{array}$ \\
\hline
\end{tabular}




\section{Material: IN72 Weld Overlay}

\section{Summary of Findings, (continued)}

\begin{tabular}{|c|c|}
\hline Issue & Discussion \\
\hline $\begin{array}{l}\text { Wall } \\
\text { Thickness } \\
\text { Loss Versus } \\
\text { Average Metal } \\
\text { Temperature }\end{array}$ & $\begin{array}{l}\text { - The chart in Figure L-10 plots the maximum wall thickness lost } \\
\text { against the calculated average metal temperature for the two IN72 } \\
\text { Weld Overlay samples in Section C. The data from the Section A } \\
\text { samples also are plotted. Note that these data do not take into account } \\
\text { the additional wall thickness that would be lost due to internal weld } \\
\text { cracking. } \\
\text { The hot corrosion rate for the Section C samples was slightly lower } \\
\text { than the rate for Section A samples, but both were found to be very } \\
\text { low. Again it was noted that the rate was higher at the lower exposure } \\
\text { temperature, as is consistent with the grit blasted surface images } \\
\text { shown in Figures L-6 and L-7. }\end{array}$ \\
\hline $\begin{array}{l}\text { Wall } \\
\text { Thickness } \\
\text { Loss as a } \\
\text { Function of } \\
\text { Time and } \\
\text { Location }\end{array}$ & $\begin{array}{l}\text { - The chart in Figure L-11 plots metal loss as a function of time for each } \\
\text { sample location for both Sections A and C, with samples from Section } \\
\text { C having experienced generally longer exposure times. Note again that } \\
\text { these data do not take into account the additional wall thickness that } \\
\text { would be lost due to internal weld cracking. } \\
\text { The chart shows a straight line fit to the maximum metal loss data } \\
\text { points for Samples Q-R and FF-GG for both Sections A and C. } \\
\text { Evaluation of the rate of metal loss data depicted in Figure L-10 } \\
\text { suggests that a straight line fit would not likely be appropriate for } \\
\text { these samples in that the rate of metal loss decreased from Section A } \\
\text { to Section C for both samples under consideration. }\end{array}$ \\
\hline $\begin{array}{l}\text { Metallurgical } \\
\text { Analysis - } \\
\text { SEM }\end{array}$ & $\begin{array}{l}\text { - Sample FF-GG was selected for a detailed SEM/EDS assessment of } \\
\text { the constituents and structure in the vicinity of the external and } \\
\text { internal surfaces. This sample was selected for evaluation due to its } \\
\text { higher rate of corrosion based on diameter measurements. } \\
\text { - Figure L-12 shows back-scattered electron images (BEI) of the } \\
\text { external surface of Sample FF-GG. This mode of imaging was chosen } \\
\text { because it highlights differences in atomic weight. } \\
\text { - Figures L-12a and b show BEI of the external scale/metal interface for } \\
\text { Sample FF-GG. Also shown in this image are the scale-lined weld } \\
\text { cracks that have intersected corrosion that is progressing inward from } \\
\text { the external surface. } \\
\text { The numbers on Figure L-12b show the locations of EDS analyses to } \\
\text { further characterize these regions. The results of these are provided in } \\
\text { a later section of this report. } \\
\text { Figure L-12c provides the appearance of a shallow pit found on the } \\
\text { surface of Sample FF-GG. These were found typically to be under a } \\
\text { local build-up of deposit on the tube surface. }\end{array}$ \\
\hline
\end{tabular}


Summary of Findings, (continued)

\section{Material: IN72 Weld Overlay}

\begin{tabular}{|c|c|}
\hline Issue & Discussion \\
\hline $\begin{array}{l}\text { Metallurgical } \\
\text { Analysis - } \\
\text { SEM, } \\
\text { Continued. }\end{array}$ & $\begin{array}{l}\text { - Based on examination the images in Figure L-12, five distinct regions } \\
\text { are in evidence. These regions are, starting within the metal and } \\
\text { working out: } \\
\text { Base metal - Oxalic acid etch faintly delineates grain } \\
\text { boundaries, carbides, and inclusions. } \\
\text { Dark-Etching Band Parallel to the Scale/Metal Interface - } \\
\text { This region is clearly indicated by the arrow associated with } \\
\text { EDS analysis point \#5 in Figure L-12b. Analysis reported later } \\
\text { will suggest that this region has suffered internal oxidation } \\
\text { (6.23\% oxygen). } \\
\text { Light-Etching Band at the Scale/Metal Interface - This region } \\
\text { is clearly indicated by the arrow associated with the EDS } \\
\text { analysis point \#4 in Figure L-12b. Analysis reported later will } \\
\text { suggest that this region is primarily unaffected tube metal with } \\
\text { a lesser oxygen content (1.83\% oxygen). } \\
\text { Tightly adherent scale - this is seen continuous along the } \\
\text { surface underneath the surface deposit. EDS analysis will later } \\
\text { show that this is predominantly a chromium-rich oxide. } \\
\text { Thick and very porous deposit/scale conglomerate - many } \\
\text { spherical particles are noted along with what appears to be } \\
\text { islands of scale (metal oxides). } \\
\text { Figure L-13 shows the appearance of the internal metal surface for } \\
\text { Sample FF-GG. No oxide scale is in evidence. }\end{array}$ \\
\hline $\begin{array}{l}\text { Metallurgical } \\
\text { Analysis - } \\
\text { Weld Cross } \\
\text { Section. }\end{array}$ & $\begin{array}{l}\text { - Figure L-14 shows a longitudinal profile (at the } 12 \text { o'clock orientation) } \\
\text { of the IN72 Weld Overlay samples adjacent to the welds in Section C. } \\
\text { The welds in question are those identified as welds "R" and "FF", see } \\
\text { Figure L-1 for locations } \\
\text { Evaluation of the images in Figure L-14 suggests that the Inconel } 625 \\
\text { weld filler metal was less resistant to corrosion than the adjacent IN72 } \\
\text { weld overlay. It is believed that dissolution of the Inconel } 625 \text { filler } \\
\text { metal is the principle reason for the local loss of cross section at the } \\
\text { weld at location "R". This suggests that IN72 filler metal may have } \\
\text { been a better choice at this location. }\end{array}$ \\
\hline
\end{tabular}


Summary of Findings, (continued)

\section{Material: IN72 Weld Overlay}

\begin{tabular}{|c|c|}
\hline Issue & Discussion \\
\hline $\begin{array}{l}\text { EDS Analysis } \\
\text { Results }\end{array}$ & $\begin{array}{l}\text { - The table in Figure L-15 provides a summary of the EDS analyses of } \\
\text { the seven locations numbered in Figure L-12 b. Also shown in this } \\
\text { table is a summary of the spectrographic analysis reported earlier, see } \\
\text { Figure L-2. } \\
\text { - Review of this table suggests the following: } \\
\text { - Area 1: Bulk deposit/scale analysis - Alkali metals are present. } \\
\text { Sulfur also is present in an increased amount compared to the } \\
\text { base metal. An iron oxide is likely, as are aluminum and silicon } \\
\text { oxides. } \\
\text { - Area 2: Nodule in bulk deposit - Iron-, aluminum-, and silicon- } \\
\text { rich oxides are indicated. Again the corrosive combination of } \\
\text { alkali metals and sulfur also are in evidence. } \\
\text { - Areas 3: Adherent scale- The analysis suggest a chromium and } \\
\text { nickel rich oxide. Again a significant amount of corrosive species } \\
\text { are also indicated, particularly sulfur. } \\
\text { - Area 4: Light etching band at scale/metal interface - The analysis } \\
\text { suggest that this is a narrow zone of base metal showing some } \\
\text { oxygen ingress. } \\
\text { Areas 5: Dark etching band parallel to scale/metal interface - The } \\
\text { analysis suggests ingress of oxygen and formation of a } \\
\text { chromium-bearing oxide at this location. } \\
\text { - Area 6: Scale within corrosion deposit within weld crack - The } \\
\text { crack is shown to be lined with a chromium-rich oxide. } \\
\text { Area 7: Bulk analysis:- Given its limitations this EDAX analysis } \\
\text { compares favorably with that of the reference spectrographic } \\
\text { analysis below it. }\end{array}$ \\
\hline
\end{tabular}




\section{Gas Flow}

Direction

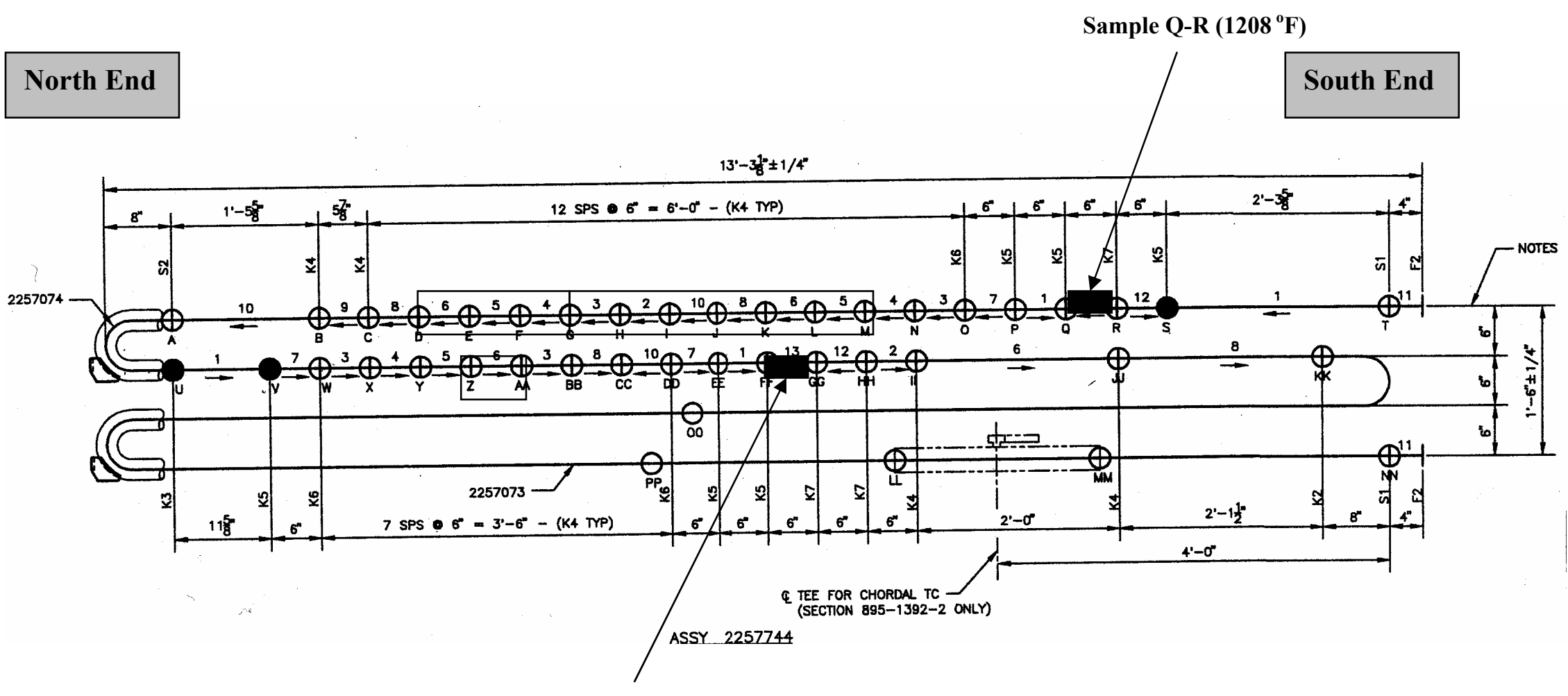

Sample FF-GG $\left(1079{ }^{\circ} \mathrm{F}\right)$

Figure L-1: Schematic Drawing Showing Position of IN72 Weld Overlay Tube Samples Within Test Section C

1. Drawing shown in same relative orientation as per the actual installation.

2. This group of tube samples was removed from service in November 2001 - highlighted in red.

3. This group of tube samples was removed from service in June 2002 - highlighted in yellow. 
Figure L-2 Semi-Quantitative Composition Verification for Inco 72 / 800 HT (Weld clad duplex tubing)

Description:

Source:

Reference Composition:
Nominal $45 \mathrm{Cr} / 55 \mathrm{Ni}$ alloy cladding for oxidation/corrosion protection. Incoloy $800 \mathrm{HT}$ substrate provides creep strength.

Special Metals Corporation (SMC)

Filler wire "nominal" chemistry - Per SMC literature.

\begin{tabular}{|c|c|c|c|c|}
\hline Element & $\begin{array}{c}\text { Reference } \\
\text { Composition for } \\
\text { Weld Cladding }\end{array}$ & $\begin{array}{c}\text { Composition } \\
\text { Heat UKL8138 }\end{array}$ & $\begin{array}{c}\text { Tube Sample } \\
\text { Q-R }\end{array}$ & $\begin{array}{c}\text { Tube Sample } \\
\text { FF-GG }\end{array}$ \\
\hline $\mathrm{Ni}$ & 55 & 54.96 & $\mathrm{~N} / \mathrm{A}$ & 53.0 \\
\hline $\mathrm{Cr}$ & 44 & 43.30 & N/A & 42.9 \\
\hline $\mathrm{Fe}$ & 0.2 & 0.43 & N/A & 3.21 \\
\hline $\mathrm{Mo}$ & - & - & N/A & - \\
\hline $\mathrm{Co}$ & - & - & N/A & - \\
\hline $\mathrm{C}$ & 0.05 & 0.02 & N/A & - \\
\hline $\mathrm{N}$ & - & - & N/A & - \\
\hline $\mathrm{B}$ & - & - & N/A & - \\
\hline $\mathrm{Mn}$ & 0.1 & 0.15 & N/A & - \\
\hline $\mathrm{Si}$ & 0.1 & 0.20 & N/A & 0.26 \\
\hline $\mathrm{Al}$ & - & 0.66 & N/A & - \\
\hline $\mathrm{Ti}$ & 0.6 & - & N/A & 0.61 \\
\hline $\mathrm{Al}+\mathrm{Ti}$ & - & - & N/A & - \\
\hline $\mathrm{Nb}$ & - & - & N/A & - \\
\hline $\mathrm{Ta}$ & - & - & N/A & - \\
\hline $\mathrm{Nb}+\mathrm{Ta}$ & - & - & N/A & - \\
\hline $\mathrm{V}$ & - & - & N/A & - \\
\hline $\mathrm{W}$ & - & - & N/A & - \\
\hline $\mathrm{Cu}$ & 0.2 & - & N/A & - \\
\hline $\mathrm{Sn}$ & - & 0.005 & N/A & - \\
\hline $\mathrm{P}$ & - & 0.003 & N/A & - \\
\hline $\mathrm{S}$ & 0.008 & - & N/A & - \\
\hline $\mathrm{O} 2$ & - & -0.50 & N/A & - \\
\hline $\mathrm{Others}$ & $<0.50$ & & & \\
\hline & & & - & \\
\hline
\end{tabular}

N/A = Sample not analyzed. Note: Reference composition is for filler wire, not as-deposited. 


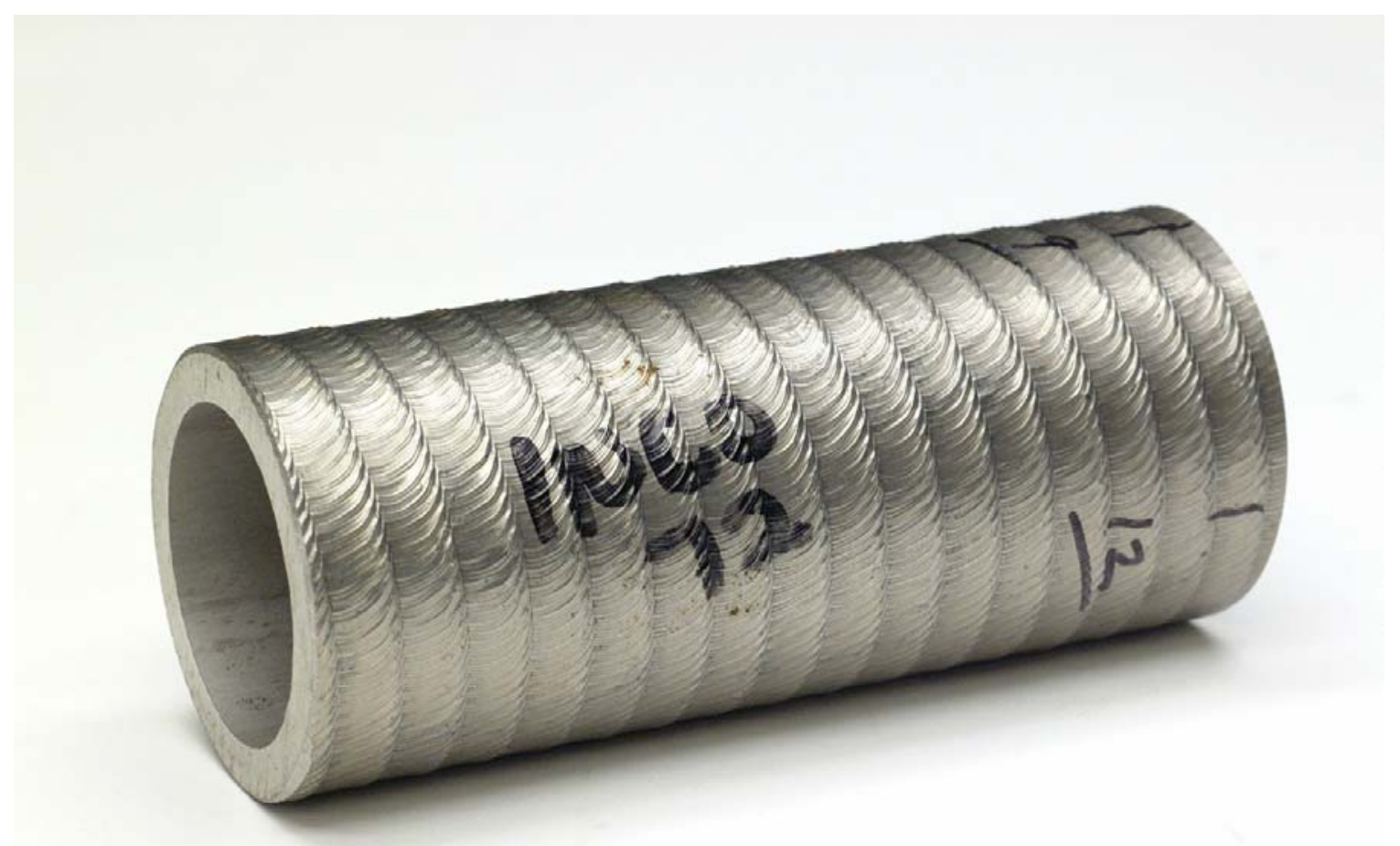

Figure L-3: Appearance of IN72 Weld Overlay Tube Sample Prior to Service 


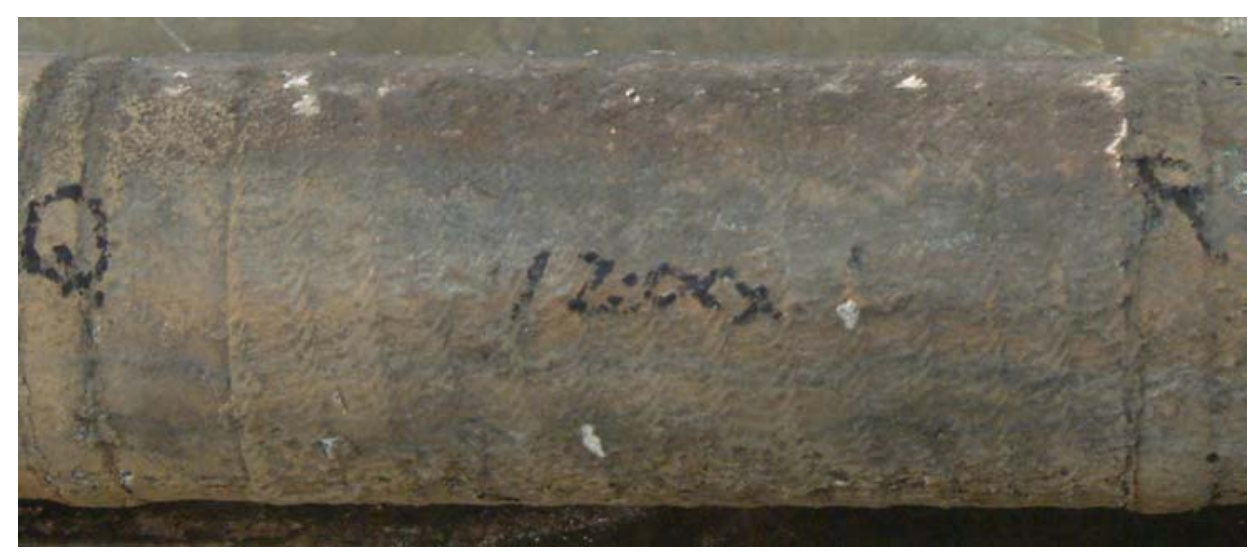

12 O'Clock - Facing Gas Flow

(Note: 3 o'clock is up in this image)

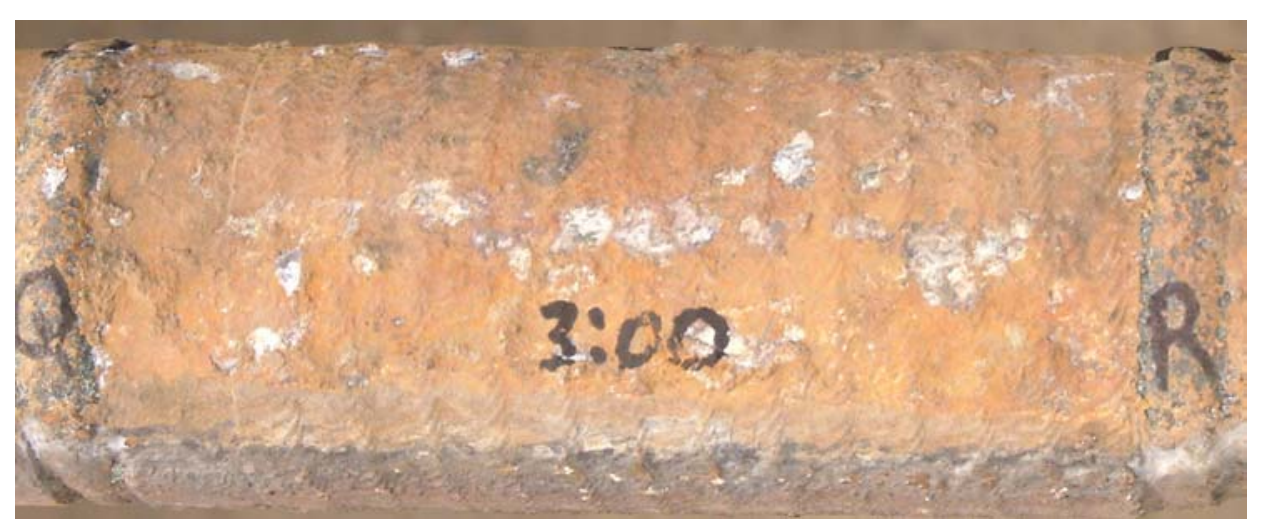

3 O'Clock

(Note: 6 o'clock is up in this image)

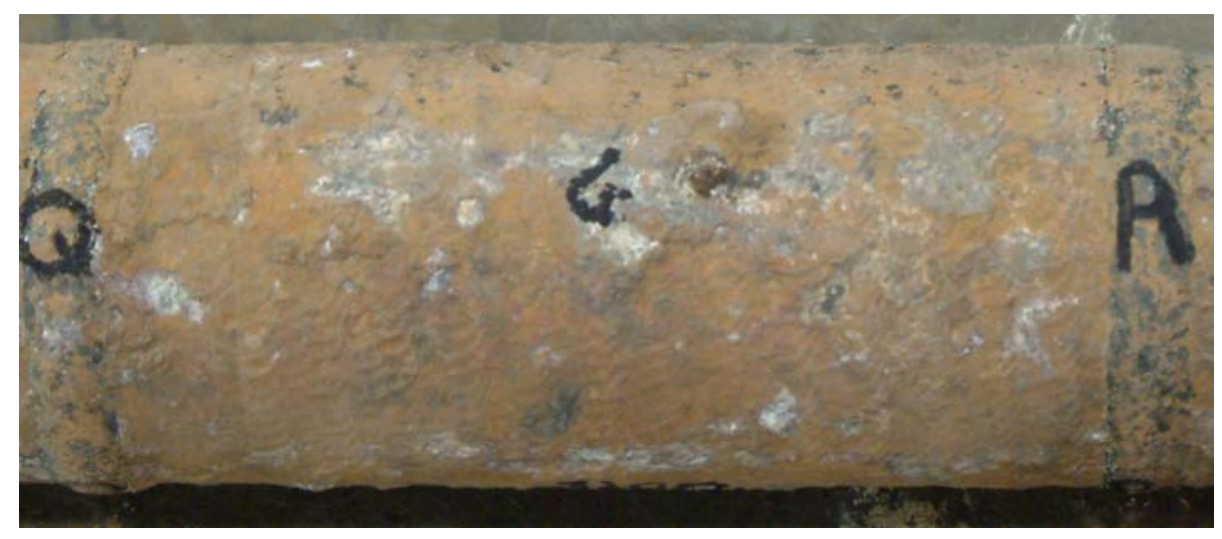

6 O'Clock - Opposite Gas Flow

Figure L-4: As-Received Appearance of Sample Q-R (IN72 Weld Overlay)

(Average surface metal temperature: $\mathbf{1 2 0 8}^{\circ} \mathrm{F}$ ) 


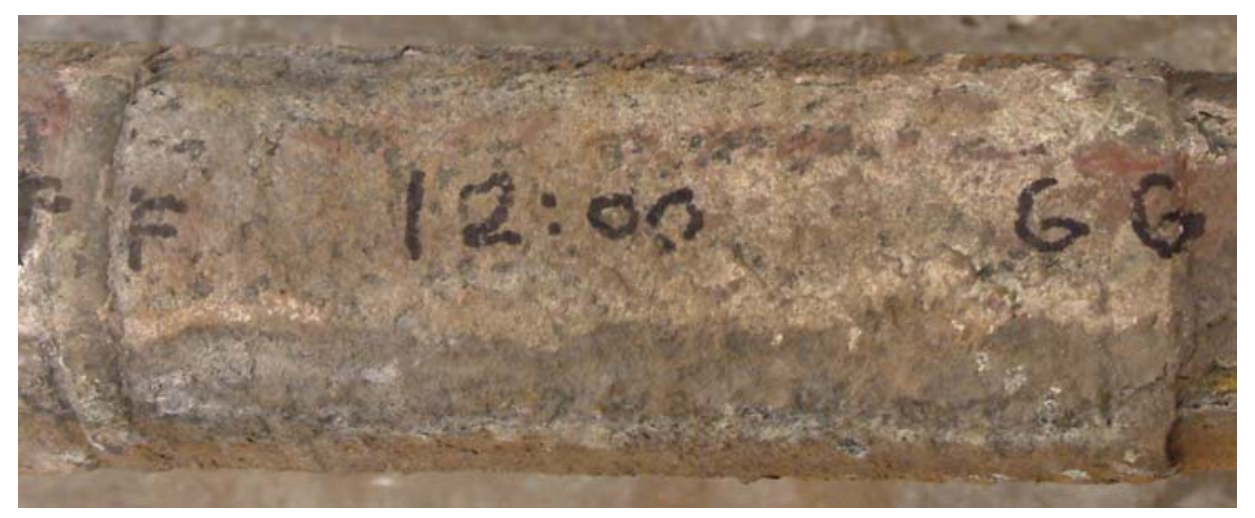

12 O'Clock - Facing Gas Flow

(Note: 3 o'clock is up in this image)

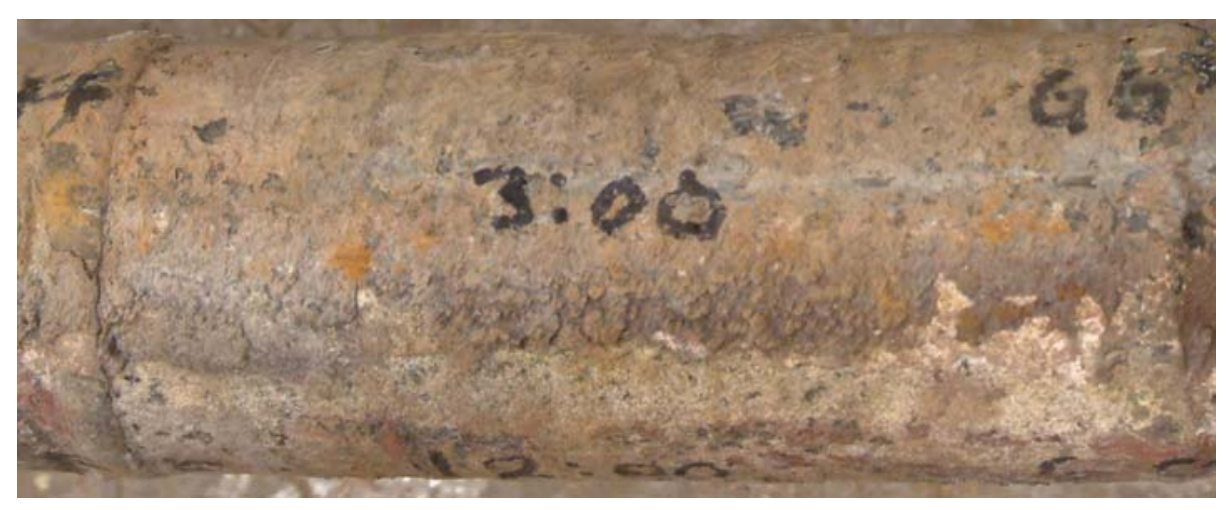

3 O'Clock

(Note: 6 o'clock is up in this image)

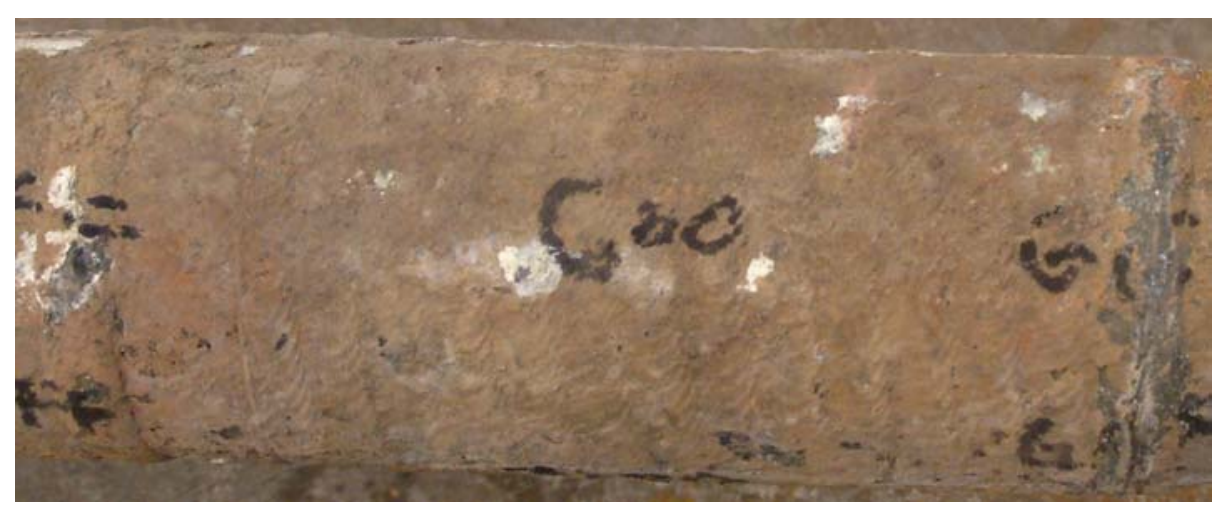

6 O'Clock - Opposite Gas Flow

Figure L-5 As-Received Appearance of Sample FF-GG (IN72 Weld Overlay)

(Average surface metal temperature: $1079^{\circ} \mathrm{F}$ ) 
12 O'Clock

(N/A mils lost)

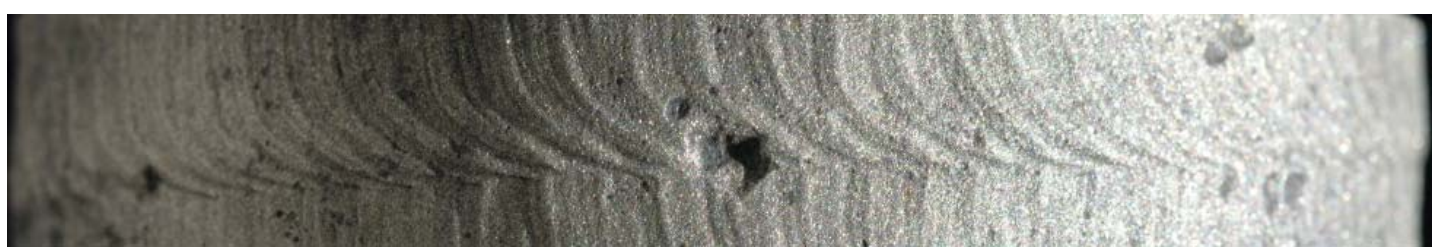

3 O'Clock

(0 mils lost)

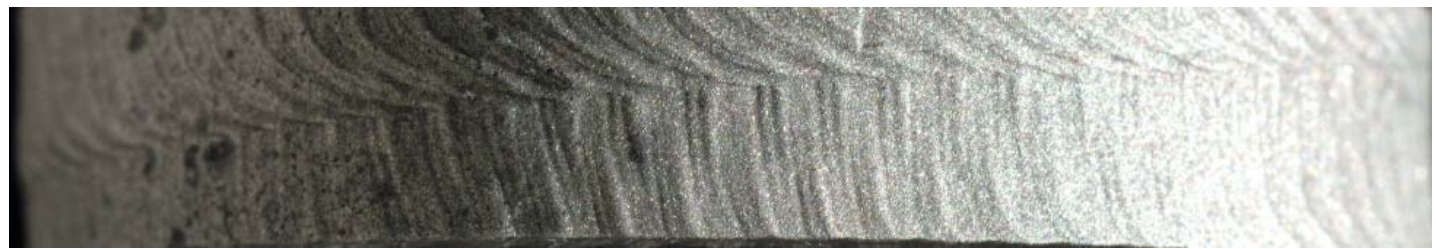

6 O'Clock

(0 mils lost)

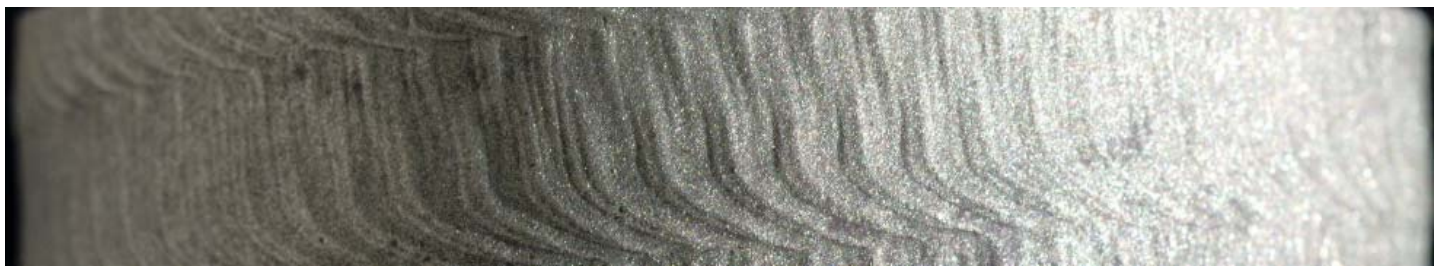

Figure L-6: OD Surface Appearance After Grit Blast

Material: IN72 Weld Overlay

Sample: Q-R 
12 O'Clock

(N/A mils lost)

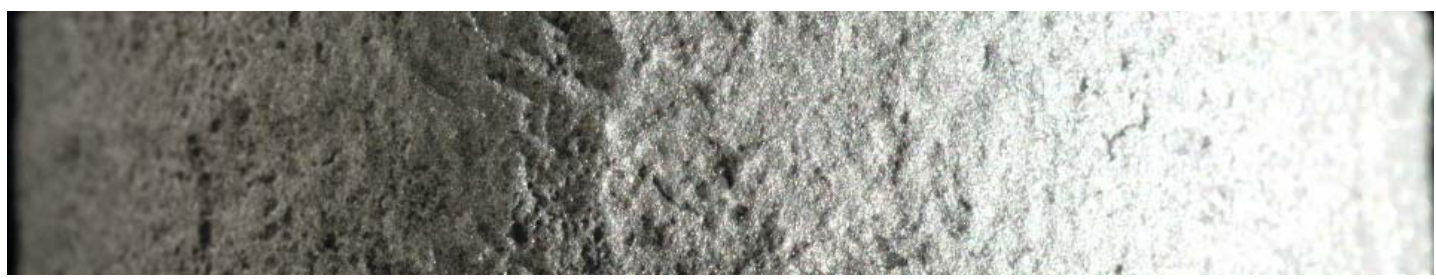

3 O'Clock

(8 mils lost)

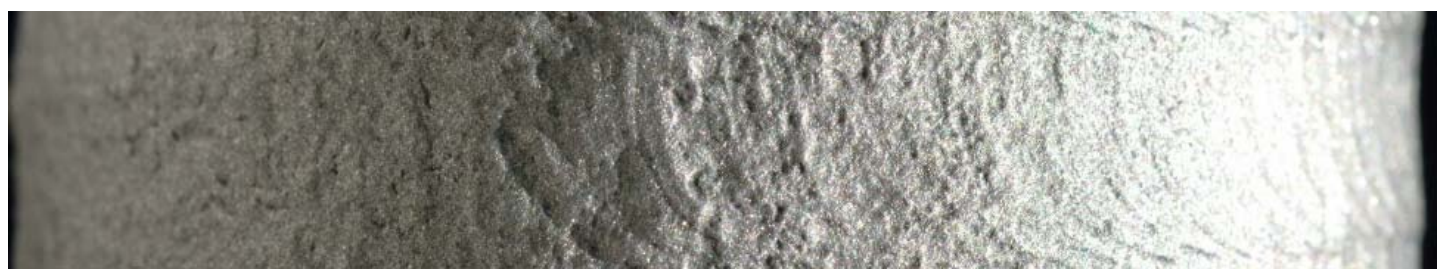

6 O'Clock

(0 mils lost)

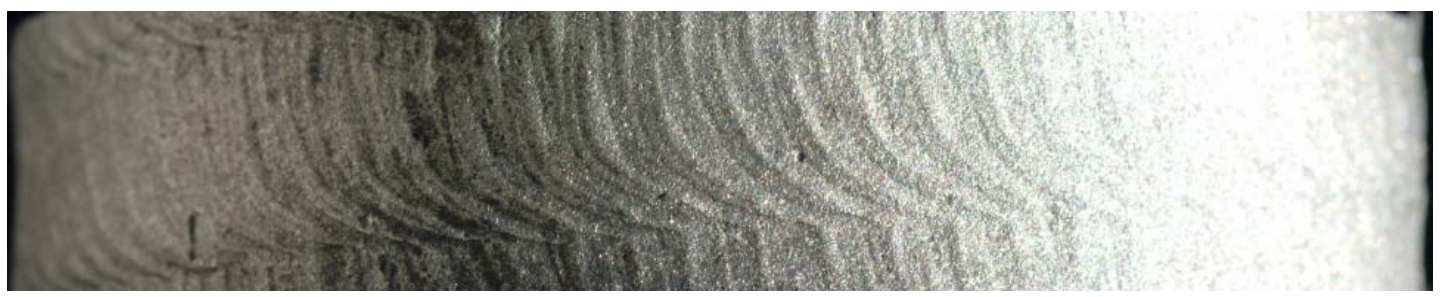

Figure L-7: OD Surface Appearance After Grit Blast

Material: IN72 Weld Overlay

Sample: FF-GG 


\section{Material:}

Sample Location:

Maximum Rate:

Maximum Loss Orientation:

Average Surface Metal Temperature:
IN72 Weld Overlay

Q-R

$6 \mathrm{mils} / \mathrm{yr}$

$7: 30$ o'clock

$1208^{\circ} \mathrm{F}$

\section{O’Clock}

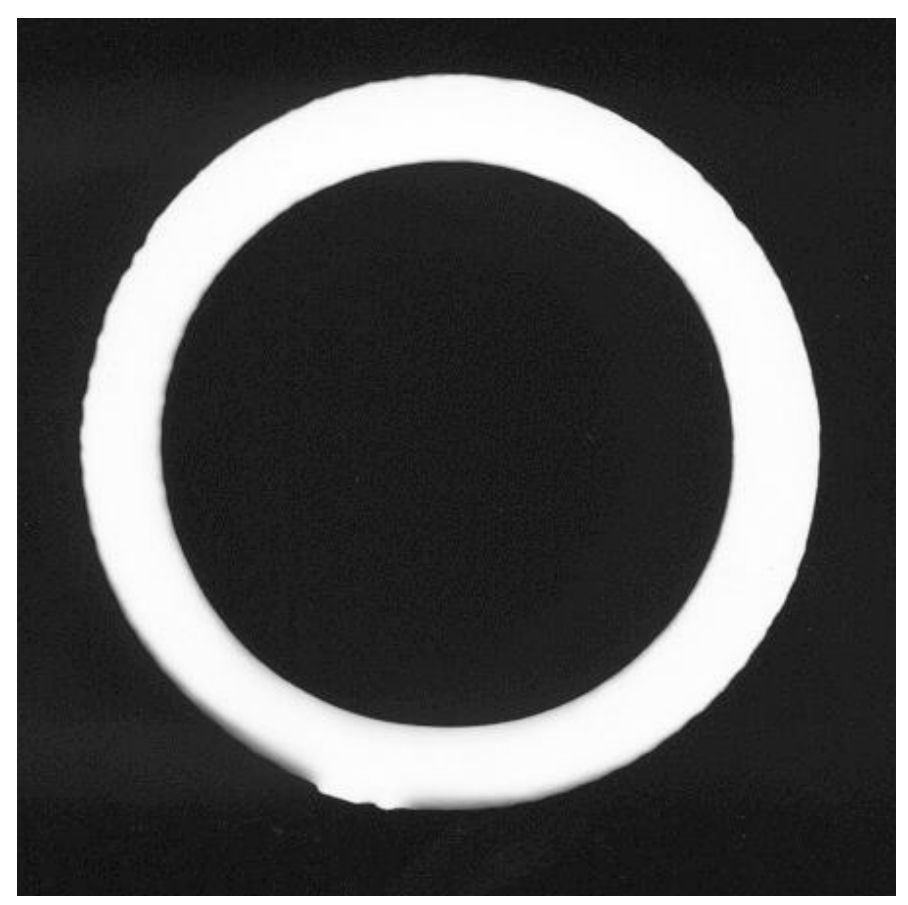

3 O’Clock

Approx. 1.2X

\section{Wall Reduction by Position (in.)}

\begin{tabular}{|l|c|c|c|c|c|c|c|c|c|c|}
\hline & $\begin{array}{c}12 \\
\text { o'clock }\end{array}$ & $\mathbf{1 : 3 0}$ & $\mathbf{3 : 0 0}$ & $\mathbf{4 : 3 0}$ & $\mathbf{6 : 0 0}$ & $\mathbf{7 : 3 0}$ & $\mathbf{9 : 0 0}$ & $\mathbf{1 0 : 3 0}$ & AVG & $\begin{array}{c}\text { Max }^{*} \\
\text { Loss }\end{array}$ \\
\hline Before & 0.293 & 0.286 & 0.285 & 0.287 & 0.292 & 0.297 & 0.299 & 0.298 & 0.292 & 0.289 \\
\hline After & N/A & 0.282 & 0.285 & 0.289 & 0.294 & 0.298 & 0.300 & 0.283 & & 0.283 \\
\hline Delta & N/A & 0.004 & 0.000 & -0.002 & -0.002 & -0.001 & -0.001 & 0.015 & & 0.015 \\
\hline
\end{tabular}

Figure L-8: Silhouette and Wall Thickness Measurements for Sample Q-R

* Measurement taken at "maximum loss orientation" noted above. The average

"before" wall thickness was used to calculate the "delta". 


\section{Material:}

Sample Location:

Maximum Rate:

Maximum Loss Orientation:

Average Surface Metal Temperature:
IN72 Weld Overlay

FF-GG

14 mils/yr

Approx. 10 o'clock

$1079^{\circ} \mathrm{F}$

\section{O’Clock}

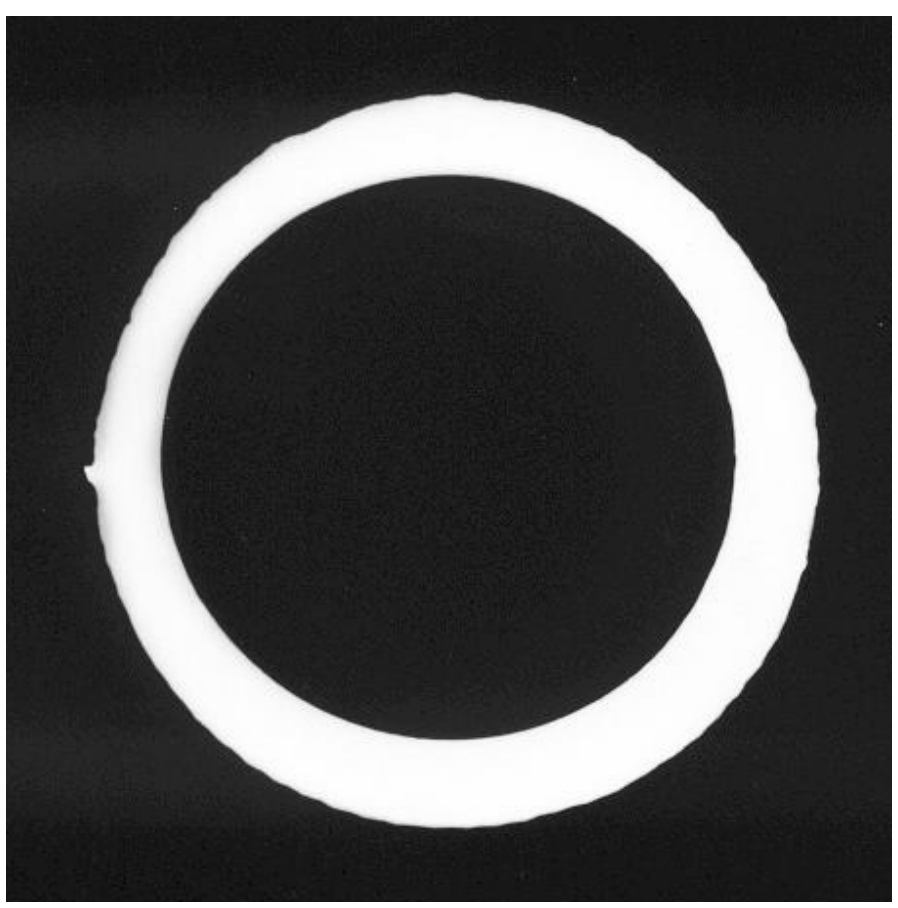

3 O’Clock

Approx. 1.2X

Wall Reduction by Position (in.)

\begin{tabular}{|l|c|c|c|c|c|c|c|c|c|c|}
\hline & $\begin{array}{c}\mathbf{1 2} \\
\text { o'clock }\end{array}$ & $\mathbf{1 : 3 0}$ & $\mathbf{3 : 0 0}$ & $\mathbf{4 : 3 0}$ & $\mathbf{6 : 0 0}$ & $\mathbf{7 : 3 0}$ & $\mathbf{9 : 0 0}$ & $\mathbf{1 0 : 3 0}$ & AVG & $\begin{array}{c}\text { Max* } \\
\text { Loss }\end{array}$ \\
\hline Before & 0.293 & 0.292 & 0.288 & 0.289 & 0.289 & 0.295 & 0.296 & 0.294 & 0.292 & 0.292 \\
\hline After & N/A & 0.263 & 0.280 & 0.292 & 0.290 & 0.288 & 0.282 & N/A & & 0.256 \\
\hline Delta & N/A & 0.029 & 0.008 & -0.003 & -0.001 & 0.007 & 0.014 & N/A & & 0.036 \\
\hline
\end{tabular}

Figure L-9: Silhouette and Wall Thickness Measurements for Sample FF-GG

* Measurement taken at "maximum loss orientation" noted above. The average

"before" wall thickness was used to calculate the "delta". 


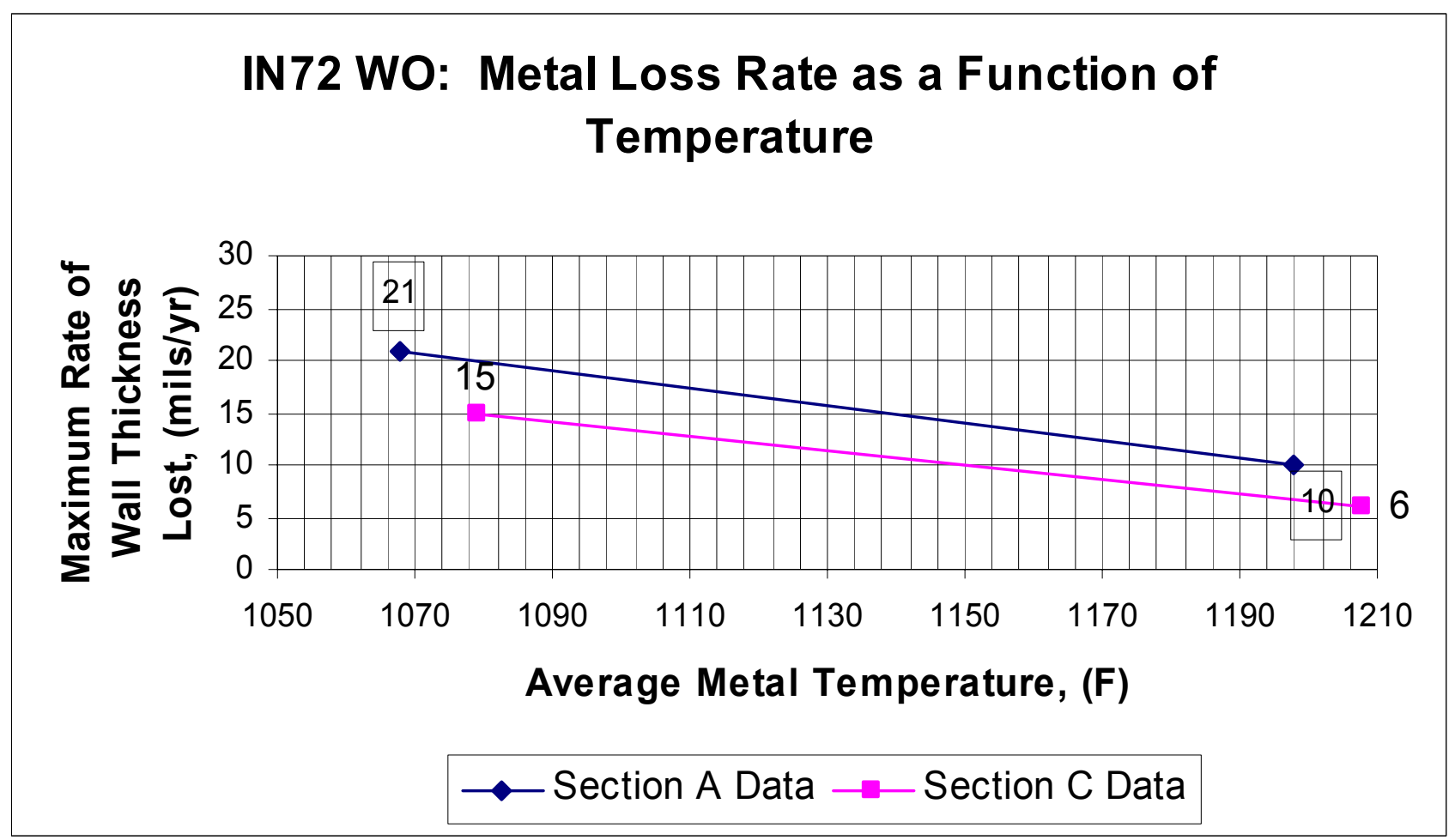

Figure L-10: Metal Loss Rate as a Function of Average Surface Metal Temperature

Material: IN72 Weld Overlay 


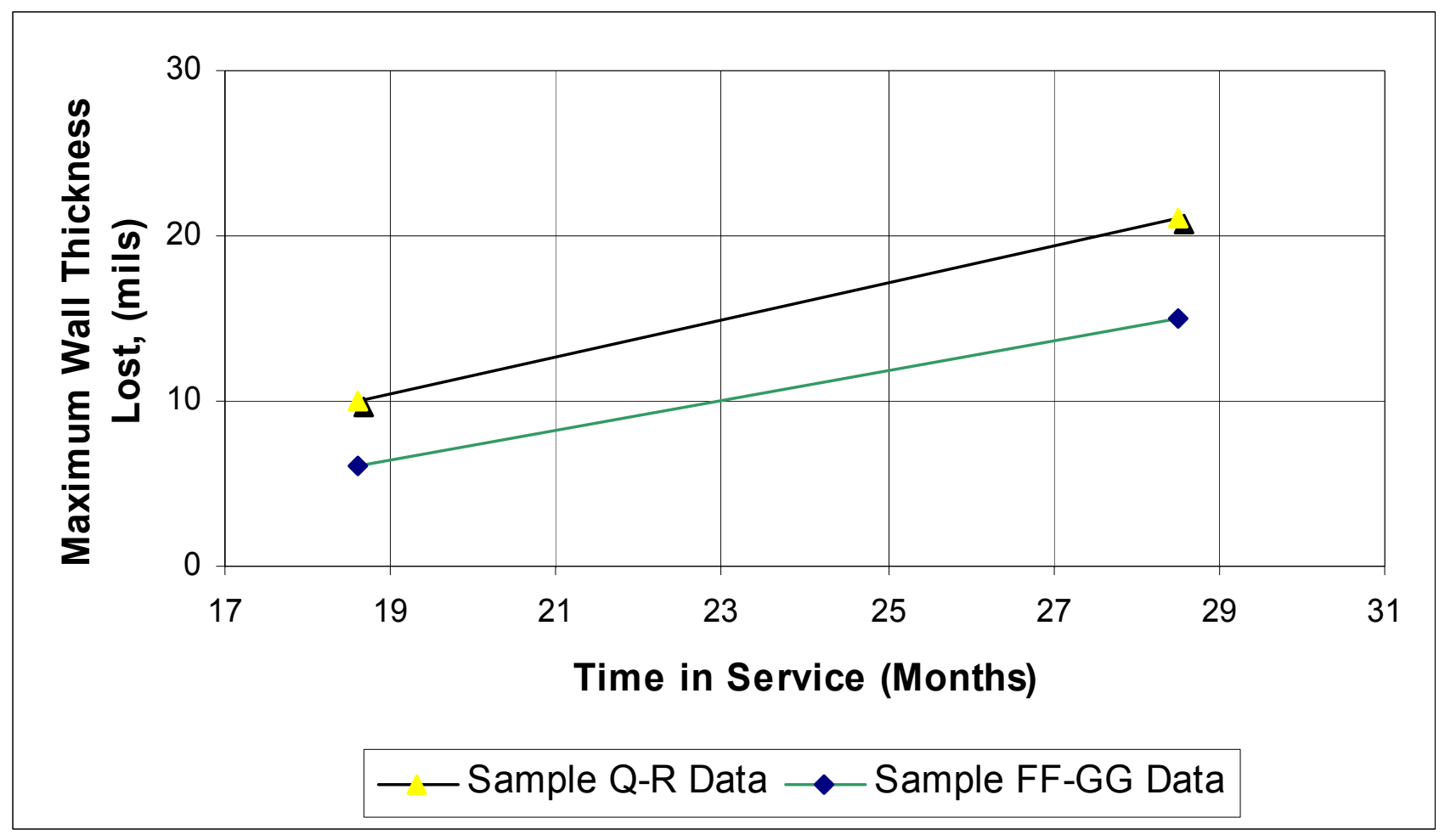

Figure L-11: Metal Loss as a Function of Time

Material: IN72 Weld Overlay 


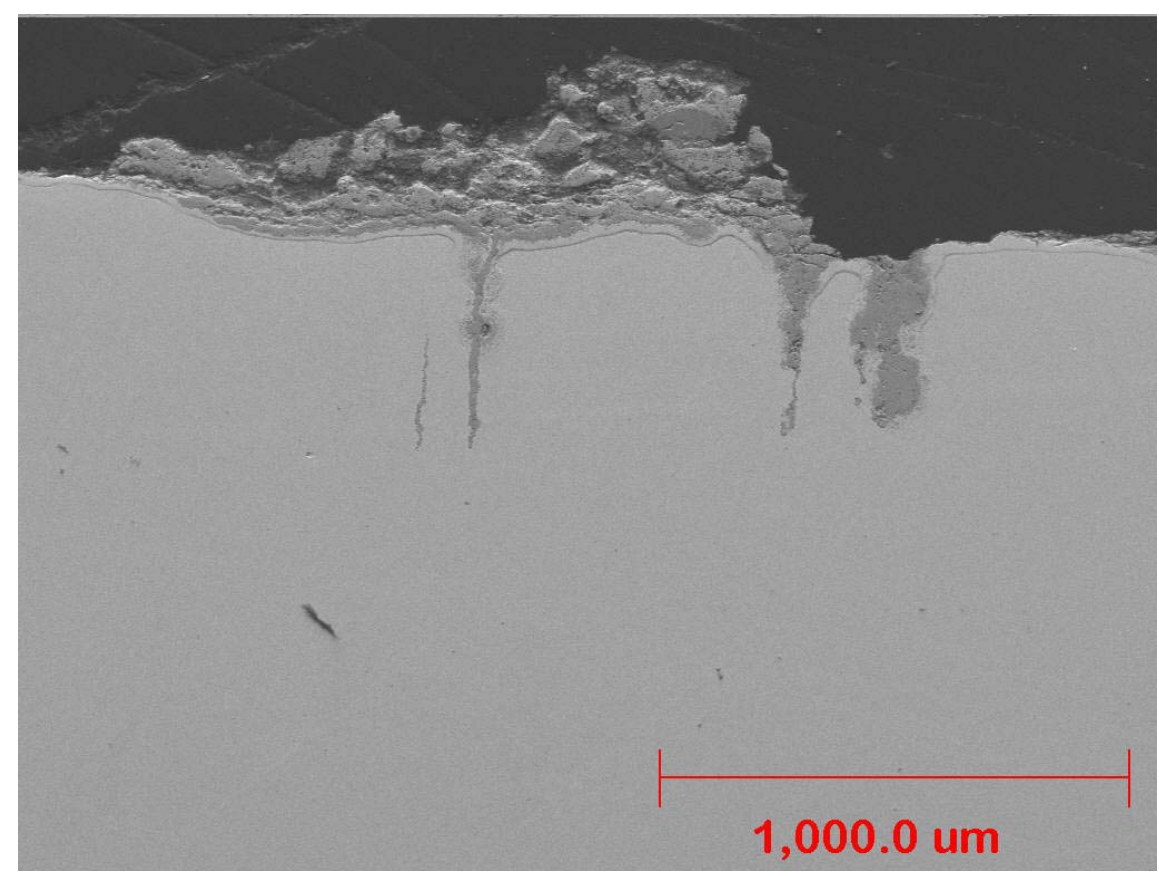

(a) External Scale/Metal Interface (BEI - Low Magnification)

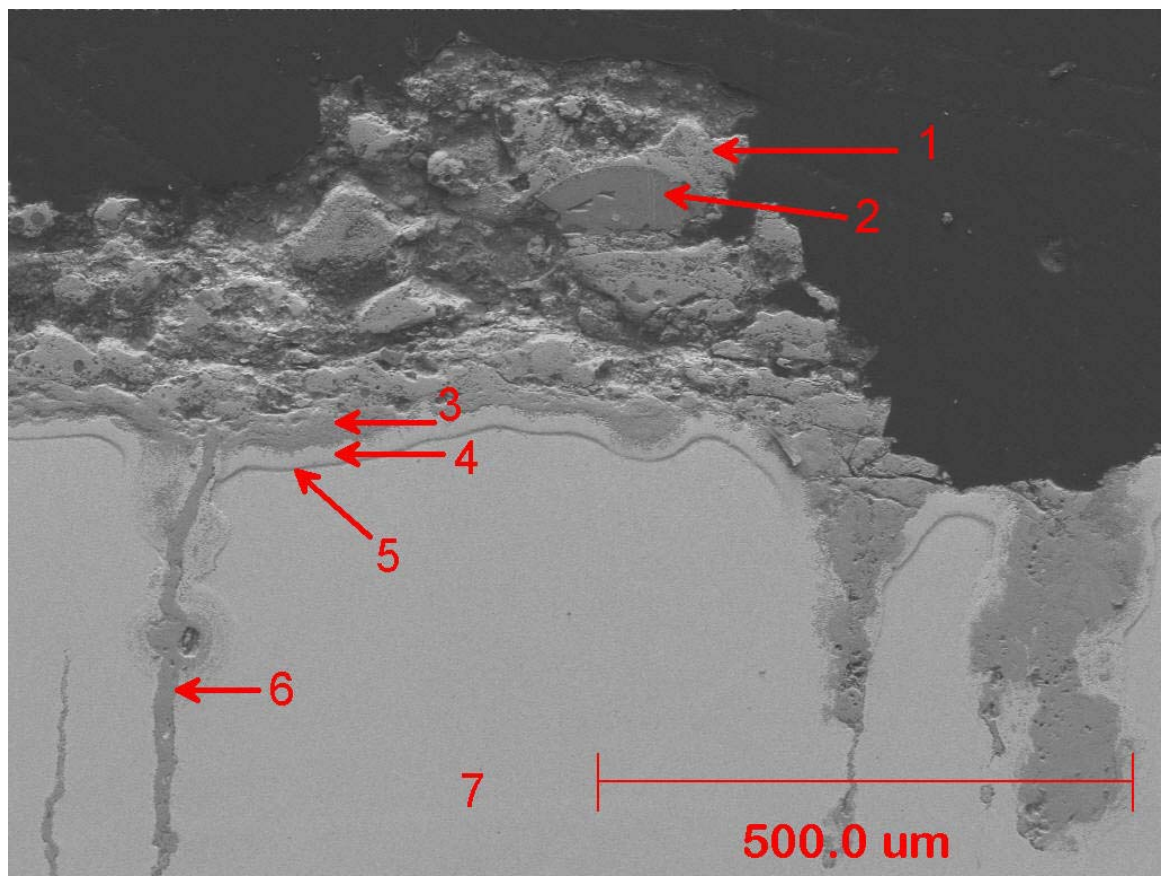

(b) External Scale/Metal Interface (BEI - Intermediate Magnification)

Figure L-12: Microstructure: IN72 Weld Overlay

Note: Numbers on photos indicate distinct areas characterized by the visual assessment and EDS analyses that follow. 


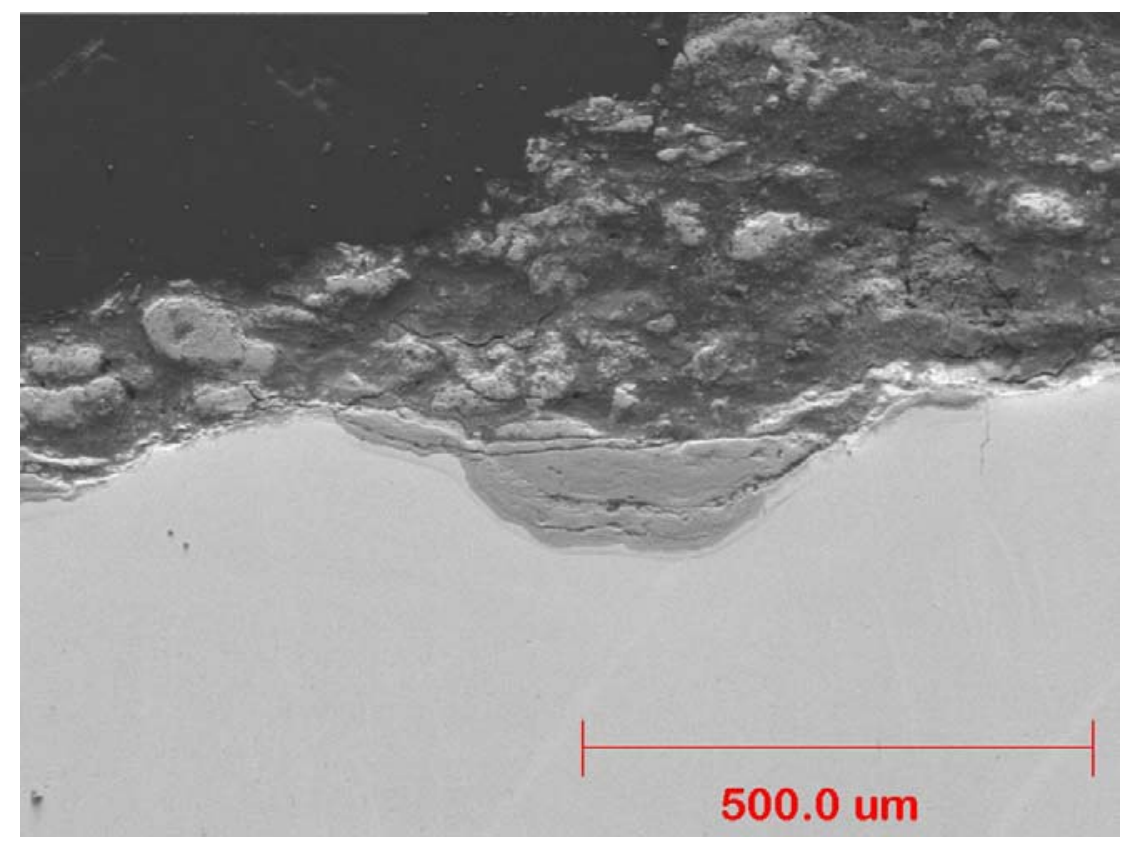

(c) Pit at External Scale/Metal Interface (BEI - Intermediate Magnification)

Figure L-12 (Cont.): Microstructure: IN72 Weld Overlay 


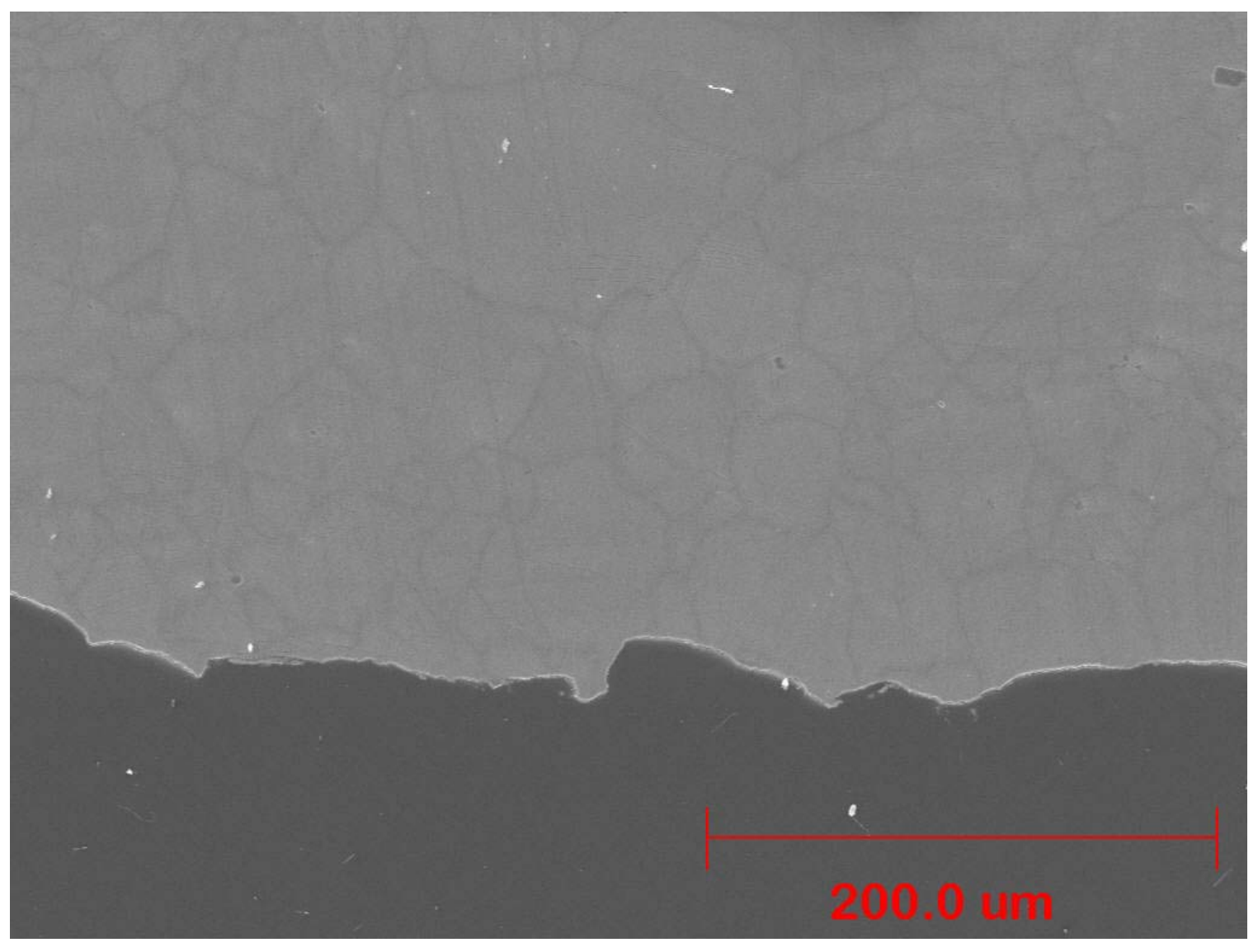

Internal Surface Appearance

Figure L-13: Microstructure: IN72 Weld Overlay 


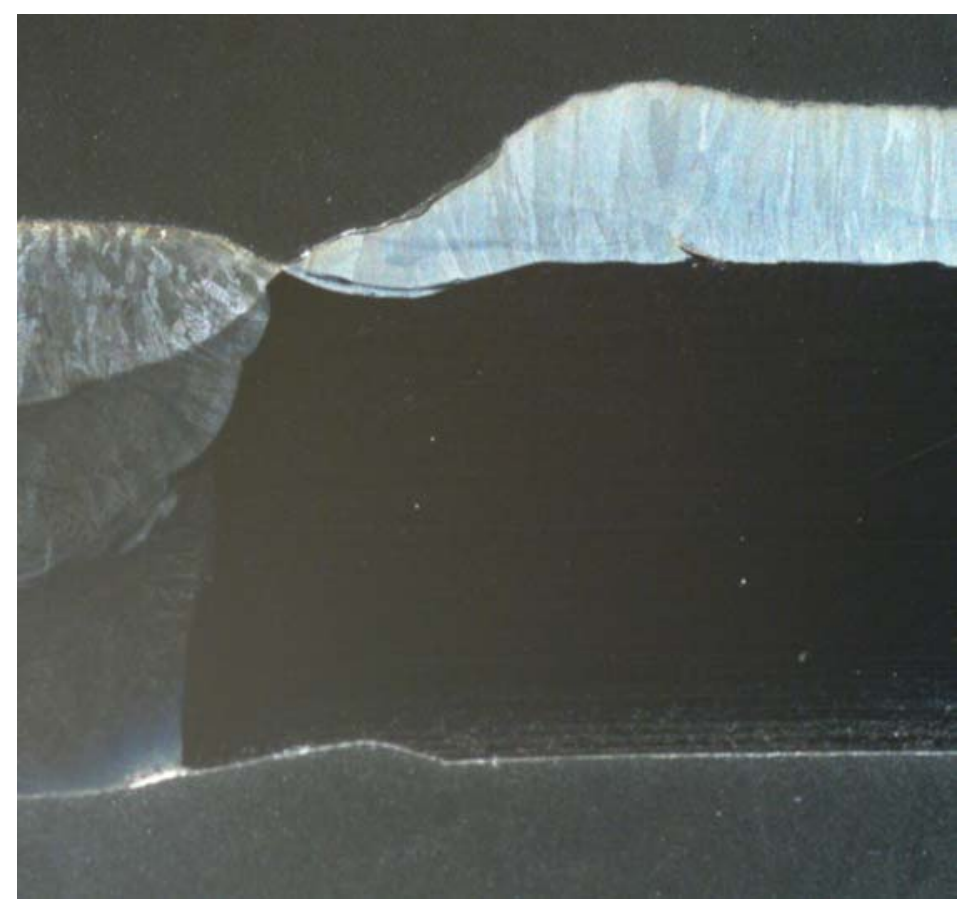

(a) IN72 Weld Overlay Adjacent to Weld "R"

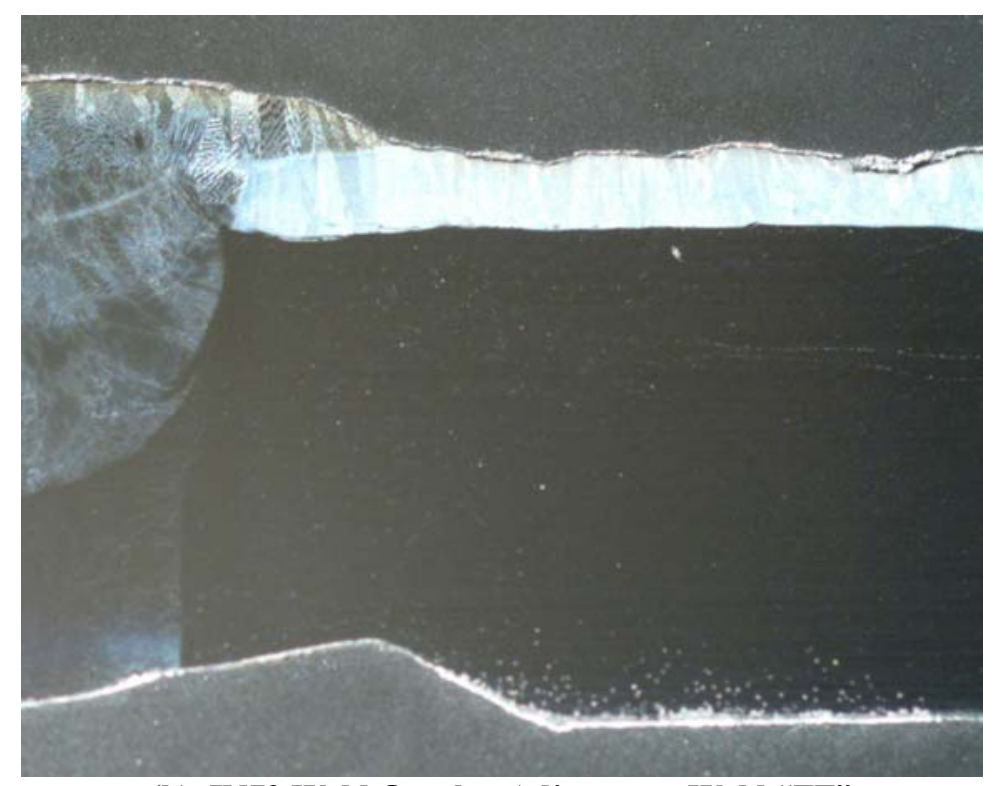

(b) IN72 Weld Overlay Adjacent to Weld "FF"

Figure L-14: Longitudinal Cross-Sections: IN72 Weld Overlay Adjacent to Welds 
Figure L-15: EDS Analysis of Deposit/Scale/Metal Interface for IN72 Weld Overlay (Sample FF-GG)

\begin{tabular}{|c|c|c|c|c|c|c|c|c|c|c|c|c|c|c|c|c|c|c|}
\hline Area & Description & $\mathbf{N i}$ & $\mathrm{Cr}$ & $\mathbf{F e}$ & Co & Mn & $\mathbf{V}$ & $\mathbf{T i}$ & Al & $\mathbf{S i}$ & $\mathrm{C}$ & $\mathbf{O}$ & $\mathrm{Ca}$ & $\mathbf{K}$ & $\mathbf{N a}$ & Mg & $\mathbf{P}$ & $\mathbf{S}$ \\
\hline 1 & $\begin{array}{l}\text { Bulk deposit } \\
\text { analysis }\end{array}$ & -- & - & 80.8 & -- & - & - & - & 1.29 & 1.93 & -- & 15.7 & - & 0.28 & -- & -- & -- & -- \\
\hline 2 & $\begin{array}{l}\text { Nodule in } \\
\text { bulk deposit }\end{array}$ & -- & -- & 32.9 & -- & -- & -- & 0.51 & 10.1 & 19.3 & -- & 25.0 & 1.94 & 4.39 & 0.61 & 0.30 & -- & 4.93 \\
\hline 3 & $\begin{array}{l}\text { Adherent } \\
\text { scale analysis }\end{array}$ & 18.6 & 54.0 & 0.88 & -- & -- & -- & 1.45 & 0.90 & 0.42 & -- & 15.1 & 0.15 & 0.35 & -- & -- & -- & 8.01 \\
\hline 4 & $\begin{array}{l}\text { Light etching } \\
\text { band parallel } \\
\text { to scale } \\
\text { interface }\end{array}$ & 53.5 & 41.6 & 2.07 & -- & -- & - & 0.43 & 0.18 & 0.31 & -- & 1.83 & -- & -- & -- & -- & -- & -- \\
\hline 5 & $\begin{array}{l}\text { Dark etching } \\
\text { band parallel } \\
\text { to scale } \\
\text { interface }\end{array}$ & 39.7 & 46.7 & 1.64 & -- & -- & -- & 0.54 & 5.16 & -- & -- & 6.23 & -- & -- & -- & -- & -- & -- \\
\hline 6 & $\begin{array}{l}\text { Scale within } \\
\text { weld crack. }\end{array}$ & 1.43 & 73.2 & & -- & -- & -- & -- & -- & 0.18 & -- & 18.7 & -- & -- & -- & -- & -- & 6.22 \\
\hline 7 & $\begin{array}{l}\text { Bulk overlay } \\
\text { analysis }\end{array}$ & 53.0 & 42.9 & 3.21 & -- & -- & -- & 0.61 & -- & 0.26 & -- & -- & -- & -- & -- & -- & -- & -- \\
\hline & $\begin{array}{l}\text { Reference } \\
\text { bulk analysis }\end{array}$ & 54.8 & 41.8 & 1.85 & 0.09 & 0.10 & 0.01 & 0.59 & 0.24 & 0.19 & 0.03 & -- & -- & -- & -- & -- & -- & 0.019 \\
\hline
\end{tabular}

